\title{
Meteorological, Stream-Discharge, and Water- Quality Data for 1986 through 1991 from Two Small Basins in Central Nevada
}

by P.W. McKinley and T.A. Oliver

U.S. GEOLOGICAL SURVEY

Open-File Report 93-651

Prepared in cooperation with the

NEVADA FIELD OFFICE

U.S. DEPARTMENT OF ENERGY

Interagency Agreement DE-Al08-92NV10874

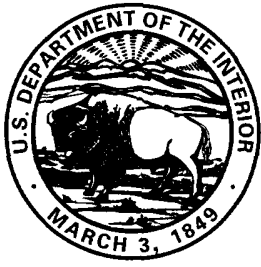




\section{U.S. DEPARTMENT OF THE INTERIOR \\ BRUCE BABBITT, Secretary}

U.S. GEOLOGICAL SURVEY

Robert M. Hirsch, Acting Director

The use of trade, product, industry, or firm names is for descriptive purposes only and does not imply endorsement by the U.S. Government.

For additional information write to:

Copies of this report can be purchased from:

Chief, Hydrologic Investigations Program

Yucca Mountain Project Branch

U.S. Geological Survey

U.S. Geological Survey

Box 25046, MS 421

Earth Science Information Center

Denver Federal Center

Open-File Reports Section

Denver, CO 80225

Box 25286, MS 517

Denver Federal Center

Denver, CO 80225 


\section{CONTENTS}

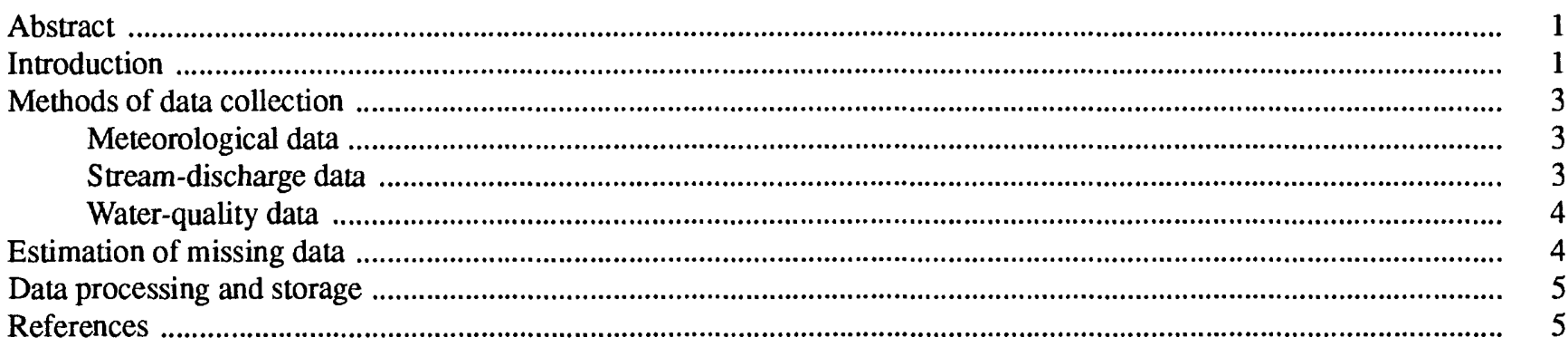

\section{DATA COLLECTION STATIONS FOR WHICH RECORDS ARE INCLUDED IN THIS REPORT}

Records from 3 Springs Basin

Kawich Peak near Warm Springs, Nev.

Daily mean air temperatures for 1989-91.

Daily maximum air temperatures for 1989-91

Daily minimum air temperatures for 1989-91

Daily mean soil temperatures for 1989-91

Daily total solar radiation for 1991

Daily mean relative humidity for 1991

Daily total precipitation for $1989-91$.

Precipitation water quality for 1989-91.

3 Springs near Ledge Spring, near Warm Springs, Nev................................................................................. 28

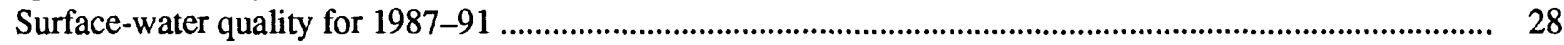

3 Springs Creek near 3 Spring \#2, near Warm Springs, Nev. .................................................................... 30

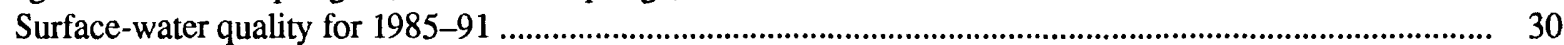

3 Springs Creek near 3 Spring \#3, near Warm Springs, Nev. ....................................................................... 33

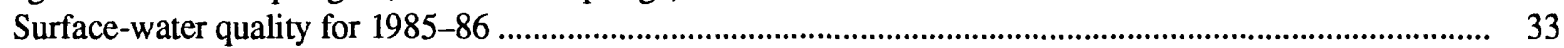

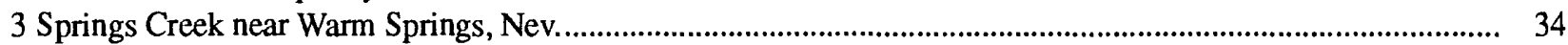

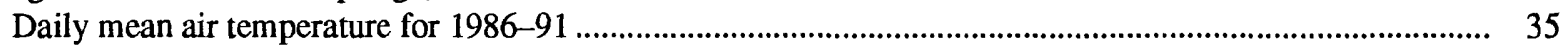

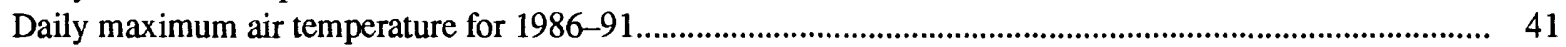

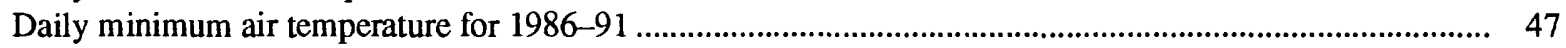

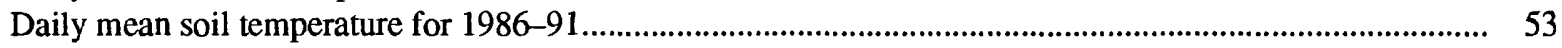

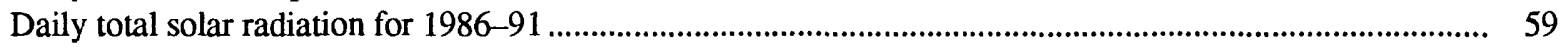

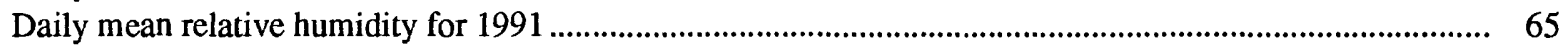

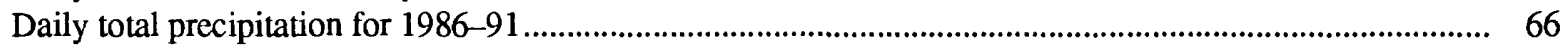

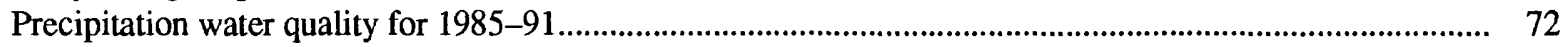

Water-quality analysis from the unsaturated-zone lysimeter for 1986-91 _................................................ 75

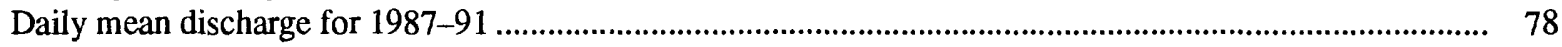

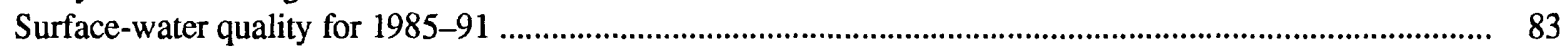

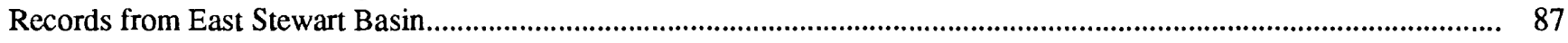

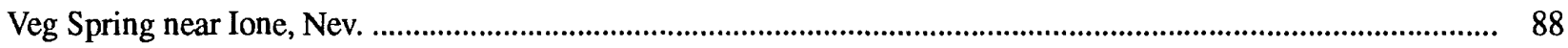

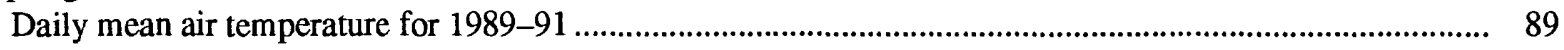

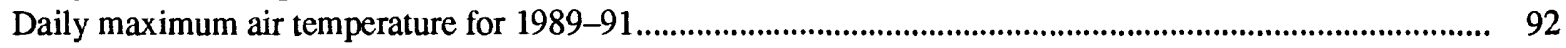

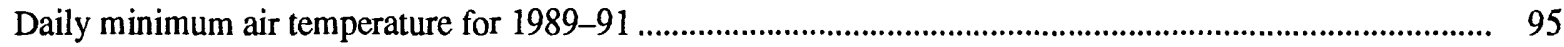

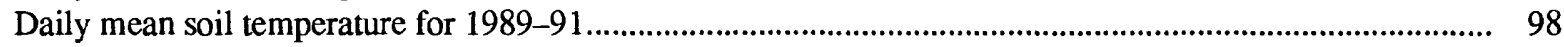

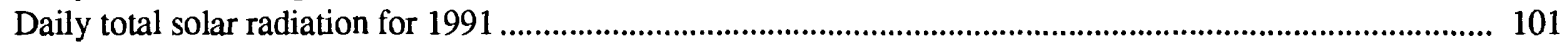

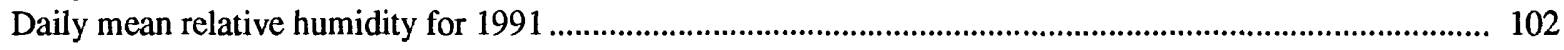

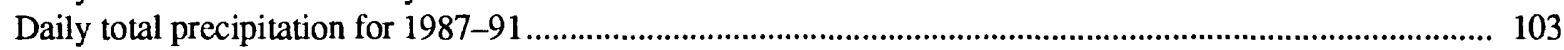

Water-quality analysis from the unsaturated-zone lysimeter \#1 for 1988-91 ............................................. 108

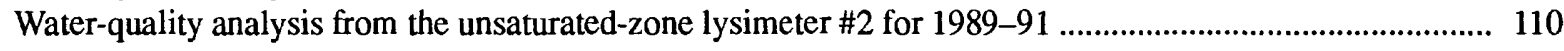

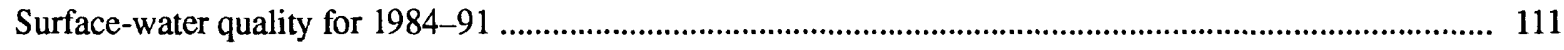




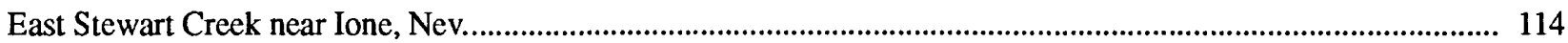

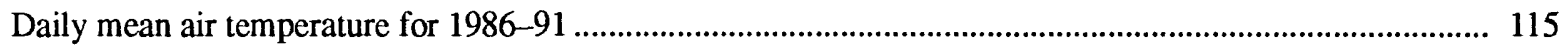

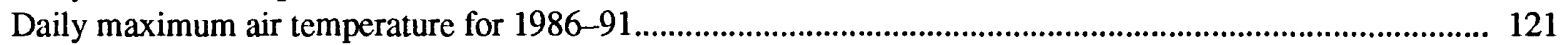

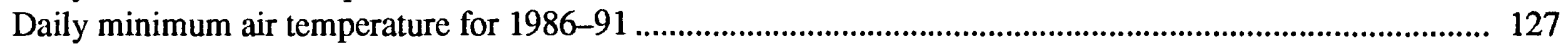

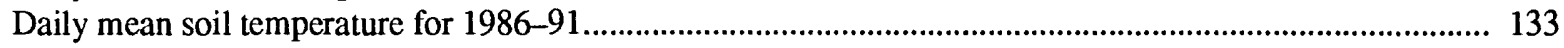

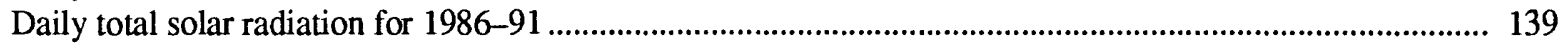

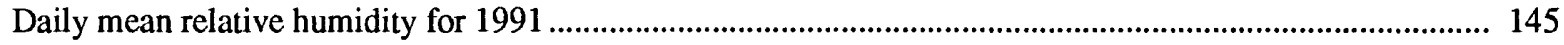

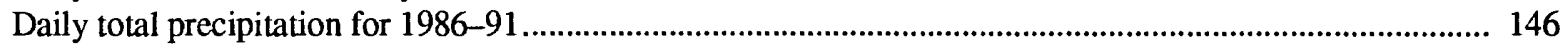

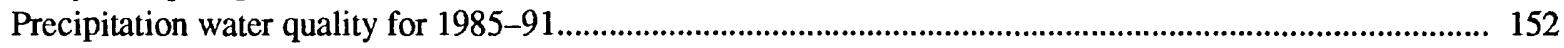

Water-quality analysis from the unsaturated-zone lysimeter for 1986-91 ............................................... 155

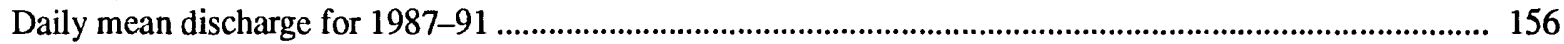

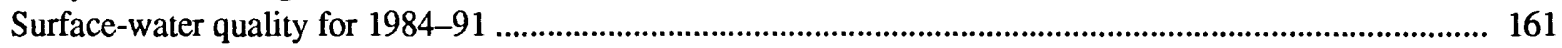

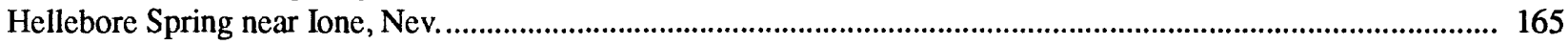

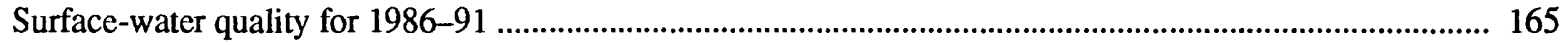

\section{FIGURE}

1. Map showing location of study areas in Nevada ............................................................................................. 2

\section{TABLE}

1. Physical characteristics of 3 Springs and East Stewart Basins and study sites.................................................... 3

\section{CONVERSION FACTORS AND VERTICAL DATUM}

\begin{tabular}{rll}
\hline Multiply & By & To obtain \\
\hline calorie $(\mathrm{cal})$ & 4.184 & joule \\
cubic foot per second $\left(\mathrm{ft}^{3} / \mathrm{s}\right)$ & 0.02832 & cubic meter per second \\
foot $(\mathrm{ft})$ & 0.3048 & meter \\
inch (in) & 2.54 & centimeter \\
mile $(\mathrm{mi})$ & 1.609 & kilometer \\
square foot $\left(\mathrm{ft}^{2}\right)$ & 0.09294 & square meter \\
square mile $\left(\mathrm{mi}^{2}\right)$ & 2.59 & square kilometer \\
\hline
\end{tabular}

Degree Celsius $\left({ }^{\circ} \mathrm{C}\right)$ may be converted to degree Fahrenheit $\left({ }^{\circ} \mathrm{F}\right)$ by using the following equation:

$$
{ }^{\circ} \mathrm{F}=9 / 5\left({ }^{\circ} \mathrm{C}\right)+32 \text {. }
$$

Sea level: In this report "sea level" refers to the National Geodetic Vertical Datum of 1929 (NGVD of 1929)--a geodetic datum derived from a general adjustment of the first-order level nets of both the United States and Canada, formerly called Sea Level Datum of 1929. 


\title{
Meteorological, Stream-Discharge, and Water-Quality Data for 1986 through 1991 from Two Small Basins in Central Nevada
}

\author{
By Patrick W. McKinley and Thomas A. Oliver
}

\begin{abstract}
Two small basins, measuring less than 2 square miles, were studied to determine the volume of precipitation available for recharge to the ground water. The semiarid 3 Springs Basin is located to the east of Kawich Peak in the Kawich Range east of Tonopah, Nevada. Stewart Basin is a subalpine drainage basin north of Arc Dome in the Toiyabe Range north of Tonopah, Nevada. This publication presents the meteorological, stream-discharge, and water-quality data collected during the study. Meteorological data collected include air temperature, soil temperature, solar radiation, and relative humidity. Stream-discharge data were collected from the surface-water outlet of each basin. Water-quality data are chemical analyses of water samples collected from surfaceand ground-water sources.

Data were collected throughout the two basins. Each basin has a meteorological station located in the lower and upper reaches of the basin. Hydrologic records include stream-discharge and water-quality data from the lower meteorological site and water-quality data from springs within the basins. Meteorological data are available from the lower sites from the winter of 1986 through the fall of 1991. Periods of data collection were shorter for additional sites in the basin.
\end{abstract}

\section{INTRODUCTION}

The U.S. Geological Survey (USGS), in cooperation with the U.S. Department of Energy, is investigating the volcanic tuffs of Yucca Mountain, Nevada, for their suitability as storage sites for nuclear waste. Other characteristics of the Yucca Mountain environment, such as the current arid climatic conditions and the deep water table, are desirable for the confinement of the radionuclides. The longevity of current climatic and hydrologic conditions is questionable and not characteristic of the climatic past. Quantifying potential recharge under cooler and wetter climatic conditions is important to the design of the potential repository and the modeling of future hydrologic conditions.

As part of the characterization of the Yucca Mountain site, the analog recharge project has been designed to determine possible recharge to the ground water under cooler and wetter climatic conditions. For the study, two small basins that are geologically similar to the recharge area for Yucca Mountain were chosen.

This report presents the meteorological, streamdischarge, and water-quality data collected from the two study basins. The structure of the data tabulated in this report is similar to that of a USGS annual data report (Frisbie, et al., 1982). Modifications to the data report format have been made to enhance the usefulness of this report. A list, by page number, of datacollection stations and type of data collected is included in the Table of Contents. Tables of the data follow the References.

The 3 Springs Basin is in the Kawich Range east of Kawich Peak (fig. 1). Some basin statistics are listed in table 1. Vegetation is dominated by a piñon-juniper forest, but includes large areas of willows, mountain mahogany, and sage. The 3 Springs Creek is an intermittent creek at the basin outlet and is occasionally dry for the whole year. The major source of recharge is spring runoff; although, occasionally summer rain or heavy snow in the fall which then quickly melt are sufficient to cause the creek to flow.

East Stewart Basin is in the Toiyabe Range north of Arc Dome (fig. 1). A subalpine forest containing aspen, mountain mahogany, and limber pine covers most of the basin. East Stewart Basin has a perennial stream that is fed by snowmelt from April through early to mid-June and by springs during the remainder of the year. 


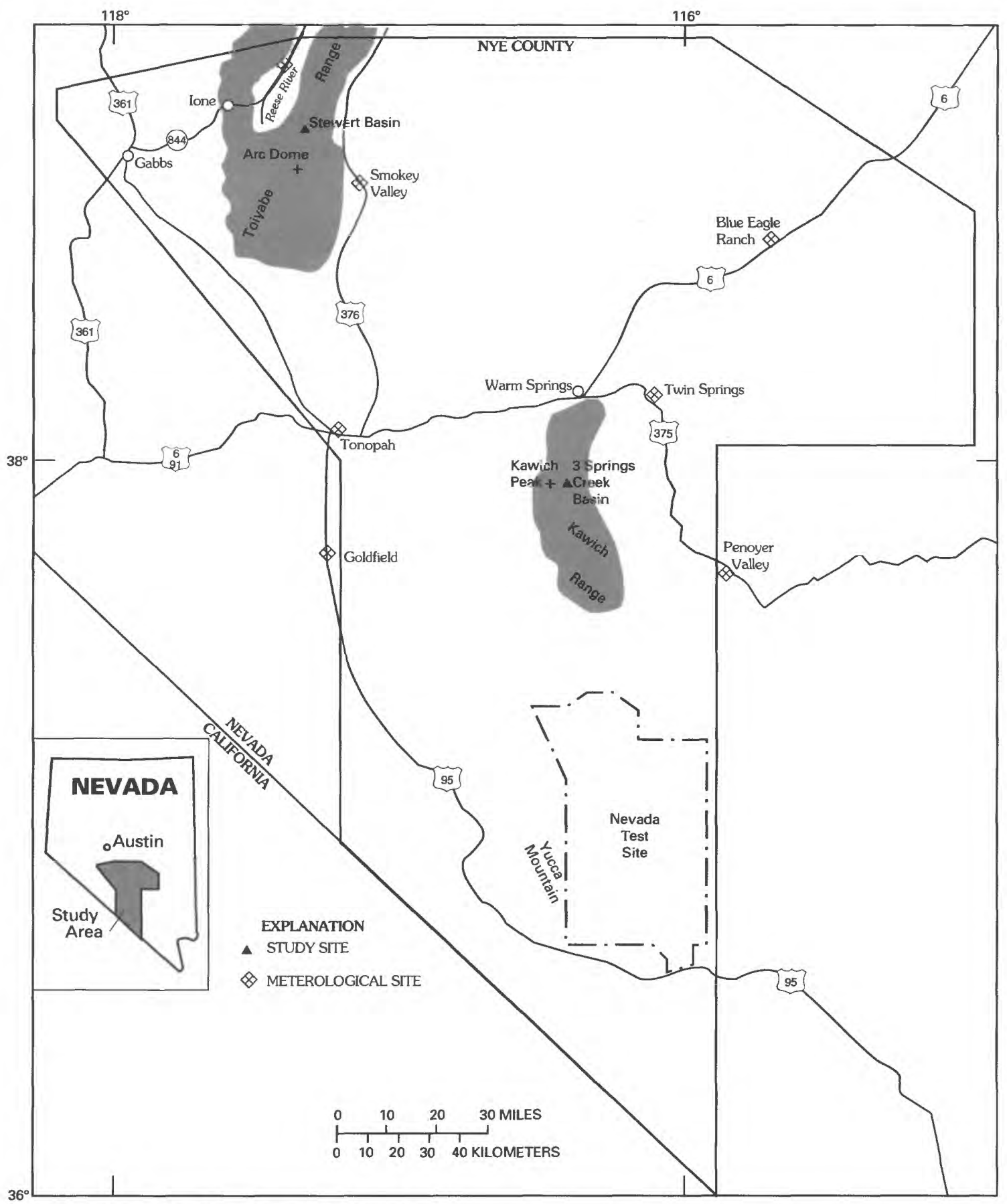

Figure 1. Location of study areas in Nevada. 
Table 1. Physical characteristics of 3 Springs and East Stewart Basins and study sites

[Locations and altitudes were taken from USGS 7 1/2" Kawich Peak NW, Nevada, provisional edition 1987, and 7 1/2" South Toiyabe Peak, Nevada, 1979; $\mathrm{mi}^{2}$, square miles; ft, feet]

\begin{tabular}{|c|c|c|c|c|}
\hline \multirow{2}{*}{ Basin or site name } & \multicolumn{2}{|c|}{ Location } & \multirow{2}{*}{$\begin{array}{l}\text { Drainage area } \\
\qquad\left(\mathrm{mi}^{2}\right)\end{array}$} & \multirow{2}{*}{$\begin{array}{l}\text { Altitude } \\
\text { (ft) }\end{array}$} \\
\hline & Latitude & Longitude & & \\
\hline 1) 3 Springs Basin & & & 1.62 & \\
\hline 2) 3 Springs Base ${ }^{1}$ & $37^{\circ} 57^{\prime} 38^{\prime \prime}$ & $116^{\circ} 25^{\prime} 20^{\prime \prime}$ & & 7,070 \\
\hline 3) Kawich Peak ${ }^{2}$ & $37^{\circ} 57^{\prime} 19^{\prime \prime}$ & $116^{\circ} 26^{\prime} 57^{\prime \prime}$ & & 9,040 \\
\hline 4) East Stewart Basin & & & 0.36 & \\
\hline 5) Stewart Base & $38^{\circ} 53^{\prime} 23^{\prime \prime}$ & $117^{\circ} 21^{\prime} 37^{\prime \prime}$ & & 9,455 \\
\hline 6) Veg Spring & $38^{\circ} 53^{\prime} 04^{\prime \prime}$ & $117^{\circ} 21^{\prime} 13^{\prime \prime}$ & & 10,240 \\
\hline
\end{tabular}

${ }^{1} 3$ Springs Base refers to the study site at 3 Springs Creek near Warm Springs, Nev.

${ }^{2}$ Kawich Peak refers to the study site at Kawich Peak near Warm Springs, Nev., and not the actual Kawich Peak.

${ }^{3}$ Stewart Base refers to the study site at East Stewart Creek near Ione, Nev.

\section{METHODS OF DATA COLLECTION}

\section{Meteorological Data}

Meteorological data were collected using Campbell Scientific Instrument Co. (CSI) electronic dataloggers and the following probes: air temperature was measured by CSI 107 temperature probes before November 1991, air temperature and relative humidity were measured by CSI 207 humidity probes after November 1990, soil temperature was measured by CSI 107 temperature probes, and solar radiation was measured by Li-Cor Co. LI200SB probes.

Belfort weighing rain gages with Alter windshields were used to measure precipitation. Gages were mounted on adjustable platforms so they could be kept above the snow level. Precipitation was trapped by a film of oil to decrease evaporation in the summer. During the winter, an antifreeze solution was used to melt and retain the snowfall. Time and amount of precipitation were recorded on a strip chart. Two gages were located in each basin-one at a low altitude in the basin and one in the higher part of the basin. The gage locations and altitudes are listed in table 1.

Air temperature, soil temperature, and relative humidity were measured every 30 seconds and averaged over a 24 -hour period. Solar radiation was measured over a 30 -second interval and was totaled for a 24-hour period. Air temperature, soil temperature, and relative humidity are reported as daily average values. Precipitation and solar radiation are reported as daily total values.
Calibrations of the meteorological measurement probes were at regular intervals and when possible field checked during each site visit. The CSI 107 temperature probes used to measure soil temperatures were very stable; probe measurements rarely varying more than $0.2^{\circ} \mathrm{C}$ from the calibration standard. Relative humidity values reported are considered poor because the annual calibrations were generally off the standard by approximately 10 percent. Manufacturer specifications for the CSI 207 humidity probe indicated the probe was reliable between 15 through 85 percent relative humidity. The temperature measurements by the CSI 207 humidity probe were equivalent to the CSI 107 probe measurements discussed above. Solar radiation measurements by the LI200SB probes were calibrated every two years; the manufactory estimated a decline in sensitivity of 2 percent year was a good estimate. Radiation values were not corrected for the sensitivity decline. Calibration of raingages was checked in the field annually using Belfort calibration weights. Raingage accuracy was approximately 0.5 percent of full scale. More information about the measurements can be found in the Remarks section immediately proceeding records for each site.

\section{Stream-Discharge Data}

Stream discharge was monitored using a V-notch weir and a Parshall flume for most of the record presented. The gage height of the weir pool was monitored by a datalogger connected to a potentiometerstilling well system. Gage heights were read by the 
datalogger every 30 seconds and averaged over a 30 -minute period. In addition to the weir, starting on October 1, 1988, 3 Springs Creek was equipped with a 6-in flume and Stewart Creek with a 9-in flume.

Because 3 Springs Creek is generally dry and low flows are significant to understanding the basin hydrology, discharge at 3 Springs Creek is expressed to three decimal places to indicate the presence of flow. The additional decimal place does not infer the record accuracy has increased. The water level in the flumes was monitored every 15 minutes by an automatic digital recorder and stilling well system. Additional information about the record is located in the Remarks section associated with the data tables at the back of the report.

\section{Water-Quality Data}

Precipitation samples obtained from the rain gages during quarterly field trips were analyzed for major anions and cations; the stable isotopes, $\mathrm{O}^{18}$ and deuterium; and the physical properties, $\mathrm{pH}$ and specific conductance. Concentrations of other elements and compounds were measured on an intermittent basis. Precipitation samples represent integrated soluble dryplus-wetfall. The collection equipment and operation are described by Voegeli and Claussen (1971, p. 3-4).

Surface-water samples were collected quarterly and analyzed for the same constituents and properties as were precipitation samples. Water temperatures reported for the surface-water samples were measured in the stream. Because of the small size of the streams, samples were dipped from the main flow of the stream; the samples are referred to as grab samples.

Unsaturated-zone samples were collected from lysimeters located just below the root zone. Water samples were usually collected twice each year-one in the late spring, to represent the infiltration resulting from snowmelt; and one in early fall, to represent summer rains. Springs also were sampled and were considered to represent the shallow ground water. The samples were analyzed for major anions and cations; the stable isotopes $\mathrm{O}^{18}$ and deuterium; and the physical properties, temperature, $\mathrm{pH}$, and specific conductance.

Samples generally were filtered within 24 hours of sampling. All samples were then analyzed at the USGS National Water Quality Laboratory in Arvada, Colorado.

\section{ESTIMATION OF MISSING DATA}

Meteorological data "lost" because of equipment failure and human error were estimated by stepforward regression. Data from before and after the period of missing record were regressed with data from other meteorological stations in central Nevada. Multiple regression equations were developed using data from the analog sites and (or) National Oceanic and Atmospheric Administration, National Weather Service (NOAA/NWS) stations at Tonopah, Goldfield, Twin Springs, Austin, Blue Eagle Ranch, Smokey Valley, Reese River, and Penoyer Valley. Estimates of humidity were not considered possible with available data.

Daily average air temperatures at the analog sites usually correlated best with temperatures at meteorological sites at similar altitudes. Summer air temperatures correlated well, but temperature inversions made winter estimates difficult.

Daily average soil temperatures were available only at the analog sites. Estimates of missing data for periods greater than 2 days were made by regression with available soil-temperature data. Soil temperatures change at a much slower rate than air temperatures; therefore, interpolation was used to estimate one or two missing daily values between known daily values.

Solar-radiation curves were developed from the measured insolation at each site and used to estimate the missing daily values. For periods of less than a day, the Bird and Hulstrum (1981) clear-sky model was used to calculate estimates. If only a few readings were missing and clouds were present, simple interpolation between known values was used. The use of the three methods was necessary because there was no single method that provided the best estimate for all circumstances.

A second precipitation recorder was installed in the East Stewart Basin at Veg Spring on October 9, 1986 , and in the 3 Springs Basin at Kawich Peak on September 24, 1988. Two precipitation gages in each basin enabled precipitation estimates to be made from data collected from within the study basins. When precipitation data from the basins was not available, data from nearby NOAA/NWS stations were used to estimate total daily precipitation. Unlike air- and soiltemperature records, precipitation records correlate poorly for central Nevada, eliminating regression as a viable means of estimating lost precipitation data. Clock failure was the cause of lost precipitation record, but the amount of precipitation was available from the strip chart; only the timing of the precipitation was estimated. Humidity measurements at the analog sites and precipitation records at NOAA/NWS stations were used to determine the timing of precipitation. Estimated daily precipitation values for the analog site were calculated as noted in equation 1 :

estimated daily precipitation $=\frac{(P)(T a)}{T b}$ 
where $P$ is the known daily precipitation at a NOAA/NWS station, $T a$ is the total precipitation at the analog site for the period to be estimated, and $T b$ is the total precipitation at the NOAA/NWS station for the period to be estimated.

Estimated daily discharges usually were from low-flow periods. The stilling well intake attached to the flume is located slightly above the bottom of the flume, thus very low flows are undetected. Periods when the flow approaches zero are common at 3 Springs Creek, an intermittent creek. During a period of undetected flow but when there is indication that flow exists (for example, flow is noted by an observer), daily discharges are reported as estimated. Record lost because of gage malfunction was estimated using peak or recession trends and meteorological data.

\section{DATA PROCESSING AND STORAGE}

All time-sequence data were stored as unit values in the USGS National Water Information System (NWIS) data base, which provides calculation of daily, monthly, and annual means or totals. The NWIS software tabling routines were used to organize the data for this report. More information on the NWIS data base was reported by Dempster (1990).

Meteorological data, except for precipitation, were transferred electronically to NWIS and then were processed by and stored in NWIS. Precipitation data were stored in NWIS, but the original data were recorded on strip charts and then were manually translated and input into NWIS.

Surface-water-data computations were made according to methods described by Rantz (1982). Unit values, 30-minute average gage heights from the weir and 15-minute instantaneous gage heights from the flume, were input into NWIS electronically. Datum and shift corrections were applied to the gage heights to correct for erroneous gage operation (datum) or for changes in the control (shift). Daily mean stage was computed by NWIS from the unit values; daily mean discharge was computed from the stage-discharge relation (rating curve) and the daily mean stage.

The standard stage-discharge relation noted in equation 2, (U.S. Bureau of Reclamation, 1984):

$$
Q=2.49 H^{2.48}
$$

was used for conversion of stage to discharge from the $90^{\circ} \mathrm{V}$-notch thin-plate weirs. $Q$ is the discharge, in cubic feet per second; and $H$ is the stage, gage height, or head of the weir, in feet. Flume stage-discharge relations (ratings) were developed from actual laboratory tests (Dodge, 1990). Variations from standard flume dimensions and stream approach conditions accounted for the differences between the standard Parshall flume rating and the developed rating.

\section{REFERENCES}

Bird, R.E., and Hulstrum, R.L., 1981, Simplified clear-sky model for direct and diffuse insolation on horizontal surface: Solar Energy Research Institute, TR-642-761, 47 p. (NNA.931124.0013)

Dempster, G.R., complier, 1990, National water information system user's manual, volume 2, chapter 3, Automated data processing system: U.S. Geological Survey OpenFile Report 90-116, variously paginated.

(NNA.931124.0014)

Dodge, R.A., 1990, Effects of mountain stream topography on the accuracy of small Parshall flumes: Bureau of Reclamation, Report R-90-03, 15 p. (NNA.931109.0002)

Frisbie, H.R., La Camera, R.J., Rick, M.M., and Wood, D.B., 1982, Water Resources Data, Nevada, Water Year 1982, U.S. Geological Survey Water-Data Report NV-82-1, 360 p. (NNA.940202.0111)

Rantz, S.E., 1982, Measurement and computation of streamflow, volume 2-Computation of discharge: U.S. Geological Survey Water-Supply Paper 2175, p. 285-631. (NNA.931109.0003)

U.S. Bureau of Reclamation, 1984, Water measurement manual (2nd ed.): U.S. Bureau of Reclamation, p. 270. (NNA.931026.0002)

Voegelli, P.T., Sr., and Claassen, H.C., 1971, Radiochemical analyses of water from selected streams, wells, springs, and precipitation collected prior to reentry drilling, Project Rulison: U.S. Geological Survey Open-File Report USGS-474-101, p. 3-4. (NNA.931201.0115) 


\section{RECORDS FROM 3 SPRINGS BASIN}


KAWICH PEAK NEAR WARM SPRINGS, NV

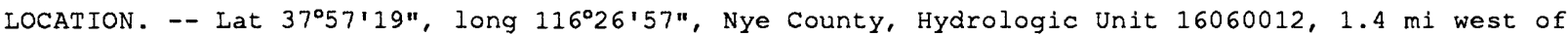
the 3 springs Creek surface-water equipment, $3.9 \mathrm{mi}$ west of Eden Ranch in the Reveille Valley, $16.8 \mathrm{mi}$ south of Warm Springs at the intersection of US Highway 6 and Nevada Highway 375 .

PERIOD OF RECORD. -- October 1988 to current year.

REMARKS.-- The precipitation water-quality data for september 25 , 1991 was not reported because the sample was contaminated. Values for the daily maximum and minimum air temperature were obtained from the 40 minute average values. Estimated periods of daily record are considered poor.

PERIOD OF DAILY RECORD. --

AIR TEMPERATURE: October 1988 to current year.

SOIL TEMPERATURE: October 1988 to current year.

SOLAR RADIATION: November 1990 to current year.

RELATIVE HUMIDITY: October 1990 to current year.

TOTAL PRECIPITATION: October 1988 to current year.

EXTREMES FOR PERIOD OF RECORD. --

AIR TEMPERATURE: Maximum daily, 26. $6^{\circ} \mathrm{C}$, July 4, 1991; minimum daily, $-25.5^{\circ} \mathrm{C}$, Feb. 6 , 1989. See remarks for more information.

SOIL TEMPERATURE: Maximum daily mean, $20.0^{\circ} \mathrm{C}$, Aug 25, 1991; minimum daily mean, $-6.1^{\circ} \mathrm{C}$,

DeC. 30, 1990 .

SOLAR RADIATION: Maximum daily total, $799 \mathrm{cal} / \mathrm{cm}^{2}$, June 22-23, 1991; minimum daily total, $25 \mathrm{cal} / \mathrm{cm}^{2}$, Dec. 13, 1990 .

RELATIVE HUMIDITY: Maximum daily mean, 100 pct, Mar. 4, 1991; minimum daily mean, 15 pct, July 4 and 5, 1991, and Aug. 9, 1991.

TOTAL PRECIPITATION: Maximum daily, 1.45 in, May 29, 1990; no precipitation, most days of record. 


\section{SPRINGS BASIN}

KAWICH PEAK NEAR WARM SPRINGS, NV

DAILY MEAN AIR TEMPERATURE, WATER YEAR OCTOBER 1988 TO SEPTEMBER 1989 DEGREES CELSIUS

\begin{tabular}{|c|c|c|c|c|c|c|c|c|c|c|c|c|}
\hline DAY & OCT & NOV & DEC & JAN & FEB & MAR & APR & MAY & JUN & JUL & AUG & SEP \\
\hline $\begin{array}{l}1 \\
2 \\
3 \\
4 \\
5\end{array}$ & $\begin{array}{l}13.9 \\
14.0 \\
13.0 \\
12.4 \\
10.5\end{array}$ & $\begin{array}{l}7.3 \\
5.5 \\
8.0 \\
6.3 \\
8.6\end{array}$ & $\begin{array}{l}\text { e } 5.1 \\
\text { e } 4.0 \\
\text { e2 } 21 \\
\text { e2 } 26 \\
\text { e2 } 2.7\end{array}$ & $\begin{array}{l}-9.2 \\
-2.7 \\
-1.8 \\
-2.9 \\
-6.7\end{array}$ & $\begin{array}{r}-3.7 \\
-6.6 \\
-8.2 \\
-13.8 \\
-20.9\end{array}$ & $\begin{array}{r}.8 \\
-3.0 \\
-12.6 \\
-6.7 \\
2.7\end{array}$ & $\begin{array}{l}2.1 \\
3.1 \\
3.0 \\
5.1 \\
8.1\end{array}$ & $\begin{array}{r}8.0 \\
8.5 \\
9.8 \\
10.3 \\
12.9\end{array}$ & $\begin{array}{r}\text { e7.0 } \\
9.1 \\
7.0 \\
6.0 \\
6.5\end{array}$ & $\begin{array}{l}15.3 \\
16.8 \\
18.4 \\
18.4 \\
20.1\end{array}$ & $\begin{array}{l}16.1 \\
15.3 \\
15.3 \\
15.6 \\
16.8\end{array}$ & $\begin{array}{l}13.2 \\
14.7 \\
14.9 \\
15.0 \\
16.2\end{array}$ \\
\hline $\begin{array}{r}6 \\
7 \\
8 \\
9 \\
10\end{array}$ & $\begin{array}{l}6.1 \\
7.4 \\
9.3 \\
9.7 \\
7.2\end{array}$ & $\begin{array}{r}5.5 \\
3.7 \\
.2 \\
.2 \\
.1\end{array}$ & $\begin{array}{r}\mathrm{e} 1.8 \\
\mathrm{e}-4.9 \\
\mathrm{e}-4.8 \\
-2.1 \\
1.5\end{array}$ & $\begin{array}{r}-14.8 \\
-14.3 \\
-10.7 \\
-4.2 \\
-3.9\end{array}$ & $\begin{array}{r}-21.3 \\
-17.3 \\
-10.7 \\
-4.5 \\
-6.2\end{array}$ & $\begin{array}{l}4.4 \\
3.3 \\
4.2 \\
5.3 \\
5.4\end{array}$ & $\begin{array}{l}12.2 \\
12.8 \\
10.9 \\
11.0 \\
11.9\end{array}$ & $\begin{array}{r}14.0 \\
14.4 \\
13.7 \\
9.1 \\
1.3\end{array}$ & $\begin{array}{l}9.0 \\
9.7 \\
9.5 \\
8.9 \\
9.2\end{array}$ & $\begin{array}{l}21.1 \\
21.7 \\
20.8 \\
18.2 \\
16.3\end{array}$ & $\begin{array}{l}17.5 \\
16.4 \\
13.1 \\
12.0 \\
13.0\end{array}$ & $\begin{array}{r}14.4 \\
8.4 \\
8.7 \\
11.8 \\
11.8\end{array}$ \\
\hline $\begin{array}{l}11 \\
12 \\
13 \\
14 \\
15\end{array}$ & $\begin{array}{l}7.1 \\
6.6 \\
7.5 \\
5.9 \\
7.9\end{array}$ & $\begin{array}{r}-1.1 \\
1.5 \\
.5 \\
-6.6 \\
-6.3\end{array}$ & $\begin{array}{r}3.8 \\
5.4 \\
5.7 \\
-3.0 \\
-12.2\end{array}$ & $\begin{array}{r}-13.9 \\
-10.1 \\
-5.1 \\
-8.0 \\
-6.7\end{array}$ & $\begin{array}{r}-8.4 \\
-10.6 \\
-12.2 \\
-10.0 \\
-6.3\end{array}$ & $\begin{array}{r}5.2 \\
5.2 \\
.0 \\
-1.6 \\
1.1\end{array}$ & $\begin{array}{r}5.6 \\
4.4 \\
6.9 \\
10.1 \\
9.9\end{array}$ & $\begin{array}{r}-2.0 \\
-1.1 \\
-2.0 \\
-1.2 \\
1.2\end{array}$ & $\begin{array}{l}10.4 \\
11.5 \\
15.6 \\
18.2 \\
18.6\end{array}$ & $\begin{array}{l}14.2 \\
13.6 \\
15.2 \\
17.7 \\
17.7\end{array}$ & $\begin{array}{l}13.2 \\
15.6 \\
17.3 \\
18.1 \\
17.9\end{array}$ & $\begin{array}{r}9.9 \\
8.0 \\
9.6 \\
12.2 \\
13.3\end{array}$ \\
\hline $\begin{array}{l}16 \\
17 \\
18 \\
19 \\
20\end{array}$ & $\begin{array}{r}10.4 \\
11.7 \\
12.2 \\
11.5 \\
9.4\end{array}$ & $\begin{array}{r}-5.3 \\
-8.2 \\
-10.2 \\
-4.3 \\
-3.4\end{array}$ & $\begin{array}{l}-6.4 \\
-3.5 \\
-5.8 \\
-7.4 \\
-6.9\end{array}$ & $\begin{array}{r}-2.3 \\
.9 \\
.0 \\
.5 \\
-.7\end{array}$ & $\begin{array}{r}-3.4 \\
-.4 \\
-.8 \\
-2.9 \\
-4.4\end{array}$ & $\begin{array}{r}-1.0 \\
-1.8 \\
2.6 \\
.1 \\
-4.2\end{array}$ & $\begin{array}{l}10.1 \\
11.8 \\
12.1 \\
12.5 \\
11.6\end{array}$ & $\begin{array}{r}4.7 \\
8.7 \\
9.2 \\
4.7 \\
10.7\end{array}$ & $\begin{array}{l}13.9 \\
15.5 \\
18.5 \\
18.1 \\
13.1\end{array}$ & $\begin{array}{l}16.3 \\
17.4 \\
19.5 \\
20.5 \\
20.5\end{array}$ & $\begin{array}{l}16.8 \\
14.6 \\
13.6 \\
14.0 \\
12.7\end{array}$ & $\begin{array}{r}10.8 \\
6.9 \\
3.7 \\
2.8 \\
7.3\end{array}$ \\
\hline $\begin{array}{l}21 \\
22 \\
23 \\
24 \\
25\end{array}$ & $\begin{array}{r}10.8 \\
11.5 \\
10.7 \\
10.7 \\
9.0\end{array}$ & $\begin{array}{r}-1.2 \\
1.5 \\
-2.6 \\
-10.1 \\
-8.4\end{array}$ & $\begin{array}{r}-10.2 \\
-10.7 \\
-12.5 \\
-9.3 \\
-14.3\end{array}$ & $\begin{array}{r}-.6 \\
-3.2 \\
-5.0 \\
-7.2 \\
-9.3\end{array}$ & $\begin{array}{l}.5 \\
2.3 \\
3.7 \\
4.5 \\
4.8\end{array}$ & $\begin{array}{r}3.1 \\
3.4 \\
2.7 \\
2.5 \\
-3.2\end{array}$ & $\begin{array}{r}8.7 \\
3.9 \\
1.0 \\
-5.3 \\
-4.5\end{array}$ & $\begin{array}{r}10.2 \\
11.7 \\
7.0 \\
\text { e2.0 } \\
\text { e2 } 2.8\end{array}$ & $\begin{array}{r}7.3 \\
12.3 \\
11.1 \\
6.9 \\
7.8\end{array}$ & $\begin{array}{l}16.7 \\
17.8 \\
17.0 \\
16.5 \\
17.9\end{array}$ & $\begin{array}{r}12.4 \\
13.8 \\
10.8 \\
7.0 \\
10.5\end{array}$ & $\begin{array}{r}9.3 \\
10.0 \\
12.9 \\
14.1 \\
13.1\end{array}$ \\
\hline $\begin{array}{l}26 \\
27 \\
28 \\
29 \\
30 \\
31\end{array}$ & $\begin{array}{l}9.8 \\
8.6 \\
6.6 \\
5.9 \\
5.8 \\
8.0\end{array}$ & $\begin{array}{r}-8.4 \\
.9 \\
-.8 \\
\mathrm{e}-1.1 \\
\mathrm{e} .7 \\
---\end{array}$ & $\begin{array}{r}-16.7 \\
-14.1 \\
-15.5 \\
-9.7 \\
-4.0 \\
-10.1\end{array}$ & $\begin{array}{r}-6.5 \\
-2.5 \\
-2.1 \\
.0 \\
4.2 \\
2.8\end{array}$ & $\begin{array}{l}4.0 \\
-.5 \\
-.2 \\
--- \\
--- \\
---\end{array}$ & $\begin{array}{r}-4.9 \\
-.1 \\
4.2 \\
1.5 \\
3.6 \\
5.6\end{array}$ & $\begin{array}{r}-2.5 \\
-3.4 \\
-1.6 \\
1.7 \\
6.0 \\
---\end{array}$ & $\begin{array}{r}\text { e7.3 } \\
\text { e11.1 } \\
\text { e6.3 } \\
\text { e-.3 } \\
\mathrm{e}-1.1 \\
\mathrm{e} 3.9\end{array}$ & $\begin{array}{c}13.1 \\
11.1 \\
10.6 \\
12.8 \\
13.4 \\
-.-\end{array}$ & $\begin{array}{l}19.0 \\
18.0 \\
17.3 \\
17.9 \\
18.3 \\
17.8\end{array}$ & $\begin{array}{l}12.9 \\
14.0 \\
14.6 \\
15.0 \\
13.0 \\
12.0\end{array}$ & $\begin{array}{r}13.1 \\
12.1 \\
12.9 \\
11.4 \\
7.3 \\
---\end{array}$ \\
\hline $\begin{array}{l}\text { MEAN } \\
\text { MAX } \\
\text { MIN } \\
\text { MED }\end{array}$ & $\begin{array}{r}9.4 \\
14.0 \\
5.8 \\
9.4\end{array}$ & $\begin{array}{r}-.9 \\
8.6 \\
-10.2 \\
-.4\end{array}$ & $\begin{array}{r}-4.8 \\
5.7 \\
-16.7 \\
-4.9\end{array}$ & $\begin{array}{r}-4.7 \\
4.2 \\
-14.8 \\
-3.9\end{array}$ & $\begin{array}{r}-5.5 \\
4.8 \\
-21.3 \\
-4.4\end{array}$ & $\begin{array}{r}.9 \\
5.6 \\
-12.6 \\
2.5\end{array}$ & $\begin{array}{r}6.0 \\
12.8 \\
-5.3 \\
6.4\end{array}$ & $\begin{array}{r}6.3 \\
14.4 \\
-2.0 \\
7.3\end{array}$ & $\begin{array}{r}11.4 \\
18.6 \\
6.0 \\
10.9\end{array}$ & $\begin{array}{l}17.9 \\
21.7 \\
13.6 \\
17.8\end{array}$ & $\begin{array}{r}14.2 \\
18.1 \\
7.0 \\
14.0\end{array}$ & $\begin{array}{r}11.0 \\
16.2 \\
2.8 \\
11.8\end{array}$ \\
\hline
\end{tabular}

$\begin{array}{lllllll}\text { WTR YR } 1989 \text { MEAN } 5.2 & \text { MAX } 21.7 & \text { MIN } & -21.3 & \text { MED } & 6.3\end{array}$

e Estimated 


\section{SPRINGS BASIN}

KAWICH PEAK NEAR WARM SPRINGS, NV

DAILY MEAN AIR TEMPERATURE, WATER YEAR OCTOBER 1989 TO SEPTEMBER 1990 DEGREES CELSIUS

\begin{tabular}{|c|c|c|c|c|c|c|c|c|c|c|c|c|}
\hline DAY & OCT & NOV & $\mathrm{DEC}$ & JAN & FEB & MAR & $\mathrm{APR}$ & MAY & JUN & JUL & AUG & SEP \\
\hline 1 & 7.2 & -2.2 & -2.0 & -4.0 & -10.0 & 1.5 & 3.6 & -.1 & 2.4 & 20.2 & 17.2 & 15.8 \\
\hline 2 & 4.9 & 1.0 & 2.8 & -9.6 & -9.3 & 1.7 & 3.6 & 3.5 & 10.2 & 16.7 & 16.6 & 15.3 \\
\hline 3 & .6 & 3.2 & 4.2 & -9.5 & -6.0 & 1.2 & 3.7 & 4.9 & 15.1 & 14.5 & 18.1 & 15.2 \\
\hline 4 & 2.7 & 6.2 & 6.1 & -6.0 & -8.7 & -.5 & 2.4 & 8.4 & 16.5 & 15.2 & 19.0 & 13.4 \\
\hline 5 & 4.7 & 4.8 & 5.2 & -3.0 & -5.1 & -6.1 & 3.5 & 10.9 & 14.6 & 16.6 & 19.4 & 13.8 \\
\hline 6 & 7.6 & 2.0 & -.2 & -1.0 & -5.8 & -4.7 & 2.8 & 12.7 & 12.4 & 16.6 & 20.0 & 13.9 \\
\hline 7 & 6.4 & .1 & 3.3 & 1.1 & -11.2 & -.4 & 2.0 & 10.1 & 14.3 & 16.1 & 18.4 & 15.6 \\
\hline 8 & 8.7 & 2.0 & 4.7 & 4.2 & -8.7 & -.3 & -2.2 & 5.4 & 15.9 & 15.0 & 19.9 & 17.0 \\
\hline 9 & 10.4 & 5.6 & 2.3 & 6.8 & -3.6 & .9 & 2.3 & 6.9 & 12.6 & 15.5 & 17.7 & 17.9 \\
\hline 10 & 11.6 & 7.2 & -8.4 & 7.3 & .3 & -.4 & 5.0 & 5.7 & 7.1 & 15.2 & 16.3 & 18.1 \\
\hline 11 & 11.7 & 7.9 & -9.5 & 5.1 & 1.8 & -10.1 & 8.0 & 4.5 & 9.1 & 18.0 & 15.9 & 18.5 \\
\hline 12 & 11.7 & 6.8 & -.4 & .1 & -.5 & -12.5 & 7.8 & 6.8 & 8.7 & 19.6 & 14.9 & 18.6 \\
\hline 13 & 11.4 & 5.7 & -1.4 & -3.2 & -10.2 & -11.0 & 9.4 & 6.9 & 8.7 & 17.5 & 16.6 & 17.4 \\
\hline 14 & 8.9 & 3.0 & 2.3 & -5.7 & -19.0 & -4.3 & 11.5 & 6.7 & 6.0 & 14.7 & 15.8 & 16.2 \\
\hline 15 & 6.1 & 3.3 & 2.6 & -7.5 & -14.0 & -4.0 & 9.5 & 3.4 & 5.4 & 14.8 & 11.4 & 14.6 \\
\hline 16 & 4.9 & 4.1 & -1.5 & -8.1 & -11.7 & .2 & 4.3 & 5.8 & 8.3 & 13.0 & 12.7 & 12.8 \\
\hline 17 & 5.6 & .7 & -2.0 & -8.3 & -8.0 & 2.8 & -.8 & 8.0 & 11.8 & 15.5 & 13.6 & 10.3 \\
\hline 18 & 7.4 & 3.2 & -3.7 & -9.3 & -10.3 & 2.7 & .8 & 6.7 & 10.8 & 16.7 & 10.9 & 8.3 \\
\hline 19 & 8.1 & 7.9 & -2.5 & -8.4 & -9.6 & 5.2 & 4.9 & 4.3 & 11.5 & 17.1 & 6.8 & 7.2 \\
\hline 20 & 6.5 & 8.9 & -.2 & -6.3 & -2.4 & 6.0 & 3.6 & 6.8 & 14.9 & 18.1 & 7.2 & 9.1 \\
\hline 21 & 2.4 & 6.4 & -1.4 & -2.9 & -2.6 & 6.5 & 2.4 & 10.3 & 17.8 & 18.8 & 10.7 & 7.6 \\
\hline 22 & 2.3 & 4.6 & 2.1 & -.3 & 1.2 & 6.2 & 4.4 & 9.9 & 19.1 & 19.1 & 12.8 & 7.9 \\
\hline 23 & 3.1 & 3.6 & 3.2 & -.7 & 3.0 & 5.4 & .7 & 7.4 & 17.6 & 18.6 & 13.8 & 5.9 \\
\hline 24 & 2.4 & 1.4 & 2.0 & -4.6 & 3.1 & 5.2 & -1.4 & -.8 & 17.7 & 15.9 & 12.7 & 7.3 \\
\hline 25 & -4.4 & -1.1 & 1.8 & -1.6 & 2.6 & 6.3 & 3.0 & 6.0 & 18.1 & 15.3 & 9.9 & 9.0 \\
\hline 26 & -2.1 & -7.0 & 1.7 & -5.0 & 1.8 & 5.3 & 6.0 & 7.5 & 18.2 & 14.9 & 9.9 & 9.0 \\
\hline 27 & .3 & -9.6 & .5 & -9.1 & .2 & 1.0 & 8.3 & 6.5 & 17.7 & 17.0 & 12.8 & 7.6 \\
\hline 28 & -7.0 & -6.0 & $-4 \cdot 3$ & $-4 \cdot 3$ & 1.6 & -3.5 & 6.4 & -.2 & 18.2 & 18.6 & 15.4 & 5.7 \\
\hline 29 & -5.8 & -2.9 & -7.1 & $-3 \cdot 3$ & --- & -3.0 & -3.0 & 3.7 & 18.9 & 19.2 & 17.2 & 7.2 \\
\hline 30 & .8 & -.8 & -1.8 & -5.2 & --- & -.4 & -3.2 & 4.7 & 19.8 & 19.1 & 16.6 & 9.7 \\
\hline 31 & 2.0 & --- & .7 & -8.3 & --- & 2.5 & --- & 3.0 & --- & 18.4 & 15.2 & --- \\
\hline MEAN & 4.6 & 2.3 & .0 & -3.6 & -5.0 & .0 & 3.6 & 6.0 & 13.3 & 16.8 & 14.7 & 12.2 \\
\hline MAX & 11.7 & 8.9 & 6.1 & 7.3 & 3.1 & 6.5 & 11.5 & 12.7 & 19.8 & 20.2 & 20.0 & 18.6 \\
\hline MIN & -7.0 & -9.6 & -9.5 & -9.6 & -19.0 & -12.5 & -3.2 & -.8 & 2.4 & 13.0 & 6.8 & 5.7 \\
\hline MED & 4.9 & 3.2 & .5 & -4.3 & -5.5 & .9 & 3.6 & 6.5 & 14.4 & 16.7 & 15.4 & 13.1 \\
\hline
\end{tabular}

CAL YR 1989 MEAN 5.4 MAX 21.7 MIN -21.3 MED 5.3 WTR YR 1990 MEAN 5.5 MAX 20.2 MIN -19.0 MED 5.2 
3 SPRINGS BASIN

KAWICH PEAK NEAR WARM SPRINGS, NV

DAILY MEAN AIR TEMPERATURE, WATER YEAR OCTOBER 1990 TO SEPTEMBER 1991 DEGREES CELSIUS

\begin{tabular}{|c|c|c|c|c|c|c|c|c|c|c|c|c|}
\hline DAY & $\mathrm{OCT}$ & NOV & $\mathrm{DEC}$ & JAN & FEB & MAR & APR & MAY & JUN & JUL & AUG & SEP \\
\hline $\begin{array}{l}1 \\
2 \\
3 \\
4 \\
5\end{array}$ & $\begin{array}{r}11.5 \\
10.5 \\
9.1 \\
11.3 \\
10.9\end{array}$ & $\begin{array}{r}-3.3 \\
-8.7 \\
-3.2 \\
3.2 \\
-.8\end{array}$ & $\begin{array}{r}-2.7 \\
-4.0 \\
-2.9 \\
2.4 \\
.8\end{array}$ & $\begin{array}{r}-.2 \\
-.5 \\
-2.3 \\
-3.0 \\
-2.9\end{array}$ & $\begin{array}{r}1.2 \\
-1.4 \\
.7 \\
3.3 \\
.5\end{array}$ & $\begin{array}{r}-4.1 \\
-4.8 \\
-1.4 \\
.5 \\
-5.1\end{array}$ & $\begin{array}{r}-1.1 \\
1.9 \\
3.9 \\
7.7 \\
7.5\end{array}$ & $\begin{array}{r}.0 \\
-4.4 \\
-1.7 \\
2.5 \\
6.2\end{array}$ & $\begin{array}{r}4.6 \\
9.4 \\
12.4 \\
12.4 \\
10.1\end{array}$ & $\begin{array}{l}15.2 \\
18.2 \\
19.7 \\
21.0 \\
21.3\end{array}$ & $\begin{array}{l}13.0 \\
14.2 \\
14.6 \\
14.9 \\
14.0\end{array}$ & $\begin{array}{l}15.3 \\
16.8 \\
15.6 \\
15.2 \\
12.9\end{array}$ \\
\hline $\begin{array}{r}6 \\
7 \\
8 \\
9 \\
10\end{array}$ & $\begin{array}{l}8.1 \\
1.1 \\
.0 \\
6.5 \\
9.6\end{array}$ & $\begin{array}{r}-9.0 \\
-4.4 \\
2.9 \\
5.6 \\
7.7\end{array}$ & $\begin{array}{l}-.5 \\
1.2 \\
2.8 \\
4.4 \\
2.3\end{array}$ & $\begin{array}{l}-2.9 \\
-3.2 \\
-5.4 \\
-7.2 \\
-2.7\end{array}$ & $\begin{array}{r}-.3 \\
.8 \\
1.2 \\
1.2 \\
.8\end{array}$ & $\begin{array}{l}-7.7 \\
-6.4 \\
-1.2 \\
-1.5 \\
-2.3\end{array}$ & $\begin{array}{r}6.8 \\
.6 \\
-1.5 \\
3.7 \\
-7.1\end{array}$ & $\begin{array}{r}6.8 \\
9.2 \\
9.5 \\
-4.8 \\
-4.5\end{array}$ & $\begin{array}{l}11.2 \\
13.3 \\
12.8 \\
14.2 \\
16.3\end{array}$ & $\begin{array}{l}19.3 \\
16.8 \\
11.8 \\
13.3 \\
15.4\end{array}$ & $\begin{array}{l}13.7 \\
14.3 \\
16.6 \\
18.2 \\
17.0\end{array}$ & $\begin{array}{r}8.2 \\
7.3 \\
10.7 \\
8.2 \\
5.5\end{array}$ \\
\hline $\begin{array}{l}11 \\
12 \\
13 \\
14 \\
15\end{array}$ & $\begin{array}{r}6.8 \\
8.6 \\
10.1 \\
11.0 \\
10.9\end{array}$ & $\begin{array}{l}7.3 \\
7.0 \\
7.5 \\
4.9 \\
2.7\end{array}$ & $\begin{array}{r}-.7 \\
-3.6 \\
-6.9 \\
-9.4 \\
-7.5\end{array}$ & $\begin{array}{r}.3 \\
1.1 \\
-.5 \\
-1.4 \\
-3.3\end{array}$ & $\begin{array}{l}2.0 \\
2.8 \\
2.1 \\
2.6 \\
3.2\end{array}$ & $\begin{array}{l}-8.7 \\
-4.0 \\
-5.5 \\
-5.5 \\
-7.7\end{array}$ & $\begin{array}{r}-9.2 \\
-5.8 \\
-1.1 \\
2.4 \\
1.6\end{array}$ & $\begin{array}{r}-1.7 \\
3.5 \\
4.1 \\
-.4 \\
3.7\end{array}$ & $\begin{array}{l}16.8 \\
16.1 \\
13.8 \\
12.2 \\
12.5\end{array}$ & $\begin{array}{l}16.7 \\
18.3 \\
18.9 \\
17.3 \\
16.0\end{array}$ & $\begin{array}{l}12.6 \\
13.8 \\
15.1 \\
15.8 \\
15.0\end{array}$ & $\begin{array}{r}5.9 \\
8.5 \\
10.9 \\
11.8 \\
10.6\end{array}$ \\
\hline $\begin{array}{l}16 \\
17 \\
18 \\
19 \\
20\end{array}$ & $\begin{array}{r}8.2 \\
6.4 \\
5.3 \\
-.7 \\
-2.7\end{array}$ & $\begin{array}{r}3.7 \\
3.0 \\
1.4 \\
-1.4 \\
-6.3\end{array}$ & $\begin{array}{r}-10.9 \\
-7.4 \\
-4.4 \\
-12.3 \\
-19.8\end{array}$ & $\begin{array}{r}-6.7 \\
-3.1 \\
.0 \\
-2.0 \\
-8.4\end{array}$ & $\begin{array}{r}-.9 \\
-5.3 \\
-5.6 \\
.9 \\
4.9\end{array}$ & $\begin{array}{l}-5.4 \\
-4.9 \\
-2.9 \\
-5.5 \\
-7.6\end{array}$ & $\begin{array}{r}-.5 \\
-1.5 \\
.6 \\
3.1 \\
1.8\end{array}$ & $\begin{array}{r}8.2 \\
1.0 \\
-2.5 \\
.2 \\
3.1\end{array}$ & $\begin{array}{l}14.5 \\
15.4 \\
14.6 \\
11.4 \\
11.4\end{array}$ & $\begin{array}{l}15.2 \\
16.2 \\
16.6 \\
14.4 \\
13.3\end{array}$ & $\begin{array}{l}15.9 \\
16.7 \\
16.6 \\
17.2 \\
17.0\end{array}$ & $\begin{array}{l}11.5 \\
13.6 \\
15.5 \\
15.0 \\
15.0\end{array}$ \\
\hline $\begin{array}{l}21 \\
22 \\
23 \\
24 \\
25\end{array}$ & $\begin{array}{l}3.7 \\
6.5 \\
7.1 \\
8.6 \\
9.6\end{array}$ & $\begin{array}{r}-5.0 \\
1.9 \\
7.0 \\
6.6 \\
2.3\end{array}$ & $\begin{array}{r}-21.3 \\
-18.5 \\
-14.9 \\
-8.2 \\
-7.4\end{array}$ & $\begin{array}{r}-10.3 \\
-3.6 \\
-2.3 \\
-3.2 \\
-3.6\end{array}$ & $\begin{array}{l}4.5 \\
3.3 \\
1.6 \\
-.5 \\
1.7\end{array}$ & $\begin{array}{l}-6.5 \\
-4.4 \\
-4.7 \\
-2.7 \\
-7.0\end{array}$ & $\begin{array}{r}.4 \\
-.5 \\
2.4 \\
3.2 \\
-3.7\end{array}$ & $\begin{array}{r}2.1 \\
4.1 \\
9.9 \\
11.5 \\
10.6\end{array}$ & $\begin{array}{r}11.1 \\
12.0 \\
11.8 \\
9.3 \\
8.7\end{array}$ & $\begin{array}{l}14.9 \\
15.1 \\
17.1 \\
17.6 \\
17.4\end{array}$ & $\begin{array}{l}17.8 \\
19.1 \\
19.1 \\
18.8 \\
17.5\end{array}$ & $\begin{array}{l}15.0 \\
13.7 \\
14.6 \\
13.3 \\
14.5\end{array}$ \\
\hline $\begin{array}{l}26 \\
27 \\
28 \\
29 \\
30 \\
31\end{array}$ & $\begin{array}{r}9.8 \\
10.3 \\
9.4 \\
7.5 \\
6.8 \\
4.3\end{array}$ & $\begin{array}{r}-9.8 \\
-6.0 \\
-3.7 \\
1.1 \\
-. .2 \\
---\end{array}$ & $\begin{array}{r}-8.2 \\
-2.0 \\
-3.5 \\
-12.7 \\
-4.5 \\
-1.0\end{array}$ & $\begin{array}{r}-3.9 \\
-3.2 \\
-4.7 \\
-6.8 \\
.3 \\
.5\end{array}$ & $\begin{array}{r}.5 \\
-.9 \\
-3.8 \\
--- \\
---\end{array}$ & $\begin{array}{r}-6.8 \\
-6.5 \\
-1.6 \\
-.3 \\
.2 \\
2.1\end{array}$ & $\begin{array}{r}-2.3 \\
-2.7 \\
-.3 \\
1.5 \\
2.3 \\
-.-\end{array}$ & $\begin{array}{r}9.4 \\
5.6 \\
7.9 \\
9.2 \\
1.5 \\
.9\end{array}$ & $\begin{array}{r}8.8 \\
5.5 \\
7.6 \\
7.2 \\
11.3 \\
-.-\end{array}$ & $\begin{array}{l}18.0 \\
18.9 \\
18.9 \\
19.2 \\
18.1 \\
13.8\end{array}$ & $\begin{array}{l}15.1 \\
12.3 \\
12.4 \\
15.5 \\
17.3 \\
13.2\end{array}$ & $\begin{array}{r}12.6 \\
9.7 \\
8.9 \\
8.7 \\
10.2 \\
\ldots\end{array}$ \\
\hline $\begin{array}{l}\text { MEAN } \\
\text { MAX } \\
\text { MIN } \\
\text { MED }\end{array}$ & $\begin{array}{r}7.3 \\
11.5 \\
-2.7 \\
8.2\end{array}$ & $\begin{array}{r}.5 \\
7.7 \\
-9.8 \\
1.7\end{array}$ & $\begin{array}{r}-5.8 \\
4.4 \\
-21.3 \\
-4.4\end{array}$ & $\begin{array}{r}-3.1 \\
1.1 \\
-10.3 \\
-3.0\end{array}$ & $\begin{array}{r}.8 \\
4.9 \\
-5.6 \\
1.0\end{array}$ & $\begin{array}{r}-4.2 \\
2.1 \\
-8.7 \\
-4.8\end{array}$ & $\begin{array}{r}.5 \\
7.7 \\
-9.2 \\
.6\end{array}$ & $\begin{array}{r}3.6 \\
11.5 \\
-4.8 \\
3.5\end{array}$ & $\begin{array}{r}11.6 \\
16.8 \\
4.6 \\
11.9\end{array}$ & $\begin{array}{l}16.9 \\
21.3 \\
11.8 \\
17.1\end{array}$ & $\begin{array}{l}15.6 \\
19.1 \\
12.3 \\
15.5\end{array}$ & $\begin{array}{r}11.8 \\
16.8 \\
5.5 \\
12.2\end{array}$ \\
\hline
\end{tabular}

CAL YR 1990 MEAN 5.1 MAX 20.2 MIN -21.3 MED 5.7

WTR YR 1991 MEAN 4.6 MAX $21.3 \quad$ MIN $-21.3 \quad$ MED 3.3 
3 SPRINGS BASIN

KAWICH PEAK NEAR WARM SPRINGS, NV DAILY MAXIMUM AIR TEMPERATURE, WATER YEAR OCTOBER 1988 TO SEPTEMBER 1989 DEGREES CELSIUS

\begin{tabular}{|c|c|c|c|c|c|c|c|c|c|c|c|c|}
\hline DAY & OCT & NOV & DEC & JAN & FEB & MAR & $\mathrm{APR}$ & MAY & JUN & JUL & AUG & SEP \\
\hline $\begin{array}{l}1 \\
2 \\
3 \\
4 \\
5\end{array}$ & $\begin{array}{l}18.7 \\
18.0 \\
16.7 \\
16.5 \\
13.7\end{array}$ & $\begin{array}{r}10.4 \\
8.7 \\
11.7 \\
9.5 \\
12.7\end{array}$ & $\begin{array}{l}--- \\
--- \\
--- \\
--- \\
---\end{array}$ & $\begin{array}{r}-4.6 \\
.7 \\
1.4 \\
-.6 \\
-3.3\end{array}$ & $\begin{array}{r}-2.1 \\
-5.2 \\
-6.4 \\
-10.1 \\
-18.1\end{array}$ & $\begin{array}{r}4.7 \\
-1.2 \\
-8.1 \\
-1.2 \\
6.7\end{array}$ & $\begin{array}{r}5.3 \\
7.7 \\
7.1 \\
10.2 \\
13.8\end{array}$ & $\begin{array}{l}13.0 \\
12.6 \\
13.9 \\
15.4 \\
18.2\end{array}$ & $\begin{array}{r}--- \\
13.2 \\
10.4 \\
10.8 \\
9.5\end{array}$ & $\begin{array}{l}20.1 \\
21.5 \\
23.2 \\
22.8 \\
24.2\end{array}$ & $\begin{array}{l}20.0 \\
19.5 \\
19.3 \\
19.7 \\
21.5\end{array}$ & $\begin{array}{l}17.6 \\
19.5 \\
19.6 \\
20.3 \\
20.5\end{array}$ \\
\hline $\begin{array}{r}6 \\
7 \\
8 \\
9 \\
10\end{array}$ & $\begin{array}{l}10.1 \\
12.2 \\
13.1 \\
12.4 \\
10.7\end{array}$ & $\begin{array}{l}8.5 \\
5.4 \\
2.3 \\
4.2 \\
2.8\end{array}$ & $\begin{array}{l}--- \\
--- \\
--- \\
2.2 \\
5.6\end{array}$ & $\begin{array}{r}-13.4 \\
-11.1 \\
-6.5 \\
-2.5 \\
1.7\end{array}$ & $\begin{array}{r}-18.9 \\
-13.8 \\
-5.3 \\
-3.3 \\
-3.9\end{array}$ & $\begin{array}{l}8.4 \\
6.8 \\
6.7 \\
8.7 \\
8.8\end{array}$ & $\begin{array}{l}16.5 \\
17.3 \\
15.9 \\
15.4 \\
16.4\end{array}$ & $\begin{array}{r}18.0 \\
18.4 \\
18.3 \\
12.8 \\
5.9\end{array}$ & $\begin{array}{l}15.0 \\
14.4 \\
13.3 \\
15.0 \\
14.5\end{array}$ & $\begin{array}{l}25.7 \\
26.3 \\
25.1 \\
21.5 \\
20.5\end{array}$ & $\begin{array}{l}22.6 \\
23.1 \\
19.2 \\
16.0 \\
18.4\end{array}$ & $\begin{array}{l}19.2 \\
13.4 \\
14.5 \\
16.1 \\
16.6\end{array}$ \\
\hline $\begin{array}{l}11 \\
12 \\
13 \\
14 \\
15\end{array}$ & $\begin{array}{r}11.0 \\
10.6 \\
11.0 \\
9.0 \\
11.7\end{array}$ & $\begin{array}{r}2.0 \\
4.9 \\
3.6 \\
-.7 \\
-4.6\end{array}$ & $\begin{array}{r}7.0 \\
10.2 \\
9.4 \\
2.3 \\
-9.0\end{array}$ & $\begin{array}{r}-11.6 \\
-5.8 \\
-1.3 \\
-5.6 \\
-2.9\end{array}$ & $\begin{array}{l}-3.4 \\
-8.0 \\
-7.9 \\
-4.7 \\
-2.1\end{array}$ & $\begin{array}{l}9.3 \\
9.6 \\
2.6 \\
2.0 \\
5.4\end{array}$ & $\begin{array}{r}10.8 \\
8.6 \\
13.1 \\
15.0 \\
13.3\end{array}$ & $\begin{array}{r}-1.1 \\
2.5 \\
.6 \\
.6 \\
4.8\end{array}$ & $\begin{array}{l}16.1 \\
16.3 \\
19.6 \\
22.5 \\
22.1\end{array}$ & $\begin{array}{l}18.4 \\
18.1 \\
20.8 \\
22.7 \\
21.6\end{array}$ & $\begin{array}{l}17.4 \\
20.0 \\
21.4 \\
22.0 \\
21.3\end{array}$ & $\begin{array}{l}13.9 \\
12.1 \\
15.3 \\
17.2 \\
18.6\end{array}$ \\
\hline $\begin{array}{l}16 \\
17 \\
18 \\
19 \\
20\end{array}$ & $\begin{array}{l}14.7 \\
16.1 \\
16.6 \\
15.1 \\
13.4\end{array}$ & $\begin{array}{r}-2.6 \\
-6.1 \\
-7.3 \\
-1.5 \\
-.9\end{array}$ & $\begin{array}{r}-4.1 \\
-.6 \\
-4.2 \\
-6.6 \\
-5.9\end{array}$ & $\begin{array}{l}1.7 \\
5.6 \\
4.8 \\
2.3 \\
4.6\end{array}$ & $\begin{array}{r}-.3 \\
3.9 \\
2.3 \\
-1.1 \\
-1.5\end{array}$ & $\begin{array}{l}4.5 \\
3.3 \\
4.7 \\
3.0 \\
1.6\end{array}$ & $\begin{array}{l}15.1 \\
16.7 \\
17.3 \\
16.7 \\
15.3\end{array}$ & $\begin{array}{r}8.7 \\
12.8 \\
14.1 \\
11.5 \\
15.5\end{array}$ & $\begin{array}{l}17.6 \\
21.0 \\
23.0 \\
22.3 \\
17.6\end{array}$ & $\begin{array}{l}20.7 \\
22.5 \\
24.2 \\
24.8 \\
24.3\end{array}$ & $\begin{array}{l}20.7 \\
17.7 \\
17.8 \\
18.0 \\
17.1\end{array}$ & $\begin{array}{r}14.4 \\
9.4 \\
8.3 \\
6.5 \\
11.7\end{array}$ \\
\hline $\begin{array}{l}21 \\
22 \\
23 \\
24 \\
25\end{array}$ & $\begin{array}{l}15.2 \\
15.3 \\
16.3 \\
14.9 \\
13.3\end{array}$ & $\begin{array}{r}3.1 \\
4.5 \\
1.3 \\
-7.2 \\
-6.8\end{array}$ & $\begin{array}{l}-4.7 \\
-9.3 \\
-9.3 \\
-5.9 \\
-7.5\end{array}$ & $\begin{array}{r}3.6 \\
-1.5 \\
-2.4 \\
-4.2 \\
-7.1\end{array}$ & $\begin{array}{l}3.7 \\
5.1 \\
7.7 \\
7.8 \\
8.3\end{array}$ & $\begin{array}{l}8.8 \\
7.2 \\
5.7 \\
6.2 \\
-.4\end{array}$ & $\begin{array}{r}12.7 \\
9.0 \\
5.3 \\
-.3 \\
1.0\end{array}$ & $\begin{array}{r}14.5 \\
16.2 \\
12.8 \\
---\end{array}$ & $\begin{array}{r}12.8 \\
17.0 \\
15.1 \\
9.7 \\
13.4\end{array}$ & $\begin{array}{l}21.6 \\
22.3 \\
23.1 \\
20.8 \\
23.4\end{array}$ & $\begin{array}{l}17.3 \\
17.8 \\
16.0 \\
12.6 \\
15.5\end{array}$ & $\begin{array}{l}13.7 \\
15.0 \\
17.9 \\
18.5 \\
16.3\end{array}$ \\
\hline $\begin{array}{l}26 \\
27 \\
28 \\
29 \\
30 \\
31\end{array}$ & $\begin{array}{r}13.5 \\
13.2 \\
9.8 \\
9.3 \\
11.0 \\
12.6\end{array}$ & $\begin{array}{r}-4.8 \\
4.2 \\
1.4 \\
--- \\
--- \\
---\end{array}$ & $\begin{array}{r}-15.1 \\
-9.8 \\
-13.2 \\
-3.4 \\
.7 \\
-7.2\end{array}$ & $\begin{array}{r}-3.2 \\
.8 \\
.7 \\
3.1 \\
8.3 \\
6.3\end{array}$ & $\begin{array}{l}6.6 \\
1.8 \\
3.5 \\
--- \\
--- \\
---\end{array}$ & $\begin{array}{r}-2.1 \\
4.3 \\
8.5 \\
4.6 \\
8.2 \\
9.9\end{array}$ & $\begin{array}{r}.9 \\
.6 \\
3.4 \\
7.5 \\
9.5 \\
---\end{array}$ & $\begin{array}{l}--- \\
--- \\
--- \\
--- \\
--- \\
---\end{array}$ & $\begin{array}{r}17.3 \\
14.5 \\
14.9 \\
17.0 \\
17.3 \\
-.-\end{array}$ & $\begin{array}{l}22.8 \\
21.9 \\
21.7 \\
22.2 \\
22.2 \\
21.7\end{array}$ & $\begin{array}{l}17.5 \\
18.4 \\
19.7 \\
19.0 \\
17.7 \\
17.8\end{array}$ & $\begin{array}{r}16.3 \\
14.8 \\
16.9 \\
15.0 \\
11.0 \\
-\end{array}$ \\
\hline MAX & 18.7 & --- & --- & 8.3 & 8.3 & 9.9 & 17.3 & -- & --- & 26.3 & 23.1 & 20.5 \\
\hline
\end{tabular}


3 SPRINGS BASIN

KAWICH PEAK NEAR WARM SPRINGS, NV

DAILY MAXIMUM AIR TEMPERATURE, WATER YEAR OCTOBER 1989 TO SEPTEMBER 1990 DEGREES CELSIUS

\begin{tabular}{|c|c|c|c|c|c|c|c|c|c|c|c|c|}
\hline DAY & OCT & NOV & DEC & JAN & EEB & MAR & APR & MAY & JUN & JUL & AUG & SEP \\
\hline $\begin{array}{l}1 \\
2 \\
3 \\
4 \\
5\end{array}$ & $\begin{array}{r}12.1 \\
9.4 \\
4.1 \\
6.7 \\
10.4\end{array}$ & $\begin{array}{l}2.4 \\
4.4 \\
7.1 \\
8.9 \\
8.6\end{array}$ & $\begin{array}{r}-.1 \\
6.6 \\
8.0 \\
10.7 \\
7.6\end{array}$ & $\begin{array}{r}-.5 \\
-7.4 \\
-6.4 \\
-3.1 \\
-1.1\end{array}$ & $\begin{array}{r}-8.4 \\
-5.9 \\
-2.3 \\
-7.3 \\
-.9\end{array}$ & $\begin{array}{r}4.6 \\
4.8 \\
5.1 \\
2.7 \\
-4.5\end{array}$ & $\begin{array}{l}8.7 \\
8.1 \\
8.4 \\
6.4 \\
8.1\end{array}$ & $\begin{array}{r}5.7 \\
7.6 \\
9.2 \\
13.7 \\
15.7\end{array}$ & $\begin{array}{r}8.7 \\
15.5 \\
20.1 \\
21.4 \\
18.5\end{array}$ & $\begin{array}{l}24.0 \\
21.4 \\
18.9 \\
20.2 \\
21.2\end{array}$ & $\begin{array}{l}22.0 \\
21.0 \\
22.8 \\
24.0 \\
24.2\end{array}$ & $\begin{array}{l}19.8 \\
19.4 \\
19.0 \\
17.5 \\
18.8\end{array}$ \\
\hline $\begin{array}{r}6 \\
7 \\
8 \\
9 \\
10\end{array}$ & $\begin{array}{r}11.2 \\
9.7 \\
14.3 \\
16.0 \\
16.5\end{array}$ & $\begin{array}{r}6.1 \\
3.5 \\
5.7 \\
8.9 \\
11.2\end{array}$ & $\begin{array}{r}1.8 \\
6.1 \\
8.7 \\
4.5 \\
-2.1\end{array}$ & $\begin{array}{r}2.3 \\
3.0 \\
6.0 \\
9.9 \\
11.6\end{array}$ & $\begin{array}{r}-4.0 \\
-6.7 \\
-4.1 \\
.4 \\
3.1\end{array}$ & $\begin{array}{l}-.5 \\
3.3 \\
2.4 \\
4.8 \\
2.8\end{array}$ & $\begin{array}{r}7.1 \\
6.2 \\
-.9 \\
7.7 \\
10.9\end{array}$ & $\begin{array}{l}16.6 \\
15.0 \\
10.2 \\
12.9 \\
10.3\end{array}$ & $\begin{array}{l}15.6 \\
18.8 \\
21.1 \\
16.4 \\
11.0\end{array}$ & $\begin{array}{l}20.9 \\
20.3 \\
18.4 \\
20.0 \\
18.2\end{array}$ & $\begin{array}{l}24.4 \\
22.2 \\
24.3 \\
23.4 \\
21.9\end{array}$ & $\begin{array}{l}19.5 \\
21.2 \\
22.0 \\
22.4 \\
23.0\end{array}$ \\
\hline $\begin{array}{l}11 \\
12 \\
13 \\
14 \\
15\end{array}$ & $\begin{array}{r}16.0 \\
16.2 \\
15.2 \\
12.7 \\
9.3\end{array}$ & $\begin{array}{r}12.7 \\
10.2 \\
8.7 \\
6.0 \\
6.5\end{array}$ & $\begin{array}{r}-1.9 \\
3.6 \\
1.4 \\
4.7 \\
6.4\end{array}$ & $\begin{array}{r}8.5 \\
2.1 \\
-2.2 \\
-3.8 \\
-4.8\end{array}$ & $\begin{array}{r}6.9 \\
2.0 \\
-3.2 \\
-17.0 \\
-9.4\end{array}$ & $\begin{array}{r}-6.4 \\
-10.0 \\
-8.2 \\
-.3 \\
.3\end{array}$ & $\begin{array}{l}11.8 \\
11.8 \\
14.4 \\
15.8 \\
14.0\end{array}$ & $\begin{array}{r}7.9 \\
11.8 \\
11.9 \\
10.1 \\
7.9\end{array}$ & $\begin{array}{r}13.8 \\
13.3 \\
13.3 \\
11.4 \\
8.8\end{array}$ & $\begin{array}{l}23.1 \\
24.2 \\
23.4 \\
19.9 \\
19.9\end{array}$ & $\begin{array}{l}20.9 \\
19.8 \\
21.0 \\
20.8 \\
16.3\end{array}$ & $\begin{array}{l}23.5 \\
23.3 \\
22.2 \\
20.0 \\
19.2\end{array}$ \\
\hline $\begin{array}{l}16 \\
17 \\
18 \\
19 \\
20\end{array}$ & $\begin{array}{r}9.8 \\
9.9 \\
12.7 \\
12.7 \\
10.2\end{array}$ & $\begin{array}{r}6.8 \\
3.1 \\
8.3 \\
13.9 \\
13.1\end{array}$ & $\begin{array}{r}2.1 \\
1.1 \\
-1.0 \\
.5 \\
3.0\end{array}$ & $\begin{array}{l}-7.6 \\
-7.5 \\
-5.7 \\
-3.4 \\
-3.1\end{array}$ & $\begin{array}{r}-8.8 \\
-6.7 \\
-7.9 \\
-6.6 \\
1.3\end{array}$ & $\begin{array}{l}4.1 \\
5.7 \\
6.4 \\
8.3 \\
8.6\end{array}$ & $\begin{array}{l}9.4 \\
1.6 \\
4.1 \\
9.0 \\
7.9\end{array}$ & $\begin{array}{r}11.8 \\
13.2 \\
11.3 \\
6.8 \\
12.0\end{array}$ & $\begin{array}{l}13.7 \\
16.5 \\
15.6 \\
16.4 \\
20.7\end{array}$ & $\begin{array}{l}17.7 \\
20.3 \\
22.3 \\
21.9 \\
22.4\end{array}$ & $\begin{array}{l}17.0 \\
17.4 \\
15.9 \\
13.0 \\
12.6\end{array}$ & $\begin{array}{l}17.0 \\
13.6 \\
11.8 \\
10.4 \\
13.8\end{array}$ \\
\hline $\begin{array}{l}21 \\
22 \\
23 \\
24 \\
25\end{array}$ & $\begin{array}{r}4.2 \\
5.5 \\
5.7 \\
6.2 \\
-1.7\end{array}$ & $\begin{array}{l}9.6 \\
9.2 \\
7.4 \\
5.4 \\
2.5\end{array}$ & $\begin{array}{l}1.0 \\
6.4 \\
8.8 \\
5.7 \\
6.1\end{array}$ & $\begin{array}{r}2.1 \\
2.8 \\
1.2 \\
-1.1 \\
2.3\end{array}$ & $\begin{array}{l}-.6 \\
4.5 \\
8.5 \\
7.6 \\
6.2\end{array}$ & $\begin{array}{r}10.2 \\
10.1 \\
9.1 \\
10.0 \\
10.2\end{array}$ & $\begin{array}{l}6.4 \\
8.4 \\
5.3 \\
2.4 \\
9.2\end{array}$ & $\begin{array}{r}15.1 \\
14.7 \\
12.7 \\
5.2 \\
11.6\end{array}$ & $\begin{array}{l}23.4 \\
23.1 \\
21.5 \\
21.7 \\
22.6\end{array}$ & $\begin{array}{l}23.2 \\
23.9 \\
22.6 \\
20.2 \\
19.6\end{array}$ & $\begin{array}{l}15.5 \\
18.1 \\
18.8 \\
16.6 \\
14.5\end{array}$ & $\begin{array}{r}10.2 \\
12.7 \\
9.0 \\
11.5 \\
13.2\end{array}$ \\
\hline $\begin{array}{l}26 \\
27 \\
28 \\
29 \\
30 \\
31\end{array}$ & $\begin{array}{r}3.1 \\
4.5 \\
-3.4 \\
-1.2 \\
7.3 \\
6.0\end{array}$ & $\begin{array}{r}-2.2 \\
-7.1 \\
-2.8 \\
.4 \\
3.2 \\
-.--\end{array}$ & $\begin{array}{r}6.2 \\
5.0 \\
-1.7 \\
-4.6 \\
.5 \\
4.7\end{array}$ & $\begin{array}{r}-.8 \\
-4.9 \\
-2.5 \\
-1.1 \\
-3.1 \\
-5.4\end{array}$ & $\begin{array}{l}5.7 \\
4.5 \\
5.2 \\
--- \\
--- \\
---\end{array}$ & $\begin{array}{r}9.8 \\
4.5 \\
-1.6 \\
.3 \\
3.4 \\
6.8\end{array}$ & $\begin{array}{r}10.7 \\
12.8 \\
11.8 \\
2.8 \\
1.4 \\
-\end{array}$ & $\begin{array}{r}11.1 \\
11.1 \\
.6 \\
7.3 \\
9.0 \\
7.3\end{array}$ & $\begin{array}{r}22.3 \\
22.1 \\
23.0 \\
22.6 \\
24.2 \\
-.-\end{array}$ & $\begin{array}{l}20.2 \\
22.1 \\
23.4 \\
24.0 \\
23.5 \\
22.7\end{array}$ & $\begin{array}{l}14.4 \\
17.8 \\
20.8 \\
21.4 \\
20.6 \\
19.9\end{array}$ & $\begin{array}{r}13.6 \\
12.7 \\
10.4 \\
12.0 \\
15.1 \\
-\end{array}$ \\
\hline MAX & 16.5 & 13.9 & 10.7 & 11.6 & 8.5 & 10.2 & 15.8 & 16.6 & 24.2 & 24.2 & 24.4 & 23.5 \\
\hline
\end{tabular}

WTR YR $1990 \quad$ MAX 24.4 
3 SPRINGS BASIN

KAWICH PEAK NEAR WARM SPRINGS, NV

DAILY MAXIMUM AIR TEMPERATURE, WATER YEAR OCTOBER 1990 TO SEPTEMBER 1991 DEGREES CELSIUS

\begin{tabular}{|c|c|c|c|c|c|c|c|c|c|c|c|c|}
\hline DAY & OCT & NOV & DEC & JAN & FEB & MAR & APR & MAY & JUN & JUL & AUG & SEP \\
\hline $\begin{array}{l}1 \\
2 \\
3 \\
4 \\
5\end{array}$ & $\begin{array}{l}16.8 \\
14.8 \\
15.4 \\
17.0 \\
15.3\end{array}$ & $\begin{array}{r}-.6 \\
-5.3 \\
1.6 \\
8.2 \\
2.2\end{array}$ & $\begin{array}{l}.0 \\
.0 \\
2.3 \\
7.3 \\
4.1\end{array}$ & $\begin{array}{r}4.7 \\
6.3 \\
-.2 \\
.1 \\
1.8\end{array}$ & $\begin{array}{r}6.4 \\
.5 \\
4.7 \\
7.4 \\
3.8\end{array}$ & $\begin{array}{r}-2.8 \\
-1.3 \\
1.0 \\
2.1 \\
-.8\end{array}$ & $\begin{array}{r}1.8 \\
8.5 \\
10.0 \\
12.7 \\
10.7\end{array}$ & $\begin{array}{r}4.7 \\
-1.0 \\
3.0 \\
9.0 \\
12.8\end{array}$ & $\begin{array}{r}8.9 \\
15.0 \\
17.8 \\
16.4 \\
15.0\end{array}$ & $\begin{array}{l}21.5 \\
24.5 \\
25.7 \\
26.6 \\
26.0\end{array}$ & $\begin{array}{l}18.2 \\
19.8 \\
19.8 \\
18.3 \\
17.8\end{array}$ & $\begin{array}{l}21.0 \\
22.6 \\
21.0 \\
21.3 \\
16.5\end{array}$ \\
\hline $\begin{array}{r}6 \\
7 \\
8 \\
9 \\
10\end{array}$ & $\begin{array}{r}13.1 \\
5.1 \\
7.4 \\
13.2 \\
14.4\end{array}$ & $\begin{array}{r}-6.4 \\
1.8 \\
7.4 \\
11.1 \\
13.5\end{array}$ & $\begin{array}{r}6.6 \\
7.9 \\
10.5 \\
9.4 \\
6.2\end{array}$ & $\begin{array}{r}2.6 \\
.9 \\
-.9 \\
-4.2 \\
1.3\end{array}$ & $\begin{array}{l}4.0 \\
6.1 \\
6.6 \\
6.8 \\
6.3\end{array}$ & $\begin{array}{r}-1.9 \\
.5 \\
1.9 \\
2.3 \\
2.1\end{array}$ & $\begin{array}{r}11.8 \\
3.6 \\
5.3 \\
8.8 \\
-3.5\end{array}$ & $\begin{array}{r}10.1 \\
15.6 \\
14.7 \\
-.5 \\
.0\end{array}$ & $\begin{array}{l}15.8 \\
18.1 \\
18.7 \\
19.4 \\
22.4\end{array}$ & $\begin{array}{l}24.9 \\
21.9 \\
16.7 \\
19.0 \\
21.0\end{array}$ & $\begin{array}{l}18.4 \\
19.2 \\
22.8 \\
22.8 \\
21.5\end{array}$ & $\begin{array}{r}9.6 \\
12.0 \\
14.5 \\
13.3 \\
9.7\end{array}$ \\
\hline $\begin{array}{l}11 \\
12 \\
13 \\
14 \\
15\end{array}$ & $\begin{array}{l}11.8 \\
14.1 \\
13.8 \\
16.0 \\
16.0\end{array}$ & $\begin{array}{r}11.9 \\
13.0 \\
11.1 \\
9.3 \\
5.5\end{array}$ & $\begin{array}{r}2.0 \\
-1.0 \\
-5.0 \\
-3.7 \\
-3.8\end{array}$ & $\begin{array}{l}5.2 \\
4.4 \\
2.9 \\
1.6 \\
-.8\end{array}$ & $\begin{array}{l}6.5 \\
5.7 \\
6.4 \\
8.7 \\
7.6\end{array}$ & $\begin{array}{r}-5.3 \\
1.9 \\
-2.0 \\
.2 \\
-4.4\end{array}$ & $\begin{array}{r}-4.3 \\
-2.3 \\
4.4 \\
6.6 \\
6.1\end{array}$ & $\begin{array}{r}3.5 \\
10.1 \\
9.2 \\
3.7 \\
11.1\end{array}$ & $\begin{array}{l}22.2 \\
21.2 \\
18.3 \\
16.3 \\
17.8\end{array}$ & $\begin{array}{l}21.9 \\
23.8 \\
22.9 \\
21.4 \\
20.0\end{array}$ & $\begin{array}{l}18.7 \\
18.9 \\
20.9 \\
20.4 \\
19.4\end{array}$ & $\begin{array}{l}10.8 \\
13.4 \\
16.6 \\
16.3 \\
16.8\end{array}$ \\
\hline $\begin{array}{l}16 \\
17 \\
18 \\
19 \\
20\end{array}$ & $\begin{array}{r}12.9 \\
11.8 \\
6.4 \\
4.3 \\
2.1\end{array}$ & $\begin{array}{r}7.7 \\
7.4 \\
4.7 \\
.1 \\
-3.3\end{array}$ & $\begin{array}{r}-8.0 \\
-4.1 \\
-1.3 \\
-7.7 \\
-17.2\end{array}$ & $\begin{array}{r}-2.2 \\
.2 \\
5.4 \\
3.1 \\
-6.2\end{array}$ & $\begin{array}{r}1.4 \\
-2.4 \\
-1.6 \\
5.8 \\
10.2\end{array}$ & $\begin{array}{r}-.4 \\
-.9 \\
.9 \\
-3.7 \\
-5.7\end{array}$ & $\begin{array}{l}4.4 \\
2.5 \\
5.7 \\
7.6 \\
4.3\end{array}$ & $\begin{array}{r}13.7 \\
6.5 \\
2.0 \\
5.8 \\
9.5\end{array}$ & $\begin{array}{l}19.9 \\
19.9 \\
19.2 \\
16.3 \\
16.5\end{array}$ & $\begin{array}{l}19.3 \\
20.7 \\
20.2 \\
18.7 \\
18.3\end{array}$ & $\begin{array}{l}21.5 \\
22.1 \\
21.1 \\
23.4 \\
22.4\end{array}$ & $\begin{array}{l}18.3 \\
19.8 \\
21.2 \\
20.2 \\
20.8\end{array}$ \\
\hline $\begin{array}{l}21 \\
22 \\
23 \\
24 \\
25\end{array}$ & $\begin{array}{r}9.4 \\
10.9 \\
13.5 \\
14.5 \\
14.9\end{array}$ & $\begin{array}{r}-1.4 \\
8.2 \\
10.6 \\
12.2 \\
4.6\end{array}$ & $\begin{array}{r}-17.2 \\
-15.4 \\
-10.1 \\
-2.7 \\
-3.1\end{array}$ & $\begin{array}{r}-6.3 \\
.3 \\
2.6 \\
.0 \\
1.4\end{array}$ & $\begin{array}{l}9.5 \\
7.7 \\
6.2 \\
4.6 \\
7.1\end{array}$ & $\begin{array}{r}-2.4 \\
1.6 \\
-1.1 \\
1.6 \\
-4.8\end{array}$ & $\begin{array}{r}4.7 \\
3.8 \\
7.8 \\
8.3 \\
-1.4\end{array}$ & $\begin{array}{r}5.5 \\
8.4 \\
16.1 \\
17.5 \\
15.9\end{array}$ & $\begin{array}{l}15.0 \\
17.8 \\
16.2 \\
14.2 \\
13.0\end{array}$ & $\begin{array}{l}21.1 \\
20.5 \\
22.8 \\
22.7 \\
22.3\end{array}$ & $\begin{array}{l}23.4 \\
23.7 \\
23.6 \\
23.2 \\
21.5\end{array}$ & $\begin{array}{l}19.5 \\
20.1 \\
19.5 \\
19.6 \\
19.7\end{array}$ \\
\hline $\begin{array}{l}26 \\
27 \\
28 \\
29 \\
30 \\
31\end{array}$ & $\begin{array}{r}16.1 \\
16.2 \\
13.6 \\
10.9 \\
11.2 \\
8.0\end{array}$ & $\begin{array}{r}-4.7 \\
-2.1 \\
.9 \\
6.4 \\
3.6 \\
----\end{array}$ & $\begin{array}{r}-5.3 \\
2.7 \\
.3 \\
-9.6 \\
-.4 \\
3.9\end{array}$ & $\begin{array}{r}1.6 \\
.8 \\
-1.1 \\
-2.0 \\
6.1 \\
4.9\end{array}$ & $\begin{array}{r}6.4 \\
3.3 \\
-3.2 \\
--- \\
--- \\
---\end{array}$ & $\begin{array}{r}-.6 \\
-4.1 \\
4.5 \\
4.9 \\
7.0 \\
7.0\end{array}$ & $\begin{array}{l}3.4 \\
1.5 \\
7.1 \\
5.7 \\
8.3 \\
---\end{array}$ & $\begin{array}{r}13.5 \\
10.8 \\
13.3 \\
14.2 \\
7.3 \\
5.1\end{array}$ & $\begin{array}{r}14.2 \\
7.2 \\
12.4 \\
11.6 \\
18.4 \\
\ldots-.\end{array}$ & $\begin{array}{l}22.8 \\
23.8 \\
25.8 \\
24.2 \\
24.5 \\
17.5\end{array}$ & $\begin{array}{l}19.0 \\
15.7 \\
19.0 \\
21.4 \\
22.8 \\
17.9\end{array}$ & $\begin{array}{r}18.1 \\
13.6 \\
13.9 \\
13.8 \\
16.7 \\
---\end{array}$ \\
\hline MAX & 17.0 & 13.5 & 10.5 & 6.3 & 10.2 & 7.0 & 12.7 & 17.5 & 22.4 & 26.6 & 23.7 & 22.6 \\
\hline
\end{tabular}

CAL YR 1990 MAX 24.4

WTR YR 1991 MAX 26.6 
3 SPRINGS BASIN

KAWICH PEAK NEAR WARM SPRINGS, NV

DAILY MINIMUM AIR TEMPERATURE, WATER YEAR OCTOBER 1988 TO SEPTEMBER 1989 DEGREES CELSIUS

\begin{tabular}{|c|c|c|c|c|c|c|c|c|c|c|c|c|}
\hline DAY & OCT & NOV & DEC & JAN & FEB & MAR & $\mathrm{APR}$ & MAY & JUN & JUL & AUG & SEP \\
\hline $\begin{array}{l}1 \\
2 \\
3 \\
4 \\
5\end{array}$ & $\begin{array}{r}9.1 \\
9.0 \\
10.2 \\
7.4 \\
5.4\end{array}$ & $\begin{array}{l}4.5 \\
3.6 \\
4.9 \\
3.5 \\
5.8\end{array}$ & $\begin{array}{l}--- \\
--- \\
--- \\
--- \\
---\end{array}$ & $\begin{array}{r}-11.6 \\
-9.0 \\
-4.1 \\
-4.8 \\
-13.5\end{array}$ & $\begin{array}{r}-5.7 \\
-7.7 \\
-10.0 \\
-19.0 \\
-25.3\end{array}$ & $\begin{array}{r}-1.9 \\
-7.4 \\
-15.2 \\
-14.0 \\
-3.6\end{array}$ & $\begin{array}{r}-1.3 \\
.2 \\
-.5 \\
.7 \\
1.0\end{array}$ & $\begin{array}{l}3.5 \\
2.0 \\
5.8 \\
6.4 \\
6.4\end{array}$ & $\begin{array}{l}--- \\
4.8 \\
2.1 \\
2.3 \\
3.1\end{array}$ & $\begin{array}{r}9.2 \\
12.1 \\
13.6 \\
11.0 \\
15.2\end{array}$ & $\begin{array}{l}12.7 \\
11.1 \\
11.7 \\
11.6 \\
12.0\end{array}$ & $\begin{array}{r}9.3 \\
10.0 \\
8.5 \\
8.5 \\
12.6\end{array}$ \\
\hline $\begin{array}{r}6 \\
7 \\
8 \\
9 \\
10\end{array}$ & $\begin{array}{l}4.3 \\
2.6 \\
3.9 \\
7.4 \\
4.9\end{array}$ & $\begin{array}{r}3.5 \\
1.2 \\
-2.8 \\
-3.4 \\
-2.5\end{array}$ & $\begin{array}{r}--- \\
--- \\
-5.9 \\
-2.1\end{array}$ & $\begin{array}{r}-16.5 \\
-16.5 \\
-16.0 \\
-7.0 \\
-14.4\end{array}$ & $\begin{array}{r}-25.5 \\
-22.0 \\
-16.4 \\
-5.5 \\
-10.8\end{array}$ & $\begin{array}{r}1.3 \\
.6 \\
2.0 \\
3.0 \\
2.9\end{array}$ & $\begin{array}{l}8.1 \\
6.2 \\
4.3 \\
6.5 \\
8.1\end{array}$ & $\begin{array}{r}8.5 \\
10.4 \\
8.6 \\
3.8 \\
-1.1\end{array}$ & $\begin{array}{l}2.7 \\
6.0 \\
5.7 \\
4.7 \\
3.9\end{array}$ & $\begin{array}{l}16.8 \\
18.2 \\
17.9 \\
14.7 \\
10.5\end{array}$ & $\begin{array}{r}13.1 \\
9.7 \\
8.8 \\
8.5 \\
8.1\end{array}$ & $\begin{array}{l}8.7 \\
4.8 \\
4.0 \\
6.2 \\
8.2\end{array}$ \\
\hline $\begin{array}{l}11 \\
12 \\
13 \\
14 \\
15\end{array}$ & $\begin{array}{l}3.1 \\
1.6 \\
5.1 \\
2.2 \\
2.9\end{array}$ & $\begin{array}{l}-4.3 \\
-1.2 \\
-1.9 \\
-9.7 \\
-9.6\end{array}$ & $\begin{array}{r}1.0 \\
1.8 \\
.7 \\
-14.5 \\
-15.5\end{array}$ & $\begin{array}{r}-15.5 \\
-14.9 \\
-8.1 \\
-11.5 \\
-12.2\end{array}$ & $\begin{array}{r}-11.8 \\
-13.6 \\
-14.8 \\
-15.8 \\
-9.2\end{array}$ & $\begin{array}{r}1.3 \\
1.6 \\
-3.9 \\
-5.1 \\
-5.5\end{array}$ & $\begin{array}{r}-.3 \\
.3 \\
.8 \\
3.5 \\
7.0\end{array}$ & $\begin{array}{l}-3.7 \\
-4.2 \\
-3.1 \\
-2.5 \\
-1.7\end{array}$ & $\begin{array}{r}5.4 \\
6.4 \\
9.4 \\
13.3 \\
13.6\end{array}$ & $\begin{array}{r}11.4 \\
9.7 \\
9.0 \\
11.9 \\
13.9\end{array}$ & $\begin{array}{r}9.3 \\
11.1 \\
11.5 \\
13.4 \\
13.6\end{array}$ & $\begin{array}{l}6.1 \\
4.3 \\
3.4 \\
8.2 \\
6.9\end{array}$ \\
\hline $\begin{array}{l}16 \\
17 \\
18 \\
19 \\
20\end{array}$ & $\begin{array}{l}7.2 \\
6.9 \\
6.8 \\
6.6 \\
5.3\end{array}$ & $\begin{array}{r}-9.6 \\
-10.3 \\
-12.2 \\
-7.7 \\
-5.2\end{array}$ & $\begin{array}{l}-9.9 \\
-5.7 \\
-7.7 \\
-8.5 \\
-8.2\end{array}$ & $\begin{array}{l}-8.3 \\
-3.6 \\
-3.7 \\
-2.0 \\
-4.0\end{array}$ & $\begin{array}{l}-6.4 \\
-5.7 \\
-2.5 \\
-5.6 \\
-6.9\end{array}$ & $\begin{array}{r}-7.5 \\
-7.6 \\
.0 \\
-7.0 \\
-9.9\end{array}$ & $\begin{array}{l}2.8 \\
5.7 \\
6.0 \\
8.5 \\
8.7\end{array}$ & $\begin{array}{r}.2 \\
3.7 \\
2.5 \\
-2.5 \\
6.4\end{array}$ & $\begin{array}{r}10.2 \\
7.2 \\
12.3 \\
13.7 \\
3.7\end{array}$ & $\begin{array}{r}9.6 \\
10.1 \\
14.3 \\
14.5 \\
12.0\end{array}$ & $\begin{array}{r}13.4 \\
12.0 \\
9.0 \\
10.7 \\
7.6\end{array}$ & $\begin{array}{r}7.1 \\
2.9 \\
-1.2 \\
-.4 \\
3.1\end{array}$ \\
\hline $\begin{array}{l}21 \\
22 \\
23 \\
24 \\
25\end{array}$ & $\begin{array}{l}5.4 \\
6.9 \\
5.3 \\
6.4 \\
5.2\end{array}$ & $\begin{array}{r}-4.1 \\
-2.1 \\
-10.6 \\
-11.8 \\
-10.6\end{array}$ & $\begin{array}{l}-13.1 \\
-12.0 \\
-14.6 \\
-12.4 \\
-16.9\end{array}$ & $\begin{array}{r}-3.2 \\
-4.7 \\
-9.0 \\
-10.0 \\
-11.2\end{array}$ & $\begin{array}{r}-2.7 \\
-1.0 \\
.5 \\
2.5 \\
1.8\end{array}$ & $\begin{array}{r}-3.4 \\
.3 \\
-.2 \\
-2.2 \\
-7.8\end{array}$ & $\begin{array}{r}3.3 \\
-.4 \\
-2.8 \\
-10.0 \\
-9.1\end{array}$ & $\begin{array}{r}5.4 \\
7.2 \\
.5 \\
--- \\
---\end{array}$ & $\begin{array}{l}1.3 \\
6.2 \\
6.0 \\
3.4 \\
3.0\end{array}$ & $\begin{array}{l}11.6 \\
11.9 \\
12.0 \\
12.3 \\
11.1\end{array}$ & $\begin{array}{r}6.1 \\
10.1 \\
4.4 \\
1.5 \\
3.8\end{array}$ & $\begin{array}{r}4.3 \\
5.5 \\
6.5 \\
11.0 \\
10.2\end{array}$ \\
\hline $\begin{array}{l}26 \\
27 \\
28 \\
29 \\
30 \\
31\end{array}$ & $\begin{array}{r}5.4 \\
4.4 \\
4.8 \\
3.9 \\
.7 \\
3.0\end{array}$ & $\begin{array}{r}-10.2 \\
-5.1 \\
-4.9 \\
--- \\
--- \\
---\end{array}$ & $\begin{array}{r}-17.8 \\
-17.6 \\
-18.2 \\
-17.2 \\
-9.4 \\
-11.8\end{array}$ & $\begin{array}{r}-9.1 \\
-8.1 \\
-4.5 \\
-3.2 \\
1.3 \\
-2.9\end{array}$ & $\begin{array}{r}.1 \\
-2.9 \\
-3.7 \\
--- \\
---\end{array}$ & $\begin{array}{r}-7.5 \\
-4.7 \\
-1.8 \\
-1.2 \\
-.8 \\
1.0\end{array}$ & $\begin{array}{r}-5.2 \\
-6.9 \\
-6.9 \\
-4.1 \\
2.5 \\
---\end{array}$ & $\begin{array}{l}--- \\
--- \\
--- \\
--- \\
--- \\
---\end{array}$ & $\begin{array}{l}7.6 \\
7.8 \\
5.1 \\
8.3 \\
9.5 \\
---\end{array}$ & $\begin{array}{l}15.7 \\
14.2 \\
12.4 \\
13.6 \\
15.0 \\
14.5\end{array}$ & $\begin{array}{r}9.2 \\
10.0 \\
7.9 \\
12.1 \\
8.7 \\
5.3\end{array}$ & $\begin{array}{r}10.2 \\
10.1 \\
9.8 \\
7.7 \\
3.0 \\
-.-\end{array}$ \\
\hline MIN & .7 & -- & -- & -16.5 & -25.5 & -15.2 & -10.0 & --- & --- & 9.0 & 1.5 & -1.2 \\
\hline
\end{tabular}


3 SPRINGS BASIN

KAWICH PEAK NEAR WARM SPRINGS, NV

DAILY MINIMUM AIR TEMPERATURE, WATER YEAR OCTOBER 1989 TO SEPTEMBER 1990 DEGREES CELSIUS

\begin{tabular}{|c|c|c|c|c|c|c|c|c|c|c|c|c|}
\hline DAY & OCT & NOV & DEC & JAN & FEB & MAR & APR & MAY & JUN & JUL & AUG & SEP \\
\hline $\begin{array}{l}1 \\
2 \\
3 \\
4 \\
5\end{array}$ & $\begin{array}{r}.1 \\
-.6 \\
-2.5 \\
-.9 \\
-.1\end{array}$ & $\begin{array}{r}-6.7 \\
-3.6 \\
.3 \\
3.1 \\
1.2\end{array}$ & $\begin{array}{r}-4.6 \\
-1.6 \\
-.6 \\
1.7 \\
-.1\end{array}$ & $\begin{array}{r}-7.7 \\
-14.5 \\
-14.1 \\
-8.1 \\
-4.5\end{array}$ & $\begin{array}{r}-11.4 \\
-12.9 \\
-9.7 \\
-10.0 \\
-11.1\end{array}$ & $\begin{array}{r}-.1 \\
-1.4 \\
-2.4 \\
-5.9 \\
-7.3\end{array}$ & $\begin{array}{r}-1.8 \\
-.8 \\
-1.7 \\
-1.4 \\
-2.2\end{array}$ & $\begin{array}{r}-6.1 \\
-.4 \\
-.1 \\
2.9 \\
6.1\end{array}$ & $\begin{array}{r}-4.1 \\
4.6 \\
10.0 \\
10.2 \\
10.2\end{array}$ & $\begin{array}{r}16.5 \\
13.1 \\
8.9 \\
10.5 \\
12.1\end{array}$ & $\begin{array}{l}12.2 \\
11.0 \\
13.4 \\
15.2 \\
13.4\end{array}$ & $\begin{array}{r}12.2 \\
10.7 \\
11.7 \\
9.7 \\
9.9\end{array}$ \\
\hline $\begin{array}{r}6 \\
7 \\
8 \\
9 \\
10\end{array}$ & $\begin{array}{l}4.1 \\
1.2 \\
3.3 \\
4.9 \\
7.0\end{array}$ & $\begin{array}{r}-1.2 \\
-2.2 \\
-2.7 \\
1.7 \\
4.5\end{array}$ & $\begin{array}{r}-2.6 \\
.9 \\
1.4 \\
-1.7 \\
-13.8\end{array}$ & $\begin{array}{r}-5.2 \\
-1.0 \\
1.7 \\
4.3 \\
4.1\end{array}$ & $\begin{array}{r}-7.1 \\
-17.1 \\
-15.7 \\
-7.6 \\
-2.4\end{array}$ & $\begin{array}{l}-8.5 \\
-4.4 \\
-2.2 \\
-2.0 \\
-5.2\end{array}$ & $\begin{array}{r}.2 \\
-1.9 \\
-3.3 \\
-1.7 \\
-2.0\end{array}$ & $\begin{array}{r}8.9 \\
6.1 \\
.1 \\
-1.7 \\
2.0\end{array}$ & $\begin{array}{l}8.3 \\
9.3 \\
8.7 \\
6.1 \\
4.3\end{array}$ & $\begin{array}{l}12.6 \\
11.9 \\
11.5 \\
11.3 \\
12.9\end{array}$ & $\begin{array}{l}15.1 \\
13.4 \\
16.0 \\
13.3 \\
10.0\end{array}$ & $\begin{array}{r}9.4 \\
9.8 \\
11.2 \\
12.3 \\
13.4\end{array}$ \\
\hline $\begin{array}{l}11 \\
12 \\
13 \\
14 \\
15\end{array}$ & $\begin{array}{l}8.4 \\
7.1 \\
8.9 \\
6.4 \\
2.1\end{array}$ & $\begin{array}{r}3.8 \\
4.3 \\
2.5 \\
-.4 \\
.7\end{array}$ & $\begin{array}{r}-14.5 \\
-3.1 \\
-4.0 \\
-.1 \\
-2.9\end{array}$ & $\begin{array}{r}2.0 \\
-2.1 \\
-3.8 \\
-7.4 \\
-8.9\end{array}$ & $\begin{array}{r}-2.8 \\
-4.2 \\
-19.0 \\
-20.5 \\
-19.5\end{array}$ & $\begin{array}{r}-12.1 \\
-14.3 \\
-14.5 \\
-9.5 \\
-7.4\end{array}$ & $\begin{array}{l}3.3 \\
3.8 \\
5.3 \\
8.4 \\
5.5\end{array}$ & $\begin{array}{r}1.3 \\
-.7 \\
.7 \\
.8 \\
-1.4\end{array}$ & $\begin{array}{r}1.8 \\
3.7 \\
1.4 \\
.8 \\
2.2\end{array}$ & $\begin{array}{l}12.5 \\
14.3 \\
14.2 \\
11.2 \\
10.2\end{array}$ & $\begin{array}{r}9.2 \\
11.0 \\
11.1 \\
12.0 \\
8.1\end{array}$ & $\begin{array}{l}14.8 \\
13.9 \\
12.0 \\
13.2 \\
10.7\end{array}$ \\
\hline $\begin{array}{l}16 \\
17 \\
18 \\
19 \\
20\end{array}$ & $\begin{array}{l}.1 \\
2.5 \\
2.8 \\
3.6 \\
3.8\end{array}$ & $\begin{array}{r}1.3 \\
-2.1 \\
-3.6 \\
3.5 \\
4.5\end{array}$ & $\begin{array}{l}-5.0 \\
-4.2 \\
-7.6 \\
-6.4 \\
-3.7\end{array}$ & $\begin{array}{r}-8.7 \\
-9.2 \\
-12.6 \\
-12.0 \\
-9.3\end{array}$ & $\begin{array}{r}-14.7 \\
-9.3 \\
-12.8 \\
-12.3 \\
-7.0\end{array}$ & $\begin{array}{r}-4.7 \\
.7 \\
.0 \\
2.4 \\
4.5\end{array}$ & $\begin{array}{r}-1.3 \\
-2.6 \\
-1.6 \\
.9 \\
-.6\end{array}$ & $\begin{array}{r}-1.0 \\
.4 \\
.2 \\
1.8 \\
.4\end{array}$ & $\begin{array}{l}1.0 \\
7.2 \\
5.8 \\
6.0 \\
9.4\end{array}$ & $\begin{array}{l}10.3 \\
11.5 \\
12.0 \\
11.5 \\
12.6\end{array}$ & $\begin{array}{r}8.8 \\
9.7 \\
5.4 \\
1.2 \\
.4\end{array}$ & $\begin{array}{l}7.1 \\
6.1 \\
4.3 \\
3.7 \\
4.9\end{array}$ \\
\hline $\begin{array}{l}21 \\
22 \\
23 \\
24 \\
25\end{array}$ & $\begin{array}{r}1.0 \\
-.3 \\
1.5 \\
-1.6 \\
-7.3\end{array}$ & $\begin{array}{r}2.7 \\
.0 \\
.4 \\
-3.0 \\
-3.3\end{array}$ & $\begin{array}{r}-4.1 \\
-.5 \\
-.7 \\
-.7 \\
-2.0\end{array}$ & $\begin{array}{l}-8.6 \\
-3.3 \\
-4.0 \\
-7.7 \\
-5.5\end{array}$ & $\begin{array}{l}-4.3 \\
-2.5 \\
-1.7 \\
-1.1 \\
-1.1\end{array}$ & $\begin{array}{r}3.7 \\
1.8 \\
1.3 \\
-1.0 \\
.3\end{array}$ & $\begin{array}{r}.0 \\
-.6 \\
-3.4 \\
-4.7 \\
-3.1\end{array}$ & $\begin{array}{r}5.1 \\
4.7 \\
-5.0 \\
-6.3 \\
-1.7\end{array}$ & $\begin{array}{l}10.1 \\
15.0 \\
13.5 \\
13.4 \\
13.5\end{array}$ & $\begin{array}{l}13.4 \\
13.1 \\
14.1 \\
11.5 \\
10.4\end{array}$ & $\begin{array}{l}6.3 \\
5.7 \\
7.8 \\
9.5 \\
5.0\end{array}$ & $\begin{array}{l}5.6 \\
5.2 \\
3.9 \\
3.5 \\
5.9\end{array}$ \\
\hline $\begin{array}{l}26 \\
27 \\
28 \\
29 \\
30 \\
31\end{array}$ & $\begin{array}{r}-9.1 \\
-5.5 \\
-10.5 \\
-10.8 \\
-6.4 \\
-4.6\end{array}$ & $\begin{array}{r}-10.6 \\
-11.6 \\
-10.6 \\
-6.7 \\
-6.2 \\
---\end{array}$ & $\begin{array}{l}-2.2 \\
-3.1 \\
-7.3 \\
-9.3 \\
-4.4 \\
-2.9\end{array}$ & $\begin{array}{r}-12.6 \\
-14.3 \\
-7.2 \\
-6.0 \\
-9.5 \\
-10.3\end{array}$ & $\begin{array}{r}-2.2 \\
-2.7 \\
-2.9 \\
--- \\
--- \\
---\end{array}$ & $\begin{array}{r}2.0 \\
-3.8 \\
-5.1 \\
-6.4 \\
-5.3 \\
-1.0\end{array}$ & $\begin{array}{r}2.2 \\
2.9 \\
-4.8 \\
-7.0 \\
-7.9 \\
---\end{array}$ & $\begin{array}{r}3.5 \\
.6 \\
-1.1 \\
.4 \\
-.4 \\
-.8\end{array}$ & $\begin{array}{r}13.7 \\
11.9 \\
10.8 \\
15.1 \\
15.5 \\
---\end{array}$ & $\begin{array}{r}7.7 \\
10.7 \\
12.6 \\
13.9 \\
15.1 \\
14.8\end{array}$ & $\begin{array}{r}4.9 \\
8.2 \\
10.8 \\
13.5 \\
12.4 \\
11.3\end{array}$ & $\begin{array}{l}6.0 \\
2.9 \\
3.4 \\
3.2 \\
6.0 \\
---\end{array}$ \\
\hline MIN & -10.8 & -11.6 & -14.5 & -14.5 & -20.5 & -14.5 & -7.9 & -6.3 & -4.1 & 7.7 & .4 & 2.9 \\
\hline
\end{tabular}

WTR YR 1990 MIN -20.5 
3 SPRINGS BASIN

KAWICH PEAK NEAR WARM SPRINGS, NV

DAILY MINIMUM AIR TEMPERATURE, WATER YEAR OCTOBER 1990 TO SEPTEMBER 1991 DEGREES CELSIUS

\begin{tabular}{|c|c|c|c|c|c|c|c|c|c|c|c|c|}
\hline DAY & $\mathrm{OCT}$ & NOV & DEC & JAN & FEB & MAR & APR & MAY & JUN & JUL & AUG & SEP \\
\hline $\begin{array}{l}1 \\
2 \\
3 \\
4 \\
5\end{array}$ & $\begin{array}{l}5.4 \\
7.0 \\
5.2 \\
5.0 \\
7.6\end{array}$ & $\begin{array}{r}-9.4 \\
-12.3 \\
-9.2 \\
-1.5 \\
-8.7\end{array}$ & $\begin{array}{l}-6.7 \\
-7.2 \\
-6.5 \\
-3.1 \\
-4.0\end{array}$ & $\begin{array}{l}-5.2 \\
-4.9 \\
-4.2 \\
-6.1 \\
-6.6\end{array}$ & $\begin{array}{l}-2.2 \\
-3.2 \\
-3.5 \\
-2.3 \\
-2.7\end{array}$ & $\begin{array}{r}-6.4 \\
-8.8 \\
-4.4 \\
-1.1 \\
-10.5\end{array}$ & $\begin{array}{r}-2.5 \\
-5.9 \\
-3.4 \\
.3 \\
5.4\end{array}$ & $\begin{array}{l}-6.7 \\
-6.4 \\
-7.2 \\
-3.3 \\
-1.8\end{array}$ & $\begin{array}{l}.7 \\
5.3 \\
6.1 \\
8.6 \\
4.4\end{array}$ & $\begin{array}{r}8.5 \\
11.5 \\
14.4 \\
14.3 \\
14.6\end{array}$ & $\begin{array}{r}9.8 \\
8.5 \\
8.0 \\
11.3 \\
10.6\end{array}$ & $\begin{array}{r}10.3 \\
13.0 \\
11.4 \\
9.9 \\
9.5\end{array}$ \\
\hline $\begin{array}{r}6 \\
7 \\
8 \\
9 \\
10\end{array}$ & $\begin{array}{r}3.5 \\
-3.8 \\
-4.8 \\
-.1 \\
4.8\end{array}$ & $\begin{array}{r}-11.4 \\
-11.0 \\
.1 \\
-.3 \\
1.4\end{array}$ & $\begin{array}{l}-4.6 \\
-3.6 \\
-1.7 \\
-1.1 \\
-1.1\end{array}$ & $\begin{array}{r}-6.0 \\
-6.2 \\
-9.3 \\
-10.2 \\
-6.0\end{array}$ & $\begin{array}{l}-3.8 \\
-3.4 \\
-3.7 \\
-3.3 \\
-4.3\end{array}$ & $\begin{array}{r}-12.6 \\
-12.4 \\
-4.7 \\
-5.2 \\
-5.9\end{array}$ & $\begin{array}{r}.7 \\
-3.8 \\
-7.7 \\
-2.8 \\
-13.1\end{array}$ & $\begin{array}{r}4.7 \\
3.1 \\
.1 \\
-7.4 \\
-8.3\end{array}$ & $\begin{array}{l}6.2 \\
6.9 \\
5.5 \\
7.2 \\
8.4\end{array}$ & $\begin{array}{r}14.6 \\
13.7 \\
8.8 \\
9.5 \\
9.2\end{array}$ & $\begin{array}{r}7.8 \\
10.0 \\
10.3 \\
12.4 \\
13.2\end{array}$ & $\begin{array}{r}6.9 \\
5.2 \\
8.0 \\
3.0 \\
.9\end{array}$ \\
\hline $\begin{array}{l}11 \\
12 \\
13 \\
14 \\
15\end{array}$ & $\begin{array}{l}1.2 \\
1.0 \\
6.6 \\
7.1 \\
7.2\end{array}$ & $\begin{array}{r}3.4 \\
2.6 \\
4.3 \\
3.0 \\
.8\end{array}$ & $\begin{array}{r}-2.3 \\
-5.5 \\
-11.5 \\
-15.2 \\
-10.7\end{array}$ & $\begin{array}{l}-3.4 \\
-3.6 \\
-2.7 \\
-4.1 \\
-8.0\end{array}$ & $\begin{array}{r}-3.5 \\
.4 \\
-.8 \\
-3.1 \\
-.1\end{array}$ & $\begin{array}{r}-12.0 \\
-8.3 \\
-8.5 \\
-8.6 \\
-9.4\end{array}$ & $\begin{array}{r}-14.0 \\
-8.8 \\
-7.5 \\
-2.7 \\
-3.2\end{array}$ & $\begin{array}{l}-8.4 \\
-3.7 \\
-3.1 \\
-3.6 \\
-1.7\end{array}$ & $\begin{array}{r}10.2 \\
11.4 \\
8.8 \\
8.1 \\
4.4\end{array}$ & $\begin{array}{l}10.2 \\
12.3 \\
13.8 \\
13.6 \\
12.4\end{array}$ & $\begin{array}{r}8.9 \\
10.4 \\
8.8 \\
11.0 \\
10.6\end{array}$ & $\begin{array}{l}1.1 \\
4.6 \\
3.5 \\
7.1 \\
6.0\end{array}$ \\
\hline $\begin{array}{l}16 \\
17 \\
18 \\
19 \\
20\end{array}$ & $\begin{array}{r}1.0 \\
-.4 \\
3.8 \\
-6.7 \\
-7.4\end{array}$ & $\begin{array}{r}.4 \\
-.3 \\
-1.3 \\
-4.0 \\
-9.0\end{array}$ & $\begin{array}{r}-12.9 \\
-12.2 \\
-7.6 \\
-18.9 \\
-23.5\end{array}$ & $\begin{array}{r}-10.2 \\
-5.6 \\
-3.6 \\
-7.0 \\
-11.4\end{array}$ & $\begin{array}{r}-3.4 \\
-8.5 \\
-10.0 \\
-3.9 \\
.7\end{array}$ & $\begin{array}{l}-9.5 \\
-7.9 \\
-5.9 \\
-8.2 \\
-9.6\end{array}$ & $\begin{array}{l}-6.1 \\
-5.5 \\
-5.2 \\
-1.3 \\
-1.4\end{array}$ & $\begin{array}{l}.1 \\
-4.3 \\
-6.3 \\
-4.1 \\
-3.2\end{array}$ & $\begin{array}{r}9.5 \\
10.4 \\
10.4 \\
6.0 \\
6.2\end{array}$ & $\begin{array}{r}11.0 \\
12.0 \\
12.6 \\
10.2 \\
8.2\end{array}$ & $\begin{array}{r}8.9 \\
10.1 \\
11.3 \\
12.0 \\
9.3\end{array}$ & $\begin{array}{r}5.5 \\
7.0 \\
8.3 \\
10.2 \\
8.7\end{array}$ \\
\hline $\begin{array}{l}21 \\
22 \\
23 \\
24 \\
25\end{array}$ & $\begin{array}{r}-2.0 \\
3.3 \\
1.1 \\
3.3 \\
4.4\end{array}$ & $\begin{array}{r}-8.7 \\
-4.1 \\
4.2 \\
.2 \\
-3.4\end{array}$ & $\begin{array}{l}-23.5 \\
-23.1 \\
-18.2 \\
-15.7 \\
-11.0\end{array}$ & $\begin{array}{r}-14.2 \\
-8.0 \\
-7.4 \\
-6.5 \\
-7.9\end{array}$ & $\begin{array}{r}-1.2 \\
.3 \\
-3.8 \\
-4.3 \\
-4.5\end{array}$ & $\begin{array}{r}-9.5 \\
-10.8 \\
-6.7 \\
-6.4 \\
-9.8\end{array}$ & $\begin{array}{l}-3.6 \\
-3.8 \\
-3.7 \\
-1.8 \\
-6.8\end{array}$ & $\begin{array}{r}-1.2 \\
.5 \\
4.5 \\
6.7 \\
5.3\end{array}$ & $\begin{array}{l}6.9 \\
3.8 \\
7.1 \\
4.7 \\
4.0\end{array}$ & $\begin{array}{r}9.4 \\
8.6 \\
9.6 \\
11.0 \\
12.9\end{array}$ & $\begin{array}{l}10.2 \\
14.0 \\
15.1 \\
14.7 \\
13.6\end{array}$ & $\begin{array}{l}9.0 \\
8.2 \\
8.9 \\
8.4 \\
7.8\end{array}$ \\
\hline $\begin{array}{l}26 \\
27 \\
28 \\
29 \\
30 \\
31\end{array}$ & $\begin{array}{l}4.9 \\
5.2 \\
6.7 \\
5.2 \\
4.1 \\
-.3\end{array}$ & $\begin{array}{r}-12.4 \\
-10.0 \\
-7.7 \\
-4.7 \\
-6.5 \\
---\end{array}$ & $\begin{array}{r}-11.4 \\
-6.9 \\
-9.3 \\
-15.9 \\
-10.5 \\
-5.4\end{array}$ & $\begin{array}{r}-8.7 \\
-6.0 \\
-12.9 \\
-14.8 \\
-5.8 \\
-3.3\end{array}$ & $\begin{array}{r}-5.5 \\
-4.4 \\
-4.8 \\
--- \\
--- \\
---\end{array}$ & $\begin{array}{r}-10.6 \\
-8.1 \\
-7.2 \\
-4.3 \\
-5.8 \\
-1.6\end{array}$ & $\begin{array}{l}-8.2 \\
-6.9 \\
-6.3 \\
-3.9 \\
-5.4 \\
---\end{array}$ & $\begin{array}{r}3.2 \\
.4 \\
1.4 \\
3.8 \\
-4.0 \\
-3.7\end{array}$ & $\begin{array}{l}1.8 \\
4.1 \\
3.0 \\
2.7 \\
2.1 \\
---\end{array}$ & $\begin{array}{l}13.4 \\
14.8 \\
12.7 \\
13.0 \\
14.1 \\
10.3\end{array}$ & $\begin{array}{r}11.9 \\
6.1 \\
5.3 \\
9.2 \\
12.4 \\
9.5\end{array}$ & $\begin{array}{l}8.5 \\
6.5 \\
5.0 \\
5.3 \\
4.5 \\
-.-\end{array}$ \\
\hline MIN & -7.4 & -12.4 & -23.5 & -14.8 & -10.0 & -12.6 & -14.0 & -8.4 & .7 & 8.2 & 5.3 & .9 \\
\hline
\end{tabular}

CAL YR 1990 MIN -23.5

WTR YR 1991 MIN -23.5 
3 SPRINGS BASIN

KAWICH PEAK NEAR WARM SPRINGS, NV

DAILY MEAN SOIL TEMPERATURE, WATER YEAR OCTOBER 1988 TO SEPTEMBER 1989 DEGREES CEISIUS

\begin{tabular}{|c|c|c|c|c|c|c|c|c|c|c|c|c|}
\hline DAY & OCT & NOV & $\mathrm{DEC}$ & JAN & FEB & MAR & APR & MAY & JUN & JUL & AUG & SEP \\
\hline $\begin{array}{l}1 \\
2 \\
3 \\
4 \\
5\end{array}$ & $\begin{array}{l}9.6 \\
10.0 \\
10.2 \\
10.2 \\
10.1\end{array}$ & $\begin{array}{l}5.8 \\
5.6 \\
5.8 \\
5.8 \\
5.6\end{array}$ & $\begin{array}{l}e-1.2 \\
e-1.2 \\
e-1.3 \\
e-1.4 \\
e-1.4\end{array}$ & $\begin{array}{l}-1.2 \\
-1.2 \\
-1.3 \\
-1.3 \\
-1.2\end{array}$ & $\begin{array}{r}-1.0 \\
-.8 \\
-.7 \\
-.8 \\
-1.0\end{array}$ & $\begin{array}{r}-.1 \\
.0 \\
.0 \\
-.1 \\
-.2\end{array}$ & $\begin{array}{l}2.9 \\
3.1 \\
3.1 \\
3.6 \\
4.3\end{array}$ & $\begin{array}{l}6.1 \\
7.0 \\
8.1 \\
9.1 \\
9.3\end{array}$ & $\begin{array}{r}\text { e } 7.5 \\
9.3 \\
9.1 \\
7.8 \\
6.9\end{array}$ & $\begin{array}{l}13.5 \\
14.2 \\
14.8 \\
15.3 \\
15.6\end{array}$ & $\begin{array}{l}17.2 \\
16.8 \\
16.6 \\
16.6 \\
16.6\end{array}$ & $\begin{array}{l}13.7 \\
13.9 \\
14.2 \\
13.6 \\
14.1\end{array}$ \\
\hline $\begin{array}{r}6 \\
7 \\
8 \\
9 \\
10\end{array}$ & $\begin{array}{l}9.2 \\
7.8 \\
7.3 \\
7.9 \\
7.7\end{array}$ & $\begin{array}{l}5.8 \\
5.0 \\
4.4 \\
3.6 \\
3.5\end{array}$ & $\begin{array}{r}e-1.3 \\
e-1.0 \\
e-1.1 \\
-1.1 \\
-1.1\end{array}$ & $\begin{array}{l}-1.0 \\
-1.0 \\
-1.0 \\
-1.1 \\
-1.1\end{array}$ & $\begin{array}{l}-1.1 \\
-1.2 \\
-1.4 \\
-1.6 \\
-1.7\end{array}$ & $\begin{array}{r}-.1 \\
.0 \\
.0 \\
.0 \\
.1\end{array}$ & $\begin{array}{l}5.0 \\
5.8 \\
6.6 \\
7.1 \\
7.5\end{array}$ & $\begin{array}{r}9.6 \\
10.1 \\
10.6 \\
10.0 \\
8.3\end{array}$ & $\begin{array}{l}7.9 \\
9.2 \\
9.1 \\
8.9 \\
9.3\end{array}$ & $\begin{array}{l}16.1 \\
16.4 \\
16.6 \\
16.6 \\
15.9\end{array}$ & $\begin{array}{l}16.8 \\
16.7 \\
15.8 \\
14.5 \\
14.3\end{array}$ & $\begin{array}{l}14.0 \\
13.7 \\
13.0 \\
12.5 \\
12.4\end{array}$ \\
\hline $\begin{array}{l}11 \\
12 \\
13 \\
14 \\
15\end{array}$ & $\begin{array}{l}7.1 \\
7.5 \\
7.4 \\
7.6 \\
7.1\end{array}$ & $\begin{array}{l}2.8 \\
2.4 \\
2.5 \\
2.7 \\
2.4\end{array}$ & $\begin{array}{r}-1.0 \\
-.8 \\
-.6 \\
-.4 \\
-.8\end{array}$ & $\begin{array}{l}-1.0 \\
-1.0 \\
-1.1 \\
-1.2 \\
-1.3\end{array}$ & $\begin{array}{l}-1.7 \\
-1.6 \\
-1.6 \\
-1.6 \\
-1.7\end{array}$ & $\begin{array}{l}.1 \\
.1 \\
.1 \\
.1 \\
.1\end{array}$ & $\begin{array}{l}7.6 \\
7.0 \\
6.8 \\
6.9 \\
7.9\end{array}$ & $\begin{array}{l}5.9 \\
4.5 \\
3.5 \\
3.0 \\
2.6\end{array}$ & $\begin{array}{l}10.1 \\
10.3 \\
10.9 \\
12.1 \\
13.6\end{array}$ & $\begin{array}{l}15.4 \\
14.9 \\
14.7 \\
14.7 \\
15.4\end{array}$ & $\begin{array}{l}14.5 \\
15.0 \\
15.3 \\
15.6 \\
15.9\end{array}$ & $\begin{array}{r}12.2 \\
11.9 \\
9.8 \\
10.1 \\
10.5\end{array}$ \\
\hline $\begin{array}{l}16 \\
17 \\
18 \\
19 \\
20\end{array}$ & $\begin{array}{l}7.5 \\
7.5 \\
7.3 \\
8.0 \\
7.4\end{array}$ & $\begin{array}{l}2.1 \\
1.9 \\
1.7 \\
1.3 \\
1.0\end{array}$ & $\begin{array}{r}-1.2 \\
-1.1 \\
-.9 \\
-.9 \\
-1.0\end{array}$ & $\begin{array}{l}-1.4 \\
-1.5 \\
-1.4 \\
-1.4 \\
-1.3\end{array}$ & $\begin{array}{l}-1.7 \\
-1.7 \\
-1.7 \\
-1.6 \\
-1.5\end{array}$ & $\begin{array}{l}.1 \\
.1 \\
.1 \\
.1 \\
.0\end{array}$ & $\begin{array}{l}8.1 \\
8.9 \\
9.3 \\
9.4 \\
9.6\end{array}$ & $\begin{array}{l}2.2 \\
2.2 \\
4.7 \\
6.3 \\
7.5\end{array}$ & $\begin{array}{l}13.9 \\
13.8 \\
14.3 \\
14.9 \\
14.7\end{array}$ & $\begin{array}{l}15.5 \\
15.8 \\
16.3 \\
16.4 \\
16.8\end{array}$ & $\begin{array}{l}15.8 \\
15.5 \\
15.1 \\
15.1 \\
14.8\end{array}$ & $\begin{array}{r}10.3 \\
10.1 \\
8.4 \\
7.7 \\
7.7\end{array}$ \\
\hline $\begin{array}{l}21 \\
22 \\
23 \\
24 \\
25\end{array}$ & $\begin{array}{l}7.0 \\
7.2 \\
6.8 \\
6.5 \\
5.8\end{array}$ & $\begin{array}{r}.8 \\
.9 \\
1.1 \\
.9 \\
.1\end{array}$ & $\begin{array}{l}-1.0 \\
-1.0 \\
-1.1 \\
-1.2 \\
-1.2\end{array}$ & $\begin{array}{l}-1.2 \\
-1.2 \\
-1.2 \\
-1.1 \\
-1.2\end{array}$ & $\begin{array}{r}-1.4 \\
-1.4 \\
-1.3 \\
-1.0 \\
-.6\end{array}$ & $\begin{array}{r}.0 \\
.3 \\
.5 \\
1.0 \\
.8\end{array}$ & $\begin{array}{l}9.5 \\
8.7 \\
8.1 \\
6.9 \\
5.6\end{array}$ & $\begin{array}{r}8.8 \\
9.4 \\
9.5 \\
\text { e } 8.3 \\
\text { e } 8.2\end{array}$ & $\begin{array}{l}13.5 \\
13.2 \\
13.3 \\
12.6 \\
11.4\end{array}$ & $\begin{array}{l}16.6 \\
16.1 \\
16.5 \\
16.2 \\
15.3\end{array}$ & $\begin{array}{l}14.1 \\
14.5 \\
14.6 \\
13.8 \\
12.9\end{array}$ & $\begin{array}{r}7.7 \\
8.5 \\
8.6 \\
9.7 \\
10.2\end{array}$ \\
\hline $\begin{array}{l}26 \\
27 \\
28 \\
29 \\
30 \\
31\end{array}$ & $\begin{array}{l}5.5 \\
5.8 \\
6.1 \\
6.3 \\
5.6 \\
4.9\end{array}$ & $\begin{array}{r}.1 \\
.1 \\
.2 \\
\mathrm{e}-. .5 \\
\mathrm{e}-1.0 \\
---\end{array}$ & $\begin{array}{l}-1.1 \\
-1.1 \\
-1.2 \\
-1.3 \\
-1.4 \\
-1.3\end{array}$ & $\begin{array}{l}-1.3 \\
-1.5 \\
-1.6 \\
-1.6 \\
-1.5 \\
-1.2\end{array}$ & $\begin{array}{l}-.4 \\
-.2 \\
-.1 \\
--- \\
--- \\
---\end{array}$ & $\begin{array}{r}.3 \\
.2 \\
.6 \\
1.2 \\
1.7 \\
2.3\end{array}$ & $\begin{array}{l}4.7 \\
3.3 \\
2.6 \\
3.3 \\
4.5 \\
\ldots .-\end{array}$ & $\begin{array}{l}\text { e } 8.8 \\
\text { e } 9.8 \\
\text { e } 9.9 \\
\text { e } 8.7 \\
\text { e } 7.1 \\
\text { e6.3 }\end{array}$ & $\begin{array}{r}11.7 \\
12.4 \\
12.3 \\
12.7 \\
13.0 \\
---\end{array}$ & $\begin{array}{l}15.9 \\
16.5 \\
16.9 \\
17.0 \\
17.2 \\
17.4\end{array}$ & $\begin{array}{l}13.4 \\
13.9 \\
14.0 \\
14.3 \\
14.2 \\
13.7\end{array}$ & $\begin{array}{r}10.6 \\
10.6 \\
10.5 \\
10.3 \\
9.9 \\
\ldots .-\end{array}$ \\
\hline $\begin{array}{l}\text { MEAN } \\
\text { MAX } \\
\text { MIN } \\
\text { MED }\end{array}$ & $\begin{array}{r}7.5 \\
10.2 \\
4.9 \\
7.4\end{array}$ & $\begin{array}{r}2.5 \\
5.8 \\
-1.0 \\
2.2\end{array}$ & $\begin{array}{r}-1.1 \\
-.4 \\
-1.4 \\
-1.1\end{array}$ & $\begin{array}{l}-1.2 \\
-1.0 \\
-1.6 \\
-1.2\end{array}$ & $\begin{array}{r}-1.2 \\
-.1 \\
-1.7 \\
-1.4\end{array}$ & $\begin{array}{r}.3 \\
2.3 \\
-.2 \\
.1\end{array}$ & $\begin{array}{l}6.3 \\
9.6 \\
2.6 \\
6.8\end{array}$ & $\begin{array}{r}7.3 \\
10.6 \\
2.2 \\
8.2\end{array}$ & $\begin{array}{r}11.3 \\
14.9 \\
6.9 \\
11.9\end{array}$ & $\begin{array}{l}15.9 \\
17.4 \\
13.5 \\
16.1\end{array}$ & $\begin{array}{l}15.1 \\
17.2 \\
12.9 \\
15.0\end{array}$ & $\begin{array}{r}11.0 \\
14.2 \\
7.7 \\
10.5\end{array}$ \\
\hline
\end{tabular}

WTR YR 1989 MEAN 6.2 MAX $17.4 \quad$ MIN -1.7 MED 6.5 
3 SPRINGS BASIN

KAWICH PEAK NEAR WARM SPRINGS, NV

DAILY MEAN SOIL TEMPERATURE, WATER YEAR OCTOBER 1989 TO SEPTEMBER 1990 DEGREES CELSIUS

\begin{tabular}{|c|c|c|c|c|c|c|c|c|c|c|c|c|}
\hline DAY & $\mathrm{OCT}$ & NOV & DEC & JAN & FEB & MAR & $A P R$ & MAY & JUN & JUL & AUG & SEP \\
\hline $\begin{array}{l}1 \\
2 \\
3 \\
4 \\
5\end{array}$ & $\begin{array}{l}8.5 \\
8.4 \\
7.4 \\
6.4 \\
5.7\end{array}$ & $\begin{array}{r}-1.0 \\
-1.6 \\
-.6 \\
.6 \\
1.5\end{array}$ & $\begin{array}{l}-3.0 \\
-3.5 \\
-3.4 \\
-3.0 \\
-2.1\end{array}$ & $\begin{array}{l}-3.2 \\
-3.1 \\
-4.0 \\
-4.4 \\
-4.0\end{array}$ & $\begin{array}{l}-2.4 \\
-2.4 \\
-2.4 \\
-2.5 \\
-2.4\end{array}$ & $\begin{array}{l}-.3 \\
-.2 \\
-.2 \\
-.1 \\
-.1\end{array}$ & $\begin{array}{r}.6 \\
1.5 \\
2.3 \\
2.2 \\
2.7\end{array}$ & $\begin{array}{l}3.8 \\
4.6 \\
5.4 \\
6.5 \\
7.9\end{array}$ & $\begin{array}{r}6.2 \\
7.5 \\
9.5 \\
10.9 \\
11.9\end{array}$ & $\begin{array}{l}16.5 \\
16.5 \\
15.7 \\
15.7 \\
15.9\end{array}$ & $\begin{array}{l}17.2 \\
16.7 \\
17.1 \\
17.0 \\
16.7\end{array}$ & $\begin{array}{l}14.4 \\
14.5 \\
14.7 \\
14.7 \\
14.6\end{array}$ \\
\hline $\begin{array}{r}6 \\
7 \\
8 \\
9 \\
10\end{array}$ & $\begin{array}{l}6.0 \\
6.3 \\
6.1 \\
6.0 \\
6.1\end{array}$ & $\begin{array}{r}1.4 \\
1.0 \\
.0 \\
.5 \\
1.6\end{array}$ & $\begin{array}{l}-1.5 \\
-1.5 \\
-1.6 \\
-1.2 \\
-2.1\end{array}$ & $\begin{array}{l}-4.2 \\
-3.5 \\
-2.2 \\
-1.4 \\
-1.0\end{array}$ & $\begin{array}{l}-2.3 \\
-2.1 \\
-2.0 \\
-2.0 \\
-1.9\end{array}$ & $\begin{array}{l}.0 \\
.0 \\
.0 \\
.0 \\
.0\end{array}$ & $\begin{array}{l}3.2 \\
2.7 \\
1.9 \\
1.2 \\
2.2\end{array}$ & $\begin{array}{l}8.8 \\
9.2 \\
8.8 \\
8.0 \\
8.4\end{array}$ & $\begin{array}{l}11.8 \\
12.4 \\
12.8 \\
12.6 \\
10.6\end{array}$ & $\begin{array}{l}16.2 \\
16.3 \\
16.2 \\
16.2 \\
15.4\end{array}$ & $\begin{array}{l}17.0 \\
16.8 \\
16.9 \\
16.6 \\
16.4\end{array}$ & $\begin{array}{l}14.2 \\
13.8 \\
14.0 \\
14.5 \\
14.5\end{array}$ \\
\hline $\begin{array}{l}11 \\
12 \\
13 \\
14 \\
15\end{array}$ & $\begin{array}{l}7.1 \\
7.5 \\
8.1 \\
8.1 \\
7.1\end{array}$ & $\begin{array}{l}1.5 \\
2.5 \\
2.3 \\
2.0 \\
1.7\end{array}$ & $\begin{array}{l}-3.9 \\
-3.7 \\
-2.9 \\
-2.8 \\
-1.7\end{array}$ & $\begin{array}{r}-.7 \\
-.7 \\
-.8 \\
-1.2 \\
-1.9\end{array}$ & $\begin{array}{l}-1.6 \\
-1.3 \\
-1.1 \\
-1.2 \\
-1.8\end{array}$ & $\begin{array}{l}.1 \\
.1 \\
.1 \\
.1 \\
.1\end{array}$ & $\begin{array}{l}3.4 \\
4.3 \\
5.4 \\
6.2 \\
6.9\end{array}$ & $\begin{array}{l}8.4 \\
8.2 \\
8.9 \\
8.9 \\
8.7\end{array}$ & $\begin{array}{r}9.7 \\
10.2 \\
10.6 \\
11.0 \\
9.9\end{array}$ & $\begin{array}{l}14.5 \\
14.6 \\
15.6 \\
14.7 \\
12.6\end{array}$ & $\begin{array}{l}15.9 \\
15.5 \\
14.8 \\
15.3 \\
14.8\end{array}$ & $\begin{array}{l}14.6 \\
14.7 \\
14.6 \\
14.6 \\
14.1\end{array}$ \\
\hline $\begin{array}{l}16 \\
17 \\
18 \\
19 \\
20\end{array}$ & $\begin{array}{l}5.9 \\
5.4 \\
4.7 \\
4.6 \\
5.6\end{array}$ & $\begin{array}{r}1.4 \\
1.5 \\
.6 \\
.2 \\
.6\end{array}$ & $\begin{array}{l}-1.9 \\
-2.7 \\
-3.0 \\
-3.8 \\
-3.9\end{array}$ & $\begin{array}{l}-2.3 \\
-2.2 \\
-2.0 \\
-1.9 \\
-1.9\end{array}$ & $\begin{array}{l}-2.4 \\
-2.6 \\
-2.6 \\
-2.5 \\
-2.3\end{array}$ & $\begin{array}{l}.0 \\
.1 \\
.1 \\
.1 \\
.1\end{array}$ & $\begin{array}{l}6.5 \\
5.0 \\
3.8 \\
3.6 \\
4.0\end{array}$ & $\begin{array}{l}8.6 \\
9.1 \\
9.2 \\
8.6 \\
8.4\end{array}$ & $\begin{array}{r}9.7 \\
11.0 \\
12.0 \\
12.4 \\
13.1\end{array}$ & $\begin{array}{l}12.9 \\
13.4 \\
14.5 \\
15.2 \\
15.8\end{array}$ & $\begin{array}{l}13.7 \\
13.8 \\
13.8 \\
13.1 \\
11.8\end{array}$ & $\begin{array}{l}13.1 \\
12.5 \\
12.1 \\
10.4 \\
10.5\end{array}$ \\
\hline $\begin{array}{l}21 \\
22 \\
23 \\
24 \\
25\end{array}$ & $\begin{array}{l}5.6 \\
5.7 \\
5.9 \\
5.2 \\
4.2\end{array}$ & $\begin{array}{l}1.7 \\
1.1 \\
1.1 \\
1.6 \\
1.2\end{array}$ & $\begin{array}{l}-3.5 \\
-2.9 \\
-3.1 \\
-3.0 \\
-3.3\end{array}$ & $\begin{array}{l}-2.0 \\
-2.1 \\
-2.0 \\
-1.9 \\
-2.0\end{array}$ & $\begin{array}{l}-2.2 \\
-2.0 \\
-1.8 \\
-1.4 \\
-1.0\end{array}$ & $\begin{array}{l}.0 \\
.1 \\
.1 \\
.1 \\
.2\end{array}$ & $\begin{array}{l}3.7 \\
3.9 \\
3.7 \\
3.0 \\
3.1\end{array}$ & $\begin{array}{r}9.6 \\
10.5 \\
10.4 \\
8.7 \\
7.5\end{array}$ & $\begin{array}{l}13.6 \\
14.4 \\
14.8 \\
14.9 \\
15.3\end{array}$ & $\begin{array}{l}16.3 \\
16.3 \\
16.9 \\
16.7 \\
16.0\end{array}$ & $\begin{array}{l}12.2 \\
12.4 \\
12.8 \\
13.4 \\
13.1\end{array}$ & $\begin{array}{r}10.4 \\
10.5 \\
10.1 \\
9.0 \\
9.1\end{array}$ \\
\hline $\begin{array}{l}26 \\
27 \\
28 \\
29 \\
30 \\
31\end{array}$ & $\begin{array}{r}2.7 \\
1.3 \\
.6 \\
-.7 \\
-1.3 \\
-1.3\end{array}$ & $\begin{array}{r}.9 \\
-1.3 \\
-2.5 \\
-3.1 \\
-3.3 \\
---\end{array}$ & $\begin{array}{l}-3.7 \\
-3.3 \\
-3.2 \\
-3.4 \\
-3.5 \\
-3.2\end{array}$ & $\begin{array}{l}-2.0 \\
-2.1 \\
-2.3 \\
-2.3 \\
-2.3 \\
-2.2\end{array}$ & $\begin{array}{l}-.7 \\
-.5 \\
-.4 \\
--- \\
--- \\
---\end{array}$ & $\begin{array}{l}.4 \\
.5 \\
.3 \\
.1 \\
.0 \\
.1\end{array}$ & $\begin{array}{l}4.6 \\
5.8 \\
6.5 \\
5.3 \\
4.4 \\
---\end{array}$ & $\begin{array}{l}8.7 \\
8.7 \\
6.1 \\
3.7 \\
3.9 \\
5.1\end{array}$ & $\begin{array}{r}15.5 \\
15.4 \\
15.2 \\
15.6 \\
16.0 \\
---\end{array}$ & $\begin{array}{l}15.9 \\
15.8 \\
16.5 \\
17.0 \\
17.1 \\
17.2\end{array}$ & $\begin{array}{l}12.6 \\
12.7 \\
13.3 \\
14.2 \\
14.7 \\
14.6\end{array}$ & $\begin{array}{l}8.9 \\
8.6 \\
8.5 \\
8.7 \\
8.8 \\
---\end{array}$ \\
\hline $\begin{array}{l}\text { MEAN } \\
\text { MAX } \\
\text { MIN } \\
\text { MED }\end{array}$ & $\begin{array}{r}5.1 \\
8.5 \\
-1.3 \\
5.9\end{array}$ & $\begin{array}{r}.5 \\
2.5 \\
-3.3 \\
1.0\end{array}$ & $\begin{array}{l}-2.9 \\
-1.2 \\
-3.9 \\
-3.0\end{array}$ & $\begin{array}{r}-2.3 \\
-.7 \\
-4.4 \\
-2.1\end{array}$ & $\begin{array}{r}-1.9 \\
-.4 \\
-2.6 \\
-2.0\end{array}$ & $\begin{array}{r}.1 \\
.5 \\
-.3 \\
.1\end{array}$ & $\begin{array}{r}3.8 \\
6.9 \\
.6 \\
3.7\end{array}$ & $\begin{array}{r}7.8 \\
10.5 \\
3.7 \\
8.6\end{array}$ & $\begin{array}{r}12.2 \\
16.0 \\
6.2 \\
12.2\end{array}$ & $\begin{array}{l}15.7 \\
17.2 \\
12.6 \\
15.9\end{array}$ & $\begin{array}{l}14.7 \\
17.2 \\
11.8 \\
14.7\end{array}$ & $\begin{array}{r}12.3 \\
14.7 \\
8.5 \\
13.4\end{array}$ \\
\hline
\end{tabular}

CAL YR 1989 MEAN 5.7 MAX 17.4 MIN -3.9 MED 5.6 WTR YR 1990 MEAN 5.5 MAX 17.2 MIN -4.4 MED 4.6 
3 SPRINGS BASIN

KAWICH PEAK NEAR WARM SPRINGS, NV

DAILY MEAN SOIL TEMPERATURE, WATER YEAR OCTOBER 1990 TO SEPTEMBER 1991 DEGREES CELSIUS

\begin{tabular}{|c|c|c|c|c|c|c|c|c|c|c|c|c|}
\hline DAY & OCT & NOV & $\mathrm{DEC}$ & JAN & FEB & MAR & APR & MAY & JUN & JUL & AUG & SEP \\
\hline $\begin{array}{l}1 \\
2 \\
3 \\
4 \\
5\end{array}$ & $\begin{array}{l}8.6 \\
9.0 \\
9.0 \\
8.3 \\
9.2\end{array}$ & $\begin{array}{r}3.6 \\
2.2 \\
.6 \\
.3 \\
1.2\end{array}$ & $\begin{array}{l}-2.5 \\
-2.8 \\
-3.0 \\
-3.0 \\
-2.1\end{array}$ & $\begin{array}{l}-5.5 \\
-5.6 \\
-5.0 \\
-4.1 \\
-3.7\end{array}$ & $\begin{array}{l}-5.0 \\
-4.5 \\
-4.3 \\
-3.1 \\
-2.2\end{array}$ & $\begin{array}{l}-1.1 \\
-1.1 \\
-1.1 \\
-1.0 \\
-.5\end{array}$ & $\begin{array}{l}.0 \\
.1 \\
.1 \\
.2 \\
.2\end{array}$ & $\begin{array}{r}.4 \\
.5 \\
.4 \\
.5 \\
1.5\end{array}$ & $\begin{array}{l}5.9 \\
7.3 \\
8.1 \\
8.9 \\
9.8\end{array}$ & $\begin{array}{l}12.8 \\
14.0 \\
15.2 \\
15.6 \\
16.2\end{array}$ & $\begin{array}{l}16.1 \\
15.9 \\
15.7 \\
15.4 \\
15.3\end{array}$ & $\begin{array}{l}18.1 \\
18.3 \\
18.0 \\
17.3 \\
17.0\end{array}$ \\
\hline $\begin{array}{r}6 \\
7 \\
8 \\
9 \\
10\end{array}$ & $\begin{array}{l}9.2 \\
8.4 \\
6.1 \\
5.2 \\
5.3\end{array}$ & $\begin{array}{r}.1 \\
-1.4 \\
.1 \\
1.2 \\
1.2\end{array}$ & $\begin{array}{l}-2.4 \\
-3.3 \\
-3.7 \\
-3.5 \\
-2.5\end{array}$ & $\begin{array}{l}-4.1 \\
-4.2 \\
-4.2 \\
-4.3 \\
-4.2\end{array}$ & $\begin{array}{l}-2.2 \\
-2.4 \\
-3.0 \\
-3.3 \\
-3.1\end{array}$ & $\begin{array}{l}-.2 \\
-.1 \\
-.2 \\
-.2 \\
-.4\end{array}$ & $\begin{array}{l}.3 \\
.3 \\
.3 \\
.3 \\
.3\end{array}$ & $\begin{array}{l}3.1 \\
4.4 \\
6.1 \\
3.8 \\
2.3\end{array}$ & $\begin{array}{l}10.5 \\
11.2 \\
11.5 \\
11.4 \\
12.2\end{array}$ & $\begin{array}{l}16.9 \\
16.8 \\
15.6 \\
14.8 \\
15.1\end{array}$ & $\begin{array}{l}15.7 \\
15.9 \\
16.2 \\
16.7 \\
17.2\end{array}$ & $\begin{array}{l}16.3 \\
15.5 \\
15.1 \\
14.8 \\
13.7\end{array}$ \\
\hline $\begin{array}{l}11 \\
12 \\
13 \\
14 \\
15\end{array}$ & $\begin{array}{l}6.4 \\
5.5 \\
6.8 \\
7.6 \\
7.8\end{array}$ & $\begin{array}{l}1.3 \\
1.8 \\
2.7 \\
3.3 \\
3.2\end{array}$ & $\begin{array}{l}-2.2 \\
-2.0 \\
-2.1 \\
-2.9 \\
-3.8\end{array}$ & $\begin{array}{l}-3.8 \\
-3.2 \\
-2.8 \\
-2.5 \\
-2.5\end{array}$ & $\begin{array}{l}-3.1 \\
-2.3 \\
-1.9 \\
-1.5 \\
-1.3\end{array}$ & $\begin{array}{l}-.3 \\
-.4 \\
-.3 \\
-.5 \\
-.5\end{array}$ & $\begin{array}{l}.3 \\
.3 \\
.3 \\
.3 \\
.3\end{array}$ & $\begin{array}{l}1.5 \\
2.2 \\
3.3 \\
2.4 \\
1.6\end{array}$ & $\begin{array}{l}13.0 \\
13.7 \\
14.0 \\
13.7 \\
13.1\end{array}$ & $\begin{array}{l}15.1 \\
15.8 \\
16.6 \\
16.6 \\
16.5\end{array}$ & $\begin{array}{l}17.1 \\
16.4 \\
16.5 \\
17.1 \\
17.4\end{array}$ & $\begin{array}{l}12.9 \\
12.8 \\
13.0 \\
13.6 \\
13.7\end{array}$ \\
\hline $\begin{array}{l}16 \\
17 \\
18 \\
19 \\
20\end{array}$ & $\begin{array}{l}7.8 \\
6.6 \\
6.8 \\
5.7 \\
3.1\end{array}$ & $\begin{array}{l}2.7 \\
3.2 \\
2.4 \\
2.0 \\
1.7\end{array}$ & $\begin{array}{l}-3.7 \\
-4.0 \\
-3.7 \\
-3.9 \\
-4.3\end{array}$ & $\begin{array}{l}-3.1 \\
-3.4 \\
-3.0 \\
-2.9 \\
-3.4\end{array}$ & $\begin{array}{l}-.8 \\
-1.0 \\
-2.3 \\
-2.6 \\
-2.1\end{array}$ & $\begin{array}{l}-.4 \\
-.3 \\
-.3 \\
-.3 \\
-.3\end{array}$ & $\begin{array}{l}.3 \\
.3 \\
.3 \\
.4 \\
.4\end{array}$ & $\begin{array}{l}2.2 \\
3.4 \\
2.5 \\
3.4 \\
4.2\end{array}$ & $\begin{array}{l}13.6 \\
14.0 \\
14.0 \\
13.7 \\
13.2\end{array}$ & $\begin{array}{l}16.2 \\
16.3 \\
16.3 \\
16.3 \\
16.0\end{array}$ & $\begin{array}{l}17.9 \\
18.0 \\
18.1 \\
18.0 \\
18.4\end{array}$ & $\begin{array}{l}13.3 \\
13.3 \\
14.0 \\
14.5 \\
14.4\end{array}$ \\
\hline $\begin{array}{l}21 \\
22 \\
23 \\
24 \\
25\end{array}$ & $\begin{array}{l}2.0 \\
2.6 \\
2.9 \\
3.2 \\
3.9\end{array}$ & $\begin{array}{l}.8 \\
.5 \\
.6 \\
.5 \\
.3\end{array}$ & $\begin{array}{l}-4.9 \\
-5.4 \\
-5.7 \\
-5.9 \\
-5.4\end{array}$ & $\begin{array}{l}-4.5 \\
-5.0 \\
-4.4 \\
-4.8 \\
-5.2\end{array}$ & $\begin{array}{l}-1.9 \\
-1.3 \\
-1.2 \\
-1.5 \\
-2.0\end{array}$ & $\begin{array}{l}-.3 \\
-.3 \\
-.3 \\
-.3 \\
-.2\end{array}$ & $\begin{array}{l}.4 \\
.4 \\
.4 \\
.4 \\
.4\end{array}$ & $\begin{array}{l}4.1 \\
3.8 \\
5.6 \\
7.3 \\
8.5\end{array}$ & $\begin{array}{l}12.6 \\
12.5 \\
13.1 \\
13.1 \\
12.7\end{array}$ & $\begin{array}{l}16.1 \\
15.6 \\
15.8 \\
16.7 \\
17.3\end{array}$ & $\begin{array}{l}18.6 \\
19.0 \\
19.5 \\
19.9 \\
20.0\end{array}$ & $\begin{array}{l}15.1 \\
15.1 \\
15.1 \\
15.1 \\
13.2\end{array}$ \\
\hline $\begin{array}{l}26 \\
27 \\
28 \\
29 \\
30 \\
31\end{array}$ & $\begin{array}{l}4.8 \\
4.2 \\
5.2 \\
5.2 \\
5.0 \\
5.0\end{array}$ & $\begin{array}{r}-.3 \\
-1.4 \\
-1.9 \\
-2.2 \\
-1.6 \\
---\end{array}$ & $\begin{array}{l}-5.2 \\
-5.2 \\
-4.7 \\
-5.2 \\
-6.1 \\
-5.5\end{array}$ & $\begin{array}{l}-5.9 \\
-5.5 \\
-5.2 \\
-5.9 \\
-5.4 \\
-5.2\end{array}$ & $\begin{array}{r}-2.3 \\
-2.0 \\
-1.1 \\
--- \\
--- \\
---\end{array}$ & $\begin{array}{l}-.2 \\
-.2 \\
-.2 \\
-.2 \\
-.2 \\
-.1\end{array}$ & $\begin{array}{r}.4 \\
.4 \\
.4 \\
.4 \\
.3 \\
-.--\end{array}$ & $\begin{array}{l}9.1 \\
8.6 \\
8.5 \\
9.2 \\
8.2 \\
6.0\end{array}$ & $\begin{array}{r}12.7 \\
11.5 \\
11.1 \\
11.2 \\
11.6 \\
-.-\end{array}$ & $\begin{array}{l}17.6 \\
17.6 \\
17.3 \\
17.1 \\
17.3 \\
16.7\end{array}$ & $\begin{array}{l}19.9 \\
19.2 \\
18.1 \\
18.4 \\
18.8 \\
18.5\end{array}$ & $\begin{array}{r}10.8 \\
10.3 \\
10.1 \\
9.2 \\
8.5 \\
---\end{array}$ \\
\hline $\begin{array}{l}\text { MEAN } \\
\text { MAX } \\
\text { MIN } \\
\text { MED }\end{array}$ & $\begin{array}{l}6.0 \\
9.2 \\
2.0 \\
5.7\end{array}$ & $\begin{array}{r}1.0 \\
3.6 \\
-2.2 \\
1.0\end{array}$ & $\begin{array}{l}-3.9 \\
-2.0 \\
-6.1 \\
-3.7\end{array}$ & $\begin{array}{l}-4.3 \\
-2.5 \\
-5.9 \\
-4.2\end{array}$ & $\begin{array}{r}-2.3 \\
-.8 \\
-5.0 \\
-2.2\end{array}$ & $\begin{array}{r}-.4 \\
-.1 \\
-1.1 \\
-.3\end{array}$ & $\begin{array}{l}.3 \\
.4 \\
.0 \\
.3\end{array}$ & $\begin{array}{r}4.1 \\
9.2 \\
.4 \\
3.4\end{array}$ & $\begin{array}{r}11.8 \\
14.0 \\
5.9 \\
12.5\end{array}$ & $\begin{array}{l}16.1 \\
17.6 \\
12.8 \\
16.3\end{array}$ & $\begin{array}{l}17.6 \\
20.0 \\
15.3 \\
17.9\end{array}$ & $\begin{array}{r}14.1 \\
18.3 \\
8.5 \\
14.2\end{array}$ \\
\hline
\end{tabular}

CAL YR 1990 MEAN 5.5 MAX 17.2 MIN -6.1 MED 4.6

WTR YR 1991 MEAN 5.1 MAX 20.0 MIN -6.1 MED 2.2 
3 SPRINGS BASIN

KAWICH PEAK NEAR WARM SPRINGS, NV

DAILY TOTAL SOLAR RADIATION, INCIDENTAL, WATER YEAR OCTOBER 1990 TO SEPTEMBER 1991 CALORIES PER SQUARE CENTIMETER

\begin{tabular}{|c|c|c|c|c|c|c|c|c|c|c|c|c|}
\hline DAY & OCT & NOV & DEC & JAN & FEB & MAR & $\mathrm{APR}$ & MAY & JUN & JUL & AUG & SEP \\
\hline 1 & --- & --- & 294 & 239 & 354 & 103 & 136 & 674 & 300 & 785 & 277 & 592 \\
\hline 2 & --- & --- & 289 & 293 & 150 & 465 & 595 & 275 & 478 & 783 & 472 & 597 \\
\hline 3 & --- & --- & 278 & 52 & 345 & 269 & 626 & 422 & 544 & 786 & 718 & 341 \\
\hline 4 & --- & --- & 283 & 76 & 360 & 45 & 567 & 732 & 595 & 783 & 707 & 385 \\
\hline 5 & --- & --- & 282 & 221 & 283 & 291 & 399 & 692 & 776 & 770 & 671 & 229 \\
\hline 6 & --- & --- & 288 & 214 & 377 & 461 & 463 & 607 & 786 & 629 & 725 & 105 \\
\hline 7 & --- & --- & 264 & 199 & 385 & 507 & 650 & 661 & 788 & 517 & 678 & 185 \\
\hline 8 & --- & 313 & 285 & 281 & 381 & 514 & 650 & 593 & 693 & 228 & 720 & 317 \\
\hline 9 & --- & 358 & 282 & 110 & 392 & 383 & 640 & 290 & 574 & 707 & 726 & 386 \\
\hline 10 & --- & 360 & 277 & 284 & 394 & 453 & 659 & 539 & 702 & 769 & 669 & 458 \\
\hline 11 & --- & 339 & 205 & 242 & 368 & 440 & 588 & 609 & 611 & 711 & 363 & 325 \\
\hline 12 & --- & 334 & 126 & 248 & 371 & 485 & 367 & 746 & 773 & 768 & 446 & 386 \\
\hline 13 & --- & 311 & 25 & 248 & 373 & 285 & 661 & 411 & 782 & 686 & 658 & 577 \\
\hline 14 & --- & 303 & 273 & 295 & 404 & 386 & 577 & 508 & 796 & 771 & 585 & 572 \\
\hline 15 & --- & 157 & 274 & 260 & 289 & 186 & 653 & 752 & 729 & 766 & 676 & 588 \\
\hline 16 & --- & 161 & 190 & 306 & 170 & 497 & 676 & 750 & 784 & 759 & 689 & 589 \\
\hline 17 & --- & 316 & 257 & 226 & 262 & 455 & 658 & 718 & 701 & 725 & 526 & 581 \\
\hline 18 & --- & 198 & 238 & 311 & 430 & 220 & 677 & 481 & 532 & 602 & 496 & 569 \\
\hline 19 & --- & 108 & 63 & 316 & 417 & 180 & 587 & 685 & 795 & 741 & 678 & 545 \\
\hline 20 & --- & 306 & 167 & 242 & 438 & 190 & 298 & 696 & 754 & 752 & 680 & 485 \\
\hline 21 & --- & 312 & 209 & 327 & 389 & 277 & 606 & 371 & 600 & 767 & 683 & 549 \\
\hline 22 & --- & 311 & 279 & 325 & 423 & 532 & 317 & 404 & 799 & 495 & 661 & 556 \\
\hline 23 & --- & 315 & 273 & 281 & 446 & 356 & 426 & 657 & 799 & 762 & 619 & 543 \\
\hline 24 & --- & 316 & 277 & 332 & 460 & 538 & 540 & 561 & 774 & 726 & 610 & 546 \\
\hline 25 & --- & 308 & 278 & 341 & 466 & 182 & 547 & 629 & 638 & 745 & 596 & 541 \\
\hline 26 & --- & 292 & 273 & 317 & 462 & 484 & 642 & 774 & 706 & 675 & 634 & 373 \\
\hline 27 & --- & 305 & 279 & 345 & 266 & 224 & 717 & 651 & 150 & 549 & 551 & 288 \\
\hline 28 & --- & 302 & 283 & 347 & 86 & 606 & 718 & 761 & 698 & 569 & 659 & 466 \\
\hline 29 & --- & 299 & 281 & 353 & --- & 603 & 723 & 678 & 532 & 518 & 654 & 252 \\
\hline 30 & --- & 210 & 282 & 350 & --- & 609 & 684 & 423 & 763 & 480 & 658 & 432 \\
\hline 31 & --- & --- & 281 & 265 & --- & 544 & --- & 613 & --- & 322 & 484 & --- \\
\hline TOTAL & --- & --- & 7635 & 8246 & 9941 & 11770 & 17047 & 18363 & 19952 & 20646 & 18969 & 13358 \\
\hline
\end{tabular}


3 SPRINGS BASIN

KAWICH PEAK NEAR WARM SPRINGS, NV

DAILY MEAN RELATIVE HUMIDITY, WATER YEAR OCTOBER 1990 TO SEPTEMBER 1991 PERCENT

\begin{tabular}{|c|c|c|c|c|c|c|c|c|c|c|c|c|}
\hline DAY & $\mathrm{OCT}$ & NOV & $\mathrm{DEC}$ & JAN & FEB & MAR & APR & MAY & JUN & JUL & AUG & SEP \\
\hline 1 & 34 & 83 & 30 & 22 & 21 & 98 & 75 & 45 & 91 & 28 & 79 & 44 \\
\hline 2 & 36 & 66 & 26 & 22 & 22 & 97 & 56 & 90 & 68 & 21 & 65 & 33 \\
\hline 3 & 32 & 27 & 33 & 68 & 40 & 97 & 32 & 69 & 43 & 18 & 47 & 40 \\
\hline 4 & 30 & 35 & 30 & 98 & 45 & 100 & 26 & 39 & 36 & 15 & 22 & 37 \\
\hline 5 & 35 & 53 & 32 & 59 & 48 & 75 & 28 & 34 & 34 & 15 & 19 & 50 \\
\hline 6 & 42 & 70 & 22 & 37 & 65 & 60 & 26 & 36 & 28 & 27 & 21 & 91 \\
\hline 7 & 60 & 42 & 21 & 59 & 39 & 60 & 22 & 40 & 19 & 43 & 19 & 93 \\
\hline 8 & 28 & 68 & 20 & 61 & 33 & 29 & 33 & 36 & 19 & 81 & 18 & 66 \\
\hline 9 & 20 & 41 & 20 & 91 & 32 & 30 & 25 & 81 & 23 & 75 & 15 & 54 \\
\hline 10 & 18 & 18 & 20 & 67 & 36 & 44 & 52 & 71 & 21 & 44 & 27 & 62 \\
\hline 11 & 22 & 19 & 34 & 63 & 29 & 75 & 64 & 56 & 20 & 25 & 74 & 71 \\
\hline 12 & 19 & 19 & 75 & 60 & 29 & 45 & 82 & 47 & 24 & 23 & 65 & 57 \\
\hline 13 & 19 & 20 & 96 & 76 & 57 & 68 & 54 & 51 & 25 & 21 & 59 & 41 \\
\hline 14 & 20 & 27 & 62 & 60 & 60 & 91 & 40 & 88 & 18 & 18 & 55 & 30 \\
\hline 15 & 21 & 33 & 49 & 68 & 51 & 93 & 45 & 53 & 19 & 20 & 44 & 19 \\
\hline 16 & 26 & 33 & 80 & 64 & 82 & 89 & 30 & 34 & 18 & 17 & 43 & 18 \\
\hline 17 & 25 & 56 & 69 & 80 & 70 & 72 & 41 & 42 & 17 & 24 & 27 & 18 \\
\hline 18 & 85 & 56 & 57 & 66 & 53 & 57 & 37 & 80 & 17 & 25 & 27 & 19 \\
\hline 19 & 85 & 69 & 86 & 64 & 51 & 84 & 29 & 71 & 22 & 26 & 23 & 21 \\
\hline 20 & 32 & 75 & 81 & 50 & 21 & 94 & 33 & 56 & 17 & 24 & 19 & 29 \\
\hline 21 & 23 & 54 & 76 & 46 & 20 & 94 & 55 & 75 & 18 & 20 & 18 & 25 \\
\hline 22 & 32 & 37 & 37 & 30 & 22 & 93 & 75 & 75 & 18 & 23 & 18 & 21 \\
\hline 23 & 34 & 19 & 33 & 29 & 31 & 84 & 61 & 47 & 18 & 17 & 28 & 18 \\
\hline 24 & 30 & 19 & 34 & 29 & 27 & 60 & 58 & 43 & 22 & 18 & 32 & 18 \\
\hline 25 & 25 & 22 & 39 & 25 & 21 & 88 & 70 & 40 & 31 & 20 & 40 & 18 \\
\hline 26 & 20 & 63 & 52 & 26 & 25 & 78 & 66 & 21 & 42 & 18 & 49 & 44 \\
\hline 27 & 19 & 26 & 23 & 25 & 47 & 94 & 40 & 32 & 49 & 18 & 30 & 71 \\
\hline 28 & 19 & 28 & 25 & 26 & 98 & 50 & 33 & 24 & 55 & 21 & 18 & 77 \\
\hline 29 & 22 & 23 & 39 & 29 & --- & 48 & 32 & 24 & 58 & 20 & 16 & 74 \\
\hline 30 & 32 & 31 & 23 & 21 & --- & 51 & 26 & 69 & 45 & 28 & 19 & 60 \\
\hline 31 & 33 & --- & 22 & 21 & --- & 35 & --- & 80 & --- & 66 & 57 & --- \\
\hline MEAN & 32 & 41 & 43 & 50 & 42 & 72 & 45 & 53 & 31 & 28 & 35 & 44 \\
\hline MAX & 85 & 83 & 96 & 98 & 98 & 100 & 82 & 90 & 91 & 81 & 79 & 93 \\
\hline MIN & 18 & 18 & 20 & 21 & 20 & 29 & 22 & 21 & 17 & 15 & 15 & 18 \\
\hline MED & 28 & 34 & 34 & 59 & 38 & 75 & 40 & 47 & 22 & 21 & 27 & 40 \\
\hline
\end{tabular}

WTR YR 1991 MEAN 43 MAX 100 MIN 15 MED 35 
3 SPRINGS BASIN

KAWICH PEAK NEAR WARM SPRINGS, NV

DAILY TOTAL PRECIPITATION, WATER YEAR OCTOBER 1988 TO SEPTEMBER 1989 INCHES

\begin{tabular}{|c|c|c|c|c|c|c|c|c|c|c|c|c|}
\hline DAY & OCT & NOV & $\mathrm{DEC}$ & JAN & FEB & MAR & $\mathrm{APR}$ & MAY & JUN & JUL & AUG & SEP \\
\hline 1 & .00 & .00 & .00 & .00 & .00 & .00 & .00 & .00 & .00 & .00 & .00 & .00 \\
\hline 2 & .00 & .00 & .00 & .00 & .00 & .45 & .00 & .00 & .00 & .00 & .00 & .00 \\
\hline 3 & .00 & .00 & .00 & .00 & .00 & .00 & .00 & .00 & .35 & .00 & .00 & .00 \\
\hline 4 & .00 & .00 & .00 & .10 & .75 & .00 & .00 & .00 & .13 & .00 & .00 & .00 \\
\hline 5 & .00 & .00 & .00 & .05 & .00 & .00 & .00 & .00 & .00 & .00 & .00 & .00 \\
\hline 6 & .00 & .00 & .00 & .35 & .00 & .00 & .00 & .00 & .00 & .00 & .00 & .00 \\
\hline 7 & .00 & .00 & .00 & .00 & .00 & .00 & .00 & .00 & .03 & .00 & .00 & .00 \\
\hline 8 & .00 & .00 & .00 & .00 & .40 & .00 & .00 & .00 & .15 & .00 & .47 & .00 \\
\hline 9 & .00 & .00 & .00 & .00 & .20 & .00 & .00 & .00 & .75 & .00 & .39 & .00 \\
\hline 10 & .00 & .00 & .00 & .00 & .18 & .00 & .00 & .05 & .07 & .00 & .00 & .00 \\
\hline 11 & .00 & .00 & .00 & .00 & .00 & .00 & .00 & 1.30 & .17 & .00 & .21 & .00 \\
\hline 12 & .00 & .00 & .00 & .00 & .07 & .00 & .00 & .00 & .00 & .00 & .00 & .00 \\
\hline 13 & .00 & .00 & .00 & .00 & .00 & .00 & .00 & 1.00 & .00 & .00 & .00 & .00 \\
\hline 14 & .00 & .78 & .00 & .00 & .00 & .00 & .00 & .10 & .00 & .00 & .00 & .00 \\
\hline 15 & .00 & .00 & .12 & .00 & .00 & .00 & .00 & .10 & .00 & .00 & .00 & .00 \\
\hline 16 & .00 & .00 & .03 & .00 & .00 & .00 & .00 & .00 & .00 & .00 & .00 & .00 \\
\hline 17 & .00 & .25 & .00 & .00 & .00 & .00 & .00 & .00 & .00 & .00 & .00 & .10 \\
\hline 18 & .00 & .00 & .06 & .00 & .00 & .00 & .00 & .00 & .00 & .00 & .00 & .00 \\
\hline 19 & .00 & .00 & .09 & .00 & .00 & .00 & .00 & .00 & .00 & .00 & .00 & .00 \\
\hline 20 & .00 & .00 & .00 & .00 & .00 & .00 & .00 & .00 & .00 & .00 & .00 & .00 \\
\hline 21 & .00 & .00 & .98 & .00 & .00 & .00 & .00 & .00 & .00 & .00 & .00 & .00 \\
\hline 22 & .00 & .00 & .00 & .00 & .00 & .00 & .00 & .00 & .00 & .06 & .00 & .00 \\
\hline 23 & .00 & .25 & .15 & .00 & .00 & .00 & .00 & .00 & .00 & .00 & .00 & .00 \\
\hline 24 & .00 & .00 & .12 & .00 & .00 & .00 & .07 & .00 & .00 & .00 & .00 & .00 \\
\hline 25 & .00 & .28 & .44 & .00 & .00 & .47 & .00 & .00 & .35 & .11 & .00 & .00 \\
\hline 26 & .00 & .00 & .00 & .00 & .00 & .03 & .00 & .00 & .00 & .00 & .00 & .00 \\
\hline 27 & .00 & .00 & .03 & .00 & .00 & .00 & .15 & .00 & .00 & .00 & .00 & .00 \\
\hline 28 & .00 & .00 & .03 & .00 & .00 & .00 & .00 & .00 & .00 & .00 & .00 & .00 \\
\hline 29 & .00 & .00 & .00 & .00 & --- & .00 & .00 & .00 & .00 & .00 & .00 & .00 \\
\hline 30 & .00 & .00 & .00 & .00 & --- & .00 & .00 & .50 & .00 & .00 & .00 & .00 \\
\hline 31 & .00 & $-\cdots$ & .02 & .00 & --- & .00 & --- & .00 & -- & .00 & .00 & --- \\
\hline TOTAL & 0.00 & 1.56 & 2.07 & 0.50 & 1.60 & 0.95 & 0.22 & 3.05 & 2.00 & 0.17 & 1.07 & 0.10 \\
\hline
\end{tabular}

WTR YR 1989 TOTAL 13.29 
3 SPRINGS BASIN

KAWICH PEAK NEAR WARM SPRINGS, NV

DAILY TOTAL PRECIPITATION, WATER YEAR OCTOBER 1989 TO SEPTEMBER 1990 INCHES

\begin{tabular}{|c|c|c|c|c|c|c|c|c|c|c|c|c|}
\hline DAY & OCT & NOV & $\mathrm{DEC}$ & JAN & FEB & MAR & APR & MAY & JUN & JUL & AUG & SEP \\
\hline $\begin{array}{l}1 \\
2 \\
3 \\
4 \\
5\end{array}$ & $\begin{array}{l}.00 \\
.00 \\
.00 \\
.00 \\
.00\end{array}$ & $\begin{array}{l}.00 \\
.00 \\
.00 \\
.00 \\
.00\end{array}$ & $\begin{array}{l}.00 \\
.00 \\
.00 \\
.00 \\
.00\end{array}$ & $\begin{array}{l}.10 \\
.08 \\
.00 \\
.00 \\
.00\end{array}$ & $\begin{array}{l}.50 \\
.00 \\
.00 \\
.20 \\
.00\end{array}$ & $\begin{array}{l}.02 \\
.00 \\
.00 \\
.75 \\
.70\end{array}$ & $\begin{array}{l}.00 \\
.00 \\
.00 \\
.10 \\
.05\end{array}$ & $\begin{array}{l}.00 \\
.00 \\
.00 \\
.00 \\
.00\end{array}$ & $\begin{array}{l}.00 \\
.00 \\
.00 \\
.00 \\
.00\end{array}$ & $\begin{array}{l}.00 \\
.00 \\
.00 \\
.00 \\
.00\end{array}$ & $\begin{array}{l}.00 \\
.00 \\
.00 \\
.00 \\
.00\end{array}$ & $\begin{array}{l}.00 \\
.00 \\
.00 \\
.00 \\
.00\end{array}$ \\
\hline $\begin{array}{r}6 \\
7 \\
8 \\
9 \\
10\end{array}$ & $\begin{array}{l}.00 \\
.00 \\
.00 \\
.00 \\
.00\end{array}$ & $\begin{array}{l}.00 \\
.00 \\
.00 \\
.00 \\
.00\end{array}$ & $\begin{array}{l}.00 \\
.00 \\
.00 \\
.00 \\
.00\end{array}$ & $\begin{array}{l}.00 \\
.00 \\
.00 \\
.00 \\
.00\end{array}$ & $\begin{array}{l}.05 \\
.25 \\
.00 \\
.00 \\
.00\end{array}$ & $\begin{array}{l}.00 \\
.00 \\
.00 \\
.00 \\
.00\end{array}$ & $\begin{array}{l}.00 \\
.35 \\
.35 \\
.00 \\
.00\end{array}$ & $\begin{array}{l}.00 \\
.00 \\
.00 \\
.00 \\
.00\end{array}$ & $\begin{array}{l}.00 \\
.00 \\
.00 \\
.00 \\
.58\end{array}$ & $\begin{array}{l}.00 \\
.00 \\
.00 \\
.00 \\
.00\end{array}$ & $\begin{array}{l}.00 \\
.00 \\
.00 \\
.01 \\
.03\end{array}$ & $\begin{array}{l}.00 \\
.00 \\
.00 \\
.00 \\
.00\end{array}$ \\
\hline $\begin{array}{l}11 \\
12 \\
13 \\
14 \\
15\end{array}$ & $\begin{array}{l}.00 \\
.00 \\
.00 \\
.00 \\
.00\end{array}$ & $\begin{array}{l}.00 \\
.00 \\
.00 \\
.00 \\
.00\end{array}$ & $\begin{array}{l}.00 \\
.00 \\
.00 \\
.00 \\
.00\end{array}$ & $\begin{array}{l}.00 \\
.00 \\
.57 \\
.24 \\
.00\end{array}$ & $\begin{array}{l}.00 \\
.00 \\
.07 \\
.00 \\
.00\end{array}$ & $\begin{array}{l}.48 \\
.10 \\
.00 \\
.00 \\
.00\end{array}$ & $\begin{array}{l}.00 \\
.00 \\
.00 \\
.00 \\
.00\end{array}$ & $\begin{array}{l}.00 \\
.00 \\
.00 \\
.00 \\
.00\end{array}$ & $\begin{array}{l}.05 \\
.00 \\
.00 \\
.00 \\
.00\end{array}$ & $\begin{array}{l}.00 \\
.00 \\
.02 \\
.73 \\
.00\end{array}$ & $\begin{array}{l}.19 \\
.00 \\
.00 \\
.00 \\
.22\end{array}$ & $\begin{array}{l}.00 \\
.00 \\
.00 \\
.00 \\
.00\end{array}$ \\
\hline $\begin{array}{l}16 \\
17 \\
18 \\
19 \\
20\end{array}$ & $\begin{array}{l}.00 \\
.00 \\
.00 \\
.00 \\
.00\end{array}$ & $\begin{array}{l}.00 \\
.00 \\
.00 \\
.00 \\
.00\end{array}$ & $\begin{array}{l}.00 \\
.00 \\
.00 \\
.00 \\
.00\end{array}$ & $\begin{array}{l}.38 \\
.65 \\
.06 \\
.00 \\
.00\end{array}$ & $\begin{array}{l}.13 \\
.35 \\
.60 \\
.00 \\
.00\end{array}$ & $\begin{array}{l}.00 \\
.00 \\
.00 \\
.00 \\
.00\end{array}$ & $\begin{array}{l}.00 \\
.12 \\
.23 \\
.00 \\
.20\end{array}$ & $\begin{array}{l}.00 \\
.00 \\
.00 \\
.00 \\
.00\end{array}$ & $\begin{array}{l}.00 \\
.00 \\
.00 \\
.00 \\
.00\end{array}$ & $\begin{array}{l}.42 \\
.00 \\
.00 \\
.00 \\
.00\end{array}$ & $\begin{array}{l}.20 \\
.00 \\
.00 \\
.00 \\
.00\end{array}$ & $\begin{array}{l}.00 \\
.00 \\
.00 \\
.00 \\
.00\end{array}$ \\
\hline $\begin{array}{l}21 \\
22 \\
23 \\
24 \\
25\end{array}$ & $\begin{array}{l}.00 \\
.00 \\
.00 \\
.00 \\
.15\end{array}$ & $\begin{array}{l}.00 \\
.00 \\
.00 \\
.00 \\
.13\end{array}$ & $\begin{array}{l}.00 \\
.00 \\
.00 \\
.00 \\
.00\end{array}$ & $\begin{array}{l}.00 \\
.00 \\
.00 \\
.00 \\
.00\end{array}$ & $\begin{array}{l}.00 \\
.00 \\
.00 \\
.00 \\
.00\end{array}$ & $\begin{array}{l}.00 \\
.00 \\
.00 \\
.00 \\
.00\end{array}$ & $\begin{array}{l}.05 \\
.00 \\
.27 \\
.00 \\
.00\end{array}$ & $\begin{array}{l}.00 \\
.00 \\
.00 \\
.18 \\
.00\end{array}$ & $\begin{array}{l}.00 \\
.00 \\
.00 \\
.00 \\
.00\end{array}$ & $\begin{array}{l}.00 \\
.00 \\
.00 \\
.00 \\
.00\end{array}$ & $\begin{array}{l}.00 \\
.00 \\
.00 \\
.00 \\
.00\end{array}$ & $\begin{array}{l}.00 \\
.00 \\
.00 \\
.00 \\
.00\end{array}$ \\
\hline $\begin{array}{l}26 \\
27 \\
28 \\
29 \\
30 \\
31\end{array}$ & $\begin{array}{l}.00 \\
.00 \\
.00 \\
.00 \\
.00 \\
.00\end{array}$ & $\begin{array}{l}.03 \\
.00 \\
.00 \\
.00 \\
.00 \\
----\end{array}$ & $\begin{array}{l}.00 \\
.00 \\
.00 \\
.00 \\
.00 \\
.00\end{array}$ & $\begin{array}{l}.00 \\
.00 \\
.00 \\
.00 \\
.00 \\
.00\end{array}$ & $\begin{array}{l}.00 \\
.04 \\
.00 \\
--- \\
--- \\
---\end{array}$ & $\begin{array}{l}.00 \\
.00 \\
.05 \\
.00 \\
.00 \\
.00\end{array}$ & $\begin{array}{l}.00 \\
.00 \\
.03 \\
.00 \\
.00 \\
-.--\end{array}$ & $\begin{array}{r}.00 \\
.27 \\
.20 \\
1.45 \\
.00 \\
.00\end{array}$ & $\begin{array}{l}.00 \\
.00 \\
.00 \\
.00 \\
.00 \\
----\end{array}$ & $\begin{array}{l}.00 \\
.00 \\
.00 \\
.00 \\
.00 \\
.00\end{array}$ & $\begin{array}{l}.00 \\
.00 \\
.00 \\
.00 \\
.00 \\
.00\end{array}$ & $\begin{array}{l}.00 \\
.00 \\
.00 \\
.00 \\
.00 \\
-.--\end{array}$ \\
\hline TOTAI & 0.15 & 0.16 & 0.00 & 2.08 & 2.19 & 2.10 & 1.75 & 2.10 & 0.63 & 1.17 & 0.65 & 0.00 \\
\hline
\end{tabular}

CAL YR 1989 TOTAL 9.97

WTR YR 1990 TOTAL 12.98 
3 SPRINGS BASIN

KAWICH PEAK NEAR WARM SPRINGS, NV

DAILY TOTAL PRECIPITATION, WATER YEAR OCTOBER 1990 TO SEPTEMBER 1991 INCHES

\begin{tabular}{|c|c|c|c|c|c|c|c|c|c|c|c|c|}
\hline DAY & OCT & NOV & DEC & JAN & FEB & MAR & APR & MAY & JUN & JUL & AUG & SEP \\
\hline 1 & .00 & .10 & .00 & .00 & .00 & .68 & .15 & .18 & .00 & .00 & e. 23 & .00 \\
\hline 2 & .00 & .00 & .00 & .00 & .00 & .00 & .00 & .15 & .00 & .00 & e. 19 & e.03 \\
\hline 3 & .00 & .00 & .00 & .40 & .00 & .05 & .00 & .00 & .00 & .00 & e. 21 & e. 21 \\
\hline 4 & .00 & .00 & .00 & .05 & .00 & .07 & .00 & .00 & .00 & .00 & .00 & .00 \\
\hline 5 & .00 & .00 & .00 & .00 & .00 & .00 & .00 & .00 & .00 & .00 & .00 & .00 \\
\hline 6 & .00 & .00 & .00 & .00 & .00 & .02 & .00 & .00 & .00 & .00 & .00 & e. 25 \\
\hline 7 & .00 & .00 & .00 & .00 & .00 & .00 & .00 & .00 & .00 & .00 & .00 & e. 34 \\
\hline 8 & .00 & .00 & .00 & .00 & .00 & .00 & .00 & .00 & .00 & e. 97 & .00 & e. 02 \\
\hline 9 & .00 & .00 & .00 & .00 & .00 & .00 & .00 & .60 & .00 & e. 14 & .00 & e. 03 \\
\hline 10 & .00 & .00 & .00 & .00 & .00 & .35 & .00 & .00 & .00 & .00 & .00 & .00 \\
\hline 11 & .00 & .00 & .00 & .00 & .00 & .00 & .00 & .00 & .00 & .00 & e. 05 & e. 02 \\
\hline 12 & .00 & .00 & .00 & .00 & .00 & .00 & .07 & .00 & .00 & .00 & .00 & e. 02 \\
\hline 13 & .00 & .00 & .20 & .00 & .00 & .70 & .00 & e. 50 & .00 & .00 & .00 & e. 01 \\
\hline 14 & .00 & .00 & .00 & .00 & .00 & .00 & .00 & .00 & .00 & .00 & .00 & .00 \\
\hline 15 & .00 & .00 & .05 & .05 & .15 & .65 & .00 & .00 & .00 & .00 & .00 & .00 \\
\hline 16 & .00 & .00 & .00 & .00 & .00 & .00 & .00 & .00 & .00 & .00 & .00 & .00 \\
\hline 17 & .00 & .00 & .00 & .00 & .00 & .00 & .00 & .00 & .00 & .00 & .00 & .00 \\
\hline 18 & .24 & .00 & .00 & .00 & .00 & .00 & .00 & e. 20 & .00 & .00 & .00 & .00 \\
\hline 19 & .15 & .50 & .12 & .00 & .00 & .60 & .00 & .00 & .00 & .00 & .00 & .00 \\
\hline 20 & .00 & .15 & .03 & .00 & .00 & .65 & .00 & e. 70 & .00 & .00 & .00 & .00 \\
\hline 21 & .00 & .00 & .00 & .00 & .00 & .17 & .00 & .00 & .00 & .00 & .00 & .00 \\
\hline 22 & .00 & .00 & .00 & .00 & .00 & .23 & .40 & .00 & .00 & .00 & .00 & .00 \\
\hline 23 & .00 & .00 & .00 & .00 & .00 & .00 & .00 & .00 & .00 & .00 & .00 & .00 \\
\hline 24 & .00 & .00 & .00 & .00 & .00 & .00 & .05 & .00 & .00 & .00 & .00 & .00 \\
\hline 25 & .00 & .20 & .00 & .00 & .00 & .00 & .17 & .00 & .00 & .00 & .00 & .00 \\
\hline 26 & .00 & .00 & .00 & .00 & .00 & .25 & .00 & .00 & .00 & .00 & .00 & .20 \\
\hline 27 & .00 & .00 & .00 & .00 & .42 & .15 & .00 & .00 & .00 & .00 & .00 & .23 \\
\hline 28 & .00 & .00 & .00 & .00 & .99 & .50 & .00 & .00 & .00 & .00 & .00 & .00 \\
\hline 29 & .00 & .00 & .00 & .00 & --- & .00 & .00 & .00 & .00 & .00 & .00 & .17 \\
\hline 30 & .00 & .00 & .00 & .00 & --- & .00 & .00 & .00 & .00 & e. 01 & .00 & .00 \\
\hline 31 & .00 & --- & .00 & .00 & --- & .00 & --- & e. 25 & --- & e. 64 & e. 67 & --- \\
\hline TOTAL & 0.39 & 0.95 & 0.40 & 0.50 & 1.56 & 5.07 & 0.84 & 2.58 & 0.00 & 1.76 & 1.35 & 1.53 \\
\hline
\end{tabular}

CAL YR 1990 TOTAL 14.41

WTR YR 1991 TOTAL 16.93

e Estimated 
3 SPRINGS BASIN

KAWICH PEAK NEAR WARM SPRINGS, NV

PRECIPITATION WATER QUALITY, WATER YEARS OCTOBER 1988 TO SEPTEMBER 1991

DATE

SPE-

CIFIC

CON-

$\mathrm{CON}-$

DUCT-

DUCT-

ANCE

TIME

$(\mu \mathrm{S} / \mathrm{CM}) \quad(\mu \mathrm{S} / \mathrm{CM})$

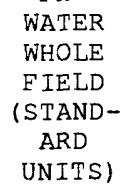

PH
WATER
WHOLE
LAB
(STAND-
ARD
UNITS)

ALKA-

INTTY CALCIUM

MAGNE-

SIUM, SODIUM

DIS- DIS-

(MG/L SOLVED SOLVED SOLVED

AS (MG/L (MG/L (MG/L

CACO3) AS CA) AS MG) AS NA)

FEB 1989

$23 \ldots$

0915

12

13

\begin{abstract}
6.6
\end{abstract}
6.6

3.0

0.90

0.10

1.0

$12 \ldots$

0800

29

6.3

7.1

8.0

1.6

0.05

4.4

$24 \ldots$

0930

SEP

$12 \ldots$

1345

NOV

$08 \ldots$

JAN 1990

$30 . .$.

1530

APR

$04 \ldots$

MAY

$22 \ldots$

JUL

$10 \ldots$

SEP

$22 \ldots$

JAN 1991

$30 \ldots$

JUN

$04 \ldots$

SEP

$25 \ldots$

1115

0725

1515

1920

1320

0830

1700

6.0

5.5

2.0

0.40

0.30

0.25

$16 \quad 16$

6.0

$6.8 \quad-$

1.3

0.18

0.60

6.1

$6.5 \quad 4.0$

1.5

0.12

0.90

5.7

$6.1 \quad 2.0$

0.72

0.04

0.40

$14 \quad 16$

6.5

$\begin{array}{ll}6.6 & 3.6\end{array}$

0.95

0.05

1.3

5.8

$5.7<0.5$

0.60

0.05

0.30

5.7

6.41 .3

0.47

0.05

0.30

5.5

$5.6<0.5$

0.88

0.09

0.20

6.1

$\begin{array}{ll}5.8 & 0.9\end{array}$

0.59

0.13

0.50

6.5

$5.7<0.5$

0.61

0.06

0.60

1215

POTAS-

$\begin{array}{ccl}\text { SIUM, } & \text { SULFATE } & \text { RIDE, } \\ \text { DIS- } & \text { DIS- } & \text { DIS- } \\ \text { SOLVED } & \text { SOLVED } & \text { SOLVED } \\ \text { (MG/L } & \text { (MG/L } & \text { (MG/L }\end{array}$

(MS $K$

AS SO4)

AS CL)
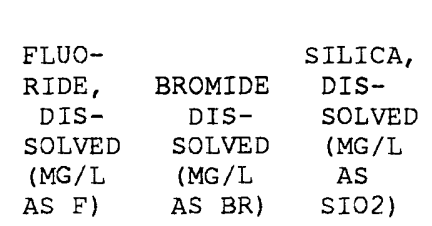

NITRO- PHOS-

GEN, PHORUS

NITRATE ORTHO,

DIS- DIS-

SOLVED SOLVED

(MG/L (MG/L

FEB 1989

$23 \ldots$

0.16
0.38
0.08
--
--
--
--

\title{
1.1
}

0.65

$0.03<0.010$

0.05

0.240

$<0.010$

$12 \ldots$

MAY

$24 \ldots$

SEP

$12 \ldots$

NOV

$08 \ldots$

JAN 1990

$30 \ldots$

APR

$04 \ldots$

MAY

$22 \ldots$

JUL

$10 \ldots$

$<0.10$

3.2

1.7

$0.05<0.010$

0.17

$0.290<0.010$

0.50

0.18

$<0.01$

$<0.010$

0.07

0.140

$<0.010$

$\begin{array}{ll}1.6 & 0.71\end{array}$

$0.01<0.010$

0.56

$<0.010$

$<0.010$

$\begin{array}{ll}1.6 & 0.57\end{array}$

$0.04<0.010$

0.05

0.720

$<0.010$

0.56

0.20

$0.03<0.010$

0.04

0.380

$<0.010$

1.7

0.46

$0.03<0.010$

$<0.01$

$0.460<0.010$

0.86

0.17

$0.02<0.010$

$<0.10$

0.610

$<0.010$

0.20

0.52

0.26

$0.02<0.010$

$<0.01$

0.260

$<0.010$

22 ...

JAN 1991

0.10

0.79

0.18

$0.02<0.010$

0.10

$0.440<0.010$

0.20

0.63

0.96

$0.02<0.010$

$<0.10$

$0.280<0.010$

$<0.10$

0.92

0.22

$0.02<0.010$

$<0.10$

$0.200<0.010$ 


\section{SPRINGS BASIN}

KAWICH PEAK NEAR WARM SPRINGS, NV

PRECIPITATION WATER QUALITY, WATER YEARS OCTOBER 1988 TO SEPTEMBER 1991

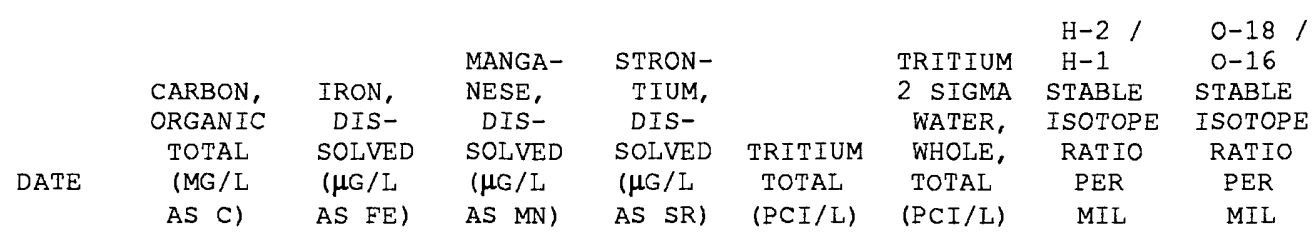

\begin{tabular}{|c|c|c|c|c|c|c|c|c|}
\hline $\begin{array}{c}\mathrm{FEB} 1989 \\
23 \ldots \\
\mathrm{APR}\end{array}$ & 1.6 & 3 & 1 & 10 & -- & -- & -109.0 & -15.05 \\
\hline${ }_{M A Y}^{12} \ldots$ & -- & 3 & 1 & 30 & -- & -- & -133.5 & -17.75 \\
\hline$\underset{\operatorname{SEP}}{24 \ldots}$ & -- & 5 & -- & 3 & -- & -- & -135.0 & -18.40 \\
\hline${ }_{\text {Nov }}^{12} \ldots$ & -- & $<3$ & 20 & -- & -- & -- & -50.0 & -7.25 \\
\hline $\begin{array}{l}08 \\
\text { JAN } 1990\end{array}$ & -- & $<3$ & $<1$ & -- & -- & -- & -81.5 & -12.05 \\
\hline $\begin{array}{l}30 \ldots \\
A P R\end{array}$ & -- & 3 & 6 & -- & 19 & 2.0 & -93.0 & -13.25 \\
\hline $\begin{array}{l}04 \ldots \\
M A Y\end{array}$ & -- & 2 & 5 & -- & -- & -- & -115.0 & -16.10 \\
\hline$\underset{\text { JUL }}{22 \ldots}$ & -- & 5 & $<5$ & -- & -- & -- & -83.5 & -12.05 \\
\hline $\operatorname{sEP}^{10} \cdots$ & -- & $<2$ & $<1$ & -- & -- & -- & -139.0 & -18.55 \\
\hline JAN 22.991 & -- & 2 & 4 & -- & -- & -- & -91.0 & $-13 \cdot 30$ \\
\hline$\underset{\text { JUN }}{30} \cdots$ & -- & $<3$ & 14 & -- & -- & -- & -95.0 & $-13 \cdot 65$ \\
\hline $\begin{array}{l}04 \ldots \\
\mathrm{SEP}\end{array}$ & -- & 4 & 6 & -- & -- & -- & -125.0 & -17.15 \\
\hline 25 & -- & -- & -- & -- & -- & -- & -- & -- \\
\hline
\end{tabular}




\section{SPRINGS BASIN}

3 SPRINGS CREEK NEAR LEDGE SPRING, NEAR WARM SPRINGS, NV

LOCATION. - Lat 37\%57'42", long 116 $26^{\prime} 23^{\prime \prime}$, Nye County, Hydrologic Unit 16060012, 1.1 mi upstream from the 3 springs Creek surface-water equipment, $3.6 \mathrm{mi}$ upstream from Eden Ranch in the Reveille Valley, $16.3 \mathrm{mi}$ south of Warm Springs at the intersection of Us Highway 6 and Nevada Highway 375 .

DRAINAGE AREA. -- $0.78 \mathrm{mi}^{2}$.

PERIOD OF RECORD. -- September 1987 to current year.

SURFACE-WATER QUALITY, WATER YEARS OCTOBER 1986 TO SEPTEMBER 1991

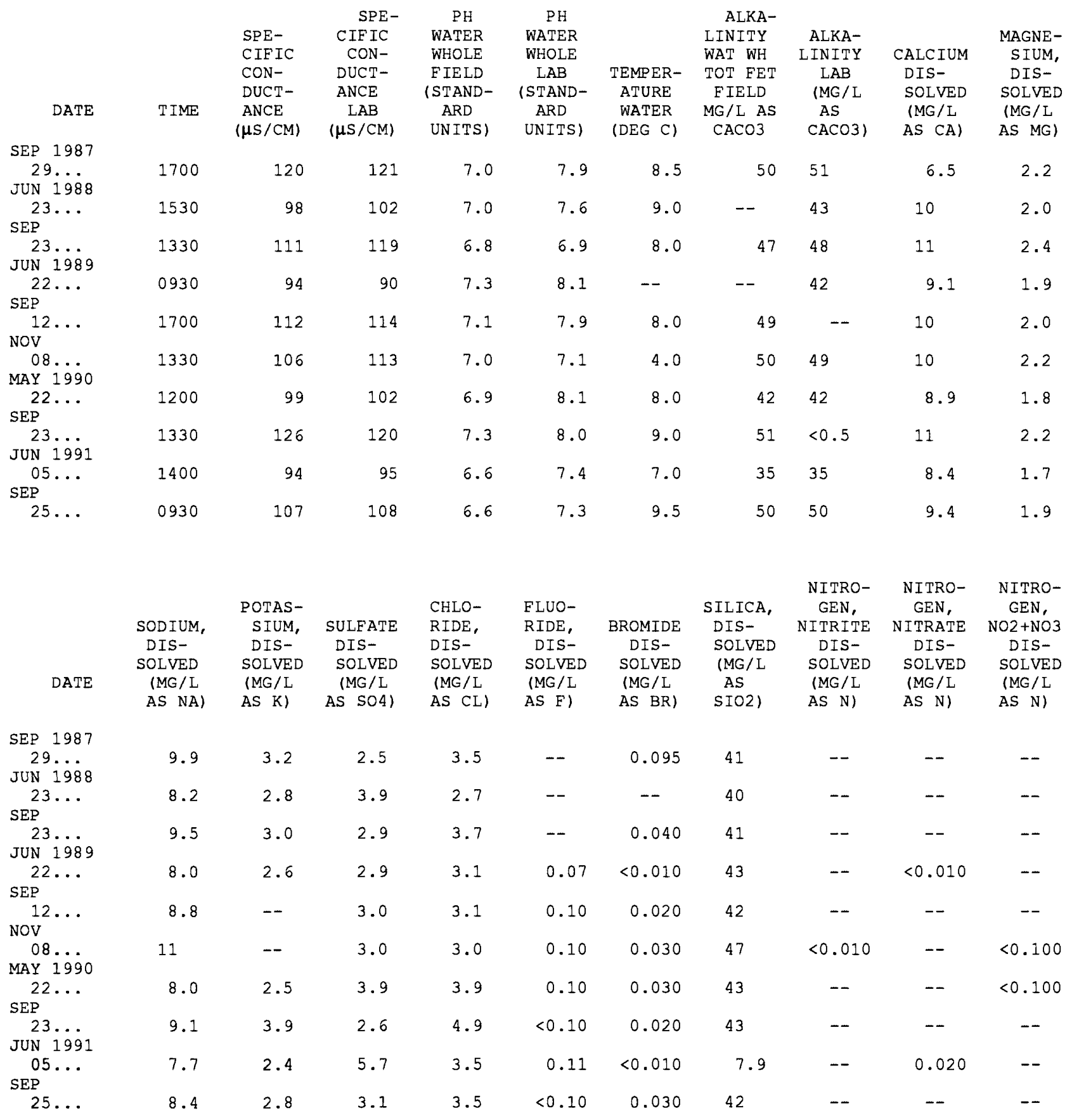


3 SPRINGS BASIN

3 SPRINGS CREEK NEAR LEDGE SPRING, NEAR WARM SPRINGS, NV SURFACE-WATER QUALITY, WATER YEARS OCTOBER 1986 TO SEPTEMBER 1991

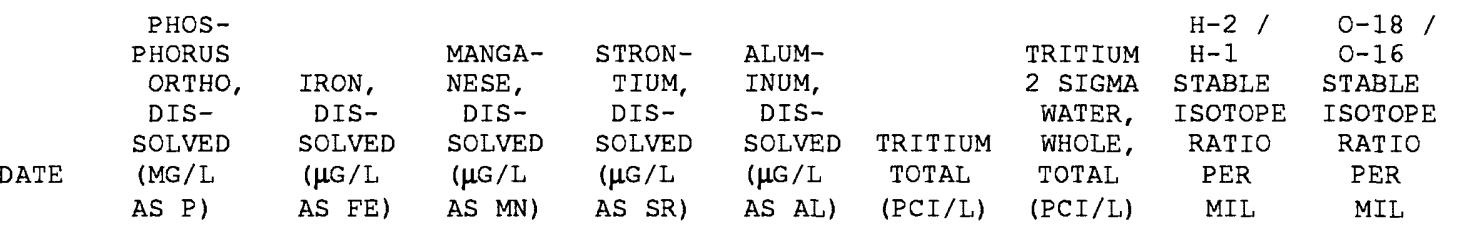

SEP 1987

$29 \ldots$

JUN 1988

$23 \ldots$

SEP

$23 \ldots$

JUN 1989

$22 \ldots$

SEP

$12 \ldots$

NOV

$08 \ldots$

MAY 1990

$22 \ldots$

SEP

$23 \ldots$

JUN 1991

$05 \ldots$

SEP

$25 \ldots$

--
--
-0.010
0.030
--
$<0.010$
--

$\begin{array}{ll}- & \\ <1 & \\ <1 & \\ 2 & \\ <1 & -- \\ <1 & - \\ 7 & - \\ <1 & -\end{array}$

91

$82 \quad--$

$98<10$

48

$-$

$-$

$-$

$-$

$-$

$-$

$-$ $\begin{array}{lll}\ldots & -103.5 & -14.00 \\ - & -104.5 & -14.20 \\ 6.4 & -103.5 & -14.15 \\ - & -105.0 & -14.15 \\ \ldots & -106.0 & -14.10 \\ - & -104.5 & -14.05 \\ - & -103.0 & -14.10 \\ - & -102.0 & -14.00 \\ -- & -106.0 & -14.30 \\ - & -104.0 & -13.90\end{array}$ 
3 SPRINGS BASIN

3 SPRINGS CREEK NEAR 3 SPRING \#2, NEAR WARM SPRINGS, NV

LOCATION. -- Lat 37057'36", long 116\%25'50", Nye County, Hydrologic Unit 16060012, 0.5 mi upstream from the 3 Springs Creek surface-water equipment, $0.7 \mathrm{mi}$ above the confluence of 3 springs Creek with Eden Creek, $3.2 \mathrm{mi}$ upstream from Eden Ranch in the Reveille valley, $16.2 \mathrm{mi}$ south of Warm Springs at the intersection of US Highway 6 and Nevada Highway 375.

DRAINAGE AREA. -- $1.19 \mathrm{mi}^{2}$.

PERIOD OF RECORD. -- July 1985 to current year.

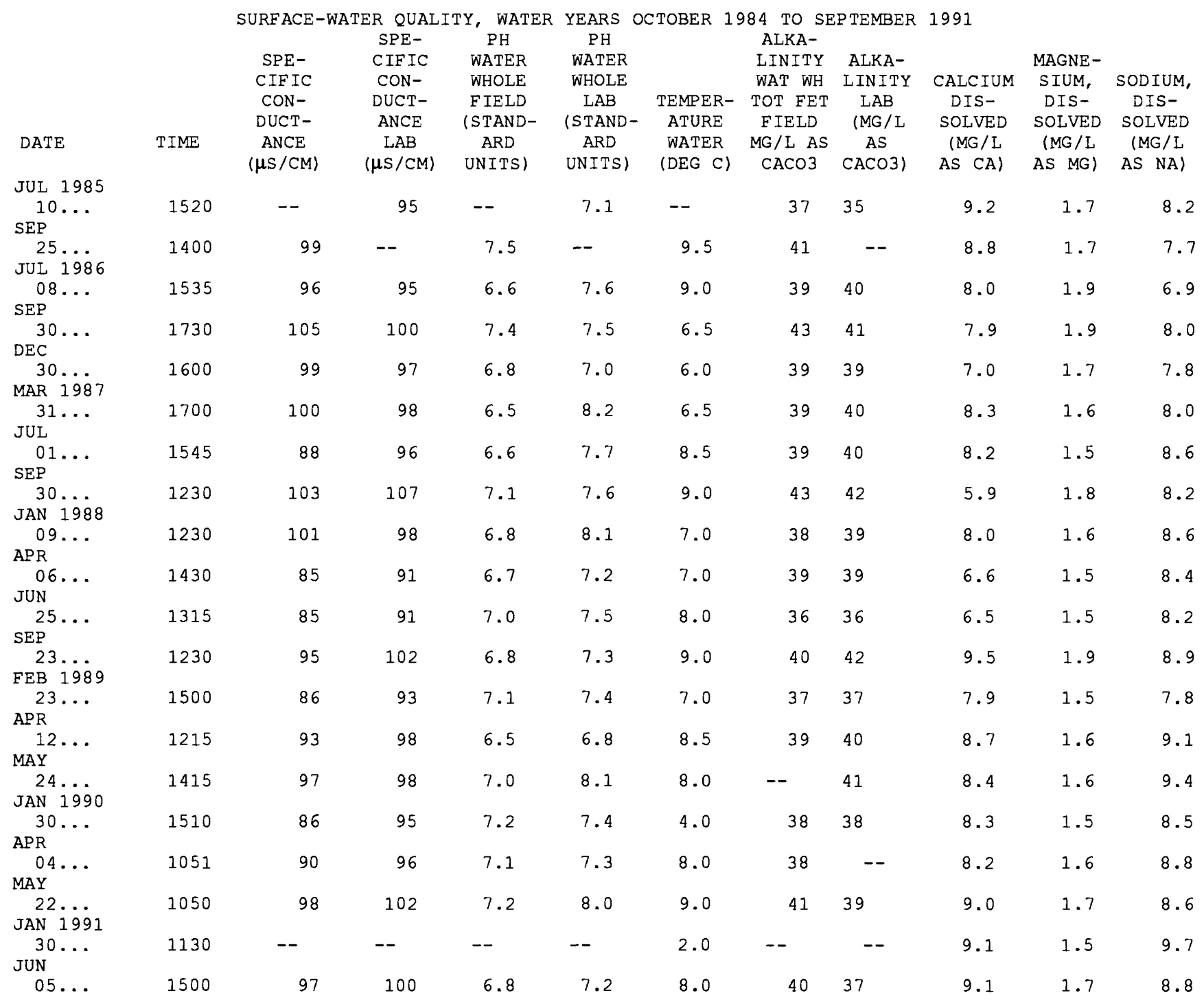


3 SPRINGS BASIN

3 SPRINGS CREEK NEAR 3 SPRING \#2, NEAR WARM SPRINGS, NV

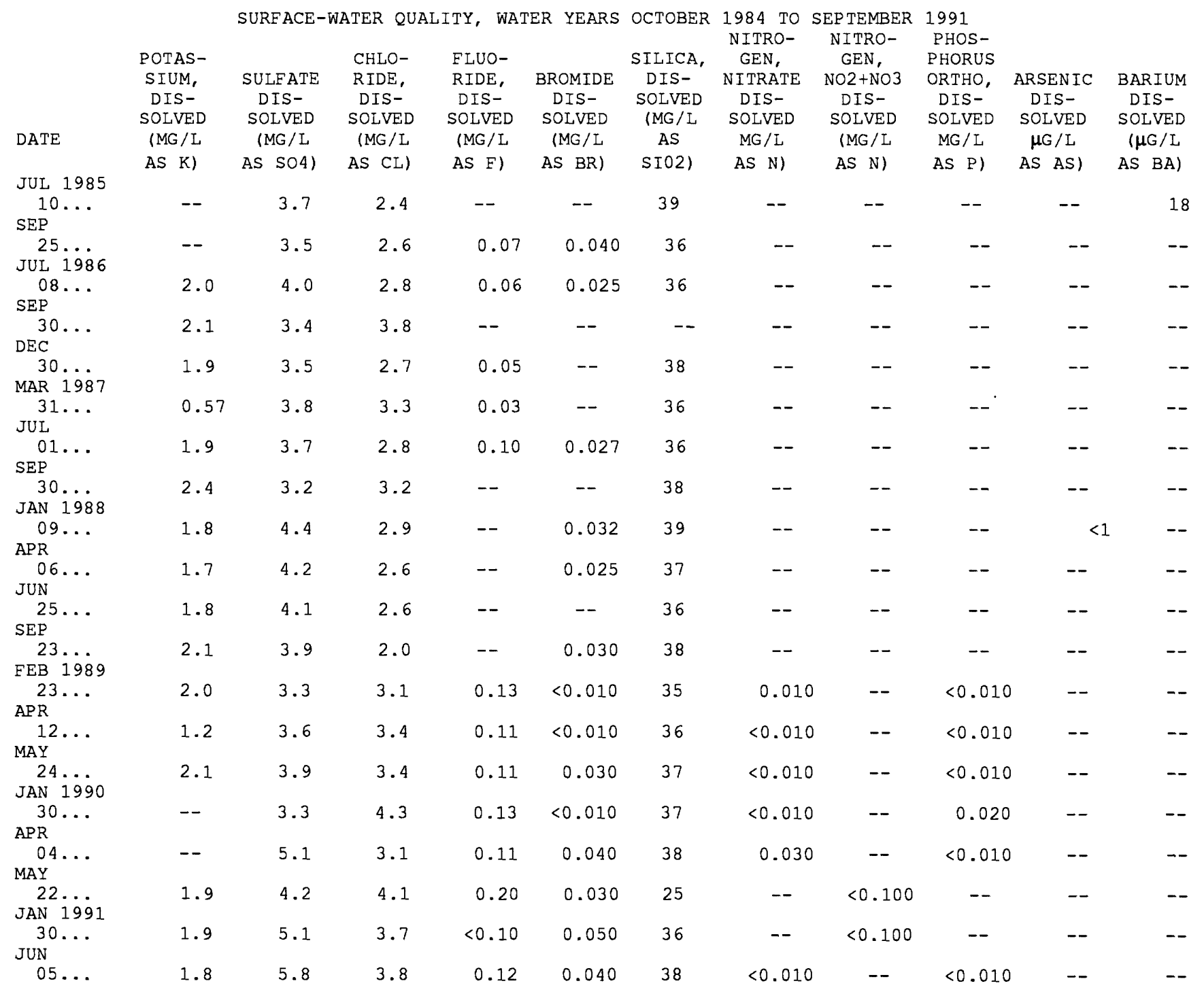




\section{SPRINGS BASIN}

3 SPRINGS CREEK NEAR 3 SPRING \#2, NEAR WARM SPRINGS, NV SURFACE-WATER QUALITY, WATER YEARS OCTOBER 1984 TO SEPTEMBER 1991

DATE

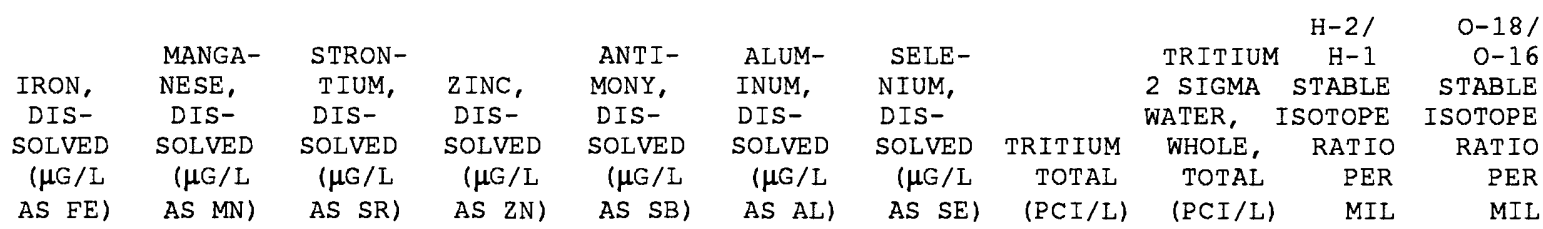

JUL 1985

$10 \ldots$

SEP

$25 \ldots$

JUL 1986

$08 \ldots$

SEP

$30 \ldots$

DEC

$30 \ldots$

MAR 1987

$31 \ldots$

JUL

$01 \ldots$

SEP

$30 \ldots$

JAN 1988

$09 \ldots$

APR

$06 .$.

JUN

25 ...

SEP

$23 .$.

FEB 1989

$23 \ldots$

APR

$12 \ldots$

MAY

$24 \ldots$

JAN 1990

$30 \ldots$

APR

$04 \ldots$

MAY

$22 \ldots$

JAN 1991

30 ...

JUN

$05 .$.

\begin{tabular}{|c|c|c|c|c|c|c|c|c|c|c|}
\hline 4 & 1 & 75 & 33 & -- & -- & -- & -- & -- & -112.0 & -13.89 \\
\hline 20 & 23 & 77 & -- & -- & -- & -- & -- & -- & -101.5 & -13.85 \\
\hline 9 & -- & 71 & -- & -- & -- & -- & -- & -- & -100.5 & -13.75 \\
\hline$<10$ & -- & 170 & -- & -- & -- & -- & -- & -- & -100.0 & -10.25 \\
\hline 7 & -- & 73 & -- & -- & -- & -- & -- & -- & -100.0 & -13.80 \\
\hline 36 & -- & 8 & -- & -- & -- & -- & -- & -- & -102.0 & -13.85 \\
\hline$<3$ & -- & 72 & -- & -- & -- & -- & 53 & 6.4 & -103.5 & -13.95 \\
\hline 5 & -- & 77 & -- & -- & -- & -- & -- & -- & $-102 \cdot 5$ & -13.95 \\
\hline$<3$ & -- & 81 & -- & $<1$ & $<10$ & $<1$ & 51 & 5.0 & -103.5 & -14.05 \\
\hline$<3$ & -- & 77 & -- & -- & -- & -- & -- & -- & -104.0 & -13.95 \\
\hline$<3$ & -- & 67 & -- & -- & -- & -- & -- & -- & -103.0 & -14.05 \\
\hline 5 & -- & 79 & -- & -- & $<10$ & -- & 54 & 5.8 & -102.0 & -14.00 \\
\hline 5 & $<1$ & 50 & -- & -- & -- & -- & 47 & 4.0 & -103.5 & -13.95 \\
\hline$<3$ & $<1$ & 60 & -- & -- & -- & -- & -- & -- & -101.5 & -14.00 \\
\hline$<3$ & -- & 78 & -- & -- & -- & -- & -- & -- & -106.0 & -14.00 \\
\hline$<3$ & $<1$ & -- & -- & -- & -- & -- & 44 & 4.0 & -105.0 & -13.90 \\
\hline 120 & 7 & -- & -- & -- & -- & -- & -- & -- & -103.0 & -14.05 \\
\hline 34 & 7 & -- & -- & -- & -- & -- & -- & -- & -102.0 & -13.95 \\
\hline 76 & 4 & -- & -- & -- & -- & -- & -- & -- & -104.0 & -14.00 \\
\hline 8 & 2 & -- & -- & -- & -- & -- & 41 & 3.0 & -106.0 & -14.30 \\
\hline
\end{tabular}




\section{SPRINGS BASIN}

3 SPRINGS CREEK NEAR 3 SPRING \#3, NEAR WARM SPRINGS, NV

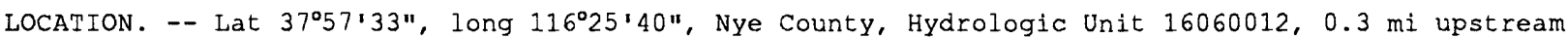
from the 3 springs Creek surface-water equipment, $0.5 \mathrm{mi}$ above the confluence of 3 springs Creek with Eden Creek, $3.0 \mathrm{mi}$ upstream from Eden Ranch in the Reveille Valley, $16.2 \mathrm{mi}$ south of Warm springs at the intersection of US Highway 6 and Nevada Highway 375.

DRAINAGE AREA. -- $1.25 \mathrm{mi}^{2}$.

PERIOD OF RECORD. -- January 1985 to March 1986.

SURFACE-WATER QUALITY, WATER YEARS OCTOBER 1984 TO SEPTEMBER 1986

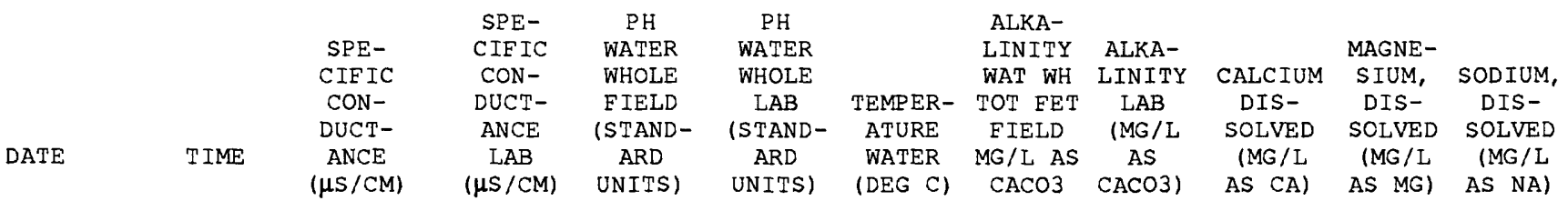

\begin{tabular}{|c|c|c|c|c|c|c|c|c|c|c|c|}
\hline $\begin{array}{c}\text { JAN } 1985 \\
19 \ldots \\
\text { APR }\end{array}$ & 1215 & -- & -- & -- & -- & -- & 42 & -- & 9.4 & 1.8 & 8.7 \\
\hline${ }_{\text {JUL }}^{03} \ldots$ & 1245 & -- & -- & -- & -- & -- & 33 & -- & $<5.0$ & 1.4 & 5.5 \\
\hline $\begin{array}{c}10 \ldots \\
\text { JAN } 1986\end{array}$ & 1634 & -- & 98 & -- & 7.7 & -- & 40 & -- & 9.4 & 1.7 & 8.6 \\
\hline $\operatorname{MAR}_{M}^{11} \cdots$ & 1530 & 49 & 11 & 7.1 & 7.3 & 5.0 & 38 & 1.0 & 8.0 & 1.5 & 7.7 \\
\hline $20 \ldots$ & 1400 & 85 & 84 & 8.1 & 8.0 & 7.0 & 30 & 31 & 6.7 & 1.4 & 7.0 \\
\hline
\end{tabular}

\begin{tabular}{|c|c|c|c|c|c|c|c|c|c|c|c|}
\hline 1 & $\begin{array}{l}\text { POTAS- } \\
\text { SIUM, } \\
\text { DIS- } \\
\text { SOLVED } \\
\text { (MG / L }\end{array}$ & $\begin{array}{l}\text { SULFATE } \\
\text { DIS - } \\
\text { SOLVED } \\
\text { (MG/L }\end{array}$ & $\begin{array}{l}\text { CHLO- } \\
\text { RIDE, } \\
\text { DIS- } \\
\text { SOLVED } \\
\text { (MG / L }\end{array}$ & $\begin{array}{l}\text { FLUO } \\
\text { RIDE, } \\
\text { DIS- } \\
\text { SOLVED } \\
\text { (MG / L }\end{array}$ & $\begin{array}{l}\text { BROMIDE } \\
\text { DIS- } \\
\text { SOLVED } \\
\text { (MG / L }\end{array}$ & $\begin{array}{l}\text { SILICA, } \\
\text { DIS- } \\
\text { SOLVED } \\
\text { (MG/L } \\
\text { AS }\end{array}$ & $\begin{array}{l}\text { BARIUM } \\
\text { DIS- } \\
\text { SOLVED } \\
\{\mu G / L\end{array}$ & $\begin{array}{l}\text { BERYL- } \\
\text { LIUM, } \\
\text { DIS- } \\
\text { SOLVED } \\
(\mu \mathrm{G} / \mathrm{L}\end{array}$ & $\begin{array}{l}\text { CADMIUM } \\
\text { DIS- } \\
\text { SOLVED } \\
(\mu G / L\end{array}$ & $\begin{array}{l}\text { COBALT } \\
\text { DIS- } \\
\text { SOLVED } \\
(\mu G / L\end{array}$ & $\begin{array}{c}\text { COPPER, } \\
\text { DIS- } \\
\text { SOLVED } \\
(\mu G / L\end{array}$ \\
\hline & AS $K$ ) & AS SO4) & AS CL) & AS F) & AS BR) & SI02) & AS BA) & AS BE) & AS CD) & AS $\mathrm{CO})$ & AS CU) \\
\hline
\end{tabular}

\begin{tabular}{|c|c|c|c|c|c|c|}
\hline $\begin{array}{c}\text { JAN } 1985 \\
19 \ldots\end{array}$ & 2.2 & 2.7 & 2.9 & 0.07 & 0.031 & 36 \\
\hline APR & & & & & & \\
\hline $03 \ldots$ & 2.2 & 4.3 & 2.9 & 0.09 & -- & 37 \\
\hline $\begin{array}{l}10 \ldots \\
\text { JAN } 1986\end{array}$ & -- & 3.6 & 2.5 & 0.07 & -- & 40 \\
\hline $11 \ldots$ & -- & 4.3 & 3.0 & 0.07 & -- & 36 \\
\hline $20 \ldots$ & 1.8 & 5.0 & 2.6 & 0.08 & -- & 36 \\
\hline
\end{tabular}

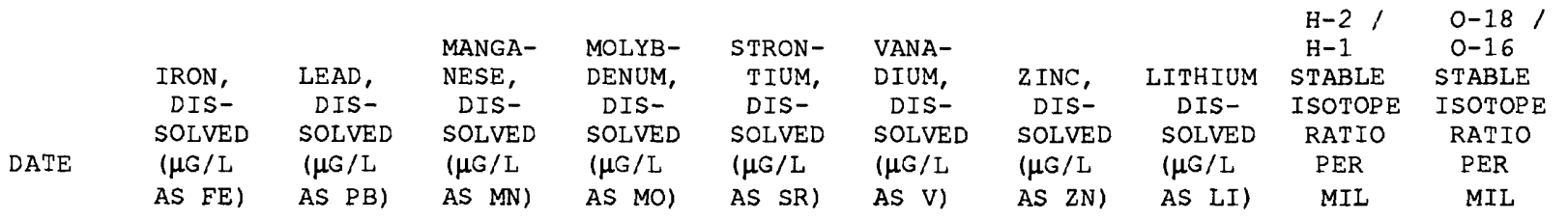

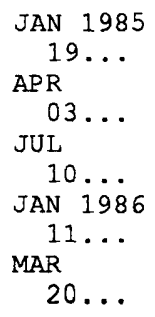

$\begin{aligned}-- & -- \\ - & -- \\ 4 & -- \\ <3 & <10 \\ <10 & --\end{aligned}$

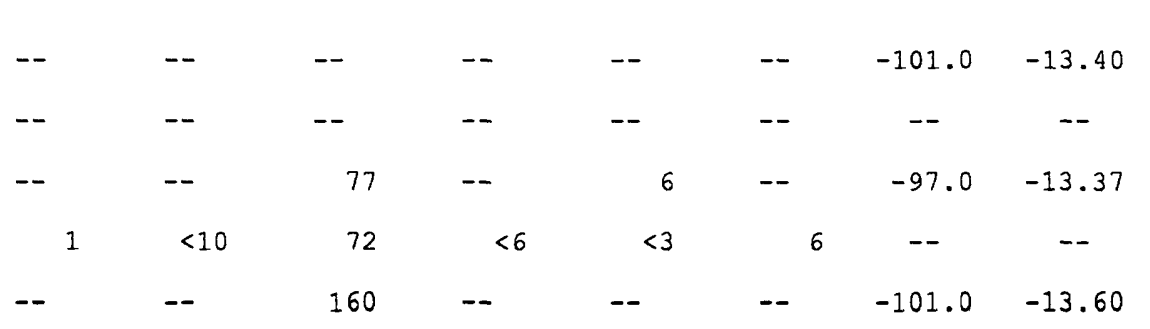




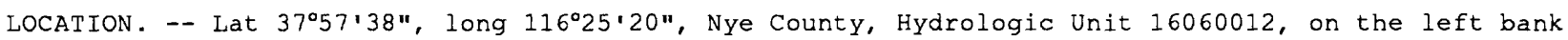
$0.2 \mathrm{mi}$ above the confluence of 3 springs Creek with Eden Creek, 2.7 mi upstream from Eden Ranch in the Reveille Valley, $16.1 \mathrm{mi}$ south of Warm springs at the intersection of US Highway 6 and Nevada Highway 375.

DRAINAGE AREA. - $1.62 \mathrm{mi}^{2}$.

PERIOD OF RECORD. -- October 1985 to current year.

GAGE. -- Two water-stage recorders on a 6 in Parshall flume and a standard 90 degree v-notch weir, and a crest stage gage. The flume is considered the primary gage. See remarks for more information.

REMARKS.-- The weather equipment is located $0.1 \mathrm{mi}$ downstream from the surface-water equipment. The surface-water-quality samples are taken upstream from the Parshall flume. The unsaturated-zone water-quality samples are taken approximately $0.1 \mathrm{mi}$ north and uphill from the meteorological site. Daily discharges for 1987 and 1988 are determined from data collected at the v-notch weir. During the 1987 water year, the discharge values are estimated because inadequate measurements were made to determine the stage-discharge relationship. Daily discharges for water year 1988 are considered fair because of an unstable stage-discharge relationship. The discharge data were lost from January 6, 1988 to April 6, 1988. This period was estimated from weather records and discharge measurements made on January 6, 1988 and April 6, 1988. Daily discharge values for water years 1989, 1990, and 1991 are determined from the 6 in Parshall flume. Accuracy of measurement by the flume diminishes below a discharge of $0.16 \mathrm{ft}^{3} / \mathrm{s}$. Any daily discharges below $0.16 \mathrm{ft}^{3} / \mathrm{s} \mathrm{reported}$ during the years 1989 and 1991 are considered fair to poor; discharges above $0.16 \mathrm{ft}^{3} / \mathrm{s}$ are considered good to excellent. No flow was recorded or observed during the 1990 water year. The precipitation water-quality sample on July 10, 1985 was lost. During visits when the unsaturatedzone lysimeter was checked and found empty, the container was cleaned and replaced. Estimated periods of daily record are considered poor. Values for the daily maximum and minimum air temperature were obtained from the 40 minute average values.

PERIOD OF DAILY RECORD. --

AIR TEMPERATURE: October 1985 to current year.

SOIL TEMPERATURE: October 1985 to current year.

SOLAR RADIATION: October 1985 to current year.

RELATIVE HUMIDITY: October 1990 to current year.

TOTAL PRECIPITATION: October 1985 to current year.

DISCHARGE: October 1986 to current year.

EXTREMES FOR PERIOD OF RECORD. --

AIR TEMPERATURE: Maximum daily, $32.2^{\circ} \mathrm{C}$, July 7, 1989 and July 4, 1991; minimum daily, $-28.8^{\circ} \mathrm{C}$, Feb. 6, 1989. See remarks for more information.

SOIL TEMPERATURE: Maximum daily mean, $31.7^{\circ} \mathrm{C}$, July 7, 1990; minimum daily mean, $-7.4^{\circ} \mathrm{C}, \mathrm{DeC}^{3} 30$, 1990.

SOLAR RADIATION: Maximum daily total, $827 \mathrm{cal} / \mathrm{cm}^{2}$, June 18, 1989; minimum daily total, $9 \mathrm{cal} / \mathrm{cm}^{2}$, Nov. 11, 1985 and Jan. 17, 1990.

RELATIVE HUMIDITY: Maximum daily mean, 99 pct, Jan 4, 1991, and Feb. 4, 1991; minimum daily mean, 12 pet, July 5, 1991.

TOTAL, PRECIPITATION: Maximum daily, 1.50 in, Nov. 1, 1987; no precipitation, most days of record. DISCHARGE: Maximum daily mean, $2.1 \mathrm{ft}^{3} / \mathrm{s}, \mathrm{Apr} .29-30,1988$; no flow, most days of record. 
3 SPRINGS BASIN

3 SPRINGS CREEK NEAR WARM SPRINGS, NV

DAILY MEAN AIR TEMPERATURE, WATER YEAR OCTOBER 1985 TO SEPTEMBER 1986 DEGREES CELSIUS

\begin{tabular}{|c|c|c|c|c|c|c|c|c|c|c|c|c|}
\hline DAY & $\mathrm{OCT}$ & NOV & DEC & JAN & FEB & MAR & APR & MAY & JUN & JUL & AUG & SEP \\
\hline $\begin{array}{l}1 \\
2 \\
3 \\
4 \\
5\end{array}$ & $\begin{array}{r}\text { e10.4 } \\
\text { e } 9.5 \\
\text { e12.2 } \\
\text { e12.7 } \\
\text { e12.7 }\end{array}$ & $\begin{array}{l}1.3 \\
4.3 \\
7.6 \\
9.0 \\
5.8\end{array}$ & $\begin{array}{r}.2 \\
.2 \\
-.4 \\
-1.3 \\
1.3\end{array}$ & $\begin{array}{r}1.4 \\
.1 \\
.1 \\
.1 \\
2.4\end{array}$ & $\begin{array}{r}1.0 \\
2.4 \\
.5 \\
-2.3 \\
-3.2\end{array}$ & $\begin{array}{r}11.3 \\
4.5 \\
5.9 \\
5.5 \\
6.0\end{array}$ & $\begin{array}{r}7.0 \\
-1.1 \\
1.8 \\
5.1 \\
5.7\end{array}$ & $\begin{array}{r}14.6 \\
15.1 \\
12.6 \\
2.3 \\
4.6\end{array}$ & $\begin{array}{l}19.0 \\
18.4 \\
19.4 \\
19.8 \\
18.3\end{array}$ & $\begin{array}{l}23.0 \\
24.1 \\
23.4 \\
22.8 \\
16.5\end{array}$ & $\begin{array}{l}24.3 \\
25.1 \\
25.3 \\
23.3 \\
23.4\end{array}$ & $\begin{array}{l}16.1 \\
15.5 \\
15.8 \\
17.9 \\
19.9\end{array}$ \\
\hline $\begin{array}{r}6 \\
7 \\
8 \\
9 \\
10\end{array}$ & $\begin{array}{r}\text { e13.3 } \\
\text { e } 9.5 \\
\text { e } 8.4 \\
e .5 \\
\text { e3.7 }\end{array}$ & $\begin{array}{r}2.0 \\
6.6 \\
7.8 \\
-1.3 \\
-.5\end{array}$ & $\begin{array}{r}-1.5 \\
-5.9 \\
-5.9 \\
-8.6 \\
-9.0\end{array}$ & $\begin{array}{r}.7 \\
-4.3 \\
-3.9 \\
.5 \\
1.0\end{array}$ & $\begin{array}{r}-7.5 \\
-9.4 \\
-8.9 \\
-10.4 \\
-4.6\end{array}$ & $\begin{array}{r}7.2 \\
7.1 \\
4.2 \\
.7 \\
-.3\end{array}$ & $\begin{array}{r}1.9 \\
.3 \\
2.4 \\
6.4 \\
6.7\end{array}$ & $\begin{array}{r}.9 \\
2.0 \\
3.2 \\
6.8 \\
10.5\end{array}$ & $\begin{array}{l}18.3 \\
17.2 \\
13.0 \\
13.1 \\
13.8\end{array}$ & $\begin{array}{l}19.0 \\
18.7 \\
19.6 \\
20.5 \\
19.7\end{array}$ & $\begin{array}{l}24.6 \\
24.5 \\
24.0 \\
24.0 \\
19.6\end{array}$ & $\begin{array}{r}22.9 \\
21.0 \\
17.9 \\
13.8 \\
8.9\end{array}$ \\
\hline $\begin{array}{l}11 \\
12 \\
13 \\
14 \\
15\end{array}$ & $\begin{array}{l}6.8 \\
6.9 \\
1.6 \\
2.4 \\
4.0\end{array}$ & $\begin{array}{r}-5.3 \\
-8.9 \\
-10.8 \\
-9.5 \\
-4.2\end{array}$ & $\begin{array}{r}-15.8 \\
-12.7 \\
-9.2 \\
-4.8 \\
-4.3\end{array}$ & $\begin{array}{r}.6 \\
1.3 \\
.0 \\
1.6 \\
1.6\end{array}$ & $\begin{array}{r}.1 \\
-.8 \\
2.0 \\
3.9 \\
1.4\end{array}$ & $\begin{array}{r}.9 \\
-.2 \\
-2.6 \\
-2.4 \\
-.5\end{array}$ & $\begin{array}{l}9.0 \\
6.2 \\
-.3 \\
3.4 \\
8.0\end{array}$ & $\begin{array}{r}8.6 \\
7.8 \\
10.2 \\
11.1 \\
11.1\end{array}$ & $\begin{array}{l}18.7 \\
17.9 \\
18.6 \\
21.2 \\
18.7\end{array}$ & $\begin{array}{l}21.9 \\
23.2 \\
22.9 \\
22.9 \\
16.0\end{array}$ & $\begin{array}{l}20.6 \\
23.2 \\
21.1 \\
22.2 \\
23.8\end{array}$ & $\begin{array}{l}12.9 \\
15.6 \\
14.8 \\
13.3 \\
10.8\end{array}$ \\
\hline $\begin{array}{l}16 \\
17 \\
18 \\
19 \\
20\end{array}$ & $\begin{array}{r}5.8 \\
5.8 \\
7.3 \\
10.2 \\
11.5\end{array}$ & $\begin{array}{r}-1.4 \\
-4.4 \\
-9.1 \\
-12.2 \\
-2.1\end{array}$ & $\begin{array}{r}-4.8 \\
-4.2 \\
-1.7 \\
-.6 \\
-1.4\end{array}$ & $\begin{array}{l}2.2 \\
2.4 \\
1.8 \\
5.3 \\
1.8\end{array}$ & $\begin{array}{r}2.4 \\
4.5 \\
4.9 \\
4.7 \\
.4\end{array}$ & $\begin{array}{r}-2.7 \\
-2.9 \\
-1.5 \\
-.3 \\
3.2\end{array}$ & $\begin{array}{r}1.9 \\
.3 \\
1.2 \\
4.5 \\
9.3\end{array}$ & $\begin{array}{r}8.5 \\
8.7 \\
14.4 \\
19.0 \\
17.5\end{array}$ & $\begin{array}{l}21.5 \\
21.5 \\
20.0 \\
15.6 \\
17.5\end{array}$ & $\begin{array}{l}14.8 \\
16.0 \\
18.4 \\
20.4 \\
20.0\end{array}$ & $\begin{array}{l}22.8 \\
22.8 \\
21.0 \\
19.0 \\
17.7\end{array}$ & $\begin{array}{r}10.6 \\
12.2 \\
10.2 \\
9.5 \\
5.4\end{array}$ \\
\hline $\begin{array}{l}21 \\
22 \\
23 \\
24 \\
25\end{array}$ & $\begin{array}{l}4.3 \\
-.7 \\
7.6 \\
8.4 \\
9.0\end{array}$ & $\begin{array}{r}-2.3 \\
1.8 \\
2.0 \\
2.0 \\
.7\end{array}$ & $\begin{array}{r}-2.0 \\
-1.1 \\
-.7 \\
-.8 \\
-1.8\end{array}$ & $\begin{array}{r}-3.1 \\
.7 \\
1.6 \\
-3.5 \\
-2.0\end{array}$ & $\begin{array}{l}.8 \\
3.1 \\
5.6 \\
5.6 \\
6.9\end{array}$ & $\begin{array}{l}6.4 \\
8.9 \\
9.4 \\
9.5 \\
5.0\end{array}$ & $\begin{array}{r}13.1 \\
14.7 \\
10.0 \\
6.6 \\
6.6\end{array}$ & $\begin{array}{r}11.7 \\
5.5 \\
12.1 \\
13.9 \\
18.0\end{array}$ & $\begin{array}{l}18.6 \\
20.5 \\
20.6 \\
22.0 \\
20.5\end{array}$ & $\begin{array}{l}17.0 \\
13.7 \\
13.8 \\
15.6 \\
16.9\end{array}$ & $\begin{array}{l}17.2 \\
18.7 \\
20.6 \\
21.7 \\
17.8\end{array}$ & $\begin{array}{l}6.9 \\
8.6 \\
7.2 \\
6.5 \\
2.5\end{array}$ \\
\hline $\begin{array}{l}26 \\
27 \\
28 \\
29 \\
30 \\
31\end{array}$ & $\begin{array}{l}5.5 \\
8.9 \\
9.0 \\
7.3 \\
9.8 \\
2.0\end{array}$ & $\begin{array}{r}-1.7 \\
3.0 \\
3.4 \\
-.6 \\
-2.6 \\
---\end{array}$ & $\begin{array}{r}-1.8 \\
-1.4 \\
-1.3 \\
.7 \\
2.3 \\
.5\end{array}$ & $\begin{array}{r}-.8 \\
.7 \\
5.4 \\
7.6 \\
2.1 \\
2.4\end{array}$ & $\begin{array}{l}6.3 \\
6.1 \\
8.6 \\
--- \\
--- \\
---\end{array}$ & $\begin{array}{r}6.7 \\
8.8 \\
10.1 \\
9.5 \\
10.8 \\
7.6\end{array}$ & $\begin{array}{r}-.1 \\
7.2 \\
12.2 \\
9.5 \\
10.3 \\
-.--\end{array}$ & $\begin{array}{l}22.0 \\
22.0 \\
20.0 \\
18.0 \\
17.9 \\
19.0\end{array}$ & $\begin{array}{r}22.5 \\
24.1 \\
21.7 \\
18.9 \\
21.0 \\
---\end{array}$ & $\begin{array}{l}16.1 \\
19.7 \\
20.0 \\
18.1 \\
19.6 \\
22.5\end{array}$ & $\begin{array}{l}18.0 \\
15.1 \\
17.2 \\
20.7 \\
19.6 \\
16.9\end{array}$ & $\begin{array}{l}4.8 \\
5.7 \\
3.1 \\
4.5 \\
7.6 \\
---\end{array}$ \\
\hline $\begin{array}{l}\text { MEAN } \\
\text { MAX } \\
\text { MIN } \\
\text { MED }\end{array}$ & $\begin{array}{r}7.3 \\
13.3 \\
-.7 \\
7.6\end{array}$ & $\begin{array}{r}-.7 \\
9.0 \\
-12.2 \\
-.6\end{array}$ & $\begin{array}{r}-2.9 \\
2.3 \\
-15.8 \\
-1.4\end{array}$ & $\begin{array}{r}.9 \\
7.6 \\
-4.3 \\
1.0\end{array}$ & $\begin{array}{r}.9 \\
8.6 \\
-10.4 \\
1.7\end{array}$ & $\begin{array}{r}4.4 \\
11.3 \\
-2.9 \\
5.5\end{array}$ & $\begin{array}{r}5.7 \\
14.7 \\
-1.1 \\
6.3\end{array}$ & $\begin{array}{r}11.9 \\
22.0 \\
.9 \\
11.7\end{array}$ & $\begin{array}{l}19.1 \\
24.1 \\
13.0 \\
19.0\end{array}$ & $\begin{array}{l}19.3 \\
24.1 \\
13.7 \\
19.6\end{array}$ & $\begin{array}{l}21.2 \\
25.3 \\
15.1 \\
21.1\end{array}$ & $\begin{array}{r}11.4 \\
22.9 \\
2.5 \\
10.7\end{array}$ \\
\hline
\end{tabular}

WTR YR 1986 MEAN 8.2 MAX 25.3 MIN -15.8 MED 7.0

e Estimated 


\section{SPRINGS BASIN}

3 SPRINGS CREEK NEAR WARM SPRINGS, NV

DAILY MEAN AIR TEMPERATURE, WATER YEAR OCTOBER 1986 TO SEPTEMBER 1987 DEGREES CELSIUS

\begin{tabular}{|c|c|c|c|c|c|c|c|c|c|c|c|c|}
\hline DAY & $\mathrm{OCT}$ & NOV & DEC & JAN & FEB & MAR & APR & MAY & JUN & JUL & AUG & SEP \\
\hline $\begin{array}{l}1 \\
2 \\
3 \\
4 \\
5\end{array}$ & $\begin{array}{l}4.0 \\
2.1 \\
2.9 \\
3.9 \\
7.0\end{array}$ & $\begin{array}{l}2.7 \\
5.1 \\
8.8 \\
4.9 \\
7.4\end{array}$ & $\begin{array}{r}-1.8 \\
.2 \\
.3 \\
3.3 \\
4.4\end{array}$ & $\begin{array}{r}1.9 \\
-.7 \\
3.6 \\
-.4 \\
-4.3\end{array}$ & $\begin{array}{r}1.5 \\
4.7 \\
.6 \\
-3.7 \\
-2.3\end{array}$ & $\begin{array}{r}-1.5 \\
1.3 \\
3.2 \\
6.3 \\
5.8\end{array}$ & $\begin{array}{l}6.1 \\
8.5 \\
4.0 \\
2.1 \\
4.0\end{array}$ & $\begin{array}{r}8.4 \\
4.6 \\
7.3 \\
9.7 \\
11.7\end{array}$ & $\begin{array}{l}12.5 \\
13.0 \\
15.6 \\
18.3 \\
15.3\end{array}$ & $\begin{array}{l}\text { e18.7 } \\
\text { e17.8 } \\
\text { e17.8 } \\
\text { e17.8 } \\
\text { e18.7 }\end{array}$ & $\begin{array}{l}23.5 \\
23.2 \\
22.0 \\
24.1 \\
24.8\end{array}$ & $\begin{array}{l}20.6 \\
19.9 \\
17.9 \\
16.4 \\
17.4\end{array}$ \\
\hline $\begin{array}{r}6 \\
7 \\
8 \\
9 \\
10\end{array}$ & $\begin{array}{r}6.6 \\
8.6 \\
9.1 \\
9.1 \\
10.3\end{array}$ & $\begin{array}{r}.4 \\
-3.1 \\
-2.7 \\
-2.3 \\
-1.6\end{array}$ & $\begin{array}{r}.2 \\
-1.8 \\
-3.6 \\
-7.7 \\
-7.5\end{array}$ & $\begin{array}{r}-7.6 \\
-8.3 \\
-13.9 \\
-11.3 \\
-6.8\end{array}$ & $\begin{array}{l}.0 \\
1.8 \\
1.1 \\
1.8 \\
2.9\end{array}$ & $\begin{array}{r}2.7 \\
2.2 \\
2.0 \\
.9 \\
1.9\end{array}$ & $\begin{array}{l}4.1 \\
5.3 \\
7.2 \\
6.9 \\
8.2\end{array}$ & $\begin{array}{l}13.0 \\
11.9 \\
11.8 \\
12.6 \\
11.9\end{array}$ & $\begin{array}{l}11.1 \\
13.7 \\
12.8 \\
13.2 \\
16.3\end{array}$ & $\begin{array}{r}\text { e17.8 } \\
\text { e19.5 } \\
\text { e19.5 } \\
21.7 \\
21.1\end{array}$ & $\begin{array}{l}23.3 \\
21.4 \\
18.1 \\
20.6 \\
23.7\end{array}$ & $\begin{array}{l}13.4 \\
14.6 \\
18.5 \\
18.1 \\
18.4\end{array}$ \\
\hline $\begin{array}{l}11 \\
12 \\
13 \\
14 \\
15\end{array}$ & $\begin{array}{l}3.8 \\
2.6 \\
3.9 \\
5.4 \\
7.7\end{array}$ & $\begin{array}{r}.3 \\
1.1 \\
4.3 \\
7.2 \\
6.4\end{array}$ & $\begin{array}{l}-5.6 \\
-2.2 \\
-2.1 \\
-1.9 \\
-2.0\end{array}$ & $\begin{array}{r}-4.1 \\
-5.2 \\
-5.4 \\
-9.3 \\
-13.1\end{array}$ & $\begin{array}{l}2.9 \\
4.2 \\
3.3 \\
-.9 \\
1.0\end{array}$ & $\begin{array}{r}4.4 \\
6.9 \\
2.6 \\
1.2 \\
-2.1\end{array}$ & $\begin{array}{r}9.0 \\
1.7 \\
6.6 \\
10.9 \\
12.5\end{array}$ & $\begin{array}{l}12.5 \\
12.9 \\
14.1 \\
14.3 \\
12.0\end{array}$ & $\begin{array}{l}17.0 \\
20.3 \\
18.8 \\
19.9 \\
15.2\end{array}$ & $\begin{array}{l}18.1 \\
19.7 \\
20.2 \\
21.7 \\
22.3\end{array}$ & $\begin{array}{l}22.3 \\
17.9 \\
19.8 \\
16.4 \\
15.5\end{array}$ & $\begin{array}{l}17.4 \\
16.1 \\
11.6 \\
16.2 \\
16.6\end{array}$ \\
\hline $\begin{array}{l}16 \\
17 \\
18 \\
19 \\
20\end{array}$ & $\begin{array}{r}8.8 \\
7.7 \\
1.1 \\
.5 \\
1.8\end{array}$ & $\begin{array}{l}5.4 \\
7.6 \\
5.2 \\
4.6 \\
4.6\end{array}$ & $\begin{array}{r}-2.5 \\
-3.9 \\
-1.1 \\
-.6 \\
-2.7\end{array}$ & $\begin{array}{r}-12.5 \\
-8.9 \\
-6.9 \\
-7.9 \\
-10.6\end{array}$ & $\begin{array}{l}-2.4 \\
-3.0 \\
-4.8 \\
-6.8 \\
-4.9\end{array}$ & $\begin{array}{r}-.5 \\
1.8 \\
2.5 \\
-4.8 \\
-4.2\end{array}$ & $\begin{array}{r}14.2 \\
16.3 \\
7.7 \\
-.6 \\
3.1\end{array}$ & $\begin{array}{r}9.6 \\
10.1 \\
11.3 \\
10.0 \\
8.1\end{array}$ & $\begin{array}{l}14.4 \\
17.5 \\
16.2 \\
16.7 \\
19.5\end{array}$ & $\begin{array}{l}17.1 \\
14.1 \\
10.4 \\
14.1 \\
13.2\end{array}$ & $\begin{array}{l}17.5 \\
20.2 \\
21.4 \\
22.8 \\
21.9\end{array}$ & $\begin{array}{l}13.6 \\
11.0 \\
15.3 \\
14.8 \\
14.0\end{array}$ \\
\hline $\begin{array}{l}21 \\
22 \\
23 \\
24 \\
25\end{array}$ & $\begin{array}{l}2.8 \\
4.5 \\
6.9 \\
7.3 \\
7.1\end{array}$ & $\begin{array}{r}4.5 \\
-2.0 \\
-2.0 \\
3.0 \\
.7\end{array}$ & $\begin{array}{l}-5.8 \\
-4.7 \\
-3.1 \\
-5.3 \\
-7.2\end{array}$ & $\begin{array}{r}-5.5 \\
2.3 \\
-.8 \\
-1.4 \\
1.9\end{array}$ & $\begin{array}{l}-5.6 \\
-2.7 \\
-5.9 \\
-8.6 \\
-9.6\end{array}$ & $\begin{array}{r}-3.9 \\
-3.7 \\
-3.1 \\
-.8 \\
-.2\end{array}$ & $\begin{array}{r}7.1 \\
11.3 \\
14.0 \\
12.8 \\
13.3\end{array}$ & $\begin{array}{r}3.8 \\
6.3 \\
9.4 \\
10.4 \\
4.6\end{array}$ & $\begin{array}{l}20.1 \\
13.4 \\
16.9 \\
20.0 \\
21.6\end{array}$ & $\begin{array}{l}13.4 \\
15.4 \\
17.8 \\
21.2 \\
22.0\end{array}$ & $\begin{array}{l}21.0 \\
20.6 \\
19.5 \\
18.4 \\
16.4\end{array}$ & $\begin{array}{l}14.1 \\
14.8 \\
14.6 \\
13.9 \\
16.5\end{array}$ \\
\hline $\begin{array}{l}26 \\
27 \\
28 \\
29 \\
30 \\
31\end{array}$ & $\begin{array}{r}9.7 \\
12.9 \\
10.2 \\
11.3 \\
10.4 \\
3.3\end{array}$ & $\begin{array}{r}-1.2 \\
5.8 \\
7.9 \\
.9 \\
-4.9 \\
---\end{array}$ & $\begin{array}{l}-4.7 \\
-2.8 \\
-4.0 \\
-2.7 \\
-5.0 \\
-2.1\end{array}$ & $\begin{array}{r}9.1 \\
7.5 \\
-.1 \\
-2.0 \\
-1.3 \\
-2.2\end{array}$ & $\begin{array}{r}-9.0 \\
-9.9 \\
-5.6 \\
--- \\
--- \\
---\end{array}$ & $\begin{array}{r}1.5 \\
-2.8 \\
-2.6 \\
-2.3 \\
1.0 \\
4.9\end{array}$ & $\begin{array}{r}12.9 \\
11.7 \\
12.5 \\
9.8 \\
10.4 \\
-.\end{array}$ & $\begin{array}{r}2.5 \\
7.0 \\
7.4 \\
8.8 \\
12.5 \\
16.1\end{array}$ & $\begin{array}{r}22.0 \\
21.2 \\
19.3 \\
17.8 \\
19.6 \\
---\end{array}$ & $\begin{array}{l}22.0 \\
19.5 \\
20.3 \\
20.9 \\
20.4 \\
22.8\end{array}$ & $\begin{array}{l}14.9 \\
17.1 \\
20.8 \\
19.7 \\
19.0 \\
19.9\end{array}$ & $\begin{array}{r}16.0 \\
\mathrm{e} 13.1 \\
\mathrm{e} 11.9 \\
\mathrm{e} 13.1 \\
\mathrm{e} 14.2 \\
\\
.\end{array}$ \\
\hline $\begin{array}{l}\text { MEAN } \\
\text { MAX } \\
\text { MIN } \\
\text { MED }\end{array}$ & $\begin{array}{r}6.2 \\
12.9 \\
.5 \\
6.9\end{array}$ & $\begin{array}{r}2.6 \\
8.8 \\
-4.9 \\
3.7\end{array}$ & $\begin{array}{r}-2.8 \\
4.4 \\
-7.7 \\
-2.7\end{array}$ & $\begin{array}{r}-4.0 \\
9.1 \\
-13.9 \\
-4.3\end{array}$ & $\begin{array}{r}-2.1 \\
4.7 \\
-9.9 \\
-2.4\end{array}$ & $\begin{array}{r}.7 \\
6.9 \\
-4.8 \\
1.2\end{array}$ & $\begin{array}{r}8.5 \\
16.3 \\
-.6 \\
8.4\end{array}$ & $\begin{array}{r}9.9 \\
16.1 \\
2.5 \\
10.1\end{array}$ & $\begin{array}{l}17.0 \\
22.0 \\
11.1 \\
17.0\end{array}$ & $\begin{array}{l}18.6 \\
22.8 \\
10.4 \\
19.5\end{array}$ & $\begin{array}{l}20.2 \\
24.8 \\
14.9 \\
20.6\end{array}$ & $\begin{array}{l}15.5 \\
20.6 \\
11.0 \\
15.0\end{array}$ \\
\hline
\end{tabular}

CAL YR 1986 MEAN 8.4 MAX 25.3 MIN -10.4 MED 6.9 WTR YR 1987 MEAN 7.6 MAX $24.8 \quad$ MIN -13.9 MED 7.3

e Estimated 
3 SPRINGS BASIN

3 SPRINGS CREEK NEAR WARM SPRINGS, NV

DAILY MEAN AIR TEMPERATURE, WATER YEAR OCTOBER 1987 TO SEPTEMBER 1988 DEGREES CELSIUS

\begin{tabular}{|c|c|c|c|c|c|c|c|c|c|c|c|c|}
\hline DAY & OCT & NOV & DEC & JAN & EEB & MAR & APR & MAY & JUN & JUL & AUG & SEP \\
\hline $\begin{array}{l}1 \\
2 \\
3 \\
4 \\
5\end{array}$ & $\begin{array}{l}13.7 \\
14.5 \\
14.5 \\
14.8 \\
13.2\end{array}$ & $\begin{array}{l}5.9 \\
4.4 \\
3.1 \\
6.0 \\
2.8\end{array}$ & $\begin{array}{r}.8 \\
1.0 \\
3.5 \\
6.0 \\
-.2\end{array}$ & $\begin{array}{r}-10.2 \\
-6.6 \\
-3.0 \\
-1.2 \\
-2.1\end{array}$ & $\begin{array}{l}-6.6 \\
-5.8 \\
-9.1 \\
-7.1 \\
-5.8\end{array}$ & $\begin{array}{r}.9 \\
.4 \\
2.0 \\
2.3 \\
3.8\end{array}$ & $\begin{array}{r}3.8 \\
7.9 \\
10.5 \\
8.3 \\
7.0\end{array}$ & $\begin{array}{r}-1.8 \\
3.0 \\
7.4 \\
8.1 \\
3.4\end{array}$ & $\begin{array}{l}13.6 \\
16.8 \\
21.3 \\
19.9 \\
11.6\end{array}$ & $\begin{array}{l}22.9 \\
23.9 \\
23.6 \\
21.0 \\
20.0\end{array}$ & $\begin{array}{l}22.2 \\
21.7 \\
18.9 \\
19.0 \\
17.1\end{array}$ & $\begin{array}{l}18.7 \\
18.9 \\
18.6 \\
19.4 \\
19.9\end{array}$ \\
\hline $\begin{array}{r}6 \\
7 \\
8 \\
9 \\
10\end{array}$ & $\begin{array}{l}15.1 \\
17.0 \\
14.0 \\
15.2 \\
12.1\end{array}$ & $\begin{array}{l}2.4 \\
3.6 \\
2.5 \\
2.6 \\
1.7\end{array}$ & $\begin{array}{r}1.1 \\
-.8 \\
-2.4 \\
4.9 \\
7.4\end{array}$ & $\begin{array}{r}-5.6 \\
-4.7 \\
-3.4 \\
1.5 \\
4.7\end{array}$ & $\begin{array}{r}-3.9 \\
-1.5 \\
2.7 \\
3.0 \\
.5\end{array}$ & $\begin{array}{r}5.9 \\
-1.4 \\
1.8 \\
2.0 \\
-5.6\end{array}$ & $\begin{array}{r}13.8 \\
13.7 \\
.8 \\
2.6 \\
7.7\end{array}$ & $\begin{array}{r}-1.1 \\
.6 \\
4.6 \\
9.4 \\
10.7\end{array}$ & $\begin{array}{r}8.0 \\
7.3 \\
9.4 \\
15.1 \\
16.8\end{array}$ & $\begin{array}{l}20.4 \\
20.6 \\
23.7 \\
22.2 \\
22.3\end{array}$ & $\begin{array}{l}15.1 \\
14.5 \\
15.5 \\
16.9 \\
20.0\end{array}$ & $\begin{array}{l}22.0 \\
22.2 \\
22.0 \\
18.4 \\
16.2\end{array}$ \\
\hline $\begin{array}{l}11 \\
12 \\
13 \\
14 \\
15\end{array}$ & $\begin{array}{r}11.9 \\
9.5 \\
7.4 \\
6.2 \\
7.8\end{array}$ & $\begin{array}{r}2.4 \\
3.8 \\
4.5 \\
.0 \\
-2.9\end{array}$ & $\begin{array}{r}-1.1 \\
-9.4 \\
-9.9 \\
-11.6 \\
-9.4\end{array}$ & $\begin{array}{r}-2.5 \\
-10.3 \\
-3.2 \\
1.4 \\
.1\end{array}$ & $\begin{array}{r}-1.1 \\
1.8 \\
2.2 \\
-3.2 \\
3.5\end{array}$ & $\begin{array}{r}-6.7 \\
-6.3 \\
-3.7 \\
.8 \\
-.6\end{array}$ & $\begin{array}{r}11.5 \\
12.5 \\
9.3 \\
3.3 \\
1.3\end{array}$ & $\begin{array}{l}13.2 \\
16.8 \\
13.7 \\
13.0 \\
17.8\end{array}$ & $\begin{array}{l}15.8 \\
12.7 \\
13.7 \\
16.6 \\
18.8\end{array}$ & $\begin{array}{l}21.8 \\
21.7 \\
22.6 \\
23.7 \\
24.0\end{array}$ & $\begin{array}{l}18.1 \\
16.9 \\
18.5 \\
19.6 \\
16.8\end{array}$ & $\begin{array}{r}8.5 \\
5.2 \\
8.4 \\
10.6 \\
14.8\end{array}$ \\
\hline $\begin{array}{l}16 \\
17 \\
18 \\
19 \\
20\end{array}$ & $\begin{array}{l}7.2 \\
8.8 \\
7.8 \\
5.8 \\
6.7\end{array}$ & $\begin{array}{r}3.3 \\
2.9 \\
-1.2 \\
-.9 \\
4.1\end{array}$ & $\begin{array}{l}-5.8 \\
-4.1 \\
-4.7 \\
-5.5 \\
-8.4\end{array}$ & $\begin{array}{r}-4.9 \\
-5.5 \\
-11.3 \\
-15.9 \\
-9.4\end{array}$ & $\begin{array}{l}-3.1 \\
-3.2 \\
-3.2 \\
-3.9 \\
-1.1\end{array}$ & $\begin{array}{l}-.8 \\
-.4 \\
2.0 \\
6.6 \\
8.4\end{array}$ & $\begin{array}{l}4.2 \\
5.7 \\
5.0 \\
5.9 \\
1.1\end{array}$ & $\begin{array}{r}17.1 \\
9.6 \\
8.4 \\
9.2 \\
10.5\end{array}$ & $\begin{array}{l}20.5 \\
15.0 \\
16.7 \\
16.1 \\
17.0\end{array}$ & $\begin{array}{l}22.9 \\
22.1 \\
22.6 \\
20.2 \\
21.4\end{array}$ & $\begin{array}{l}17.1 \\
19.0 \\
20.8 \\
20.5 \\
19.1\end{array}$ & $\begin{array}{r}18.1 \\
13.9 \\
5.9 \\
12.4 \\
9.6\end{array}$ \\
\hline $\begin{array}{l}21 \\
22 \\
23 \\
24 \\
25\end{array}$ & $\begin{array}{l}9.9 \\
9.2 \\
7.3 \\
5.1 \\
4.8\end{array}$ & $\begin{array}{r}-.2 \\
-1.8 \\
-2.9 \\
-.9 \\
-3.6\end{array}$ & $\begin{array}{r}2.6 \\
.4 \\
-10.8 \\
-12.5 \\
-11.7\end{array}$ & $\begin{array}{r}-5.9 \\
-7.9 \\
-.1 \\
-5.5 \\
-2.8\end{array}$ & $\begin{array}{r}-.8 \\
1.0 \\
.7 \\
3.1 \\
2.6\end{array}$ & $\begin{array}{r}9.3 \\
6.0 \\
10.7 \\
4.1 \\
7.1\end{array}$ & $\begin{array}{r}.0 \\
.9 \\
-.2 \\
4.5 \\
5.4\end{array}$ & $\begin{array}{l}13.6 \\
18.2 \\
17.4 \\
18.6 \\
18.8\end{array}$ & $\begin{array}{l}17.3 \\
15.1 \\
18.0 \\
19.7 \\
18.8\end{array}$ & $\begin{array}{l}25.5 \\
22.8 \\
21.0 \\
19.8 \\
20.2\end{array}$ & $\begin{array}{l}18.5 \\
17.3 \\
20.8 \\
18.5 \\
19.1\end{array}$ & $\begin{array}{r}5.2 \\
8.0 \\
11.6 \\
14.9 \\
15.3\end{array}$ \\
\hline $\begin{array}{l}26 \\
27 \\
28 \\
29 \\
30 \\
31\end{array}$ & $\begin{array}{l}6.1 \\
9.3 \\
9.5 \\
6.4 \\
6.0 \\
4.0\end{array}$ & $\begin{array}{l}-4.6 \\
-5.0 \\
-4.7 \\
-4.8 \\
-2.8 \\
---\end{array}$ & $\begin{array}{r}-7.3 \\
-7.6 \\
-4.7 \\
-4.8 \\
-8.8 \\
-13.5\end{array}$ & $\begin{array}{r}-.5 \\
2.4 \\
4.8 \\
3.1 \\
-1.4 \\
-2.9\end{array}$ & $\begin{array}{l}3.0 \\
2.8 \\
4.8 \\
3.4 \\
--. \\
---\end{array}$ & $\begin{array}{r}8.6 \\
5.7 \\
-4.5 \\
2.1 \\
-2.0 \\
-.5\end{array}$ & $\begin{array}{r}7.2 \\
11.9 \\
11.5 \\
11.9 \\
4.3 \\
-\end{array}$ & $\begin{array}{r}17.1 \\
14.4 \\
14.1 \\
1.3 \\
1.5 \\
8.6\end{array}$ & $\begin{array}{r}17.8 \\
23.1 \\
23.3 \\
20.6 \\
19.3 \\
-. .\end{array}$ & $\begin{array}{l}21.0 \\
20.1 \\
19.1 \\
21.2 \\
20.5 \\
20.7\end{array}$ & $\begin{array}{l}18.3 \\
18.6 \\
19.9 \\
20.0 \\
19.1 \\
18.9\end{array}$ & $\begin{array}{r}15.6 \\
12.4 \\
9.2 \\
11.6 \\
13.4 \\
---\end{array}$ \\
\hline $\begin{array}{l}\text { MEAN } \\
\text { MAX } \\
\text { MIN } \\
\text { MED }\end{array}$ & $\begin{array}{r}9.7 \\
17.0 \\
4.0 \\
9.2\end{array}$ & $\begin{array}{r}.7 \\
6.0 \\
-5.0 \\
2.0\end{array}$ & $\begin{array}{r}-4.1 \\
7.4 \\
-13.5 \\
-4.7\end{array}$ & $\begin{array}{r}-3.5 \\
4.8 \\
-15.9 \\
-3.0\end{array}$ & $\begin{array}{r}-.8 \\
4.8 \\
-9.1 \\
-.8\end{array}$ & $\begin{array}{r}1.9 \\
10.7 \\
-6.7 \\
2.0\end{array}$ & $\begin{array}{r}6.4 \\
13.8 \\
-.2 \\
5.8\end{array}$ & $\begin{array}{r}10.2 \\
18.8 \\
-1.8 \\
10.5\end{array}$ & $\begin{array}{r}16.5 \\
23.3 \\
7.3 \\
16.8\end{array}$ & $\begin{array}{l}21.8 \\
25.5 \\
19.1 \\
21.7\end{array}$ & $\begin{array}{l}18.6 \\
22.2 \\
14.5 \\
18.9\end{array}$ & $\begin{array}{r}14.0 \\
22.2 \\
5.2 \\
14.3\end{array}$ \\
\hline
\end{tabular}

CAL YR 1987 MEAN 7.6 $\quad$ MAX 24.8 $\quad$ MIN -13.9 MED 7.5 WTR YR 1988

MEAN 7.6 MAX 25.5 MIN -15.9 MED 7.1 
3 SPRINGS BASIN

3 SPRINGS CREEK NEAR WARM SPRINGS, NV

DAILY MEAN AIR TEMPERATURE, WATER YEAR OCTOBER 1988 TO SEPTEMBER 1989 DEGREES CELSIUS

\begin{tabular}{|c|c|c|c|c|c|c|c|c|c|c|c|c|}
\hline DAY & OCT & NOV & $\mathrm{DEC}$ & JAN & FEB & MAR & APR & MAY & JUN & JUL & AUG & SEP \\
\hline $\begin{array}{l}1 \\
2 \\
3 \\
4 \\
5\end{array}$ & $\begin{array}{l}15.1 \\
18.3 \\
16.9 \\
16.7 \\
15.6\end{array}$ & $\begin{array}{r}12.9 \\
10.3 \\
13.8 \\
10.6 \\
9.3\end{array}$ & $\begin{array}{l}-1.4 \\
-1.6 \\
-1.9 \\
-2.5 \\
-1.9\end{array}$ & $\begin{array}{r}-10.3 \\
-7.9 \\
-3.4 \\
-.6 \\
-3.9\end{array}$ & $\begin{array}{r}2.1 \\
-1.1 \\
-2.7 \\
-10.5 \\
-18.5\end{array}$ & $\begin{array}{r}4.2 \\
2.5 \\
-6.9 \\
-5.1 \\
3.8\end{array}$ & $\begin{array}{l}6.4 \\
9.0 \\
8.8 \\
7.4 \\
9.3\end{array}$ & $\begin{array}{l}12.8 \\
11.0 \\
13.2 \\
12.4 \\
16.0\end{array}$ & $\begin{array}{r}9.8 \\
11.8 \\
10.8 \\
9.8 \\
9.7\end{array}$ & $\begin{array}{l}19.4 \\
21.9 \\
23.5 \\
23.3 \\
21.9\end{array}$ & $\begin{array}{l}21.9 \\
20.9 \\
21.0 \\
21.1 \\
22.2\end{array}$ & $\begin{array}{l}18.5 \\
20.2 \\
18.2 \\
17.6 \\
20.2\end{array}$ \\
\hline $\begin{array}{r}6 \\
7 \\
8 \\
9 \\
10\end{array}$ & $\begin{array}{r}8.7 \\
8.6 \\
10.0 \\
10.0 \\
9.3\end{array}$ & $\begin{array}{l}9.1 \\
4.9 \\
4.4 \\
2.6 \\
4.3\end{array}$ & $\begin{array}{r}.9 \\
-1.9 \\
-2.3 \\
-3.0 \\
-1.8\end{array}$ & $\begin{array}{r}-10.6 \\
-12.3 \\
-9.5 \\
1.6 \\
1.3\end{array}$ & $\begin{array}{r}-21.5 \\
-18.7 \\
-12.2 \\
-4.0 \\
-2.6\end{array}$ & $\begin{array}{r}7.7 \\
9.0 \\
10.2 \\
11.3 \\
11.3\end{array}$ & $\begin{array}{l}12.0 \\
13.6 \\
12.0 \\
11.2 \\
13.4\end{array}$ & $\begin{array}{r}16.9 \\
17.5 \\
17.1 \\
12.6 \\
7.3\end{array}$ & $\begin{array}{l}12.0 \\
12.9 \\
12.9 \\
12.2 \\
12.5\end{array}$ & $\begin{array}{l}21.8 \\
22.6 \\
23.5 \\
22.2 \\
19.7\end{array}$ & $\begin{array}{l}22.0 \\
19.6 \\
17.0 \\
15.6 \\
16.6\end{array}$ & $\begin{array}{l}18.0 \\
13.7 \\
12.0 \\
13.5 \\
13.2\end{array}$ \\
\hline $\begin{array}{l}11 \\
12 \\
13 \\
14 \\
15\end{array}$ & $\begin{array}{r}9.9 \\
10.2 \\
13.2 \\
9.0 \\
8.0\end{array}$ & $\begin{array}{r}.8 \\
6.0 \\
6.4 \\
-2.1 \\
-4.5\end{array}$ & $\begin{array}{r}-2.1 \\
1.0 \\
2.7 \\
1.7 \\
-7.7\end{array}$ & $\begin{array}{r}-10.4 \\
-11.5 \\
-9.8 \\
-10.3 \\
-10.3\end{array}$ & $\begin{array}{r}-6.7 \\
-8.6 \\
-11.5 \\
-11.9 \\
-8.2\end{array}$ & $\begin{array}{r}11.0 \\
10.7 \\
4.7 \\
1.6 \\
3.9\end{array}$ & $\begin{array}{r}7.7 \\
5.8 \\
8.0 \\
13.1 \\
13.9\end{array}$ & $\begin{array}{r}1.0 \\
3.6 \\
.8 \\
2.1 \\
5.3\end{array}$ & $\begin{array}{l}14.7 \\
13.9 \\
18.0 \\
20.5 \\
23.7\end{array}$ & $\begin{array}{l}19.5 \\
18.5 \\
17.9 \\
21.6 \\
23.1\end{array}$ & $\begin{array}{l}17.0 \\
20.1 \\
21.8 \\
21.8 \\
22.7\end{array}$ & $\begin{array}{r}12.8 \\
9.7 \\
10.8 \\
13.3 \\
16.2\end{array}$ \\
\hline $\begin{array}{l}16 \\
17 \\
18 \\
19 \\
20\end{array}$ & $\begin{array}{r}9.9 \\
11.4 \\
13.1 \\
11.9 \\
8.6\end{array}$ & $\begin{array}{l}-2.4 \\
-2.8 \\
-6.5 \\
-6.5 \\
-4.8\end{array}$ & $\begin{array}{l}-6.4 \\
-1.3 \\
-2.5 \\
-4.2 \\
-2.9\end{array}$ & $\begin{array}{l}-6.7 \\
-4.2 \\
-3.5 \\
-2.5 \\
-2.1\end{array}$ & $\begin{array}{r}-4.6 \\
-1.0 \\
3.2 \\
2.4 \\
-3.4\end{array}$ & $\begin{array}{l}4.7 \\
2.0 \\
5.1 \\
4.9 \\
-.5\end{array}$ & $\begin{array}{l}12.7 \\
15.4 \\
15.2 \\
17.7 \\
17.2\end{array}$ & $\begin{array}{r}8.3 \\
12.5 \\
14.5 \\
7.2 \\
13.6\end{array}$ & $\begin{array}{l}18.0 \\
18.1 \\
21.6 \\
23.7 \\
18.9\end{array}$ & $\begin{array}{l}20.9 \\
21.5 \\
23.5 \\
24.4 \\
25.9\end{array}$ & $\begin{array}{l}22.3 \\
20.1 \\
19.1 \\
18.8 \\
17.8\end{array}$ & $\begin{array}{r}14.4 \\
12.3 \\
8.2 \\
6.1 \\
9.2\end{array}$ \\
\hline $\begin{array}{l}21 \\
22 \\
23 \\
24 \\
25\end{array}$ & $\begin{array}{r}11.4 \\
11.3 \\
10.0 \\
9.4 \\
8.5\end{array}$ & $\begin{array}{r}.9 \\
5.6 \\
3.0 \\
-5.7 \\
-3.1\end{array}$ & $\begin{array}{l}-4.5 \\
-6.3 \\
-8.9 \\
-4.4 \\
-9.6\end{array}$ & $\begin{array}{r}-1.2 \\
2.0 \\
-1.2 \\
-10.3 \\
-11.6\end{array}$ & $\begin{array}{r}-1.6 \\
5.0 \\
9.2 \\
9.9 \\
9.2\end{array}$ & $\begin{array}{l}5.1 \\
7.2 \\
7.3 \\
8.0 \\
2.2\end{array}$ & $\begin{array}{r}14.7 \\
9.4 \\
5.9 \\
-.3 \\
-.1\end{array}$ & $\begin{array}{r}15.1 \\
17.0 \\
12.7 \\
8.3 \\
7.1\end{array}$ & $\begin{array}{l}11.0 \\
14.4 \\
14.7 \\
11.0 \\
11.7\end{array}$ & $\begin{array}{l}19.9 \\
21.2 \\
20.1 \\
18.8 \\
20.4\end{array}$ & $\begin{array}{l}15.9 \\
19.4 \\
16.1 \\
10.2 \\
12.9\end{array}$ & $\begin{array}{l}10.1 \\
10.5 \\
14.3 \\
15.5 \\
16.2\end{array}$ \\
\hline $\begin{array}{l}26 \\
27 \\
28 \\
29 \\
30 \\
31\end{array}$ & $\begin{array}{r}10.5 \\
11.1 \\
12.5 \\
11.1 \\
6.9 \\
9.8\end{array}$ & $\begin{array}{r}-4.9 \\
-5.1 \\
1.1 \\
-5.0 \\
-2.9 \\
---\end{array}$ & $\begin{array}{r}-16.6 \\
-15.6 \\
-15.1 \\
-14.8 \\
-3.5 \\
-5.9\end{array}$ & $\begin{array}{r}-8.9 \\
-6.6 \\
-4.9 \\
.0 \\
1.0 \\
2.7\end{array}$ & $\begin{array}{r}6.7 \\
1.7 \\
.3 \\
--- \\
--- \\
---\end{array}$ & $\begin{array}{r}-.2 \\
5.2 \\
9.9 \\
5.5 \\
6.2 \\
10.0\end{array}$ & $\begin{array}{r}1.5 \\
-.2 \\
2.0 \\
5.3 \\
10.8 \\
-\ldots\end{array}$ & $\begin{array}{r}10.4 \\
15.6 \\
12.1 \\
4.9 \\
3.2 \\
7.4\end{array}$ & $\begin{array}{r}16.2 \\
16.6 \\
16.3 \\
18.5 \\
19.0 \\
\ldots \ldots\end{array}$ & $\begin{array}{l}23.7 \\
23.4 \\
22.9 \\
23.6 \\
24.1 \\
23.4\end{array}$ & $\begin{array}{l}17.7 \\
19.3 \\
18.2 \\
20.7 \\
18.2 \\
15.8\end{array}$ & $\begin{array}{c}17.6 \\
17.4 \\
16.1 \\
16.7 \\
11.7 \\
---\end{array}$ \\
\hline $\begin{array}{l}\text { MEAN } \\
\text { MAX } \\
\text { MIN } \\
\text { MED }\end{array}$ & $\begin{array}{r}11.2 \\
18.3 \\
6.9 \\
10.2\end{array}$ & $\begin{array}{r}1.7 \\
13.8 \\
-6.5 \\
1.0\end{array}$ & $\begin{array}{r}-4.7 \\
2.7 \\
-16.6 \\
-2.9\end{array}$ & $\begin{array}{r}-5.4 \\
2.7 \\
-12.3 \\
-4.9\end{array}$ & $\begin{array}{r}-3.6 \\
9.9 \\
-21.5 \\
-2.7\end{array}$ & $\begin{array}{r}5.2 \\
11.3 \\
-6.9 \\
5.1\end{array}$ & $\begin{array}{r}9.3 \\
17.7 \\
-.3 \\
9.3\end{array}$ & $\begin{array}{r}10.3 \\
17.5 \\
.8 \\
12.1\end{array}$ & $\begin{array}{r}15.2 \\
23.7 \\
9.7 \\
14.5\end{array}$ & $\begin{array}{l}21.9 \\
25.9 \\
17.9 \\
21.9\end{array}$ & $\begin{array}{l}18.8 \\
22.7 \\
10.2 \\
19.3\end{array}$ & $\begin{array}{r}14.1 \\
20.2 \\
6.1 \\
14.0\end{array}$ \\
\hline
\end{tabular}

CAL YR 1988 MEAN 7.8 MAX 25.5 MIN -16.6 MED 8.4 WTR YR 1989 MEAN 7.9 MAX 25.9 MIN -21.5 MED 9.8 
3 SPRINGS BASIN

3 SPRINGS CREEK NEAR WARM SPRINGS, NV

DAILY MEAN AIR TEMPERATURE, WATER YEAR OCTOBER 1989 TO SEPTEMBER 1990 DEGREES CELSIUS

\begin{tabular}{|c|c|c|c|c|c|c|c|c|c|c|c|c|}
\hline DAY & OCT & NOV & DEC & JAN & FEB & MAR & APR & MAY & JUN & JUL & AUG & SEP \\
\hline $\begin{array}{l}1 \\
2 \\
3 \\
4 \\
5\end{array}$ & $\begin{array}{r}9.2 \\
10.1 \\
3.9 \\
3.5 \\
5.3\end{array}$ & $\begin{array}{r}-3.2 \\
-1.9 \\
1.6 \\
5.3 \\
5.6\end{array}$ & $\begin{array}{r}-4.2 \\
-4.5 \\
-2.4 \\
.5 \\
6.3\end{array}$ & $\begin{array}{r}1.1 \\
-5.4 \\
-12.0 \\
-8.1 \\
-6.3\end{array}$ & $\begin{array}{l}-6.4 \\
-9.2 \\
-6.2 \\
-4.3 \\
-8.5\end{array}$ & $\begin{array}{r}6.2 \\
5.8 \\
5.4 \\
2.9 \\
-1.7\end{array}$ & $\begin{array}{l}6.3 \\
7.5 \\
5.7 \\
5.8 \\
7.1\end{array}$ & $\begin{array}{r}5.3 \\
7.7 \\
9.3 \\
11.5 \\
12.8\end{array}$ & $\begin{array}{r}6.3 \\
13.7 \\
19.8 \\
18.8 \\
16.1\end{array}$ & $\begin{array}{l}25.4 \\
21.7 \\
18.4 \\
20.7 \\
22.2\end{array}$ & $\begin{array}{l}21.2 \\
19.4 \\
22.9 \\
23.2 \\
22.3\end{array}$ & $\begin{array}{l}20.4 \\
20.3 \\
19.9 \\
18.4 \\
18.4\end{array}$ \\
\hline $\begin{array}{r}6 \\
7 \\
8 \\
9 \\
10\end{array}$ & $\begin{array}{r}10.0 \\
6.5 \\
8.0 \\
9.7 \\
13.8\end{array}$ & $\begin{array}{l}2.6 \\
-.4 \\
-.8 \\
2.9 \\
4.5\end{array}$ & $\begin{array}{r}.1 \\
-1.7 \\
2.5 \\
3.9 \\
-5.9\end{array}$ & $\begin{array}{r}-4.2 \\
3.2 \\
6.9 \\
6.9 \\
4.3\end{array}$ & $\begin{array}{r}-2.8 \\
-8.6 \\
-11.3 \\
-4.6 \\
-.4\end{array}$ & $\begin{array}{r}-2.4 \\
.8 \\
5.3 \\
6.0 \\
5.3\end{array}$ & $\begin{array}{l}6.0 \\
6.2 \\
1.6 \\
5.2 \\
7.0\end{array}$ & $\begin{array}{r}17.6 \\
15.1 \\
9.5 \\
8.7 \\
10.6\end{array}$ & $\begin{array}{l}15.2 \\
16.6 \\
18.9 \\
17.7 \\
12.3\end{array}$ & $\begin{array}{l}22.1 \\
21.5 \\
19.5 \\
20.3 \\
17.5\end{array}$ & $\begin{array}{l}22.1 \\
22.7 \\
23.4 \\
20.9 \\
20.0\end{array}$ & $\begin{array}{l}18.3 \\
18.8 \\
20.0 \\
21.8 \\
21.4\end{array}$ \\
\hline $\begin{array}{l}11 \\
12 \\
13 \\
14 \\
15\end{array}$ & $\begin{array}{r}15.3 \\
15.5 \\
15.4 \\
11.8 \\
7.0\end{array}$ & $\begin{array}{r}8.9 \\
10.9 \\
7.6 \\
4.1 \\
-1.1\end{array}$ & $\begin{array}{r}-9.7 \\
-6.8 \\
-5.0 \\
-5.0 \\
-.4\end{array}$ & $\begin{array}{r}4.4 \\
5.4 \\
1.4 \\
-1.7 \\
-5.4\end{array}$ & $\begin{array}{r}1.4 \\
4.9 \\
-5.0 \\
-14.4 \\
-12.0\end{array}$ & $\begin{array}{l}-6.2 \\
-9.4 \\
-9.3 \\
-2.4 \\
-1.1\end{array}$ & $\begin{array}{r}10.7 \\
9.3 \\
10.5 \\
16.3 \\
14.2\end{array}$ & $\begin{array}{r}9.0 \\
10.0 \\
11.1 \\
11.9 \\
6.6\end{array}$ & $\begin{array}{r}12.8 \\
13.7 \\
12.1 \\
11.0 \\
8.6\end{array}$ & $\begin{array}{l}20.4 \\
23.8 \\
22.1 \\
18.9 \\
17.7\end{array}$ & $\begin{array}{l}18.8 \\
18.1 \\
18.0 \\
20.4 \\
14.8\end{array}$ & $\begin{array}{l}21.5 \\
22.3 \\
21.9 \\
21.4 \\
19.5\end{array}$ \\
\hline $\begin{array}{l}16 \\
17 \\
18 \\
19 \\
20\end{array}$ & $\begin{array}{r}4.9 \\
5.5 \\
6.5 \\
9.9 \\
11.9\end{array}$ & $\begin{array}{r}1.5 \\
1.1 \\
.4 \\
3.0 \\
5.6\end{array}$ & $\begin{array}{r}-4.7 \\
-.4 \\
-2.4 \\
-5.8 \\
-4.6\end{array}$ & $\begin{array}{r}-3.8 \\
-5.0 \\
-7.3 \\
-10.5 \\
-11.1\end{array}$ & $\begin{array}{l}-6.3 \\
-3.3 \\
-6.3 \\
-7.1 \\
-5.5\end{array}$ & $\begin{array}{r}.4 \\
3.7 \\
3.8 \\
8.3 \\
10.7\end{array}$ & $\begin{array}{l}8.8 \\
4.4 \\
5.2 \\
8.6 \\
8.3\end{array}$ & $\begin{array}{r}9.2 \\
13.3 \\
11.7 \\
8.6 \\
10.4\end{array}$ & $\begin{array}{l}11.9 \\
16.2 \\
15.9 \\
13.8 \\
16.9\end{array}$ & $\begin{array}{l}17.3 \\
18.6 \\
19.5 \\
20.0 \\
21.0\end{array}$ & $\begin{array}{l}17.4 \\
18.7 \\
16.1 \\
11.0 \\
10.7\end{array}$ & $\begin{array}{r}16.0 \\
13.9 \\
12.5 \\
9.6 \\
11.4\end{array}$ \\
\hline $\begin{array}{l}21 \\
22 \\
23 \\
24 \\
25\end{array}$ & $\begin{array}{r}8.0 \\
7.7 \\
8.7 \\
7.9 \\
.9\end{array}$ & $\begin{array}{l}4.2 \\
3.8 \\
5.6 \\
5.7 \\
1.5\end{array}$ & $\begin{array}{l}-4.1 \\
-3.5 \\
-1.2 \\
-1.5 \\
-2.0\end{array}$ & $\begin{array}{r}-7.1 \\
-5.4 \\
1.1 \\
-6.0 \\
-2.7\end{array}$ & $\begin{array}{r}-2.9 \\
-1.0 \\
1.1 \\
3.0 \\
2.9\end{array}$ & $\begin{array}{l}9.4 \\
7.9 \\
7.0 \\
5.9 \\
8.5\end{array}$ & $\begin{array}{l}6.8 \\
7.9 \\
5.6 \\
4.2 \\
7.8\end{array}$ & $\begin{array}{r}15.4 \\
14.8 \\
13.0 \\
3.9 \\
9.1\end{array}$ & $\begin{array}{l}20.7 \\
23.5 \\
23.0 \\
22.8 \\
22.2\end{array}$ & $\begin{array}{l}21.4 \\
22.2 \\
23.1 \\
21.1 \\
18.4\end{array}$ & $\begin{array}{l}14.2 \\
14.6 \\
16.9 \\
17.9 \\
15.2\end{array}$ & $\begin{array}{r}10.2 \\
11.4 \\
9.5 \\
10.2 \\
12.3\end{array}$ \\
\hline $\begin{array}{l}26 \\
27 \\
28 \\
29 \\
30 \\
31\end{array}$ & $\begin{array}{r}-.6 \\
2.2 \\
-2.9 \\
-4.3 \\
-3.1 \\
-1.4\end{array}$ & $\begin{array}{l}-2.4 \\
-8.4 \\
-8.5 \\
-5.0 \\
-4.3 \\
---\end{array}$ & $\begin{array}{r}-.8 \\
1.3 \\
-.6 \\
-3.2 \\
-5.0 \\
-1.4\end{array}$ & $\begin{array}{r}-1.3 \\
-7.7 \\
-2.8 \\
1.3 \\
.4 \\
-5.3\end{array}$ & $\begin{array}{l}3.5 \\
1.8 \\
3.2 \\
--- \\
--- \\
---\end{array}$ & $\begin{array}{r}10.5 \\
4.7 \\
.5 \\
1.5 \\
2.4 \\
4.0\end{array}$ & $\begin{array}{r}10.9 \\
12.3 \\
11.7 \\
2.2 \\
2.2 \\
---\end{array}$ & $\begin{array}{r}12.3 \\
11.3 \\
4.2 \\
9.4 \\
9.6 \\
8.5\end{array}$ & $\begin{array}{l}22.9 \\
19.9 \\
21.5 \\
24.3 \\
24.3 \\
-\ldots\end{array}$ & $\begin{array}{l}17.4 \\
19.6 \\
23.1 \\
23.6 \\
23.9 \\
23.5\end{array}$ & $\begin{array}{l}15.2 \\
18.0 \\
17.8 \\
21.5 \\
21.5 \\
18.2\end{array}$ & $\begin{array}{r}13.8 \\
11.1 \\
8.8 \\
10.9 \\
11.0 \\
---\end{array}$ \\
\hline $\begin{array}{l}\text { MEAN } \\
\text { MAX } \\
\text { MIN } \\
\text { MED }\end{array}$ & $\begin{array}{r}6.7 \\
15.5 \\
-4.3 \\
7.7\end{array}$ & $\begin{array}{r}1.7 \\
10.9 \\
-8.5 \\
2.1\end{array}$ & $\begin{array}{r}-2.3 \\
6.3 \\
-9.7 \\
-2.4\end{array}$ & $\begin{array}{r}-2.7 \\
6.9 \\
-12.0 \\
-3.8\end{array}$ & $\begin{array}{r}-3.7 \\
4.9 \\
-14.4 \\
-4.5\end{array}$ & $\begin{array}{r}3.0 \\
10.7 \\
-9.4 \\
4.0\end{array}$ & $\begin{array}{r}7.5 \\
16.3 \\
1.6 \\
7.1\end{array}$ & $\begin{array}{r}10.4 \\
17.6 \\
3.9 \\
10.0\end{array}$ & $\begin{array}{r}17.1 \\
24.3 \\
6.3 \\
16.7\end{array}$ & $\begin{array}{l}20.8 \\
25.4 \\
17.3 \\
21.0\end{array}$ & $\begin{array}{l}18.5 \\
23.4 \\
10.7 \\
18.2\end{array}$ & $\begin{array}{r}15.9 \\
22.3 \\
8.8 \\
17.1\end{array}$ \\
\hline
\end{tabular}

CAL YR 1989 MEAN 7.7 MAX 25.9 MIN -21.5 MED 8.3

WTR YR 1990 MEAN 7.8 MAX 25.4 MIN -14.4 MED 7.8 
3 SPRINGS BASIN

3 SPRINGS CREEK NEAR WARM SPRINGS, NV

DAILY MEAN AIR TEMPERATURE, WATER YEAR OCTOBER 1990 TO SEPTEMBER 1991 DEGREES CELSIUS

\begin{tabular}{|c|c|c|c|c|c|c|c|c|c|c|c|c|}
\hline DAY & OCT & NOV & DEC & JAN & $\mathrm{FEB}$ & MAR & APR & MAY & JUN & JUL & AUG & SEP \\
\hline $\begin{array}{l}1 \\
2 \\
3 \\
4 \\
5\end{array}$ & $\begin{array}{r}14.2 \\
14.3 \\
9.2 \\
14.2 \\
16.6\end{array}$ & $\begin{array}{r}.5 \\
-3.7 \\
-2.3 \\
3.5 \\
4.7\end{array}$ & $\begin{array}{r}-1.2 \\
-2.2 \\
-4.2 \\
-.8 \\
.9\end{array}$ & $\begin{array}{l}-4.3 \\
-4.4 \\
-3.0 \\
-2.2 \\
-5.0\end{array}$ & $\begin{array}{r}-1.2 \\
.9 \\
.6 \\
2.6 \\
4.1\end{array}$ & $\begin{array}{r}.0 \\
1.3 \\
4.6 \\
5.0 \\
.7\end{array}$ & $\begin{array}{r}2.3 \\
4.5 \\
4.6 \\
10.0 \\
13.2\end{array}$ & $\begin{array}{r}5.6 \\
.9 \\
2.2 \\
5.4 \\
10.3\end{array}$ & $\begin{array}{r}8.5 \\
12.6 \\
17.1 \\
17.8 \\
14.8\end{array}$ & $\begin{array}{l}17.5 \\
20.3 \\
22.0 \\
23.7 \\
25.6\end{array}$ & $\begin{array}{l}\text { e16.1 } \\
\text { e18.5 } \\
\text { e18.8 } \\
\text { e19.3 } \\
\text { e18.3 }\end{array}$ & $\begin{array}{l}\text { e21. } 4 \\
\text { e20.4 } \\
\text { e19.0 } \\
\text { e18.5 } \\
\text { e16.8 }\end{array}$ \\
\hline $\begin{array}{r}6 \\
7 \\
8 \\
9 \\
10\end{array}$ & $\begin{array}{r}12.8 \\
4.9 \\
1.5 \\
5.2 \\
8.6\end{array}$ & $\begin{array}{r}-3.5 \\
-2.6 \\
5.9 \\
2.9 \\
3.5\end{array}$ & $\begin{array}{r}-4.9 \\
-3.6 \\
-2.2 \\
-.4 \\
6.3\end{array}$ & $\begin{array}{l}-6.1 \\
-4.2 \\
-5.9 \\
-5.0 \\
-5.0\end{array}$ & $\begin{array}{r}-.2 \\
.4 \\
1.0 \\
2.2 \\
1.2\end{array}$ & $\begin{array}{r}-4.2 \\
-4.8 \\
-1.9 \\
1.0 \\
3.4\end{array}$ & $\begin{array}{r}12.5 \\
5.2 \\
.7 \\
6.5 \\
-1.3\end{array}$ & $\begin{array}{r}12.1 \\
12.6 \\
15.1 \\
-.4 \\
-.3\end{array}$ & $\begin{array}{l}16.4 \\
17.0 \\
15.5 \\
16.0 \\
18.6\end{array}$ & $\begin{array}{l}24.5 \\
22.4 \\
16.5 \\
17.2 \\
19.2\end{array}$ & $\begin{array}{l}\text { e17.7 } \\
\text { e2 } 0.7 \\
\text { e20.0 } \\
\text { e22 } 2.1 \\
\text { e21. }\end{array}$ & $\begin{array}{r}\text { e15.3 } \\
\text { e13.3 } \\
\text { e15.4 } \\
\text { e12.0 } \\
\text { e } 6.7\end{array}$ \\
\hline $\begin{array}{l}11 \\
12 \\
13 \\
14 \\
15\end{array}$ & $\begin{array}{r}7.0 \\
8.9 \\
13.1 \\
11.9 \\
15.0\end{array}$ & $\begin{array}{r}6.1 \\
6.3 \\
9.9 \\
10.8 \\
5.9\end{array}$ & $\begin{array}{r}5.2 \\
1.1 \\
-2.2 \\
-9.6 \\
-6.7\end{array}$ & $\begin{array}{r}-2.0 \\
.0 \\
.9 \\
.5 \\
2.0\end{array}$ & $\begin{array}{l}3.3 \\
4.8 \\
4.2 \\
2.8 \\
6.1\end{array}$ & $\begin{array}{r}-3.4 \\
-1.5 \\
-.6 \\
-.9 \\
-3.7\end{array}$ & $\begin{array}{r}-3.6 \\
-1.2 \\
.9 \\
6.0 \\
6.5\end{array}$ & $\begin{array}{l}2.4 \\
7.7 \\
9.4 \\
4.0 \\
6.5\end{array}$ & $\begin{array}{l}20.3 \\
21.8 \\
19.4 \\
15.8 \\
15.9\end{array}$ & $\begin{array}{l}21.3 \\
22.5 \\
24.6 \\
23.3 \\
22.2\end{array}$ & $\begin{array}{l}\text { e14.2 } \\
\text { e19.7 } \\
\text { e22.1 } \\
\text { e20.5 } \\
\text { e } 19.7\end{array}$ & $\begin{array}{r}\mathrm{e} 7.7 \\
\mathrm{e} 10.1 \\
\mathrm{e} 12.6 \\
\mathrm{e} 13.7 \\
\mathrm{e} 11.0\end{array}$ \\
\hline $\begin{array}{l}16 \\
17 \\
18 \\
19 \\
20\end{array}$ & $\begin{array}{r}12.5 \\
7.9 \\
10.9 \\
3.0 \\
-1.4\end{array}$ & $\begin{array}{r}5.1 \\
6.7 \\
4.8 \\
3.8 \\
-3.0\end{array}$ & $\begin{array}{r}-8.6 \\
-8.4 \\
.6 \\
-6.8 \\
-15.7\end{array}$ & $\begin{array}{l}-3.5 \\
-1.7 \\
-2.4 \\
-2.4 \\
-6.3\end{array}$ & $\begin{array}{r}4.4 \\
-.6 \\
-3.1 \\
.5 \\
2.5\end{array}$ & $\begin{array}{r}-2.8 \\
.6 \\
2.7 \\
-.9 \\
-4.0\end{array}$ & $\begin{array}{l}3.5 \\
2.2 \\
3.6 \\
8.2 \\
7.1\end{array}$ & $\begin{array}{r}11.8 \\
6.7 \\
2.9 \\
4.5 \\
7.0\end{array}$ & $\begin{array}{l}19.9 \\
18.7 \\
20.5 \\
16.0 \\
15.2\end{array}$ & $\begin{array}{l}21.3 \\
22.2 \\
22.3 \\
20.0 \\
18.3\end{array}$ & $\begin{array}{l}\text { e19.0 } \\
\text { e } 21.3 \\
\text { e21.7 } \\
\text { e } 21.1 \\
\text { e } 21.2\end{array}$ & $\begin{array}{l}\text { e13.6 } \\
\text { e16.3 } \\
\text { e18. } \\
\text { e16.9 } \\
\text { e17.7 }\end{array}$ \\
\hline $\begin{array}{l}21 \\
22 \\
23 \\
24 \\
25\end{array}$ & $\begin{array}{r}2.1 \\
6.2 \\
6.7 \\
8.0 \\
10.3\end{array}$ & $\begin{array}{r}-3.8 \\
-1.3 \\
2.4 \\
3.5 \\
7.7\end{array}$ & $\begin{array}{r}-21.0 \\
-21.6 \\
-19.5 \\
-14.2 \\
-9.5\end{array}$ & $\begin{array}{l}-9.6 \\
-7.8 \\
-5.8 \\
-2.1 \\
-5.6\end{array}$ & $\begin{array}{l}5.7 \\
7.7 \\
3.2 \\
-.5 \\
-.5\end{array}$ & $\begin{array}{r}-2.4 \\
-1.6 \\
1.0 \\
2.8 \\
-1.6\end{array}$ & $\begin{array}{l}4.1 \\
2.0 \\
5.2 \\
8.5 \\
2.0\end{array}$ & $\begin{array}{r}5.8 \\
7.0 \\
11.5 \\
15.1 \\
14.4\end{array}$ & $\begin{array}{l}14.9 \\
16.4 \\
17.7 \\
15.1 \\
13.8\end{array}$ & $\begin{array}{l}20.3 \\
19.7 \\
22.7 \\
22.9 \\
23.2\end{array}$ & $\begin{array}{l}\mathrm{e} 22.4 \\
\mathrm{e} 23.2 \\
\mathrm{e} 22.8 \\
\mathrm{e} 22.2 \\
\mathrm{e} 24.0\end{array}$ & $\begin{array}{l}\text { e1 } 8.3 \\
\text { e1 } 4.9 \\
\text { e15.1 } \\
\text { e1 } 14.9 \\
\text { e16.3 }\end{array}$ \\
\hline $\begin{array}{l}26 \\
27 \\
28 \\
29 \\
30 \\
31\end{array}$ & $\begin{array}{r}9.9 \\
11.8 \\
15.0 \\
13.3 \\
12.4 \\
9.6\end{array}$ & $\begin{array}{l}-5.5 \\
-9.4 \\
-7.3 \\
-2.6 \\
-1.0 \\
---\end{array}$ & $\begin{array}{r}-8.9 \\
-5.9 \\
1.3 \\
-10.8 \\
-13.0 \\
-6.9\end{array}$ & $\begin{array}{r}-5.1 \\
-4.0 \\
-.4 \\
-9.8 \\
-5.5 \\
-2.4\end{array}$ & $\begin{array}{l}2.3 \\
3.4 \\
-.1 \\
--- \\
--- \\
---\end{array}$ & $\begin{array}{r}-2.5 \\
-2.8 \\
-.2 \\
1.8 \\
2.0 \\
3.6\end{array}$ & $\begin{array}{l}2.9 \\
2.6 \\
2.0 \\
5.5 \\
5.9 \\
----\end{array}$ & $\begin{array}{r}14.3 \\
9.5 \\
11.0 \\
13.0 \\
7.1 \\
6.3\end{array}$ & $\begin{array}{r}14.2 \\
11.4 \\
13.4 \\
11.8 \\
13.9 \\
-.-\end{array}$ & $\begin{array}{r}23.3 \\
24.5 \\
22.7 \\
22.9 \\
22.9 \\
\text { e20.1 }\end{array}$ & $\begin{array}{l}\mathrm{e} 21.2 \\
\mathrm{e} 20.2 \\
\mathrm{e} 12.9 \\
\mathrm{e} 18.1 \\
\mathrm{e} 21.0 \\
\mathrm{e} 18.2\end{array}$ & $\begin{array}{r}14.9 \\
13.6 \\
12.2 \\
10.2 \\
11.9 \\
---\end{array}$ \\
\hline $\begin{array}{l}\text { MEAN } \\
\text { MAX } \\
\text { MIN } \\
\text { MED }\end{array}$ & $\begin{array}{r}9.5 \\
16.6 \\
-1.4 \\
9.9\end{array}$ & $\begin{array}{r}1.6 \\
10.8 \\
-9.4 \\
3.2\end{array}$ & $\begin{array}{r}-6.2 \\
6.3 \\
-21.6 \\
-5.9\end{array}$ & $\begin{array}{r}-3.8 \\
2.0 \\
-9.8 \\
-4.2\end{array}$ & $\begin{array}{r}2.1 \\
7.7 \\
-3.1 \\
2.2\end{array}$ & $\begin{array}{r}-.3 \\
5.0 \\
-4.8 \\
-.6\end{array}$ & $\begin{array}{r}4.4 \\
13.2 \\
-3.6 \\
4.3\end{array}$ & $\begin{array}{r}7.8 \\
15.1 \\
-.4 \\
7.0\end{array}$ & $\begin{array}{r}16.0 \\
21.8 \\
8.5 \\
15.9\end{array}$ & $\begin{array}{l}21.7 \\
25.6 \\
16.5 \\
22.3\end{array}$ & $\begin{array}{l}20.0 \\
24.0 \\
12.9 \\
20.5\end{array}$ & $\begin{array}{r}14.6 \\
21.4 \\
6.7 \\
14.9\end{array}$ \\
\hline
\end{tabular}

CAL YR 1990 MEAN 7.7 MAX 25.4 MIN -21.6 MED 8.5 WTR YR 1991 MEAN 7.3 MAX 25.6 MIN -21.6 MED 6.1

e Estimated 
3 SPRINGS BASIN

3 SPRINGS CREEK NEAR WARM SPRINGS, NV

DAILY MAXIMUM AIR TEMPERATURE, WATER YEAR OCTOBER 1985 TO SEPTEMBER 1986 DEGREES CELSIUS

\begin{tabular}{|c|c|c|c|c|c|c|c|c|c|c|c|c|}
\hline DAY & OCT & NOV & DEC & JAN & FEB & MAR & APR & MAY & JUN & JUL & AUG & SEP \\
\hline $\begin{array}{l}1 \\
2 \\
3 \\
4 \\
5\end{array}$ & $\begin{array}{l}--- \\
--- \\
--- \\
--- \\
---\end{array}$ & $\begin{array}{l}11.2 \\
15.4 \\
18.0 \\
14.1 \\
11.9\end{array}$ & $\begin{array}{r}4.3 \\
.9 \\
6.0 \\
7.4 \\
5.7\end{array}$ & $\begin{array}{l}8.2 \\
7.5 \\
8.4 \\
2.4 \\
6.1\end{array}$ & $\begin{array}{l}6.3 \\
5.2 \\
4.8 \\
1.0 \\
3.1\end{array}$ & $\begin{array}{r}16.6 \\
9.4 \\
13.4 \\
16.7 \\
14.7\end{array}$ & $\begin{array}{r}12.5 \\
3.1 \\
9.2 \\
10.5 \\
10.4\end{array}$ & $\begin{array}{r}18.5 \\
19.2 \\
16.8 \\
7.6 \\
11.2\end{array}$ & $\begin{array}{l}26.9 \\
24.7 \\
26.4 \\
25.5 \\
21.8\end{array}$ & $\begin{array}{l}27.7 \\
29.2 \\
28.6 \\
27.2 \\
22.6\end{array}$ & $\begin{array}{l}29.2 \\
29.4 \\
29.9 \\
29.8 \\
28.1\end{array}$ & $\begin{array}{l}23.7 \\
24.1 \\
25.4 \\
26.7 \\
28.0\end{array}$ \\
\hline $\begin{array}{r}6 \\
7 \\
8 \\
9 \\
10\end{array}$ & $\begin{array}{l}--- \\
--- \\
--- \\
--- \\
---\end{array}$ & $\begin{array}{r}12.1 \\
15.5 \\
11.4 \\
3.4 \\
3.8\end{array}$ & $\begin{array}{r}7.8 \\
8.8 \\
-1.1 \\
-3.5 \\
-6.7\end{array}$ & $\begin{array}{r}5.2 \\
2.3 \\
6.8 \\
12.0 \\
12.5\end{array}$ & $\begin{array}{r}-3.7 \\
-2.7 \\
-4.2 \\
-3.6 \\
3.1\end{array}$ & $\begin{array}{r}14.2 \\
13.0 \\
7.5 \\
4.5 \\
1.1\end{array}$ & $\begin{array}{r}6.6 \\
4.1 \\
7.0 \\
12.7 \\
12.5\end{array}$ & $\begin{array}{r}5.8 \\
8.1 \\
8.7 \\
14.1 \\
14.8\end{array}$ & $\begin{array}{l}22.5 \\
21.0 \\
19.3 \\
21.0 \\
22.2\end{array}$ & $\begin{array}{l}23.0 \\
22.3 \\
23.7 \\
25.5 \\
26.5\end{array}$ & $\begin{array}{l}28.8 \\
28.5 \\
29.3 \\
29.4 \\
25.5\end{array}$ & $\begin{array}{l}26.9 \\
25.0 \\
21.6 \\
18.2 \\
16.6\end{array}$ \\
\hline $\begin{array}{l}11 \\
12 \\
13 \\
14 \\
15\end{array}$ & $\begin{array}{r}13.8 \\
11.4 \\
7.0 \\
10.6 \\
14.7\end{array}$ & $\begin{array}{r}-1.1 \\
-5.4 \\
-3.9 \\
1.6 \\
7.4\end{array}$ & $\begin{array}{r}-6.7 \\
-1.9 \\
.3 \\
6.0 \\
5.7\end{array}$ & $\begin{array}{r}11.5 \\
11.7 \\
10.1 \\
8.8 \\
4.2\end{array}$ & $\begin{array}{r}5.2 \\
.9 \\
4.9 \\
6.1 \\
2.5\end{array}$ & $\begin{array}{l}4.9 \\
2.6 \\
1.2 \\
3.2 \\
3.8\end{array}$ & $\begin{array}{r}14.3 \\
11.8 \\
5.5 \\
9.9 \\
12.2\end{array}$ & $\begin{array}{l}14.8 \\
15.5 \\
18.3 \\
16.8 \\
16.7\end{array}$ & $\begin{array}{l}25.1 \\
24.0 \\
25.7 \\
25.3 \\
25.8\end{array}$ & $\begin{array}{l}29.1 \\
28.9 \\
27.2 \\
26.9 \\
20.6\end{array}$ & $\begin{array}{l}26.5 \\
27.0 \\
27.6 \\
28.2 \\
27.7\end{array}$ & $\begin{array}{l}20.1 \\
19.8 \\
18.5 \\
17.7 \\
14.7\end{array}$ \\
\hline $\begin{array}{l}16 \\
17 \\
18 \\
19 \\
20\end{array}$ & $\begin{array}{l}17.4 \\
17.2 \\
17.5 \\
17.0 \\
15.2\end{array}$ & $\begin{array}{r}6.7 \\
1.3 \\
-4.2 \\
-4.4 \\
6.1\end{array}$ & $\begin{array}{r}4.5 \\
7.0 \\
9.5 \\
11.3 \\
11.3\end{array}$ & $\begin{array}{r}7.8 \\
10.0 \\
12.2 \\
11.5 \\
6.6\end{array}$ & $\begin{array}{l}5.1 \\
6.6 \\
7.0 \\
8.5 \\
5.3\end{array}$ & $\begin{array}{r}.4 \\
1.9 \\
4.7 \\
8.3 \\
12.3\end{array}$ & $\begin{array}{r}4.6 \\
4.6 \\
7.8 \\
13.6 \\
18.3\end{array}$ & $\begin{array}{l}15.7 \\
18.3 \\
22.6 \\
22.8 \\
20.8\end{array}$ & $\begin{array}{l}25.6 \\
25.7 \\
24.5 \\
23.3 \\
24.4\end{array}$ & $\begin{array}{l}19.9 \\
22.4 \\
23.3 \\
26.7 \\
27.3\end{array}$ & $\begin{array}{l}27.2 \\
27.8 \\
24.4 \\
25.2 \\
24.2\end{array}$ & $\begin{array}{l}16.1 \\
16.7 \\
14.3 \\
13.2 \\
12.7\end{array}$ \\
\hline $\begin{array}{l}21 \\
22 \\
23 \\
24 \\
25\end{array}$ & $\begin{array}{r}10.5 \\
7.7 \\
16.0 \\
17.4 \\
17.5\end{array}$ & $\begin{array}{l}3.4 \\
7.8 \\
5.5 \\
4.3 \\
4.9\end{array}$ & $\begin{array}{r}10.5 \\
12.2 \\
11.9 \\
9.3 \\
9.3\end{array}$ & $\begin{array}{l}3.0 \\
7.0 \\
7.7 \\
5.6 \\
8.1\end{array}$ & $\begin{array}{r}7.6 \\
10.1 \\
12.7 \\
16.3 \\
18.4\end{array}$ & $\begin{array}{l}13.7 \\
14.9 \\
14.5 \\
13.7 \\
13.0\end{array}$ & $\begin{array}{l}20.1 \\
18.6 \\
14.1 \\
11.7 \\
12.9\end{array}$ & $\begin{array}{l}17.2 \\
15.6 \\
19.6 \\
22.9 \\
25.5\end{array}$ & $\begin{array}{l}25.7 \\
27.2 \\
27.8 \\
27.2 \\
28.2\end{array}$ & $\begin{array}{l}23.2 \\
17.2 \\
18.3 \\
21.4 \\
22.9\end{array}$ & $\begin{array}{l}25.8 \\
26.1 \\
27.0 \\
25.0 \\
23.9\end{array}$ & $\begin{array}{r}14.2 \\
15.6 \\
11.5 \\
9.1 \\
6.9\end{array}$ \\
\hline $\begin{array}{l}26 \\
27 \\
28 \\
29 \\
30 \\
31\end{array}$ & $\begin{array}{l}17.2 \\
18.0 \\
16.1 \\
16.6 \\
15.1 \\
10.8\end{array}$ & $\begin{array}{l}7.7 \\
7.2 \\
5.1 \\
2.1 \\
2.4 \\
---\end{array}$ & $\begin{array}{r}9.8 \\
10.6 \\
11.4 \\
5.3 \\
5.7 \\
6.1\end{array}$ & $\begin{array}{r}10.4 \\
12.7 \\
14.3 \\
10.8 \\
3.3 \\
4.1\end{array}$ & $\begin{array}{r}16.8 \\
16.1 \\
17.2 \\
-.- \\
- \\
-\end{array}$ & $\begin{array}{l}16.2 \\
17.6 \\
17.0 \\
14.8 \\
16.2 \\
12.7\end{array}$ & $\begin{array}{r}5.1 \\
16.5 \\
18.7 \\
15.9 \\
17.7 \\
---\end{array}$ & $\begin{array}{l}25.8 \\
26.4 \\
25.3 \\
25.5 \\
25.5 \\
26.7\end{array}$ & $\begin{array}{r}29.5 \\
28.6 \\
26.5 \\
25.5 \\
26.1 \\
---\end{array}$ & $\begin{array}{l}22.4 \\
24.1 \\
24.8 \\
25.5 \\
27.5 \\
28.8\end{array}$ & $\begin{array}{l}25.4 \\
18.4 \\
23.6 \\
25.0 \\
24.0 \\
23.9\end{array}$ & $\begin{array}{r}10.6 \\
9.8 \\
9.5 \\
12.7 \\
13.8 \\
-.-\end{array}$ \\
\hline MAX & --- & 18.0 & 12.2 & 14.3 & 18.4 & 17.6 & 20.1 & 26.7 & 29.5 & 29.2 & 29.9 & 28.0 \\
\hline
\end{tabular}


3 SPRINGS BASIN

3 SPRINGS CREEK NEAR WARM SPRINGS, NV

DAILY MAXIMUM AIR TEMPERATURE, WATER YEAR OCTOBER 1986 TO SEPTEMBER 1987 DEGREES CELSIUS

\begin{tabular}{|c|c|c|c|c|c|c|c|c|c|c|c|c|}
\hline DAY & OCT & NOV & DEC & JAN & FEB & MAR & APR & MAY & JUN & JUL & AUG & SEP \\
\hline $\begin{array}{l}1 \\
2 \\
3 \\
4 \\
5\end{array}$ & $\begin{array}{r}9.1 \\
3.6 \\
9.7 \\
12.0 \\
14.7\end{array}$ & $\begin{array}{r}6.5 \\
9.9 \\
13.6 \\
13.1 \\
16.6\end{array}$ & $\begin{array}{r}10.5 \\
11.7 \\
11.5 \\
8.7 \\
6.1\end{array}$ & $\begin{array}{r}6.9 \\
4.1 \\
5.8 \\
2.5 \\
-1.5\end{array}$ & $\begin{array}{r}11.9 \\
7.6 \\
3.5 \\
5.0 \\
7.7\end{array}$ & $\begin{array}{r}6.7 \\
8.7 \\
11.2 \\
12.4 \\
8.5\end{array}$ & $\begin{array}{r}15.3 \\
15.9 \\
8.7 \\
5.2 \\
10.0\end{array}$ & $\begin{array}{l}12.7 \\
12.1 \\
14.7 \\
18.6 \\
20.3\end{array}$ & $\begin{array}{l}22.2 \\
22.3 \\
24.2 \\
24.1 \\
21.8\end{array}$ & $\begin{array}{l}--- \\
--- \\
--- \\
--- \\
---\end{array}$ & $\begin{array}{l}27.9 \\
28.6 \\
29.3 \\
28.0 \\
29.8\end{array}$ & $\begin{array}{l}26.5 \\
24.0 \\
23.1 \\
21.3 \\
22.1\end{array}$ \\
\hline $\begin{array}{r}6 \\
7 \\
8 \\
9 \\
10\end{array}$ & $\begin{array}{l}15.9 \\
16.7 \\
17.6 \\
17.3 \\
18.3\end{array}$ & $\begin{array}{l}8.4 \\
1.4 \\
3.4 \\
7.2 \\
6.4\end{array}$ & $\begin{array}{r}2.7 \\
1.4 \\
6.1 \\
-1.0 \\
2.4\end{array}$ & $\begin{array}{r}-3.9 \\
-5.1 \\
-5.5 \\
-1.8 \\
5.9\end{array}$ & $\begin{array}{l}8.5 \\
8.0 \\
8.2 \\
5.9 \\
6.1\end{array}$ & $\begin{array}{l}4.3 \\
5.5 \\
7.9 \\
5.9 \\
7.1\end{array}$ & $\begin{array}{l}11.5 \\
13.3 \\
14.9 \\
15.5 \\
15.8\end{array}$ & $\begin{array}{l}21.2 \\
19.6 \\
19.1 \\
18.9 \\
19.8\end{array}$ & $\begin{array}{l}15.1 \\
19.5 \\
18.7 \\
20.8 \\
23.0\end{array}$ & $\begin{array}{c}--- \\
--- \\
26.8 \\
25.6 \\
25.1\end{array}$ & $\begin{array}{l}27.3 \\
27.8 \\
26.1 \\
27.5 \\
27.9\end{array}$ & $\begin{array}{l}21.0 \\
22.3 \\
23.8 \\
24.5 \\
24.2\end{array}$ \\
\hline $\begin{array}{l}11 \\
12 \\
13 \\
14 \\
15\end{array}$ & $\begin{array}{r}7.6 \\
10.0 \\
14.4 \\
16.5 \\
17.1\end{array}$ & $\begin{array}{l}10.4 \\
10.5 \\
11.8 \\
10.4 \\
11.1\end{array}$ & $\begin{array}{l}4.6 \\
7.5 \\
6.1 \\
7.0 \\
4.1\end{array}$ & $\begin{array}{r}8.9 \\
5.9 \\
.2 \\
1.5 \\
-9.4\end{array}$ & $\begin{array}{l}6.4 \\
7.3 \\
6.0 \\
3.7 \\
5.0\end{array}$ & $\begin{array}{r}10.0 \\
12.5 \\
5.9 \\
6.9 \\
-.2\end{array}$ & $\begin{array}{r}12.9 \\
9.7 \\
15.6 \\
16.7 \\
20.3\end{array}$ & $\begin{array}{l}19.6 \\
20.7 \\
21.6 \\
22.1 \\
16.9\end{array}$ & $\begin{array}{l}23.3 \\
25.7 \\
26.2 \\
25.6 \\
19.3\end{array}$ & $\begin{array}{l}25.4 \\
27.2 \\
27.7 \\
29.3 \\
27.7\end{array}$ & $\begin{array}{l}27.5 \\
26.3 \\
24.0 \\
20.6 \\
22.0\end{array}$ & $\begin{array}{l}24.2 \\
21.8 \\
16.9 \\
21.6 \\
22.2\end{array}$ \\
\hline $\begin{array}{l}16 \\
17 \\
18 \\
19 \\
20\end{array}$ & $\begin{array}{r}15.8 \\
11.9 \\
3.2 \\
2.8 \\
8.0\end{array}$ & $\begin{array}{r}10.7 \\
13.9 \\
7.4 \\
10.3 \\
12.7\end{array}$ & $\begin{array}{l}3.5 \\
4.5 \\
3.7 \\
1.7 \\
1.1\end{array}$ & $\begin{array}{r}-9.1 \\
-2.9 \\
1.8 \\
-3.8 \\
-4.5\end{array}$ & $\begin{array}{r}1.9 \\
1.9 \\
-.9 \\
-2.5 \\
.6\end{array}$ & $\begin{array}{r}3.1 \\
8.6 \\
7.7 \\
-2.7 \\
.3\end{array}$ & $\begin{array}{r}22.0 \\
20.0 \\
15.5 \\
5.9 \\
12.2\end{array}$ & $\begin{array}{l}12.6 \\
16.3 \\
17.1 \\
16.0 \\
12.7\end{array}$ & $\begin{array}{l}21.0 \\
22.0 \\
22.3 \\
24.1 \\
24.4\end{array}$ & $\begin{array}{l}22.0 \\
18.3 \\
15.5 \\
17.8 \\
17.4\end{array}$ & $\begin{array}{l}25.0 \\
27.0 \\
28.0 \\
26.8 \\
25.7\end{array}$ & $\begin{array}{l}21.2 \\
21.2 \\
22.5 \\
23.7 \\
23.7\end{array}$ \\
\hline $\begin{array}{l}21 \\
22 \\
23 \\
24 \\
25\end{array}$ & $\begin{array}{l}10.9 \\
12.7 \\
12.7 \\
15.2 \\
16.0\end{array}$ & $\begin{array}{r}8.7 \\
4.2 \\
4.2 \\
12.2 \\
6.7\end{array}$ & $\begin{array}{l}1.7 \\
2.5 \\
2.3 \\
3.2 \\
-.7\end{array}$ & $\begin{array}{l}5.9 \\
6.9 \\
3.5 \\
5.7 \\
9.9\end{array}$ & $\begin{array}{r}2.8 \\
3.7 \\
-2.9 \\
-5.1 \\
-6.4\end{array}$ & $\begin{array}{r}-.8 \\
1.3 \\
.6 \\
3.7 \\
4.5\end{array}$ & $\begin{array}{l}16.8 \\
19.6 \\
18.3 \\
19.1 \\
20.2\end{array}$ & $\begin{array}{r}10.7 \\
13.5 \\
16.2 \\
15.4 \\
8.0\end{array}$ & $\begin{array}{l}24.4 \\
21.6 \\
25.2 \\
27.2 \\
28.0\end{array}$ & $\begin{array}{l}17.4 \\
21.2 \\
24.0 \\
26.3 \\
26.6\end{array}$ & $\begin{array}{l}24.8 \\
25.2 \\
24.4 \\
23.2 \\
23.5\end{array}$ & $\begin{array}{l}23.5 \\
22.9 \\
21.3 \\
22.2 \\
22.8\end{array}$ \\
\hline $\begin{array}{l}26 \\
27 \\
28 \\
29 \\
30 \\
31\end{array}$ & $\begin{array}{r}16.8 \\
17.2 \\
16.2 \\
16.3 \\
15.1 \\
7.0\end{array}$ & $\begin{array}{r}9.3 \\
11.3 \\
11.6 \\
5.6 \\
4.6 \\
---\end{array}$ & $\begin{array}{l}2.8 \\
2.9 \\
3.4 \\
5.5 \\
5.3 \\
6.4\end{array}$ & $\begin{array}{r}12.6 \\
10.2 \\
4.3 \\
3.4 \\
2.7 \\
5.1\end{array}$ & $\begin{array}{r}-2.2 \\
-.5 \\
3.4 \\
--- \\
--- \\
---\end{array}$ & $\begin{array}{r}9.4 \\
1.1 \\
4.8 \\
4.9 \\
11.5 \\
13.7\end{array}$ & $\begin{array}{r}19.7 \\
19.1 \\
18.3 \\
14.0 \\
14.0 \\
---\end{array}$ & $\begin{array}{r}4.1 \\
12.3 \\
13.6 \\
15.0 \\
18.2 \\
22.1\end{array}$ & $\begin{array}{r}27.2 \\
27.5 \\
26.8 \\
23.5 \\
26.6 \\
---\end{array}$ & $\begin{array}{l}26.7 \\
23.9 \\
25.7 \\
25.3 \\
26.0 \\
27.1\end{array}$ & $\begin{array}{l}24.3 \\
24.2 \\
24.4 \\
25.1 \\
26.0 \\
27.5\end{array}$ & $\begin{array}{r}22.5 \\
--- \\
--- \\
-- \\
-- \\
--\end{array}$ \\
\hline MAX & 18.3 & 16.6 & 11.7 & 12.6 & 11.9 & 13.7 & 22.0 & 22.1 & 28.0 & --- & 29.8 & --- \\
\hline
\end{tabular}

CAL YR 1986 MAX 29.9 
3 SPRINGS BASIN

3 SPRINGS CREEK NEAR WARM SPRINGS, NV

DAILY MAXIMUM AIR TEMPERATURE, WATER YEAR OCTOBER 1987 TO SEPTEMBER 1988 DEGREES CELSIUS

\begin{tabular}{|c|c|c|c|c|c|c|c|c|c|c|c|c|}
\hline DAY & $\mathrm{OCT}$ & NOV & $\mathrm{DEC}$ & JAN & FEB & MAR & APR & MAY & JUN & JUL & AUG & SEP \\
\hline $\begin{array}{l}1 \\
2 \\
3 \\
4 \\
5\end{array}$ & $\begin{array}{l}23.1 \\
23.8 \\
23.7 \\
24.6 \\
23.6\end{array}$ & $\begin{array}{r}8.5 \\
5.7 \\
6.0 \\
10.4 \\
5.2\end{array}$ & $\begin{array}{r}6.3 \\
7.1 \\
11.0 \\
7.9 \\
3.7\end{array}$ & $\begin{array}{r}-3.5 \\
-.3 \\
-1.0 \\
3.7 \\
.1\end{array}$ & $\begin{array}{r}-2.8 \\
-2.0 \\
-.3 \\
2.5 \\
4.3\end{array}$ & $\begin{array}{r}5.6 \\
5.0 \\
8.9 \\
8.9 \\
11.1\end{array}$ & $\begin{array}{l}12.9 \\
15.1 \\
14.9 \\
14.2 \\
17.4\end{array}$ & $\begin{array}{r}2.7 \\
11.7 \\
13.6 \\
10.6 \\
8.5\end{array}$ & $\begin{array}{l}18.6 \\
24.2 \\
25.3 \\
23.1 \\
14.3\end{array}$ & $\begin{array}{l}27.6 \\
28.0 \\
26.8 \\
24.4 \\
24.5\end{array}$ & $\begin{array}{l}28.1 \\
26.7 \\
26.0 \\
27.1 \\
22.0\end{array}$ & $\begin{array}{l}26.3 \\
27.6 \\
27.6 \\
27.8 \\
27.3\end{array}$ \\
\hline $\begin{array}{r}6 \\
7 \\
8 \\
9 \\
10\end{array}$ & $\begin{array}{l}24.0 \\
22.7 \\
21.3 \\
21.0 \\
19.8\end{array}$ & $\begin{array}{l}4.2 \\
9.3 \\
9.0 \\
9.9 \\
9.0\end{array}$ & $\begin{array}{r}4.8 \\
2.3 \\
2.5 \\
10.6 \\
13.7\end{array}$ & $\begin{array}{r}.4 \\
.8 \\
.1 \\
6.7 \\
9.7\end{array}$ & $\begin{array}{r}7.9 \\
7.8 \\
9.8 \\
11.3 \\
4.9\end{array}$ & $\begin{array}{r}9.5 \\
4.1 \\
10.9 \\
8.1 \\
-2.5\end{array}$ & $\begin{array}{r}18.9 \\
18.2 \\
7.0 \\
12.4 \\
17.4\end{array}$ & $\begin{array}{r}4.7 \\
6.1 \\
10.5 \\
15.8 \\
18.9\end{array}$ & $\begin{array}{l}12.4 \\
12.6 \\
16.6 \\
20.2 \\
20.8\end{array}$ & $\begin{array}{l}24.8 \\
27.0 \\
28.2 \\
29.3 \\
29.3\end{array}$ & $\begin{array}{l}23.1 \\
23.2 \\
24.1 \\
25.2 \\
25.1\end{array}$ & $\begin{array}{l}26.8 \\
26.5 \\
26.1 \\
23.6 \\
23.0\end{array}$ \\
\hline $\begin{array}{l}11 \\
12 \\
13 \\
14 \\
15\end{array}$ & $\begin{array}{l}20.0 \\
14.6 \\
13.5 \\
14.4 \\
16.6\end{array}$ & $\begin{array}{r}12.3 \\
12.6 \\
7.8 \\
3.5 \\
1.5\end{array}$ & $\begin{array}{r}4.6 \\
-6.9 \\
-7.2 \\
-7.8 \\
-4.7\end{array}$ & $\begin{array}{r}2.7 \\
-2.8 \\
5.4 \\
9.0 \\
3.4\end{array}$ & $\begin{array}{r}7.4 \\
11.1 \\
8.5 \\
5.9 \\
10.6\end{array}$ & $\begin{array}{r}-1.8 \\
1.9 \\
5.2 \\
8.3 \\
3.7\end{array}$ & $\begin{array}{r}19.5 \\
18.2 \\
13.9 \\
7.7 \\
3.8\end{array}$ & $\begin{array}{l}21.8 \\
23.1 \\
18.8 \\
21.5 \\
23.7\end{array}$ & $\begin{array}{l}19.4 \\
19.9 \\
22.2 \\
24.2 \\
25.9\end{array}$ & $\begin{array}{l}27.3 \\
28.2 \\
27.8 \\
28.0 \\
28.4\end{array}$ & $\begin{array}{l}24.2 \\
23.2 \\
22.5 \\
24.2 \\
23.4\end{array}$ & $\begin{array}{l}15.1 \\
10.4 \\
16.1 \\
19.9 \\
22.2\end{array}$ \\
\hline $\begin{array}{l}16 \\
17 \\
18 \\
19 \\
20\end{array}$ & $\begin{array}{l}16.8 \\
17.9 \\
17.5 \\
15.1 \\
16.2\end{array}$ & $\begin{array}{r}7.2 \\
6.5 \\
6.7 \\
10.3 \\
10.3\end{array}$ & $\begin{array}{r}-3.8 \\
-2.5 \\
.5 \\
-1.6 \\
.8\end{array}$ & $\begin{array}{r}.2 \\
-2.7 \\
-6.8 \\
-6.6 \\
.5\end{array}$ & $\begin{array}{r}4.0 \\
3.6 \\
-.5 \\
3.3 \\
8.9\end{array}$ & $\begin{array}{r}4.6 \\
7.6 \\
11.9 \\
15.2 \\
14.7\end{array}$ & $\begin{array}{r}7.6 \\
9.1 \\
10.2 \\
7.4 \\
3.6\end{array}$ & $\begin{array}{l}20.5 \\
15.1 \\
15.7 \\
16.2 \\
19.0\end{array}$ & $\begin{array}{l}25.0 \\
21.0 \\
23.7 \\
24.3 \\
25.0\end{array}$ & $\begin{array}{l}30.1 \\
30.7 \\
31.4 \\
28.7 \\
30.3\end{array}$ & $\begin{array}{l}25.0 \\
26.6 \\
27.9 \\
26.8 \\
24.1\end{array}$ & $\begin{array}{l}21.9 \\
21.7 \\
14.9 \\
19.6 \\
14.1\end{array}$ \\
\hline $\begin{array}{l}21 \\
22 \\
23 \\
24 \\
25\end{array}$ & $\begin{array}{r}16.0 \\
13.3 \\
9.5 \\
9.8 \\
12.0\end{array}$ & $\begin{array}{l}4.7 \\
4.3 \\
3.8 \\
5.9 \\
-.4\end{array}$ & $\begin{array}{r}5.2 \\
5.1 \\
-7.1 \\
-10.2 \\
-7.1\end{array}$ & $\begin{array}{r}-.1 \\
-.7 \\
7.4 \\
1.9 \\
8.3\end{array}$ & $\begin{array}{r}9.0 \\
11.3 \\
10.4 \\
10.7 \\
11.3\end{array}$ & $\begin{array}{l}13.8 \\
13.0 \\
16.7 \\
13.1 \\
15.2\end{array}$ & $\begin{array}{r}4.5 \\
4.6 \\
2.5 \\
10.1 \\
11.6\end{array}$ & $\begin{array}{l}22.5 \\
23.1 \\
22.9 \\
22.2 \\
22.3\end{array}$ & $\begin{array}{l}25.1 \\
23.3 \\
27.1 \\
25.7 \\
24.4\end{array}$ & $\begin{array}{l}29.5 \\
28.5 \\
26.5 \\
26.2 \\
27.8\end{array}$ & $\begin{array}{l}24.0 \\
23.3 \\
26.3 \\
23.1 \\
25.2\end{array}$ & $\begin{array}{r}9.7 \\
19.1 \\
19.0 \\
19.9 \\
20.1\end{array}$ \\
\hline $\begin{array}{l}26 \\
27 \\
28 \\
29 \\
30 \\
31\end{array}$ & $\begin{array}{r}13.8 \\
14.5 \\
13.5 \\
10.4 \\
9.6 \\
6.4\end{array}$ & $\begin{array}{l}.7 \\
3.3 \\
1.9 \\
3.0 \\
3.5 \\
\ldots \ldots\end{array}$ & $\begin{array}{r}-1.2 \\
-.2 \\
1.4 \\
-2.7 \\
-3.7 \\
-4.6\end{array}$ & $\begin{array}{r}8.8 \\
11.1 \\
8.3 \\
5.0 \\
3.1 \\
2.0\end{array}$ & $\begin{array}{r}11.5 \\
6.7 \\
8.1 \\
7.5 \\
\ldots \\
-. .-\end{array}$ & $\begin{array}{r}17.6 \\
10.9 \\
1.0 \\
10.4 \\
1.3 \\
5.7\end{array}$ & $\begin{array}{r}14.4 \\
15.2 \\
14.5 \\
16.2 \\
10.3 \\
-\end{array}$ & $\begin{array}{r}21.2 \\
19.5 \\
20.6 \\
11.1 \\
6.6 \\
15.2\end{array}$ & $\begin{array}{r}25.1 \\
27.3 \\
27.7 \\
26.7 \\
26.7 \\
\ldots .-\end{array}$ & $\begin{array}{l}27.6 \\
26.5 \\
26.7 \\
28.3 \\
27.6 \\
25.9\end{array}$ & $\begin{array}{l}22.3 \\
26.0 \\
25.6 \\
26.2 \\
25.4 \\
25.6\end{array}$ & $\begin{array}{r}21.1 \\
18.8 \\
18.0 \\
21.9 \\
23.7 \\
\ldots\end{array}$ \\
\hline MAX & 24.6 & 12.6 & 13.7 & 11.1 & 11.5 & 17.6 & 19.5 & 23.7 & 27.7 & 31.4 & 28.1 & 27.8 \\
\hline
\end{tabular}

WTR YR 1988 MAX 31.4 
3 SPRINGS BASIN

3 SPRINGS CREEK NEAR WARM SPRINGS, NV DAILY MAXIMUM AIR TEMPERATURE, WATER YEAR OCTOBER 1988 TO SEPTEMBER 1989 DEGREES CELSIUS

\begin{tabular}{|c|c|c|c|c|c|c|c|c|c|c|c|c|}
\hline DAY & OCT & NOV & DEC & JAN & FEB & MAR & APR & MAY & JUN & JUL & AUG & SEP \\
\hline $\begin{array}{l}1 \\
2 \\
3 \\
4 \\
5\end{array}$ & $\begin{array}{l}24.6 \\
24.6 \\
22.7 \\
22.6 \\
20.1\end{array}$ & $\begin{array}{l}16.7 \\
15.7 \\
18.0 \\
16.3 \\
18.0\end{array}$ & $\begin{array}{r}10.1 \\
9.0 \\
6.8 \\
7.7 \\
10.8\end{array}$ & $\begin{array}{r}-1.9 \\
2.5 \\
6.1 \\
4.8 \\
-1.2\end{array}$ & $\begin{array}{r}3.5 \\
1.6 \\
-.8 \\
-5.9 \\
-11.1\end{array}$ & $\begin{array}{r}10.9 \\
5.5 \\
-2.2 \\
.8 \\
11.5\end{array}$ & $\begin{array}{l}11.3 \\
14.0 \\
14.0 \\
16.2 \\
19.0\end{array}$ & $\begin{array}{l}18.0 \\
18.5 \\
20.0 \\
20.4 \\
23.3\end{array}$ & $\begin{array}{l}17.1 \\
18.8 \\
15.7 \\
17.1 \\
15.6\end{array}$ & $\begin{array}{l}25.8 \\
27.0 \\
28.7 \\
28.6 \\
30.2\end{array}$ & $\begin{array}{l}25.6 \\
24.6 \\
24.6 \\
25.1 \\
27.1\end{array}$ & $\begin{array}{l}23.2 \\
24.6 \\
25.0 \\
25.5 \\
25.7\end{array}$ \\
\hline $\begin{array}{r}6 \\
7 \\
8 \\
9 \\
10\end{array}$ & $\begin{array}{l}16.6 \\
18.7 \\
19.9 \\
19.3 \\
17.5\end{array}$ & $\begin{array}{r}15.6 \\
11.7 \\
9.2 \\
9.5 \\
9.1\end{array}$ & $\begin{array}{r}11.2 \\
1.0 \\
2.3 \\
5.5 \\
7.2\end{array}$ & $\begin{array}{r}-6.4 \\
-5.5 \\
-2.3 \\
5.2 \\
8.3\end{array}$ & $\begin{array}{r}-10.9 \\
-8.3 \\
-8.1 \\
-.1 \\
2.6\end{array}$ & $\begin{array}{l}14.2 \\
12.8 \\
12.8 \\
15.2 \\
15.0\end{array}$ & $\begin{array}{l}22.4 \\
23.1 \\
21.5 \\
21.8 \\
22.4\end{array}$ & $\begin{array}{l}23.4 \\
24.2 \\
23.5 \\
17.8 \\
12.0\end{array}$ & $\begin{array}{l}19.5 \\
19.4 \\
19.0 \\
20.8 \\
19.5\end{array}$ & $\begin{array}{l}31.4 \\
32.2 \\
31.0 \\
27.3 \\
26.2\end{array}$ & $\begin{array}{l}28.0 \\
28.2 \\
24.7 \\
21.6 \\
23.9\end{array}$ & $\begin{array}{l}24.3 \\
19.2 \\
19.6 \\
21.8 \\
22.2\end{array}$ \\
\hline $\begin{array}{l}11 \\
12 \\
13 \\
14 \\
15\end{array}$ & $\begin{array}{l}16.9 \\
16.8 \\
17.8 \\
15.3 \\
17.8\end{array}$ & $\begin{array}{r}8.0 \\
11.0 \\
9.7 \\
4.1 \\
3.0\end{array}$ & $\begin{array}{r}8.4 \\
11.4 \\
13.8 \\
8.0 \\
-5.4\end{array}$ & $\begin{array}{r}-5.7 \\
-4.0 \\
-.3 \\
-.6 \\
-.9\end{array}$ & $\begin{array}{r}-.5 \\
-1.4 \\
-4.0 \\
-1.5 \\
2.6\end{array}$ & $\begin{array}{r}15.1 \\
15.6 \\
9.1 \\
8.4 \\
11.3\end{array}$ & $\begin{array}{l}16.0 \\
14.2 \\
18.0 \\
20.2 \\
19.2\end{array}$ & $\begin{array}{r}2.6 \\
8.9 \\
2.8 \\
5.8 \\
10.9\end{array}$ & $\begin{array}{l}21.4 \\
21.7 \\
25.3 \\
27.6 \\
27.7\end{array}$ & $\begin{array}{l}23.5 \\
23.8 \\
25.2 \\
28.3 \\
27.2\end{array}$ & $\begin{array}{l}22.8 \\
24.9 \\
27.4 \\
27.5 \\
26.8\end{array}$ & $\begin{array}{l}19.3 \\
17.3 \\
20.0 \\
22.0 \\
23.3\end{array}$ \\
\hline $\begin{array}{l}16 \\
17 \\
18 \\
19 \\
20\end{array}$ & $\begin{array}{l}20.7 \\
22.3 \\
23.2 \\
21.3 \\
19.5\end{array}$ & $\begin{array}{l}3.7 \\
-.5 \\
-.1 \\
2.1 \\
5.1\end{array}$ & $\begin{array}{r}1.9 \\
4.4 \\
1.6 \\
-.8 \\
.0\end{array}$ & $\begin{array}{l}4.2 \\
8.2 \\
7.3 \\
8.5 \\
8.4\end{array}$ & $\begin{array}{l}4.7 \\
7.4 \\
7.9 \\
6.4 \\
4.2\end{array}$ & $\begin{array}{r}10.6 \\
9.0 \\
10.3 \\
9.8 \\
7.3\end{array}$ & $\begin{array}{l}20.6 \\
21.7 \\
22.6 \\
21.8 \\
21.0\end{array}$ & $\begin{array}{l}15.1 \\
18.5 \\
19.9 \\
16.2 \\
20.3\end{array}$ & $\begin{array}{l}23.5 \\
26.4 \\
28.5 \\
28.0 \\
24.2\end{array}$ & $\begin{array}{l}26.0 \\
28.4 \\
29.8 \\
30.5 \\
30.2\end{array}$ & $\begin{array}{l}26.0 \\
23.4 \\
23.1 \\
23.4 \\
22.2\end{array}$ & $\begin{array}{l}19.5 \\
14.6 \\
13.9 \\
11.7 \\
17.7\end{array}$ \\
\hline $\begin{array}{l}21 \\
22 \\
23 \\
24 \\
25\end{array}$ & $\begin{array}{l}22.0 \\
21.5 \\
21.5 \\
20.6 \\
19.6\end{array}$ & $\begin{array}{r}7.9 \\
11.2 \\
7.3 \\
-1.4 \\
.9\end{array}$ & $\begin{array}{r}-.7 \\
-1.7 \\
-2.2 \\
-2.2 \\
-3.3\end{array}$ & $\begin{array}{r}8.1 \\
4.6 \\
3.7 \\
-2.6 \\
-1.1\end{array}$ & $\begin{array}{r}8.1 \\
12.4 \\
14.5 \\
13.5 \\
14.1\end{array}$ & $\begin{array}{r}14.5 \\
13.1 \\
11.5 \\
12.0 \\
5.2\end{array}$ & $\begin{array}{r}18.7 \\
14.3 \\
10.6 \\
5.1 \\
6.5\end{array}$ & $\begin{array}{l}19.7 \\
21.4 \\
19.1 \\
13.3 \\
15.1\end{array}$ & $\begin{array}{l}18.2 \\
23.3 \\
20.2 \\
14.5 \\
18.4\end{array}$ & $\begin{array}{l}27.1 \\
28.2 \\
28.6 \\
26.0 \\
28.2\end{array}$ & $\begin{array}{l}22.5 \\
23.3 \\
21.3 \\
18.3 \\
20.7\end{array}$ & $\begin{array}{l}19.2 \\
20.0 \\
23.0 \\
23.2 \\
21.9\end{array}$ \\
\hline $\begin{array}{l}26 \\
27 \\
28 \\
29 \\
30 \\
31\end{array}$ & $\begin{array}{l}20.6 \\
18.7 \\
15.8 \\
15.9 \\
17.0 \\
18.7\end{array}$ & $\begin{array}{r}.4 \\
5.4 \\
7.2 \\
4.2 \\
7.8 \\
---\end{array}$ & $\begin{array}{r}-9.0 \\
-8.9 \\
-8.0 \\
-5.7 \\
4.6 \\
-1.0\end{array}$ & $\begin{array}{r}3.8 \\
6.0 \\
3.5 \\
7.9 \\
13.3 \\
12.6\end{array}$ & $\begin{array}{r}13.0 \\
8.7 \\
9.5 \\
--- \\
--- \\
---\end{array}$ & $\begin{array}{r}4.5 \\
11.0 \\
15.0 \\
11.1 \\
14.1 \\
15.8\end{array}$ & $\begin{array}{r}6.8 \\
6.3 \\
9.1 \\
12.2 \\
14.8 \\
-.--\end{array}$ & $\begin{array}{r}18.3 \\
19.9 \\
17.1 \\
13.0 \\
8.6 \\
14.1\end{array}$ & $\begin{array}{r}22.7 \\
20.5 \\
21.0 \\
22.9 \\
22.9 \\
---\end{array}$ & $\begin{array}{l}28.1 \\
27.4 \\
27.1 \\
27.6 \\
28.3 \\
27.0\end{array}$ & $\begin{array}{l}22.7 \\
23.7 \\
25.0 \\
24.8 \\
22.9 \\
22.7\end{array}$ & $\begin{array}{r}21.5 \\
20.3 \\
21.9 \\
20.0 \\
16.6 \\
-.--\end{array}$ \\
\hline MAX & 24.6 & 18.0 & 13.8 & 13.3 & 14.5 & 15.8 & 23.1 & 24.2 & 28.5 & 32.2 & 28.2 & 25.7 \\
\hline
\end{tabular}

CAL YR 1988 MAX 31.4

WTR YR 1989 MAX 32.2 
3 SPRINGS BASIN

3 SPRINGS CREEK NEAR WARM SPRINGS, NV

DAILY MAXIMUM AIR TEMPERATURE, WATER YEAR OCTOBER 1989 TO SEPTEMBER 1990 DEGREES CELSIUS

\begin{tabular}{|c|c|c|c|c|c|c|c|c|c|c|c|c|}
\hline DAY & OCT & NOV & DEC & JAN & FEB & MAR & APR & MAY & JUN & JUL & AUG & SEP \\
\hline $\begin{array}{l}1 \\
2 \\
3 \\
4 \\
5\end{array}$ & $\begin{array}{r}17.5 \\
14.3 \\
9.6 \\
11.8 \\
14.8\end{array}$ & $\begin{array}{r}5.9 \\
8.9 \\
11.6 \\
15.0 \\
14.1\end{array}$ & $\begin{array}{r}4.7 \\
7.4 \\
9.0 \\
12.6 \\
13.7\end{array}$ & $\begin{array}{r}4.0 \\
-2.9 \\
-4.1 \\
1.5 \\
2.8\end{array}$ & $\begin{array}{r}-2.8 \\
-2.8 \\
1.5 \\
-1.9 \\
-.3\end{array}$ & $\begin{array}{r}10.0 \\
10.1 \\
11.0 \\
9.0 \\
1.2\end{array}$ & $\begin{array}{l}13.7 \\
13.7 \\
14.0 \\
11.9 \\
13.9\end{array}$ & $\begin{array}{l}11.1 \\
12.8 \\
14.7 \\
19.0 \\
20.7\end{array}$ & $\begin{array}{l}13.7 \\
20.6 \\
25.1 \\
26.6 \\
23.9\end{array}$ & $\begin{array}{l}29.5 \\
26.7 \\
23.8 \\
25.5 \\
26.7\end{array}$ & $\begin{array}{l}27.1 \\
26.5 \\
28.0 \\
29.0 \\
29.6\end{array}$ & $\begin{array}{l}24.9 \\
24.5 \\
24.2 \\
22.4 \\
22.8\end{array}$ \\
\hline $\begin{array}{r}6 \\
7 \\
8 \\
9 \\
10\end{array}$ & $\begin{array}{l}16.9 \\
15.3 \\
18.8 \\
20.7 \\
21.4\end{array}$ & $\begin{array}{r}10.8 \\
7.5 \\
9.5 \\
14.1 \\
15.2\end{array}$ & $\begin{array}{r}6.3 \\
8.1 \\
11.9 \\
10.0 \\
3.6\end{array}$ & $\begin{array}{r}5.0 \\
8.8 \\
12.0 \\
15.1 \\
12.7\end{array}$ & $\begin{array}{r}.7 \\
-2.1 \\
-2.0 \\
2.0 \\
7.3\end{array}$ & $\begin{array}{r}4.1 \\
8.5 \\
8.2 \\
10.5 \\
9.0\end{array}$ & $\begin{array}{r}12.3 \\
11.6 \\
4.6 \\
13.0 \\
15.5\end{array}$ & $\begin{array}{l}21.3 \\
20.6 \\
15.9 \\
17.8 \\
14.9\end{array}$ & $\begin{array}{l}21.2 \\
24.0 \\
26.0 \\
21.6 \\
16.7\end{array}$ & $\begin{array}{l}26.2 \\
25.3 \\
23.3 \\
24.9 \\
24.2\end{array}$ & $\begin{array}{l}29.3 \\
27.8 \\
29.1 \\
28.0 \\
26.4\end{array}$ & $\begin{array}{l}23.7 \\
25.4 \\
26.6 \\
27.1 \\
27.7\end{array}$ \\
\hline $\begin{array}{l}11 \\
12 \\
13 \\
14 \\
15\end{array}$ & $\begin{array}{l}21.3 \\
21.6 \\
20.3 \\
17.4 \\
14.6\end{array}$ & $\begin{array}{r}17.2 \\
15.2 \\
14.9 \\
10.0 \\
6.5\end{array}$ & $\begin{array}{r}-3.9 \\
2.8 \\
3.6 \\
4.8 \\
9.3\end{array}$ & $\begin{array}{r}12.8 \\
7.2 \\
3.1 \\
1.8 \\
.0\end{array}$ & $\begin{array}{r}7.6 \\
7.8 \\
1.6 \\
-11.2 \\
-4.0\end{array}$ & $\begin{array}{r}-.6 \\
-5.2 \\
-2.6 \\
4.8 \\
5.7\end{array}$ & $\begin{array}{l}16.7 \\
17.3 \\
19.5 \\
20.2 \\
19.0\end{array}$ & $\begin{array}{l}13.9 \\
16.6 \\
16.4 \\
15.4 \\
13.4\end{array}$ & $\begin{array}{l}19.1 \\
18.8 \\
18.7 \\
16.6 \\
13.4\end{array}$ & $\begin{array}{l}28.7 \\
29.7 \\
27.8 \\
25.1 \\
23.9\end{array}$ & $\begin{array}{l}25.6 \\
24.7 \\
25.8 \\
25.3 \\
20.1\end{array}$ & $\begin{array}{l}28.9 \\
28.2 \\
26.9 \\
25.2 \\
24.0\end{array}$ \\
\hline $\begin{array}{l}16 \\
17 \\
18 \\
19 \\
20\end{array}$ & $\begin{array}{l}14.5 \\
14.7 \\
17.9 \\
17.8 \\
15.7\end{array}$ & $\begin{array}{r}10.8 \\
8.1 \\
10.9 \\
15.1 \\
16.3\end{array}$ & $\begin{array}{l}4.3 \\
6.5 \\
4.5 \\
3.6 \\
5.4\end{array}$ & $\begin{array}{r}-2.3 \\
-4.1 \\
-2.6 \\
-.8 \\
-.1\end{array}$ & $\begin{array}{r}-3.2 \\
-.6 \\
-3.6 \\
-2.4 \\
3.6\end{array}$ & $\begin{array}{r}8.5 \\
10.8 \\
12.2 \\
14.1 \\
14.3\end{array}$ & $\begin{array}{r}13.7 \\
6.9 \\
9.8 \\
14.1 \\
12.7\end{array}$ & $\begin{array}{l}16.6 \\
17.9 \\
16.5 \\
12.4 \\
17.0\end{array}$ & $\begin{array}{l}18.6 \\
21.7 \\
20.7 \\
21.7 \\
25.5\end{array}$ & $\begin{array}{l}22.2 \\
24.9 \\
28.0 \\
27.3 \\
28.3\end{array}$ & $\begin{array}{l}22.0 \\
22.4 \\
20.7 \\
17.8 \\
17.3\end{array}$ & $\begin{array}{l}21.7 \\
18.5 \\
17.0 \\
15.6 \\
18.9\end{array}$ \\
\hline $\begin{array}{l}21 \\
22 \\
23 \\
24 \\
25\end{array}$ & $\begin{array}{r}9.7 \\
11.3 \\
11.5 \\
12.0 \\
3.5\end{array}$ & $\begin{array}{r}13.9 \\
12.3 \\
13.1 \\
10.9 \\
7.1\end{array}$ & $\begin{array}{l}4.0 \\
7.1 \\
9.8 \\
8.7 \\
8.7\end{array}$ & $\begin{array}{r}2.3 \\
7.3 \\
7.4 \\
.6 \\
4.9\end{array}$ & $\begin{array}{r}4.1 \\
9.1 \\
11.1 \\
11.6 \\
11.4\end{array}$ & $\begin{array}{l}16.0 \\
16.1 \\
15.2 \\
15.0 \\
15.3\end{array}$ & $\begin{array}{r}11.8 \\
13.1 \\
11.1 \\
8.8 \\
14.6\end{array}$ & $\begin{array}{l}20.1 \\
19.2 \\
18.0 \\
11.0 \\
16.3\end{array}$ & $\begin{array}{l}28.5 \\
28.7 \\
27.2 \\
27.2 \\
28.0\end{array}$ & $\begin{array}{l}28.6 \\
29.4 \\
27.6 \\
25.0 \\
24.3\end{array}$ & $\begin{array}{l}20.1 \\
22.9 \\
23.3 \\
21.2 \\
19.5\end{array}$ & $\begin{array}{l}15.8 \\
17.6 \\
12.5 \\
16.2 \\
18.8\end{array}$ \\
\hline $\begin{array}{l}26 \\
27 \\
28 \\
29 \\
30 \\
31\end{array}$ & $\begin{array}{r}8.0 \\
10.0 \\
1.9 \\
3.7 \\
8.4 \\
9.7\end{array}$ & $\begin{array}{r}2.0 \\
-2.6 \\
1.2 \\
4.6 \\
5.8 \\
---\end{array}$ & $\begin{array}{r}9.3 \\
8.9 \\
3.8 \\
.5 \\
2.4 \\
9.4\end{array}$ & $\begin{array}{r}4.2 \\
-2.4 \\
3.5 \\
5.1 \\
2.9 \\
-1.3\end{array}$ & $\begin{array}{r}10.7 \\
9.0 \\
10.0 \\
- \\
- \\
-\end{array}$ & $\begin{array}{r}15.0 \\
10.6 \\
4.6 \\
6.7 \\
9.2 \\
12.3\end{array}$ & $\begin{array}{r}16.7 \\
18.4 \\
18.1 \\
8.2 \\
7.1 \\
---\end{array}$ & $\begin{array}{r}16.0 \\
16.4 \\
5.3 \\
13.5 \\
14.9 \\
12.9\end{array}$ & $\begin{array}{r}27.8 \\
27.3 \\
28.3 \\
28.3 \\
29.2 \\
-\ldots\end{array}$ & $\begin{array}{l}25.1 \\
27.1 \\
28.0 \\
28.8 \\
28.0 \\
27.7\end{array}$ & $\begin{array}{l}19.9 \\
22.7 \\
25.7 \\
26.2 \\
25.2 \\
25.2\end{array}$ & $\begin{array}{r}18.9 \\
17.9 \\
16.2 \\
17.2 \\
20.4 \\
-\end{array}$ \\
\hline MAX & 21.6 & 17.2 & 13.7 & 15.1 & 11.6 & 16.1 & 20.2 & 21.3 & 29.2 & 29.7 & 29.6 & 28.9 \\
\hline
\end{tabular}

CAL YR 1989 MAX 32.2

WTR YR 1990 MAX 29.7 
3 SPRINGS BASIN

3 SPRINGS CREEK NEAR WARM SPRINGS, NV

DAILY MAXIMUM AIR TEMPERATURE, WATER YEAR OCTOBER 1990 TO SEPTEMBER 1991 DEGREES CELSIUS

\begin{tabular}{|c|c|c|c|c|c|c|c|c|c|c|c|c|}
\hline DAY & $\mathrm{OCT}$ & NOV & DEC & JAN & FEB & MAR & APR & MAY & JUN & JUL & AUG & SEP \\
\hline 1 & 22.0 & 4.1 & 6.8 & 8.2 & 11.4 & .9 & 7.0 & 10.5 & 14.2 & 27.1 & --- & --- \\
\hline 2 & 20.7 & .9 & 5.1 & 7.1 & 5.9 & 7.6 & 11.6 & 5.7 & 20.0 & 29.8 & -- & --- \\
\hline 3 & 19.9 & 7.6 & 5.2 & .4 & 9.4 & 7.9 & 14.2 & 9.2 & 22.2 & 30.5 & -- & -- \\
\hline 4 & 22.3 & 13.4 & 10.6 & .9 & 12.2 & 7.1 & 17.6 & 14.2 & 21.3 & 32.2 & -- & --- \\
\hline 5 & 20.5 & 10.0 & 9.0 & 5.1 & 9.6 & 3.9 & 16.6 & 18.0 & 19.6 & 31.8 & --- & --- \\
\hline 6 & 18.8 & -.5 & 6.5 & 2.0 & 9.7 & 2.3 & 17.3 & 16.4 & 20.8 & 29.3 & -- & --- \\
\hline 7 & 11.6 & 4.9 & 9.5 & -.5 & 11.5 & 4.2 & 10.0 & 20.4 & 23.2 & 28.2 & -- & -- \\
\hline 8 & 11.7 & 13.1 & 10.5 & 1.0 & 12.1 & 7.4 & 10.3 & 19.7 & 23.4 & 22.6 & --- & --- \\
\hline 9 & 17.7 & 14.4 & 12.2 & -.7 & 12.5 & 7.7 & 15.0 & 5.3 & 24.6 & 25.4 & -- & -- \\
\hline 10 & 19.4 & 17.5 & 12.2 & 4.4 & 11.9 & 8.0 & 2.1 & 5.6 & 27.3 & 25.6 & -- & -- \\
\hline 11 & 16.6 & 17.1 & 7.7 & 5.4 & 12.1 & 1.5 & 1.5 & 8.5 & 27.9 & 27.7 & -- & -- \\
\hline 12 & 19.7 & 17.7 & 4.2 & 9.6 & 11.5 & 5.5 & 2.6 & 14.9 & 26.0 & 29.5 & -- & -- \\
\hline 13 & 20.2 & 16.6 & .4 & 9.5 & 11.6 & 3.4 & 9.9 & 14.5 & 23.3 & 29.0 & -- & \\
\hline 14 & 21.2 & 14.9 & -2.2 & 8.2 & 12.9 & 3.6 & 12.4 & 10.7 & 21.8 & 27.5 & -- & --- \\
\hline 15 & 21.7 & 9.8 & 1.9 & 6.6 & 13.1 & -1.3 & 12.1 & 15.6 & 22.9 & 26.6 & --- & --- \\
\hline 16 & 18.4 & 11.6 & -2.8 & 2.8 & 7.3 & 4.3 & 9.5 & 19.1 & 24.6 & 25.7 & -- & -- \\
\hline 17 & 16.6 & 13.1 & 1.2 & 5.4 & 2.2 & 4.7 & 8.2 & 11.6 & 25.5 & 26.6 & -- & -- \\
\hline 18 & 12.2 & 10.3 & 4.6 & 6.4 & 4.4 & 6.4 & 11.0 & 8.4 & 24.6 & 26.2 & --- & --- \\
\hline 19 & 9.7 & 6.2 & -2.2 & 8.5 & 11.9 & 1.8 & 13.2 & 10.3 & 21.5 & 24.6 & --- & -- \\
\hline 20 & 7.4 & .7 & -11.0 & -.4 & 14.2 & -1.0 & 9.9 & 14.6 & 21.5 & 23.6 & --- & \\
\hline 21 & 13.4 & 4.5 & -12.7 & -2.1 & 14.5 & 2.0 & 10.8 & 11.0 & 20.3 & 25.9 & -- & -- \\
\hline 22 & 16.9 & 9.3 & -13.2 & 3.6 & 13.0 & 4.2 & 8.6 & 13.9 & 23.0 & 26.3 & -- & -- \\
\hline 23 & 18.6 & 16.3 & -8.6 & 5.5 & 11.7 & 4.9 & 13.1 & 20.8 & 22.3 & 28.0 & --- & $\ldots$ \\
\hline 24 & 20.0 & 16.1 & -2.7 & 5.9 & 9.9 & 7.4 & 14.1 & 21.9 & 19.1 & 28.3 & -- & -- \\
\hline 25 & 20.0 & 10.7 & -.8 & 6.0 & 11.5 & 1.0 & 4.8 & 20.8 & 18.1 & 27.3 & -- & $\cdots$ \\
\hline 26 & 19.6 & 1.2 & -1.0 & 6.2 & 11.4 & 3.0 & 9.6 & 18.4 & 19.9 & 28.2 & --- & 23.4 \\
\hline 27 & 21.3 & .2 & 3.4 & 6.2 & 9.0 & 1.7 & 8.3 & 16.2 & 13.1 & 29.8 & --- & 17.8 \\
\hline 28 & 19.2 & 3.5 & 6.9 & 4.5 & 1.0 & 9.3 & 11.5 & 18.0 & 18.4 & 30.8 & -- & 19.7 \\
\hline 29 & 16.5 & 8.0 & -4.5 & -.4 & -- & 8.5 & 12.7 & 18.7 & 17.9 & 30.2 & --- & 19.4 \\
\hline 30 & 16.9 & 7.8 & -1.0 & 9.3 & -- & 11.7 & 13.9 & 12.7 & 23.4 & 29.8 & --- & 22.0 \\
\hline 31 & 13.3 & -- & 5.0 & 8.4 & -- & 11.8 & --- & 12.5 & -- & $-\cdots$ & -- & --- \\
\hline MAX & 22.3 & 17.7 & 12.2 & 9.6 & 14.5 & 11.8 & 17.6 & 21.9 & 27.9 & -- & --- & --- \\
\hline
\end{tabular}

CAL YR $1990 \quad$ MAX 29.7 
3 SPRINGS BASIN

3 SPRINGS CREEK NEAR WARM SPRINGS, NV

DAILY MINIMUM AIR TEMPERATURE, WATER YEAR OCTOBER 1985 TO SEPTEMBER 1986 DEGREES CELSIUS

\begin{tabular}{|c|c|c|c|c|c|c|c|c|c|c|c|c|}
\hline DAY & OCT & NOV & DEC & JAN & FEB & MAR & $\mathrm{APR}$ & MAY & JUN & JUL & AUG & SEP \\
\hline $\begin{array}{l}1 \\
2 \\
3 \\
4 \\
5\end{array}$ & $\begin{array}{l}--- \\
--- \\
--- \\
--- \\
---\end{array}$ & $\begin{array}{r}-4.9 \\
-2.2 \\
-1.1 \\
1.4 \\
-2.3\end{array}$ & $\begin{array}{r}-3.7 \\
-.4 \\
-5.5 \\
-6.1 \\
-4.7\end{array}$ & $\begin{array}{r}-4.2 \\
-7.1 \\
-4.6 \\
-2.3 \\
.6\end{array}$ & $\begin{array}{r}-2.8 \\
.4 \\
-3.6 \\
-6.3 \\
-9.7\end{array}$ & $\begin{array}{r}6.8 \\
.3 \\
.0 \\
-1.3 \\
-.9\end{array}$ & $\begin{array}{r}-1.5 \\
-4.8 \\
-5.2 \\
-3.4 \\
-.9\end{array}$ & $\begin{array}{r}10.9 \\
11.0 \\
5.6 \\
-2.5 \\
-4.4\end{array}$ & $\begin{array}{r}7.1 \\
10.3 \\
10.6 \\
9.8 \\
14.3\end{array}$ & $\begin{array}{r}13.8 \\
17.7 \\
12.2 \\
14.8 \\
5.8\end{array}$ & $\begin{array}{l}15.0 \\
20.1 \\
20.6 \\
11.5 \\
16.4\end{array}$ & $\begin{array}{l}6.2 \\
7.1 \\
6.1 \\
6.7 \\
8.2\end{array}$ \\
\hline $\begin{array}{r}6 \\
7 \\
8 \\
9 \\
10\end{array}$ & $\begin{array}{l}--- \\
--- \\
--- \\
--- \\
---\end{array}$ & $\begin{array}{r}-4.3 \\
-2.3 \\
2.4 \\
-6.7 \\
-6.9\end{array}$ & $\begin{array}{r}-8.5 \\
-5.8 \\
-12.0 \\
-15.3 \\
-12.0\end{array}$ & $\begin{array}{r}-7.5 \\
-10.9 \\
-11.3 \\
-4.9 \\
-4.2\end{array}$ & $\begin{array}{l}-12.3 \\
-14.4 \\
-14.7 \\
-16.1 \\
-15.8\end{array}$ & $\begin{array}{r}.7 \\
-.4 \\
-1.3 \\
-3.7 \\
-1.7\end{array}$ & $\begin{array}{r}-1.0 \\
-1.8 \\
-2.1 \\
.2 \\
-1.4\end{array}$ & $\begin{array}{r}-2.0 \\
-3.0 \\
-2.2 \\
-2.5 \\
6.6\end{array}$ & $\begin{array}{r}13.9 \\
11.2 \\
5.2 \\
2.5 \\
2.9\end{array}$ & $\begin{array}{r}15.2 \\
14.8 \\
13.2 \\
10.9 \\
8.9\end{array}$ & $\begin{array}{l}20.9 \\
20.3 \\
13.2 \\
14.0 \\
12.3\end{array}$ & $\begin{array}{r}19.4 \\
14.9 \\
13.9 \\
7.1 \\
3.1\end{array}$ \\
\hline $\begin{array}{l}11 \\
12 \\
13 \\
14 \\
15\end{array}$ & $\begin{array}{r}-1.0 \\
2.0 \\
-2.7 \\
-3.0 \\
-3.4\end{array}$ & $\begin{array}{r}-8.8 \\
-15.9 \\
-17.1 \\
-17.3 \\
-12.4\end{array}$ & $\begin{array}{l}-22.5 \\
-20.6 \\
-14.9 \\
-12.4 \\
-10.1\end{array}$ & $\begin{array}{r}-4.9 \\
-3.4 \\
-5.3 \\
-5.0 \\
-.2\end{array}$ & $\begin{array}{r}-4.7 \\
-2.9 \\
-.1 \\
1.0 \\
.4\end{array}$ & $\begin{array}{l}-2.3 \\
-6.1 \\
-6.0 \\
-7.2 \\
-3.1\end{array}$ & $\begin{array}{r}1.2 \\
-2.3 \\
-4.5 \\
-4.5 \\
3.6\end{array}$ & $\begin{array}{l}-.5 \\
-.1 \\
-.8 \\
2.4 \\
4.4\end{array}$ & $\begin{array}{r}7.1 \\
9.6 \\
6.5 \\
10.3 \\
6.1\end{array}$ & $\begin{array}{r}11.3 \\
13.1 \\
14.7 \\
18.8 \\
6.1\end{array}$ & $\begin{array}{r}10.5 \\
16.6 \\
9.1 \\
12.8 \\
20.8\end{array}$ & $\begin{array}{r}2.3 \\
10.4 \\
11.3 \\
9.7 \\
6.1\end{array}$ \\
\hline $\begin{array}{l}16 \\
17 \\
18 \\
19 \\
20\end{array}$ & $\begin{array}{r}-2.0 \\
-1.9 \\
-1.5 \\
.0 \\
9.7\end{array}$ & $\begin{array}{r}-7.0 \\
-10.6 \\
-19.6 \\
-20.5 \\
-12.0\end{array}$ & $\begin{array}{r}-9.6 \\
-11.0 \\
-7.5 \\
-5.7 \\
-7.2\end{array}$ & $\begin{array}{l}-2.8 \\
-3.2 \\
-3.2 \\
-3.0 \\
-5.6\end{array}$ & $\begin{array}{r}-.1 \\
2.6 \\
2.9 \\
.4 \\
-2.6\end{array}$ & $\begin{array}{r}-6.8 \\
-8.3 \\
-10.7 \\
-7.9 \\
-4.6\end{array}$ & $\begin{array}{r}-2.7 \\
-4.5 \\
-3.1 \\
-4.9 \\
-.1\end{array}$ & $\begin{array}{r}-1.1 \\
-1.2 \\
.7 \\
13.6 \\
13.2\end{array}$ & $\begin{array}{r}17.2 \\
18.0 \\
11.1 \\
4.9 \\
5.1\end{array}$ & $\begin{array}{r}6.2 \\
5.2 \\
14.3 \\
9.6 \\
10.3\end{array}$ & $\begin{array}{l}17.4 \\
16.1 \\
14.7 \\
10.5 \\
11.7\end{array}$ & $\begin{array}{r}1.4 \\
8.6 \\
5.8 \\
5.8 \\
-2.8\end{array}$ \\
\hline $\begin{array}{l}21 \\
22 \\
23 \\
24 \\
25\end{array}$ & $\begin{array}{r}-7.5 \\
-9.8 \\
1.1 \\
.4 \\
1.4\end{array}$ & $\begin{array}{r}-6.6 \\
-4.2 \\
-3.7 \\
-.6 \\
-4.4\end{array}$ & $\begin{array}{l}-8.5 \\
-7.4 \\
-6.7 \\
-5.5 \\
-7.1\end{array}$ & $\begin{array}{l}-7.1 \\
-6.6 \\
-7.0 \\
-9.1 \\
-7.9\end{array}$ & $\begin{array}{l}-3.9 \\
-2.5 \\
-1.7 \\
-.5 \\
-.7\end{array}$ & $\begin{array}{r}-2.5 \\
-.9 \\
.8 \\
3.3 \\
-2.0\end{array}$ & $\begin{array}{r}2.2 \\
5.7 \\
4.9 \\
2.2 \\
-2.5\end{array}$ & $\begin{array}{r}.7 \\
-4.5 \\
-.3 \\
2.8 \\
4.7\end{array}$ & $\begin{array}{r}5.3 \\
7.4 \\
8.6 \\
12.0 \\
10.8\end{array}$ & $\begin{array}{r}10.0 \\
8.6 \\
10.9 \\
8.8 \\
7.2\end{array}$ & $\begin{array}{r}9.4 \\
10.1 \\
11.1 \\
17.6 \\
12.7\end{array}$ & $\begin{array}{r}-2.6 \\
-1.4 \\
.3 \\
4.5 \\
-1.4\end{array}$ \\
\hline $\begin{array}{l}26 \\
27 \\
28 \\
29 \\
30 \\
31\end{array}$ & $\begin{array}{r}-1.9 \\
-.4 \\
1.6 \\
-.5 \\
2.5 \\
-3.1\end{array}$ & $\begin{array}{r}-8.5 \\
-2.4 \\
1.2 \\
-4.0 \\
-6.0 \\
---\end{array}$ & $\begin{array}{l}-6.6 \\
-7.6 \\
-6.1 \\
-5.9 \\
-2.1 \\
-3.5\end{array}$ & $\begin{array}{r}-6.6 \\
-6.0 \\
-3.5 \\
1.4 \\
.5 \\
1.2\end{array}$ & $\begin{array}{r}-1.0 \\
-1.0 \\
-.8 \\
--- \\
--- \\
---\end{array}$ & $\begin{array}{r}-1.7 \\
-.1 \\
1.6 \\
5.4 \\
4.8 \\
1.2\end{array}$ & $\begin{array}{r}-4.5 \\
-5.2 \\
3.0 \\
2.3 \\
-.9 \\
--\end{array}$ & $\begin{array}{r}18.0 \\
14.4 \\
11.2 \\
7.6 \\
8.5 \\
8.5\end{array}$ & $\begin{array}{r}11.3 \\
21.0 \\
12.9 \\
9.3 \\
10.7 \\
-\ldots\end{array}$ & $\begin{array}{r}5.7 \\
15.3 \\
14.7 \\
7.5 \\
8.3 \\
9.9\end{array}$ & $\begin{array}{r}12.1 \\
10.0 \\
9.5 \\
15.7 \\
13.7 \\
6.8\end{array}$ & $\begin{array}{r}-2.3 \\
.5 \\
-2.0 \\
-4.3 \\
-.5 \\
-.--\end{array}$ \\
\hline MIN & --- & -20.5 & -22.5 & -11.3 & -16.1 & -10.7 & -5.2 & -4.5 & 2.5 & 5.2 & 6.8 & $-4 \cdot 3$ \\
\hline
\end{tabular}


3 SPRINGS BASIN

3 SPRINGS CREEK NEAR WARM SPRINGS, NV

DAIIY MINIMUM AIR TEMPERATURE, WATER YEAR OCTOBER 1986 TO SEPTEMBER 1987 DEGREES CELSIUS

\begin{tabular}{|c|c|c|c|c|c|c|c|c|c|c|c|c|}
\hline DAY & OCT & NOV & DEC & JAN & FEB & MAR & APR & MAY & JUN & JUL & AUG & SEP \\
\hline $\begin{array}{l}1 \\
2 \\
3 \\
4 \\
5\end{array}$ & $\begin{array}{r}-1.1 \\
.5 \\
-1.5 \\
-2.7 \\
-1.0\end{array}$ & $\begin{array}{r}-2.2 \\
.6 \\
1.7 \\
-1.4 \\
-2.3\end{array}$ & $\begin{array}{r}-8.9 \\
-5.9 \\
-5.8 \\
-2.7 \\
2.7\end{array}$ & $\begin{array}{r}-6.5 \\
-7.1 \\
1.9 \\
-3.2 \\
-9.7\end{array}$ & $\begin{array}{r}-6.2 \\
-1.5 \\
-4.7 \\
-10.1 \\
-9.4\end{array}$ & $\begin{array}{r}-9.1 \\
-4.9 \\
-3.5 \\
-1.6 \\
2.4\end{array}$ & $\begin{array}{r}-2.8 \\
-1.6 \\
-1.5 \\
.0 \\
-2.6\end{array}$ & $\begin{array}{r}.3 \\
-3.0 \\
-1.2 \\
-.2 \\
1.7\end{array}$ & $\begin{array}{r}2.0 \\
1.9 \\
3.5 \\
10.7 \\
9.8\end{array}$ & $\begin{array}{l}--- \\
--- \\
--- \\
--- \\
---\end{array}$ & $\begin{array}{l}17.5 \\
14.3 \\
10.8 \\
20.7 \\
20.0\end{array}$ & $\begin{array}{r}10.0 \\
14.4 \\
9.9 \\
7.8 \\
11.1\end{array}$ \\
\hline $\begin{array}{r}6 \\
7 \\
8 \\
9 \\
10\end{array}$ & $\begin{array}{r}-1.5 \\
1.0 \\
2.5 \\
2.3 \\
1.2\end{array}$ & $\begin{array}{r}-4.0 \\
-8.3 \\
-8.3 \\
-10.9 \\
-5.8\end{array}$ & $\begin{array}{r}-2.6 \\
-5.1 \\
-10.4 \\
-13.4 \\
-15.1\end{array}$ & $\begin{array}{l}-12.8 \\
-15.0 \\
-19.1 \\
-18.0 \\
-13.4\end{array}$ & $\begin{array}{r}-6.4 \\
-2.4 \\
-3.1 \\
-1.3 \\
-.2\end{array}$ & $\begin{array}{r}.9 \\
-.1 \\
-.5 \\
-2.6 \\
-3.9\end{array}$ & $\begin{array}{l}-2.4 \\
-2.6 \\
-1.2 \\
-.8 \\
-.9\end{array}$ & $\begin{array}{l}2.1 \\
4.8 \\
4.2 \\
4.3 \\
3.9\end{array}$ & $\begin{array}{l}7.6 \\
6.4 \\
5.6 \\
4.2 \\
5.4\end{array}$ & $\begin{array}{l}--- \\
--- \\
19.6 \\
18.1 \\
16.2\end{array}$ & $\begin{array}{l}19.4 \\
11.7 \\
11.5 \\
10.7 \\
19.1\end{array}$ & $\begin{array}{l}6.8 \\
4.7 \\
9.2 \\
6.6 \\
8.3\end{array}$ \\
\hline $\begin{array}{l}11 \\
12 \\
13 \\
14 \\
15\end{array}$ & $\begin{array}{r}-.9 \\
-2.2 \\
-4.8 \\
-2.6 \\
-2.3\end{array}$ & $\begin{array}{r}-6.5 \\
-4.8 \\
-4.8 \\
5.4 \\
-.3\end{array}$ & $\begin{array}{r}-11.9 \\
-10.4 \\
-8.0 \\
-8.5 \\
-8.8\end{array}$ & $\begin{array}{l}-11.2 \\
-12.0 \\
-13.6 \\
-18.8 \\
-19.2\end{array}$ & $\begin{array}{r}-1.2 \\
-.7 \\
-2.1 \\
-3.5 \\
-3.0\end{array}$ & $\begin{array}{l}-.4 \\
-1.4 \\
-4.1 \\
-4.4 \\
-3.8\end{array}$ & $\begin{array}{r}.7 \\
-3.4 \\
-5.1 \\
.6 \\
5.2\end{array}$ & $\begin{array}{l}3.9 \\
6.8 \\
5.3 \\
7.4 \\
7.4\end{array}$ & $\begin{array}{r}7.6 \\
14.0 \\
11.6 \\
9.1 \\
6.6\end{array}$ & $\begin{array}{r}9.1 \\
9.7 \\
12.1 \\
10.5 \\
14.2\end{array}$ & $\begin{array}{r}15.9 \\
9.8 \\
16.5 \\
10.2 \\
4.9\end{array}$ & $\begin{array}{l}7.4 \\
5.9 \\
2.1 \\
7.2 \\
6.0\end{array}$ \\
\hline $\begin{array}{l}16 \\
17 \\
18 \\
19 \\
20\end{array}$ & $\begin{array}{r}-1.7 \\
-.9 \\
-1.9 \\
-1.1 \\
-2.7\end{array}$ & $\begin{array}{r}-2.6 \\
2.5 \\
1.1 \\
.1 \\
-1.6\end{array}$ & $\begin{array}{l}-7.6 \\
-8.4 \\
-8.2 \\
-2.3 \\
-7.9\end{array}$ & $\begin{array}{l}-16.2 \\
-14.1 \\
-14.6 \\
-11.7 \\
-15.7\end{array}$ & $\begin{array}{r}-8.1 \\
-7.9 \\
-9.5 \\
-12.5 \\
-10.7\end{array}$ & $\begin{array}{r}-3.6 \\
-4.9 \\
-4.1 \\
-8.7 \\
-11.9\end{array}$ & $\begin{array}{l}.6 \\
12.1 \\
-4.0 \\
-6.1 \\
-4.8\end{array}$ & $\begin{array}{l}7.0 \\
5.1 \\
3.8 \\
1.6 \\
-.5\end{array}$ & $\begin{array}{r}2.3 \\
12.8 \\
8.1 \\
4.4 \\
10.0\end{array}$ & $\begin{array}{r}13.0 \\
5.8 \\
4.6 \\
10.3 \\
9.8\end{array}$ & $\begin{array}{r}4.2 \\
7.6 \\
9.0 \\
19.9 \\
19.7\end{array}$ & $\begin{array}{r}4.3 \\
.9 \\
5.8 \\
4.6 \\
3.5\end{array}$ \\
\hline $\begin{array}{l}21 \\
22 \\
23 \\
24 \\
25\end{array}$ & $\begin{array}{r}-2.2 \\
-1.2 \\
-1.1 \\
-.1 \\
.9\end{array}$ & $\begin{array}{l}-4.2 \\
-9.2 \\
-8.2 \\
-5.3 \\
-5.2\end{array}$ & $\begin{array}{r}-10.4 \\
-10.3 \\
-9.7 \\
-12.1 \\
-12.6\end{array}$ & $\begin{array}{r}-13.8 \\
-4.9 \\
-8.0 \\
-7.6 \\
-4.7\end{array}$ & $\begin{array}{r}-14.2 \\
-10.9 \\
-9.3 \\
-13.2 \\
-16.2\end{array}$ & $\begin{array}{r}-7.9 \\
-10.2 \\
-9.2 \\
-3.1 \\
-4.7\end{array}$ & $\begin{array}{r}-2.8 \\
.2 \\
7.1 \\
3.2 \\
5.6\end{array}$ & $\begin{array}{r}-1.7 \\
-.8 \\
-.1 \\
.8 \\
2.0\end{array}$ & $\begin{array}{r}13.8 \\
3.1 \\
3.8 \\
7.5 \\
9.3\end{array}$ & $\begin{array}{r}9.2 \\
8.5 \\
6.7 \\
16.8 \\
17.7\end{array}$ & $\begin{array}{r}18.2 \\
14.9 \\
11.9 \\
8.6 \\
6.4\end{array}$ & $\begin{array}{l}4.2 \\
6.1 \\
6.0 \\
6.7 \\
8.0\end{array}$ \\
\hline $\begin{array}{l}26 \\
27 \\
28 \\
29 \\
30 \\
31\end{array}$ & $\begin{array}{r}.5 \\
8.7 \\
.6 \\
3.5 \\
4.5 \\
.1\end{array}$ & $\begin{array}{r}-8.9 \\
-3.4 \\
5.6 \\
-4.4 \\
-10.7 \\
---\end{array}$ & $\begin{array}{r}-12.0 \\
-7.8 \\
-9.1 \\
-9.0 \\
-10.6 \\
-9.8\end{array}$ & $\begin{array}{r}5.6 \\
4.5 \\
-6.1 \\
-5.8 \\
-5.5 \\
-9.0\end{array}$ & $\begin{array}{r}-16.9 \\
-19.3 \\
-13.4 \\
--- \\
--- \\
---\end{array}$ & $\begin{array}{l}-7.8 \\
-9.1 \\
-8.7 \\
-9.1 \\
-8.8 \\
-4.4\end{array}$ & $\begin{array}{l}7.1 \\
3.9 \\
5.8 \\
6.7 \\
5.2 \\
---\end{array}$ & $\begin{array}{l}.7 \\
2.8 \\
2.3 \\
1.0 \\
1.9 \\
9.6\end{array}$ & $\begin{array}{r}13.8 \\
13.5 \\
10.2 \\
10.2 \\
8.1 \\
-.-\end{array}$ & $\begin{array}{l}14.0 \\
14.1 \\
11.0 \\
14.4 \\
11.7 \\
17.8\end{array}$ & $\begin{array}{r}3.6 \\
6.0 \\
17.5 \\
11.1 \\
10.0 \\
9.9\end{array}$ & $\begin{array}{l}6.6 \\
--- \\
--- \\
--- \\
--- \\
---\end{array}$ \\
\hline MIN & -4.8 & -10.9 & -15.1 & -19.2 & -19.3 & -11.9 & -6.1 & -3.0 & 1.9 & -- & 3.6 & -- \\
\hline
\end{tabular}

CAL YR 1986 MIN -16.1 
3 SPRINGS BASIN

3 SPRINGS CREEK NEAR WARM SPRINGS, NV

DAILY MINIMUM AIR TEMPERATURE, WATER YEAR OCTOBER 1987 TO SEPTEMBER 1988 DEGREES CELSIUS

\begin{tabular}{|c|c|c|c|c|c|c|c|c|c|c|c|c|}
\hline DAY & OCT & NOV & DEC & JAN & $\mathrm{FEB}$ & MAR & APR & MAY & JUN & JUL & AUG & SEP \\
\hline $\begin{array}{l}1 \\
2 \\
3 \\
4 \\
5\end{array}$ & $\begin{array}{l}3.7 \\
3.5 \\
6.5 \\
5.8 \\
4.4\end{array}$ & $\begin{array}{r}4.4 \\
1.3 \\
.3 \\
1.0 \\
.2\end{array}$ & $\begin{array}{r}-6.3 \\
-2.7 \\
-3.6 \\
.8 \\
-3.5\end{array}$ & $\begin{array}{r}-17.0 \\
-13.4 \\
-5.2 \\
-4.2 \\
-3.8\end{array}$ & $\begin{array}{l}-12.3 \\
-12.5 \\
-15.5 \\
-14.0 \\
-13.0\end{array}$ & $\begin{array}{l}-1.8 \\
-2.7 \\
-5.1 \\
-2.7 \\
-2.9\end{array}$ & $\begin{array}{r}-6.5 \\
-2.5 \\
5.3 \\
-1.5 \\
-3.6\end{array}$ & $\begin{array}{r}-6.3 \\
-6.9 \\
-1.6 \\
5.4 \\
-4.1\end{array}$ & $\begin{array}{r}6.7 \\
5.7 \\
17.0 \\
15.2 \\
6.2\end{array}$ & $\begin{array}{l}15.0 \\
19.3 \\
20.5 \\
17.8 \\
15.2\end{array}$ & $\begin{array}{r}11.9 \\
16.0 \\
11.2 \\
9.2 \\
9.9\end{array}$ & $\begin{array}{l}9.7 \\
9.9 \\
8.8 \\
9.6 \\
9.7\end{array}$ \\
\hline $\begin{array}{r}6 \\
7 \\
8 \\
9 \\
10\end{array}$ & $\begin{array}{l}3.7 \\
7.9 \\
4.9 \\
4.6 \\
4.1\end{array}$ & $\begin{array}{r}.2 \\
-.3 \\
-1.7 \\
-1.6 \\
-2.2\end{array}$ & $\begin{array}{r}-3.4 \\
-9.0 \\
-9.5 \\
-2.6 \\
1.2\end{array}$ & $\begin{array}{r}-11.4 \\
-12.1 \\
-8.1 \\
-5.9 \\
-. .5\end{array}$ & $\begin{array}{r}-11.9 \\
-8.9 \\
-4.7 \\
-6.3 \\
-3.8\end{array}$ & $\begin{array}{r}1.6 \\
-6.6 \\
-8.4 \\
-6.5 \\
-8.5\end{array}$ & $\begin{array}{r}2.8 \\
7.1 \\
-4.6 \\
-7.2 \\
-3.2\end{array}$ & $\begin{array}{r}-5.2 \\
-5.0 \\
-3.6 \\
3.0 \\
.9\end{array}$ & $\begin{array}{r}3.8 \\
1.8 \\
.8 \\
4.7 \\
12.5\end{array}$ & $\begin{array}{r}17.1 \\
9.0 \\
17.8 \\
11.1 \\
10.7\end{array}$ & $\begin{array}{r}7.1 \\
5.4 \\
7.2 \\
7.6 \\
11.4\end{array}$ & $\begin{array}{r}19.0 \\
13.5 \\
17.7 \\
13.5 \\
7.0\end{array}$ \\
\hline $\begin{array}{l}11 \\
12 \\
13 \\
14 \\
15\end{array}$ & $\begin{array}{r}2.5 \\
3.2 \\
1.8 \\
-1.7 \\
-1.2\end{array}$ & $\begin{array}{r}-3.0 \\
-2.4 \\
.9 \\
-5.4 \\
-8.9\end{array}$ & $\begin{array}{r}-9.8 \\
-11.5 \\
-11.4 \\
-16.3 \\
-15.1\end{array}$ & $\begin{array}{r}-13.5 \\
-15.4 \\
-12.1 \\
-7.4 \\
-5.7\end{array}$ & $\begin{array}{r}-7.2 \\
-8.4 \\
-7.7 \\
-10.3 \\
-6.1\end{array}$ & $\begin{array}{r}-12.7 \\
-13.8 \\
-11.2 \\
-8.7 \\
-5.1\end{array}$ & $\begin{array}{r}.2 \\
1.1 \\
4.0 \\
.5 \\
.2\end{array}$ & $\begin{array}{l}3.2 \\
6.2 \\
4.2 \\
2.9 \\
7.1\end{array}$ & $\begin{array}{l}8.2 \\
4.3 \\
3.4 \\
6.3 \\
7.3\end{array}$ & $\begin{array}{l}10.5 \\
11.5 \\
14.0 \\
19.8 \\
20.0\end{array}$ & $\begin{array}{r}7.3 \\
6.3 \\
14.0 \\
16.0 \\
8.2\end{array}$ & $\begin{array}{r}.7 \\
.9 \\
-.4 \\
.2 \\
2.8\end{array}$ \\
\hline $\begin{array}{l}16 \\
17 \\
18 \\
19 \\
20\end{array}$ & $\begin{array}{r}-1.4 \\
1.3 \\
.0 \\
-.8 \\
-1.5\end{array}$ & $\begin{array}{l}-2.5 \\
-2.5 \\
-5.3 \\
-6.7 \\
-5.2\end{array}$ & $\begin{array}{r}-9.2 \\
-6.1 \\
-9.8 \\
-13.9 \\
-15.8\end{array}$ & $\begin{array}{r}-12.7 \\
-6.9 \\
-20.3 \\
-22.2 \\
-19.2\end{array}$ & $\begin{array}{r}-7.4 \\
-10.4 \\
-5.3 \\
-11.4 \\
-7.8\end{array}$ & $\begin{array}{l}-4.5 \\
-7.0 \\
-6.4 \\
-3.9 \\
-1.5\end{array}$ & $\begin{array}{r}1.4 \\
-.1 \\
-1.4 \\
3.5 \\
-.8\end{array}$ & $\begin{array}{r}13.1 \\
4.2 \\
-.5 \\
.7 \\
.7\end{array}$ & $\begin{array}{r}10.3 \\
8.2 \\
8.7 \\
10.4 \\
9.6\end{array}$ & $\begin{array}{r}14.2 \\
10.9 \\
10.7 \\
10.0 \\
9.0\end{array}$ & $\begin{array}{r}4.6 \\
8.8 \\
11.1 \\
14.2 \\
13.5\end{array}$ & $\begin{array}{r}15.1 \\
4.1 \\
-2.1 \\
-1.0 \\
3.9\end{array}$ \\
\hline $\begin{array}{l}21 \\
22 \\
23 \\
24 \\
25\end{array}$ & $\begin{array}{r}2.4 \\
5.2 \\
4.3 \\
.9 \\
-.3\end{array}$ & $\begin{array}{l}-4.8 \\
-5.7 \\
-6.8 \\
-8.4 \\
-8.0\end{array}$ & $\begin{array}{r}-2.7 \\
-6.5 \\
-13.7 \\
-14.9 \\
-17.8\end{array}$ & $\begin{array}{r}-10.9 \\
-15.6 \\
-8.9 \\
-10.1 \\
-10.3\end{array}$ & $\begin{array}{l}-8.2 \\
-6.4 \\
-7.2 \\
-3.0 \\
-4.3\end{array}$ & $\begin{array}{r}2.1 \\
-1.9 \\
4.2 \\
-3.2 \\
-1.1\end{array}$ & $\begin{array}{r}-3.9 \\
-1.8 \\
-2.7 \\
-3.0 \\
-.7\end{array}$ & $\begin{array}{r}1.4 \\
10.2 \\
7.6 \\
12.8 \\
15.2\end{array}$ & $\begin{array}{r}9.8 \\
10.0 \\
9.9 \\
12.2 \\
13.3\end{array}$ & $\begin{array}{l}18.6 \\
15.0 \\
14.9 \\
11.9 \\
12.4\end{array}$ & $\begin{array}{l}12.2 \\
10.6 \\
12.1 \\
14.3 \\
12.0\end{array}$ & $\begin{array}{r}1.3 \\
.0 \\
1.6 \\
11.1 \\
11.2\end{array}$ \\
\hline $\begin{array}{l}26 \\
27 \\
28 \\
29 \\
30 \\
31\end{array}$ & $\begin{array}{r}.2 \\
4.4 \\
4.4 \\
1.4 \\
2.3 \\
.6\end{array}$ & $\begin{array}{r}-9.1 \\
-10.4 \\
-10.5 \\
-9.4 \\
-8.4 \\
---\end{array}$ & $\begin{array}{r}-14.5 \\
-13.2 \\
-12.6 \\
-6.6 \\
-17.7 \\
-19.4\end{array}$ & $\begin{array}{r}-7.5 \\
-2.5 \\
-2.3 \\
.1 \\
-5.0 \\
-9.6\end{array}$ & $\begin{array}{r}-3.4 \\
-.9 \\
1.2 \\
-1.5 \\
--- \\
---\end{array}$ & $\begin{array}{l}-1.1 \\
-7.1 \\
-9.3 \\
-9.5 \\
-5.9 \\
-8.1\end{array}$ & $\begin{array}{r}-2.8 \\
6.3 \\
8.9 \\
3.9 \\
-4.3 \\
---\end{array}$ & $\begin{array}{r}10.0 \\
5.8 \\
3.1 \\
-2.8 \\
-3.5 \\
-2.0\end{array}$ & $\begin{array}{r}9.1 \\
18.3 \\
16.4 \\
11.0 \\
7.4 \\
---\end{array}$ & $\begin{array}{l}11.1 \\
11.3 \\
11.0 \\
11.8 \\
12.1 \\
13.0\end{array}$ & $\begin{array}{r}13.5 \\
9.6 \\
11.2 \\
11.4 \\
10.2 \\
11.1\end{array}$ & $\begin{array}{l}8.3 \\
3.5 \\
-.3 \\
3.0 \\
4.9 \\
-\end{array}$ \\
\hline MIN & -1.5 & -10.5 & -19.4 & -22.2 & -15.5 & -13.8 & -7.2 & -6.9 & .8 & 9.0 & 4.6 & -2.1 \\
\hline
\end{tabular}

WTR YR 1988 MIN -22.2 


\section{SPRINGS BASIN}

3 SPRINGS CREEK NEAR WARM SPRINGS, NV DAILY MINIMUM AIR TEMPERATURE, WATER YEAR OCTOBER 1988 TO SEPTEMBER 1989 DEGREES CELSIUS

\begin{tabular}{|c|c|c|c|c|c|c|c|c|c|c|c|c|}
\hline DAY & $\mathrm{OCT}$ & NOV & DEC & JAN & FEB & MAR & APR & MAY & JUN & JUL & AUG & SEP \\
\hline $\begin{array}{l}1 \\
2 \\
3 \\
4 \\
5\end{array}$ & $\begin{array}{l}4.3 \\
7.0 \\
7.9 \\
6.9 \\
7.7\end{array}$ & $\begin{array}{r}10.2 \\
3.3 \\
10.6 \\
2.5 \\
-.3\end{array}$ & $\begin{array}{l}-8.5 \\
-7.2 \\
-8.0 \\
-7.9 \\
-8.6\end{array}$ & $\begin{array}{r}-15.0 \\
-15.5 \\
-10.3 \\
-4.8 \\
-7.8\end{array}$ & $\begin{array}{r}.2 \\
-3.0 \\
-5.9 \\
-18.4 \\
-25.8\end{array}$ & $\begin{array}{r}-5.6 \\
-1.5 \\
-11.1 \\
-14.2 \\
-5.4\end{array}$ & $\begin{array}{r}-1.8 \\
5.9 \\
2.5 \\
-1.0 \\
-1.9\end{array}$ & $\begin{array}{r}5.1 \\
.7 \\
4.0 \\
2.5 \\
5.5\end{array}$ & $\begin{array}{l}-.3 \\
3.3 \\
5.6 \\
4.1 \\
1.0\end{array}$ & $\begin{array}{r}7.7 \\
15.0 \\
18.3 \\
14.9 \\
10.3\end{array}$ & $\begin{array}{l}18.6 \\
17.0 \\
17.8 \\
17.6 \\
17.7\end{array}$ & $\begin{array}{r}9.7 \\
15.2 \\
5.8 \\
3.9 \\
12.6\end{array}$ \\
\hline $\begin{array}{r}6 \\
7 \\
8 \\
9 \\
10\end{array}$ & $\begin{array}{r}2.2 \\
.9 \\
1.7 \\
1.0 \\
3.9\end{array}$ & $\begin{array}{l}-1.1 \\
-2.9 \\
-2.2 \\
-4.6 \\
-3.1\end{array}$ & $\begin{array}{l}-6.3 \\
-5.5 \\
-7.0 \\
-9.7 \\
-6.6\end{array}$ & $\begin{array}{r}-17.9 \\
-18.9 \\
-19.8 \\
-1.6 \\
-11.3\end{array}$ & $\begin{array}{r}-28.8 \\
-27.4 \\
-21.1 \\
-8.7 \\
-11.3\end{array}$ & $\begin{array}{r}.3 \\
5.6 \\
7.6 \\
8.6 \\
8.6\end{array}$ & $\begin{array}{l}1.2 \\
2.9 \\
3.1 \\
-.2 \\
-.7\end{array}$ & $\begin{array}{l}6.2 \\
8.1 \\
5.7 \\
6.4 \\
1.4\end{array}$ & $\begin{array}{l}1.9 \\
6.1 \\
3.7 \\
6.0 \\
4.5\end{array}$ & $\begin{array}{r}9.7 \\
10.9 \\
13.4 \\
14.1 \\
8.8\end{array}$ & $\begin{array}{r}14.3 \\
11.5 \\
11.3 \\
10.0 \\
9.6\end{array}$ & $\begin{array}{l}8.9 \\
5.3 \\
3.1 \\
3.4 \\
4.8\end{array}$ \\
\hline $\begin{array}{l}11 \\
12 \\
13 \\
14 \\
15\end{array}$ & $\begin{array}{r}2.8 \\
3.0 \\
10.2 \\
1.6 \\
-. .7\end{array}$ & $\begin{array}{r}-6.7 \\
-2.2 \\
3.9 \\
-10.1 \\
-12.9\end{array}$ & $\begin{array}{r}-8.8 \\
-4.6 \\
-3.6 \\
-9.1 \\
-10.7\end{array}$ & $\begin{array}{l}-17.0 \\
-17.5 \\
-15.6 \\
-16.9 \\
-17.3\end{array}$ & $\begin{array}{l}-13.7 \\
-15.0 \\
-18.8 \\
-20.6 \\
-16.4\end{array}$ & $\begin{array}{r}7.0 \\
2.5 \\
.1 \\
-5.1 \\
-5.9\end{array}$ & $\begin{array}{r}-1.0 \\
-1.9 \\
-2.0 \\
1.7 \\
5.0\end{array}$ & $\begin{array}{r}-.4 \\
-1.3 \\
-.2 \\
-.1 \\
.7\end{array}$ & $\begin{array}{r}5.0 \\
3.7 \\
8.5 \\
8.3 \\
15.1\end{array}$ & $\begin{array}{r}13.9 \\
11.5 \\
8.0 \\
10.8 \\
17.9\end{array}$ & $\begin{array}{l}10.4 \\
14.4 \\
11.7 \\
13.7 \\
15.7\end{array}$ & $\begin{array}{l}6.0 \\
1.9 \\
1.0 \\
3.0 \\
5.2\end{array}$ \\
\hline $\begin{array}{l}16 \\
17 \\
18 \\
19 \\
20\end{array}$ & $\begin{array}{r}1.0 \\
3.1 \\
2.1 \\
2.6 \\
.1\end{array}$ & $\begin{array}{r}-10.4 \\
-7.7 \\
-12.6 \\
-14.0 \\
-11.2\end{array}$ & $\begin{array}{r}-11.7 \\
-8.4 \\
-7.0 \\
-9.0 \\
-8.5\end{array}$ & $\begin{array}{r}-13.4 \\
-11.2 \\
-9.8 \\
-8.7 \\
-7.8\end{array}$ & $\begin{array}{r}-11.5 \\
-8.9 \\
-3.6 \\
-5.3 \\
-9.1\end{array}$ & $\begin{array}{l}-3.6 \\
-7.1 \\
-1.2 \\
-2.3 \\
-7.9\end{array}$ & $\begin{array}{r}1.4 \\
4.6 \\
3.3 \\
11.4 \\
13.9\end{array}$ & $\begin{array}{r}1.7 \\
1.9 \\
3.7 \\
-1.8 \\
1.7\end{array}$ & $\begin{array}{r}10.3 \\
6.3 \\
8.7 \\
18.9 \\
8.5\end{array}$ & $\begin{array}{r}8.9 \\
9.3 \\
11.4 \\
13.3 \\
15.3\end{array}$ & $\begin{array}{r}19.1 \\
17.4 \\
15.1 \\
11.4 \\
9.6\end{array}$ & $\begin{array}{r}7.5 \\
7.2 \\
.0 \\
.5 \\
1.0\end{array}$ \\
\hline $\begin{array}{l}21 \\
22 \\
23 \\
24 \\
25\end{array}$ & $\begin{array}{r}.5 \\
3.3 \\
1.3 \\
1.2 \\
.7\end{array}$ & $\begin{array}{r}-7.5 \\
-1.4 \\
-5.2 \\
-10.6 \\
-5.7\end{array}$ & $\begin{array}{r}-8.6 \\
-14.1 \\
-15.6 \\
-7.1 \\
-18.5\end{array}$ & $\begin{array}{r}-8.5 \\
-1.5 \\
-8.9 \\
-18.2 \\
-19.7\end{array}$ & $\begin{array}{r}-9.5 \\
-3.3 \\
4.9 \\
7.1 \\
5.2\end{array}$ & $\begin{array}{r}-6.6 \\
-2.1 \\
-.4 \\
2.4 \\
-2.4\end{array}$ & $\begin{array}{r}9.4 \\
4.7 \\
-1.7 \\
-9.1 \\
-9.7\end{array}$ & $\begin{array}{r}6.5 \\
10.1 \\
5.9 \\
3.6 \\
-2.2\end{array}$ & $\begin{array}{l}3.8 \\
1.3 \\
5.0 \\
7.3 \\
6.0\end{array}$ & $\begin{array}{l}12.2 \\
11.0 \\
11.3 \\
11.6 \\
10.4\end{array}$ & $\begin{array}{r}5.4 \\
16.0 \\
8.7 \\
1.0 \\
1.4\end{array}$ & $\begin{array}{r}.5 \\
1.1 \\
3.5 \\
6.1 \\
8.5\end{array}$ \\
\hline $\begin{array}{l}26 \\
27 \\
28 \\
29 \\
30 \\
31\end{array}$ & $\begin{array}{r}1.8 \\
1.5 \\
10.4 \\
4.0 \\
-.3 \\
.6\end{array}$ & $\begin{array}{r}-10.6 \\
-11.5 \\
-8.3 \\
-10.5 \\
-8.6 \\
---\end{array}$ & $\begin{array}{r}-24.1 \\
-25.4 \\
-20.8 \\
-22.8 \\
-10.7 \\
-9.7\end{array}$ & $\begin{array}{r}-15.9 \\
-13.7 \\
-13.0 \\
-7.6 \\
-4.4 \\
-5.4\end{array}$ & $\begin{array}{r}-.5 \\
-4.5 \\
-6.4 \\
--- \\
--- \\
---\end{array}$ & $\begin{array}{r}-3.5 \\
-2.6 \\
4.7 \\
-2.1 \\
-1.2 \\
2.4\end{array}$ & $\begin{array}{r}-3.9 \\
-5.6 \\
-6.4 \\
-2.9 \\
4.0 \\
---\end{array}$ & $\begin{array}{r}-1.5 \\
7.7 \\
6.4 \\
-2.3 \\
-.6 \\
-1.0\end{array}$ & $\begin{array}{r}4.8 \\
13.7 \\
8.2 \\
13.7 \\
14.7 \\
-\ldots-\end{array}$ & $\begin{array}{l}16.0 \\
18.7 \\
18.4 \\
19.3 \\
20.1 \\
20.4\end{array}$ & $\begin{array}{r}7.5 \\
15.6 \\
6.2 \\
17.5 \\
13.7 \\
3.7\end{array}$ & $\begin{array}{r}11.0 \\
15.0 \\
9.7 \\
13.4 \\
2.4 \\
--\end{array}$ \\
\hline MIN & -.7 & -14.0 & -25.4 & -19.8 & -28.8 & -14.2 & -9.7 & -2.3 & -.3 & 7.7 & 1.0 & .0 \\
\hline
\end{tabular}

CAL YR 1988 MIN -25.4

WTR YR 1989 MIN -28.8 
3 SPRINGS BASIN

3 SPRINGS CREEK NEAR WARM SPRINGS, NV

DAILY MINIMUM AIR TEMPERATURE, WATER YEAR OCTOBER 1989 TO SEPTEMBER 1990 DEGREES CELSIUS

\begin{tabular}{|c|c|c|c|c|c|c|c|c|c|c|c|c|}
\hline DAY & $\mathrm{OCT}$ & NOV & DEC & JAN & FEB & MAR & APR & MAY & JUN & JUL & AUG & SEP \\
\hline $\begin{array}{l}1 \\
2 \\
3 \\
4 \\
5\end{array}$ & $\begin{array}{r}-2.4 \\
3.4 \\
-.6 \\
-3.4 \\
-2.9\end{array}$ & $\begin{array}{r}-10.0 \\
-10.2 \\
-6.9 \\
-4.4 \\
-2.4\end{array}$ & $\begin{array}{r}-10.5 \\
-11.5 \\
-9.2 \\
-6.5 \\
-2.5\end{array}$ & $\begin{array}{r}-3.2 \\
-12.2 \\
-19.1 \\
-16.1 \\
-12.0\end{array}$ & $\begin{array}{r}-12.7 \\
-15.3 \\
-15.6 \\
-6.4 \\
-17.1\end{array}$ & $\begin{array}{r}1.9 \\
.9 \\
.0 \\
-2.4 \\
-4.8\end{array}$ & $\begin{array}{r}-3.3 \\
-.6 \\
-2.3 \\
-.4 \\
-.4\end{array}$ & $\begin{array}{r}-1.0 \\
-1.1 \\
-.1 \\
4.1 \\
1.5\end{array}$ & $\begin{array}{r}-.6 \\
1.8 \\
12.2 \\
10.6 \\
5.6\end{array}$ & $\begin{array}{r}21.4 \\
17.4 \\
7.9 \\
16.2 \\
17.8\end{array}$ & $\begin{array}{r}12.6 \\
9.4 \\
18.9 \\
15.2 \\
11.8\end{array}$ & $\begin{array}{l}12.7 \\
16.2 \\
15.4 \\
15.0 \\
12.6\end{array}$ \\
\hline $\begin{array}{r}6 \\
7 \\
8 \\
9 \\
10\end{array}$ & $\begin{array}{r}.3 \\
-2.1 \\
-1.6 \\
.8 \\
2.3\end{array}$ & $\begin{array}{l}-5.0 \\
-5.9 \\
-8.1 \\
-4.9 \\
-2.2\end{array}$ & $\begin{array}{r}-6.6 \\
-6.6 \\
-5.7 \\
-2.2 \\
-11.0\end{array}$ & $\begin{array}{r}-10.7 \\
-5.2 \\
-.3 \\
-1.2 \\
-2.6\end{array}$ & $\begin{array}{r}-9.0 \\
-21.2 \\
-23.0 \\
-12.1 \\
-5.3\end{array}$ & $\begin{array}{r}-7.5 \\
-9.3 \\
3.3 \\
2.0 \\
.4\end{array}$ & $\begin{array}{r}1.8 \\
.5 \\
-.7 \\
-.9 \\
-2.1\end{array}$ & $\begin{array}{r}13.4 \\
7.0 \\
-2.7 \\
-3.3 \\
7.3\end{array}$ & $\begin{array}{r}5.0 \\
8.2 \\
6.9 \\
10.3 \\
5.7\end{array}$ & $\begin{array}{l}18.5 \\
18.1 \\
15.0 \\
13.2 \\
10.8\end{array}$ & $\begin{array}{l}11.2 \\
13.9 \\
15.0 \\
12.6 \\
12.0\end{array}$ & $\begin{array}{r}12.0 \\
9.3 \\
9.1 \\
12.2 \\
11.4\end{array}$ \\
\hline $\begin{array}{l}11 \\
12 \\
13 \\
14 \\
15\end{array}$ & $\begin{array}{r}7.8 \\
4.8 \\
7.0 \\
5.1 \\
.1\end{array}$ & $\begin{array}{r}-.5 \\
.8 \\
-2.1 \\
-7.1 \\
-9.0\end{array}$ & $\begin{array}{r}-14.7 \\
-14.1 \\
-10.6 \\
-12.1 \\
-8.5\end{array}$ & $\begin{array}{r}-2.1 \\
3.2 \\
.1 \\
-7.5 \\
-11.6\end{array}$ & $\begin{array}{r}-6.5 \\
.9 \\
-14.7 \\
-21.6 \\
-22.2\end{array}$ & $\begin{array}{r}-10.5 \\
-13.8 \\
-17.1 \\
-8.0 \\
-6.6\end{array}$ & $\begin{array}{r}1.3 \\
1.3 \\
.2 \\
13.4 \\
8.6\end{array}$ & $\begin{array}{r}1.3 \\
-1.0 \\
1.7 \\
5.2 \\
-1.3\end{array}$ & $\begin{array}{l}3.5 \\
7.3 \\
1.0 \\
3.6 \\
2.2\end{array}$ & $\begin{array}{l}10.1 \\
13.5 \\
17.8 \\
14.0 \\
11.1\end{array}$ & $\begin{array}{r}10.7 \\
11.2 \\
8.0 \\
12.1 \\
10.6\end{array}$ & $\begin{array}{l}10.6 \\
13.6 \\
12.7 \\
18.5 \\
16.4\end{array}$ \\
\hline $\begin{array}{l}16 \\
17 \\
18 \\
19 \\
20\end{array}$ & $\begin{array}{r}-1.8 \\
-2.1 \\
-2.0 \\
-1.0 \\
8.5\end{array}$ & $\begin{array}{l}-5.1 \\
-3.3 \\
-6.4 \\
-5.0 \\
-2.0\end{array}$ & $\begin{array}{r}-10.5 \\
-10.6 \\
-9.4 \\
-11.9 \\
-11.9\end{array}$ & $\begin{array}{r}-5.2 \\
-6.4 \\
-14.1 \\
-15.8 \\
-18.1\end{array}$ & $\begin{array}{r}-9.0 \\
-5.5 \\
-8.3 \\
-12.3 \\
-16.4\end{array}$ & $\begin{array}{r}-8.9 \\
-2.0 \\
-3.3 \\
-1.1 \\
4.0\end{array}$ & $\begin{array}{r}2.7 \\
1.0 \\
2.5 \\
.0 \\
1.8\end{array}$ & $\begin{array}{r}-2.0 \\
5.9 \\
3.9 \\
-.3 \\
-1.5\end{array}$ & $\begin{array}{l}1.1 \\
7.5 \\
9.4 \\
4.5 \\
5.8\end{array}$ & $\begin{array}{l}13.4 \\
12.4 \\
10.7 \\
10.3 \\
10.6\end{array}$ & $\begin{array}{r}9.1 \\
15.3 \\
10.8 \\
3.2 \\
.4\end{array}$ & $\begin{array}{l}3.8 \\
6.6 \\
5.6 \\
1.8 \\
1.7\end{array}$ \\
\hline $\begin{array}{l}21 \\
22 \\
23 \\
24 \\
25\end{array}$ & $\begin{array}{r}6.1 \\
4.7 \\
7.0 \\
3.8 \\
-3.0\end{array}$ & $\begin{array}{r}-2.8 \\
-4.7 \\
-3.8 \\
.2 \\
-5.2\end{array}$ & $\begin{array}{l}-9.9 \\
-9.7 \\
-7.2 \\
-7.5 \\
-8.9\end{array}$ & $\begin{array}{r}-14.6 \\
-14.1 \\
-9.6 \\
-12.6 \\
-11.3\end{array}$ & $\begin{array}{l}-9.0 \\
-8.6 \\
-7.2 \\
-4.2 \\
-3.1\end{array}$ & $\begin{array}{r}2.9 \\
.7 \\
-1.2 \\
-3.4 \\
-2.2\end{array}$ & $\begin{array}{r}.2 \\
-1.5 \\
1.7 \\
.8 \\
.5\end{array}$ & $\begin{array}{r}10.4 \\
6.6 \\
-.9 \\
-1.5 \\
-3.3\end{array}$ & $\begin{array}{r}9.3 \\
15.1 \\
17.1 \\
15.4 \\
13.9\end{array}$ & $\begin{array}{r}10.5 \\
10.4 \\
15.2 \\
14.5 \\
7.5\end{array}$ & $\begin{array}{r}5.0 \\
5.4 \\
5.4 \\
15.2 \\
11.1\end{array}$ & $\begin{array}{l}4.2 \\
5.7 \\
5.9 \\
4.0 \\
4.3\end{array}$ \\
\hline $\begin{array}{l}26 \\
27 \\
28 \\
29 \\
30 \\
31\end{array}$ & $\begin{array}{r}-8.2 \\
-6.8 \\
-9.2 \\
-11.5 \\
-11.3 \\
-9.9\end{array}$ & $\begin{array}{r}-11.6 \\
-14.2 \\
-15.6 \\
-10.5 \\
-10.3 \\
-\end{array}$ & $\begin{array}{r}-8.2 \\
-5.4 \\
-6.1 \\
-9.2 \\
-10.7 \\
-10.5\end{array}$ & $\begin{array}{r}-12.5 \\
-16.6 \\
-13.4 \\
-3.4 \\
-4.3 \\
-13.0\end{array}$ & $\begin{array}{r}-2.2 \\
-3.2 \\
-4.6 \\
--- \\
--- \\
-\end{array}$ & $\begin{array}{r}7.3 \\
-2.5 \\
-4.5 \\
-3.7 \\
-4.9 \\
-3.9\end{array}$ & $\begin{array}{r}5.3 \\
2.9 \\
.4 \\
-2.1 \\
-2.7 \\
---\end{array}$ & $\begin{array}{l}6.2 \\
5.0 \\
3.4 \\
4.7 \\
3.4 \\
4.0\end{array}$ & $\begin{array}{r}15.0 \\
8.2 \\
8.0 \\
19.2 \\
15.9 \\
---\end{array}$ & $\begin{array}{r}6.5 \\
6.6 \\
12.0 \\
12.7 \\
19.4 \\
19.8\end{array}$ & $\begin{array}{r}10.6 \\
13.6 \\
6.4 \\
14.0 \\
18.0 \\
7.0\end{array}$ & $\begin{array}{l}7.8 \\
2.4 \\
3.0 \\
5.0 \\
2.0 \\
---\end{array}$ \\
\hline MIN & -11.5 & -15.6 & -14.7 & -19.1 & -23.0 & -17.1 & -3.3 & -3.3 & -.6 & 6.5 & .4 & 1.7 \\
\hline $\begin{array}{l}\text { CAL } \\
\text { WTR }\end{array}$ & $\begin{array}{l}1989 \\
1990\end{array}$ & $\begin{array}{l}\text { MIN }-2 \\
\text { MIN }-2\end{array}$ & & & & & & & & & & \\
\hline
\end{tabular}


3 SPRINGS BASIN

3 SPRINGS CREEK NEAR WARM SPRINGS, NV

DAILY MINIMUM AIR TEMPERATURE, WATER YEAR OCTOBER 1990 TO SEPTEMBER 1991 DEGREES CELSIUS

\begin{tabular}{|c|c|c|c|c|c|c|c|c|c|c|c|c|}
\hline DAY & OCT & NOV & DEC & JAN & FEB & MAR & $\mathrm{APR}$ & MAY & JUN & JUL & AUG & SEP \\
\hline $\begin{array}{l}1 \\
2 \\
3 \\
4 \\
5\end{array}$ & $\begin{array}{r}5.4 \\
5.6 \\
.5 \\
2.8 \\
13.7\end{array}$ & $\begin{array}{r}-4.2 \\
-8.6 \\
-11.2 \\
-7.5 \\
-3.4\end{array}$ & $\begin{array}{r}-9.8 \\
-8.8 \\
-10.5 \\
-7.4 \\
-8.3\end{array}$ & $\begin{array}{r}-10.6 \\
-11.2 \\
-5.3 \\
-6.8 \\
-10.1\end{array}$ & $\begin{array}{l}-9.0 \\
-5.4 \\
-6.1 \\
-5.9 \\
-2.8\end{array}$ & $\begin{array}{r}-1.3 \\
-6.7 \\
1.8 \\
3.4 \\
-5.0\end{array}$ & $\begin{array}{l}-1.9 \\
-4.1 \\
-5.2 \\
-1.5 \\
11.2\end{array}$ & $\begin{array}{r}-2.4 \\
-2.7 \\
-5.1 \\
-3.1 \\
-.9\end{array}$ & $\begin{array}{r}3.9 \\
4.1 \\
10.0 \\
14.7 \\
10.0\end{array}$ & $\begin{array}{r}5.0 \\
8.6 \\
10.7 \\
12.8 \\
14.8\end{array}$ & $\begin{array}{l}--- \\
--- \\
--- \\
--- \\
---\end{array}$ & $\begin{array}{l}--- \\
--- \\
--- \\
---\end{array}$ \\
\hline $\begin{array}{r}6 \\
7 \\
8 \\
9 \\
10\end{array}$ & $\begin{array}{r}6.1 \\
-3.2 \\
-6.6 \\
-4.2 \\
-2.1\end{array}$ & $\begin{array}{l}-6.3 \\
-7.7 \\
-1.0 \\
-2.7 \\
-4.7\end{array}$ & $\begin{array}{r}-10.6 \\
-10.1 \\
-8.8 \\
-7.2 \\
1.4\end{array}$ & $\begin{array}{l}-11.6 \\
-11.0 \\
-10.7 \\
-11.8 \\
-11.5\end{array}$ & $\begin{array}{l}-6.1 \\
-7.5 \\
-6.9 \\
-4.8 \\
-6.1\end{array}$ & $\begin{array}{r}-11.6 \\
-11.9 \\
-9.7 \\
-8.7 \\
-.3\end{array}$ & $\begin{array}{r}6.0 \\
-2.6 \\
-7.9 \\
-4.9 \\
-8.2\end{array}$ & $\begin{array}{r}2.4 \\
.7 \\
6.1 \\
-4.5 \\
-5.4\end{array}$ & $\begin{array}{r}10.8 \\
7.1 \\
4.2 \\
5.4 \\
7.4\end{array}$ & $\begin{array}{r}17.0 \\
17.9 \\
11.8 \\
10.8 \\
9.8\end{array}$ & $\begin{array}{l}--- \\
--- \\
--- \\
--- \\
---\end{array}$ & $\begin{array}{l}--- \\
--- \\
--- \\
---\end{array}$ \\
\hline $\begin{array}{l}11 \\
12 \\
13 \\
14 \\
15\end{array}$ & $\begin{array}{r}-1.4 \\
-2.8 \\
4.1 \\
.1 \\
4.3\end{array}$ & $\begin{array}{r}-1.3 \\
-1.5 \\
.2 \\
9.1 \\
1.0\end{array}$ & $\begin{array}{r}2.4 \\
-2.3 \\
-8.4 \\
-15.5 \\
-15.9\end{array}$ & $\begin{array}{l}-7.5 \\
-6.0 \\
-5.0 \\
-6.5 \\
-3.8\end{array}$ & $\begin{array}{l}-5.5 \\
-1.7 \\
-2.9 \\
-4.4 \\
-1.4\end{array}$ & $\begin{array}{r}-8.3 \\
-10.0 \\
-4.8 \\
-4.4 \\
-5.9\end{array}$ & $\begin{array}{r}-9.0 \\
-4.6 \\
-8.1 \\
-3.3 \\
-.6\end{array}$ & $\begin{array}{r}-5.8 \\
-2.7 \\
.4 \\
-.6 \\
-1.9\end{array}$ & $\begin{array}{r}9.5 \\
17.7 \\
14.5 \\
7.7 \\
2.5\end{array}$ & $\begin{array}{l}11.7 \\
10.7 \\
17.5 \\
19.1 \\
18.7\end{array}$ & $\begin{array}{l}--- \\
--- \\
--- \\
--- \\
---\end{array}$ & $\begin{array}{l}--- \\
--- \\
--- \\
--- \\
---\end{array}$ \\
\hline $\begin{array}{l}16 \\
17 \\
18 \\
19 \\
20\end{array}$ & $\begin{array}{r}.0 \\
-3.5 \\
8.1 \\
-3.1 \\
-8.4\end{array}$ & $\begin{array}{r}-.6 \\
-. .4 \\
-1.3 \\
.0 \\
-12.1\end{array}$ & $\begin{array}{r}-15.1 \\
-15.7 \\
-2.4 \\
-13.9 \\
-23.5\end{array}$ & $\begin{array}{r}-9.8 \\
-10.7 \\
-7.9 \\
-9.0 \\
-11.9\end{array}$ & $\begin{array}{r}.0 \\
-5.1 \\
-9.0 \\
-7.8 \\
-5.1\end{array}$ & $\begin{array}{r}-12.2 \\
-2.5 \\
-.6 \\
-4.3 \\
-9.7\end{array}$ & $\begin{array}{r}-5.6 \\
-4.8 \\
-7.1 \\
-.1 \\
-2.2\end{array}$ & $\begin{array}{r}-.3 \\
.2 \\
-1.5 \\
-1.5 \\
-2.8\end{array}$ & $\begin{array}{r}12.5 \\
8.0 \\
16.2 \\
6.6 \\
3.1\end{array}$ & $\begin{array}{l}16.8 \\
18.0 \\
16.2 \\
13.2 \\
11.0\end{array}$ & $\begin{array}{l}--- \\
--- \\
--- \\
--- \\
---\end{array}$ & $\begin{array}{l}--- \\
--- \\
--- \\
--- \\
---\end{array}$ \\
\hline $\begin{array}{l}21 \\
22 \\
23 \\
24 \\
25\end{array}$ & $\begin{array}{r}-5.6 \\
-2.2 \\
-1.0 \\
-.6 \\
1.6\end{array}$ & $\begin{array}{r}-12.7 \\
-7.8 \\
-7.0 \\
-5.0 \\
2.5\end{array}$ & $\begin{array}{l}-27.2 \\
-27.7 \\
-26.7 \\
-22.0 \\
-16.8\end{array}$ & $\begin{array}{r}-15.4 \\
-15.1 \\
-12.6 \\
-9.8 \\
-13.8\end{array}$ & $\begin{array}{r}-4.2 \\
-.7 \\
-3.2 \\
-8.1 \\
-9.3\end{array}$ & $\begin{array}{r}-6.1 \\
-11.4 \\
-1.5 \\
-1.6 \\
-5.2\end{array}$ & $\begin{array}{r}-2.3 \\
-3.5 \\
-3.6 \\
2.3 \\
-2.2\end{array}$ & $\begin{array}{r}-.8 \\
.1 \\
1.4 \\
7.5 \\
5.4\end{array}$ & $\begin{array}{r}4.0 \\
4.0 \\
13.5 \\
11.0 \\
6.6\end{array}$ & $\begin{array}{r}15.3 \\
9.9 \\
14.1 \\
12.9 \\
19.1\end{array}$ & $\begin{array}{l}--- \\
--- \\
--- \\
---\end{array}$ & $\begin{array}{l}--- \\
--- \\
--- \\
--- \\
---\end{array}$ \\
\hline $\begin{array}{l}26 \\
27 \\
28 \\
29 \\
30 \\
31\end{array}$ & $\begin{array}{r}2.1 \\
.1 \\
12.9 \\
11.3 \\
8.7 \\
5.3\end{array}$ & $\begin{array}{r}-15.4 \\
-17.5 \\
-14.6 \\
-10.7 \\
-7.7 \\
---\end{array}$ & $\begin{array}{r}-15.5 \\
-15.7 \\
-4.2 \\
-21.6 \\
-21.8 \\
-14.0\end{array}$ & $\begin{array}{r}-12.7 \\
-11.3 \\
-8.3 \\
-19.0 \\
-14.2 \\
-9.9\end{array}$ & $\begin{array}{r}-8.2 \\
-3.1 \\
-1.7 \\
--- \\
--- \\
---\end{array}$ & $\begin{array}{l}-6.9 \\
-7.1 \\
-9.0 \\
-5.7 \\
-7.0 \\
-5.2\end{array}$ & $\begin{array}{r}-2.6 \\
-3.7 \\
-7.1 \\
-3.9 \\
-6.0 \\
----\end{array}$ & $\begin{array}{r}8.2 \\
3.4 \\
-1.2 \\
3.7 \\
.3 \\
-.1\end{array}$ & $\begin{array}{l}7.8 \\
9.9 \\
9.1 \\
5.2 \\
2.0 \\
---\end{array}$ & $\begin{array}{c}17.0 \\
19.5 \\
13.2 \\
12.0 \\
16.6 \\
-\end{array}$ & $\begin{array}{l}--- \\
--- \\
--- \\
--- \\
--- \\
---\end{array}$ & $\begin{array}{l}8.7 \\
8.4 \\
6.6 \\
4.8 \\
3.5 \\
---\end{array}$ \\
\hline MIN & -8.4 & -17.5 & -27.7 & -19.0 & -9.3 & -12.2 & -9.0 & -5.8 & 2.0 & -- & --- & -- \\
\hline
\end{tabular}

CAL YR 1990 MIN -27.7 
3 SPRINGS BASIN

3 SPRINGS CREEK NEAR WARM SPRINGS, NV

DAILY MEAN SOIL TEMPERATURE, WATER YEAR OCTOBER 1985 TO SEPTEMBER 1986 DEGREES CELSIUS

\begin{tabular}{|c|c|c|c|c|c|c|c|c|c|c|c|c|}
\hline DAY & OCT & NOV & DEC & JAN & FEB & MAR & APR & MAY & JUN & JUL & AUG & SEP \\
\hline $\begin{array}{l}1 \\
2 \\
3 \\
4 \\
5\end{array}$ & $\begin{array}{l}--- \\
--- \\
--- \\
--- \\
---\end{array}$ & $\begin{array}{l}6.9 \\
6.5 \\
6.7 \\
7.0 \\
7.3\end{array}$ & $\begin{array}{l}1.5 \\
1.5 \\
1.5 \\
1.5 \\
1.5\end{array}$ & $\begin{array}{l}.5 \\
.5 \\
.6 \\
.6 \\
.6\end{array}$ & $\begin{array}{l}.6 \\
.5 \\
.6 \\
.6 \\
.6\end{array}$ & $\begin{array}{l}6.7 \\
6.8 \\
6.7 \\
6.9 \\
6.8\end{array}$ & $\begin{array}{l}9.2 \\
7.7 \\
6.8 \\
6.9 \\
7.4\end{array}$ & $\begin{array}{l}11.5 \\
12.2 \\
12.7 \\
12.1 \\
10.7\end{array}$ & $\begin{array}{l}19.1 \\
19.3 \\
18.7 \\
18.8 \\
19.1\end{array}$ & $\begin{array}{l}24.3 \\
24.8 \\
25.2 \\
25.5 \\
24.8\end{array}$ & $\begin{array}{l}22.9 \\
23.6 \\
24.2 \\
24.5 \\
24.6\end{array}$ & $\begin{array}{l}19.8 \\
19.9 \\
20.0 \\
20.1 \\
20.3\end{array}$ \\
\hline $\begin{array}{r}6 \\
7 \\
8 \\
9 \\
10\end{array}$ & $\begin{array}{l}--- \\
--- \\
--- \\
--- \\
---\end{array}$ & $\begin{array}{l}6.8 \\
6.2 \\
6.7 \\
6.0 \\
4.6\end{array}$ & $\begin{array}{l}1.4 \\
1.4 \\
1.3 \\
1.3 \\
1.2\end{array}$ & $\begin{array}{l}.6 \\
.6 \\
.6 \\
.5 \\
.4\end{array}$ & $\begin{array}{l}.6 \\
.6 \\
.6 \\
.6 \\
.6\end{array}$ & $\begin{array}{l}6.8 \\
6.5 \\
6.1 \\
4.7 \\
4.0\end{array}$ & $\begin{array}{l}7.1 \\
5.8 \\
4.7 \\
5.6 \\
6.7\end{array}$ & $\begin{array}{r}10.4 \\
9.6 \\
9.3 \\
9.3 \\
10.4\end{array}$ & $\begin{array}{l}19.6 \\
19.9 \\
19.9 \\
19.4 \\
19.4\end{array}$ & $\begin{array}{l}24.4 \\
24.5 \\
24.2 \\
24.3 \\
24.2\end{array}$ & $\begin{array}{l}24.4 \\
24.9 \\
25.1 \\
25.4 \\
24.4\end{array}$ & $\begin{array}{l}20.8 \\
21.3 \\
21.3 \\
20.9 \\
20.0\end{array}$ \\
\hline $\begin{array}{l}11 \\
12 \\
13 \\
14 \\
15\end{array}$ & $\begin{array}{l}8.0 \\
8.5 \\
8.0 \\
7.2 \\
6.8\end{array}$ & $\begin{array}{l}3.9 \\
3.3 \\
3.0 \\
2.7 \\
2.5\end{array}$ & $\begin{array}{l}1.1 \\
1.1 \\
1.0 \\
1.0 \\
1.0\end{array}$ & $\begin{array}{l}.4 \\
.4 \\
.4 \\
.4 \\
.4\end{array}$ & $\begin{array}{l}.6 \\
.5 \\
.6 \\
.6 \\
.6\end{array}$ & $\begin{array}{l}3.3 \\
3.4 \\
3.0 \\
2.6 \\
2.8\end{array}$ & $\begin{array}{l}7.3 \\
8.1 \\
7.3 \\
7.3 \\
7.9\end{array}$ & $\begin{array}{l}10.6 \\
11.3 \\
11.7 \\
12.1 \\
12.8\end{array}$ & $\begin{array}{l}19.7 \\
20.2 \\
19.8 \\
20.2 \\
20.6\end{array}$ & $\begin{array}{l}24.5 \\
25.1 \\
25.5 \\
25.6 \\
25.0\end{array}$ & $\begin{array}{l}22.2 \\
22.7 \\
22.4 \\
22.8 \\
23.7\end{array}$ & $\begin{array}{l}19.3 \\
19.2 \\
19.0 \\
18.7 \\
18.4\end{array}$ \\
\hline $\begin{array}{l}16 \\
17 \\
18 \\
19 \\
20\end{array}$ & $\begin{array}{l}7.0 \\
7.4 \\
7.7 \\
8.3 \\
9.1\end{array}$ & $\begin{array}{l}2.4 \\
2.2 \\
2.1 \\
2.0 \\
1.8\end{array}$ & $\begin{array}{l}.9 \\
.9 \\
.9 \\
.9 \\
.8\end{array}$ & $\begin{array}{l}.4 \\
.4 \\
.4 \\
.5 \\
.5\end{array}$ & $\begin{array}{l}.6 \\
.7 \\
.7 \\
.5 \\
.6\end{array}$ & $\begin{array}{l}2.5 \\
2.3 \\
2.2 \\
2.1 \\
3.0\end{array}$ & $\begin{array}{l}7.7 \\
7.0 \\
7.0 \\
7.4 \\
8.5\end{array}$ & $\begin{array}{l}13.0 \\
13.0 \\
13.4 \\
14.6 \\
15.5\end{array}$ & $\begin{array}{l}21.1 \\
21.6 \\
22.0 \\
22.0 \\
21.9\end{array}$ & $\begin{array}{l}21.3 \\
21.1 \\
21.6 \\
22.1 \\
22.5\end{array}$ & $\begin{array}{l}24.0 \\
24.1 \\
23.9 \\
23.3 \\
23.4\end{array}$ & $\begin{array}{l}18.0 \\
17.7 \\
17.6 \\
17.3 \\
16.8\end{array}$ \\
\hline $\begin{array}{l}21 \\
22 \\
23 \\
24 \\
25\end{array}$ & $\begin{array}{l}9.2 \\
7.3 \\
6.2 \\
7.1 \\
7.7\end{array}$ & $\begin{array}{l}1.7 \\
1.6 \\
1.5 \\
1.5 \\
1.5\end{array}$ & $\begin{array}{l}.8 \\
.8 \\
.7 \\
.6 \\
.6\end{array}$ & $\begin{array}{l}.6 \\
.6 \\
.6 \\
.6 \\
.6\end{array}$ & $\begin{array}{r}.8 \\
1.1 \\
1.7 \\
2.7 \\
4.1\end{array}$ & $\begin{array}{l}4.4 \\
5.5 \\
6.3 \\
6.7 \\
6.9\end{array}$ & $\begin{array}{r}9.7 \\
10.7 \\
10.8 \\
10.3 \\
10.1\end{array}$ & $\begin{array}{l}15.8 \\
15.1 \\
14.8 \\
15.3 \\
15.9\end{array}$ & $\begin{array}{l}22.0 \\
22.3 \\
22.6 \\
22.9 \\
23.4\end{array}$ & $\begin{array}{l}21.8 \\
19.7 \\
18.5 \\
18.2 \\
18.4\end{array}$ & $\begin{array}{l}21.9 \\
21.1 \\
20.8 \\
21.6 \\
21.5\end{array}$ & $\begin{array}{l}16.0 \\
15.6 \\
15.7 \\
14.9 \\
13.9\end{array}$ \\
\hline $\begin{array}{l}26 \\
27 \\
28 \\
29 \\
30 \\
31\end{array}$ & $\begin{array}{l}7.8 \\
7.6 \\
8.1 \\
7.9 \\
8.1 \\
7.9\end{array}$ & $\begin{array}{l}1.5 \\
1.5 \\
1.4 \\
1.4 \\
1.4 \\
---\end{array}$ & $\begin{array}{l}.6 \\
.5 \\
.4 \\
.4 \\
.4 \\
.4\end{array}$ & $\begin{array}{l}.6 \\
.5 \\
.5 \\
.5 \\
.6 \\
.6\end{array}$ & $\begin{array}{l}5.2 \\
5.8 \\
6.2 \\
--- \\
--- \\
---\end{array}$ & $\begin{array}{l}7.3 \\
7.9 \\
8.1 \\
8.3 \\
8.8 \\
9.2\end{array}$ & $\begin{array}{r}9.5 \\
9.1 \\
10.2 \\
10.9 \\
10.9 \\
---\end{array}$ & $\begin{array}{l}17.0 \\
17.9 \\
18.3 \\
17.8 \\
18.4 \\
18.8\end{array}$ & $\begin{array}{r}23.6 \\
24.0 \\
24.2 \\
23.8 \\
24.0 \\
---\end{array}$ & $\begin{array}{l}19.2 \\
19.9 \\
20.8 \\
21.3 \\
21.6 \\
22.2\end{array}$ & $\begin{array}{l}20.2 \\
19.9 \\
18.7 \\
19.4 \\
20.1 \\
20.1\end{array}$ & $\begin{array}{r}12.1 \\
11.8 \\
11.5 \\
11.3 \\
11.7 \\
---\end{array}$ \\
\hline $\begin{array}{l}\text { MEAN } \\
\text { MAX } \\
\text { MIN } \\
\text { MED }\end{array}$ & $\begin{array}{l}--- \\
--- \\
--- \\
---\end{array}$ & $\begin{array}{l}3.5 \\
7.3 \\
1.4 \\
2.4\end{array}$ & $\begin{array}{r}1.0 \\
1.5 \\
.4 \\
.9\end{array}$ & $\begin{array}{l}.5 \\
.6 \\
.4 \\
.5\end{array}$ & $\begin{array}{r}1.4 \\
6.2 \\
.5 \\
.6\end{array}$ & $\begin{array}{l}5.4 \\
9.2 \\
2.1 \\
6.3\end{array}$ & $\begin{array}{r}8.2 \\
10.9 \\
4.7 \\
7.7\end{array}$ & $\begin{array}{r}13.6 \\
18.8 \\
9.3 \\
13.0\end{array}$ & $\begin{array}{l}21.2 \\
24.2 \\
18.7 \\
20.8\end{array}$ & $\begin{array}{l}22.6 \\
25.6 \\
18.2 \\
22.5\end{array}$ & $\begin{array}{l}22.6 \\
25.4 \\
18.7 \\
22.9\end{array}$ & $\begin{array}{l}17.4 \\
21.3 \\
11.3 \\
18.2\end{array}$ \\
\hline
\end{tabular}


3 SPRINGS BASIN

3 SPRINGS CREEK NEAR WARM SPRINGS, NV

DAILY MEAN SOIL TEMPERATURE, WATER YEAR OCTOBER 1986 TO SEPTEMBER 1987 DEGREES CELSIUS

\begin{tabular}{|c|c|c|c|c|c|c|c|c|c|c|c|c|}
\hline DAY & OCT & NOV & DEC & JAN & FEB & MAR & APR & MAY & JUN & JUL & AUG & SEP \\
\hline $\begin{array}{l}1 \\
2 \\
3 \\
4 \\
5\end{array}$ & $\begin{array}{r}11.9 \\
10.5 \\
9.7 \\
9.9 \\
10.2\end{array}$ & $\begin{array}{l}8.1 \\
7.4 \\
7.2 \\
7.2 \\
7.0\end{array}$ & $\begin{array}{l}.9 \\
.6 \\
.5 \\
.5 \\
.7\end{array}$ & $\begin{array}{r}-1.2 \\
-1.0 \\
-1.0 \\
-.8 \\
-.6\end{array}$ & $\begin{array}{l}.3 \\
.4 \\
.5 \\
.5 \\
.4\end{array}$ & $\begin{array}{l}.3 \\
.3 \\
.3 \\
.4 \\
.4\end{array}$ & $\begin{array}{l}5.9 \\
6.9 \\
7.3 \\
6.3 \\
6.0\end{array}$ & $\begin{array}{l}11.9 \\
11.5 \\
11.6 \\
12.1 \\
12.6\end{array}$ & $\begin{array}{l}13.6 \\
14.2 \\
14.8 \\
15.5 \\
15.7\end{array}$ & $\begin{array}{l}\text { e19.3 } \\
\text { e19.8 } \\
\text { e20.4 } \\
\text { e20.9 } \\
\text { e } 21.0\end{array}$ & $\begin{array}{l}22.8 \\
23.4 \\
23.5 \\
23.3 \\
23.2\end{array}$ & $\begin{array}{l}21.1 \\
21.0 \\
20.7 \\
20.7 \\
20.2\end{array}$ \\
\hline $\begin{array}{r}6 \\
7 \\
8 \\
9 \\
10\end{array}$ & $\begin{array}{l}10.6 \\
11.0 \\
11.1 \\
11.0 \\
10.9\end{array}$ & $\begin{array}{l}6.7 \\
4.9 \\
4.1 \\
3.5 \\
3.2\end{array}$ & $\begin{array}{r}1.0 \\
1.2 \\
.9 \\
.4 \\
-.3\end{array}$ & $\begin{array}{l}-.4 \\
-.3 \\
-.3 \\
-.3 \\
-.3\end{array}$ & $\begin{array}{l}.3 \\
.3 \\
.4 \\
.5 \\
.5\end{array}$ & $\begin{array}{l}.4 \\
.4 \\
.4 \\
.5 \\
.8\end{array}$ & $\begin{array}{l}6.7 \\
7.5 \\
7.8 \\
8.5 \\
9.2\end{array}$ & $\begin{array}{l}13.2 \\
13.5 \\
12.9 \\
12.4 \\
12.5\end{array}$ & $\begin{array}{l}15.1 \\
14.4 \\
14.7 \\
14.1 \\
14.9\end{array}$ & $\begin{array}{r}\mathrm{e} 21.0 \\
\mathrm{e} 21.2 \\
22.2 \\
22.7 \\
23.0\end{array}$ & $\begin{array}{l}23.8 \\
24.1 \\
23.5 \\
22.5 \\
22.7\end{array}$ & $\begin{array}{l}20.2 \\
18.5 \\
18.3 \\
18.5 \\
18.7\end{array}$ \\
\hline $\begin{array}{l}11 \\
12 \\
13 \\
14 \\
15\end{array}$ & $\begin{array}{r}10.9 \\
10.1 \\
9.6 \\
9.5 \\
9.6\end{array}$ & $\begin{array}{l}3.2 \\
3.3 \\
3.4 \\
4.0 \\
4.7\end{array}$ & $\begin{array}{r}-.9 \\
-1.2 \\
-1.3 \\
-1.2 \\
-1.3\end{array}$ & $\begin{array}{l}-.4 \\
-.5 \\
-.5 \\
-.4 \\
-.5\end{array}$ & $\begin{array}{l}.6 \\
.7 \\
.8 \\
.8 \\
.9\end{array}$ & $\begin{array}{l}1.5 \\
2.9 \\
4.0 \\
4.0 \\
3.6\end{array}$ & $\begin{array}{r}9.4 \\
9.1 \\
9.1 \\
9.8 \\
10.3\end{array}$ & $\begin{array}{l}12.6 \\
13.1 \\
13.4 \\
13.6 \\
13.5\end{array}$ & $\begin{array}{l}15.6 \\
16.2 \\
16.6 \\
16.2 \\
16.7\end{array}$ & $\begin{array}{l}22.9 \\
22.6 \\
22.4 \\
22.1 \\
21.8\end{array}$ & $\begin{array}{l}23.3 \\
23.3 \\
22.0 \\
21.5 \\
20.5\end{array}$ & $\begin{array}{r}18.5 \\
18.9 \\
18.4 \\
\mathrm{e} 18.0 \\
\mathrm{e} 17.5\end{array}$ \\
\hline $\begin{array}{l}16 \\
17 \\
18 \\
19 \\
20\end{array}$ & $\begin{array}{r}9.8 \\
10.2 \\
9.4 \\
7.8 \\
7.0\end{array}$ & $\begin{array}{l}4.7 \\
4.9 \\
5.6 \\
5.5 \\
4.9\end{array}$ & $\begin{array}{r}-1.2 \\
-1.2 \\
-1.4 \\
-1.1 \\
-.7\end{array}$ & $\begin{array}{l}-.6 \\
-. .7 \\
-.7 \\
-.8 \\
-.8\end{array}$ & $\begin{array}{r}1.0 \\
1.0 \\
.9 \\
.9 \\
.8\end{array}$ & $\begin{array}{l}2.7 \\
2.3 \\
2.6 \\
2.9 \\
2.6\end{array}$ & $\begin{array}{l}10.9 \\
11.9 \\
12.4 \\
11.4 \\
10.7\end{array}$ & $\begin{array}{l}12.8 \\
12.4 \\
12.7 \\
13.1 \\
13.2\end{array}$ & $\begin{array}{l}16.7 \\
17.0 \\
17.4 \\
17.6 \\
18.0\end{array}$ & $\begin{array}{l}20.9 \\
19.5 \\
18.7 \\
18.7 \\
18.2\end{array}$ & $\begin{array}{l}20.2 \\
20.7 \\
21.4 \\
22.0 \\
22.1\end{array}$ & $\begin{array}{l}\text { e } 17.0 \\
\text { e} 16.5 \\
\text { e16.5 } \\
\text { e16.5 } \\
\text { e } 16.5\end{array}$ \\
\hline $\begin{array}{l}21 \\
22 \\
23 \\
24 \\
25\end{array}$ & $\begin{array}{l}7.3 \\
7.5 \\
7.6 \\
7.8 \\
8.1\end{array}$ & $\begin{array}{l}4.9 \\
4.0 \\
2.9 \\
2.5 \\
2.4\end{array}$ & $\begin{array}{l}-.5 \\
-.5 \\
-.5 \\
-.4 \\
-.5\end{array}$ & $\begin{array}{l}-.9 \\
-.9 \\
-.8 \\
-.6 \\
-.5\end{array}$ & $\begin{array}{l}.7 \\
.6 \\
.5 \\
.5 \\
.5\end{array}$ & $\begin{array}{l}2.5 \\
2.0 \\
1.9 \\
2.1 \\
2.9\end{array}$ & $\begin{array}{l}10.9 \\
11.4 \\
12.1 \\
12.3 \\
12.7\end{array}$ & $\begin{array}{l}12.4 \\
11.2 \\
11.2 \\
11.9 \\
11.8\end{array}$ & $\begin{array}{l}18.5 \\
18.6 \\
18.5 \\
18.9 \\
19.3\end{array}$ & $\begin{array}{l}17.7 \\
18.1 \\
18.9 \\
19.9 \\
20.9\end{array}$ & $\begin{array}{l}22.3 \\
22.6 \\
22.6 \\
22.5 \\
21.9\end{array}$ & $\begin{array}{l}\text { e} 16.5 \\
\text { e } 16.5 \\
\text { e16.0 } \\
\text { e15.5 } \\
\text { e } 15.5\end{array}$ \\
\hline $\begin{array}{l}26 \\
27 \\
28 \\
29 \\
30 \\
31\end{array}$ & $\begin{array}{l}8.3 \\
8.9 \\
9.2 \\
9.4 \\
9.9 \\
9.4\end{array}$ & $\begin{array}{l}1.9 \\
1.5 \\
1.9 \\
2.3 \\
1.6 \\
---\end{array}$ & $\begin{array}{r}-.6 \\
-.6 \\
-.6 \\
-.6 \\
-.7 \\
-1.1\end{array}$ & $\begin{array}{r}-.4 \\
-.2 \\
-.1 \\
.1 \\
.2 \\
.3\end{array}$ & $\begin{array}{r}.5 \\
.5 \\
.4 \\
--- \\
--- \\
---\end{array}$ & $\begin{array}{l}3.5 \\
4.0 \\
3.9 \\
3.9 \\
4.1 \\
4.9\end{array}$ & $\begin{array}{c}13.2 \\
13.0 \\
12.4 \\
11.9 \\
11.6 \\
-\end{array}$ & $\begin{array}{r}10.3 \\
9.4 \\
10.6 \\
11.0 \\
11.8 \\
12.7\end{array}$ & $\begin{array}{r}19.3 \\
19.3 \\
19.7 \\
19.4 \\
18.7 \\
-\end{array}$ & $\begin{array}{l}21.5 \\
22.0 \\
21.0 \\
21.4 \\
21.9 \\
22.3\end{array}$ & $\begin{array}{l}21.4 \\
21.2 \\
20.9 \\
20.8 \\
20.7 \\
20.9\end{array}$ & $\begin{array}{l}\text { e} 16.0 \\
\text { e16.0 } \\
\text { e16.0 } \\
\text { e16.0 } \\
\text { e16.3 }\end{array}$ \\
\hline $\begin{array}{l}\text { MEAN } \\
\text { MAX } \\
\text { MIN } \\
\text { MED }\end{array}$ & $\begin{array}{r}9.5 \\
11.9 \\
7.0 \\
9.6\end{array}$ & $\begin{array}{l}4.3 \\
8.1 \\
1.5 \\
4.1\end{array}$ & $\begin{array}{r}-.4 \\
1.2 \\
-1.4 \\
-.6\end{array}$ & $\begin{array}{r}-.5 \\
.3 \\
-1.2 \\
-.5\end{array}$ & $\begin{array}{r}.6 \\
1.0 \\
.3 \\
.5\end{array}$ & $\begin{array}{r}2.2 \\
4.9 \\
.3 \\
2.5\end{array}$ & $\begin{array}{r}10.0 \\
13.2 \\
5.9 \\
10.5\end{array}$ & $\begin{array}{r}12.2 \\
13.6 \\
9.4 \\
12.4\end{array}$ & $\begin{array}{l}16.8 \\
19.7 \\
13.6 \\
16.7\end{array}$ & $\begin{array}{l}20.8 \\
23.0 \\
17.7 \\
21.0\end{array}$ & $\begin{array}{l}22.2 \\
24.1 \\
20.2 \\
22.3\end{array}$ & $\begin{array}{l}17.7 \\
21.1 \\
15.5 \\
17.2\end{array}$ \\
\hline
\end{tabular}

CAL YR 1986 MEAN 10.6 MAX 25.6 MIN -1.4 MED 9.3 WTR YR 1987 MEAN 9.7 MAX 24.1 MIN $-1.4 \quad$ MED 9.8

e Estimated 
3 SPRINGS BASIN

3 SPRINGS CREEK NEAR WARM SPRINGS, NV

DAILY MEAN SOIL TEMPERATURE, WATER YEAR OCTOBER 1987 TO SEPTEMBER 1988 DEGREES CELSIUS

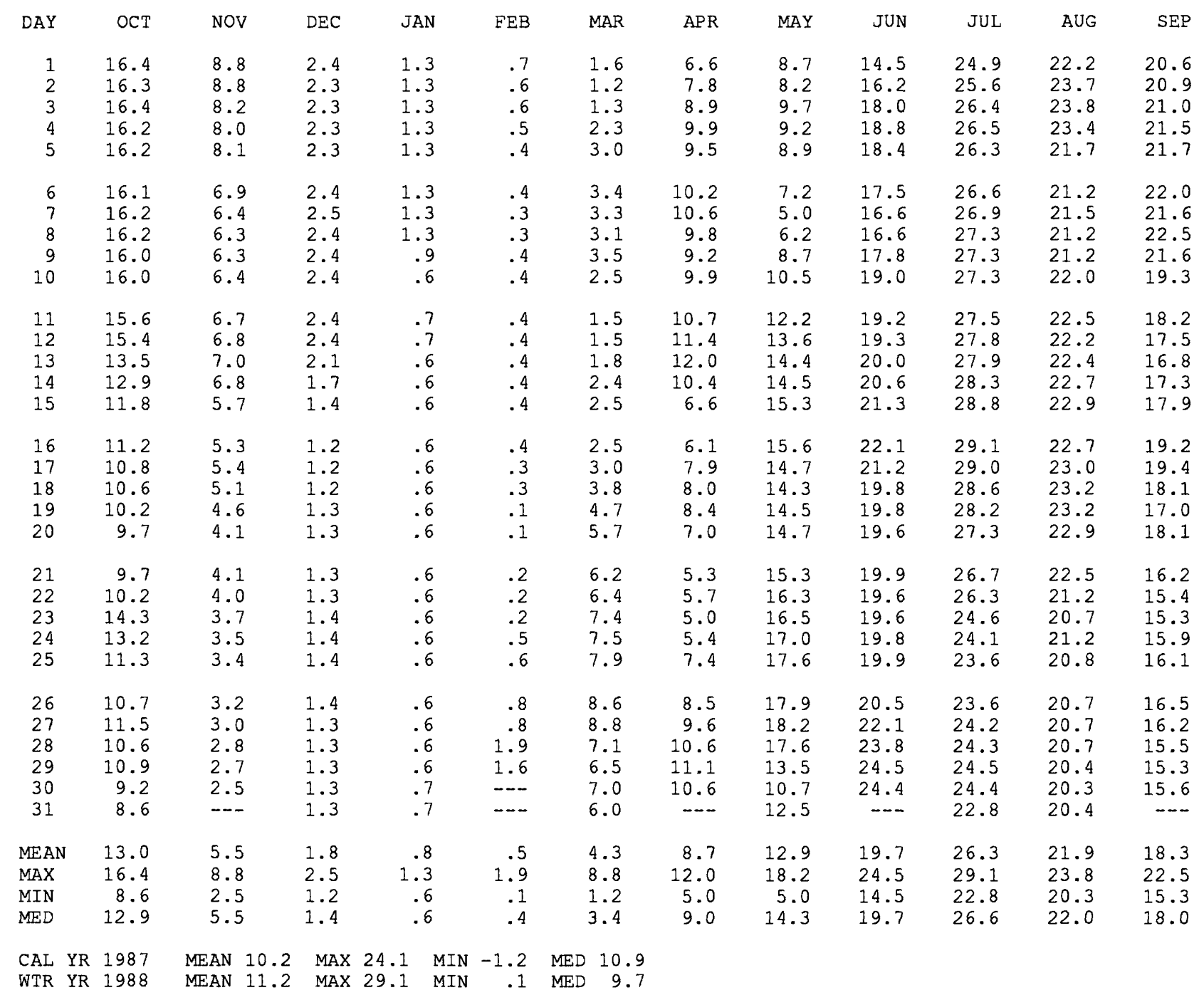


3 SPRINGS BASIN

3 SPRINGS CREEK NEAR WARM SPRINGS, NV

DAILY MEAN SOIL TEMPERATURE, WATER YEAR OCTOBER 1988 TO SEPTEMBER 1989 DEGREES CELSIUS

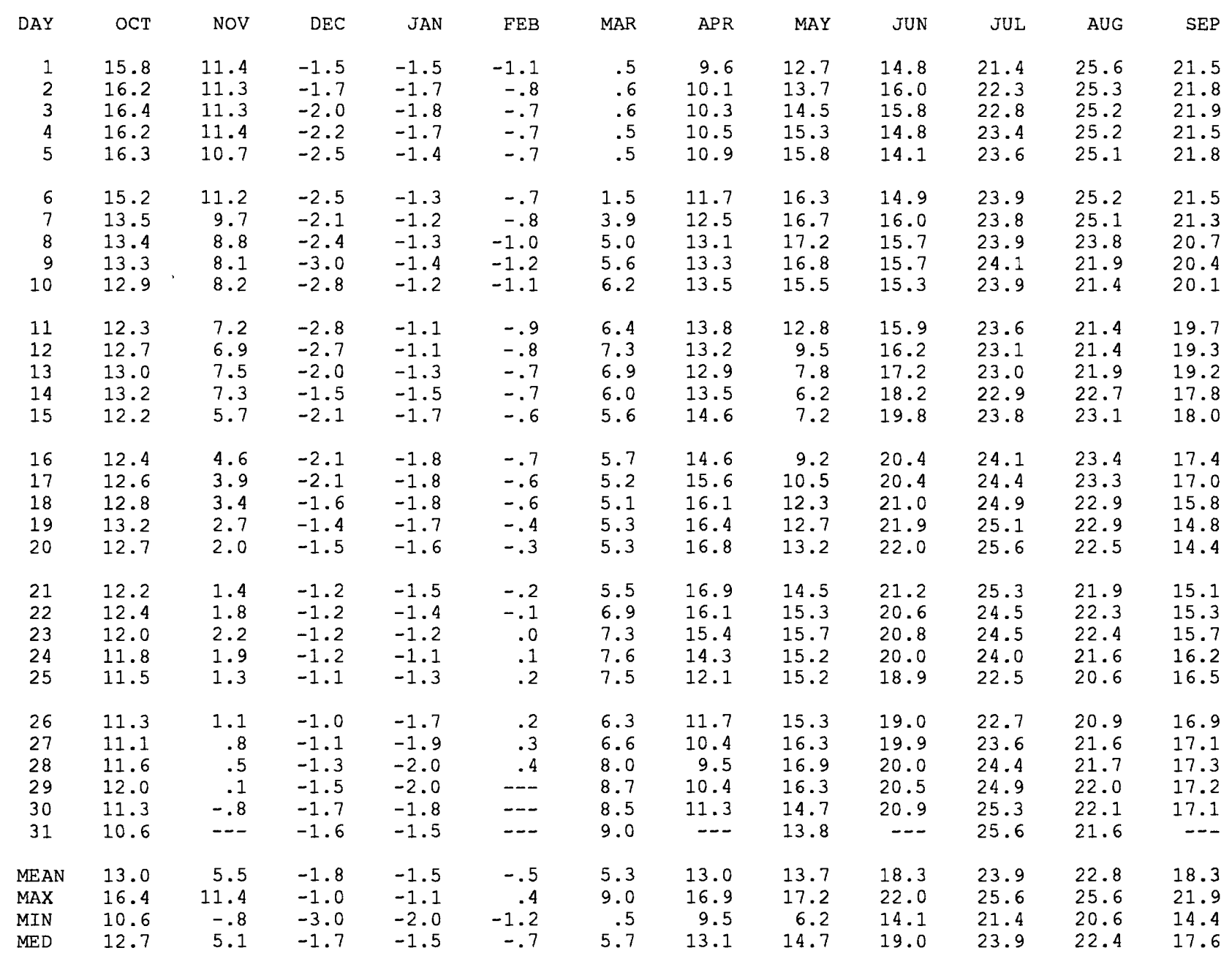

CAL YR 1988 MEAN 10.9 MAX 29.1 MIN $-3.0 \quad$ MED 10.6 WTR YR 1989 MEAN 10.9 MAX 25.6 MIN $-3.0 \quad$ MED 12.4 
3 SPRINGS BASIN

3 SPRINGS CREEK NEAR WARM SPRINGS, NV

DAILY MEAN SOIL TEMPERATURE, WATER YEAR OCTOBER 1989 TO SEPTEMBER 1990 DEGREES CELSIUS

\begin{tabular}{|c|c|c|c|c|c|c|c|c|c|c|c|c|}
\hline DAY & OCT & NOV & $\mathrm{DEC}$ & JAN & FEB & MAR & $A P R$ & MAY & JUN & JUL & AUG & SEP \\
\hline 1 & 15.7 & 4.1 & -1.7 & -2.0 & -1.2 & .6 & 8.4 & 12.2 & 16.4 & 30.7 & 29.1 & 22.8 \\
\hline 2 & 15.7 & 3.6 & -1.9 & -1.5 & -1.1 & 1.5 & 9.3 & 12.7 & 17.9 & 31.1 & 28.7 & 23.3 \\
\hline 3 & 14.4 & 3.9 & -1.9 & -2.3 & -1.3 & 3.3 & 10.0 & 13.4 & 20.5 & 30.1 & 28.9 & 23.5 \\
\hline 4 & 12.9 & 5.0 & -1.3 & -3.4 & -1.3 & 3.8 & 9.7 & 14.5 & 21.7 & 30.3 & 28.7 & 23.5 \\
\hline 5 & 12.6 & 5.7 & -.4 & -3.7 & -1.1 & 2.5 & 9.8 & 15.4 & 22.3 & 30.9 & 28.9 & 22.7 \\
\hline 6 & 12.8 & 5.7 & .4 & -3.8 & -.9 & 1.6 & 10.5 & 16.7 & 22.3 & 31.4 & 29.3 & 21.2 \\
\hline 7 & 12.9 & 5.0 & -.8 & -2.9 & -.6 & 1.5 & 9.6 & 17.4 & 22.6 & 31.7 & 29.5 & 20.4 \\
\hline 8 & 12.7 & 4.0 & -.6 & -1.0 & -.5 & 2.1 & 7.9 & 17.3 & 23.1 & 31.5 & 29.3 & 21.3 \\
\hline 9 & 12.9 & 4.3 & -.2 & -.3 & -.5 & 3.4 & 7.8 & 16.3 & 22.6 & 31.3 & 28.6 & 22.5 \\
\hline 10 & 13.2 & 5.1 & -.8 & -.2 & -.4 & 3.9 & 8.9 & 16.6 & 20.0 & 30.0 & 28.8 & 23.3 \\
\hline 11 & 14.1 & 5.7 & -2.8 & -.1 & .0 & 2.7 & 10.3 & 16.9 & 18.1 & 28.2 & 27.1 & 23.4 \\
\hline 12 & 14.4 & 6.9 & -3.6 & .5 & .3 & 1.8 & 10.8 & 16.7 & 19.6 & 28.4 & 25.8 & 24.0 \\
\hline 13 & 14.8 & 6.6 & -2.8 & .9 & .5 & 1.5 & 11.8 & 17.4 & 20.3 & 29.4 & 24.9 & 24.4 \\
\hline 14 & 14.9 & 6.5 & -3.3 & .8 & .5 & 1.3 & 12.9 & 17.4 & 20.6 & 28.9 & 25.2 & 25.1 \\
\hline 15 & 13.4 & 4.5 & -2.8 & -.2 & .1 & 1.4 & 13.8 & 17.4 & 18.8 & 25.2 & 23.0 & 25.1 \\
\hline 16 & 12.4 & 4.5 & -2.2 & -1.1 & -.2 & 1.9 & 13.2 & 17.2 & 18.5 & 22.8 & 20.1 & 24.0 \\
\hline 17 & 11.7 & 4.7 & -3.1 & -.7 & -.3 & 3.0 & 12.1 & 18.2 & 20.5 & 21.8 & 20.0 & 23.0 \\
\hline 18 & 11.5 & 4.2 & -2.9 & -.4 & -.2 & 4.3 & 11.1 & 18.4 & 21.9 & 23.3 & 20.7 & 22.1 \\
\hline 19 & 11.4 & 4.0 & -3.3 & -.3 & -.1 & 5.2 & 10.4 & 18.3 & 22.6 & 24.0 & 20.9 & 19.8 \\
\hline 20 & 12.3 & 4.5 & -3.6 & -.5 & -.1 & 6.8 & 10.7 & 17.7 & 23.2 & 25.1 & 19.6 & 19.2 \\
\hline 21 & 12.2 & 4.7 & -3.2 & -.8 & -.1 & 7.1 & 10.4 & 19.4 & 24.2 & 25.8 & 20.2 & 18.7 \\
\hline 22 & 11.6 & 4.2 & -3.2 & -.9 & .0 & 7.5 & 11.0 & 20.5 & 25.5 & 25.9 & 20.7 & 17.3 \\
\hline 23 & 12.1 & 4.4 & -2.7 & -.9 & .2 & 7.5 & 11.4 & 20.8 & 26.5 & 26.9 & 21.2 & 15.7 \\
\hline 24 & 11.4 & 5.3 & -2.4 & -.8 & .3 & 7.4 & 10.5 & 19.3 & 26.9 & 26.9 & 22.0 & 13.3 \\
\hline 25 & 9.7 & 4.3 & -2.5 & -1.1 & .4 & 7.8 & 10.9 & 18.0 & 27.4 & 26.3 & 21.8 & 13.3 \\
\hline 26 & 7.4 & 4.2 & -2.6 & -1.0 & .4 & 8.6 & 12.8 & 19.3 & 28.0 & 26.1 & 21.3 & 13.4 \\
\hline 27 & 7.1 & 1.1 & -2.0 & -1.3 & .5 & 8.6 & 13.8 & 19.0 & 27.9 & 25.9 & 21.5 & 13.6 \\
\hline 28 & 6.7 & -.9 & -1.5 & -1.8 & .5 & 7.3 & 14.7 & 15.4 & 28.1 & 27.0 & 21.6 & 12.9 \\
\hline 29 & 5.2 & -1.5 & -1.0 & -1.5 & -- & 6.6 & 13.1 & 14.7 & 28.8 & 27.9 & 22.4 & 14.0 \\
\hline 30 & 4.6 & -1.5 & -2.3 & -1.2 & -- & 7.3 & 12.7 & 15.5 & 29.4 & 28.4 & 23.4 & 14.0 \\
\hline 31 & 4.3 & --- & -2.5 & -1.1 & --- & 7.7 & --- & 15.3 & --- & 28.7 & 23.0 & \\
\hline MEAN & 11.6 & 4.1 & -2.1 & -1.1 & -.2 & 4.4 & 11.0 & 16.9 & 22.9 & 27.8 & 24.4 & 20.0 \\
\hline MAX & 15.7 & 6.9 & .4 & .9 & .5 & 8.6 & 14.7 & 20.8 & 29.4 & 31.7 & 29.5 & 25.1 \\
\hline MIN & 4.3 & -1.5 & -3.6 & -3.8 & -1.3 & .6 & 7.8 & 12.2 & 16.4 & 21.8 & 19.6 & 12.9 \\
\hline MED & 12.4 & 4.4 & -2.4 & -1.0 & -.1 & 3.8 & 10.7 & 17.3 & 22.4 & 28.2 & 23.0 & 21.7 \\
\hline
\end{tabular}

CAL YR 1989 MEAN 10.6 MAX 25.6 MIN -3.6 MED 12.2

WTR YR 1990 MEAN 11.7 MAX 31.7 MIN -3.8 MED 11.7 
3 SPRINGS BASIN

3 SPRINGS CREEK NEAR WARM SPRINGS, NV

DAILY MEAN SOIL TEMPERATURE, WATER YEAR OCTOBER 1990 TO SEPTEMBER 1991 DEGREES CELSIUS

\begin{tabular}{|c|c|c|c|c|c|c|c|c|c|c|c|c|}
\hline DAY & OCT & NOV & DEC & JAN & FEB & MAR & APR & MAY & JUN & JUL & AUG & SEP \\
\hline $\begin{array}{l}1 \\
2 \\
3 \\
4 \\
5\end{array}$ & $\begin{array}{l}14.4 \\
15.0 \\
14.1 \\
14.1 \\
15.4\end{array}$ & $\begin{array}{l}7.3 \\
4.5 \\
3.1 \\
3.3 \\
4.9\end{array}$ & $\begin{array}{l}-1.8 \\
-2.0 \\
-2.6 \\
-2.3 \\
-1.7\end{array}$ & $\begin{array}{l}-6.5 \\
-6.0 \\
-5.1 \\
-4.0 \\
-3.7\end{array}$ & $\begin{array}{l}-4.2 \\
-3.6 \\
-3.7 \\
-1.9 \\
-1.0\end{array}$ & $\begin{array}{r}1.5 \\
1.1 \\
1.0 \\
.9 \\
1.7\end{array}$ & $\begin{array}{l}1.2 \\
2.7 \\
4.0 \\
5.3 \\
6.7\end{array}$ & $\begin{array}{r}10.8 \\
9.0 \\
7.6 \\
8.9 \\
10.5\end{array}$ & $\begin{array}{l}12.8 \\
13.3 \\
14.5 \\
15.8 \\
16.9\end{array}$ & $\begin{array}{l}20.4 \\
21.7 \\
22.8 \\
23.6 \\
24.5\end{array}$ & $\begin{array}{l}\text { e23.9 } \\
\text { e } 23.6 \\
\text { e } 23.4 \\
\text { e } 23.1 \\
\text { e22. }\end{array}$ & $\begin{array}{l}\text { e2 } 6.2 \\
\text { e2 } 6.4 \\
\text { e2 } 6.1 \\
\text { e } 25.3 \\
\text { e2 } 4.9\end{array}$ \\
\hline $\begin{array}{r}6 \\
7 \\
8 \\
9 \\
10\end{array}$ & $\begin{array}{l}15.2 \\
14.4 \\
11.5 \\
10.7 \\
10.9\end{array}$ & $\begin{array}{l}3.5 \\
2.3 \\
3.3 \\
4.0 \\
3.5\end{array}$ & $\begin{array}{l}-2.5 \\
-3.4 \\
-3.5 \\
-3.4 \\
-2.7\end{array}$ & $\begin{array}{l}-4.0 \\
-3.8 \\
-3.2 \\
-3.3 \\
-3.5\end{array}$ & $\begin{array}{l}-.7 \\
-.5 \\
-.5 \\
-.5 \\
-.5\end{array}$ & $\begin{array}{l}1.2 \\
1.5 \\
1.6 \\
1.2 \\
1.7\end{array}$ & $\begin{array}{l}7.5 \\
6.8 \\
5.9 \\
6.3 \\
6.5\end{array}$ & $\begin{array}{r}12.2 \\
13.0 \\
14.3 \\
11.1 \\
8.7\end{array}$ & $\begin{array}{l}17.5 \\
18.1 \\
18.3 \\
18.3 \\
18.5\end{array}$ & $\begin{array}{l}24.8 \\
24.6 \\
22.5 \\
21.2 \\
21.5\end{array}$ & $\begin{array}{l}\text { e23. } 4 \\
\text { e } 23.6 \\
\text { e } 24.0 \\
\text { e } 24.6 \\
\text { e } 25.1\end{array}$ & $\begin{array}{l}\text { e2 } 4.1 \\
\text { e23.2 } \\
\text { e22.7 } \\
\text { e22 } 4 \\
\text { e2 } 1.1\end{array}$ \\
\hline $\begin{array}{l}11 \\
12 \\
13 \\
14 \\
15\end{array}$ & $\begin{array}{l}11.1 \\
10.5 \\
11.5 \\
11.6 \\
12.6\end{array}$ & $\begin{array}{l}3.6 \\
4.3 \\
4.7 \\
6.4 \\
6.3\end{array}$ & $\begin{array}{r}-1.2 \\
-.7 \\
-.6 \\
-1.2 \\
-2.5\end{array}$ & $\begin{array}{l}-3.3 \\
-2.8 \\
-2.6 \\
-2.5 \\
-2.1\end{array}$ & $\begin{array}{r}-.4 \\
-.3 \\
.1 \\
.6 \\
1.2\end{array}$ & $\begin{array}{r}1.2 \\
.8 \\
.7 \\
.7 \\
.7\end{array}$ & $\begin{array}{l}5.4 \\
4.9 \\
5.9 \\
6.7 \\
7.8\end{array}$ & $\begin{array}{r}8.5 \\
10.1 \\
10.9 \\
9.1 \\
9.7\end{array}$ & $\begin{array}{l}19.4 \\
20.5 \\
21.1 \\
20.8 \\
20.1\end{array}$ & $\begin{array}{l}21.6 \\
23.2 \\
24.4 \\
24.8 \\
25.0\end{array}$ & $\begin{array}{l}\text { e25.0 } \\
\text { e } 24.2 \\
\text { e } 24.3 \\
\text { e } 25.0 \\
\text { e2 } 25.4\end{array}$ & $\begin{array}{l}\text { e20.2 } \\
\text { e20.0 } \\
\text { e20.3 } \\
\text { e } 21.0 \\
\text { e21. } 1\end{array}$ \\
\hline $\begin{array}{l}16 \\
17 \\
18 \\
19 \\
20\end{array}$ & $\begin{array}{r}13.1 \\
11.3 \\
11.3 \\
9.1 \\
6.2\end{array}$ & $\begin{array}{l}5.4 \\
6.2 \\
5.5 \\
5.1 \\
4.2\end{array}$ & $\begin{array}{l}-2.7 \\
-3.7 \\
-3.3 \\
-3.3 \\
-3.6\end{array}$ & $\begin{array}{l}-1.7 \\
-2.8 \\
-2.7 \\
-2.4 \\
-2.4\end{array}$ & $\begin{array}{r}2.3 \\
.8 \\
.1 \\
.1 \\
.6\end{array}$ & $\begin{array}{l}.6 \\
.6 \\
.6 \\
.6 \\
.6\end{array}$ & $\begin{array}{l}7.9 \\
7.9 \\
8.1 \\
9.3 \\
9.0\end{array}$ & $\begin{array}{l}11.5 \\
12.4 \\
11.6 \\
11.8 \\
12.2\end{array}$ & $\begin{array}{l}21.0 \\
21.1 \\
20.9 \\
20.9 \\
20.4\end{array}$ & $\begin{array}{l}24.8 \\
24.7 \\
24.9 \\
24.8 \\
24.3\end{array}$ & $\begin{array}{l}\text { e25. } 9 \\
\text { e26.1 } \\
\text { e26. } 2 \\
\text { e26.1 } \\
\text { e2 } 6.5\end{array}$ & $\begin{array}{l}\text { e20.6 } \\
\text { e20.6 } \\
\text { e21. } 4 \\
\text { e22.0 } \\
\text { e21.9 }\end{array}$ \\
\hline $\begin{array}{l}21 \\
22 \\
23 \\
24 \\
25\end{array}$ & $\begin{array}{l}5.5 \\
6.1 \\
6.8 \\
7.4 \\
7.9\end{array}$ & $\begin{array}{r}2.4 \\
1.2 \\
.7 \\
.6 \\
.8\end{array}$ & $\begin{array}{l}-4.9 \\
-6.0 \\
-6.7 \\
-6.9 \\
-6.5\end{array}$ & $\begin{array}{l}-4.0 \\
-5.4 \\
-5.3 \\
-4.9 \\
-5.0\end{array}$ & $\begin{array}{l}1.3 \\
3.0 \\
3.2 \\
2.8 \\
2.2\end{array}$ & $\begin{array}{l}.6 \\
.6 \\
.6 \\
.6 \\
.6\end{array}$ & $\begin{array}{l}8.7 \\
7.5 \\
7.2 \\
8.8 \\
8.4\end{array}$ & $\begin{array}{l}11.4 \\
10.4 \\
12.1 \\
13.9 \\
14.9\end{array}$ & $\begin{array}{l}19.7 \\
19.9 \\
20.9 \\
21.0 \\
20.4\end{array}$ & $\begin{array}{l}24.2 \\
23.6 \\
24.1 \\
25.0 \\
25.5\end{array}$ & $\begin{array}{l}\text { e2 } 6.8 \\
\text { e } 27.2 \\
\text { e } 27.8 \\
\text { e } 28.3 \\
\text { e2 } 8.4\end{array}$ & $\begin{array}{l}\text { e22.7 } \\
\text { e22.7 } \\
\text { e } 22.7 \\
\text { e } 22.7 \\
\text { e2 } 0.5\end{array}$ \\
\hline $\begin{array}{l}26 \\
27 \\
28 \\
29 \\
30 \\
31\end{array}$ & $\begin{array}{l}8.5 \\
8.0 \\
9.4 \\
9.5 \\
9.5 \\
9.6\end{array}$ & $\begin{array}{r}1.0 \\
.2 \\
-1.1 \\
-1.6 \\
-1.2 \\
---\end{array}$ & $\begin{array}{l}-6.1 \\
-6.2 \\
-5.2 \\
-5.6 \\
-7.4 \\
-7.0\end{array}$ & $\begin{array}{l}-5.2 \\
-5.1 \\
-4.6 \\
-5.5 \\
-6.1 \\
-5.0\end{array}$ & $\begin{array}{l}2.4 \\
2.8 \\
2.4 \\
--- \\
--- \\
---\end{array}$ & $\begin{array}{r}.5 \\
.5 \\
.5 \\
.5 \\
.7 \\
1.4\end{array}$ & $\begin{array}{l}8.4 \\
8.9 \\
8.7 \\
9.5 \\
9.8 \\
---\end{array}$ & $\begin{array}{l}16.0 \\
15.4 \\
15.0 \\
15.9 \\
15.1 \\
13.1\end{array}$ & $\begin{array}{l}20.4 \\
18.5 \\
17.7 \\
18.5 \\
19.0 \\
\ldots .-\end{array}$ & $\begin{array}{r}25.7 \\
25.6 \\
24.8 \\
24.4 \\
24.5 \\
\mathrm{e} 24.6\end{array}$ & $\begin{array}{l}\text { e28.3 } \\
\text { e2 } 2.5 \\
\text { e26. } \\
\text { e26.5 } \\
\text { e27.0 } \\
\text { e26. } 6\end{array}$ & $\begin{array}{c}16.0 \\
15.2 \\
15.0 \\
14.0 \\
13.6 \\
---\end{array}$ \\
\hline $\begin{array}{l}\text { MEAN } \\
\text { MAX } \\
\text { MIN } \\
\text { MED }\end{array}$ & $\begin{array}{r}10.7 \\
15.4 \\
5.5 \\
10.9\end{array}$ & $\begin{array}{r}3.1 \\
7.3 \\
-1.6 \\
3.5\end{array}$ & $\begin{array}{r}-3.8 \\
-.6 \\
-7.4 \\
-3.4\end{array}$ & $\begin{array}{l}-4.0 \\
-1.7 \\
-6.5 \\
-4.0\end{array}$ & $\begin{array}{r}.3 \\
3.2 \\
-4.2 \\
.1\end{array}$ & $\begin{array}{r}.9 \\
1.7 \\
.5 \\
.7\end{array}$ & $\begin{array}{l}7.1 \\
9.8 \\
1.2 \\
7.5\end{array}$ & $\begin{array}{r}11.8 \\
16.0 \\
7.6 \\
11.6\end{array}$ & $\begin{array}{l}18.9 \\
21.1 \\
12.8 \\
19.5\end{array}$ & $\begin{array}{l}23.9 \\
25.7 \\
20.4 \\
24.5\end{array}$ & $\begin{array}{l}25.6 \\
28.4 \\
22.9 \\
25.9\end{array}$ & $\begin{array}{l}21.2 \\
26.4 \\
13.6 \\
21.7\end{array}$ \\
\hline
\end{tabular}

CAL YR 1990 MEAN 11.4 MAX 31.7 MIN -7.4 MED 10.9

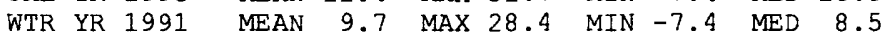

e Estimated 
3 SPRINGS BASIN

3 SPRINGS CREEK NEAR WARM SPRINGS, NV

DAILY TOTAL SOLAR RADIATION, INCIDENTAL, WATER YEAR OCTOBER 1985 TO SEPTEMBER 1986 CALORIES PER SQUARE CENTIMETER

\begin{tabular}{|c|c|c|c|c|c|c|c|c|c|c|c|c|}
\hline DAY & OCT & NOV & $\mathrm{DEC}$ & JAN & FEB & MAR & $\mathrm{APR}$ & MAY & JUN & JUL & AUG & SEP \\
\hline 1 & e 485 & 318 & 167 & 195 & 258 & 359 & 394 & 621 & 741 & 741 & 731 & 597 \\
\hline 2 & e 480 & 317 & 60 & 218 & 211 & 166 & 550 & 690 & 455 & 721 & 724 & 609 \\
\hline 3 & e 475 & 327 & 206 & 202 & 244 & 414 & 532 & 677 & 579 & 705 & 716 & 612 \\
\hline 4 & e 470 & 178 & 216 & 56 & 204 & 412 & 541 & 663 & 653 & 622 & 713 & 589 \\
\hline 5 & e 466 & 306 & 157 & 142 & 289 & 443 & 473 & 542 & 761 & 682 & 574 & 553 \\
\hline 6 & e461 & 301 & 204 & 222 & 203 & 413 & 249 & 438 & 765 & 761 & 697 & 581 \\
\hline 7 & e 456 & 299 & 190 & 228 & 237 & 302 & 164 & 617 & 707 & 684 & 700 & 599 \\
\hline 8 & e451 & 287 & 187 & 196 & 52 & 65 & 349 & 510 & 717 & 765 & 705 & 537 \\
\hline 9 & e 447 & 294 & 187 & 210 & 178 & 410 & 486 & 722 & 771 & 703 & 681 & 555 \\
\hline 10 & e 443 & 235 & 52 & 232 & 335 & 149 & 352 & 543 & 770 & 693 & 335 & 583 \\
\hline 11 & 420 & 9 & 69 & 228 & 213 & 390 & 549 & 726 & 774 & 744 & 616 & 544 \\
\hline 12 & 257 & 12 & 141 & 240 & 162 & 323 & 444 & 681 & 643 & 720 & 415 & 498 \\
\hline 13 & 420 & 36 & 191 & 230 & 137 & 188 & 631 & 665 & 647 & 726 & 577 & 455 \\
\hline 14 & 417 & 38 & 220 & 125 & 52 & 347 & 556 & 599 & 734 & 626 & 651 & 561 \\
\hline 15 & 411 & 283 & 214 & 192 & 50 & 262 & 477 & 673 & 722 & 392 & 654 & 561 \\
\hline 16 & 406 & 235 & 214 & 161 & 254 & 146 & 456 & 740 & 773 & 738 & 678 & 519 \\
\hline 17 & 399 & 214 & 215 & 215 & 193 & 360 & 456 & 722 & 786 & 740 & 678 & 424 \\
\hline 18 & 393 & 270 & 215 & 249 & 70 & 519 & 640 & 743 & 767 & 742 & 351 & 454 \\
\hline 19 & 384 & 263 & 215 & 219 & 156 & 521 & 654 & 723 & 768 & 753 & 591 & 432 \\
\hline 20 & 281 & 236 & 217 & 262 & 379 & 509 & 633 & 681 & 770 & 545 & 380 & 525 \\
\hline 21 & 140 & 266 & 219 & 161 & 392 & 522 & 562 & 715 & 773 & 285 & 509 & 531 \\
\hline 22 & 267 & 256 & 220 & 141 & 315 & 537 & 586 & 726 & 773 & 227 & 465 & 517 \\
\hline 23 & 365 & 132 & 215 & 241 & 385 & 452 & 462 & 660 & 688 & 385 & 637 & 319 \\
\hline 24 & 361 & 105 & 212 & 264 & 404 & 418 & 412 & 721 & 707 & 480 & 562 & 330 \\
\hline 25 & 356 & 124 & 209 & 279 & 414 & 541 & 615 & 743 & 765 & 712 & 324 & 245 \\
\hline 26 & 354 & 248 & 216 & 280 & 413 & 545 & 599 & 738 & 743 & 640 & 495 & 491 \\
\hline 27 & 343 & 220 & 210 & 284 & 415 & 557 & 667 & 729 & 689 & 728 & 160 & 322 \\
\hline 28 & 273 & 84 & 212 & 260 & 425 & 340 & 639 & 477 & 604 & 744 & 538 & 405 \\
\hline 29 & 333 & 48 & 142 & 253 & -- & 306 & 686 & 707 & 774 & 728 & 620 & 477 \\
\hline 30 & 286 & 214 & 93 & 35 & -- & 465 & 647 & 758 & 766 & 732 & 626 & 474 \\
\hline 31 & 330 & -- & 147 & 175 & -- & 368 & --- & 710 & -- & 737 & 533 & -- \\
\hline TOTAL & 11830 & 6155 & 5632 & 6395 & 7040 & 11749 & 15461 & 20660 & 21585 & 20201 & 17636 & 14899 \\
\hline
\end{tabular}

e Estimated 
3 SPRINGS BASIN

3 SPRINGS CREEK NEAR WARM SPRINGS, NV

DAILY TOTAL SOLAR RADIATION, INCIDENTAL, WATER YEAR OCTOBER 1986 TO SEPTEMBER 1987 CALORIES PER SQUARE CENTIMETER

\begin{tabular}{|c|c|c|c|c|c|c|c|c|c|c|c|c|}
\hline DAY & OCT & NoV & DEC & JAN & FEB & MAR & APR & MAY & JUN & JUL & AUG & SEP \\
\hline 1 & 227 & 324 & 239 & 163 & 309 & 436 & 601 & 563 & 764 & e 776 & 722 & 403 \\
\hline 2 & 96 & 321 & 239 & 200 & 149 & 430 & 603 & 637 & 764 & e 775 & 714 & 359 \\
\hline 3 & 425 & 312 & 234 & 183 & 150 & 440 & 189 & 699 & 763 & e 774 & 535 & 482 \\
\hline 4 & 456 & 317 & 73 & 66 & 320 & 416 & 108 & 711 & 647 & e 773 & 389 & 348 \\
\hline 5 & 453 & 315 & 117 & 16 & 325 & 110 & 333 & 709 & 486 & e 772 & 608 & 482 \\
\hline 6 & 439 & 191 & 113 & 17 & 325 & 115 & 599 & 689 & 270 & e 771 & 603 & 332 \\
\hline 7 & 362 & 253 & 224 & 15 & 242 & 120 & 486 & 408 & 708 & e 770 & 639 & 440 \\
\hline 8 & 319 & 304 & 221 & 33 & 213 & 290 & 531 & 436 & 406 & 730 & 536 & 534 \\
\hline 9 & 333 & 297 & 223 & 32 & 143 & 219 & 623 & 379 & 690 & 687 & 681 & 486 \\
\hline 10 & 337 & 294 & 221 & 225 & 167 & 304 & 481 & 496 & 688 & 657 & 683 & 443 \\
\hline 11 & 430 & 293 & 226 & 248 & 134 & 457 & 448 & 447 & 735 & 556 & 617 & 571 \\
\hline 12 & 431 & 292 & 226 & 250 & 184 & 421 & 628 & 536 & 771 & 559 & 477 & 495 \\
\hline 13 & 427 & 288 & 163 & 238 & 106 & 493 & 640 & 566 & 480 & 561 & 621 & 454 \\
\hline 14 & 426 & 272 & 211 & 252 & 359 & 320 & 516 & 451 & 700 & 503 & 499 & 387 \\
\hline 15 & 419 & 277 & 190 & 178 & 304 & 270 & 556 & 265 & 778 & 281 & 667 & 536 \\
\hline 16 & 403 & 238 & 190 & 227 & 354 & 399 & 652 & 194 & 774 & 248 & 686 & 532 \\
\hline 17 & 326 & 251 & 213 & 253 & 382 & 491 & 652 & 450 & 778 & 578 & 679 & 536 \\
\hline 18 & 88 & 116 & 121 & 259 & 190 & 349 & 592 & 657 & 767 & 738 & 683 & 539 \\
\hline 19 & 59 & 241 & 60 & 157 & 290 & 248 & 657 & 688 & 762 & 454 & 551 & 540 \\
\hline 20 & 307 & 261 & 59 & 265 & 390 & 445 & 671 & 524 & 753 & 301 & 492 & 533 \\
\hline 21 & 375 & 249 & 210 & 272 & 387 & 133 & 681 & 430 & 750 & 690 & 658 & 515 \\
\hline 22 & 370 & 197 & 194 & 189 & 393 & 474 & 687 & 489 & 771 & 741 & 633 & 519 \\
\hline 23 & 251 & 238 & 190 & 210 & 82 & 266 & 580 & 667 & 743 & 744 & 628 & 341 \\
\hline 24 & 348 & 253 & 191 & 248 & 324 & 368 & 574 & 605 & 769 & 750 & 596 & 350 \\
\hline 25 & 360 & 249 & 215 & 270 & 126 & 487 & 617 & 439 & 564 & 751 & 527 & 496 \\
\hline 26 & 309 & 246 & 200 & 292 & 421 & 539 & 498 & 175 & 539 & 726 & 639 & 498 \\
\hline 27 & 357 & 199 & 236 & 227 & 426 & 568 & 510 & 615 & 642 & 423 & 436 & e 503 \\
\hline 28 & 278 & 186 & 222 & 269 & 413 & 575 & 350 & 454 & 564 & 644 & 434 & e499 \\
\hline 29 & 325 & 191 & 167 & 230 & -- & 585 & 234 & 567 & 411 & 704 & 457 & e 494 \\
\hline 30 & 288 & 241 & 223 & 131 & --- & 586 & 639 & 662 & 620 & 723 & 490 & e 489 \\
\hline 31 & 315 & $\cdots$ & 157 & 301 & --- & 565 & $\cdots$ & 754 & $\ldots$ & 728 & 542 & $\ldots$ \\
\hline TOTAL & 10339 & 7706 & 5768 & 5916 & 7608 & 11919 & 15936 & 16362 & 19857 & 19888 & 18122 & 14136 \\
\hline $\begin{array}{l}\text { CAL YR } \\
\text { WTR YR }\end{array}$ & $\begin{array}{l}1986 \\
1987\end{array}$ & $\begin{array}{l}\text { TOTAL } \\
\text { TOTAL }\end{array}$ & $\begin{array}{l}9439 \\
3557\end{array}$ & & & & & & & & & \\
\hline
\end{tabular}

e Estimated 
3 SPRINGS BASIN

3 SPRINGS CREEK NEAR WARM SPRINGS, NV

DAILY TOTAL SOLAR RADIATION, INCIDENTAL, WATER YEAR OCTOBER 1987 TO SEPTEMBER 1988 CALORIES PER SQUARE CENTIMETER

\begin{tabular}{|c|c|c|c|c|c|c|c|c|c|c|c|c|}
\hline DAY & OCT & NOV & DEC & JAN & FEB & MAR & APR & MAY & JUN & JUL & AUG & SEP \\
\hline $\begin{array}{l}1 \\
2 \\
3 \\
4 \\
5\end{array}$ & $\begin{array}{l}478 \\
490 \\
421 \\
458 \\
450\end{array}$ & $\begin{array}{r}\text { e334 } \\
87 \\
129 \\
196 \\
57\end{array}$ & $\begin{array}{r}\text { e2 } 44 \\
\text { e2 } 43 \\
\text { e241 } \\
\text { e239 } \\
39\end{array}$ & $\begin{array}{l}\text { e234 } \\
\text { e235 } \\
\text { e237 } \\
\text { e238 } \\
\text { e239 }\end{array}$ & $\begin{array}{l}152 \\
150 \\
304 \\
317 \\
321\end{array}$ & $\begin{array}{l}306 \\
166 \\
417 \\
371 \\
340\end{array}$ & $\begin{array}{l}592 \\
577 \\
461 \\
560 \\
610\end{array}$ & $\begin{array}{l}581 \\
650 \\
665 \\
227 \\
631\end{array}$ & $\begin{array}{l}597 \\
742 \\
776 \\
744 \\
787\end{array}$ & $\begin{array}{r}619 \\
434 \\
\text { e } 774 \\
\text { e } 773 \\
\text { e } 772\end{array}$ & $\begin{array}{l}\text { e } 732 \\
\text { e } 729 \\
\text { e } 726 \\
\text { e } 722 \\
\text { e } 719\end{array}$ & $\begin{array}{l}\text { e } 623 \\
\text { e } 619 \\
\text { e } 615 \\
\text { e } 607 \\
\text { e } 603\end{array}$ \\
\hline $\begin{array}{r}6 \\
7 \\
8 \\
9 \\
10\end{array}$ & $\begin{array}{l}445 \\
445 \\
430 \\
419 \\
422\end{array}$ & $\begin{array}{r}66 \\
285 \\
292 \\
234 \\
279\end{array}$ & $\begin{array}{r}157 \\
\text { e236 } \\
\text { e235 } \\
143 \\
\text { e233 }\end{array}$ & $\begin{array}{l}\text { e2 } 40 \\
\text { e241 } \\
\text { e243 } \\
\text { e2 } 44 \\
\text { e2 } 46\end{array}$ & $\begin{array}{l}319 \\
316 \\
324 \\
317 \\
333\end{array}$ & $\begin{array}{l}307 \\
454 \\
422 \\
386 \\
294\end{array}$ & $\begin{array}{l}474 \\
414 \\
619 \\
647 \\
645\end{array}$ & $\begin{array}{l}638 \\
373 \\
669 \\
615 \\
663\end{array}$ & $\begin{array}{l}788 \\
628 \\
787 \\
792 \\
709\end{array}$ & $\begin{array}{l}\text { e } 771 \\
\text { e } 770 \\
\text { e } 770 \\
\text { e } 769 \\
\text { e } 768\end{array}$ & $\begin{array}{l}\text { e } 716 \\
\text { e } 713 \\
\text { e } 709 \\
\text { e } 706 \\
\text { e } 703\end{array}$ & $\begin{array}{l}\text { e599 } \\
\text { e } 595 \\
\text { e590 } \\
\text { e586 } \\
\text { e582 }\end{array}$ \\
\hline $\begin{array}{l}11 \\
12 \\
13 \\
14 \\
15\end{array}$ & $\begin{array}{l}426 \\
149 \\
316 \\
411 \\
411\end{array}$ & $\begin{array}{r}\text { e2 } 94 \\
\text { e291 } \\
\text { e287 } \\
131 \\
160\end{array}$ & $\begin{array}{r}109 \\
\mathrm{e} 232 \\
166 \\
\mathrm{e} 232 \\
\mathrm{e} 232\end{array}$ & $\begin{array}{l}\text { e2 } 46 \\
\text { e248 } \\
\text { e250 } \\
\text { e } 252 \\
\text { e } 255\end{array}$ & $\begin{array}{l}338 \\
346 \\
347 \\
343 \\
260\end{array}$ & $\begin{array}{l}350 \\
490 \\
499 \\
489 \\
401\end{array}$ & $\begin{array}{r}625 \\
580 \\
409 \\
185 \\
55\end{array}$ & $\begin{array}{l}682 \\
741 \\
746 \\
749 \\
750\end{array}$ & $\begin{array}{l}648 \\
689 \\
781 \\
680 \\
761\end{array}$ & $\begin{array}{l}\text { e767 } \\
\text { e766 } \\
\text { e765 } \\
\text { e764 } \\
\text { e763 }\end{array}$ & $\begin{array}{l}\text { e } 700 \\
\text { e } 696 \\
\text { e } 693 \\
\text { e } 689 \\
\text { e } 686\end{array}$ & $\begin{array}{l}\text { e } 577 \\
\text { e572 } \\
\text { e567 } \\
\text { e} 562 \\
\text { e } 557\end{array}$ \\
\hline $\begin{array}{l}16 \\
17 \\
18 \\
19 \\
20\end{array}$ & $\begin{array}{l}401 \\
396 \\
399 \\
388 \\
384\end{array}$ & $\begin{array}{r}\text { e277 } \\
125 \\
268 \\
271 \\
157\end{array}$ & $\begin{array}{r}\text { e232 } \\
10 \\
\text { e232 } \\
\text { e232 } \\
\text { e232 }\end{array}$ & $\begin{array}{l}\text { e258 } \\
\text { e260 } \\
\text { e263 } \\
\text { e2 } 66 \\
\text { e2 } 69\end{array}$ & $\begin{array}{l}324 \\
365 \\
212 \\
377 \\
388\end{array}$ & $\begin{array}{l}470 \\
522 \\
521 \\
532 \\
533\end{array}$ & $\begin{array}{l}155 \\
313 \\
459 \\
237 \\
166\end{array}$ & $\begin{array}{l}642 \\
393 \\
674 \\
761 \\
769\end{array}$ & $\begin{array}{l}761 \\
353 \\
589 \\
411 \\
553\end{array}$ & $\begin{array}{l}\text { e } 762 \\
\text { e } 761 \\
\text { e } 761 \\
\text { e } 760 \\
\text { e } 759\end{array}$ & $\begin{array}{l}\text { e } 682 \\
\text { e } 679 \\
\text { e } 675 \\
\text { e } 672 \\
\text { e } 668\end{array}$ & $\begin{array}{l}\text { e } 552 \\
\text { e547 } \\
\text { e } 542 \\
\text { e } 537 \\
\text { e } 532\end{array}$ \\
\hline $\begin{array}{l}21 \\
22 \\
23 \\
24 \\
25\end{array}$ & $\begin{array}{l}305 \\
156 \\
203 \\
148 \\
330\end{array}$ & $\begin{array}{l}215 \\
147 \\
239 \\
247 \\
155\end{array}$ & $\begin{array}{l}\text { e232 } \\
\text { e233 } \\
\text { e234 } \\
\text { e234 } \\
\text { e235 }\end{array}$ & $\begin{array}{l}\text { e } 272 \\
\text { e2 } 74 \\
\text { e } 277 \\
\text { e } 280 \\
\text { e } 283\end{array}$ & $\begin{array}{l}365 \\
404 \\
381 \\
403 \\
408\end{array}$ & $\begin{array}{l}445 \\
531 \\
524 \\
545 \\
557\end{array}$ & $\begin{array}{l}372 \\
405 \\
319 \\
631 \\
691\end{array}$ & $\begin{array}{l}762 \\
688 \\
593 \\
727 \\
755\end{array}$ & $\begin{array}{r}584 \\
397 \\
589 \\
210 \\
15\end{array}$ & $\begin{array}{l}\text { e } 758 \\
\text { e } 756 \\
\text { e } 755 \\
\text { e } 753 \\
\text { e } 751\end{array}$ & $\begin{array}{l}\text { e } 665 \\
\text { e } 661 \\
\text { e } 657 \\
\text { e } 653 \\
\text { e } 650\end{array}$ & $\begin{array}{r}e 527 \\
e 523 \\
547 \\
547 \\
543\end{array}$ \\
\hline $\begin{array}{l}26 \\
27 \\
28 \\
29 \\
30 \\
31\end{array}$ & $\begin{array}{r}351 \\
240 \\
\text { e } 352 \\
\text { e } 347 \\
\text { e } 343 \\
\text { e } 338\end{array}$ & $\begin{array}{r}90 \\
55 \\
96 \\
74 \\
\mathrm{e} 246 \\
---\end{array}$ & $\begin{array}{l}\text { e236 } \\
\text { e237 } \\
\text { e237 } \\
\text { e238 } \\
\text { e239 } \\
\text { e2 } 40\end{array}$ & $\begin{array}{l}250 \\
224 \\
154 \\
266 \\
291 \\
271\end{array}$ & $\begin{array}{l}336 \\
124 \\
211 \\
247 \\
--- \\
---\end{array}$ & $\begin{array}{l}571 \\
566 \\
571 \\
563 \\
447 \\
589\end{array}$ & $\begin{array}{l}672 \\
531 \\
653 \\
708 \\
401 \\
---\end{array}$ & $\begin{array}{l}730 \\
745 \\
584 \\
225 \\
501 \\
614\end{array}$ & $\begin{array}{r}379 \\
671 \\
213 \\
22 \\
461 \\
---\end{array}$ & $\begin{array}{l}\text { e } 749 \\
\text { e } 746 \\
\text { e } 743 \\
\text { e } 741 \\
\text { e } 738 \\
\text { e } 735\end{array}$ & $\begin{array}{l}\text { e } 646 \\
\text { e } 642 \\
\text { e } 638 \\
\text { e } 634 \\
\text { e } 631 \\
\text { e } 627\end{array}$ & $\begin{array}{l}534 \\
500 \\
518 \\
519 \\
516 \\
---\end{array}$ \\
\hline TOTAL & 11252 & 5784 & 6514 & 7806 & 9032 & 14179 & 14166 & 19543 & 17117 & 23073 & 21119 & 16838 \\
\hline $\begin{array}{l}\text { CAL YR } \\
\text { WTR YR }\end{array}$ & $\begin{array}{l}1987 \\
1988\end{array}$ & $\begin{array}{l}\text { TOTAL } \\
\text { TOTAL }\end{array}$ & $\begin{array}{l}294 \\
423\end{array}$ & & & & & & & & & \\
\hline
\end{tabular}

e Estimated 
3 SPRINGS BASIN

3 SPRINGS CREEK NEAR WARM SPRINGS, NV

DAILY TOTAL SOLAR RADIATION, INCIDENTAL, WATER YEAR OCTOBER 1988 TO SEPTEMBER 1989 CALORIES PER SQUARE CENTIMETER

\begin{tabular}{|c|c|c|c|c|c|c|c|c|c|c|c|c|}
\hline DAY & OCT & NOV & DEC & JAN & $\mathrm{FEB}$ & MAR & $\mathrm{APR}$ & MAY & JUN & JUL & AUG & SEP \\
\hline 1 & 509 & 340 & 249 & 234 & 238 & 441 & 572 & 734 & 799 & 819 & 761 & 642 \\
\hline 2 & 502 & 307 & 248 & 238 & 249 & 71 & 485 & 674 & 802 & 823 & 746 & 655 \\
\hline 3 & 415 & 329 & 191 & 242 & 170 & 418 & 631 & 652 & 301 & 827 & 753 & 648 \\
\hline 4 & 483 & 330 & 220 & 79 & 34 & 345 & 640 & 680 & 579 & 824 & 653 & 647 \\
\hline 5 & 395 & 325 & 229 & 25 & 61 & 398 & 649 & 657 & 515 & 821 & 745 & 642 \\
\hline 6 & 256 & 324 & 168 & 210 & 71 & 358 & 658 & 505 & 728 & 820 & 603 & 639 \\
\hline 7 & 472 & 171 & 141 & 174 & 85 & 378 & 661 & 649 & 520 & 653 & 570 & 612 \\
\hline 8 & 468 & 201 & 236 & 250 & 29 & 181 & 668 & 677 & 537 & 600 & 435 & 610 \\
\hline 9 & 419 & 292 & 231 & 256 & 13 & 448 & 655 & 367 & 411 & 592 & 334 & 605 \\
\hline 10 & 269 & 196 & 241 & 220 & 160 & 308 & 662 & 440 & 567 & 804 & 595 & 487 \\
\hline 11 & 270 & 283 & 234 & 231 & 207 & 470 & 664 & 52 & 582 & 426 & 611 & 419 \\
\hline 12 & 379 & 299 & 229 & 257 & 179 & 516 & 671 & 545 & 760 & 595 & 595 & 600 \\
\hline 13 & 411 & 273 & 227 & 214 & 211 & 510 & 676 & 31 & 592 & 532 & 694 & 532 \\
\hline 14 & 266 & 50 & 231 & 251 & 372 & 520 & 627 & 269 & 805 & 706 & 714 & 594 \\
\hline 15 & 434 & 296 & 25 & 263 & 380 & 474 & 662 & 470 & 819 & 798 & 715 & 590 \\
\hline 16 & 431 & 269 & 148 & 268 & 334 & 396 & 627 & 583 & 798 & 797 & 722 & 309 \\
\hline 17 & 423 & 122 & 150 & 271 & 327 & 522 & 641 & 770 & 818 & 790 & 720 & 259 \\
\hline 18 & 420 & 264 & 105 & 273 & 337 & 234 & 692 & 776 & 827 & 797 & 708 & 465 \\
\hline 19 & 416 & 228 & 74 & 280 & 396 & 378 & 489 & 785 & 822 & 636 & 699 & 186 \\
\hline 20 & 412 & 246 & 144 & 287 & 411 & 563 & 624 & 702 & 749 & 698 & 596 & 505 \\
\hline 21 & 405 & 267 & 239 & 283 & 399 & 547 & 617 & 800 & 818 & 470 & 643 & 551 \\
\hline 22 & 401 & 247 & 218 & 154 & 344 & 556 & 671 & 721 & 819 & 689 & 686 & 545 \\
\hline 23 & 390 & 135 & 232 & 210 & 381 & 347 & 611 & 750 & 609 & 585 & 552 & 544 \\
\hline 24 & 390 & 201 & 56 & 275 & 375 & 536 & 425 & 796 & 318 & 433 & 682 & 521 \\
\hline 25 & 381 & 187 & 237 & 301 & 367 & 191 & 586 & 801 & 690 & 473 & 674 & 297 \\
\hline 26 & 377 & 261 & 234 & 304 & 424 & 315 & 429 & 798 & 715 & 678 & 663 & 385 \\
\hline 27 & 321 & 254 & 229 & 301 & 454 & 580 & 318 & 799 & 791 & 761 & 665 & 411 \\
\hline 28 & 331 & 256 & 92 & 308 & 453 & 547 & 585 & 695 & 821 & 745 & 663 & 492 \\
\hline 29 & 308 & 235 & 211 & 308 & --- & 617 & 604 & 692 & 815 & 744 & 668 & 496 \\
\hline 30 & 337 & 248 & 230 & 322 & $-\cdots$ & 610 & 483 & 436 & 822 & 744 & 668 & 497 \\
\hline 31 & 348 & --- & 182 & 320 & -- & 479 & --- & 793 & -- & 724 & 661 & $\cdots$ \\
\hline TOTAL & 12039 & 7436 & 5881 & 7609 & 7461 & 13254 & 17983 & 19099 & 20549 & 21404 & 20194 & 15385 \\
\hline CAL YR & 1988 & TOTAL & 229 & & & & & & & & & \\
\hline WTR YP & 1989 & TOTAL & 294 & & & & & & & & & \\
\hline
\end{tabular}


3 SPRINGS BASIN

3 SPRINGS CREEK NEAR WARM SPRINGS, NV

DAILY TOTAL SOLAR RADIATION, INCIDENTAL, WATER YEAR OCTOBER 1989 TO SEPTEMBER 1990 CALORIES PER SQUARE CENTIMETER

\begin{tabular}{|c|c|c|c|c|c|c|c|c|c|c|c|c|}
\hline DAY & $\mathrm{OCT}$ & NOV & DEC & JAN & FEB & MAR & APR & MAY & JUN & JUL & AUG & SEP \\
\hline 1 & 494 & 343 & 244 & 85 & 178 & 266 & 606 & 725 & 778 & 715 & 695 & 631 \\
\hline 2 & 391 & 339 & 242 & 83 & 316 & 310 & 476 & 549 & 771 & 674 & 610 & 558 \\
\hline 3 & 175 & 324 & 237 & 232 & 200 & 417 & 593 & 732 & 772 & 783 & 572 & 549 \\
\hline 4 & 471 & 329 & 232 & 232 & 35 & 325 & 330 & 735 & 767 & 789 & 508 & 498 \\
\hline 5 & 477 & 321 & 226 & 235 & 238 & 140 & 512 & 739 & 788 & 794 & 580 & 416 \\
\hline 6 & 468 & 318 & 232 & 205 & 249 & 473 & 321 & 711 & 777 & 790 & 728 & 326 \\
\hline 7 & 466 & 258 & 219 & 150 & 115 & 436 & 336 & 753 & 780 & 771 & 447 & 380 \\
\hline 8 & 461 & 313 & 154 & 238 & 326 & 291 & 184 & 756 & 780 & 643 & 610 & 594 \\
\hline 9 & 453 & 306 & 227 & 239 & 344 & 463 & 641 & 720 & 374 & 757 & 489 & 599 \\
\hline 10 & 412 & 302 & 230 & 215 & 342 & 474 & 589 & 622 & 411 & 419 & 529 & 600 \\
\hline 11 & 450 & 302 & 228 & 237 & 303 & 193 & 578 & 675 & 741 & 581 & 504 & 600 \\
\hline 12 & 441 & 300 & 215 & 190 & 269 & 235 & 647 & 713 & 800 & 467 & 414 & 601 \\
\hline 13 & 388 & 284 & 224 & 47 & 375 & 438 & 635 & 672 & 787 & 477 & 599 & 589 \\
\hline 14 & 308 & 296 & 221 & 127 & 334 & 441 & 551 & 476 & 753 & 528 & 571 & 577 \\
\hline 15 & 313 & 189 & 224 & 207 & 377 & 518 & 620 & 735 & 421 & 423 & 217 & 592 \\
\hline 16 & 415 & 292 & 210 & 80 & 300 & 444 & 404 & 737 & 749 & 226 & 654 & 577 \\
\hline 17 & 412 & 262 & 222 & 9 & 159 & 302 & 351 & 630 & 792 & 577 & 619 & 340 \\
\hline 18 & 411 & 277 & 213 & 147 & 264 & 490 & 302 & 750 & 739 & 661 & 604 & 343 \\
\hline 19 & 404 & 276 & 219 & 260 & 381 & 436 & 394 & 577 & 791 & 624 & 539 & 278 \\
\hline 20 & 346 & 275 & 192 & 279 & 355 & 340 & 302 & 752 & 726 & 741 & 656 & 513 \\
\hline 21 & 150 & 270 & 219 & 281 & 394 & 532 & 410 & 727 & 797 & 604 & 669 & 277 \\
\hline 22 & 339 & 261 & 218 & 275 & 41.9 & 450 & 572 & 720 & 797 & 728 & 653 & 341 \\
\hline 23 & 167 & 252 & 217 & 268 & 424 & 567 & 392 & 727 & 811 & 746 & 656 & 182 \\
\hline 24 & 308 & 234 & 197 & 286 & 342 & 562 & 609 & 772 & 782 & 750 & 650 & 256 \\
\hline 25 & 151 & 182 & 223 & 235 & 361 & 536 & 688 & 725 & 802 & 752 & 666 & 456 \\
\hline 26 & 366 & 197 & 216 & 296 & 385 & 582 & 693 & 538 & 802 & 749 & 662 & 477 \\
\hline 27 & 365 & 250 & 174 & 296 & 245 & 542 & 669 & 437 & 805 & 748 & 665 & 381 \\
\hline 28 & 354 & 250 & 202 & 291 & 368 & 363 & 592 & 268 & 809 & 674 & 658 & 327 \\
\hline 29 & 354 & 247 & 222 & 276 & $-\cdots$ & 558 & 505 & 521 & 798 & 742 & 626 & 433 \\
\hline 30 & 355 & 246 & 223 & 229 & $\cdots$ & 582 & 630 & 641 & 705 & 735 & 640 & 461 \\
\hline 31 & 349 & -- & 230 & 309 & $-\cdots$ & 602 & --- & 628 & -- & 740 & 648 & -- \\
\hline TOTAL & 11414 & 8295 & 6752 & 6539 & 8398 & 13308 & 15132 & 20463 & 22205 & 20408 & 18338 & 13752 \\
\hline CAL YR & 1989 & OTAL & נוs & & & & & & & & & \\
\hline WTR YR & 1990 & TOTAL & 004 & & & & & & & & & \\
\hline
\end{tabular}


3 SPRINGS BASIN

3 SPRINGS CREEK NEAR WARM SPRINGS, NV

DAILY TOTAI SOLAR RADIATION, INCIDENTAL, WATER YEAR OCTOBER 1990 TO SEPTEMBER 1991 CALORIES PER SQUARE CENTIMETER

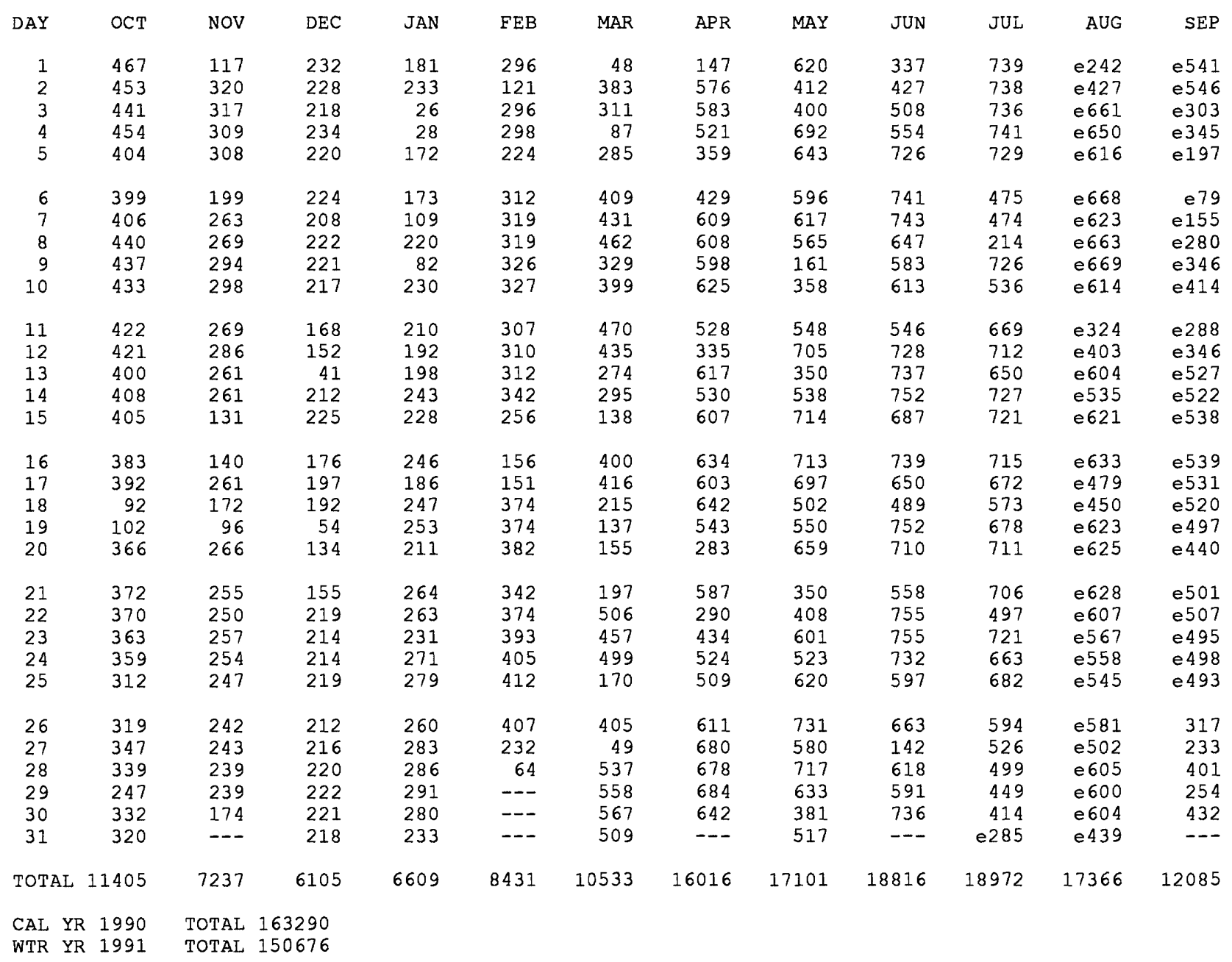

e Estimated 
3 SPRINGS BASIN

3 SPRINGS CREEK NEAR WARM SPRINGS, NV

DAILY MEAN RELATIVE HUMIDITY, WATER YEAR OCTOBER 1990 TO SEPTEMBER 1991 PERCENT

\begin{tabular}{|c|c|c|c|c|c|c|c|c|c|c|c|c|}
\hline DAY & OCT & NOV & DEC & JAN & EEB & MAR & APR & MAY & JUN & JUL & AUG & SEP \\
\hline 1 & 36 & 73 & 42 & 40 & 28 & 97 & 71 & 29 & 87 & 26 & --- & --- \\
\hline 2 & 31 & 54 & 29 & 40 & 25 & 59 & 55 & 74 & 66 & 19 & --- & --- \\
\hline 3 & 38 & 36 & 49 & 72 & 48 & 62 & 42 & 58 & 31 & 16 & --- & --- \\
\hline 4 & 31 & 38 & 45 & 99 & 57 & 92 & 33 & 37 & 22 & 13 & --- & $\cdots$ \\
\hline 5 & 25 & 39 & 39 & 91 & 45 & 55 & 19 & 29 & 22 & 12 & --- & --- \\
\hline 6 & 33 & 52 & 48 & 85 & 72 & 59 & 18 & 24 & 20 & 16 & --- & --- \\
\hline 7 & 52 & 35 & 39 & 96 & 55 & 61 & 20 & 34 & 18 & 23 & --- & --- \\
\hline 8 & 32 & 58 & 38 & 91 & 43 & 47 & 37 & 22 & 21 & 62 & --- & --- \\
\hline 9 & 26 & 65 & 30 & 92 & 37 & 34 & 29 & 79 & 27 & 70 & --- & --- \\
\hline 10 & 23 & 30 & 19 & 84 & 42 & 27 & 34 & 64 & 24 & 41 & --- & --- \\
\hline 11 & 27 & 22 & 26 & 86 & 35 & 61 & 41 & 47 & 21 & 19 & $--\infty$ & --- \\
\hline 12 & 22 & 23 & 59 & 80 & 31 & 44 & 67 & 36 & 15 & 20 & --- & --- \\
\hline 13 & 19 & 20 & 74 & 79 & 53 & 58 & 61 & 38 & 16 & 14 & $-\infty$ & --- \\
\hline 14 & 25 & 20 & 68 & 64 & 69 & 76 & 33 & 79 & 16 & 13 & $-\infty$ & -- \\
\hline 15 & 20 & 30 & 58 & 54 & 51 & 90 & 33 & 55 & 17 & 14 & --- & -- \\
\hline 16 & 20 & 44 & 71 & 61 & 63 & 76 & 25 & 32 & 14 & 14 & --- & --- \\
\hline 17 & 24 & 46 & 75 & 74 & 54 & 49 & 34 & 28 & 16 & 14 & -- & $-\cdots$ \\
\hline 18 & 65 & 49 & 44 & 88 & 50 & 39 & 37 & 62 & 14 & 15 & --- & --- \\
\hline 19 & 87 & 55 & 65 & 78 & 54 & 75 & 22 & 63 & 17 & 16 & --- & --- \\
\hline 20 & 59 & 71 & 60 & 64 & 35 & 94 & 23 & 45 & 16 & 17 & -- & -- \\
\hline 21 & 47 & 60 & 66 & 53 & 26 & 96 & 44 & 60 & 16 & 14 & --- & --- \\
\hline 22 & 47 & 63 & 64 & 52 & 20 & 72 & 72 & 69 & 16 & 16 & --- & --- \\
\hline 23 & 51 & 42 & 53 & 60 & 32 & 57 & 59 & 50 & 15 & 13 & --- & $\cdots$ \\
\hline 24 & 46 & 31 & 59 & 39 & 34 & 39 & 43 & 38 & 16 & 14 & -- & $\cdots$ \\
\hline 25 & 34 & 19 & 59 & 41 & 34 & 61 & 52 & 39 & 19 & 13 & -- & -- \\
\hline 26 & 29 & 56 & 62 & 44 & 30 & 62 & 45 & 18 & 25 & 13 & -- & 55 \\
\hline 27 & 26 & 60 & 58 & 40 & 36 & 98 & 30 & 24 & 28 & 13 & --- & 69 \\
\hline 28 & 16 & 62 & 22 & 25 & 99 & 61 & 34 & 24 & 31 & 16 & --- & 78 \\
\hline 29 & 18 & 45 & 36 & 40 & -- & 50 & 29 & 21 & 38 & 16 & --- & 85 \\
\hline 30 & 23 & 40 & 39 & 35 & --- & 54 & 25 & 49 & 36 & 20 & $-\cdots$ & 69 \\
\hline 31 & 24 & --- & 47 & 32 & -- & 43 & --- & 69 & -- & -- & --- & $-\cdots$ \\
\hline MEAN & 34 & 45 & 50 & 64 & 45 & 63 & 39 & 45 & 25 & $-\cdots$ & -- & $-\cdots$ \\
\hline MAX & 87 & 73 & 75 & 99 & 99 & 98 & 72 & 79 & 87 & $-\cdots$ & -- & -- \\
\hline MIN & 16 & 19 & 19 & 25 & 20 & 27 & 18 & 18 & 14 & --- & $-\cdots$ & -- \\
\hline MED & 29 & 44 & 49 & 64 & 43 & 61 & 34 & 39 & 20 & -- & --- & $-\cdots$ \\
\hline
\end{tabular}


3 SPRINGS BASIN

3 SPRINGS CREEK NEAR WARM SPRINGS, NV

DAILY TOTAL PRECIPITATION, WATER YEAR OCTOBER 1985 TO SEPTEMBER 1986 INCHES

\begin{tabular}{|c|c|c|c|c|c|c|c|c|c|c|c|c|}
\hline DAY & $\mathrm{OCT}$ & NOV & DEC & JAN & FEB & MAR & APR & MAY & JUN & JUL & AUG & SEP \\
\hline 1 & .00 & .00 & .00 & .00 & e.00 & e. 00 & .00 & .00 & .00 & .00 & .00 & .00 \\
\hline 2 & .00 & .00 & .20 & .00 & e. 00 & e. 19 & .00 & .00 & .00 & .00 & .00 & .00 \\
\hline 3 & .00 & .00 & .00 & .00 & e. 00 & e. 04 & .00 & .00 & .00 & .00 & .00 & .00 \\
\hline 4 & .00 & .00 & .00 & .10 & e. 00 & e. 00 & .00 & .40 & .00 & .00 & .00 & .00 \\
\hline 5 & .00 & .00 & .00 & .00 & e. 00 & .00 & .00 & .00 & .00 & .00 & .00 & .00 \\
\hline 6 & 1.20 & .00 & .00 & .00 & e. 00 & .00 & .15 & .00 & .00 & .00 & .00 & .00 \\
\hline 7 & .00 & .00 & .00 & .00 & e. 00 & .00 & .40 & .00 & .00 & .00 & .00 & .00 \\
\hline 8 & .60 & .00 & .00 & .00 & e.00 & .80 & .00 & .00 & .00 & .00 & .00 & .00 \\
\hline 9 & .30 & .00 & .00 & .00 & e. 00 & .10 & .00 & .00 & .00 & .00 & .07 & .00 \\
\hline 10 & .00 & .00 & .15 & .00 & e. 00 & .15 & .00 & .00 & .00 & .00 & .08 & .00 \\
\hline 11 & .00 & .65 & .00 & e.00 & e. 00 & .15 & .00 & .00 & .00 & .00 & .00 & .00 \\
\hline 12 & .00 & .35 & .00 & e. 00 & e.00 & .08 & .00 & .00 & .00 & .00 & .00 & .00 \\
\hline 13 & .00 & .00 & .00 & e. 00 & e. 32 & .03 & .00 & .00 & .00 & .00 & .00 & .00 \\
\hline 14 & .00 & .00 & .00 & e. 00 & e. 00 & .09 & .00 & .00 & .00 & .05 & .00 & .00 \\
\hline 15 & .00 & .00 & .00 & e. 00 & e. 56 & .10 & .00 & .00 & .00 & .74 & .00 & .00 \\
\hline 16 & .00 & .00 & .00 & e. 00 & e. 15 & .12 & .00 & .00 & .00 & .06 & .00 & .00 \\
\hline 17 & .00 & .00 & .00 & e. 00 & e. 00 & .30 & .00 & .00 & .00 & .00 & .00 & .00 \\
\hline 18 & .00 & .00 & .00 & e. 00 & e. 15 & .10 & .00 & .00 & .00 & .00 & .00 & .00 \\
\hline 19 & .00 & .00 & .00 & e. 00 & e. 00 & .00 & .00 & .00 & .00 & .00 & .00 & .00 \\
\hline 20 & .00 & .00 & .00 & e. 00 & e. 00 & .00 & .00 & .00 & .00 & .00 & .20 & .00 \\
\hline 21 & .50 & .00 & .00 & e. 00 & e. 00 & .00 & .00 & .00 & .00 & .00 & .00 & .00 \\
\hline 22 & .00 & .00 & .00 & e. 00 & e. 00 & .00 & .00 & .00 & .00 & .40 & .00 & .00 \\
\hline 23 & .00 & .00 & .00 & e. 00 & e. 00 & .00 & .00 & .00 & .00 & .00 & .00 & .00 \\
\hline 24 & .00 & .00 & .00 & e. 00 & e. 00 & .00 & .00 & .00 & .00 & .00 & .00 & .07 \\
\hline 25 & .00 & .20 & .00 & e. 00 & e. 00 & .00 & .00 & .00 & .00 & .00 & .50 & .24 \\
\hline 26 & .80 & .00 & .00 & e. 00 & e. 00 & .00 & .00 & .00 & .00 & .00 & .10 & .00 \\
\hline 27 & .00 & .00 & .00 & e. 00 & e. 00 & .00 & .00 & .00 & .00 & .00 & .20 & .00 \\
\hline 28 & .00 & .00 & .00 & e. 00 & e. 00 & .00 & .00 & .00 & .00 & .00 & .00 & .00 \\
\hline 29 & .00 & .50 & .00 & e. 00 & --- & .00 & .00 & .00 & .00 & .00 & .00 & .00 \\
\hline 30 & .00 & .10 & .00 & e. 66 & --- & .00 & .00 & .00 & .00 & .00 & .00 & .00 \\
\hline 31 & .00 & --- & .00 & e. 43 & --- & .10 & --- & .00 & --- & .00 & .00 & -- \\
\hline TOTAL & 3.40 & 1.80 & 0.35 & 1.19 & 1.18 & 2.35 & 0.55 & 0.40 & 0.00 & 1.25 & 1.15 & 0.31 \\
\hline
\end{tabular}

WTR YR 1986 TOTAL 13.93

e Estimated 
3 SPRINGS BASIN

3 SPRINGS CREEK NEAR WARM SPRINGS, NV

DAILY TOTAL PRECIPITATION, WATER YEAR OCTOBER 1986 TO SEPTEMBER 1987 INCHES

\begin{tabular}{|c|c|c|c|c|c|c|c|c|c|c|c|c|}
\hline DAY & OCT & NOV & DEC & JAN & FEB & MAR & $\mathrm{APR}$ & MAY & JUN & JUL & AUG & SEP \\
\hline 1 & .15 & .00 & .00 & .00 & .00 & .00 & .00 & .00 & .00 & .00 & .00 & .00 \\
\hline 2 & .15 & .00 & .00 & .00 & .00 & .00 & .00 & .00 & .00 & .00 & .00 & .00 \\
\hline 3 & .00 & .00 & .00 & .00 & .00 & .00 & .00 & .00 & .00 & .00 & .00 & .00 \\
\hline 4 & .00 & .00 & .00 & .47 & .00 & .00 & .00 & .00 & .00 & .00 & .00 & .00 \\
\hline 5 & .00 & .00 & .00 & .48 & .00 & .60 & .00 & .00 & .00 & .00 & .00 & .00 \\
\hline 6 & .00 & .00 & .00 & .10 & .00 & .70 & .00 & .00 & .05 & .00 & .00 & .00 \\
\hline 7 & .00 & .00 & .00 & .20 & .00 & .30 & .00 & .00 & .00 & .00 & .00 & .00 \\
\hline 8 & .00 & .00 & .00 & .00 & .00 & .30 & .00 & .00 & .00 & .00 & .00 & .00 \\
\hline 9 & .00 & .00 & .00 & .00 & .00 & .00 & .00 & .00 & .00 & .00 & .00 & .00 \\
\hline 10 & .00 & .00 & .00 & .00 & .00 & .00 & .00 & .00 & .00 & .00 & .00 & .00 \\
\hline 11 & .00 & .00 & .00 & .00 & .00 & .00 & .00 & .00 & .00 & .00 & .00 & .00 \\
\hline 12 & .00 & .00 & .00 & .00 & .00 & .00 & .00 & .00 & .00 & .00 & .00 & .00 \\
\hline 13 & .00 & .00 & .00 & .00 & .15 & .00 & .00 & .00 & .00 & .00 & .00 & .00 \\
\hline 14 & .00 & .00 & .00 & .00 & .00 & .00 & .00 & .00 & .00 & .00 & .00 & .00 \\
\hline 15 & .00 & .00 & .00 & .00 & .00 & .75 & .00 & .00 & .00 & .00 & .00 & .00 \\
\hline 16 & .00 & .00 & .00 & .00 & .00 & .00 & .00 & .45 & .00 & .00 & .00 & .00 \\
\hline 17 & .00 & .00 & .00 & .00 & .00 & .00 & .00 & .20 & .00 & .00 & .00 & .00 \\
\hline 18 & .45 & .12 & .00 & .00 & .00 & .00 & .00 & .00 & .00 & .00 & .00 & .00 \\
\hline 19 & .00 & .00 & .45 & .00 & .00 & .00 & .00 & .00 & .00 & .00 & .00 & .00 \\
\hline 20 & .00 & .00 & .30 & .00 & .00 & .00 & .00 & .00 & .00 & .00 & .00 & .00 \\
\hline 21 & .00 & .00 & .00 & .00 & .00 & .12 & .00 & .00 & .00 & .00 & .00 & .00 \\
\hline 22 & .00 & .00 & .00 & .00 & .00 & .08 & .00 & .00 & .00 & .00 & .00 & .00 \\
\hline 23 & .00 & .00 & .00 & .00 & .00 & .00 & .00 & .00 & .00 & .00 & .00 & .00 \\
\hline 24 & .00 & .00 & .00 & .00 & .75 & .00 & .00 & .00 & .00 & .00 & .00 & .00 \\
\hline 25 & .00 & .00 & .00 & .00 & .15 & .00 & .00 & .00 & .00 & .00 & .00 & .00 \\
\hline 26 & .00 & .00 & .00 & .00 & .00 & .00 & .00 & 1.25 & .00 & .00 & .00 & .00 \\
\hline 27 & .00 & .00 & .00 & .00 & .00 & .00 & .00 & .00 & .00 & .00 & .00 & .00 \\
\hline 28 & .00 & .00 & .00 & .18 & .00 & .00 & .10 & .20 & .00 & .00 & .00 & .00 \\
\hline 29 & .00 & .00 & .00 & .00 & --- & .00 & .30 & .00 & .00 & .00 & .00 & .00 \\
\hline 30 & .00 & .00 & .00 & .00 & --- & .00 & .10 & .00 & .00 & .00 & .00 & .00 \\
\hline 31 & .00 & --- & .00 & .00 & --- & .00 & --- & .00 & --- & .00 & .00 & --- \\
\hline TOTAL & 0.75 & 0.12 & 0.75 & 1.43 & 1.05 & 2.85 & 0.50 & 2.10 & 0.05 & 0.00 & 0.00 & 0.00 \\
\hline
\end{tabular}

CAL YR 1986 TOTAL 10.00

WTR YR 1987 TOTAL 9.60 
3 SPRINGS BASIN

3 SPRINGS CREEK NEAR WARM SPRINGS, NV

DAILY TOTAL PRECIPITATION, WATER YEAR OCTOBER 1987 TO SEPTEMBER 1988 INCHES

\begin{tabular}{|c|c|c|c|c|c|c|c|c|c|c|c|c|}
\hline DAY & OCT & NoV & DEC & JAN & FEB & MAR & $A P R$ & MAY & JUN & JUL & AUG & SEP \\
\hline $\begin{array}{l}1 \\
2 \\
3 \\
4 \\
5\end{array}$ & $\begin{array}{l}.00 \\
.00 \\
.00 \\
.00 \\
.00\end{array}$ & $\begin{array}{r}1.50 \\
.30 \\
.30 \\
.00 \\
.90\end{array}$ & $\begin{array}{l}.00 \\
.00 \\
.00 \\
.00 \\
.00\end{array}$ & $\begin{array}{l}.00 \\
.00 \\
.10 \\
.00 \\
.20\end{array}$ & $\begin{array}{l}.00 \\
.00 \\
.00 \\
.00 \\
.00\end{array}$ & $\begin{array}{l}.40 \\
.15 \\
.00 \\
.00 \\
.00\end{array}$ & $\begin{array}{l}.00 \\
.00 \\
.00 \\
.00 \\
.00\end{array}$ & $\begin{array}{l}.00 \\
.00 \\
.00 \\
.00 \\
.80\end{array}$ & $\begin{array}{l}.00 \\
.00 \\
.00 \\
.00 \\
.00\end{array}$ & $\begin{array}{l}.00 \\
.00 \\
.00 \\
.00 \\
.00\end{array}$ & $\begin{array}{l}.00 \\
.00 \\
.00 \\
.00 \\
.80\end{array}$ & $\begin{array}{l}.00 \\
.00 \\
.00 \\
.00 \\
.00\end{array}$ \\
\hline $\begin{array}{r}6 \\
7 \\
8 \\
9 \\
10\end{array}$ & $\begin{array}{l}.00 \\
.00 \\
.00 \\
.00 \\
.00\end{array}$ & $\begin{array}{l}.40 \\
.00 \\
.00 \\
.00 \\
.00\end{array}$ & $\begin{array}{l}.00 \\
.00 \\
.00 \\
.00 \\
.00\end{array}$ & $\begin{array}{l}.00 \\
.00 \\
.00 \\
.00 \\
.00\end{array}$ & $\begin{array}{l}.00 \\
.00 \\
.00 \\
.00 \\
.00\end{array}$ & $\begin{array}{l}.00 \\
.00 \\
.00 \\
.00 \\
.00\end{array}$ & $\begin{array}{l}.00 \\
.00 \\
.00 \\
.00 \\
.00\end{array}$ & $\begin{array}{l}.00 \\
.00 \\
.00 \\
.00 \\
.00\end{array}$ & $\begin{array}{l}.00 \\
.00 \\
.00 \\
.00 \\
.00\end{array}$ & $\begin{array}{l}.00 \\
.00 \\
.00 \\
.00 \\
.00\end{array}$ & $\begin{array}{l}.00 \\
.00 \\
.00 \\
.00 \\
.00\end{array}$ & $\begin{array}{l}.00 \\
.00 \\
.00 \\
.00 \\
.00\end{array}$ \\
\hline $\begin{array}{l}11 \\
12 \\
13 \\
14 \\
15\end{array}$ & $\begin{array}{l}.00 \\
.50 \\
.15 \\
.00 \\
.00\end{array}$ & $\begin{array}{l}.00 \\
.00 \\
.20 \\
.00 \\
.00\end{array}$ & $\begin{array}{l}.00 \\
.00 \\
.00 \\
.00 \\
.00\end{array}$ & $\begin{array}{l}.00 \\
.00 \\
.00 \\
.00 \\
.00\end{array}$ & $\begin{array}{l}.00 \\
.00 \\
.00 \\
.00 \\
.00\end{array}$ & $\begin{array}{l}.00 \\
.00 \\
.00 \\
.00 \\
.00\end{array}$ & $\begin{array}{r}.00 \\
.00 \\
.00 \\
1.00 \\
1.10\end{array}$ & $\begin{array}{l}.00 \\
.00 \\
.00 \\
.00 \\
.00\end{array}$ & $\begin{array}{l}.00 \\
.00 \\
.00 \\
.00 \\
.00\end{array}$ & $\begin{array}{l}.00 \\
.00 \\
.00 \\
.00 \\
.00\end{array}$ & $\begin{array}{l}.00 \\
.00 \\
.00 \\
.00 \\
.00\end{array}$ & $\begin{array}{l}.00 \\
.00 \\
.00 \\
.00 \\
.00\end{array}$ \\
\hline $\begin{array}{l}16 \\
17 \\
18 \\
19 \\
20\end{array}$ & $\begin{array}{l}.00 \\
.00 \\
.00 \\
.00 \\
.00\end{array}$ & $\begin{array}{l}.00 \\
.00 \\
.00 \\
.00 \\
.00\end{array}$ & $\begin{array}{l}.00 \\
.12 \\
.08 \\
.18 \\
.00\end{array}$ & $\begin{array}{l}.00 \\
.95 \\
.10 \\
.00 \\
.00\end{array}$ & $\begin{array}{l}.00 \\
.00 \\
.00 \\
.00 \\
.00\end{array}$ & $\begin{array}{l}.00 \\
.00 \\
.00 \\
.00 \\
.00\end{array}$ & $\begin{array}{l}.25 \\
.15 \\
.00 \\
.00 \\
.50\end{array}$ & $\begin{array}{l}.00 \\
.00 \\
.00 \\
.00 \\
.00\end{array}$ & $\begin{array}{l}.00 \\
.00 \\
.00 \\
.00 \\
.00\end{array}$ & $\begin{array}{l}.00 \\
.00 \\
.00 \\
.00 \\
.00\end{array}$ & $\begin{array}{l}.00 \\
.00 \\
.00 \\
.00 \\
.00\end{array}$ & $\begin{array}{l}.00 \\
.00 \\
.00 \\
.00 \\
.00\end{array}$ \\
\hline $\begin{array}{l}21 \\
22 \\
23 \\
24 \\
25\end{array}$ & $\begin{array}{l}.00 \\
.10 \\
.00 \\
.00 \\
.00\end{array}$ & $\begin{array}{l}.00 \\
.00 \\
.00 \\
.00 \\
.00\end{array}$ & $\begin{array}{l}.00 \\
.27 \\
.00 \\
.00 \\
.00\end{array}$ & $\begin{array}{l}.00 \\
.00 \\
.00 \\
.00 \\
.00\end{array}$ & $\begin{array}{l}.00 \\
.00 \\
.00 \\
.00 \\
.00\end{array}$ & $\begin{array}{l}.00 \\
.00 \\
.00 \\
.00 \\
.00\end{array}$ & $\begin{array}{l}.10 \\
.50 \\
.28 \\
.00 \\
.00\end{array}$ & $\begin{array}{l}.00 \\
.00 \\
.00 \\
.00 \\
.00\end{array}$ & $\begin{array}{l}.00 \\
.00 \\
.50 \\
.00 \\
.00\end{array}$ & $\begin{array}{l}.00 \\
.00 \\
.00 \\
.20 \\
.05\end{array}$ & $\begin{array}{l}.00 \\
.00 \\
.00 \\
.00 \\
.30\end{array}$ & $\begin{array}{l}.00 \\
.00 \\
.00 \\
.00 \\
.00\end{array}$ \\
\hline $\begin{array}{l}26 \\
27 \\
28 \\
29 \\
30 \\
31\end{array}$ & $\begin{array}{l}.00 \\
.00 \\
.00 \\
.15 \\
.00 \\
.15\end{array}$ & $\begin{array}{l}.00 \\
.00 \\
.00 \\
.00 \\
.00 \\
-.--\end{array}$ & $\begin{array}{l}.00 \\
.00 \\
.00 \\
.30 \\
.08 \\
.00\end{array}$ & $\begin{array}{l}.00 \\
.00 \\
.00 \\
.00 \\
.00 \\
.00\end{array}$ & $\begin{array}{l}.00 \\
.20 \\
.00 \\
.25 \\
--- \\
---\end{array}$ & $\begin{array}{l}.00 \\
.00 \\
.00 \\
.00 \\
.00 \\
.00\end{array}$ & $\begin{array}{l}.00 \\
.00 \\
.00 \\
.00 \\
.20 \\
---\end{array}$ & $\begin{array}{r}.00 \\
.00 \\
.00 \\
1.00 \\
.00 \\
.00\end{array}$ & $\begin{array}{l}.00 \\
.00 \\
.00 \\
.00 \\
.00 \\
---\end{array}$ & $\begin{array}{l}.00 \\
.00 \\
.00 \\
.00 \\
.00 \\
.00\end{array}$ & $\begin{array}{l}.25 \\
.00 \\
.00 \\
.00 \\
.00 \\
.00\end{array}$ & $\begin{array}{l}.00 \\
.00 \\
.00 \\
.00 \\
.00 \\
.--\end{array}$ \\
\hline TOTAL & 1.05 & 3.60 & 1.03 & 1.35 & 0.45 & 0.55 & 4.08 & 1.80 & 0.50 & 0.25 & 1.35 & 0.00 \\
\hline
\end{tabular}

CAL YR 1987 TOTAL 13.66

WTR YR 1988 TOTAL 16.01 
3 SPRINGS BASIN

3 SPRINGS CREEK NEAR WARM SPRINGS, NV

DAILY TOTAL PRECIPITATION, WATER YEAR OCTOBER 1988 TO SEPTEMBER 1989

\begin{tabular}{|c|c|c|c|c|c|c|c|c|c|c|c|c|}
\hline DAY & OCT & NOV & DEC & JAN & $\mathrm{FEB}$ & MAR & APR & MAY & JUN & JUI & AUG & SEP \\
\hline 1 & e.00 & e. 00 & e. 00 & e. 00 & .00 & .00 & .00 & .00 & .00 & .00 & .00 & .00 \\
\hline 2 & e. 00 & e. 00 & e. 00 & e. 00 & .00 & .35 & .00 & .00 & .00 & .00 & .00 & .00 \\
\hline 3 & e. 00 & e.00 & e. 00 & e. 00 & .00 & .00 & .00 & .00 & .10 & .00 & .00 & .00 \\
\hline 4 & e. 00 & e. 00 & e. 00 & e. 07 & .45 & .00 & .00 & .00 & .10 & .00 & .00 & .00 \\
\hline 5 & e. 00 & e.00 & e. 00 & e.03 & .00 & .00 & .00 & .00 & .00 & .00 & .00 & .00 \\
\hline 6 & e. 00 & e. 00 & e. 00 & e. 26 & .00 & .00 & .00 & .00 & .00 & .00 & .00 & .00 \\
\hline 7 & e. 00 & e. 00 & e. 00 & e. 00 & .00 & .00 & .00 & .00 & .10 & .00 & .00 & .00 \\
\hline 8 & e. 00 & e. 00 & e. 00 & e. 00 & .30 & .00 & .00 & .00 & .10 & .00 & .40 & .00 \\
\hline 9 & e. 00 & e.00 & e. 00 & e. 00 & .20 & .00 & .00 & .00 & .10 & .00 & .35 & .00 \\
\hline 10 & e. 00 & e. 00 & e. 00 & .00 & .25 & .00 & .00 & .00 & .55 & .00 & .00 & .00 \\
\hline 11 & e. 00 & e.00 & e. 00 & .00 & .00 & .00 & .00 & 1.00 & .00 & .00 & .45 & .00 \\
\hline 12 & e. 00 & e.00 & e.00 & .00 & .00 & .00 & .00 & .10 & .00 & .00 & .00 & .00 \\
\hline 13 & e. 00 & e. 00 & e. 00 & .00 & .00 & .00 & .00 & .75 & .00 & .00 & .00 & .00 \\
\hline 14 & e. 00 & e. 58 & e. 00 & .00 & .00 & .00 & .00 & .35 & .00 & .00 & .00 & .00 \\
\hline 15 & e. 00 & $e .00$ & e. 08 & .00 & .00 & .00 & .00 & .00 & .00 & .00 & .00 & .00 \\
\hline 16 & e. 00 & e. 00 & e. 01 & .00 & .00 & .00 & .00 & .00 & .00 & .00 & .00 & .00 \\
\hline 17 & e. 00 & e. 18 & e. 00 & .00 & .00 & .00 & .00 & .00 & .00 & .00 & .00 & .10 \\
\hline 18 & e. 00 & e.00 & e. 04 & .00 & .00 & .00 & .00 & .00 & .00 & .00 & .00 & .00 \\
\hline 19 & e. 00 & e.00 & e. 06 & .00 & .00 & .00 & .00 & .00 & .00 & .00 & .00 & .00 \\
\hline 20 & e. 00 & e.00 & e.00 & .00 & .00 & .00 & .00 & .00 & .00 & .00 & .00 & .00 \\
\hline 21 & e. 00 & e.00 & e. 74 & .00 & .00 & .00 & .00 & .00 & .00 & .00 & .00 & .00 \\
\hline 22 & e. 00 & e. 00 & e. 00 & .00 & .00 & .00 & .00 & .00 & .00 & .00 & .00 & .00 \\
\hline 23 & e. 00 & e. 18 & e. 11 & .00 & .00 & .00 & .00 & .00 & .00 & .00 & .00 & .00 \\
\hline 24 & e. 00 & e. 00 & e.09 & .00 & .00 & .00 & .00 & .00 & .00 & .00 & .00 & .00 \\
\hline 25 & e.00 & e. 20 & e. 33 & .00 & .00 & .00 & .10 & .00 & .15 & .10 & .00 & .00 \\
\hline 26 & e. 00 & e. 00 & e. 00 & .00 & .00 & .20 & .00 & .00 & .00 & .00 & .00 & .00 \\
\hline 27 & e.00 & e. 00 & e. 00 & .00 & .00 & .00 & .10 & .00 & .00 & .00 & .00 & .00 \\
\hline 28 & e. 00 & e. 00 & e. 04 & .00 & .00 & .00 & .00 & .00 & .00 & .00 & .00 & .00 \\
\hline 29 & e. 00 & e. 00 & e. 00 & .00 & -- & .00 & .00 & .00 & .00 & .00 & .00 & .00 \\
\hline 30 & e. 00 & e.00 & e.00 & .00 & - - & .00 & .00 & .20 & .00 & .00 & .00 & .00 \\
\hline 31 & e.00 & -- & e. 01 & .00 & -- & .00 & -- & .00 & -- & .00 & .00 & $\cdots$ \\
\hline TOTAL & 0.00 & 1.14 & 1.51 & 0.36 & 1.20 & 0.55 & 0.20 & 2.40 & 1.20 & 0.10 & 1.20 & 0.10 \\
\hline CAL YR & 1988 & TAL & .98 & & & & & & & & & \\
\hline WTR YR & 1989 & TOTAL & .96 & & & & & & & & & \\
\hline
\end{tabular}

e Estimated 
3 SPRINGS BASIN

3 SPRINGS CREEK NEAR WARM SPRINGS, NV

DAILY TOTAL PRECIPITATION, WATER YEAR OCTOBER 1989 TO SEPTEMBER 1990 INCHES

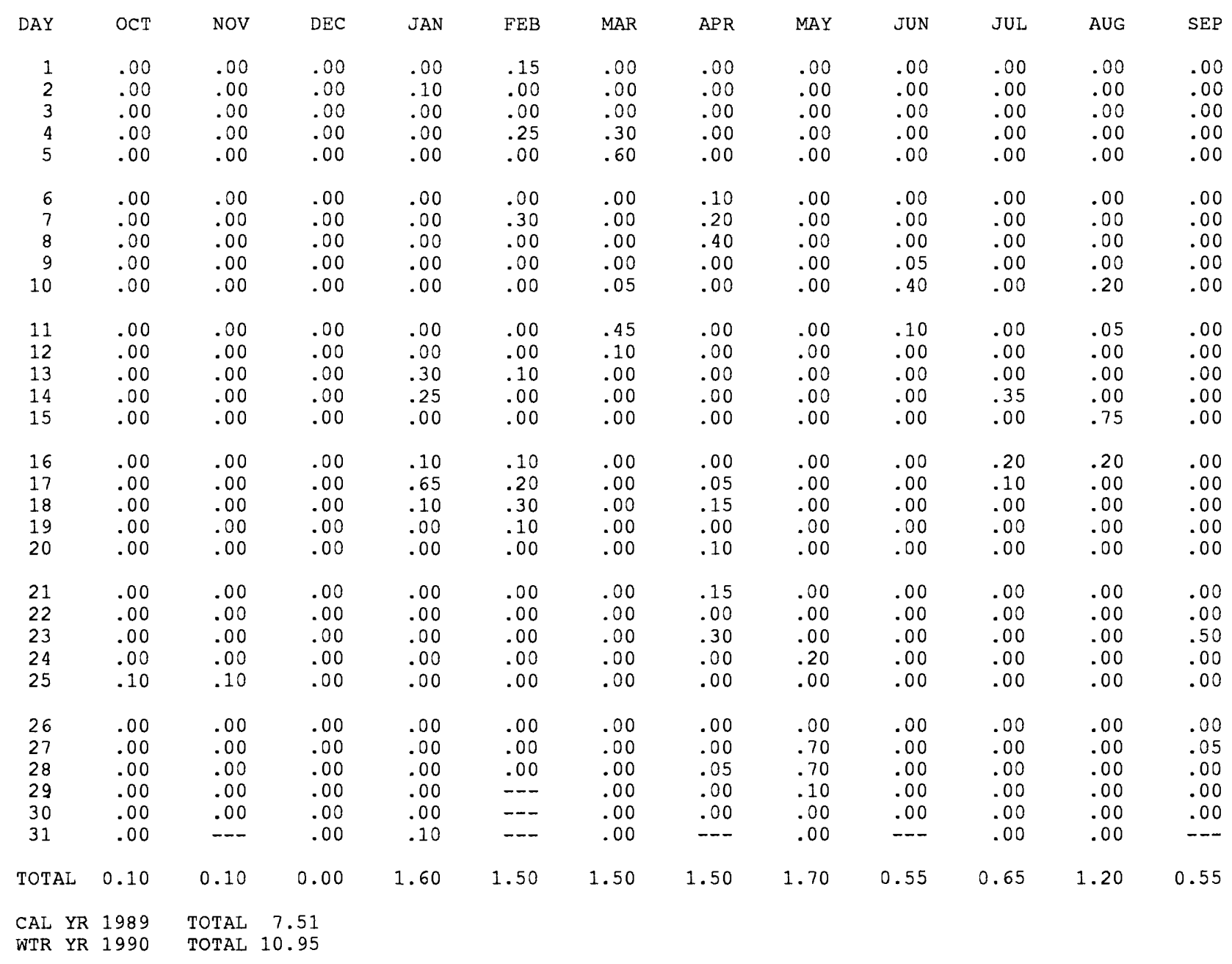


3 SPRINGS BASIN

3 SPRINGS CREEK NEAR WARM SPRINGS, NV

DAILY TOTAL PRECIPITATION, WATER YEAR OCTOBER 1990 TO SEPTEMBER 1991 INCHES

\begin{tabular}{|c|c|c|c|c|c|c|c|c|c|c|c|c|}
\hline DAY & OCT & NOV & DEC & JAN & FEB & MAR & $\mathrm{APR}$ & MAY & JUN & JUL & AUG & SEP \\
\hline 1 & .00 & .10 & .00 & .00 & .00 & e. 55 & .06 & .00 & .00 & .00 & .22 & .00 \\
\hline 2 & .00 & .00 & .00 & .00 & .00 & .00 & .04 & .25 & .00 & .00 & .18 & .04 \\
\hline 3 & .00 & .00 & .00 & .25 & .00 & e.05 & .00 & .00 & .00 & .00 & .20 & .25 \\
\hline 4 & .00 & .00 & .00 & .00 & .00 & e. 05 & .00 & .00 & .00 & .00 & .00 & .00 \\
\hline 5 & .00 & .00 & .00 & .00 & .00 & .00 & .00 & .00 & .00 & .00 & .00 & .00 \\
\hline 6 & .00 & .00 & .00 & .00 & .00 & .00 & .00 & .00 & .00 & .00 & .00 & .30 \\
\hline 7 & .00 & .00 & .00 & .00 & .00 & .00 & .00 & .00 & .00 & .00 & .00 & .40 \\
\hline 8 & .00 & .00 & .00 & .00 & .00 & .00 & .00 & .15 & .00 & .91 & .00 & .00 \\
\hline 9 & .00 & .00 & .00 & .00 & .00 & .00 & .00 & .05 & .00 & .13 & .00 & .05 \\
\hline 10 & .00 & .00 & .00 & .00 & .00 & e. 30 & .00 & .00 & .00 & .00 & .00 & .00 \\
\hline 11 & .00 & .00 & .00 & .00 & .00 & .00 & .00 & .00 & .00 & .00 & .06 & .00 \\
\hline 12 & .00 & .00 & .00 & .00 & .00 & .00 & .00 & .00 & .00 & .00 & .00 & .00 \\
\hline 13 & .00 & .00 & .10 & .00 & .00 & e. 60 & .05 & .20 & .00 & .00 & .00 & .00 \\
\hline 14 & .00 & .00 & .00 & .05 & .00 & .00 & .00 & .00 & .00 & .00 & .00 & .00 \\
\hline 15 & .00 & .00 & .05 & .10 & e. 15 & e. 55 & .00 & .00 & .00 & .00 & .00 & .00 \\
\hline 16 & .00 & .00 & .00 & .02 & .00 & .00 & .00 & .00 & .00 & .00 & .00 & .00 \\
\hline 17 & .00 & .00 & .00 & .00 & .00 & .00 & .00 & .00 & .00 & .00 & .00 & .00 \\
\hline 18 & .35 & .00 & .00 & .00 & .00 & .00 & .00 & e. 15 & .00 & .00 & .00 & .00 \\
\hline 19 & .10 & .10 & .15 & .00 & .00 & e. 50 & .00 & .00 & .00 & .00 & .00 & .00 \\
\hline 20 & .00 & .03 & .00 & .00 & .00 & e. 55 & .00 & e. 55 & .00 & .00 & .00 & .00 \\
\hline 21 & .00 & .00 & .00 & .00 & .00 & .05 & .00 & .00 & .00 & .00 & .00 & .00 \\
\hline 22 & .00 & .02 & .00 & .00 & .00 & .05 & .20 & .00 & .00 & .00 & .00 & .00 \\
\hline 23 & .00 & .00 & .00 & .00 & .00 & .00 & .02 & .00 & .00 & .00 & .00 & .00 \\
\hline 24 & .00 & .00 & .00 & .00 & .00 & .00 & .04 & .00 & .00 & .00 & .00 & .00 \\
\hline 25 & .00 & .25 & .00 & .00 & .00 & .03 & .14 & .00 & .00 & .00 & .00 & .00 \\
\hline 26 & .00 & .10 & .00 & .00 & .00 & .22 & .00 & .00 & .00 & .00 & .00 & .21 \\
\hline 27 & .00 & .00 & .00 & .00 & e. 35 & .53 & .00 & .00 & .00 & .00 & .00 & .10 \\
\hline 28 & .00 & .00 & .00 & .00 & e. 85 & .02 & .00 & .00 & .00 & .00 & .00 & .00 \\
\hline 29 & .00 & .00 & .00 & .00 & --- & .00 & .00 & .00 & .00 & .00 & .00 & .13 \\
\hline 30 & .00 & .00 & .00 & .00 & -- & .00 & .00 & .00 & .00 & .01 & .00 & .00 \\
\hline 31 & .00 & $-\cdots$ & .00 & .00 & --- & .00 & -- & e. 20 & $\cdots$ & .60 & .80 & -- \\
\hline TOTAL & 0.45 & 0.60 & 0.30 & 0.42 & 1.35 & 4.05 & 0.55 & 1.55 & 0.00 & 1.65 & 1.46 & 1.48 \\
\hline $\begin{array}{l}\text { CAL YR } \\
\text { WTR YR }\end{array}$ & $\begin{array}{l}1990 \\
1991\end{array}$ & TOTAL & & & & & & & & & & \\
\hline
\end{tabular}

e Estimated 
3 SPRINGS BASIN

3 SPRINGS CREEK NEAR WARM SPRINGS, NV

PRECIPITATION WATER QUALITY, WATER YEARS OCTOBER 1984 TO SEPTEMBER 1991

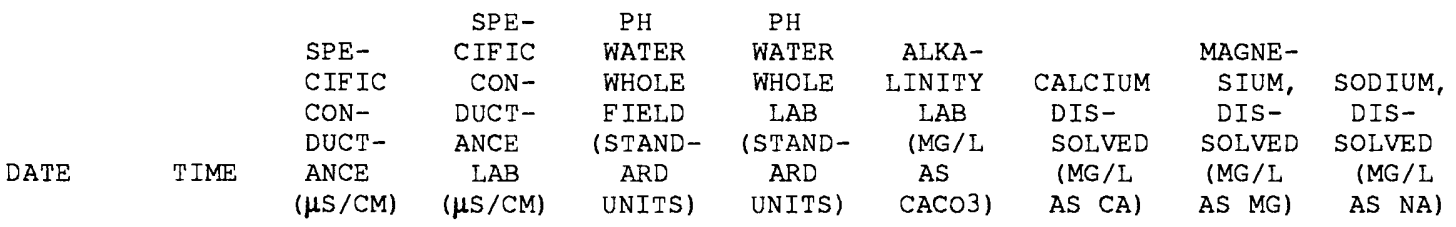

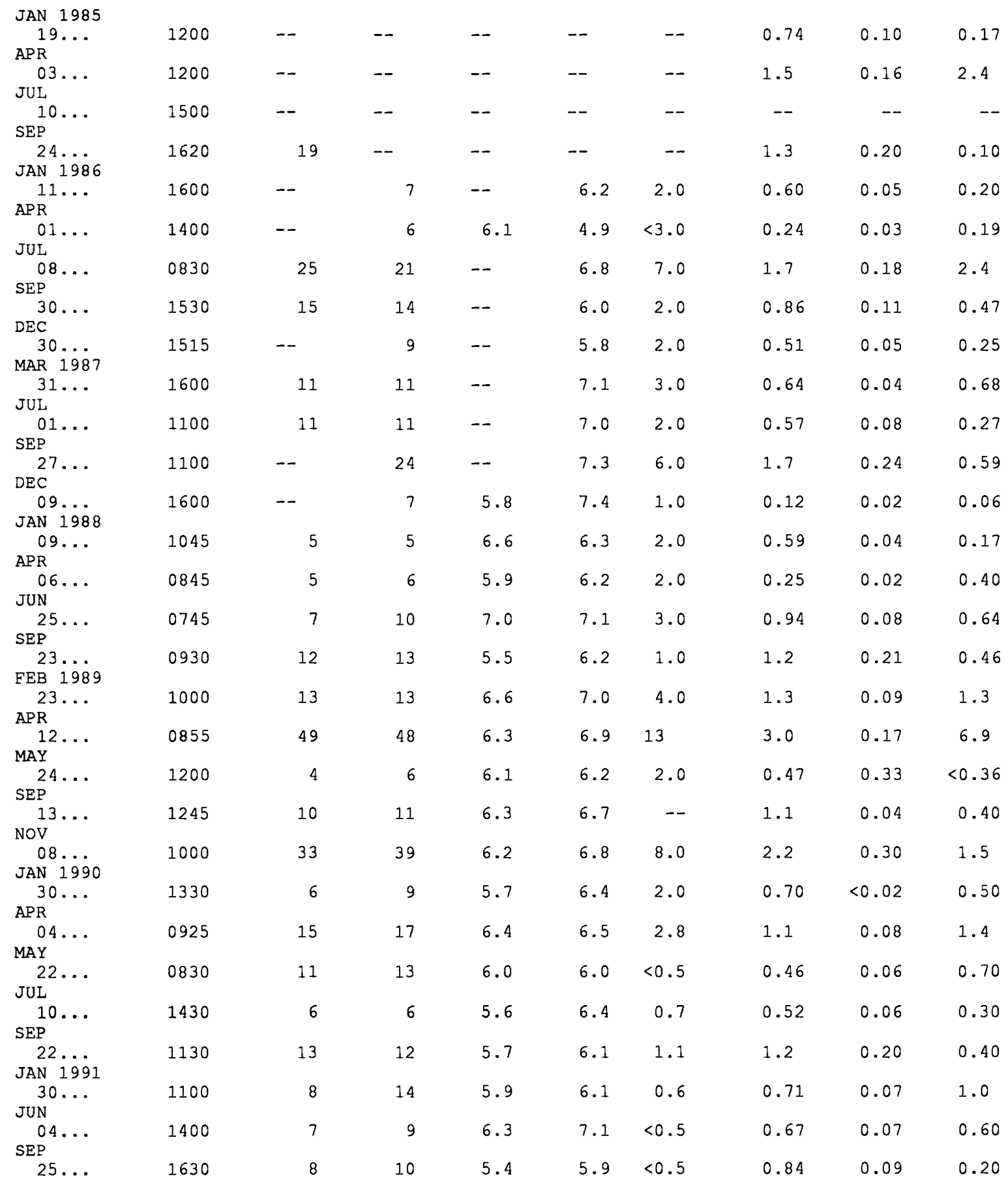


3 SPRINGS BASIN

3 SPRINGS CREEK NEAR WARM SPRINGS, NV

PRECIPITATION WATER QUALITY, WATER YEARS OCTOBER 1984 TO SEPTEMBER 1991

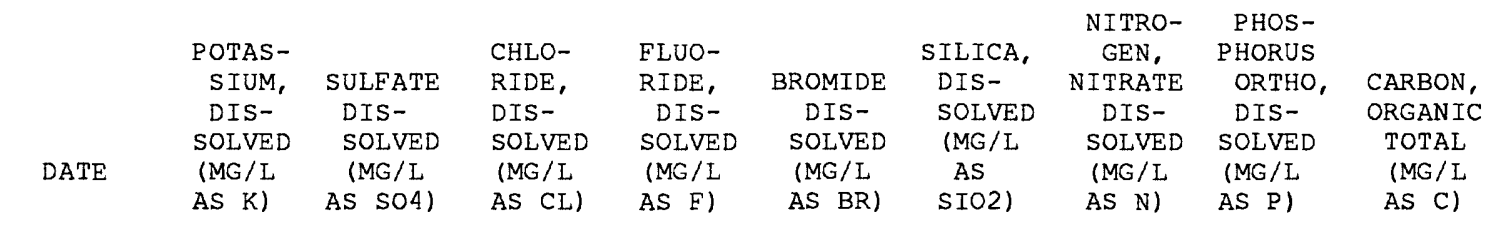

JAN 1985

$19 .$.

APR

$03 \ldots$

$0.08-0.47$

0.02

$0.010<1.0$

JUI $10 \ldots$

SEP

$24 \ldots$

JAN 1986

0.10

1.8

0.68

0.01

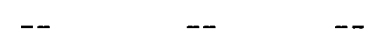

$--\quad 1.2$

0.34

$\begin{array}{ll}-- & 0.61\end{array}$

0.27

.01

$11 \ldots$

0.03

0.45

0.13

0.03

0.009

0.40

$01 \ldots$

0.70

1.4

1.3

0.02

$--$

0.10

$08 \ldots$

SEP $30 \ldots$

DEC

30 ...

MAR 1987

$31 \ldots$

JUL

$01 \ldots$

SEP

$27 \ldots$

0.15

1.2

0.52

0.06

0.98

0.13

0.72

0.21

0.02

0.23

0.82

0.59

0.14

1.0

0.26

0.03

0.01

0.48

2.0

0.67

0.02

0.014

0.10

DEC

$09 \ldots$

JAN 1988

$09 \ldots$

APR

$06 \ldots$

JUN

$25 \ldots$

SEP

$23 \ldots$

FEB 1989

$23 \ldots$

APR

$12 \ldots$

MAY

$24 \ldots$

0.02

0.28

0.11

--

--

0.07

0.27

0.16

-- $\quad 0.021$

0.93

0.39

0.24

0.10

1.1

0.43

0.30

1.7

0.55

0.24

1.3

0.62

$<0.010$

0.54

4.8

3.2

$0.05<0.10$

0.07

0.350

$<0.010$

$0.07<0.010$

0.36

0.220

$<0.010$

0.06

0.51

0.17

$0.01<0.010$

0.07

1.4

0.38

$<0.01<0.010$

0.27

0.040

$<0.010$

NOV

$08 \ldots$

$30 \ldots$

APR

$04 \ldots$

3.1

1.2

0.06

$<0.010$

0.13

1.10

0.730

-- 0.49

0.20

$0.03<0.010$

$<0.02$

0.380

$<0.010$

$2.1 \quad 0.48$

$0.02<0.010$

$<0.01$

0.450

$<0.010$

0.30

0.74

$0.02<0.010$

$<0.01$

0.670

$<0.010$

JUL

$10 \ldots$

0.20

0.50

0.19

$0.02<0.010$

$<0.10$

0.170

$<0.010$

$22 \ldots$

JAN 1991

0.10

0.99

0.25

$0.03<0.010$

0.20

0.490

$<0.010$

0.20

0.98

1.4

$0.02<0.010$

$<0.10$

0.450

$0.03<0.010$

$<0.10$

0.220

$<0.010$

$04 \ldots$

0.10

1.2

0.20

$<0.01<0.010$

0.20

$0.470<0.010$

\footnotetext{
0.010
}

0.20 


\section{SPRINGS BASIN}

3 SPRINGS CREEK NEAR WARM SPRINGS, NV

PRECIPITATION WATER QUALITY, WATER YEARS OCTOBER 1984 TO SEPTEMBER 1991

DATE

$\begin{array}{ccc} & \text { MANGA- } & \text { STRON- } \\ \text { IRON, } & \text { NESE, } & \text { TIUM, } \\ \text { DIS- } & \text { DIS- } & \text { DIS- } \\ \text { SOLVED } & \text { SOLVED } & \text { SOLVED } \\ (\mu G / L & (\mu G / L & (\mu G / L \\ \text { AS FE) } & \text { AS MN }) & \text { AS SR) }\end{array}$

$\begin{array}{ll} & \\ \text { ALUM- } & \\ \text { INUM, } & \\ \text { DIS- } & \\ \text { SOLVED } & \text { TRITIUM } \\ (\mu G / L & \text { TOTAL } \\ \text { AS AL) } & (P C I / L)\end{array}$

$\begin{array}{ccc} & \text { H-2 } / & 0-18 / \\ \text { TRITIUM } & \text { H-1 } & \text { O-16 } \\ 2 \text { SIGMA } & \text { STABLE } & \text { STABLE } \\ \text { WATER, } & \text { ISOTOPE } & \text { ISOTOPE } \\ \text { WHOLE, } & \text { RATIO } & \text { RATIO } \\ \text { TOTAL } & \text { PER } & \text { PER } \\ (\mathrm{PCI} / \mathrm{L}) & \text { MIL } & \text { MIL }\end{array}$

JAN 1985

$19 \ldots$

APR

$03 \ldots$

JUL

$10 \ldots$

SEP

$24 \ldots$

JAN 1986

$11 \ldots$

APR

$01 \ldots$

JUL

$08 \ldots$

SEP

$30 \ldots$

DEC

$30 \ldots$

MAR 1987

$31 \ldots$

JUL

$01 \ldots$

SEP

$27 \ldots$

DEC

$09 \ldots$

JAN 1988

$09 . .$.

APR

$06 .$.

JUN

$25 \ldots$

SEP

$23 \ldots$

FEB 1989

$23 \ldots$

APR

$12 \ldots$

MAY

$24 \ldots$

SEP

$13 \ldots$

NOV

$08 \ldots$

JAN 1990

$30 \ldots$

APR

$04 \ldots$.

MAY

$22 \ldots$

JUL

$10 \ldots$

SEP

$22 \ldots$

JAN 1991

$30 . .$.

JUN

$04 \ldots$.

SEP

$25 \ldots$

\begin{tabular}{|c|c|c|c|c|c|c|c|}
\hline-- & -- & -- & -- & -- & -- & -115.0 & -16.00 \\
\hline-- & -- & -- & -- & 43 & 7.0 & -113.5 & -15.79 \\
\hline-- & -- & -- & -- & -- & -- & -- & -- \\
\hline 14 & 2 & 8 & -- & -- & -- & -69.5 & -10.60 \\
\hline 4 & 17 & 6 & -- & -- & -- & -109.0 & $-14 \cdot 30$ \\
\hline$<10$ & -- & -- & -- & -- & -- & -92.0 & -13.10 \\
\hline 18 & -- & 17 & -- & -- & -- & -61.0 & -9.05 \\
\hline$<10$ & -- & 70 & -- & -- & -- & -70.0 & -9.75 \\
\hline 6 & -- & 4 & -- & -- & -- & -103.5 & -14.85 \\
\hline$<3$ & -- & 3 & -- & 56 & 6.4 & -124.0 & -16.80 \\
\hline$<3$ & -- & 4 & -- & 71 & 7.0 & -68.9 & -9.75 \\
\hline$<3$ & -- & 12 & -- & -- & -- & -74.5 & -10.05 \\
\hline 3 & -- & $<1$ & -- & -- & -- & -105.5 & -15.00 \\
\hline 5 & -- & 4 & -- & 69 & 9.0 & -163.5 & -21.85 \\
\hline 4 & 2 & 1 & -- & 37 & 4.0 & -137.0 & -18.15 \\
\hline 5 & -- & 6 & -- & 45 & 5.0 & -87.4 & -12.20 \\
\hline 12 & 20 & 7 & $<10$ & 60 & 7.0 & -60.4 & -8.55 \\
\hline 6 & 13 & $<10$ & -- & 27 & 3.0 & -111.5 & -15.25 \\
\hline$<3$ & 5 & 90 & -- & -- & -- & -138.0 & -18.10 \\
\hline 5 & -- & 4 & -- & 34 & 3.0 & -136.5 & -18.30 \\
\hline$<3$ & $<1$ & -- & -- & -- & -- & -66.0 & -9.25 \\
\hline$<3$ & 24 & -- & -- & -- & -- & -78.4 & -10.15 \\
\hline$<3$ & 5 & -- & -- & 21 & 3.0 & -105.0 & -14.75 \\
\hline 2 & 5 & -- & -- & 27 & 3.0 & -122.0 & -16.70 \\
\hline 3 & 6 & -- & -- & -- & -- & -77.5 & -10.70 \\
\hline$<3$ & 4 & -- & -- & -- & -- & -132.0 & -17.05 \\
\hline 2 & 13 & -- & -- & -- & -- & -65.0 & -9.45 \\
\hline$<3$ & 6 & -- & -- & -- & -- & -112.0 & -15.80 \\
\hline 4 & 6 & -- & -- & 34 & 2.0 & -127.0 & -17.30 \\
\hline 5 & 7 & -- & -- & -- & -- & -58.5 & -8.65 \\
\hline
\end{tabular}


3 SPRINGS BASIN

3 SPRINGS CREEK NEAR WARM SPRINGS, NV

WATER-QUALITY ANALYSIS FROM THE UNSATURATED-ZONE LYSIMETER, WATER YEARS OCTOBER 1985 TO SEPTEMBER 1991

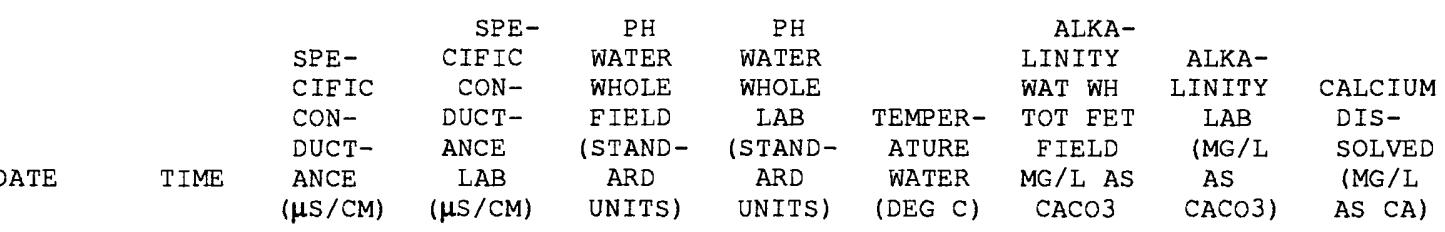

\begin{tabular}{|c|c|c|c|c|c|c|c|c|c|}
\hline $\begin{array}{l}\text { JUL } 1986 \\
08 \ldots \\
\text { OCT }\end{array}$ & 1400 & 66 & 64 & 6.8 & 7.1 & -- & 20 & 20 & 6.0 \\
\hline $\begin{array}{l}04 \\
\text { JUL } 1987\end{array}$ & ${ }^{1} 1200$ & -- & -- & -- & -- & -- & -- & -- & -- \\
\hline $\begin{array}{l}01 \ldots \\
\text { SEP }\end{array}$ & 1215 & 88 & 82 & 6.4 & 6.7 & 21.0 & 22 & 19 & 7.3 \\
\hline${ }_{D E C}^{30} \ldots$ & ${ }^{1} 1130$ & -- & -- & -- & -- & -- & -- & -- & -- \\
\hline $\begin{array}{l}09 \\
\text { APR }\end{array}$ & 1500 & 85 & 82 & 6.5 & 7.7 & 4.5 & -- & 24 & 8.6 \\
\hline$\underset{\text { JUN }}{06 \ldots}$ & 1300 & 55 & 67 & 6.8 & 6.9 & 10.5 & 24 & 26 & 5.1 \\
\hline$\stackrel{24 \ldots}{\mathrm{SEP}}$ & 1045 & 76 & 82 & 7.0 & 7.2 & 18.0 & -- & 25 & 6.3 \\
\hline FEB 19389 & 1015 & -- & -- & -- & -- & -- & -- & -- & -- \\
\hline $\begin{array}{c}23 \ldots \\
\operatorname{APR}\end{array}$ & 1300 & -- & -- & -- & -- & -- & -- & -- & -- \\
\hline $\operatorname{sEP}^{12 \cdots}$ & 1310 & -- & -- & -- & -- & -- & -- & -- & -- \\
\hline $\begin{array}{l}13 \ldots \\
\text { MAY }\end{array}$ & 1530 & -- & -- & -- & -- & -- & -- & -- & -- \\
\hline $\begin{array}{l}22 \ldots \\
\text { JUL } 1990\end{array}$ & ${ }^{1} 1000$ & -- & -- & -- & -- & -- & -- & -- & -- \\
\hline SEP & & & & & & & & & \\
\hline $\begin{array}{l}23 \\
\text { JUN } 1991\end{array}$ & ${ }^{1} 1500$ & -- & -- & -- & -- & -- & -- & -- & -- \\
\hline $\begin{array}{l}05 \ldots \\
\operatorname{SEP}\end{array}$ & 0800 & 61 & 61 & 7.0 & 7.1 & 14.5 & 17 & 15 & 6.4 \\
\hline $25 \ldots$ & 1650 & -- & -- & -- & -- & -- & -- & -- & -- \\
\hline
\end{tabular}


3 SPRINGS BASIN

3 SPRINGS CREEK NEAR WARM SPRINGS, NV

WATER-QUALITY ANALYSIS FROM THE UNSATURATED-ZONE LYSIMETER, WATER YEARS OCTOBER 1985 TO SEPTEMBER 1991

\begin{tabular}{|c|c|c|c|c|c|c|c|c|c|}
\hline$T E$ & $\begin{array}{l}\text { MAGNE- } \\
\text { SIUM, } \\
\text { DIS- } \\
\text { SOLVED } \\
\text { (MG/I }\end{array}$ & $\begin{array}{l}\text { SODIUM, } \\
\text { DIS- } \\
\text { SOLVED } \\
\text { (MG/I }\end{array}$ & $\begin{array}{l}\text { POTAS- } \\
\text { SIUM, } \\
\text { DIS- } \\
\text { SOIVED } \\
\text { (MG/L }\end{array}$ & $\begin{array}{l}\text { SULFATE } \\
\text { DIS- } \\
\text { SOLVED } \\
\text { (MG/L }\end{array}$ & $\begin{array}{l}\text { CHLO- } \\
\text { RIDE, } \\
\text { DIS- } \\
\text { SOLVED } \\
\text { (MG/I }\end{array}$ & $\begin{array}{l}\text { FIUO- } \\
\text { RIDE, } \\
\text { DIS- } \\
\text { SOLVED } \\
(M G / L\end{array}$ & $\begin{array}{c}\text { BROMIDE } \\
\text { DIS- } \\
\text { SOLVED } \\
\text { (MG/L }\end{array}$ & $\begin{array}{l}\text { SILICA, } \\
\text { DIS- } \\
\text { SOLVED } \\
\text { (MG/L } \\
\text { AS }\end{array}$ & $\begin{array}{c}\text { NITRO- } \\
\text { GEN, } \\
\text { NITRATE } \\
\text { DIS- } \\
\text { SOLVED } \\
\text { (MG/I }\end{array}$ \\
\hline & AS MG) & AS NA) & AS K) & AS SO4) & AS CL) & AS F) & AS BR) & SIO2) & AS $N$ ) \\
\hline
\end{tabular}

\begin{tabular}{|c|c|c|c|c|c|c|c|c|c|}
\hline $\begin{array}{c}\text { JUL } 1986 \\
08 \ldots\end{array}$ & 1.5 & 3.0 & 2.5 & 4.2 & 1.3 & 0.09 & $<0.010$ & 33 & -- \\
\hline OCT & & & & & & & & & \\
\hline JUL 19487 & -- & -- & -- & -- & -- & -- & -- & -- & -- \\
\hline $\operatorname{seP}_{\mathrm{SEP}} 01 \ldots$ & 1.7 & 3.3 & 1.6 & 6.6 & 3.1 & 0.15 & 0.027 & 34 & -- \\
\hline${ }_{\mathrm{DEC}}^{30}$ & -- & -- & -- & -- & -- & -- & -- & -- & -- \\
\hline APR 19988 & 1.7 & 3.0 & 2.9 & 3.5 & 1.3 & -- & 0.015 & 27 & -- \\
\hline$\underset{\text { JUN }}{06 \ldots}$ & 1.3 & 3.0 & 2.1 & 2.7 & 0.53 & -- & -- & 34 & -- \\
\hline $\operatorname{sEP}_{\operatorname{SEP}}^{24}$ & 1.9 & 3.2 & 3.0 & 4.3 & 1.8 & -- & -- & 34 & -- \\
\hline $\begin{array}{l}23 \\
\text { FEB } 1989\end{array}$ & -- & -- & -- & 1.7 & 2.8 & 0.15 & $<0.010$ & -- & -- \\
\hline$\underset{A P R}{23 \ldots}$ & 2.1 & 5.2 & 4.1 & 15 & 2.8 & 0.31 & $<0.010$ & -- & 0.690 \\
\hline $\operatorname{SEP}_{\mathrm{SE}}^{12 \ldots}$ & -- & -- & -- & 1.2 & 3.2 & 0.09 & $<0.010$ & -- & 6.60 \\
\hline$\frac{13}{M A Y} \ldots$ & -- & -- & -- & 1.0 & 1.2 & 0.08 & $<0.010$ & -- & 4.40 \\
\hline JUL 1990 & -- & -- & -- & -- & -- & -- & -- & -- & -- \\
\hline $\operatorname{SEP}_{\operatorname{SEP}}^{10 \ldots}$ & -- & -- & -- & 1.5 & 5.1 & $<0.10$ & -- & -- & 36.0 \\
\hline $\begin{array}{l}23 \ldots \\
\text { JUN } 1991\end{array}$ & -- & -- & -- & -- & -- & -- & -- & -- & -- \\
\hline $\begin{array}{c}05 \ldots \\
\operatorname{SEP}\end{array}$ & 1.4 & 3.0 & 2.1 & 6.0 & 2.1 & 0.13 & 0.030 & 35 & 0.210 \\
\hline $25 \ldots$ & -- & -- & -- & 2.3 & 4.3 & 0.12 & $<0.010$ & -- & 9.20 \\
\hline
\end{tabular}




\section{SPRINGS BASIN}

3 SPRINGS CREEK NEAR WARM SPRINGS, NV

WATER-QUALITY ANALYSIS FROM THE UNSATURATED-ZONE LYSIMETER, WATER YEARS OCTOBER 1985 TO SEPTEMBER 1991

\begin{tabular}{|c|c|c|c|c|c|c|c|}
\hline PHOS- & & & & & & $\mathrm{H}-2$ & $0-18$ \\
\hline PHORUS & & MANGA- & STRON- & & TRITIUM & $\mathrm{H}-1$ & $0-16$ \\
\hline ORTHO, & IRON, & NESE, & TIUM, & & 2 SIGMA & STABLE & STABLE \\
\hline DIS- & DIS- & DIS- & DIS- & & WATER, & ISOTOPE & ISOTOPE \\
\hline SOLVED & SOLVED & SOLVED & SOLVED & TRITIUM & WHOLE, & $\begin{array}{l}\text { RATIO } \\
\text { PER }\end{array}$ & RATIO \\
\hline AS P) & AS $\mathrm{EE}$ ) & AS MN) & AS SR) & $(\mathrm{PCI} / \mathrm{L})$ & $(\mathrm{PCI} / \mathrm{L})$ & MIL & MIL \\
\hline
\end{tabular}

\begin{tabular}{|c|c|c|c|c|c|c|c|c|}
\hline $\begin{array}{l}\text { JUL } 1986 \\
08 \ldots \\
\text { OCT }\end{array}$ & -- & 10 & -- & 41 & -- & -- & -109.5 & $-14 \cdot 20$ \\
\hline $\begin{array}{l}\text { OCT } \\
04 \ldots \\
\text { JUL } 1987\end{array}$ & -- & -- & -- & -- & -- & -- & -- & -- \\
\hline $01 \ldots$ & -- & 130 & -- & 54 & 90 & 9.6 & -107.5 & -13.20 \\
\hline SEP & & & & & & & & \\
\hline${ }_{D E C}^{30} \ldots$ & -- & -- & -- & -- & -- & -- & -- & -- \\
\hline $\begin{array}{l}09 \\
\text { APR } 1988\end{array}$ & -- & 6 & -- & 55 & 36 & 3.0 & -108.5 & -14.30 \\
\hline $\begin{array}{l}06 \ldots \\
\text { JUN }\end{array}$ & -- & $<3$ & -- & 48 & 36 & 3.0 & -112.5 & -14.55 \\
\hline$\stackrel{24 \ldots}{\mathrm{SEP}}$ & -- & 6 & -- & 55 & 46 & 4.0 & -105.5 & -13.45 \\
\hline $\begin{array}{l}23 \ldots \\
\mathrm{FEB}\end{array}$ & 0.190 & -- & -- & -- & -- & -- & -- & -- \\
\hline $\begin{array}{c}23 \cdots \\
A P R\end{array}$ & 0.470 & -- & -- & -- & -- & -- & -89.5 & -9.90 \\
\hline $\operatorname{SEP}^{12} \cdots$ & 0.210 & -- & -- & -- & -- & -- & -- & -- \\
\hline${ }_{\text {MAY }}^{13} \ldots$ & 0.590 & -- & -- & -- & -- & -- & -109.0 & -13.75 \\
\hline JUL 22 i990 & -- & -- & -- & -- & -- & -- & -- & -- \\
\hline $\operatorname{SEP}^{10 \ldots}$ & -- & -- & -- & -- & -- & -- & -98.0 & -12.55 \\
\hline$\stackrel{23}{23}{ }_{\text {JUN }} 199$ & -- & -- & -- & -- & -- & -- & -- & -- \\
\hline $\begin{array}{l}05 \ldots \\
\text { SEP }\end{array}$ & 0.080 & 5 & 12 & -- & -- & -- & -113.0 & -14.05 \\
\hline $25 \ldots$ & 1.30 & -- & -- & -- & -- & -- & -- & -- \\
\hline
\end{tabular}

1 Sample collector dry. 
3 SPRINGS BASIN

3 SPRINGS CREEK NEAR WARM SPRINGS, NV

DAILY MEAN DISCHARGE, WATER YEAR OCTOBER 1986 TO SEPTEMBER 1987 CUBIC FEET PER SECOND

\begin{tabular}{|c|c|c|c|c|c|c|c|c|c|c|c|c|}
\hline DAY & OCT & NOV & DEC & JAN & $\mathrm{FEB}$ & MAR & APR & MAY & JUN & JUL & AUG & SEP \\
\hline 1 & --- & --- & --- & --- & -- & .00 & e. 05 & e. 14 & e. 14 & .00 & .00 & .00 \\
\hline 2 & --- & --- & --- & --- & --- & .00 & e.05 & e. 14 & e. 13 & .00 & .00 & .00 \\
\hline 3 & --- & --- & -- & --- & --- & .00 & e.05 & e. 13 & e. 12 & .00 & .00 & .00 \\
\hline 4 & --- & --- & --- & --- & -- & .00 & e.05 & e. 12 & e. 12 & .00 & .00 & .00 \\
\hline 5 & --- & -- & --- & --- & --- & .00 & e. 05 & e. 12 & e. 13 & .00 & .00 & .00 \\
\hline 6 & --- & --- & --- & --- & --- & .00 & e.05 & e. 12 & e.14 & .00 & .00 & .00 \\
\hline 7 & --- & --- & --- & --- & --- & .00 & e.05 & e. 12 & e. 17 & .00 & .00 & .00 \\
\hline 8 & --- & --- & -- & --- & --- & .00 & e.05 & e. 12 & e. 17 & .00 & .00 & .00 \\
\hline 9 & --- & --- & -- & --- & --- & .00 & e.05 & e. 10 & e. 17 & .00 & .00 & .00 \\
\hline 10 & --- & --- & --- & --- & --- & .00 & e.05 & e. 10 & e. 16 & .00 & .00 & .00 \\
\hline 11 & --- & --- & --- & --- & --- & .00 & e.05 & e. 10 & e. 12 & .00 & .00 & .00 \\
\hline 12 & --- & --- & --- & --- & --- & .00 & e.05 & e. 11 & e.09 & .00 & .00 & .00 \\
\hline 13 & --- & --- & --- & --- & --- & .00 & e.05 & e.11 & e.08 & .00 & .00 & .00 \\
\hline 14 & --- & --- & --- & --- & --- & .00 & e. 05 & e.11 & e.07 & .00 & .00 & .00 \\
\hline 15 & --- & --- & --- & --- & --- & e.01 & e.05 & e. 11 & e.06 & .00 & .00 & .00 \\
\hline 16 & --- & --- & --- & --- & --- & e. 01 & e.05 & e.11 & e.06 & .00 & .00 & .00 \\
\hline 17 & --- & --- & --- & --- & -- & e.01 & e.05 & e. 12 & e.05 & .00 & .00 & .00 \\
\hline 18 & --- & --- & --- & --- & --- & e. 01 & e.05 & e. 12 & e.03 & .00 & .00 & .00 \\
\hline 19 & --- & --- & --- & --- & --- & e.02 & e.05 & e.12 & e.03 & .00 & .00 & .00 \\
\hline 20 & --- & --- & --- & --- & --- & e. 02 & e.05 & e.11 & e.02 & .00 & .00 & .00 \\
\hline 21 & -- & --- & --- & --- & -- & e.02 & e.05 & e. 10 & e. 02 & .00 & .00 & .00 \\
\hline 22 & --- & --- & --- & --- & --- & e. 02 & e.05 & e.09 & e. 02 & .00 & .00 & .00 \\
\hline 23 & --- & --- & --- & --- & --- & e.03 & e.05 & e. 08 & e.01 & .00 & .00 & .00 \\
\hline 24 & --- & --- & --- & --- & --- & e. 03 & e.05 & e. 08 & e.01 & .00 & .00 & .00 \\
\hline 25 & --- & --- & --- & --- & --- & e. 03 & e.05 & e. 09 & .00 & .00 & .00 & .00 \\
\hline 26 & --- & --- & --- & -- & --- & e. 04 & e.05 & e. 12 & .00 & .00 & .00 & .00 \\
\hline 27 & --- & -- & --- & --- & --- & e. 04 & e.05 & e. 14 & .00 & .00 & .00 & .00 \\
\hline 28 & --- & --- & --- & -- & --- & e. 04 & e.05 & e. 14 & .00 & .00 & .00 & .00 \\
\hline 29 & --- & -- & --- & --- & --- & e. 04 & e.05 & e.12 & .00 & .00 & .00 & .00 \\
\hline 30 & --- & -- & --- & --- & --- & e. 04 & e. 10 & e.12 & .00 & .00 & .00 & .00 \\
\hline 31 & --- & --- & --- & --- & --- & e.05 & --- & e.14 & --- & .00 & .00 & --- \\
\hline TOTAL & --- & --- & --- & --- & --- & 0.46 & 1.55 & 3.55 & 2.12 & 0.00 & 0.00 & 0.00 \\
\hline MEAN & --- & --- & --- & --- & --- & .015 & .052 & .11 & .071 & .000 & .000 & .000 \\
\hline $\operatorname{MAX}$ & --- & --- & --- & --- & --- & .05 & .10 & .14 & .17 & .00 & .00 & .00 \\
\hline MIN & --- & --- & -- & --- & --- & .00 & .05 & .08 & .00 & .00 & .00 & .00 \\
\hline $\mathrm{AC}-\mathrm{FT}$ & --- & --- & --- & --- & --- & .9 & 3.1 & 7.0 & 4.2 & .00 & .00 & .00 \\
\hline WTR YR & 987 & OTAL & $8 \quad$ MI & .063 & $x .17$ & MIN .00 & $A C-F T$ & & & & & \\
\hline
\end{tabular}

e Estimated 
3 SPRINGS BASIN

3 SPRINGS CREEK NEAR WARM SPRINGS, NV

DAILY MEAN DISCHARGE, WATER YEAR OCTOBER 1987 TO SEPTEMBER 1988 CUBIC FEET PER SECOND

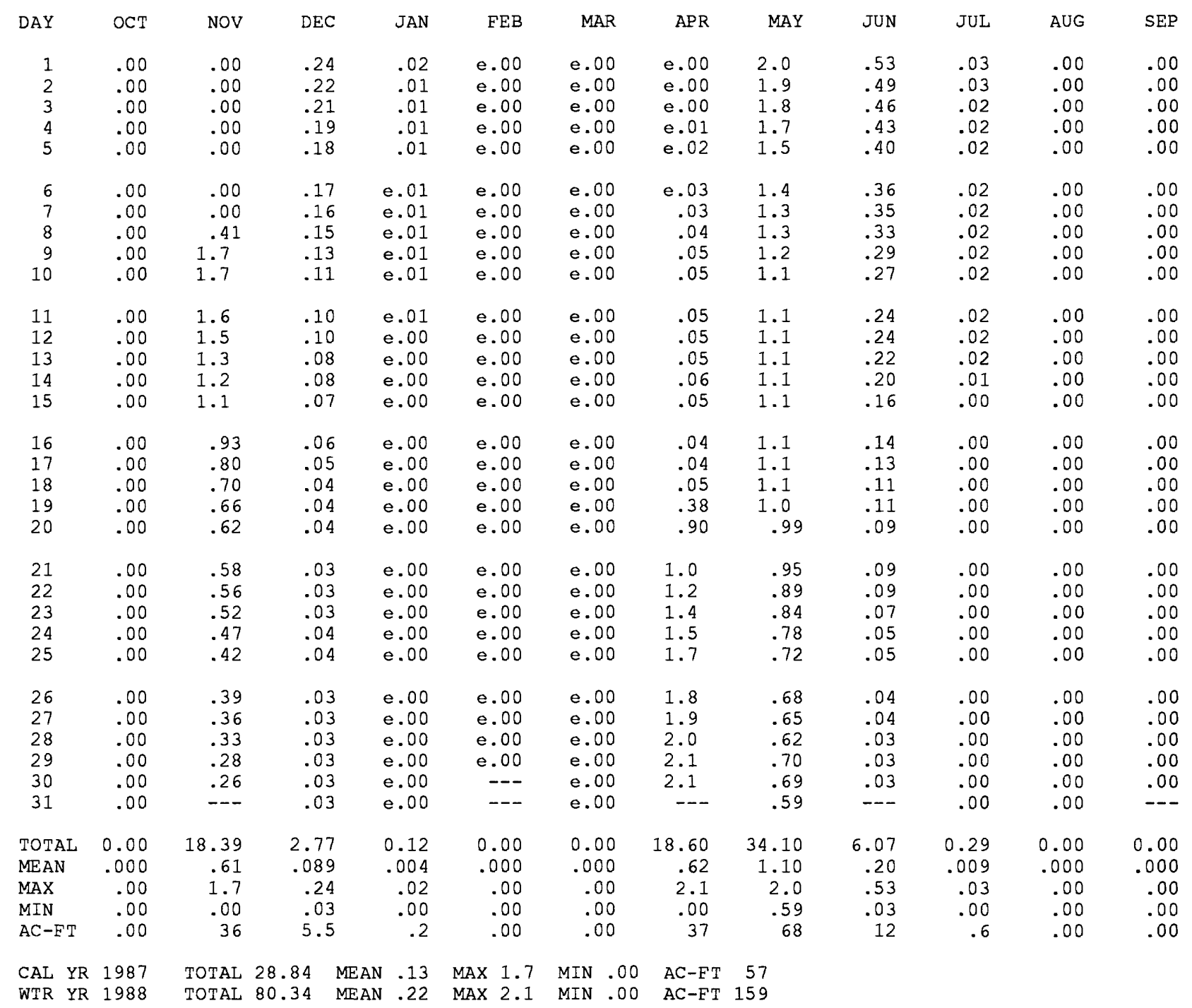

e Estimated 


\section{SPRINGS BASIN}

3 SPRINGS CREEK NEAR WARM SPRINGS, NV

DAILY MEAN DISCHARGE, WATER YEAR OCTOBER 1988 TO SEPTEMBER 1989 CUBIC FEET PER SECOND

\begin{tabular}{|c|c|c|c|c|c|c|c|c|c|c|c|c|}
\hline DAY & OCT & NOV & $\mathrm{DEC}$ & JAN & FEB & MAR & APR & MAY & JUN & JUL & AUG & SEP \\
\hline 1 & .000 & .000 & .000 & .000 & .000 & .000 & .008 & e. 001 & .000 & .000 & .000 & .000 \\
\hline 2 & .000 & .000 & .000 & .000 & .000 & .000 & .008 & e. 001 & .000 & .000 & .000 & .000 \\
\hline 3 & .000 & .000 & .000 & .000 & .000 & .000 & .008 & e.001 & .000 & .000 & .000 & .000 \\
\hline 4 & .000 & .000 & .000 & .000 & .000 & .000 & .008 & e. 001 & .000 & .000 & .000 & .000 \\
\hline 5 & .000 & .000 & .000 & .000 & .000 & .000 & .008 & e. 001 & .000 & .000 & .000 & .000 \\
\hline 6 & .000 & .000 & .000 & .000 & .000 & .000 & .008 & e. 001 & .000 & .000 & .000 & .000 \\
\hline 7 & .000 & .000 & .000 & .000 & .000 & .000 & .008 & e. 001 & .000 & .000 & .000 & .000 \\
\hline 8 & .000 & .000 & .000 & .000 & .000 & .000 & .008 & e. 001 & .000 & .000 & .000 & .000 \\
\hline 9 & .000 & .000 & .000 & .000 & .000 & .000 & .008 & e.001 & .000 & .000 & .000 & .000 \\
\hline 10 & .000 & .000 & .000 & .000 & .000 & .000 & .008 & e. 001 & .000 & .000 & .000 & .000 \\
\hline 11 & .000 & .000 & .000 & .000 & .000 & .000 & .008 & e. 001 & .000 & .000 & .000 & .000 \\
\hline 12 & .000 & .000 & .000 & .000 & .000 & .000 & .006 & e.001 & .000 & .000 & .000 & .000 \\
\hline 13 & .000 & .000 & .000 & .000 & .000 & .000 & .004 & e. 001 & .000 & .000 & .000 & .000 \\
\hline 14 & .000 & .000 & .000 & .000 & .000 & .000 & .004 & e. 001 & .000 & .000 & .000 & .000 \\
\hline 15 & .000 & .000 & .000 & .000 & .000 & .004 & .004 & e. 001 & .000 & .000 & .000 & .000 \\
\hline 16 & .000 & .000 & .000 & .000 & .000 & .012 & .004 & e. 001 & .000 & .000 & .000 & .000 \\
\hline 17 & .000 & .000 & .000 & .000 & .000 & .012 & .004 & e. 001 & .000 & .000 & .000 & .000 \\
\hline 18 & .000 & .000 & .000 & .000 & .000 & .012 & .004 & e. 001 & .000 & .000 & .000 & .000 \\
\hline 19 & .000 & .000 & .000 & .000 & .000 & .014 & .004 & e. 001 & .000 & .000 & .000 & .000 \\
\hline 20 & .000 & .000 & .000 & .000 & .000 & .012 & .004 & e. 001 & .000 & .000 & .000 & .000 \\
\hline 21 & .000 & .000 & .000 & .000 & .000 & .010 & .004 & e. 001 & .000 & .000 & .000 & .000 \\
\hline 22 & .000 & .000 & .000 & .000 & .000 & .010 & .004 & e. 001 & .000 & .000 & .000 & .000 \\
\hline 23 & .000 & .000 & .000 & .000 & .000 & .012 & .004 & e. 001 & .000 & .000 & .000 & .000 \\
\hline 24 & .000 & .000 & .000 & .000 & .000 & .012 & .004 & e. 001 & .000 & .000 & .000 & .000 \\
\hline 25 & .000 & .000 & .000 & .000 & .000 & .012 & .004 & .000 & .000 & .000 & .000 & .000 \\
\hline 26 & .000 & .000 & .000 & .000 & .000 & .010 & .004 & .000 & .000 & .000 & .000 & .000 \\
\hline 27 & .000 & .000 & .000 & .000 & .000 & .008 & e.001 & .000 & .000 & .000 & .000 & .000 \\
\hline 28 & .000 & .000 & .000 & .000 & .000 & .008 & e. 001 & .000 & .000 & .000 & .000 & .000 \\
\hline 29 & .000 & .000 & .000 & .000 & --- & .008 & e. 001 & .000 & .000 & .000 & .000 & .000 \\
\hline 30 & .000 & .000 & .000 & .000 & --- & .008 & e.001 & .000 & .000 & .000 & .000 & .000 \\
\hline 31 & .000 & --- & .000 & .000 & --- & .008 & --- & .000 & --- & .000 & .000 & --- \\
\hline TOTAL & 0.000 & 0.000 & 0.000 & 0.000 & 0.000 & 0.172 & 0.154 & 0.024 & 0.000 & 0.000 & 0.000 & 0.000 \\
\hline MEAN & .000 & .000 & .000 & .000 & .000 & .006 & .005 & .001 & .000 & .000 & .000 & .000 \\
\hline MAX & .000 & .000 & .000 & .000 & .000 & .014 & .008 & .001 & .000 & .000 & .000 & .000 \\
\hline MIN & .000 & .000 & .000 & .000 & .000 & .000 & .001 & .000 & .000 & .000 & .000 & .000 \\
\hline $\mathrm{AC}-\mathrm{ET}$ & .00 & .00 & .00 & .00 & .00 & .3 & .3 & .05 & .00 & .00 & .00 & .00 \\
\hline
\end{tabular}

$\begin{array}{llllllllllll}\text { WTR YR } 1989 & \text { TOTAL } & 0.350 & \text { MEAN } & .001 & \text { MAX } & .014 & \text { MIN } & .000 & \text { AC-ET } & .7\end{array}$

e Estimated 
3 SPRINGS BASIN

3 SPRINGS CREEK NEAR WARM SPRINGS, NV

DAILY MEAN DISCHARGE, WATER YEAR OCTOBER 1989 TO SEPTEMBER 1990 CUBIC FEET PER SECOND

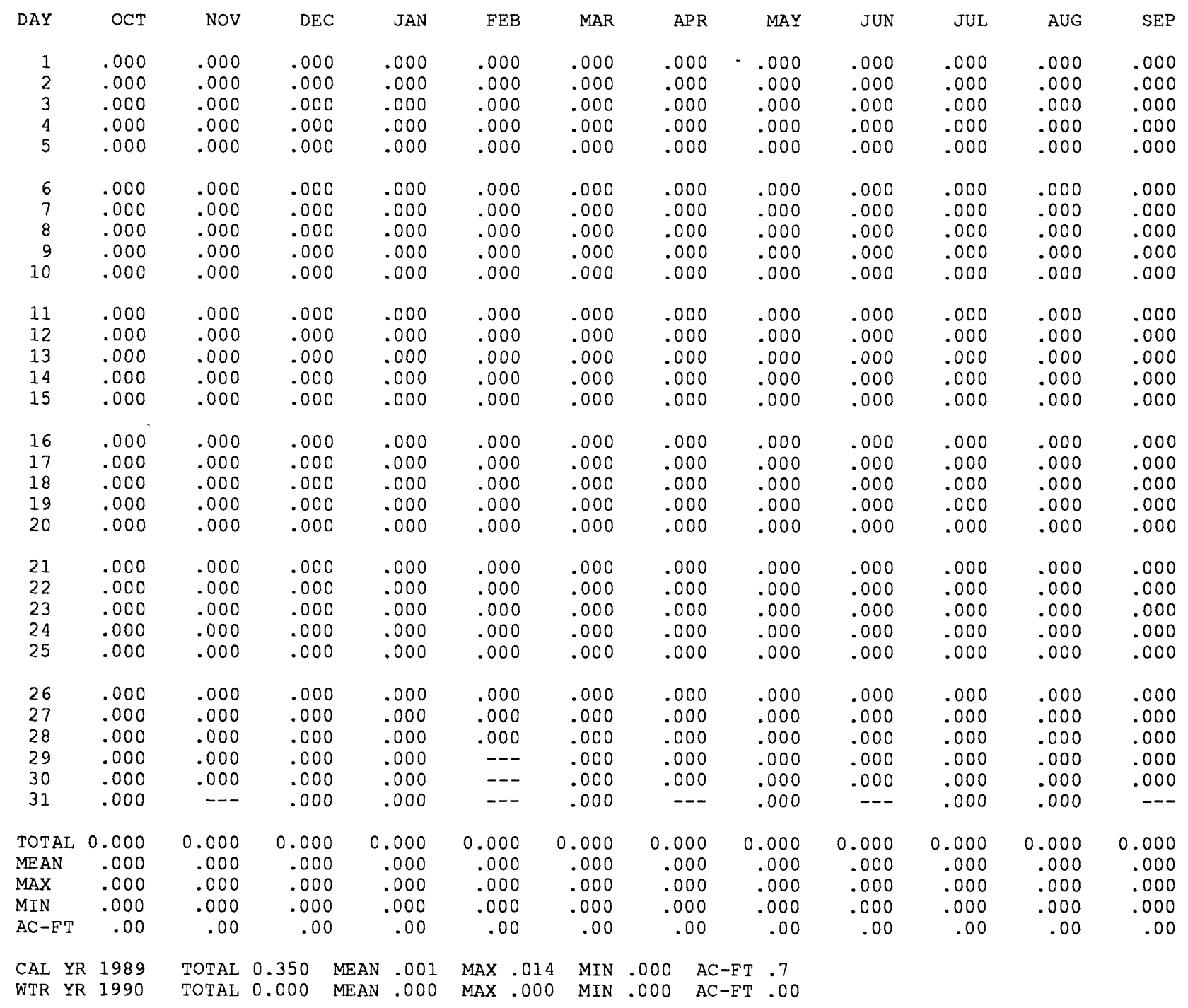


3 SPRINGS BASIN

3 SPRINGS CREEK NEAR WARM SPRINGS, NV

DAILY MEAN DISCHARGE, WATER YEAR OCTOBER 1990 TO SEPTEMBER 1991 CUBIC FEET PER SECOND

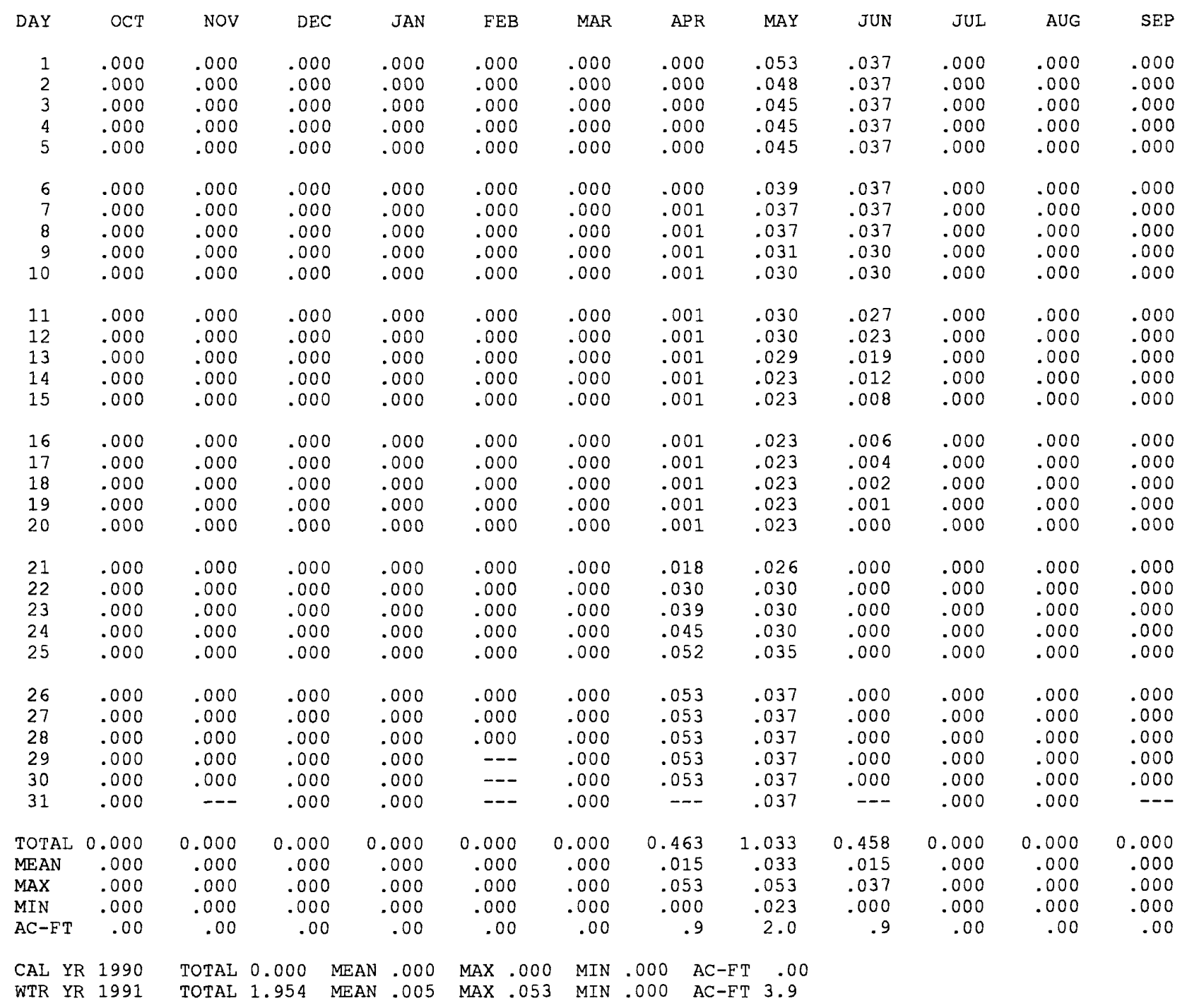


3 SPRINGS BASIN

3 SPRINGS CREEK NEAR WARM SPRINGS, NV

SURFACE-WATER QUALITY, WATER YEARS OCTOBER 1984 TO SEPTEMBER 1991

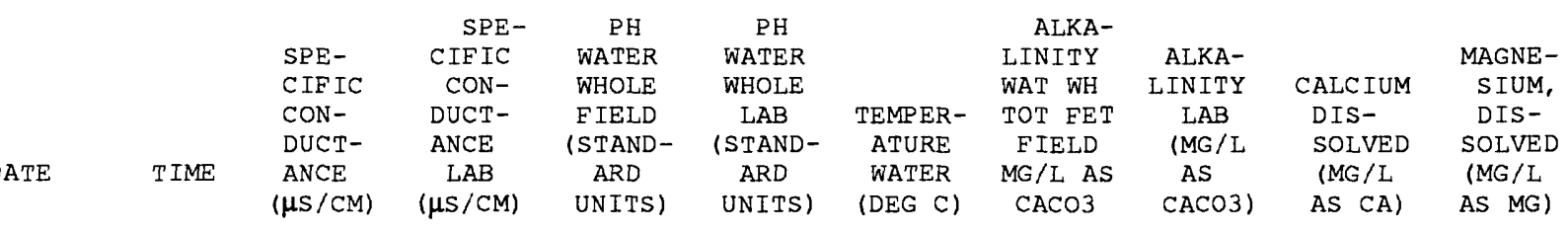

\begin{tabular}{|c|c|c|c|c|c|c|c|c|c|c|}
\hline $\begin{array}{c}\text { APR } 1985 \\
03 \ldots \\
\text { MAR } 1986\end{array}$ & -- & -- & -- & -- & -- & -- & 38 & -- & $>5.0$ & 1.5 \\
\hline $04 \ldots$ & 1500 & -- & 97 & 7.1 & 7.6 & 7.0 & 34 & 33 & 7.6 & 1.6 \\
\hline $20 \ldots$ & 1317 & 95 & 95 & 7.2 & 7.9 & 8.0 & 34 & 36 & 8.0 & 1.5 \\
\hline APR & & & & & & & & & & \\
\hline $\begin{array}{l}01 \\
\text { MAR } 1987\end{array}$ & 1510 & 91 & 91 & 7.1 & 7.6 & 7.0 & 32 & 34 & 7.5 & 1.4 \\
\hline$\underset{M A Y}{31 \ldots}$ & 1620 & 120 & 111 & 6.7 & 8.2 & 6.5 & 43 & 44 & 9.7 & 1.4 \\
\hline${ }_{\text {JUL }}^{07 \ldots}$ & 0945 & -- & 108 & -- & 7.3 & 3.0 & -- & 42 & 9.3 & 2.5 \\
\hline $\begin{array}{l}01 \ldots \\
\text { Nov }\end{array}$ & 1745 & 130 & 127 & 6.7 & 7.6 & 8.5 & 53 & 54 & 12 & 1.7 \\
\hline $\begin{array}{c}18 \ldots \\
\mathrm{DEC}\end{array}$ & 1600 & -- & 126 & -- & 8.3 & 7.5 & -- & 53 & 9.7 & 1.8 \\
\hline JAN 19988 & 1615 & 142 & 121 & 6.5 & 7.4 & 7.0 & -- & 46 & 10 & 1.6 \\
\hline $\begin{array}{c}09 . . \\
A P R\end{array}$ & 1130 & 128 & 123 & 6.8 & 7.5 & 7.0 & 50 & 50 & 10 & 1.7 \\
\hline$\underset{\text { JUN }}{06 \ldots}$ & 0945 & 101 & 112 & 6.8 & 7.2 & 6.0 & 47 & 48 & 8.7 & 1.4 \\
\hline $\begin{array}{r}25 \ldots \\
\text { APR } 1989\end{array}$ & 1430 & 109 & 118 & 7.0 & 7.5 & 8.5 & -- & 49 & 10 & 1.7 \\
\hline${ }_{M A Y}^{12} \ldots$ & 1000 & 112 & 118 & 6.6 & 7.2 & 8.0 & 49 & 49 & 11 & 1.5 \\
\hline MAY 24990 & 1215 & 122 & 108 & 6.9 & 8.2 & 8.5 & -- & 53 & 11 & 0.52 \\
\hline $\begin{array}{l}22 \\
\text { JUN } 1991\end{array}$ & 1000 & 123 & 124 & 6.8 & 7.4 & 8.0 & 50 & 48 & 11 & 1.5 \\
\hline $06 \ldots$ & 0745 & 118 & 121 & 6.7 & 7.2 & 7.5 & 46 & 46 & 11 & 1.5 \\
\hline
\end{tabular}


3 SPRINGS BASIN

3 SPRINGS CREEK NEAR WARM SPRINGS, NV SURFACE-WATER QUALITY, WATER YEARS OCTOBER 1984 TO SEPTEMBER 1991

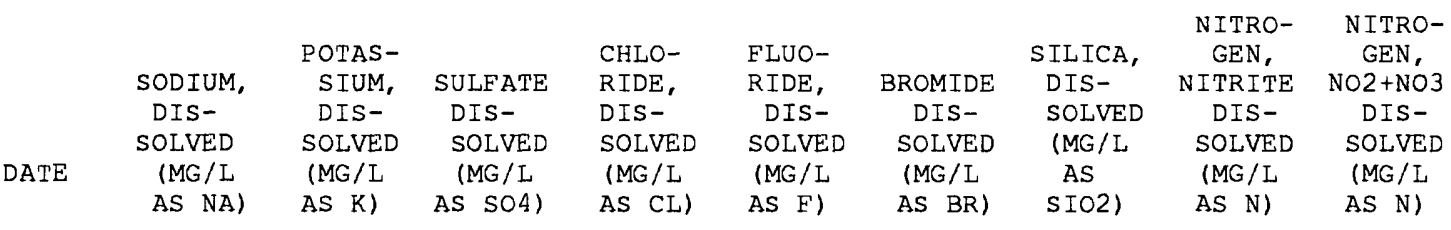

\begin{tabular}{|c|c|c|c|c|c|c|c|c|c|}
\hline $\begin{array}{c}\text { APR } 1985 \\
03 \ldots \\
\text { MAR } 1986\end{array}$ & $>5.0$ & 1.9 & 5.2 & 3.4 & 0.09 & -- & 39 & -- & -- \\
\hline $04 \ldots$ & 8.3 & 2.0 & -- & -- & -- & -- & 38 & -- & -- \\
\hline $20 \ldots$ & 7.0 & 1.6 & 5.7 & 2.8 & 0.11 & 0.039 & 38 & -- & -- \\
\hline APR & & & & & & & & & \\
\hline $\begin{array}{l}01 \\
\text { MAR } 1987\end{array}$ & 7.0 & 1.7 & 5.3 & 2.6 & 0.08 & 0.019 & 35 & -- & -- \\
\hline $\operatorname{MAY}^{31 \ldots}$ & 1.9 & 1.2 & 5.4 & 4.0 & 0.06 & -- & 39 & -- & -- \\
\hline$\underset{\text { JUL }}{07 \ldots}$ & 11 & 1.0 & 5.0 & 3.2 & 0.16 & 0.027 & 38 & -- & -- \\
\hline$\underset{\text { NOV }}{01 \ldots}$ & 12 & 1.2 & 4.8 & 3.4 & 0.11 & 0.038 & 40 & -- & -- \\
\hline${ }_{D E C}^{18 \ldots}$ & 9.7 & 2.1 & 5.8 & 3.5 & -- & 0.038 & 40 & -- & -- \\
\hline JAN 19988 & 10 & 1.3 & 5.8 & 3.5 & -- & 0.035 & 40 & -- & -- \\
\hline$\underset{A P R}{09 \ldots}$ & 9.9 & 1.3 & 5.3 & 3.5 & -- & 0.35 & 42 & -- & -- \\
\hline$\underset{\text { JUN }}{06 \ldots}$ & 11 & 1.0 & 5.4 & 3.3 & -- & 0.035 & 39 & -- & -- \\
\hline $\begin{array}{r}25 \ldots \\
A P R \\
1989\end{array}$ & 11 & 1.3 & 5.0 & 3.0 & -- & -- & 38 & -- & -- \\
\hline$\underset{\text { MAY }}{12 \ldots}$ & 13 & 1.2 & 6.0 & 3.7 & 0.10 & 0.030 & 41 & -- & $<0.100$ \\
\hline MAY 1990 & 13 & 1.5 & 5.0 & 3.8 & 0.10 & 0.030 & 40 & -- & $<0.100$ \\
\hline $\begin{array}{l}22 \ldots \\
\text { JUN } 1991\end{array}$ & 13 & 1.0 & 5.2 & 4.6 & 0.10 & 0.050 & 41 & -- & $<0.100$ \\
\hline $06 \ldots$ & 12 & 0.90 & 7.2 & 4.3 & 0.20 & 0.040 & 40 & $<0.010$ & $<0.050$ \\
\hline
\end{tabular}




\section{SPRINGS BASIN}

3 SPRINGS CREEK NEAR WARM SPRINGS, NV SURFACE-WATER QUALITY, WATER YEARS OCTOBER 1984 TO SEPTEMBER 1991

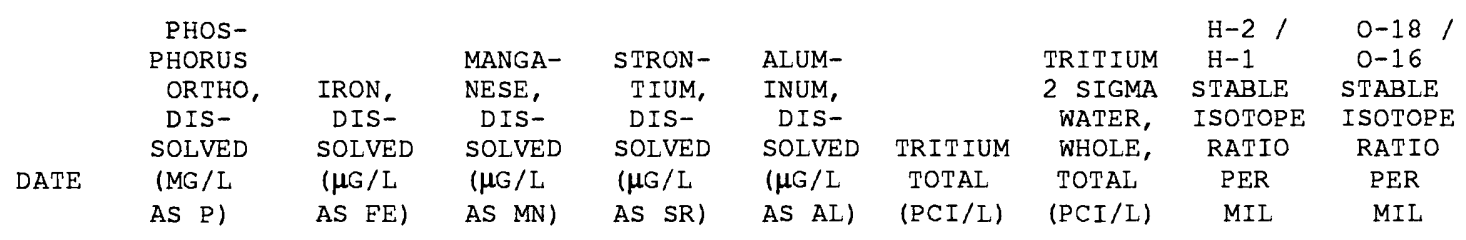

APR 1985

$03 \ldots$

MAR 1986

$04 \ldots$

$20 \ldots$

APR

$01 \ldots$

MAR 1987

$31 \ldots$

MAY

$07 \ldots$

JUL

$01 \ldots$

NoV

$18 \ldots$

DEC

$09 .$.

JAN 1988

$09 . .$.

APR

06 ...

JUN

$25 \ldots$

APR 1989

$12 \ldots$

MAY

$24 \ldots$

MAY 1990

$22 \ldots$

JUN 1991

$06 \ldots$

AS P)

AS FE)

AS MN)

AS SR)

AS AL) (PCI/L)

MIL MIL

$\begin{array}{lrlrrrrrrc}-- & -- & -- & -- & -- & -- & -- & -- & -- \\ -- & 6 & & 4 & - & -- & -- & -- & -- & -- \\ -- & <10 & -- & 260 & -- & -- & -- & -100.0 & -13.20 \\ -- & 3 & -- & 190 & -- & <200 & -- & -100.0 & -13.30 \\ -- & 3 & -- & 10 & -- & -- & -- & -101.5 & -13.70 \\ -- & <10 & -- & 110 & <10 & -- & -- & -- & -- \\ -- & <3 & -- & 110 & -- & -- & -- & -103.5 & -13.80 \\ -- & 3 & 91 & 91 & -- & -- & -- & -102.0 & -13.75 \\ -- & <3 & -- & 98 & -- & 54 & 5.0 & -105.0 & -13.80 \\ -- & <3 & -- & 110 & -- & -- & -- & -103.0 & -13.75 \\ -- & <3 & -- & 110 & -- & -- & -- & -102.0 & -13.85 \\ -- & 6 & -- & 100 & -- & -- & -- & -101.5 & -13.80 \\ -- & <3 & 2 & 90 & -- & -- & -- & -102.5 & -13.80 \\ -- & 4 & -- & 110 & -- & -- & -- & -102.5 & -13.80 \\ -- & 72 & & 1 & -- & -- & -- & -- & -102.0 & -13.70 \\ <0.010 & 7 & 2 & -- & -- & -- & -- & -104.0 & -13.90\end{array}$




\section{RECORDS FROM EAST STEWART BASIN}




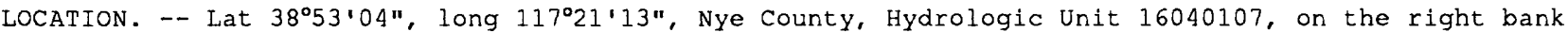
$0.5 \mathrm{mi}$ upstream from the East Stewart Creek surface-water equipment, $1.8 \mathrm{mi}$ upstream from the Columbine campground, $13.8 \mathrm{mi}$ east of Ione on state Route 21 .

PERIOD OF RECORD. -- September 1984 to current year.

REMARKS.-- The weather equipment is located approximately 200 feet southeast and uphill from Veg spring. The two unsaturated-zone water-quality samples are taken approximately 20 feet downhill from the weather equipment. Minimum air temperatures from October 7, 1989 to July 12, 1990 were not published due to intermittent probe failure. The maximum total daily precipitation was not published because the value is estimated. During visits when the unsaturated-zone lysimeter was checked and found empty, the container was cleaned and replaced. Estimated periods of daily record are considered poor. Values for the daily maximum and minimum air temperature were obtained from the 40 minute average values.

PERIOD OF DAILY RECORD. --

AIR TEMPERATURE: October 1988 to current year.

SOIL TEMPERATURE: October 1988 to current year.

SOLAR RADIATION: November 1990 to current year.

RELATIVE HUMIDITY: October 1990 to current year.

TOTAL PRECIPITATION: October 1986 to current year.

EXTREMES FOR PERIOD OF RECORD. --

AIR TEMPERATURE: Maximum daily, $23.7^{\circ} \mathrm{C}$, July 4, 1991; minimum daily, $-27.0^{\circ} \mathrm{C}$, Dec. $22,1990$. See remarks for more information.

SOIL TEMPERATURE: Maximum daily mean, 19.9 $\mathrm{C}$, Aug. 6, 1990; minimum daily mean, $-10.7^{\circ} \mathrm{C}, \mathrm{DeC}^{3} 30$, 1990 .

SOLAR RADIATION: Maximum daily total, $714 \mathrm{cal} / \mathrm{cm}^{2}$, May 15, 1991; minimum daily total, $34 \mathrm{cal} / \mathrm{cm}^{2}$, Jan. 4, 1991.

RELATIVE HUMIDITY: Maximum daily mean, 100 pct, several days of record; minimum daily

mean, 15 pct, Aug. 9, 1991.

TOTAL PRECIPITATION: Maximum daily, not available; no precipitation, most days of record. 
EAST STEWART BASIN

VEG SPRING NEAR IONE, NV

DAILY MEAN AIR TEMPERATURE, WATER YEAR OCTOBER 1988 TO SEPTEMBER 1989 DEGREES CELSIUS

\begin{tabular}{|c|c|c|c|c|c|c|c|c|c|c|c|c|}
\hline DAY & OCT & NOV & $\mathrm{DEC}$ & JAN & FEB & MAR & APR & MAY & JUN & JUL & AUG & SEP \\
\hline $\begin{array}{l}1 \\
2 \\
3 \\
4 \\
5\end{array}$ & $\begin{array}{r}11.3 \\
11.4 \\
10.7 \\
9.7 \\
7.5\end{array}$ & $\begin{array}{l}4.3 \\
1.2 \\
3.2 \\
1.7 \\
6.3\end{array}$ & $\begin{array}{r}3.3 \\
1.2 \\
-.3 \\
.3 \\
.4\end{array}$ & $\begin{array}{r}-10.8 \\
-3.0 \\
-1.1 \\
-3.9 \\
-8.7\end{array}$ & $\begin{array}{r}-5.2 \\
-10.6 \\
-12.6 \\
-19.3 \\
-23.5\end{array}$ & $\begin{array}{r}-2.2 \\
-5.7 \\
-16.4 \\
-5.3 \\
.0\end{array}$ & $\begin{array}{r}-4.5 \\
-2.8 \\
-2.6 \\
.7 \\
6.1\end{array}$ & $\begin{array}{r}3.7 \\
3.4 \\
3.9 \\
6.4 \\
10.2\end{array}$ & $\begin{array}{l}4.9 \\
7.3 \\
2.6 \\
2.4 \\
2.8\end{array}$ & $\begin{array}{l}11.9 \\
14.7 \\
16.4 \\
15.0 \\
16.1\end{array}$ & $\begin{array}{l}13.0 \\
12.2 \\
13.0 \\
13.5 \\
14.3\end{array}$ & $\begin{array}{l}10.7 \\
11.4 \\
11.7 \\
13.1 \\
12.6\end{array}$ \\
\hline $\begin{array}{r}6 \\
7 \\
8 \\
9 \\
10\end{array}$ & $\begin{array}{l}5.5 \\
5.9 \\
7.0 \\
6.8 \\
4.8\end{array}$ & $\begin{array}{r}1.2 \\
1.2 \\
-5.3 \\
-2.8 \\
-2.2\end{array}$ & $\begin{array}{r}-.7 \\
-9.0 \\
-7.2 \\
-2.0 \\
-.5\end{array}$ & $\begin{array}{r}-17.2 \\
-16.9 \\
-12.8 \\
-5.7 \\
-9.6\end{array}$ & $\begin{array}{r}-20.9 \\
-15.8 \\
-7.6 \\
-5.5 \\
-8.7\end{array}$ & $\begin{array}{r}1.2 \\
.5 \\
1.7 \\
2.5 \\
3.0\end{array}$ & $\begin{array}{l}8.2 \\
8.1 \\
6.2 \\
5.6 \\
6.4\end{array}$ & $\begin{array}{r}11.1 \\
10.2 \\
8.5 \\
4.6 \\
-2.6\end{array}$ & $\begin{array}{l}6.0 \\
6.4 \\
6.8 \\
5.5 \\
7.8\end{array}$ & $\begin{array}{l}16.5 \\
17.4 \\
15.7 \\
13.7 \\
12.0\end{array}$ & $\begin{array}{l}14.0 \\
13.2 \\
10.6 \\
10.9 \\
11.0\end{array}$ & $\begin{array}{l}9.6 \\
2.2 \\
5.7 \\
8.4 \\
8.3\end{array}$ \\
\hline $\begin{array}{l}11 \\
12 \\
13 \\
14 \\
15\end{array}$ & $\begin{array}{l}1.8 \\
2.5 \\
4.4 \\
1.1 \\
6.2\end{array}$ & $\begin{array}{r}-5.3 \\
-.2 \\
-.4 \\
-10.2 \\
-10.0\end{array}$ & $\begin{array}{r}1.0 \\
4.3 \\
2.0 \\
-8.3 \\
-14.7\end{array}$ & $\begin{array}{r}-16.5 \\
-8.0 \\
-4.9 \\
-13.1 \\
-10.0\end{array}$ & $\begin{array}{r}-10.8 \\
-12.7 \\
-14.0 \\
-11.4 \\
-5.9\end{array}$ & $\begin{array}{r}1.3 \\
.7 \\
-6.7 \\
-7.4 \\
-1.9\end{array}$ & $\begin{array}{l}1.8 \\
1.6 \\
5.5 \\
7.3 \\
4.4\end{array}$ & $\begin{array}{l}-3.4 \\
-4.3 \\
-4.3 \\
-3.1 \\
-1.7\end{array}$ & $\begin{array}{r}6.6 \\
10.2 \\
12.9 \\
14.5 \\
13.8\end{array}$ & $\begin{array}{l}12.7 \\
10.1 \\
13.2 \\
15.5 \\
14.3\end{array}$ & $\begin{array}{l}12.3 \\
13.3 \\
14.7 \\
14.3 \\
14.6\end{array}$ & $\begin{array}{r}6.4 \\
4.9 \\
8.0 \\
10.1 \\
11.4\end{array}$ \\
\hline $\begin{array}{l}16 \\
17 \\
18 \\
19 \\
20\end{array}$ & $\begin{array}{l}8.4 \\
8.8 \\
9.7 \\
7.6 \\
8.4\end{array}$ & $\begin{array}{r}-6.7 \\
-11.4 \\
-13.4 \\
-7.6 \\
-7.0\end{array}$ & $\begin{array}{l}-9.1 \\
-6.1 \\
-8.1 \\
-9.9 \\
-7.9\end{array}$ & $\begin{array}{r}-4.8 \\
-2.5 \\
.1 \\
-2.4 \\
-1.0\end{array}$ & $\begin{array}{r}-4.8 \\
-.9 \\
-1.9 \\
-7.1 \\
-8.2\end{array}$ & $\begin{array}{l}-5.7 \\
-5.7 \\
-1.8 \\
-5.2 \\
-5.9\end{array}$ & $\begin{array}{l}4.9 \\
7.1 \\
8.6 \\
9.4 \\
8.3\end{array}$ & $\begin{array}{l}1.0 \\
5.8 \\
3.2 \\
2.7 \\
6.9\end{array}$ & $\begin{array}{r}7.9 \\
11.1 \\
15.0 \\
13.9 \\
6.8\end{array}$ & $\begin{array}{l}12.1 \\
13.7 \\
16.7 \\
18.7 \\
16.6\end{array}$ & $\begin{array}{r}14.3 \\
11.8 \\
10.5 \\
10.4 \\
7.8\end{array}$ & $\begin{array}{r}9.1 \\
1.5 \\
.2 \\
1.1 \\
3.9\end{array}$ \\
\hline $\begin{array}{l}21 \\
22 \\
23 \\
24 \\
25\end{array}$ & $\begin{array}{l}9.1 \\
7.5 \\
8.9 \\
7.6 \\
7.5\end{array}$ & $\begin{array}{r}-3.7 \\
-.6 \\
-5.0 \\
-12.8 \\
-9.0\end{array}$ & $\begin{array}{l}-13 \cdot 3 \\
-12.3 \\
-14.5 \\
-10.0 \\
-17.3\end{array}$ & $\begin{array}{l}-1.5 \\
-2.9 \\
-7.8 \\
-9.8 \\
-9.2\end{array}$ & $\begin{array}{r}-2.3 \\
.7 \\
.4 \\
1.9 \\
1.3\end{array}$ & $\begin{array}{r}-.1 \\
-. .7 \\
-1.2 \\
-.1 \\
-5.3\end{array}$ & $\begin{array}{r}5.1 \\
-3.0 \\
-3.3 \\
-9.2 \\
-8.6\end{array}$ & $\begin{array}{r}5.9 \\
8.2 \\
.9 \\
-3.8 \\
-.8\end{array}$ & $\begin{array}{l}4.9 \\
8.9 \\
6.0 \\
5.4 \\
7.4\end{array}$ & $\begin{array}{l}13.7 \\
13.2 \\
12.4 \\
12.7 \\
15.1\end{array}$ & $\begin{array}{r}9.4 \\
11.6 \\
5.1 \\
2.8 \\
6.8\end{array}$ & $\begin{array}{r}6.5 \\
8.4 \\
10.0 \\
11.2 \\
9.6\end{array}$ \\
\hline $\begin{array}{l}26 \\
27 \\
28 \\
29 \\
30 \\
31\end{array}$ & $\begin{array}{l}6.9 \\
6.2 \\
5.4 \\
4.2 \\
4.2 \\
6.4\end{array}$ & $\begin{array}{r}-10.8 \\
-3.0 \\
-4.6 \\
-3.5 \\
-1.3 \\
---\end{array}$ & $\begin{array}{r}-19.0 \\
-13.8 \\
-17.4 \\
-10.1 \\
-5.6 \\
-12.1\end{array}$ & $\begin{array}{r}-5.5 \\
-4.5 \\
-4.2 \\
-1.7 \\
4.1 \\
1.3\end{array}$ & $\begin{array}{r}-1.2 \\
-5.4 \\
-4.5 \\
--- \\
--- \\
---\end{array}$ & $\begin{array}{r}-7.7 \\
-4.0 \\
-1.0 \\
-3.9 \\
-.8 \\
-.6\end{array}$ & $\begin{array}{r}-7.5 \\
-6.8 \\
-5.8 \\
-1.3 \\
2.4 \\
---\end{array}$ & $\begin{array}{r}4.8 \\
6.5 \\
.7 \\
-4.6 \\
-4.4 \\
1.1\end{array}$ & $\begin{array}{r}10.1 \\
7.9 \\
9.3 \\
11.6 \\
9.8 \\
-.-\end{array}$ & $\begin{array}{l}16.7 \\
16.0 \\
16.0 \\
16.4 \\
16.2 \\
15.7\end{array}$ & $\begin{array}{r}9.7 \\
11.3 \\
12.6 \\
13.5 \\
9.2 \\
9.0\end{array}$ & $\begin{array}{r}10.1 \\
9.2 \\
9.1 \\
7.7 \\
1.6 \\
-.-\end{array}$ \\
\hline $\begin{array}{l}\text { MEAN } \\
\text { MAX } \\
\text { MIN } \\
\text { MED }\end{array}$ & $\begin{array}{r}6.9 \\
11.4 \\
1.1 \\
7.0\end{array}$ & $\begin{array}{r}-3.9 \\
6.3 \\
-13.4 \\
-3.6\end{array}$ & $\begin{array}{r}-7.0 \\
4.3 \\
-19.0 \\
-8.1\end{array}$ & $\begin{array}{r}-6.3 \\
4.1 \\
-17.2 \\
-4.9\end{array}$ & $\begin{array}{r}-7.7 \\
1.9 \\
-23.5 \\
-6.5\end{array}$ & $\begin{array}{r}-2.7 \\
3.0 \\
-16.4 \\
-1.8\end{array}$ & $\begin{array}{r}1.7 \\
9.4 \\
-9.2 \\
3.4\end{array}$ & $\begin{array}{r}2.5 \\
11.1 \\
-4.6 \\
3.2\end{array}$ & $\begin{array}{r}8.2 \\
15.0 \\
2.4 \\
7.6\end{array}$ & $\begin{array}{l}14.7 \\
18.7 \\
10.1 \\
15.1\end{array}$ & $\begin{array}{r}11.3 \\
14.7 \\
2.8 \\
11.8\end{array}$ & $\begin{array}{r}7.8 \\
13.1 \\
.2 \\
8.7\end{array}$ \\
\hline
\end{tabular}

WTR YR 1989 MEAN 2.2 MAX 18.7 MIN -23.5 MED 2.6 
EAST STEWART BASIN

VEG SPRING NEAR IONE, NV

DAILY MEAN AIR TEMPERATURE, WATER YEAR OCTOBER 1989 TO SEPTEMBER 1990 DEGREES CELSIUS

\begin{tabular}{|c|c|c|c|c|c|c|c|c|c|c|c|c|}
\hline DAY & OCT & NOV & DEC & JAN & FEB & MAR & APR & MAY & JUN & JUL & AUG & SEP \\
\hline $\begin{array}{l}1 \\
2 \\
3 \\
4 \\
5\end{array}$ & $\begin{array}{r}4.9 \\
-.7 \\
-4.1 \\
-1.6 \\
2.5\end{array}$ & $\begin{array}{r}-.8 \\
-.3 \\
\text { e1. } \\
1.8 \\
-.2\end{array}$ & $\begin{array}{r}-.9 \\
\text { e3. } \\
\text { e3.1 } \\
\text { e2. } 9 \\
.1\end{array}$ & $\begin{array}{r}-4.7 \\
-13.4 \\
-12.2 \\
-9.5 \\
-5.0\end{array}$ & $\begin{array}{r}-12.1 \\
-11.1 \\
e-4.6 \\
-11.1 \\
-7.0\end{array}$ & $\begin{array}{r}e-3.3 \\
e-2.9 \\
e-3.4 \\
e-4.0 \\
-9.3\end{array}$ & $\begin{array}{r}\text { e. } 6 \\
\text { e1. } 1 \\
\text { e1. } 4 \\
\text { e. } 6 \\
\text { e-. } 3\end{array}$ & $\begin{array}{r}e-4.4 \\
e-1.9 \\
e-.5 \\
e 4.7 \\
7.8\end{array}$ & $\begin{array}{r}e-1.9 \\
\text { e6.6 } \\
11.3 \\
11.1 \\
\text { e8.4 }\end{array}$ & $\begin{array}{r}16.0 \\
12.2 \\
\text { e11.3 } \\
\text { e13.5 } \\
\text { e13.4 }\end{array}$ & $\begin{array}{l}12.3 \\
13.9 \\
14.6 \\
15.8 \\
17.3\end{array}$ & $\begin{array}{l}12.7 \\
13.1 \\
12.7 \\
11.8 \\
11.5\end{array}$ \\
\hline $\begin{array}{r}6 \\
7 \\
8 \\
9 \\
10\end{array}$ & $\begin{array}{r}3.5 \\
\mathrm{e} 3.7 \\
7.9 \\
9.3 \\
10.0\end{array}$ & $\begin{array}{r}-2.9 \\
\mathrm{e}-3.6 \\
\mathrm{e} .2 \\
\mathrm{e} 3.7 \\
\mathrm{e} 5.7\end{array}$ & $\begin{array}{r}-3.7 \\
\mathrm{e} .0 \\
\mathrm{e} 1.8 \\
-2.2 \\
-12.0\end{array}$ & $\begin{array}{r}-2.5 \\
-2.1 \\
.3 \\
4.1 \\
\text { e } 4.0\end{array}$ & $\begin{array}{r}-7.1 \\
-15.6 \\
-11.7 \\
e-7.5 \\
e-4.9\end{array}$ & $\begin{array}{l}e-7.6 \\
e-1.7 \\
e-2.6 \\
e-2.0 \\
e-4.8\end{array}$ & $\begin{array}{r}-.1 \\
-1.5 \\
-4.5 \\
e-2.5 \\
\text { e2.1 }\end{array}$ & $\begin{array}{l}\mathrm{e} 8.4 \\
\mathrm{e} 2.0 \\
\mathrm{e}-.7 \\
\mathrm{e} 5.0 \\
1.1\end{array}$ & $\begin{array}{r}\text { e5.8 } \\
\text { e } 8.9 \\
\text { e12.0 } \\
\text { e10.2 } \\
\text { e } 4.2\end{array}$ & $\begin{array}{r}\mathrm{e} 13.3 \\
\mathrm{e} 13.1 \\
\mathrm{e} 12.0 \\
\mathrm{e} 13.0 \\
13.6\end{array}$ & $\begin{array}{l}17.7 \\
15.5 \\
14.3 \\
14.1 \\
14.0\end{array}$ & $\begin{array}{l}11.8 \\
13.0 \\
13.8 \\
14.7 \\
15.4\end{array}$ \\
\hline $\begin{array}{l}11 \\
12 \\
13 \\
14 \\
15\end{array}$ & $\begin{array}{r}8.8 \\
\text { e } 9.2 \\
8.4 \\
5.7 \\
1.2\end{array}$ & $\begin{array}{r}6.1 \\
3.7 \\
\text { e1. } \\
-1.5 \\
1.7\end{array}$ & $\begin{array}{r}-7.1 \\
e-2.4 \\
-3.0 \\
-1.2 \\
e-1.4\end{array}$ & $\begin{array}{r}\text { e. } \\
-2.1 \\
-5.2 \\
-9.7 \\
-10.4\end{array}$ & $\begin{array}{r}e-.4 \\
-5.9 \\
-17.8 \\
-23.1 \\
-17.0\end{array}$ & $\begin{array}{r}e-13.6 \\
e-15.5 \\
e-16.3 \\
e-9.3 \\
-6.8\end{array}$ & $\begin{array}{r}\text { e1. } 3 \\
\text { e1. } 4 \\
\text { e } 6.4 \\
8.0 \\
5.9\end{array}$ & $\begin{array}{r}-.9 \\
\text { e1.9 } \\
\text { e1. } \\
\text { e. } 6 \\
\text { e-1.6 }\end{array}$ & $\begin{array}{l}\text { e } 4.0 \\
\text { e2. } 4 \\
\text { e1. } 4 \\
\text { e1. } 0 \\
\text { e. } 5\end{array}$ & $\begin{array}{r}\text { e14.9 } \\
15.4 \\
13.0 \\
12.2 \\
12.0\end{array}$ & $\begin{array}{r}11.3 \\
12.1 \\
13.9 \\
14.3 \\
9.5\end{array}$ & $\begin{array}{l}15.8 \\
16.0 \\
14.3 \\
13.5 \\
11.3\end{array}$ \\
\hline $\begin{array}{l}16 \\
17 \\
18 \\
19 \\
20\end{array}$ & $\begin{array}{l}1.8 \\
4.4 \\
6.9 \\
6.8 \\
3.8\end{array}$ & $\begin{array}{r}.1 \\
-1.7 \\
e 4.4 \\
8.8 \\
8.3\end{array}$ & $\begin{array}{r}-4.5 \\
-5.3 \\
-7.7 \\
e-5.4 \\
e-4.1\end{array}$ & $\begin{array}{r}-10.7 \\
-10.9 \\
-11.8 \\
e-10.8 \\
e-6.6\end{array}$ & $\begin{array}{r}e-13.6 \\
-13.3 \\
-15.1 \\
e-11.9 \\
e-6.1\end{array}$ & $\begin{array}{r}e-1.1 \\
e-2.0 \\
e-.6 \\
e 1.3 \\
e 1.6\end{array}$ & $\begin{array}{r}1.6 \\
-2.6 \\
-1.2 \\
\text { e1.7 } \\
\text { e. } 9\end{array}$ & $\begin{array}{l}\text { e1.5 } \\
\text { e2 } .5 \\
\text { e1.0 } \\
-1.1 \\
\text { e1.6 }\end{array}$ & $\begin{array}{l}\text { e } 4.1 \\
\text { e } 7.6 \\
\text { e2. } 3 \\
\text { e } 7.1 \\
12.0\end{array}$ & $\begin{array}{l}11.6 \\
12.1 \\
12.5 \\
12.3 \\
13.1\end{array}$ & $\begin{array}{r}10.9 \\
10.2 \\
4.4 \\
.4 \\
2.0\end{array}$ & $\begin{array}{l}8.6 \\
6.4 \\
4.0 \\
4.6 \\
5.9\end{array}$ \\
\hline $\begin{array}{l}21 \\
22 \\
23 \\
24 \\
25\end{array}$ & $\begin{array}{r}1.6 \\
1.3 \\
\text { e1.1 } \\
1.4 \\
-7.9\end{array}$ & $\begin{array}{r}3.1 \\
3.6 \\
.0 \\
-4.0 \\
-4.0\end{array}$ & $\begin{array}{r}-3.8 \\
\text { e. } 2 \\
\text { e1.0 } \\
\text { e1.0 } \\
\text { e1. } 3\end{array}$ & $\begin{array}{r}e-3.0 \\
e-3.5 \\
-6.1 \\
e-8.3 \\
e-3.2\end{array}$ & $\begin{array}{r}e-4.0 \\
e-.9 \\
2.3 \\
e .2 \\
e .0\end{array}$ & $\begin{array}{r}\text { e1. } 0 \\
\text { e1. } 4 \\
.0 \\
\text { e. } 5 \\
\text { e-. } 3\end{array}$ & $\begin{array}{r}e-1.3 \\
e-1.3 \\
-3.9 \\
e-5.5 \\
e-3.3\end{array}$ & $\begin{array}{r}\text { e5.3 } \\
5.9 \\
\text { e1.5 } \\
-4.6 \\
\text { e2.0 }\end{array}$ & $\begin{array}{r}15.3 \\
16.7 \\
15.8 \\
\mathrm{e} 15.3 \\
15.6\end{array}$ & $\begin{array}{l}14.6 \\
15.7 \\
14.4 \\
12.0 \\
10.2\end{array}$ & $\begin{array}{r}6.1 \\
8.7 \\
10.0 \\
7.8 \\
7.1\end{array}$ & $\begin{array}{l}6.4 \\
4.5 \\
2.6 \\
3.7 \\
5.4\end{array}$ \\
\hline $\begin{array}{l}26 \\
27 \\
28 \\
29 \\
30 \\
31\end{array}$ & $\begin{array}{r}-6.6 \\
-4.6 \\
-11.2 \\
-6.1 \\
.7 \\
e-1.5\end{array}$ & $\begin{array}{r}-10.3 \\
-12.2 \\
-5.2 \\
-1.8 \\
e-2.8 \\
---\end{array}$ & $\begin{array}{r}e .0 \\
e-1.1 \\
-7.3 \\
-8.8 \\
-3.9 \\
e-2.2\end{array}$ & $\begin{array}{r}-9.9 \\
-11.9 \\
-7.5 \\
-6.9 \\
-8.0 \\
-11.3\end{array}$ & $\begin{array}{r}e-1.3 \\
e-2.5 \\
e-2.0 \\
--- \\
--- \\
---\end{array}$ & $\begin{array}{r}e-1.4 \\
-5.1 \\
-7.3 \\
-6.9 \\
e-4.3 \\
e-2.6\end{array}$ & $\begin{array}{r}e-.8 \\
e 1.5 \\
e-1.7 \\
-7.0 \\
-7.3 \\
---\end{array}$ & $\begin{array}{r}e 1.7 \\
\text { e1. } \\
-1.4 \\
.1 \\
e-.3 \\
e-2.6\end{array}$ & $\begin{array}{r}13.9 \\
13.0 \\
14.2 \\
16.4 \\
\text { e16.4 } \\
\end{array}$ & $\begin{array}{l}10.6 \\
13.6 \\
14.4 \\
16.5 \\
16.6 \\
14.4\end{array}$ & $\begin{array}{r}7.5 \\
11.0 \\
12.4 \\
14.3 \\
11.3 \\
11.7\end{array}$ & $\begin{array}{l}5.4 \\
3.7 \\
2.1 \\
4.5 \\
7.6 \\
-.--\end{array}$ \\
\hline $\begin{array}{l}\text { MEAN } \\
\text { MAX } \\
\text { MIN } \\
\text { MED }\end{array}$ & $\begin{array}{r}2.0 \\
10.0 \\
-11.2 \\
1.8\end{array}$ & $\begin{array}{r}.1 \\
8.8 \\
-12.2 \\
.0\end{array}$ & $\begin{array}{r}-2.3 \\
3.9 \\
-12.0 \\
-2.2\end{array}$ & $\begin{array}{r}-6.4 \\
4.1 \\
-13.4 \\
-6.9\end{array}$ & $\begin{array}{r}-8.0 \\
2.3 \\
-23.1 \\
-7.1\end{array}$ & $\begin{array}{r}-4.2 \\
1.6 \\
-16.3 \\
-2.9\end{array}$ & $\begin{array}{r}-.3 \\
8.0 \\
-7.3 \\
-.2\end{array}$ & $\begin{array}{r}1.2 \\
8.4 \\
-4.6 \\
1.5\end{array}$ & $\begin{array}{r}9.1 \\
16.7 \\
-1.9 \\
9.5\end{array}$ & $\begin{array}{l}13.3 \\
16.6 \\
10.2 \\
13.1\end{array}$ & $\begin{array}{r}11.2 \\
17.7 \\
.4 \\
11.7\end{array}$ & $\begin{array}{r}9.2 \\
16.0 \\
2.1 \\
9.9\end{array}$ \\
\hline
\end{tabular}

CAL YR 1989 MEAN 2.5 MAX 18.7 MIN $-23.5 \quad$ MED 1.8 WTR YR 1990 MEAN 2.1 MAX 17.7 MIN -23.1 MED 1.3

e Estimated 
EAST STEWART BASIN

VEG SPRING NEAR IONE, NV

DAILY MEAN AIR TEMPERATURE, WATER YEAR OCTOBER 1990 TO SEPTEMBER 1991 DEGREES CELSIUS

\begin{tabular}{|c|c|c|c|c|c|c|c|c|c|c|c|c|}
\hline DAY & OCT & NOV & DEC & JAN & FEB & MAR & APR & MAY & JUN & JUL & AUG & SEP \\
\hline $\begin{array}{l}1 \\
2 \\
3 \\
4 \\
5\end{array}$ & $\begin{array}{r}8.9 \\
4.8 \\
5.9 \\
10.1 \\
7.8\end{array}$ & $\begin{array}{r}-8.3 \\
-11.6 \\
-4.2 \\
.6 \\
-6.5\end{array}$ & $\begin{array}{r}-7.0 \\
-8.4 \\
-2.8 \\
.4 \\
-3.2\end{array}$ & $\begin{array}{l}-1.0 \\
-1.1 \\
-2.9 \\
-4.7 \\
-3.6\end{array}$ & $\begin{array}{r}.0 \\
-1.5 \\
-3.2 \\
1.1 \\
-3.7\end{array}$ & $\begin{array}{r}-6.3 \\
-6.9 \\
-2.9 \\
-2.3 \\
-10.1\end{array}$ & $\begin{array}{r}-3.7 \\
-3.7 \\
.0 \\
5.4 \\
5.6\end{array}$ & $\begin{array}{r}-6.3 \\
-7.0 \\
-5.6 \\
-1.4 \\
4.6\end{array}$ & $\begin{array}{l}3.6 \\
7.4 \\
8.2 \\
7.2 \\
2.5\end{array}$ & $\begin{array}{l}11.7 \\
14.6 \\
16.4 \\
17.8 \\
17.2\end{array}$ & $\begin{array}{l}13.4 \\
11.4 \\
12.7 \\
12.4 \\
11.6\end{array}$ & $\begin{array}{l}11.8 \\
13.9 \\
12.4 \\
11.6 \\
10.2\end{array}$ \\
\hline $\begin{array}{r}6 \\
7 \\
8 \\
9 \\
10\end{array}$ & $\begin{array}{r}1.9 \\
-3.7 \\
-.1 \\
6.0 \\
5.8\end{array}$ & $\begin{array}{r}-12.4 \\
-5.2 \\
-.8 \\
2.9 \\
5.4\end{array}$ & $\begin{array}{r}-1.1 \\
1.1 \\
3.0 \\
4.1 \\
3.1\end{array}$ & $\begin{array}{l}-3.6 \\
-6.0 \\
-7.4 \\
-7.4 \\
-5.7\end{array}$ & $\begin{array}{l}-3.4 \\
-1.2 \\
-1.3 \\
-1.1 \\
-1.0\end{array}$ & $\begin{array}{r}-13.0 \\
-8.6 \\
-4.7 \\
-3.5 \\
-5.1\end{array}$ & $\begin{array}{r}.9 \\
-6.0 \\
-5.3 \\
-1.9 \\
-12.4\end{array}$ & $\begin{array}{r}.7 \\
4.6 \\
2.4 \\
-9.0 \\
-8.4\end{array}$ & $\begin{array}{r}7.3 \\
7.6 \\
8.9 \\
10.4 \\
12.2\end{array}$ & $\begin{array}{l}15.9 \\
15.5 \\
13.6 \\
12.2 \\
12.6\end{array}$ & $\begin{array}{l}12.0 \\
12.4 \\
14.7 \\
16.3 \\
14.9\end{array}$ & $\begin{array}{r}7.3 \\
6.4 \\
6.8 \\
1.7 \\
.6\end{array}$ \\
\hline $\begin{array}{l}11 \\
12 \\
13 \\
14 \\
15\end{array}$ & $\begin{array}{l}2.8 \\
5.9 \\
5.0 \\
7.0 \\
6.8\end{array}$ & $\begin{array}{l}5.0 \\
6.1 \\
7.3 \\
4.0 \\
1.0\end{array}$ & $\begin{array}{r}-2.2 \\
-6.2 \\
-10.7 \\
-12.2 \\
-9.7\end{array}$ & $\begin{array}{l}-2.6 \\
-2.7 \\
-4.2 \\
-5.1 \\
-7.6\end{array}$ & $\begin{array}{r}.2 \\
-2.2 \\
-2.1 \\
1.0 \\
1.2\end{array}$ & $\begin{array}{r}-12.5 \\
-8.4 \\
-7.8 \\
-8.9 \\
-10.7\end{array}$ & $\begin{array}{r}-13.0 \\
-8.0 \\
-5.7 \\
-1.5 \\
-3.9\end{array}$ & $\begin{array}{r}-5.5 \\
-.1 \\
-.5 \\
-3.3 \\
1.9\end{array}$ & $\begin{array}{r}13.5 \\
12.8 \\
9.4 \\
5.9 \\
9.3\end{array}$ & $\begin{array}{l}14.8 \\
16.4 \\
16.9 \\
15.4 \\
14.5\end{array}$ & $\begin{array}{l}13.6 \\
12.8 \\
12.3 \\
13.4 \\
10.4\end{array}$ & $\begin{array}{l}3.2 \\
4.9 \\
7.7 \\
7.2 \\
9.1\end{array}$ \\
\hline $\begin{array}{l}16 \\
17 \\
18 \\
19 \\
20\end{array}$ & $\begin{array}{r}3.1 \\
5.5 \\
2.6 \\
-5.7 \\
-5.4\end{array}$ & $\begin{array}{r}3.9 \\
-.3 \\
-.5 \\
-3.5 \\
-11.1\end{array}$ & $\begin{array}{r}-13.9 \\
-10.4 \\
-8.2 \\
-16.7 \\
-23.3\end{array}$ & $\begin{array}{r}-9.0 \\
-3.7 \\
-1.0 \\
-5.2 \\
-10.1\end{array}$ & $\begin{array}{r}-4.8 \\
-9.2 \\
-9.1 \\
-2.3 \\
.3\end{array}$ & $\begin{array}{l}-7.5 \\
-6.4 \\
-7.5 \\
-9.0 \\
-9.4\end{array}$ & $\begin{array}{r}-6.9 \\
-7.0 \\
-3.4 \\
-.4 \\
-1.9\end{array}$ & $\begin{array}{r}5.2 \\
-6.7 \\
-8.0 \\
-4.2 \\
-2.2\end{array}$ & $\begin{array}{r}10.2 \\
9.9 \\
11.2 \\
6.2 \\
5.3\end{array}$ & $\begin{array}{l}14.3 \\
14.3 \\
14.3 \\
12.1 \\
10.2\end{array}$ & $\begin{array}{l}11.8 \\
13.7 \\
14.6 \\
14.8 \\
14.9\end{array}$ & $\begin{array}{l}11.0 \\
12.1 \\
12.3 \\
12.4 \\
12.7\end{array}$ \\
\hline $\begin{array}{l}21 \\
22 \\
23 \\
24 \\
25\end{array}$ & $\begin{array}{l}3.1 \\
2.9 \\
4.9 \\
7.0 \\
8.7\end{array}$ & $\begin{array}{r}-8.6 \\
.1 \\
3.4 \\
4.4 \\
1.5\end{array}$ & $\begin{array}{r}-25.0 \\
-22.1 \\
-14.6 \\
-9.8 \\
-9.6\end{array}$ & $\begin{array}{r}-11.5 \\
-4.9 \\
-5.3 \\
-6.7 \\
-5.7\end{array}$ & $\begin{array}{r}1.5 \\
-.2 \\
-3.3 \\
-3.2 \\
-.5\end{array}$ & $\begin{array}{r}-8.9 \\
-7.9 \\
-5.5 \\
-6.1 \\
-10.3\end{array}$ & $\begin{array}{r}-4.1 \\
-3.8 \\
-.8 \\
-2.2 \\
-9.0\end{array}$ & $\begin{array}{r}-1.8 \\
3.3 \\
6.1 \\
6.2 \\
5.3\end{array}$ & $\begin{array}{l}7.1 \\
8.2 \\
8.1 \\
4.1 \\
2.6\end{array}$ & $\begin{array}{l}11.1 \\
13.6 \\
14.9 \\
13.8 \\
14.2\end{array}$ & $\begin{array}{l}16.1 \\
16.4 \\
15.9 \\
15.8 \\
14.9\end{array}$ & $\begin{array}{l}11.0 \\
11.0 \\
10.6 \\
11.7 \\
12.4\end{array}$ \\
\hline $\begin{array}{l}26 \\
27 \\
28 \\
29 \\
30 \\
31\end{array}$ & $\begin{array}{l}7.2 \\
8.8 \\
7.0 \\
5.9 \\
4.6 \\
-.4\end{array}$ & $\begin{array}{r}-14.9 \\
-10.4 \\
-4.2 \\
2.2 \\
-4.3 \\
----\end{array}$ & $\begin{array}{r}-9.8 \\
-4.8 \\
-8.4 \\
-16.9 \\
-6.3 \\
-3.2\end{array}$ & $\begin{array}{r}-6.1 \\
-8.1 \\
-9.2 \\
-9.1 \\
-2.1 \\
-.1\end{array}$ & $\begin{array}{r}-.9 \\
-2.2 \\
-4.4 \\
--- \\
--- \\
---\end{array}$ & $\begin{array}{r}-10.7 \\
-8.9 \\
-6.3 \\
-4.9 \\
-.7 \\
-.7\end{array}$ & $\begin{array}{l}-7.2 \\
-8.3 \\
-4.0 \\
-3.2 \\
-.1 \\
---\end{array}$ & $\begin{array}{r}1.4 \\
.8 \\
2.8 \\
1.8 \\
-4.3 \\
-2.8\end{array}$ & $\begin{array}{l}3.9 \\
2.1 \\
3.6 \\
1.6 \\
7.7 \\
---\end{array}$ & $\begin{array}{l}14.1 \\
15.6 \\
15.6 \\
17.0 \\
14.8 \\
11.9\end{array}$ & $\begin{array}{r}12.3 \\
7.7 \\
8.9 \\
13.5 \\
15.1 \\
13.3\end{array}$ & $\begin{array}{l}8.9 \\
6.5 \\
6.1 \\
6.7 \\
8.3 \\
---\end{array}$ \\
\hline $\begin{array}{l}\text { MEAN } \\
\text { MAX } \\
\text { MIN } \\
\text { MED }\end{array}$ & $\begin{array}{r}4.3 \\
10.1 \\
-5.7 \\
5.5\end{array}$ & $\begin{array}{r}-2.0 \\
7.3 \\
-14.9 \\
-.4\end{array}$ & $\begin{array}{r}-8.2 \\
4.1 \\
-25.0 \\
-8.4\end{array}$ & $\begin{array}{r}-5.3 \\
-.1 \\
-11.5 \\
-5.2\end{array}$ & $\begin{array}{r}-2.0 \\
1.5 \\
-9.2 \\
-1.4\end{array}$ & $\begin{array}{r}-7.2 \\
-. .7 \\
-13.0 \\
-7.5\end{array}$ & $\begin{array}{r}-3.9 \\
5.6 \\
-13.0 \\
-3.7\end{array}$ & $\begin{array}{r}-1.0 \\
6.2 \\
-9.0 \\
-.5\end{array}$ & $\begin{array}{r}7.3 \\
13.5 \\
1.6 \\
7.5\end{array}$ & $\begin{array}{l}14.4 \\
17.8 \\
10.2 \\
14.5\end{array}$ & $\begin{array}{r}13.4 \\
16.4 \\
7.7 \\
13.4\end{array}$ & $\begin{array}{r}8.9 \\
13.9 \\
.6 \\
9.6\end{array}$ \\
\hline
\end{tabular}

CAL YR 1990 MEAN 1.7 MAX 17.7 MIN -25.0 MED 1.5

WTR YR 1991 MEAN 1.6 MAX 17.8 MIN -25.0 MED .8 
EAST STEWART BASIN

VEG SPRING NEAR IONE, NV

DAILY MAXIMUM AIR TEMPERATURE, WATER YEAR OCTOBER 1988 TO SEPTEMBER 1989 DEGREES CELSIUS

\begin{tabular}{|c|c|c|c|c|c|c|c|c|c|c|c|c|}
\hline DAY & $\mathrm{OCT}$ & NOV & DEC & JAN & FEB & MAR & APR & MAY & JUN & JUL & AUG & SEP \\
\hline $\begin{array}{l}1 \\
2 \\
3 \\
4 \\
5\end{array}$ & $\begin{array}{l}15.7 \\
15.0 \\
14.7 \\
14.2 \\
10.1\end{array}$ & $\begin{array}{r}8.6 \\
2.9 \\
6.9 \\
4.5 \\
11.1\end{array}$ & $\begin{array}{l}6.3 \\
4.9 \\
2.7 \\
4.4 \\
2.0\end{array}$ & $\begin{array}{r}-5.8 \\
-1.3 \\
.9 \\
-1.1 \\
-4.4\end{array}$ & $\begin{array}{r}-1.5 \\
-8.3 \\
-10.5 \\
-16.8 \\
-20.4\end{array}$ & $\begin{array}{r}.7 \\
-1.2 \\
-9.5 \\
-.1 \\
2.6\end{array}$ & $\begin{array}{r}-2.2 \\
-1.3 \\
.2 \\
5.6 \\
10.4\end{array}$ & $\begin{array}{r}7.9 \\
7.3 \\
8.7 \\
11.5 \\
14.5\end{array}$ & $\begin{array}{r}9.5 \\
11.4 \\
6.6 \\
4.5 \\
5.5\end{array}$ & $\begin{array}{l}17.5 \\
19.7 \\
21.6 \\
20.5 \\
21.2\end{array}$ & $\begin{array}{l}17.9 \\
17.0 \\
17.8 \\
18.0 \\
19.0\end{array}$ & $\begin{array}{l}15.1 \\
16.0 \\
16.9 \\
18.2 \\
16.7\end{array}$ \\
\hline $\begin{array}{r}6 \\
7 \\
8 \\
9 \\
10\end{array}$ & $\begin{array}{r}8.9 \\
10.1 \\
10.5 \\
9.9 \\
7.4\end{array}$ & $\begin{array}{r}5.9 \\
4.6 \\
-2.2 \\
1.2 \\
.4\end{array}$ & $\begin{array}{r}2.6 \\
-7.9 \\
-3.1 \\
.4 \\
3.9\end{array}$ & $\begin{array}{r}-15.9 \\
-14.1 \\
-7.3 \\
-3.8 \\
-4.5\end{array}$ & $\begin{array}{r}-18.1 \\
-13.2 \\
-4.6 \\
-1.8 \\
-5.7\end{array}$ & $\begin{array}{l}3.3 \\
3.3 \\
3.3 \\
5.0 \\
4.8\end{array}$ & $\begin{array}{r}12.6 \\
11.5 \\
10.6 \\
9.2 \\
11.3\end{array}$ & $\begin{array}{r}15.5 \\
13.5 \\
12.5 \\
9.0 \\
.4\end{array}$ & $\begin{array}{r}11.9 \\
10.3 \\
11.4 \\
7.9 \\
12.7\end{array}$ & $\begin{array}{l}21.7 \\
23.2 \\
20.5 \\
17.5 \\
17.4\end{array}$ & $\begin{array}{l}19.6 \\
20.4 \\
14.1 \\
13.5 \\
15.5\end{array}$ & $\begin{array}{r}15.8 \\
7.2 \\
11.9 \\
13.2 \\
12.4\end{array}$ \\
\hline $\begin{array}{l}11 \\
12 \\
13 \\
14 \\
15\end{array}$ & $\begin{array}{l}4.3 \\
5.6 \\
7.2 \\
4.7 \\
9.2\end{array}$ & $\begin{array}{r}-2.3 \\
3.2 \\
2.6 \\
-7.1 \\
-8.2\end{array}$ & $\begin{array}{r}4.0 \\
7.7 \\
4.9 \\
-. .5 \\
-9.1\end{array}$ & $\begin{array}{r}-12.5 \\
-2.8 \\
-1.6 \\
-9.9 \\
-5.6\end{array}$ & $\begin{array}{r}-8.7 \\
-8.5 \\
-10.4 \\
-7.9 \\
-.2\end{array}$ & $\begin{array}{r}4.4 \\
4.7 \\
-2.9 \\
-2.9 \\
2.0\end{array}$ & $\begin{array}{r}6.2 \\
6.1 \\
10.0 \\
11.2 \\
7.4\end{array}$ & $\begin{array}{r}-1.6 \\
-.8 \\
-2.4 \\
-.4 \\
1.0\end{array}$ & $\begin{array}{l}10.9 \\
15.1 \\
17.8 \\
20.3 \\
19.5\end{array}$ & $\begin{array}{l}16.9 \\
11.8 \\
17.6 \\
20.2 \\
18.5\end{array}$ & $\begin{array}{l}16.8 \\
18.3 \\
19.8 \\
18.8 \\
19.4\end{array}$ & $\begin{array}{l}10.2 \\
10.4 \\
13.3 \\
14.6 \\
16.2\end{array}$ \\
\hline $\begin{array}{l}16 \\
17 \\
18 \\
19 \\
20\end{array}$ & $\begin{array}{l}12.3 \\
12.4 \\
13.7 \\
10.6 \\
12.2\end{array}$ & $\begin{array}{r}-4.7 \\
-9.6 \\
-10.4 \\
-.6 \\
-3.9\end{array}$ & $\begin{array}{l}-7.5 \\
-5.2 \\
-6.6 \\
-8.9 \\
-5.3\end{array}$ & $\begin{array}{r}-.8 \\
-.6 \\
3.5 \\
.4 \\
2.3\end{array}$ & $\begin{array}{r}-3.6 \\
1.2 \\
1.4 \\
-4.7 \\
-5.5\end{array}$ & $\begin{array}{r}.1 \\
-1.0 \\
-.1 \\
-2.9 \\
-.1\end{array}$ & $\begin{array}{r}9.4 \\
11.4 \\
12.9 \\
13.1 \\
12.4\end{array}$ & $\begin{array}{r}4.6 \\
11.3 \\
7.4 \\
8.4 \\
11.9\end{array}$ & $\begin{array}{l}12.7 \\
17.0 \\
20.1 \\
18.5 \\
10.8\end{array}$ & $\begin{array}{l}16.9 \\
19.7 \\
22.3 \\
23.4 \\
22.0\end{array}$ & $\begin{array}{l}18.9 \\
16.2 \\
16.1 \\
15.0 \\
11.2\end{array}$ & $\begin{array}{r}12.5 \\
5.6 \\
2.9 \\
5.6 \\
8.8\end{array}$ \\
\hline $\begin{array}{l}21 \\
22 \\
23 \\
24 \\
25\end{array}$ & $\begin{array}{l}13.0 \\
11.8 \\
12.9 \\
11.6 \\
11.1\end{array}$ & $\begin{array}{r}-1.2 \\
1.2 \\
.9 \\
-7.1 \\
-8.1\end{array}$ & $\begin{array}{r}-7.9 \\
-9.9 \\
-9.3 \\
-8.6 \\
-15.5\end{array}$ & $\begin{array}{r}2.2 \\
-1.1 \\
-5.3 \\
-6.7 \\
-6.6\end{array}$ & $\begin{array}{l}-.8 \\
4.0 \\
3.4 \\
4.2 \\
3.3\end{array}$ & $\begin{array}{l}3.5 \\
3.1 \\
1.0 \\
2.7 \\
-.5\end{array}$ & $\begin{array}{r}11.0 \\
2.0 \\
1.7 \\
-4.3 \\
-4.6\end{array}$ & $\begin{array}{r}11.9 \\
13.0 \\
4.7 \\
.9 \\
4.6\end{array}$ & $\begin{array}{r}11.0 \\
14.4 \\
10.4 \\
9.9 \\
12.8\end{array}$ & $\begin{array}{l}17.8 \\
19.5 \\
15.6 \\
16.5 \\
20.4\end{array}$ & $\begin{array}{r}14.4 \\
17.0 \\
8.2 \\
9.1 \\
12.2\end{array}$ & $\begin{array}{l}11.6 \\
13.1 \\
14.2 \\
15.2 \\
12.2\end{array}$ \\
\hline $\begin{array}{l}26 \\
27 \\
28 \\
29 \\
30 \\
31\end{array}$ & $\begin{array}{r}10.1 \\
9.9 \\
8.6 \\
7.6 \\
8.3 \\
9.8\end{array}$ & $\begin{array}{r}-8.0 \\
2.9 \\
.4 \\
-1.6 \\
-.3 \\
----\end{array}$ & $\begin{array}{r}-16.2 \\
-9.8 \\
-15.6 \\
-5.8 \\
-2.7 \\
-9.7\end{array}$ & $\begin{array}{r}-2.4 \\
-1.7 \\
-2.6 \\
.5 \\
8.3 \\
4.7\end{array}$ & $\begin{array}{r}1.0 \\
-2.8 \\
-1.0 \\
--- \\
--- \\
---\end{array}$ & $\begin{array}{r}-5.0 \\
-.9 \\
2.8 \\
-.9 \\
3.6 \\
2.0\end{array}$ & $\begin{array}{r}-4.6 \\
-4.2 \\
-2.4 \\
3.9 \\
5.1 \\
---\end{array}$ & $\begin{array}{r}10.4 \\
11.9 \\
5.0 \\
-1.2 \\
-2.3 \\
4.9\end{array}$ & $\begin{array}{r}15.3 \\
11.4 \\
14.2 \\
15.9 \\
15.2 \\
----\end{array}$ & $\begin{array}{l}21.0 \\
20.5 \\
20.1 \\
21.1 \\
20.7 \\
20.5\end{array}$ & $\begin{array}{l}14.8 \\
16.1 \\
17.8 \\
18.1 \\
14.3 \\
14.6\end{array}$ & $\begin{array}{r}13.8 \\
13.1 \\
12.6 \\
10.9 \\
4.3 \\
---\end{array}$ \\
\hline MAX & 15.7 & 11.1 & 7.7 & 8.3 & 4.2 & 5.0 & 13.1 & 15.5 & 20.3 & 23.4 & 20.4 & 18.2 \\
\hline
\end{tabular}

WTR YR 1989 MAX 23.4 
EAST STEWART BASIN

VEG SPRING NEAR IONE, NV

DAILY MAXIMUM AIR TEMPERATURE, WATER YEAR OCTOBER 1989 TO SEPTEMBER 1990 DEGREES CELSIUS

\begin{tabular}{|c|c|c|c|c|c|c|c|c|c|c|c|c|}
\hline DAY & $\mathrm{OCT}$ & NOV & $\mathrm{DEC}$ & JAN & FEB & MAR & APR & MAY & JUN & JUL & AUG & SEP \\
\hline $\begin{array}{l}1 \\
2 \\
3 \\
4 \\
5\end{array}$ & $\begin{array}{r}9.4 \\
4.1 \\
-2.4 \\
2.7 \\
7.4\end{array}$ & $\begin{array}{l}3.0 \\
2.9 \\
3.7 \\
4.8 \\
2.7\end{array}$ & $\begin{array}{l}3.1 \\
7.8 \\
5.2 \\
4.9 \\
2.6\end{array}$ & $\begin{array}{r}-1.8 \\
-10.1 \\
-9.1 \\
-6.2 \\
-2.4\end{array}$ & $\begin{array}{l}-9.7 \\
-5.4 \\
-1.4 \\
-8.0 \\
-3.7\end{array}$ & $\begin{array}{r}-1.3 \\
-1.5 \\
-.4 \\
-1.3 \\
-8.1\end{array}$ & $\begin{array}{l}6.2 \\
3.2 \\
3.7 \\
3.0 \\
3.1\end{array}$ & $\begin{array}{r}.1 \\
.4 \\
3.4 \\
10.4 \\
13.7\end{array}$ & $\begin{array}{r}3.0 \\
12.4 \\
16.8 \\
15.7 \\
13.6\end{array}$ & $\begin{array}{l}20.3 \\
16.0 \\
16.4 \\
18.7 \\
19.5\end{array}$ & $\begin{array}{l}16.7 \\
18.5 \\
19.6 \\
20.2 \\
21.5\end{array}$ & $\begin{array}{l}17.5 \\
17.3 \\
17.2 \\
15.8 \\
16.6\end{array}$ \\
\hline $\begin{array}{r}6 \\
7 \\
8 \\
9 \\
10\end{array}$ & $\begin{array}{r}7.9 \\
9.3 \\
12.0 \\
13.9 \\
13.0\end{array}$ & $\begin{array}{r}1.5 \\
.1 \\
3.8 \\
5.8 \\
9.9\end{array}$ & $\begin{array}{r}-1.2 \\
2.5 \\
3.7 \\
.0 \\
-8.7\end{array}$ & $\begin{array}{r}-.9 \\
.1 \\
2.5 \\
5.9 \\
7.0\end{array}$ & $\begin{array}{r}-5.1 \\
-11.8 \\
-8.5 \\
-5.3 \\
-1.7\end{array}$ & $\begin{array}{r}-2.7 \\
2.4 \\
.2 \\
1.5 \\
.0\end{array}$ & $\begin{array}{r}2.4 \\
3.2 \\
-3.1 \\
1.1 \\
7.0\end{array}$ & $\begin{array}{r}12.8 \\
7.5 \\
2.7 \\
8.9 \\
3.3\end{array}$ & $\begin{array}{r}10.4 \\
15.1 \\
17.5 \\
15.1 \\
7.8\end{array}$ & $\begin{array}{l}17.8 \\
19.0 \\
17.4 \\
18.3 \\
16.9\end{array}$ & $\begin{array}{l}21.9 \\
18.7 \\
18.4 \\
18.3 \\
19.1\end{array}$ & $\begin{array}{l}16.4 \\
17.8 \\
18.8 \\
19.7 \\
19.8\end{array}$ \\
\hline $\begin{array}{l}11 \\
12 \\
13 \\
14 \\
15\end{array}$ & $\begin{array}{r}12.9 \\
14.1 \\
13.2 \\
8.7 \\
4.6\end{array}$ & $\begin{array}{r}10.0 \\
6.7 \\
5.2 \\
.1 \\
4.3\end{array}$ & $\begin{array}{r}-3.2 \\
-1.1 \\
-1.3 \\
1.2 \\
1.9\end{array}$ & $\begin{array}{r}3.3 \\
-.2 \\
-3.9 \\
-8.4 \\
-9.2\end{array}$ & $\begin{array}{r}3.8 \\
-2.5 \\
-12.9 \\
-21.3 \\
-12.7\end{array}$ & $\begin{array}{r}-8.0 \\
-10.3 \\
-12.2 \\
-6.1 \\
-2.6\end{array}$ & $\begin{array}{r}4.4 \\
5.6 \\
10.1 \\
11.2 \\
9.4\end{array}$ & $\begin{array}{l}2.7 \\
8.5 \\
8.3 \\
4.1 \\
2.1\end{array}$ & $\begin{array}{l}8.6 \\
7.9 \\
8.2 \\
4.7 \\
3.3\end{array}$ & $\begin{array}{l}18.6 \\
18.7 \\
16.5 \\
16.2 \\
15.9\end{array}$ & $\begin{array}{l}16.4 \\
17.0 \\
18.6 \\
18.7 \\
12.8\end{array}$ & $\begin{array}{l}20.2 \\
20.0 \\
18.9 \\
18.0 \\
15.5\end{array}$ \\
\hline $\begin{array}{l}16 \\
17 \\
18 \\
19 \\
20\end{array}$ & $\begin{array}{r}6.0 \\
8.8 \\
11.3 \\
10.9 \\
6.6\end{array}$ & $\begin{array}{r}3.1 \\
.2 \\
7.6 \\
12.1 \\
12.5\end{array}$ & $\begin{array}{l}-2.6 \\
-3.7 \\
-5.6 \\
-3.4 \\
-1.4\end{array}$ & $\begin{array}{l}-8.6 \\
-9.8 \\
-9.5 \\
-7.2 \\
-3.0\end{array}$ & $\begin{array}{r}-11.8 \\
-10.2 \\
-12.3 \\
-8.7 \\
-1.2\end{array}$ & $\begin{array}{l}4.2 \\
1.4 \\
3.9 \\
5.8 \\
6.1\end{array}$ & $\begin{array}{r}5.3 \\
-1.0 \\
2.4 \\
5.5 \\
2.3\end{array}$ & $\begin{array}{l}7.9 \\
8.0 \\
7.3 \\
2.8 \\
7.7\end{array}$ & $\begin{array}{r}9.8 \\
14.5 \\
6.7 \\
13.8 \\
18.5\end{array}$ & $\begin{array}{l}14.8 \\
16.0 \\
16.5 \\
16.1 \\
18.2\end{array}$ & $\begin{array}{r}15.6 \\
14.9 \\
8.0 \\
3.7 \\
7.7\end{array}$ & $\begin{array}{r}13.2 \\
9.6 \\
6.8 \\
8.1 \\
9.9\end{array}$ \\
\hline $\begin{array}{l}21 \\
22 \\
23 \\
24 \\
25\end{array}$ & $\begin{array}{r}3.8 \\
6.2 \\
4.1 \\
5.9 \\
-3.7\end{array}$ & $\begin{array}{r}6.2 \\
6.9 \\
2.2 \\
-1.7 \\
-. .7\end{array}$ & $\begin{array}{l}.5 \\
2.9 \\
5.4 \\
4.4 \\
3.9\end{array}$ & $\begin{array}{r}1.2 \\
.3 \\
-3.5 \\
-3.9 \\
-1.0\end{array}$ & $\begin{array}{r}-1.2 \\
4.0 \\
6.7 \\
4.4 \\
2.4\end{array}$ & $\begin{array}{l}3.3 \\
4.2 \\
4.0 \\
2.8 \\
4.8\end{array}$ & $\begin{array}{r}2.5 \\
1.6 \\
-1.7 \\
-2.7 \\
.9\end{array}$ & $\begin{array}{r}12.2 \\
11.4 \\
8.0 \\
.2 \\
6.3\end{array}$ & $\begin{array}{l}20.9 \\
21.6 \\
20.2 \\
20.4 \\
21.1\end{array}$ & $\begin{array}{l}19.9 \\
21.1 \\
19.3 \\
16.8 \\
16.0\end{array}$ & $\begin{array}{l}11.1 \\
14.5 \\
14.3 \\
12.2 \\
12.2\end{array}$ & $\begin{array}{r}10.8 \\
7.9 \\
5.4 \\
6.6 \\
11.5\end{array}$ \\
\hline $\begin{array}{l}26 \\
27 \\
28 \\
29 \\
30 \\
31\end{array}$ & $\begin{array}{r}-2.5 \\
-1.2 \\
-8.0 \\
-2.5 \\
3.4 \\
.5\end{array}$ & $\begin{array}{r}-4.4 \\
-9.3 \\
-1.9 \\
1.6 \\
-.7 \\
---\end{array}$ & $\begin{array}{r}4.3 \\
.6 \\
-3.5 \\
-5.8 \\
-1.5 \\
-.5\end{array}$ & $\begin{array}{l}-2.7 \\
-7.5 \\
-5.3 \\
-4.5 \\
-4.7 \\
-6.3\end{array}$ & $\begin{array}{l}1.7 \\
-.7 \\
-.5 \\
--- \\
--- \\
---\end{array}$ & $\begin{array}{r}3.5 \\
-.9 \\
-4.7 \\
-3.9 \\
.3 \\
2.9\end{array}$ & $\begin{array}{r}3.1 \\
5.6 \\
3.4 \\
-3.2 \\
-4.0 \\
---\end{array}$ & $\begin{array}{r}6.4 \\
3.8 \\
-.3 \\
2.4 \\
2.8 \\
.0\end{array}$ & $\begin{array}{r}18.7 \\
18.5 \\
19.7 \\
21.5 \\
20.6 \\
-.-\end{array}$ & $\begin{array}{l}16.4 \\
19.3 \\
19.0 \\
21.3 \\
20.6 \\
18.8\end{array}$ & $\begin{array}{l}12.5 \\
16.5 \\
17.6 \\
19.1 \\
15.7 \\
17.3\end{array}$ & $\begin{array}{r}10.6 \\
8.7 \\
4.7 \\
8.8 \\
11.9 \\
\end{array}$ \\
\hline MAX & 14.1 & 12.5 & 7.8 & 7.0 & 6.7 & 6.1 & 11.2 & 13.7 & 21.6 & 21.3 & 21.9 & 20.2 \\
\hline $\begin{array}{ll}\text { CAL } & \text { YR } \\
\text { WTR } & \text { YR }\end{array}$ & $\begin{array}{l}1989 \\
1990\end{array}$ & $\begin{array}{ll}\operatorname{MAX} & 23 \\
\operatorname{MAX} & 21\end{array}$ & & & & & & & & & & \\
\hline
\end{tabular}


EAST STEWART BASIN

VEG SPRING NEAR IONE, NV

DAILY MAXIMUM AIR TEMPERATURE, WATER YEAR OCTOBER 1990 TO SEPTEMBER 1991 DEGREES CELSIUS

\begin{tabular}{|c|c|c|c|c|c|c|c|c|c|c|c|c|}
\hline DAY & OCT & NOV & DEC & JAN & FEB & MAR & APR & MAY & JUN & JUL & AUG & SEP \\
\hline $\begin{array}{l}1 \\
2 \\
3 \\
4 \\
5\end{array}$ & $\begin{array}{r}14.9 \\
9.0 \\
12.1 \\
14.8 \\
12.3\end{array}$ & $\begin{array}{r}-4.5 \\
-6.7 \\
.9 \\
5.7 \\
-2.1\end{array}$ & $\begin{array}{r}-3.8 \\
-3.2 \\
4.1 \\
4.6 \\
.9\end{array}$ & $\begin{array}{r}4.2 \\
5.0 \\
.2 \\
-3.7 \\
2.6\end{array}$ & $\begin{array}{r}6.1 \\
2.9 \\
1.9 \\
6.2 \\
.4\end{array}$ & $\begin{array}{r}-3.0 \\
-3.4 \\
-1.1 \\
-.5 \\
-6.2\end{array}$ & $\begin{array}{r}1.2 \\
1.9 \\
6.6 \\
11.5 \\
9.0\end{array}$ & $\begin{array}{r}-1.3 \\
-5.8 \\
-1.3 \\
6.1 \\
12.8\end{array}$ & $\begin{array}{r}9.4 \\
13.1 \\
12.2 \\
12.2 \\
8.4\end{array}$ & $\begin{array}{l}18.7 \\
20.9 \\
22.3 \\
23.7 \\
22.8\end{array}$ & $\begin{array}{l}19.3 \\
17.4 \\
18.9 \\
18.0 \\
17.3\end{array}$ & $\begin{array}{l}16.7 \\
19.3 \\
16.4 \\
17.0 \\
13.8\end{array}$ \\
\hline $\begin{array}{r}6 \\
7 \\
8 \\
9 \\
10\end{array}$ & $\begin{array}{r}5.5 \\
-.1 \\
5.8 \\
12.4 \\
11.1\end{array}$ & $\begin{array}{r}-10.3 \\
2.2 \\
1.9 \\
9.2 \\
11.3\end{array}$ & $\begin{array}{r}7.2 \\
5.4 \\
8.7 \\
10.0 \\
8.1\end{array}$ & $\begin{array}{r}-1.6 \\
-2.5 \\
-.8 \\
-1.6 \\
-4.0\end{array}$ & $\begin{array}{l}3.9 \\
6.1 \\
6.6 \\
6.0 \\
7.3\end{array}$ & $\begin{array}{r}-8.9 \\
-.3 \\
-.5 \\
-.4 \\
.0\end{array}$ & $\begin{array}{r}5.2 \\
-.9 \\
2.1 \\
2.2 \\
-7.9\end{array}$ & $\begin{array}{r}4.5 \\
11.5 \\
6.6 \\
-6.2 \\
-1.1\end{array}$ & $\begin{array}{l}13.8 \\
14.3 \\
15.0 \\
17.5 \\
18.6\end{array}$ & $\begin{array}{l}22.3 \\
21.2 \\
17.6 \\
16.2 \\
18.7\end{array}$ & $\begin{array}{l}18.0 \\
18.9 \\
21.3 \\
22.0 \\
20.5\end{array}$ & $\begin{array}{r}9.0 \\
10.8 \\
11.7 \\
6.6 \\
5.3\end{array}$ \\
\hline $\begin{array}{l}11 \\
12 \\
13 \\
14 \\
15\end{array}$ & $\begin{array}{r}8.5 \\
10.1 \\
9.4 \\
12.2 \\
11.7\end{array}$ & $\begin{array}{r}9.0 \\
11.8 \\
10.9 \\
8.1 \\
5.3\end{array}$ & $\begin{array}{r}2.4 \\
-1.0 \\
-7.5 \\
-6.7 \\
-5.9\end{array}$ & $\begin{array}{r}1.1 \\
.0 \\
-2.6 \\
-1.5 \\
-5.0\end{array}$ & $\begin{array}{l}6.3 \\
1.6 \\
2.1 \\
7.9 \\
4.6\end{array}$ & $\begin{array}{l}-8.6 \\
-3.2 \\
-1.7 \\
-2.0 \\
-6.5\end{array}$ & $\begin{array}{r}-9.7 \\
-3.0 \\
-.4 \\
4.4 \\
.5\end{array}$ & $\begin{array}{r}1.1 \\
8.6 \\
5.8 \\
1.9 \\
10.6\end{array}$ & $\begin{array}{l}19.0 \\
19.0 \\
14.9 \\
11.4 \\
15.7\end{array}$ & $\begin{array}{l}20.8 \\
21.9 \\
22.0 \\
21.7 \\
19.8\end{array}$ & $\begin{array}{l}19.5 \\
17.6 \\
17.2 \\
18.2 \\
14.2\end{array}$ & $\begin{array}{r}7.5 \\
10.8 \\
13.2 \\
12.5 \\
14.7\end{array}$ \\
\hline $\begin{array}{l}16 \\
17 \\
18 \\
19 \\
20\end{array}$ & $\begin{array}{r}5.3 \\
11.4 \\
5.5 \\
-2.4 \\
1.9\end{array}$ & $\begin{array}{r}7.8 \\
2.5 \\
2.5 \\
-.4 \\
-6.1\end{array}$ & $\begin{array}{r}-6.0 \\
-7.1 \\
-5.6 \\
-10.5 \\
-21.8\end{array}$ & $\begin{array}{r}-5.2 \\
.1 \\
5.8 \\
-.6 \\
-5.7\end{array}$ & $\begin{array}{r}-3.0 \\
-7.6 \\
-2.2 \\
4.7 \\
7.0\end{array}$ & $\begin{array}{r}.4 \\
-2.3 \\
-5.0 \\
-5.0 \\
-5.4\end{array}$ & $\begin{array}{r}-1.0 \\
-2.7 \\
5.3 \\
5.4 \\
5.0\end{array}$ & $\begin{array}{r}12.1 \\
.2 \\
-3.9 \\
3.0 \\
2.7\end{array}$ & $\begin{array}{l}16.2 \\
15.5 \\
15.6 \\
11.8 \\
11.9\end{array}$ & $\begin{array}{l}20.1 \\
20.3 \\
20.5 \\
16.9 \\
15.4\end{array}$ & $\begin{array}{l}18.2 \\
19.2 \\
21.0 \\
20.0 \\
20.1\end{array}$ & $\begin{array}{l}17.8 \\
18.8 \\
18.2 \\
18.0 \\
18.8\end{array}$ \\
\hline $\begin{array}{l}21 \\
22 \\
23 \\
24 \\
25\end{array}$ & $\begin{array}{r}11.0 \\
6.7 \\
10.7 \\
12.6 \\
13.7\end{array}$ & $\begin{array}{r}-5.4 \\
8.6 \\
8.3 \\
10.1 \\
5.1\end{array}$ & $\begin{array}{r}-20.1 \\
-17.0 \\
-6.9 \\
-2.8 \\
-3.1\end{array}$ & $\begin{array}{r}-6.9 \\
-1.0 \\
-.2 \\
-1.4 \\
.1\end{array}$ & $\begin{array}{l}7.0 \\
5.0 \\
3.2 \\
4.5 \\
8.0\end{array}$ & $\begin{array}{r}-5.5 \\
-1.7 \\
-1.0 \\
-.4 \\
-6.1\end{array}$ & $\begin{array}{r}.7 \\
.5 \\
6.6 \\
2.9 \\
-6.4\end{array}$ & $\begin{array}{r}3.4 \\
8.9 \\
12.9 \\
14.3 \\
10.3\end{array}$ & $\begin{array}{r}12.8 \\
15.3 \\
15.0 \\
9.3 \\
8.3\end{array}$ & $\begin{array}{l}16.9 \\
18.6 \\
21.0 \\
20.4 \\
19.7\end{array}$ & $\begin{array}{l}21.7 \\
22.4 \\
20.9 \\
21.5 \\
20.9\end{array}$ & $\begin{array}{l}16.3 \\
16.7 \\
16.2 \\
18.3 \\
18.0\end{array}$ \\
\hline $\begin{array}{l}26 \\
27 \\
28 \\
29 \\
30 \\
31\end{array}$ & $\begin{array}{r}13.3 \\
15.0 \\
12.6 \\
12.1 \\
11.2 \\
2.1\end{array}$ & $\begin{array}{r}-9.9 \\
-5.3 \\
2.4 \\
7.0 \\
-.8 \\
---\end{array}$ & $\begin{array}{r}-5.9 \\
-.1 \\
-5.6 \\
-10.3 \\
-1.3 \\
3.9\end{array}$ & $\begin{array}{r}.6 \\
-2.1 \\
-3.9 \\
-2.1 \\
3.8 \\
4.5\end{array}$ & $\begin{array}{l}5.3 \\
2.7 \\
1.1 \\
--- \\
--- \\
---\end{array}$ & $\begin{array}{r}-4.0 \\
-3.1 \\
.8 \\
1.7 \\
7.2 \\
4.6\end{array}$ & $\begin{array}{r}-2.6 \\
-3.9 \\
3.3 \\
.5 \\
7.6 \\
---\end{array}$ & $\begin{array}{r}6.5 \\
7.5 \\
9.7 \\
6.2 \\
-1.7 \\
.0\end{array}$ & $\begin{array}{r}10.5 \\
4.3 \\
8.4 \\
6.4 \\
14.8 \\
---\end{array}$ & $\begin{array}{l}19.8 \\
20.8 \\
20.1 \\
22.6 \\
20.2 \\
13.5\end{array}$ & $\begin{array}{l}17.5 \\
12.5 \\
16.1 \\
19.6 \\
20.1 \\
18.1\end{array}$ & $\begin{array}{r}13.5 \\
10.7 \\
10.0 \\
12.3 \\
14.1 \\
-\end{array}$ \\
\hline MAX & 15.0 & 11.8 & 10.0 & 5.8 & 8.0 & 7.2 & 11.5 & 14.3 & 19.0 & 23.7 & 22.4 & 19.3 \\
\hline
\end{tabular}

CAL YR 1990 MAX 21.9

WTR YR 1991 MAX 23.7 
EAST STEWART BASIN

VEG SPRING NEAR IONE, NV

DAILY MINIMUM AIR TEMPERATURE, WATER YEAR OCTOBER 1988 TO SEPTEMBER 1989 DEGREES CELSIUS

\begin{tabular}{|c|c|c|c|c|c|c|c|c|c|c|c|c|}
\hline DAY & OCT & NOV & DEC & JAN & FEB & MAR & APR & MAY & JUN & JUL & AUG & SEP \\
\hline $\begin{array}{l}1 \\
2 \\
3 \\
4 \\
5\end{array}$ & $\begin{array}{l}8.5 \\
9.3 \\
7.7 \\
7.7 \\
4.5\end{array}$ & $\begin{array}{r}.2 \\
-.2 \\
-.8 \\
-.7 \\
1.5\end{array}$ & $\begin{array}{r}-.1 \\
-1.4 \\
-1.8 \\
-1.5 \\
-.9\end{array}$ & $\begin{array}{r}-15.3 \\
-6.6 \\
-2.5 \\
-7.1 \\
-15.8\end{array}$ & $\begin{array}{l}-11.1 \\
-12.3 \\
-16.5 \\
-23.4 \\
-25.4\end{array}$ & $\begin{array}{r}-4.7 \\
-12.7 \\
-19.0 \\
-10.0 \\
-3.2\end{array}$ & $\begin{array}{r}-7.5 \\
-4.2 \\
-5.0 \\
-3.6 \\
2.2\end{array}$ & $\begin{array}{r}1.2 \\
.5 \\
.0 \\
2.3 \\
6.4\end{array}$ & $\begin{array}{r}1.2 \\
3.1 \\
.0 \\
.9 \\
.9\end{array}$ & $\begin{array}{r}7.1 \\
10.6 \\
12.2 \\
9.7 \\
12.5\end{array}$ & $\begin{array}{r}8.4 \\
8.6 \\
9.0 \\
8.7 \\
10.3\end{array}$ & $\begin{array}{l}7.3 \\
7.9 \\
7.7 \\
8.6 \\
8.6\end{array}$ \\
\hline $\begin{array}{r}6 \\
7 \\
8 \\
9 \\
10\end{array}$ & $\begin{array}{l}3.5 \\
3.0 \\
4.4 \\
4.4 \\
3.5\end{array}$ & $\begin{array}{l}-1.9 \\
-5.1 \\
-7.9 \\
-8.6 \\
-6.1\end{array}$ & $\begin{array}{r}-7.6 \\
-10.2 \\
-10.9 \\
-3.8 \\
-2.3\end{array}$ & $\begin{array}{r}-18.4 \\
-18.4 \\
-19.1 \\
-7.2 \\
-17.7\end{array}$ & $\begin{array}{r}-23.5 \\
-19.7 \\
-13.9 \\
-7.8 \\
-11.3\end{array}$ & $\begin{array}{r}-.8 \\
-1.0 \\
-.7 \\
.7 \\
1.6\end{array}$ & $\begin{array}{r}4.5 \\
5.4 \\
3.5 \\
2.8 \\
.0\end{array}$ & $\begin{array}{r}8.2 \\
7.5 \\
5.5 \\
.7 \\
-5.3\end{array}$ & $\begin{array}{l}.8 \\
3.7 \\
3.8 \\
3.0 \\
3.5\end{array}$ & $\begin{array}{r}12.1 \\
13.2 \\
12.7 \\
8.2 \\
7.2\end{array}$ & $\begin{array}{l}8.1 \\
8.8 \\
7.0 \\
8.9 \\
7.6\end{array}$ & $\begin{array}{r}2.5 \\
-1.5 \\
.8 \\
4.7 \\
5.6\end{array}$ \\
\hline $\begin{array}{l}11 \\
12 \\
13 \\
14 \\
15\end{array}$ & $\begin{array}{r}-1.2 \\
-.9 \\
-.6 \\
-2.1 \\
2.4\end{array}$ & $\begin{array}{r}-8.9 \\
-3.5 \\
-5.9 \\
-12.5 \\
-12.7\end{array}$ & $\begin{array}{r}-2.2 \\
1.3 \\
-1.1 \\
-18.4 \\
-18.2\end{array}$ & $\begin{array}{r}-17.6 \\
-13.5 \\
-9.5 \\
-15.6 \\
-14.4\end{array}$ & $\begin{array}{l}-12.7 \\
-15.0 \\
-16.9 \\
-13.9 \\
-10.8\end{array}$ & $\begin{array}{r}-1.0 \\
-3.0 \\
-10.8 \\
-11.5 \\
-5.7\end{array}$ & $\begin{array}{r}-1.5 \\
-2.4 \\
1.3 \\
4.1 \\
1.9\end{array}$ & $\begin{array}{l}-5.4 \\
-7.2 \\
-6.1 \\
-4.5 \\
-3.9\end{array}$ & $\begin{array}{r}2.4 \\
5.5 \\
8.9 \\
10.1 \\
7.3\end{array}$ & $\begin{array}{r}9.4 \\
7.9 \\
9.1 \\
10.9 \\
10.5\end{array}$ & $\begin{array}{r}8.6 \\
9.8 \\
10.8 \\
11.2 \\
11.4\end{array}$ & $\begin{array}{r}2.6 \\
.6 \\
3.5 \\
7.0 \\
7.7\end{array}$ \\
\hline $\begin{array}{l}16 \\
17 \\
18 \\
19 \\
20\end{array}$ & $\begin{array}{l}6.1 \\
6.8 \\
7.0 \\
5.7 \\
4.7\end{array}$ & $\begin{array}{r}-10.2 \\
-13.3 \\
-15.5 \\
-9.9 \\
-8.1\end{array}$ & $\begin{array}{r}-10.4 \\
-7.1 \\
-10.4 \\
-10.8 \\
-11.1\end{array}$ & $\begin{array}{l}-8.2 \\
-5.0 \\
-1.4 \\
-4.3 \\
-4.0\end{array}$ & $\begin{array}{r}-5.8 \\
-5.1 \\
-5.3 \\
-9.5 \\
-11.0\end{array}$ & $\begin{array}{r}-12.0 \\
-12.5 \\
-3.2 \\
-11.2 \\
-13.5\end{array}$ & $\begin{array}{l}.6 \\
3.5 \\
4.4 \\
6.8 \\
5.8\end{array}$ & $\begin{array}{r}-2.4 \\
1.2 \\
-3.6 \\
-3.5 \\
2.2\end{array}$ & $\begin{array}{r}3.3 \\
6.0 \\
10.8 \\
7.9 \\
-. .4\end{array}$ & $\begin{array}{r}8.4 \\
8.5 \\
11.4 \\
15.3 \\
11.7\end{array}$ & $\begin{array}{r}10.1 \\
8.0 \\
7.0 \\
6.7 \\
3.2\end{array}$ & $\begin{array}{r}5.4 \\
-1.8 \\
-2.4 \\
-1.4 \\
.6\end{array}$ \\
\hline $\begin{array}{l}21 \\
22 \\
23 \\
24 \\
25\end{array}$ & $\begin{array}{l}7.0 \\
5.0 \\
6.9 \\
5.5 \\
4.9\end{array}$ & $\begin{array}{r}-7.0 \\
-3.2 \\
-13.1 \\
-14.5 \\
-10.9\end{array}$ & $\begin{array}{l}-16.0 \\
-14.8 \\
-17.3 \\
-14.7 \\
-18.8\end{array}$ & $\begin{array}{r}-3.2 \\
-5.5 \\
-10.4 \\
-11.3 \\
-12.2\end{array}$ & $\begin{array}{r}-4.9 \\
-.9 \\
-1.4 \\
.4 \\
.0\end{array}$ & $\begin{array}{l}-2.9 \\
-3.9 \\
-3.1 \\
-1.8 \\
-9.6\end{array}$ & $\begin{array}{r}-2.9 \\
-6.5 \\
-7.0 \\
-12.4 \\
-12.3\end{array}$ & $\begin{array}{r}.4 \\
3.3 \\
-6.2 \\
-8.2 \\
-5.4\end{array}$ & $\begin{array}{r}-1.2 \\
4.3 \\
1.7 \\
1.5 \\
2.4\end{array}$ & $\begin{array}{r}10.3 \\
9.9 \\
8.7 \\
8.5 \\
10.0\end{array}$ & $\begin{array}{r}5.3 \\
8.1 \\
-1.0 \\
-3.1 \\
1.8\end{array}$ & $\begin{array}{l}2.7 \\
4.4 \\
6.5 \\
8.7 \\
6.2\end{array}$ \\
\hline $\begin{array}{l}26 \\
27 \\
28 \\
29 \\
30 \\
31\end{array}$ & $\begin{array}{l}4.4 \\
3.7 \\
3.3 \\
1.9 \\
1.2 \\
4.3\end{array}$ & $\begin{array}{r}-12.1 \\
-9.6 \\
-7.5 \\
-7.3 \\
-2.4 \\
-\end{array}$ & $\begin{array}{r}-21.3 \\
-16.9 \\
-18.9 \\
-15.3 \\
-9.8 \\
-15.0\end{array}$ & $\begin{array}{r}-8.3 \\
-6.0 \\
-6.1 \\
-5.4 \\
.6 \\
-1.5\end{array}$ & $\begin{array}{r}-5.1 \\
-8.3 \\
-7.4 \\
--- \\
--- \\
---\end{array}$ & $\begin{array}{r}-10.8 \\
-8.1 \\
-5.2 \\
-6.2 \\
-5.2 \\
-4.0\end{array}$ & $\begin{array}{r}-10.1 \\
-9.0 \\
-8.6 \\
-7.1 \\
-.6 \\
---\end{array}$ & $\begin{array}{r}-1.9 \\
2.3 \\
-5.6 \\
-6.3 \\
-6.8 \\
-3.5\end{array}$ & $\begin{array}{l}6.7 \\
4.9 \\
4.7 \\
8.4 \\
6.3 \\
---\end{array}$ & $\begin{array}{l}14.0 \\
12.4 \\
12.6 \\
12.9 \\
12.9 \\
13.0\end{array}$ & $\begin{array}{r}4.7 \\
7.4 \\
7.4 \\
10.1 \\
4.8 \\
4.2\end{array}$ & $\begin{array}{r}7.9 \\
7.1 \\
6.6 \\
4.6 \\
-1.0 \\
---\end{array}$ \\
\hline MIN & -2.1 & -15.5 & -21.3 & -19.1 & -25.4 & -19.0 & -12.4 & -8.2 & -1.2 & 7.1 & -3.1 & -2.4 \\
\hline
\end{tabular}

WTR YR 1989 MIN -25.4 
EAST STEWART BASIN

VEG SPRING NEAR IONE, NV

DAILY MINIMUM AIR TEMPERATURE, WATER YEAR OCTOBER 1989 TO SEPTEMBER 1990 DEGREES CELSIUS

\begin{tabular}{|c|c|c|c|c|c|c|c|c|c|c|c|c|}
\hline DAY & OCT & NOV & DEC & JAN & FEB & MAR & APR & MAY & JUN & JUL & AUG & SEP \\
\hline 1 & .6 & --- & --- & --- & --- & --- & --- & --- & --- & --- & 9.2 & 9.3 \\
\hline 2 & -4.4 & --- & --- & --- & --- & --- & --- & --- & --- & --- & 10.1 & 9.5 \\
\hline 3 & -5.4 & --- & --- & --- & --- & --- & --- & --- & --- & --- & 10.3 & 9.6 \\
\hline 4 & -5.3 & --- & --- & --- & --- & --- & --- & --- & --- & --- & 11.8 & 8.5 \\
\hline 5 & -1.5 & --- & --- & --- & --- & --- & --- & --- & --- & --- & 13.4 & 7.4 \\
\hline 6 & .7 & --- & --- & --- & --- & --- & --- & --- & --- & --- & 14.7 & 8.5 \\
\hline 7 & --- & --- & --- & --- & --- & --- & --- & --- & --- & --- & 13.0 & 9.6 \\
\hline 8 & --- & --- & --- & --- & --- & --- & --- & --- & --- & --- & 10.4 & 9.9 \\
\hline 9 & --- & --- & --- & --- & --- & --- & --- & --- & --- & --- & 10.9 & 10.8 \\
\hline 10 & --- & -- & --- & --- & --- & --- & --- & --- & --- & --- & 9.1 & 12.1 \\
\hline 11 & --- & --- & --- & --- & --- & --- & --- & --- & --- & --- & 7.4 & 12.8 \\
\hline 12 & --- & --- & --- & --- & --- & --- & --- & --- & --- & --- & 8.7 & 12.9 \\
\hline 13 & --- & --- & --- & --- & --- & --- & --- & --- & --- & 8.9 & 9.8 & 11.0 \\
\hline 14 & --- & --- & --- & --- & --- & --- & --- & --- & --- & 9.7 & 11.1 & 10.1 \\
\hline 15 & --- & --- & --- & --- & --- & --- & --- & --- & --- & 9.5 & 5.6 & 7.1 \\
\hline 16 & --- & --- & --- & --- & --- & --- & --- & --- & --- & 7.0 & 7.9 & 5.7 \\
\hline 17 & --- & --- & --- & --- & --- & --- & --- & --- & --- & 8.8 & 6.4 & 4.4 \\
\hline 18 & --- & --- & --- & --- & --- & --- & --- & --- & --- & 9.5 & -.4 & 1.1 \\
\hline 19 & --- & --- & --- & --- & --- & --- & --- & --- & --- & 9.4 & -1.9 & 2.4 \\
\hline 20 & --- & --- & --- & --- & --- & --- & --- & --- & --- & 9.0 & -2.1 & 2.6 \\
\hline 21 & --- & --- & --- & --- & --- & --- & --- & --- & --- & 9.9 & 2.4 & 2.8 \\
\hline 22 & --- & --- & --- & --- & --- & --- & --- & --- & --- & 12.0 & 3.8 & 2.5 \\
\hline 23 & --- & --- & --- & --- & --- & --- & --- & --- & --- & 10.4 & 6.2 & 1.1 \\
\hline 24 & --- & --- & --- & --- & --- & --- & --- & --- & --- & 7.5 & 3.9 & 1.3 \\
\hline 25 & --- & -- & --- & --- & --- & --- & --- & --- & --- & 6.0 & 3.3 & 2.6 \\
\hline 26 & --- & --- & --- & --- & --- & --- & --- & --- & --- & 5.2 & 2.9 & 1.9 \\
\hline 27 & --- & --- & --- & --- & --- & --- & --- & --- & --- & 8.3 & 6.6 & .8 \\
\hline 28 & --- & --- & --- & --- & --- & --- & --- & --- & --- & 10.5 & 8.4 & .0 \\
\hline 29 & --- & --- & --- & --- & --- & --- & --- & --- & --- & 12.3 & 10.9 & 1.0 \\
\hline 30 & --- & --- & --- & --- & --- & --- & --- & --- & --- & 13.5 & 8.2 & 4.4 \\
\hline 31 & --- & --- & --- & --- & --- & --- & --- & --- & --- & 11.6 & 7.3 & --- \\
\hline MIN & --- & --- & --- & --- & --- & --- & --- & --- & --- & --- & -2.1 & .0 \\
\hline
\end{tabular}


EAST STEWART BASIN

VEG SPRING NEAR IONE, NV

DAILY MINIMUM AIR TEMPERATURE, WATER YEAR OCTOBER 1990 TO SEPTEMBER 1991 DEGREES CELSIUS

\begin{tabular}{|c|c|c|c|c|c|c|c|c|c|c|c|c|}
\hline DAY & OCT & NoV & $\mathrm{DEC}$ & JAN & FEB & MAR & $\mathrm{APR}$ & MAY & JUN & JUL & AUG & SEP \\
\hline $\begin{array}{l}1 \\
2 \\
3 \\
4 \\
5\end{array}$ & $\begin{array}{r}5.0 \\
1.0 \\
.4 \\
6.6 \\
3.6\end{array}$ & $\begin{array}{r}-13.1 \\
-16.4 \\
-7.8 \\
-2.9 \\
-12.1\end{array}$ & $\begin{array}{r}-10.3 \\
-11.9 \\
-7.0 \\
-1.1 \\
-5.6\end{array}$ & $\begin{array}{l}-3.3 \\
-3.6 \\
-6.0 \\
-6.0 \\
-5.4\end{array}$ & $\begin{array}{l}-2.1 \\
-5.6 \\
-5.9 \\
-2.1 \\
-6.5\end{array}$ & $\begin{array}{r}-8.9 \\
-11.2 \\
-4.1 \\
-6.0 \\
-14.3\end{array}$ & $\begin{array}{r}-6.2 \\
-7.7 \\
-6.0 \\
.7 \\
3.6\end{array}$ & $\begin{array}{r}-8.2 \\
-7.8 \\
-8.4 \\
-8.3 \\
-.9\end{array}$ & $\begin{array}{r}-1.0 \\
2.9 \\
4.3 \\
.5 \\
-2.6\end{array}$ & $\begin{array}{r}5.1 \\
9.0 \\
11.0 \\
12.5 \\
12.2\end{array}$ & $\begin{array}{l}8.4 \\
6.2 \\
6.9 \\
8.8 \\
7.5\end{array}$ & $\begin{array}{r}8.4 \\
10.9 \\
9.1 \\
8.5 \\
7.2\end{array}$ \\
\hline $\begin{array}{r}6 \\
7 \\
8 \\
9 \\
10\end{array}$ & $\begin{array}{r}-2.0 \\
-6.5 \\
-5.6 \\
1.7 \\
2.3\end{array}$ & $\begin{array}{r}-13.7 \\
-13.8 \\
-3.1 \\
-.9 \\
2.7\end{array}$ & $\begin{array}{r}-4.8 \\
-.8 \\
-.2 \\
2.4 \\
.5\end{array}$ & $\begin{array}{l}-5.9 \\
-9.4 \\
-9.9 \\
-9.4 \\
-8.7\end{array}$ & $\begin{array}{l}-6.7 \\
-4.1 \\
-4.8 \\
-3.7 \\
-4.4\end{array}$ & $\begin{array}{r}-16.6 \\
-15.4 \\
-8.0 \\
-6.9 \\
-11.4\end{array}$ & $\begin{array}{r}-6.1 \\
-9.9 \\
-11.5 \\
-7.4 \\
-16.7\end{array}$ & $\begin{array}{r}-2.1 \\
-1.8 \\
-8.5 \\
-12.0 \\
-12.1\end{array}$ & $\begin{array}{l}1.7 \\
2.1 \\
3.9 \\
5.4 \\
7.3\end{array}$ & $\begin{array}{r}11.1 \\
11.7 \\
10.6 \\
9.1 \\
6.9\end{array}$ & $\begin{array}{r}7.8 \\
7.6 \\
9.0 \\
12.1 \\
11.1\end{array}$ & $\begin{array}{r}4.7 \\
4.3 \\
2.6 \\
-2.6 \\
-2.9\end{array}$ \\
\hline $\begin{array}{l}11 \\
12 \\
13 \\
14 \\
15\end{array}$ & $\begin{array}{l}-.9 \\
3.6 \\
2.7 \\
3.2 \\
4.0\end{array}$ & $\begin{array}{r}2.8 \\
3.4 \\
5.5 \\
-1.1 \\
-1.5\end{array}$ & $\begin{array}{r}-5.5 \\
-7.7 \\
-15.1 \\
-18.0 \\
-13.3\end{array}$ & $\begin{array}{r}-4.7 \\
-4.4 \\
-6.5 \\
-7.0 \\
-11.9\end{array}$ & $\begin{array}{l}-2.3 \\
-4.0 \\
-4.6 \\
-2.4 \\
-2.9\end{array}$ & $\begin{array}{l}-15.0 \\
-12.8 \\
-11.9 \\
-11.9 \\
-13.9\end{array}$ & $\begin{array}{r}-17.4 \\
-10.7 \\
-11.4 \\
-5.1 \\
-10.2\end{array}$ & $\begin{array}{r}-11.0 \\
-6.7 \\
-6.1 \\
-6.3 \\
-6.4\end{array}$ & $\begin{array}{l}8.8 \\
7.5 \\
3.1 \\
1.2 \\
3.2\end{array}$ & $\begin{array}{r}9.5 \\
12.4 \\
13.0 \\
10.9 \\
10.2\end{array}$ & $\begin{array}{l}9.8 \\
9.0 \\
9.0 \\
9.0 \\
8.1\end{array}$ & $\begin{array}{r}.7 \\
.5 \\
2.7 \\
4.0 \\
5.0\end{array}$ \\
\hline $\begin{array}{l}16 \\
17 \\
18 \\
19 \\
20\end{array}$ & $\begin{array}{r}-1.3 \\
.0 \\
-2.0 \\
-10.8 \\
-10.9\end{array}$ & $\begin{array}{r}.4 \\
-2.6 \\
-2.6 \\
-6.2 \\
-14.1\end{array}$ & $\begin{array}{r}-16.2 \\
-14.9 \\
-9.6 \\
-21.8 \\
-24.9\end{array}$ & $\begin{array}{r}-13.0 \\
-6.8 \\
-4.1 \\
-8.1 \\
-14.3\end{array}$ & $\begin{array}{r}-7.4 \\
-11.2 \\
-12.8 \\
-6.9 \\
-2.4\end{array}$ & $\begin{array}{r}-13.3 \\
-9.1 \\
-9.3 \\
-12.4 \\
-11.6\end{array}$ & $\begin{array}{r}-12.1 \\
-10.7 \\
-9.7 \\
-5.0 \\
-6.1\end{array}$ & $\begin{array}{r}.3 \\
-10.9 \\
-10.5 \\
-9.9 \\
-4.8\end{array}$ & $\begin{array}{l}5.7 \\
4.3 \\
4.7 \\
-.1 \\
-.2\end{array}$ & $\begin{array}{r}9.5 \\
10.0 \\
9.1 \\
7.1 \\
6.7\end{array}$ & $\begin{array}{r}7.6 \\
9.2 \\
11.8 \\
11.4 \\
10.7\end{array}$ & $\begin{array}{l}6.4 \\
7.2 \\
8.6 \\
8.5 \\
9.4\end{array}$ \\
\hline $\begin{array}{l}21 \\
22 \\
23 \\
24 \\
25\end{array}$ & $\begin{array}{r}-3.3 \\
1.2 \\
1.8 \\
3.7 \\
6.3\end{array}$ & $\begin{array}{r}-11.2 \\
-7.5 \\
2.1 \\
1.6 \\
-9.7\end{array}$ & $\begin{array}{l}-26.9 \\
-27.0 \\
-18.1 \\
-13.6 \\
-13.2\end{array}$ & $\begin{array}{r}-14.2 \\
-7.2 \\
-7.5 \\
-9.5 \\
-8.8\end{array}$ & $\begin{array}{l}-2.4 \\
-3.3 \\
-6.7 \\
-6.7 \\
-3.9\end{array}$ & $\begin{array}{r}-10.6 \\
-13.0 \\
-8.5 \\
-9.7 \\
-13.9\end{array}$ & $\begin{array}{r}-7.4 \\
-6.7 \\
-6.1 \\
-6.3 \\
-10.6\end{array}$ & $\begin{array}{r}-4.0 \\
-2.7 \\
1.1 \\
2.5 \\
.3\end{array}$ & $\begin{array}{r}2.6 \\
1.9 \\
3.0 \\
-.1 \\
-1.6\end{array}$ & $\begin{array}{r}5.9 \\
9.1 \\
10.8 \\
9.6 \\
9.9\end{array}$ & $\begin{array}{l}11.7 \\
12.4 \\
12.0 \\
11.9 \\
10.6\end{array}$ & $\begin{array}{l}7.8 \\
7.1 \\
7.4 \\
7.7 \\
9.3\end{array}$ \\
\hline $\begin{array}{l}26 \\
27 \\
28 \\
29 \\
30 \\
31\end{array}$ & $\begin{array}{r}5.2 \\
5.4 \\
4.9 \\
3.5 \\
1.9 \\
-4.4\end{array}$ & $\begin{array}{r}-17.9 \\
-14.0 \\
-9.7 \\
-1.4 \\
-7.0 \\
----\end{array}$ & $\begin{array}{r}-12.7 \\
-7.2 \\
-14.9 \\
-22.8 \\
-10.2 \\
-6.8\end{array}$ & $\begin{array}{r}-8.7 \\
-10.2 \\
-16.4 \\
-16.6 \\
-7.0 \\
-1.9\end{array}$ & $\begin{array}{r}-3.5 \\
-5.1 \\
-6.4 \\
--- \\
--- \\
---\end{array}$ & $\begin{array}{r}-14.6 \\
-11.4 \\
-11.7 \\
-9.3 \\
-6.7 \\
-3.8\end{array}$ & $\begin{array}{r}-11.7 \\
-13.7 \\
-10.5 \\
-7.6 \\
-6.9 \\
---\end{array}$ & $\begin{array}{l}-2.5 \\
-4.8 \\
-2.8 \\
-2.3 \\
-7.2 \\
-6.7\end{array}$ & $\begin{array}{r}-2.0 \\
-.4 \\
-.5 \\
-1.9 \\
1.3 \\
-.-\end{array}$ & $\begin{array}{r}9.6 \\
11.9 \\
13.0 \\
12.8 \\
11.6 \\
9.5\end{array}$ & $\begin{array}{r}9.0 \\
.7 \\
2.0 \\
9.2 \\
12.2 \\
9.9\end{array}$ & $\begin{array}{l}5.6 \\
3.8 \\
3.4 \\
4.1 \\
4.4 \\
---\end{array}$ \\
\hline MIN & -10.9 & -17.9 & -27.0 & -16.6 & -12.8 & -16.6 & -17.4 & -12.1 & $-2 \cdot 6$ & 5.1 & .7 & -2.9 \\
\hline
\end{tabular}

WTR YR 1991 MIN -27.0 
EAST STEWART BASIN

VEG SPRING NEAR IONE, NV

DAILY MEAN SOIL TEMPERATURE, WATER YEAR OCTOBER 1988 TO SEPTEMBER 1989 DEGREES CELSIUS

\begin{tabular}{|c|c|c|c|c|c|c|c|c|c|c|c|c|}
\hline DAY & OCT & $\mathrm{NOV}$ & $\mathrm{DEC}$ & JAN & FEB & MAR & APR & MAY & JUN & JUL & AUG & SEP \\
\hline $\begin{array}{l}1 \\
2 \\
3 \\
4 \\
5\end{array}$ & $\begin{array}{l}9.1 \\
9.3 \\
9.6 \\
9.0 \\
8.8\end{array}$ & $\begin{array}{l}3.7 \\
2.8 \\
3.3 \\
2.7 \\
2.7\end{array}$ & $\begin{array}{l}-2.7 \\
-2.8 \\
-3.1 \\
-3.4 \\
-3.6\end{array}$ & $\begin{array}{l}-3.7 \\
-3.8 \\
-3.6 \\
-3.5 \\
-3.3\end{array}$ & $\begin{array}{l}-4.1 \\
-5.2 \\
-6.1 \\
-6.8 \\
-8.2\end{array}$ & $\begin{array}{l}-4.7 \\
-4.2 \\
-4.5 \\
-4.8 \\
-4.3\end{array}$ & $\begin{array}{r}-1.0 \\
-1.0 \\
-1.4 \\
-.9 \\
-.1\end{array}$ & $\begin{array}{l}1.8 \\
3.5 \\
4.5 \\
6.1 \\
7.7\end{array}$ & $\begin{array}{l}3.8 \\
6.2 \\
6.5 \\
5.1 \\
5.3\end{array}$ & $\begin{array}{l}14.1 \\
15.2 \\
16.1 \\
16.6 \\
16.6\end{array}$ & $\begin{array}{l}18.4 \\
18.1 \\
18.1 \\
18.3 \\
18.2\end{array}$ & $\begin{array}{l}15.1 \\
14.9 \\
15.4 \\
15.8 \\
15.9\end{array}$ \\
\hline $\begin{array}{r}6 \\
7 \\
8 \\
9 \\
10\end{array}$ & $\begin{array}{l}7.9 \\
7.5 \\
7.3 \\
6.8 \\
5.7\end{array}$ & $\begin{array}{r}3.0 \\
1.8 \\
1.0 \\
.7 \\
.5\end{array}$ & $\begin{array}{l}-3.6 \\
-3.6 \\
-3.7 \\
-3.8 \\
-3.5\end{array}$ & $\begin{array}{l}-3.3 \\
-3.4 \\
-3.5 \\
-3.6 \\
-3.4\end{array}$ & $\begin{array}{r}-9.6 \\
-10.4 \\
-10.0 \\
-8.1 \\
-7.6\end{array}$ & $\begin{array}{l}-3.1 \\
-2.2 \\
-1.9 \\
-1.6 \\
-1.4\end{array}$ & $\begin{array}{l}-.1 \\
1.2 \\
2.5 \\
3.1 \\
3.0\end{array}$ & $\begin{array}{l}8.4 \\
8.3 \\
7.6 \\
6.8 \\
3.9\end{array}$ & $\begin{array}{l}6.1 \\
7.5 \\
7.6 \\
6.5 \\
6.3\end{array}$ & $\begin{array}{l}17.0 \\
17.4 \\
17.1 \\
15.7 \\
15.2\end{array}$ & $\begin{array}{l}17.8 \\
16.3 \\
14.3 \\
12.9 \\
13.0\end{array}$ & $\begin{array}{l}15.0 \\
13.4 \\
12.9 \\
13.5 \\
12.9\end{array}$ \\
\hline $\begin{array}{l}11 \\
12 \\
13 \\
14 \\
15\end{array}$ & $\begin{array}{l}4.3 \\
3.2 \\
2.5 \\
2.5 \\
3.0\end{array}$ & $\begin{array}{l}.4 \\
.3 \\
.2 \\
.2 \\
.2\end{array}$ & $\begin{array}{l}-4.1 \\
-3.9 \\
-3.3 \\
-3.9 \\
-4.8\end{array}$ & $\begin{array}{l}-3.4 \\
-3.4 \\
-3.6 \\
-3.7 \\
-4.1\end{array}$ & $\begin{array}{l}-7.6 \\
-7.2 \\
-6.6 \\
-6.2 \\
-5.9\end{array}$ & $\begin{array}{l}-1.7 \\
-1.7 \\
-1.8 \\
-2.9 \\
-3.3\end{array}$ & $\begin{array}{l}3.2 \\
3.8 \\
4.3 \\
5.2 \\
5.5\end{array}$ & $\begin{array}{l}2.5 \\
1.8 \\
1.3 \\
1.1 \\
1.0\end{array}$ & $\begin{array}{r}7.0 \\
7.5 \\
10.3 \\
12.1 \\
12.8\end{array}$ & $\begin{array}{l}15.4 \\
14.7 \\
13.9 \\
14.9 \\
16.2\end{array}$ & $\begin{array}{l}14.0 \\
16.0 \\
16.2 \\
16.7 \\
17.1\end{array}$ & $\begin{array}{l}12.3 \\
11.3 \\
12.0 \\
14.4 \\
14.0\end{array}$ \\
\hline $\begin{array}{l}16 \\
17 \\
18 \\
19 \\
20\end{array}$ & $\begin{array}{l}3.8 \\
4.3 \\
4.5 \\
4.6 \\
4.3\end{array}$ & $\begin{array}{r}.1 \\
.0 \\
-.1 \\
-.1 \\
-.1\end{array}$ & $\begin{array}{l}-5.0 \\
-4.7 \\
-4.1 \\
-3.8 \\
-3.6\end{array}$ & $\begin{array}{l}-4.4 \\
-4.5 \\
-4.5 \\
-4.4 \\
-4.5\end{array}$ & $\begin{array}{l}-5.6 \\
-5.2 \\
-4.9 \\
-4.5 \\
-4.7\end{array}$ & $\begin{array}{l}-2.3 \\
-3.9 \\
-3.3 \\
-2.7 \\
-3.7\end{array}$ & $\begin{array}{l}5.5 \\
6.1 \\
6.8 \\
7.4 \\
7.9\end{array}$ & $\begin{array}{l}.9 \\
3.1 \\
5.2 \\
5.7 \\
7.1\end{array}$ & $\begin{array}{l}12.1 \\
12.0 \\
13.5 \\
14.4 \\
13.7\end{array}$ & $\begin{array}{l}15.9 \\
16.4 \\
17.1 \\
17.4 \\
17.7\end{array}$ & $\begin{array}{l}17.3 \\
17.4 \\
16.7 \\
15.5 \\
13.8\end{array}$ & $\begin{array}{r}13.4 \\
8.9 \\
4.6 \\
4.9 \\
6.7\end{array}$ \\
\hline $\begin{array}{l}21 \\
22 \\
23 \\
24 \\
25\end{array}$ & $\begin{array}{l}4.3 \\
4.3 \\
4.3 \\
4.2 \\
4.2\end{array}$ & $\begin{array}{r}-.1 \\
-.2 \\
-.2 \\
-.8 \\
-1.4\end{array}$ & $\begin{array}{l}-3.5 \\
-3.6 \\
-4.1 \\
-4.1 \\
-3.9\end{array}$ & $\begin{array}{l}-4.6 \\
-4.5 \\
-4.2 \\
-4.3 \\
-4.9\end{array}$ & $\begin{array}{l}-5.1 \\
-4.4 \\
-3.9 \\
-3.7 \\
-3.4\end{array}$ & $\begin{array}{l}-3.5 \\
-2.0 \\
-1.7 \\
-2.1 \\
-1.7\end{array}$ & $\begin{array}{l}7.7 \\
5.8 \\
4.7 \\
2.7 \\
1.4\end{array}$ & $\begin{array}{l}7.5 \\
8.3 \\
7.4 \\
5.6 \\
5.3\end{array}$ & $\begin{array}{l}12.5 \\
12.9 \\
13.5 \\
12.4 \\
11.9\end{array}$ & $\begin{array}{l}16.4 \\
15.1 \\
13.5 \\
13.1 \\
13.6\end{array}$ & $\begin{array}{l}11.8 \\
13.5 \\
13.2 \\
12.1 \\
13.0\end{array}$ & $\begin{array}{l}7.7 \\
8.2 \\
9.1 \\
9.8 \\
9.6\end{array}$ \\
\hline $\begin{array}{l}26 \\
27 \\
28 \\
29 \\
30 \\
31\end{array}$ & $\begin{array}{l}4.2 \\
3.9 \\
3.7 \\
3.8 \\
3.3 \\
3.0\end{array}$ & $\begin{array}{l}-1.4 \\
-1.6 \\
-1.6 \\
-2.3 \\
-2.6 \\
-\end{array}$ & $\begin{array}{l}-3.9 \\
-4.1 \\
-4.2 \\
-4.1 \\
-4.0 \\
-3.8\end{array}$ & $\begin{array}{l}-5.1 \\
-5.1 \\
-5.2 \\
-5.0 \\
-4.6 \\
-4.4\end{array}$ & $\begin{array}{r}-3.2 \\
-3.6 \\
-4.4 \\
--- \\
--- \\
--\end{array}$ & $\begin{array}{l}-1.9 \\
-2.0 \\
-1.8 \\
-1.9 \\
-2.2 \\
-1.4\end{array}$ & $\begin{array}{r}.7 \\
.3 \\
.2 \\
.1 \\
.1 \\
-.-\end{array}$ & $\begin{array}{l}6.8 \\
8.2 \\
7.4 \\
4.8 \\
3.0 \\
2.1\end{array}$ & $\begin{array}{r}12.9 \\
12.2 \\
11.9 \\
13.0 \\
13.7 \\
\ldots-\end{array}$ & $\begin{array}{l}16.3 \\
17.5 \\
18.1 \\
18.4 \\
18.5 \\
18.7\end{array}$ & $\begin{array}{l}13.8 \\
14.8 \\
15.4 \\
16.0 \\
15.7 \\
15.2\end{array}$ & $\begin{array}{r}9.5 \\
10.0 \\
9.7 \\
8.9 \\
8.4 \\
-.-\end{array}$ \\
\hline $\begin{array}{l}\text { MEAN } \\
\text { MAX } \\
\text { MIN } \\
\text { MED }\end{array}$ & $\begin{array}{l}5.2 \\
9.6 \\
2.5 \\
4.3\end{array}$ & $\begin{array}{r}.4 \\
3.7 \\
-2.6 \\
.1\end{array}$ & $\begin{array}{l}-3.8 \\
-2.7 \\
-5.0 \\
-3.8\end{array}$ & $\begin{array}{l}-4.1 \\
-3.3 \\
-5.2 \\
-4.2\end{array}$ & $\begin{array}{r}-5.9 \\
-3.2 \\
-10.4 \\
-5.4\end{array}$ & $\begin{array}{l}-2.7 \\
-1.4 \\
-4.8 \\
-2.2\end{array}$ & $\begin{array}{r}2.8 \\
7.9 \\
-1.4 \\
2.8\end{array}$ & $\begin{array}{r}5.0 \\
8.4 \\
.9 \\
5.3\end{array}$ & $\begin{array}{r}10.1 \\
14.4 \\
3.8 \\
11.9\end{array}$ & $\begin{array}{l}16.1 \\
18.7 \\
13.1 \\
16.3\end{array}$ & $\begin{array}{l}15.5 \\
18.4 \\
11.8 \\
15.7\end{array}$ & $\begin{array}{r}11.3 \\
15.9 \\
4.6 \\
11.6\end{array}$ \\
\hline
\end{tabular}

WTR YR 1989 MEAN 4.2 MAX 18.7 MIN -10.4 MED 3.3 
EAST STEWART BASIN

VEG SPRING NEAR IONE, NV

DAILY MEAN SOIL TEMPERATURE, WATER YEAR OCTOBER 1989 TO SEPTEMBER 1990 DEGREES CELSIUS

\begin{tabular}{|c|c|c|c|c|c|c|c|c|c|c|c|c|}
\hline DAY & OCT & NOV & DEC & JAN & FEB & MAR & APR & MAY & JUN & JUL & AUG & SEP \\
\hline $\begin{array}{l}1 \\
2 \\
3 \\
4 \\
5\end{array}$ & $\begin{array}{l}7.2 \\
5.8 \\
3.8 \\
3.6 \\
3.5\end{array}$ & $\begin{array}{l}-1.5 \\
-1.9 \\
-1.8 \\
-1.4 \\
-1.0\end{array}$ & $\begin{array}{l}-5.5 \\
-5.2 \\
-4.9 \\
-4.5 \\
-3.8\end{array}$ & $\begin{array}{l}-4.7 \\
-4.6 \\
-4.4 \\
-4.5 \\
-4.5\end{array}$ & $\begin{array}{l}-6.9 \\
-6.5 \\
-6.3 \\
-5.9 \\
-5.7\end{array}$ & $\begin{array}{l}-4.1 \\
-3.8 \\
-3.4 \\
-3.5 \\
-3.5\end{array}$ & $\begin{array}{l}.1 \\
.2 \\
.2 \\
.2 \\
.2\end{array}$ & $\begin{array}{r}.2 \\
.2 \\
1.2 \\
3.1 \\
5.2\end{array}$ & $\begin{array}{l}.9 \\
1.3 \\
5.5 \\
8.7 \\
9.8\end{array}$ & $\begin{array}{l}17.2 \\
16.0 \\
15.9 \\
16.9 \\
17.7\end{array}$ & $\begin{array}{l}16.3 \\
16.6 \\
17.2 \\
17.2 \\
18.3\end{array}$ & $\begin{array}{l}17.4 \\
17.7 \\
18.0 \\
18.3 \\
17.8\end{array}$ \\
\hline $\begin{array}{r}6 \\
7 \\
8 \\
9 \\
10\end{array}$ & $\begin{array}{l}3.9 \\
4.2 \\
5.0 \\
6.0 \\
6.4\end{array}$ & $\begin{array}{l}-1.7 \\
-2.4 \\
-3.0 \\
-2.5 \\
-1.6\end{array}$ & $\begin{array}{l}-3.5 \\
-4.6 \\
-3.9 \\
-3.7 \\
-5.6\end{array}$ & $\begin{array}{l}-4.5 \\
-4.0 \\
-3.1 \\
-3.1 \\
-2.6\end{array}$ & $\begin{array}{l}-5.5 \\
-5.2 \\
-5.1 \\
-5.0 \\
-4.7\end{array}$ & $\begin{array}{l}-3.5 \\
-3.4 \\
-3.3 \\
-3.2 \\
-3.2\end{array}$ & $\begin{array}{l}.2 \\
.2 \\
.2 \\
.2 \\
.2\end{array}$ & $\begin{array}{l}6.0 \\
5.3 \\
5.7 \\
6.1 \\
3.9\end{array}$ & $\begin{array}{r}9.8 \\
10.3 \\
12.4 \\
12.5 \\
9.7\end{array}$ & $\begin{array}{l}18.2 \\
18.5 \\
18.8 \\
18.7 \\
17.2\end{array}$ & $\begin{array}{l}19.9 \\
18.2 \\
16.3 \\
16.4 \\
17.8\end{array}$ & $\begin{array}{l}17.6 \\
17.6 \\
18.1 \\
18.6 \\
19.0\end{array}$ \\
\hline $\begin{array}{l}11 \\
12 \\
13 \\
14 \\
15\end{array}$ & $\begin{array}{l}6.5 \\
6.3 \\
6.5 \\
6.2 \\
4.4\end{array}$ & $\begin{array}{r}-1.0 \\
-.8 \\
-1.3 \\
-2.4 \\
-2.9\end{array}$ & $\begin{array}{l}-8.0 \\
-7.5 \\
-6.9 \\
-7.1 \\
-6.3\end{array}$ & $\begin{array}{l}-2.4 \\
-2.7 \\
-3.0 \\
-3.2 \\
-4.1\end{array}$ & $\begin{array}{l}-4.5 \\
-4.2 \\
-4.9 \\
-6.3 \\
-7.3\end{array}$ & $\begin{array}{l}-3.1 \\
-3.1 \\
-3.1 \\
-3.1 \\
-3.1\end{array}$ & $\begin{array}{l}.2 \\
.2 \\
.2 \\
.2 \\
.2\end{array}$ & $\begin{array}{l}2.8 \\
4.2 \\
5.4 \\
5.9 \\
5.0\end{array}$ & $\begin{array}{l}8.1 \\
8.9 \\
8.4 \\
7.1 \\
7.2\end{array}$ & $\begin{array}{l}15.9 \\
16.3 \\
14.8 \\
13.8 \\
12.9\end{array}$ & $\begin{array}{l}14.7 \\
13.8 \\
15.9 \\
17.7 \\
12.4\end{array}$ & $\begin{array}{l}19.2 \\
19.2 \\
18.9 \\
18.7 \\
18.1\end{array}$ \\
\hline $\begin{array}{l}16 \\
17 \\
18 \\
19 \\
20\end{array}$ & $\begin{array}{l}3.5 \\
3.7 \\
4.3 \\
4.6 \\
4.0\end{array}$ & $\begin{array}{l}-2.4 \\
-2.2 \\
-3.0 \\
-1.8 \\
-1.3\end{array}$ & $\begin{array}{l}-7.1 \\
-7.4 \\
-7.8 \\
-8.3 \\
-8.1\end{array}$ & $\begin{array}{l}-4.3 \\
-4.2 \\
-4.3 \\
-4.9 \\
-5.5\end{array}$ & $\begin{array}{l}-7.5 \\
-7.4 \\
-7.1 \\
-6.6 \\
-6.2\end{array}$ & $\begin{array}{l}-3.0 \\
-2.9 \\
-2.7 \\
-2.2 \\
-1.4\end{array}$ & $\begin{array}{l}.2 \\
.2 \\
.2 \\
.2 \\
.2\end{array}$ & $\begin{array}{l}5.9 \\
7.0 \\
6.5 \\
5.8 \\
5.3\end{array}$ & $\begin{array}{r}7.6 \\
9.8 \\
9.6 \\
10.6 \\
11.8\end{array}$ & $\begin{array}{l}13.1 \\
13.7 \\
13.2 \\
12.6 \\
14.1\end{array}$ & $\begin{array}{r}12.6 \\
14.8 \\
12.4 \\
8.2 \\
8.4\end{array}$ & $\begin{array}{l}16.8 \\
15.0 \\
13.3 \\
11.5 \\
11.8\end{array}$ \\
\hline $\begin{array}{l}21 \\
22 \\
23 \\
24 \\
25\end{array}$ & $\begin{array}{l}4.4 \\
3.5 \\
3.3 \\
3.3 \\
2.6\end{array}$ & $\begin{array}{l}-1.8 \\
-2.2 \\
-1.9 \\
-1.5 \\
-1.9\end{array}$ & $\begin{array}{l}-7.6 \\
-6.7 \\
-6.0 \\
-5.4 \\
-5.7\end{array}$ & $\begin{array}{l}-5.7 \\
-5.7 \\
-5.7 \\
-6.0 \\
-5.8\end{array}$ & $\begin{array}{l}-5.9 \\
-5.5 \\
-5.3 \\
-5.0 \\
-4.8\end{array}$ & $\begin{array}{r}-.8 \\
-.5 \\
-.3 \\
-.2 \\
-.1\end{array}$ & $\begin{array}{l}.2 \\
.2 \\
.2 \\
.2 \\
.2\end{array}$ & $\begin{array}{l}6.5 \\
8.0 \\
6.3 \\
3.6 \\
3.8\end{array}$ & $\begin{array}{l}13.8 \\
15.4 \\
16.1 \\
16.5 \\
17.0\end{array}$ & $\begin{array}{l}16.3 \\
17.1 \\
17.5 \\
17.1 \\
16.4\end{array}$ & $\begin{array}{l}10.0 \\
11.5 \\
12.3 \\
12.4 \\
12.6\end{array}$ & $\begin{array}{r}12.0 \\
10.7 \\
9.0 \\
8.6 \\
8.7\end{array}$ \\
\hline $\begin{array}{l}26 \\
27 \\
28 \\
29 \\
30 \\
31\end{array}$ & $\begin{array}{r}1.8 \\
1.5 \\
.9 \\
.2 \\
-.4 \\
-.9\end{array}$ & $\begin{array}{r}-2.4 \\
-3.7 \\
-4.7 \\
-5.0 \\
-5.1 \\
---\end{array}$ & $\begin{array}{l}-5.8 \\
-5.1 \\
-5.0 \\
-4.9 \\
-5.0 \\
-5.0\end{array}$ & $\begin{array}{l}-5.9 \\
-7.8 \\
-8.6 \\
-7.5 \\
-7.1 \\
-7.2\end{array}$ & $\begin{array}{r}-4.5 \\
-4.4 \\
-4.3 \\
--- \\
---\end{array}$ & $\begin{array}{r}-.1 \\
.0 \\
.0 \\
.1 \\
.1 \\
.1\end{array}$ & $\begin{array}{r}.2 \\
.2 \\
.2 \\
.2 \\
.2 \\
---\end{array}$ & $\begin{array}{l}5.1 \\
5.3 \\
2.9 \\
1.7 \\
1.3 \\
1.1\end{array}$ & $\begin{array}{r}17.2 \\
16.5 \\
16.8 \\
17.5 \\
17.4 \\
\end{array}$ & $\begin{array}{l}16.8 \\
18.2 \\
17.2 \\
18.2 \\
19.7 \\
18.2\end{array}$ & $\begin{array}{l}13.3 \\
14.7 \\
15.5 \\
16.8 \\
17.4 \\
17.5\end{array}$ & $\begin{array}{l}7.5 \\
6.9 \\
5.6 \\
5.8 \\
6.9 \\
-\end{array}$ \\
\hline $\begin{array}{l}\text { MEAN } \\
\text { MAX } \\
\text { MIN } \\
\text { MED }\end{array}$ & $\begin{array}{r}3.9 \\
7.2 \\
-.9 \\
3.9\end{array}$ & $\begin{array}{r}-2.3 \\
-.8 \\
-5.1 \\
-1.9\end{array}$ & $\begin{array}{l}-5.9 \\
-3.5 \\
-8.3 \\
-5.6\end{array}$ & $\begin{array}{l}-4.9 \\
-2.4 \\
-8.6 \\
-4.5\end{array}$ & $\begin{array}{l}-5.7 \\
-4.2 \\
-7.5 \\
-5.5\end{array}$ & $\begin{array}{r}-2.1 \\
.1 \\
-4.1 \\
-3.0\end{array}$ & $\begin{array}{l}.2 \\
.2 \\
.1 \\
.2\end{array}$ & $\begin{array}{r}4.4 \\
8.0 \\
.2 \\
5.2\end{array}$ & $\begin{array}{r}11.1 \\
17.5 \\
.9 \\
10.0\end{array}$ & $\begin{array}{l}16.4 \\
19.7 \\
12.6 \\
16.9\end{array}$ & $\begin{array}{r}14.8 \\
19.9 \\
8.2 \\
15.5\end{array}$ & $\begin{array}{r}14.1 \\
19.2 \\
5.6 \\
17.1\end{array}$ \\
\hline
\end{tabular}

CAL YR 1989 MEAN 3.7 MAX 18.7 MIN -10.4 MED 3.0

WTR YR 1990 MEAN 3.7 MAX 19.9 MIN -8.6 MED .2 
EAST STEWART BASIN

VEG SPRING NEAR IONE, NV

DAILY MEAN SOIL TEMPERATURE, WATER YEAR OCTOBER 1990 TO SEPTEMBER 1991 DEGREES CELSIUS

\begin{tabular}{|c|c|c|c|c|c|c|c|c|c|c|c|c|}
\hline DAY & OCT & NOV & DEC & JAN & FEB & MAR & APR & MAY & JUN & JUL & AUG & SEP \\
\hline $\begin{array}{l}1 \\
2 \\
3 \\
4 \\
5\end{array}$ & $\begin{array}{l}7.8 \\
8.0 \\
7.3 \\
8.1 \\
7.8\end{array}$ & $\begin{array}{r}1.0 \\
.6 \\
.1 \\
-.2 \\
-.3\end{array}$ & $\begin{array}{l}-2.6 \\
-3.6 \\
-4.5 \\
-4.2 \\
-4.0\end{array}$ & $\begin{array}{l}-9.1 \\
-9.0 \\
-7.8 \\
-6.3 \\
-6.7\end{array}$ & $\begin{array}{l}-7.6 \\
-7.0 \\
-6.4 \\
-5.6 \\
-4.7\end{array}$ & $\begin{array}{l}-4.0 \\
-4.5 \\
-4.7 \\
-3.9 \\
-4.1\end{array}$ & $\begin{array}{l}-3.0 \\
-2.8 \\
-2.7 \\
-2.6 \\
-2.4\end{array}$ & $\begin{array}{l}-1.4 \\
-1.3 \\
-1.3 \\
-1.3 \\
-1.2\end{array}$ & $\begin{array}{l}-.1 \\
-.1 \\
-.1 \\
-.1 \\
-.1\end{array}$ & $\begin{array}{r}9.3 \\
11.4 \\
13.4 \\
14.7 \\
15.1\end{array}$ & $\begin{array}{l}13.8 \\
14.7 \\
14.0 \\
15.0 \\
14.9\end{array}$ & $\begin{array}{l}14.1 \\
13.4 \\
12.5 \\
10.9 \\
10.9\end{array}$ \\
\hline $\begin{array}{r}6 \\
7 \\
8 \\
9 \\
10\end{array}$ & $\begin{array}{l}6.8 \\
6.4 \\
4.4 \\
4.7 \\
5.2\end{array}$ & $\begin{array}{l}-1.5 \\
-2.3 \\
-1.0 \\
-1.3 \\
-1.8\end{array}$ & $\begin{array}{l}-5.1 \\
-5.0 \\
-5.1 \\
-4.8 \\
-4.3\end{array}$ & $\begin{array}{l}-7.0 \\
-6.3 \\
-6.8 \\
-7.6 \\
-7.2\end{array}$ & $\begin{array}{l}-4.5 \\
-4.9 \\
-5.2 \\
-5.4 \\
-5.5\end{array}$ & $\begin{array}{l}-5.6 \\
-6.4 \\
-6.5 \\
-6.1 \\
-6.0\end{array}$ & $\begin{array}{l}-2.2 \\
-2.0 \\
-1.9 \\
-1.8 \\
-1.8\end{array}$ & $\begin{array}{l}-.8 \\
-.5 \\
-.1 \\
-.1 \\
-.1\end{array}$ & $\begin{array}{l}-.1 \\
-.1 \\
-.1 \\
-.1 \\
-.1\end{array}$ & $\begin{array}{l}15.0 \\
15.0 \\
14.2 \\
12.8 \\
13.0\end{array}$ & $\begin{array}{l}15.5 \\
16.2 \\
16.6 \\
17.6 \\
17.8\end{array}$ & $\begin{array}{r}10.6 \\
9.9 \\
10.1 \\
7.3 \\
5.0\end{array}$ \\
\hline $\begin{array}{l}11 \\
12 \\
13 \\
14 \\
15\end{array}$ & $\begin{array}{l}5.0 \\
4.8 \\
5.3 \\
5.2 \\
5.8\end{array}$ & $\begin{array}{r}-1.4 \\
-1.3 \\
-.7 \\
-.4 \\
-.3\end{array}$ & $\begin{array}{l}-3.1 \\
-2.8 \\
-3.0 \\
-3.6 \\
-4.3\end{array}$ & $\begin{array}{l}-6.2 \\
-5.4 \\
-4.7 \\
-4.5 \\
-4.5\end{array}$ & $\begin{array}{l}-5.4 \\
-5.0 \\
-4.2 \\
-3.9 \\
-3.0\end{array}$ & $\begin{array}{l}-5.8 \\
-5.8 \\
-5.5 \\
-5.1 \\
-4.7\end{array}$ & $\begin{array}{l}-1.9 \\
-1.9 \\
-2.0 \\
-2.0 \\
-2.0\end{array}$ & $\begin{array}{l}-.1 \\
-.1 \\
-.1 \\
-.1 \\
-.1\end{array}$ & $\begin{array}{r}-.1 \\
-.1 \\
.3 \\
1.9 \\
3.5\end{array}$ & $\begin{array}{l}14.5 \\
15.6 \\
16.2 \\
16.5 \\
16.5\end{array}$ & $\begin{array}{l}16.4 \\
15.3 \\
14.2 \\
14.1 \\
13.2\end{array}$ & $\begin{array}{r}5.7 \\
7.2 \\
8.9 \\
10.4 \\
10.6\end{array}$ \\
\hline $\begin{array}{l}16 \\
17 \\
18 \\
19 \\
20\end{array}$ & $\begin{array}{l}5.7 \\
4.3 \\
4.9 \\
3.3 \\
2.3\end{array}$ & $\begin{array}{l}-.3 \\
-.3 \\
-.2 \\
-.3 \\
-.4\end{array}$ & $\begin{array}{l}-4.3 \\
-4.0 \\
-3.9 \\
-5.5 \\
-6.6\end{array}$ & $\begin{array}{l}-4.7 \\
-5.0 \\
-4.9 \\
-5.2 \\
-5.8\end{array}$ & $\begin{array}{l}-2.4 \\
-3.0 \\
-3.5 \\
-3.8 \\
-4.1\end{array}$ & $\begin{array}{l}-4.5 \\
-4.3 \\
-4.1 \\
-4.0 \\
-3.9\end{array}$ & $\begin{array}{l}-2.0 \\
-2.0 \\
-2.0 \\
-2.0 \\
-2.0\end{array}$ & $\begin{array}{l}-.1 \\
-.1 \\
-.1 \\
-.1 \\
-.1\end{array}$ & $\begin{array}{l}4.9 \\
5.8 \\
6.8 \\
6.5 \\
6.1\end{array}$ & $\begin{array}{l}16.4 \\
16.8 \\
16.8 \\
16.2 \\
14.8\end{array}$ & $\begin{array}{l}12.8 \\
13.9 \\
15.3 \\
15.9 \\
16.8\end{array}$ & $\begin{array}{l}11.5 \\
12.1 \\
12.9 \\
13.1 \\
13.0\end{array}$ \\
\hline $\begin{array}{l}21 \\
22 \\
23 \\
24 \\
25\end{array}$ & $\begin{array}{l}1.5 \\
1.4 \\
1.5 \\
2.1 \\
2.5\end{array}$ & $\begin{array}{r}-.9 \\
-1.4 \\
-1.6 \\
-2.1 \\
-2.3\end{array}$ & $\begin{array}{l}-7.9 \\
-9.3 \\
-9.7 \\
-9.6 \\
-9.0\end{array}$ & $\begin{array}{l}-6.4 \\
-6.9 \\
-6.9 \\
-6.9 \\
-8.0\end{array}$ & $\begin{array}{l}-4.2 \\
-4.0 \\
-4.4 \\
-5.1 \\
-5.5\end{array}$ & $\begin{array}{l}-3.8 \\
-3.7 \\
-3.6 \\
-3.5 \\
-3.4\end{array}$ & $\begin{array}{l}-2.0 \\
-1.9 \\
-1.9 \\
-1.8 \\
-1.7\end{array}$ & $\begin{array}{l}-.1 \\
-.1 \\
-.1 \\
-.1 \\
-.1\end{array}$ & $\begin{array}{l}7.5 \\
8.7 \\
9.5 \\
8.3 \\
6.9\end{array}$ & $\begin{array}{l}13.5 \\
13.7 \\
15.4 \\
15.3 \\
15.3\end{array}$ & $\begin{array}{l}17.7 \\
18.1 \\
17.9 \\
17.2 \\
17.3\end{array}$ & $\begin{array}{l}12.9 \\
12.7 \\
12.6 \\
12.4 \\
12.1\end{array}$ \\
\hline $\begin{array}{l}26 \\
27 \\
28 \\
29 \\
30 \\
31\end{array}$ & $\begin{array}{l}3.2 \\
3.0 \\
2.9 \\
2.9 \\
2.4 \\
1.9\end{array}$ & $\begin{array}{l}-2.3 \\
-2.2 \\
-2.2 \\
-2.2 \\
-2.0 \\
-.-\end{array}$ & $\begin{array}{r}-8.4 \\
-8.2 \\
-8.2 \\
-10.0 \\
-10.7 \\
-10.0\end{array}$ & $\begin{array}{l}-8.3 \\
-8.8 \\
-9.2 \\
-9.6 \\
-9.5 \\
-8.3\end{array}$ & $\begin{array}{r}-5.1 \\
-4.7 \\
-3.9 \\
--- \\
--- \\
--\end{array}$ & $\begin{array}{l}-3.3 \\
-3.3 \\
-3.2 \\
-3.2 \\
-3.1 \\
-3.1\end{array}$ & $\begin{array}{l}-1.7 \\
-1.7 \\
-1.7 \\
-1.6 \\
-1.5 \\
---\end{array}$ & $\begin{array}{l}-.1 \\
-.1 \\
-.1 \\
-.1 \\
-.1 \\
-.1\end{array}$ & $\begin{array}{l}6.5 \\
6.1 \\
5.9 \\
5.5 \\
6.8 \\
---\end{array}$ & $\begin{array}{l}14.5 \\
14.2 \\
14.5 \\
15.0 \\
15.0 \\
13.5\end{array}$ & $\begin{array}{l}15.8 \\
14.3 \\
13.2 \\
15.1 \\
16.4 \\
16.1\end{array}$ & $\begin{array}{r}11.3 \\
8.6 \\
6.3 \\
7.0 \\
7.7 \\
-.-\end{array}$ \\
\hline $\begin{array}{l}\text { MEAN } \\
\text { MAX } \\
\text { MIN } \\
\text { MED }\end{array}$ & $\begin{array}{l}4.5 \\
8.1 \\
1.4 \\
4.7\end{array}$ & $\begin{array}{r}-1.1 \\
1.0 \\
-2.3 \\
-1.2\end{array}$ & $\begin{array}{r}-6.0 \\
-2.6 \\
-10.7 \\
-5.0\end{array}$ & $\begin{array}{l}-6.9 \\
-4.5 \\
-9.6 \\
-6.9\end{array}$ & $\begin{array}{l}-4.7 \\
-2.4 \\
-7.6 \\
-4.7\end{array}$ & $\begin{array}{l}-4.4 \\
-3.1 \\
-6.5 \\
-4.1\end{array}$ & $\begin{array}{l}-2.0 \\
-1.5 \\
-3.0 \\
-2.0\end{array}$ & $\begin{array}{r}-.3 \\
-.1 \\
-1.4 \\
-.1\end{array}$ & $\begin{array}{l}3.5 \\
9.5 \\
-.1 \\
4.2\end{array}$ & $\begin{array}{r}14.6 \\
16.8 \\
9.3 \\
15.0\end{array}$ & $\begin{array}{l}15.6 \\
18.1 \\
12.8 \\
15.5\end{array}$ & $\begin{array}{r}10.5 \\
14.1 \\
5.0 \\
10.9\end{array}$ \\
\hline
\end{tabular}

CAL YR 1990 MEAN 3.9 MAX 19.9 MIN -10.7 MED .9

WTR YR 1991 MEAN 2.0 MAX 18.1 MIN -10.7 MED -.3 
EAST STEWART BASIN

VEG SPRING NEAR IONE, NV

DAILY TOTAL SOLAR RADIATION, INCIDENTAL, WATER YEAR OCTOBER 1990 TO SEPTEMBER 1991 CALORIES PER SQUARE CENTIMETER

\begin{tabular}{|c|c|c|c|c|c|c|c|c|c|c|c|c|}
\hline DAY & OCT & NOV & DEC & JAN & FEB & MAR & APR & MAY & JUN & JUL & AUG & SEP \\
\hline 1 & --- & --- & 132 & 134 & 197 & 181 & 206 & 236 & 449 & 538 & 556 & 239 \\
\hline 2 & --- & --- & 130 & 142 & 87 & 231 & 514 & 183 & 693 & 663 & 256 & 298 \\
\hline 3 & --- & --- & 131 & 76 & 207 & 105 & 560 & 419 & 448 & 661 & 537 & 144 \\
\hline 4 & --- & --- & 148 & 34 & 182 & 89 & 458 & 685 & 488 & 670 & 602 & 264 \\
\hline 5 & --- & --- & 131 & 116 & 158 & 202 & 488 & 652 & 690 & 591 & 546 & 202 \\
\hline 6 & --- & --- & 128 & 102 & 211 & 268 & 381 & 468 & 699 & 656 & 607 & 124 \\
\hline 7 & --- & --- & 136 & 123 & 220 & 370 & 586 & 538 & 692 & 531 & 553 & 227 \\
\hline 8 & --- & --- & 127 & 143 & 223 & 376 & 598 & 479 & 384 & 319 & 604 & 315 \\
\hline 9 & --- & 192 & 127 & 140 & 218 & 247 & 487 & 240 & 482 & 333 & 606 & 126 \\
\hline 10 & --- & 186 & 124 & 112 & 226 & 314 & 401 & 419 & 578 & 523 & 512 & 237 \\
\hline 11 & --- & 155 & 89 & 107 & 203 & 355 & 400 & 483 & 656 & 540 & 394 & 315 \\
\hline 12 & --- & 163 & 86 & 108 & 202 & 306 & 323 & 682 & 664 & 627 & 284 & 395 \\
\hline 13 & --- & 144 & 43 & 101 & 184 & 268 & 557 & 342 & 618 & 619 & 265 & 444 \\
\hline 14 & --- & 165 & 120 & 166 & 241 & 246 & 449 & 465 & 680 & 661 & 431 & 462 \\
\hline 15 & --- & 158 & 138 & 62 & 210 & 219 & 433 & 714 & 656 & 651 & 250 & 464 \\
\hline 16 & --- & 150 & 112 & 141 & 92 & 452 & 600 & 541 & 606 & 664 & 232 & 456 \\
\hline 17 & --- & 125 & 119 & 152 & 174 & 421 & 384 & 341 & 535 & 639 & 447 & 450 \\
\hline 18 & --- & 114 & 149 & 145 & 274 & 263 & 513 & 330 & 414 & 597 & 510 & 421 \\
\hline 19 & --- & 76 & 58 & 148 & 267 & 266 & 489 & 301 & 616 & 560 & 497 & 433 \\
\hline 20 & --- & 154 & 90 & 151 & 287 & 344 & 417 & 316 & 526 & 465 & 560 & 380 \\
\hline 21 & --- & 135 & 55 & 152 & 230 & 91 & 406 & 398 & 583 & 330 & 560 & 433 \\
\hline 22 & --- & 150 & 118 & 156 & 281 & 452 & 278 & 658 & 670 & 538 & 556 & 419 \\
\hline 23 & --- & 154 & 122 & 144 & 283 & 525 & 502 & 590 & 625 & 641 & 452 & 418 \\
\hline 24 & --- & 141 & 122 & 164 & 290 & 495 & 442 & 389 & 467 & 324 & 472 & 405 \\
\hline 25 & --- & 140 & 139 & 169 & 292 & 202 & 230 & 640 & 279 & 514 & 466 & 370 \\
\hline 26 & --- & 124 & 105 & 175 & 291 & 431 & 328 & 673 & 520 & 250 & 479 & 244 \\
\hline 27 & --- & 140 & 127 & 174 & 240 & 299 & 622 & 681 & 289 & 336 & 309 & 166 \\
\hline 28 & --- & 140 & 124 & 177 & 338 & 538 & 664 & 612 & 414 & 309 & 540 & 141 \\
\hline 29 & --- & 152 & 109 & 182 & --- & 540 & 677 & 433 & 309 & 526 & 530 & 259 \\
\hline 30 & --- & 142 & 123 & 191 & --- & 556 & 552 & 283 & 666 & 287 & 536 & 357 \\
\hline 31 & --- & --- & 125 & 161 & --- & 483 & --- & 350 & --- & 196 & 413 & --- \\
\hline TOTAL & --- & --- & 3587 & 4248 & 6308 & 10135 & 13945 & 14541 & 16396 & 15759 & 14562 & 9608 \\
\hline
\end{tabular}


EAST STEWART BASIN

VEG SPRING NEAR IONE, NV

DAILY MEAN RELATIVE HUMIDITY, WATER YEAR OCTOBER 1990 TO SEPTEMBER 1991 PERCENT

\begin{tabular}{|c|c|c|c|c|c|c|c|c|c|c|c|c|}
\hline DAY & OCT & NOV & DEC & JAN & FEB & MAR & APR & MAY & JUN & JUL & AUG & SEP \\
\hline 1 & 40 & 98 & 31 & 23 & 21 & 95 & 81 & 85 & 81 & 44 & 46 & 31 \\
\hline 2 & 54 & 72 & 32 & 22 & 43 & 78 & 65 & 99 & 49 & 29 & 52 & 27 \\
\hline 3 & 46 & 33 & 36 & 57 & 63 & 76 & 29 & 99 & 48 & 24 & 46 & 34 \\
\hline 4 & 29 & 46 & 22 & 82 & 49 & 99 & 33 & 66 & 44 & 17 & 26 & 37 \\
\hline 5 & 35 & 95 & 36 & 23 & 83 & 88 & 25 & 30 & 43 & 20 & 20 & 48 \\
\hline 6 & 67 & 94 & 23 & 35 & 87 & 85 & 35 & 74 & 24 & 17 & 18 & 86 \\
\hline 7 & 65 & 45 & 21 & 73 & 35 & 48 & 41 & 60 & 25 & 17 & 19 & 85 \\
\hline 8 & 22 & 91 & 20 & 50 & 29 & 27 & 38 & 50 & 24 & 26 & 16 & 67 \\
\hline 9 & 20 & 24 & 20 & 51 & 25 & 28 & 36 & 94 & 26 & 42 & 15 & 87 \\
\hline 10 & 23 & 19 & 26 & 91 & 26 & 42 & 88 & 95 & 25 & 33 & 16 & 94 \\
\hline 11 & 28 & 20 & 77 & 75 & 26 & 93 & 91 & 79 & 21 & 24 & 20 & 71 \\
\hline 12 & 20 & 19 & 98 & 86 & 39 & 76 & 97 & 69 & 20 & 20 & 31 & 64 \\
\hline 13 & 25 & 19 & 96 & 100 & 85 & 69 & 78 & 75 & 22 & 19 & 44 & 41 \\
\hline 14 & 28 & 25 & 79 & 93 & 57 & 96 & 55 & 100 & 22 & 18 & 45 & 41 \\
\hline 15 & 37 & 47 & 52 & 99 & 52 & 96 & 43 & 64 & 22 & 17 & 66 & 18 \\
\hline 16 & 37 & 30 & 90 & 70 & 100 & 62 & 47 & 39 & 20 & 16 & 70 & 18 \\
\hline 17 & 23 & 57 & 94 & 76 & 98 & 51 & 81 & 92 & 20 & 19 & 34 & 18 \\
\hline 18 & 70 & 54 & 75 & 48 & 97 & 85 & 54 & 98 & 18 & 21 & 23 & 20 \\
\hline 19 & 99 & 89 & 83 & 45 & 58 & 86 & 36 & 93 & 23 & 30 & 22 & 22 \\
\hline 20 & 54 & 94 & 80 & 47 & 23 & 87 & 52 & 84 & 20 & 27 & 19 & 19 \\
\hline 21 & 35 & 81 & 79 & 39 & 21 & 96 & 86 & 95 & 20 & 26 & 17 & 19 \\
\hline 22 & 60 & 34 & 76 & 35 & 24 & 78 & 95 & 71 & 20 & 20 & 16 & 20 \\
\hline 23 & 43 & 20 & 71 & 31 & 36 & 63 & 72 & 53 & 20 & 18 & 18 & 18 \\
\hline 24 & 35 & 19 & 41 & 36 & 25 & 62 & 62 & 59 & 32 & 24 & 19 & 18 \\
\hline 25 & 22 & 29 & 40 & 24 & 23 & 93 & 98 & 40 & 69 & 23 & 40 & 18 \\
\hline 26 & 21 & 90 & 65 & 27 & 23 & 81 & 99 & 36 & 64 & 23 & 44 & 45 \\
\hline 27 & 18 & 59 & 24 & 32 & 44 & 94 & 90 & 38 & 75 & 20 & 29 & 70 \\
\hline 28 & 19 & 31 & 34 & 34 & 86 & 59 & 46 & 30 & 74 & 21 & 19 & 70 \\
\hline 29 & 20 & 20 & 62 & 28 & --- & 62 & 44 & 41 & 91 & 18 & 17 & 66 \\
\hline 30 & 21 & 37 & 25 & 22 & --- & 47 & 24 & 100 & 54 & 26 & 16 & 50 \\
\hline 31 & 53 & --- & 24 & 21 & --- & 37 & --- & 100 & --- & 53 & 22 & --- \\
\hline MEAN & 38 & 50 & 53 & 51 & 49 & 72 & 61 & 71 & 37 & 24 & 29 & 44 \\
\hline MAX & 99 & 98 & 98 & 100 & 100 & 99 & 99 & 100 & 91 & 53 & 70 & 94 \\
\hline MIN & 18 & 19 & 20 & 21 & 21 & 27 & 24 & 30 & 18 & 16 & 15 & 18 \\
\hline MED & 35 & 41 & 41 & 45 & 41 & 78 & 54 & 74 & 24 & 21 & 22 & 39 \\
\hline
\end{tabular}

$\begin{array}{llllllll}\text { WTR YR } 1991 \text { MEAN } 48 \quad \text { MAX } 100 \quad \text { MIN } 15 & \text { MED } 41\end{array}$ 
EAST STEWART BASIN

VEG SPRING NEAR IONE, NV

DAILY TOTAL PRECIPITATION, WATER YEAR OCTOBER 1986 TO SEPTEMBER 1987 INCHES

\begin{tabular}{|c|c|c|c|c|c|c|c|c|c|c|c|c|}
\hline$D A Y$ & $\mathrm{OCT}$ & NOV & DEC & JAN & FEB & MAR & APR & MAY & JUN & JUL & AUG & SEP \\
\hline 1 & .00 & .00 & .00 & e. 05 & e. 00 & e.00 & .00 & .00 & .00 & .00 & .00 & .00 \\
\hline 2 & .00 & .00 & .00 & e. 00 & e. 21 & e. 00 & .00 & .00 & .00 & .00 & .00 & .00 \\
\hline 3 & .00 & .00 & .00 & e. 11 & e.21 & e. 00 & .35 & .00 & .00 & .00 & .00 & .00 \\
\hline 4 & .00 & .00 & .00 & e.21 & e.00 & e. 11 & .00 & .00 & .00 & .00 & .00 & .00 \\
\hline 5 & .00 & .00 & .00 & e. 11 & e. 00 & e. 21 & .00 & .00 & .00 & .00 & .00 & .00 \\
\hline 6 & .00 & .00 & .00 & e. 11 & e. 00 & e.05 & .00 & .00 & .60 & .00 & .00 & .00 \\
\hline 7 & .00 & e. 20 & .00 & e. 00 & e. 00 & e. 37 & .00 & .00 & .00 & .00 & .00 & .00 \\
\hline 8 & .00 & .00 & .00 & e. 00 & e.0o & e. 00 & .00 & .00 & .00 & .00 & .00 & .00 \\
\hline 9 & .00 & .00 & .00 & e. 00 & e.00 & e. 00 & .00 & .55 & .00 & .00 & .00 & .00 \\
\hline 10 & .00 & .00 & .00 & e. 00 & e. 00 & e. 00 & .00 & .00 & .00 & .00 & .00 & .00 \\
\hline 11 & .00 & .00 & .00 & e. 00 & e. 53 & e. 00 & .00 & .00 & .00 & .00 & .00 & .00 \\
\hline 12 & .00 & .00 & .00 & e.00 & e. 11 & e. 00 & .00 & .00 & .00 & .00 & .00 & .00 \\
\hline 13 & .00 & .00 & .00 & e. 05 & e. 53 & e. 11 & .00 & .00 & .00 & .00 & .00 & .00 \\
\hline 14 & .00 & .00 & .00 & e. 05 & e. 00 & e. 43 & .00 & .30 & .00 & .00 & .00 & .00 \\
\hline 15 & .00 & .00 & .00 & e. 00 & e. 21 & e. 96 & .00 & .95 & .00 & .00 & .00 & .00 \\
\hline 16 & .00 & .00 & .00 & e. 00 & e. 00 & e. 21 & .00 & 1.10 & .00 & .00 & .00 & .00 \\
\hline 17 & .90 & .00 & .00 & e. 00 & e.00 & e.00 & .00 & .00 & .00 & .00 & .00 & .00 \\
\hline 18 & .00 & .00 & .00 & e. 00 & e. 21 & e. 21 & .00 & .40 & .00 & .00 & .00 & .00 \\
\hline 19 & .00 & .00 & .15 & e.00 & e. 11 & e. 43 & .00 & .00 & .00 & .00 & .00 & .00 \\
\hline 20 & .00 & .00 & .00 & e.00 & e. 00 & e. 00 & .00 & .00 & .00 & .00 & .00 & .00 \\
\hline 21 & .00 & .00 & .00 & e. 00 & e. 00 & $\mathrm{e} 1.17$ & .00 & .30 & .00 & .00 & .00 & .00 \\
\hline 22 & .00 & .00 & .00 & $e .00$ & e. 00 & e. 00 & .00 & .00 & .00 & .00 & .00 & .00 \\
\hline 23 & .00 & .00 & e. 00 & e. 00 & e. 64 & e. 16 & .00 & .00 & .00 & .00 & .00 & .00 \\
\hline 24 & .00 & .00 & e. 00 & e. 00 & e.21 & e. 26 & .00 & .25 & .00 & .00 & .00 & .00 \\
\hline 25 & .00 & .00 & e. 00 & e. 00 & e. 00 & e. 00 & .00 & .68 & .00 & .00 & .00 & .00 \\
\hline 26 & .00 & .00 & e. 00 & e. 00 & e. 00 & e. 00 & .00 & .87 & .00 & .00 & .00 & .00 \\
\hline 27 & .00 & .00 & e. 00 & e. 32 & e. 00 & e.00 & .00 & .00 & .00 & .00 & .00 & .00 \\
\hline 28 & .00 & .00 & e. 05 & e. 00 & e.00 & e. 00 & .75 & .00 & .00 & .00 & .00 & .00 \\
\hline 29 & .00 & e. 35 & e. 00 & e. 00 & --- & e. 00 & .25 & .00 & .00 & .00 & .00 & .00 \\
\hline 30 & .00 & .00 & e. 00 & e. 00 & --- & e. 00 & .00 & .00 & .00 & .00 & .00 & .00 \\
\hline 31 & .00 & --- & e. 00 & e. 00 & --- & .00 & --- & .00 & --- & .00 & .00 & \\
\hline TOTAL & 0.90 & 0.55 & 0.20 & 1.01 & 2.97 & 4.68 & 1.35 & 5.40 & 0.60 & 0.00 & 0.00 & 0.00 \\
\hline
\end{tabular}

WTR YR 1987 TOTAL 17.66

e Estimated 
EAST STEWART BASIN

VEG SPRING NEAR IONE, NV

DAILY TOTAL PRECIPITATION, WATER YEAR OCTOBER 1987 TO SEPTEMBER 1988 INCHES

\begin{tabular}{|c|c|c|c|c|c|c|c|c|c|c|c|c|}
\hline DAY & OCT & NOV & DEC & JAN & FEB & MAR & $\mathrm{APR}$ & MAY & JUN & JUL & AUG & SEP \\
\hline 1 & .00 & e. 44 & .10 & e. 00 & $e .00$ & e. 32 & e. 00 & e. 40 & e. 00 & .00 & .00 & .00 \\
\hline 2 & .00 & e. 49 & .00 & e. 16 & e. 00 & e. 00 & e. 00 & e. 00 & e. 00 & .00 & .10 & .00 \\
\hline 3 & .00 & e. 55 & .00 & e2.16 & e. 00 & e. 08 & e. 00 & e. 00 & e. 00 & .00 & .00 & .00 \\
\hline 4 & .00 & .00 & .00 & e. 16 & e.00 & e.00 & e. 00 & e. 00 & $e .00$ & .00 & .00 & .00 \\
\hline 5 & .00 & .80 & .15 & e. 32 & e.00 & e. 00 & e. 00 & e. 30 & $e .00$ & .00 & .70 & .00 \\
\hline 6 & .00 & .50 & .60 & e. 16 & e. 00 & e. 00 & e.00 & el. 1.00 & e. 00 & .00 & .40 & .00 \\
\hline 7 & .00 & .10 & .00 & e. 00 & e. 00 & e. 00 & e.00 & e. 20 & e.00 & .00 & .00 & .00 \\
\hline 8 & .00 & .00 & .54 & e. 00 & e. 00 & e. 00 & e. 00 & e. 20 & e. 10 & .00 & .00 & .00 \\
\hline 9 & .00 & .00 & .00 & e. 64 & e. 00 & e. 00 & $e .00$ & e. 00 & e. 00 & .00 & .00 & .00 \\
\hline 10 & .00 & .00 & .00 & e. 32 & e. 00 & e. 00 & e.00 & e. .00 & e. 00 & .00 & .00 & .00 \\
\hline 11 & .00 & .00 & e. 16 & e. 00 & e. 00 & e. 00 & e. 00 & e. 00 & e. 00 & .00 & .00 & .00 \\
\hline 12 & .25 & .00 & e. 00 & e. 00 & e.00 & e. 00 & e. 00 & e. 00 & e.00 & .00 & .00 & .00 \\
\hline 13 & .30 & .50 & e. 00 & e. 00 & e. 00 & e. 00 & e.00 & e. 00 & e. 00 & .00 & .00 & .00 \\
\hline 14 & .00 & .10 & e. 00 & e. 00 & e. 00 & e. 00 & e. 60 & e. 00 & e.00 & .00 & .00 & .00 \\
\hline 15 & .00 & .00 & $e .00$ & e. 00 & e. 00 & e. 00 & e1.10 & e. 00 & e.00 & .00 & .00 & .00 \\
\hline 16 & .00 & .10 & e. 32 & e. 00 & e. 00 & e. 00 & e. 04 & e. 00 & e. 00 & .00 & .00 & .00 \\
\hline 17 & .00 & .05 & e. 00 & e. 32 & e. 00 & $e .00$ & e1.00 & e. 00 & e. 00 & .00 & .00 & .00 \\
\hline 18 & .00 & .00 & e. 00 & e. 00 & e. 00 & e.00 & e. 20 & e. 00 & e. 00 & .00 & .00 & .00 \\
\hline 19 & .00 & .00 & e. 16 & e.0o & e. 00 & e. 00 & e. 00 & e.00 & e.0o & .00 & .00 & .00 \\
\hline 20 & .00 & .10 & e. 00 & e. 00 & e. 00 & e.00 & e1.90 & e. 00 & e. 00 & .00 & .00 & 1.25 \\
\hline 21 & .00 & .00 & e. 24 & e. 00 & e. 00 & e. 00 & e. 60 & e. 00 & e. 20 & .00 & .20 & .20 \\
\hline 22 & .30 & .00 & e1. 12 & e. 00 & e. 00 & e.00 & e. 10 & e. 00 & e. 00 & .00 & .00 & .00 \\
\hline 23 & .30 & .00 & e. 16 & e.00 & e. 00 & e.00 & e. 60 & e. 00 & e. 00 & .00 & .00 & .00 \\
\hline 24 & .15 & .05 & e. 32 & e. 00 & e.00 & e.00 & e.00 & e. 00 & e. 00 & .25 & .00 & .00 \\
\hline 25 & .00 & .20 & e. 00 & e. 00 & e. 00 & e. 00 & e. 00 & e. 00 & e. 00 & .10 & .00 & .00 \\
\hline 26 & .00 & .00 & e. 00 & e. 00 & e. 00 & e. 00 & e. 00 & e.00 & e. 00 & .10 & .40 & .00 \\
\hline 27 & .00 & .00 & e. 00 & e. 00 & e. 32 & e. 00 & e. 00 & e. 00 & .00 & .00 & .00 & .00 \\
\hline 28 & .00 & .00 & e. 00 & e. 00 & e. 64 & e.00 & e. 00 & e. 00 & .00 & .00 & .00 & .00 \\
\hline 29 & .55 & .00 & e. 00 & e. 00 & e. 16 & e.00 & e. 00 & e. 80 & .00 & .00 & .00 & .00 \\
\hline 30 & e. 22 & .00 & e. 16 & e. 00 & -- & e. 00 & e. 00 & e. 20 & .00 & .00 & .05 & .00 \\
\hline 31 & .00 & --- & e. 00 & e. 00 & --- & e. 00 & -- & e. 00 & --- & .00 & .00 & -- \\
\hline TOTAL & 2.07 & 3.98 & 4.03 & 4.24 & 1.12 & 0.40 & 6.14 & 3.10 & 0.30 & 0.45 & 1.85 & 1.45 \\
\hline
\end{tabular}

CAL YR 1987 TOTAL 26.09

WTR YR 1988 TOTAL 29.13

e Estimated 
EAST STEWART BASIN

VEG SPRING NEAR IONE, NV

DAILY TOTAL PRECIPITATION, WATER YEAR OCTOBER 1988 TO SEPTEMBER 1989 INCHES

\begin{tabular}{|c|c|c|c|c|c|c|c|c|c|c|c|c|}
\hline DAY & OCT & NOV & DEC & JAN & FEB & MAR & APR & MAY & JUN & JUL & AUG & SEP \\
\hline 1 & .00 & .10 & .00 & .00 & e. 00 & .00 & e. 20 & .00 & .00 & .00 & .00 & .00 \\
\hline 2 & .00 & .10 & .00 & .00 & e. 00 & .75 & e. 10 & .00 & .00 & .00 & .00 & .00 \\
\hline 3 & .00 & .00 & .00 & .00 & e. 06 & .00 & .00 & .00 & .45 & .00 & .00 & .00 \\
\hline 4 & .00 & .00 & .00 & .00 & e. 13 & .00 & .00 & .00 & .35 & .00 & .00 & .00 \\
\hline 5 & .00 & .00 & .00 & .30 & e. 00 & .00 & .00 & .00 & .10 & .00 & .00 & .00 \\
\hline 6 & .00 & .00 & .00 & .05 & e. 00 & .10 & .00 & .00 & .00 & .00 & .10 & .00 \\
\hline 7 & .00 & .00 & .00 & .05 & e. 00 & .00 & .00 & .00 & .00 & .00 & .05 & .00 \\
\hline 8 & .00 & .00 & .00 & .00 & e.00 & .00 & .00 & .00 & .05 & .00 & .30 & .00 \\
\hline 9 & .00 & .00 & .00 & .15 & e. 00 & .00 & .00 & .20 & .45 & .00 & .00 & .00 \\
\hline 10 & .10 & .00 & .00 & .10 & e. 00 & .00 & .00 & .40 & .00 & .00 & .00 & .00 \\
\hline 11 & .80 & .00 & .00 & .10 & e.00 & .00 & .00 & .30 & .30 & .00 & .00 & .00 \\
\hline 12 & .00 & .00 & .00 & .00 & e. 65 & .00 & .00 & .00 & .00 & .15 & .00 & .00 \\
\hline 13 & .00 & e. 18 & .00 & .00 & e. 13 & .00 & .00 & .10 & .00 & .00 & .00 & .00 \\
\hline 14 & .00 & e. 48 & .00 & .00 & e. 00 & .00 & .00 & .25 & .00 & .00 & .00 & .00 \\
\hline 15 & .00 & .00 & .25 & .00 & e. 00 & .00 & .00 & .15 & .00 & .00 & .00 & .00 \\
\hline 16 & .00 & e. 54 & .10 & .00 & e. 00 & .00 & .00 & .00 & .00 & .00 & .00 & .00 \\
\hline 17 & .00 & e. 54 & .00 & .00 & e. 00 & .00 & .00 & .00 & .00 & .00 & .00 & 1.05 \\
\hline 18 & .00 & e. 18 & .20 & .00 & e. 00 & .00 & .00 & .00 & .00 & .00 & .00 & .00 \\
\hline 19 & .00 & .00 & .20 & .00 & e. 13 & .10 & .00 & .00 & .00 & .00 & .00 & .00 \\
\hline 20 & .00 & .00 & .20 & .00 & e. 00 & .15 & .00 & .00 & .00 & .00 & .00 & .00 \\
\hline 21 & .00 & .00 & .50 & e. 00 & e.00 & .00 & .00 & .00 & .00 & .00 & .00 & .00 \\
\hline 22 & .00 & e. 12 & .30 & e. 00 & .00 & .00 & .00 & .00 & .00 & .40 & .00 & .00 \\
\hline 23 & .00 & e. 84 & .20 & e. 00 & .00 & .00 & .00 & .00 & .00 & .10 & .05 & .00 \\
\hline 24 & .00 & e. 12 & 1.15 & e. 00 & .00 & .00 & .25 & .00 & .00 & .00 & .00 & .00 \\
\hline 25 & .00 & e. 36 & .35 & e. 00 & .00 & .50 & .00 & .00 & .00 & .00 & .00 & .00 \\
\hline 26 & .00 & e. 24 & .00 & e. 00 & .00 & .00 & .00 & .00 & .00 & .00 & .00 & .00 \\
\hline 27 & .00 & .00 & .10 & e. 00 & .00 & .00 & .20 & .00 & .00 & .00 & .00 & .00 \\
\hline 28 & .00 & e. 06 & .00 & e. 00 & .00 & .00 & .05 & .00 & .00 & .00 & .00 & .00 \\
\hline 29 & .00 & .00 & .00 & e. 00 & --- & .00 & .00 & .00 & .00 & .00 & .00 & .00 \\
\hline 30 & .00 & .00 & .10 & e. 00 & --- & .00 & .00 & .25 & .00 & .00 & .00 & .05 \\
\hline 31 & .00 & --- & .00 & e. 00 & --- & .50 & --- & .05 & --- & .00 & .00 & --- \\
\hline TOTAL & 0.90 & 3.86 & 3.65 & 0.75 & 1.10 & 2.10 & 0.80 & 1.70 & 1.70 & 0.65 & 0.50 & 1.10 \\
\hline
\end{tabular}

CAL YR 1988 TOTAL 27.46

WTR YR 1989 TOTAL 18.81

e Estimated 
EAST STEWART BASIN

VEG SPRING NEAR IONE, NV

DAILY TOTAL PRECIPITATION, WATER YEAR OCTOBER 1989 TO SEPTEMBER 1990 INCHES

\begin{tabular}{|c|c|c|c|c|c|c|c|c|c|c|c|c|}
\hline DAY & OCT & NOV & DEC & JAN & FEB & MAR & APR & MAY & JUN & JUL & AUG & SEP \\
\hline 1 & .00 & .00 & .00 & .20 & .20 & .00 & .00 & .00 & .00 & .00 & .10 & .00 \\
\hline 2 & .20 & .00 & .00 & .20 & .00 & .00 & .00 & .00 & .00 & .05 & .00 & .00 \\
\hline 3 & .10 & .00 & .00 & .00 & .00 & .00 & .00 & .00 & .00 & .00 & .00 & .00 \\
\hline 4 & .00 & .00 & .00 & .00 & .10 & .10 & .00 & .00 & .00 & .00 & .00 & .00 \\
\hline 5 & .00 & .00 & .00 & .00 & .00 & .60 & .00 & .00 & .00 & .00 & .00 & .00 \\
\hline 6 & .00 & .00 & .00 & .00 & .20 & .00 & .10 & .00 & .00 & .00 & .00 & .00 \\
\hline 7 & .00 & .00 & .00 & .00 & .20 & .00 & .30 & .00 & .00 & .00 & .00 & .05 \\
\hline 8 & .00 & .00 & .00 & .00 & .00 & .00 & 1.10 & .00 & .00 & .00 & .00 & .00 \\
\hline 9 & .00 & .00 & .00 & .00 & .00 & .00 & .00 & .00 & .30 & .00 & .00 & .00 \\
\hline 10 & .00 & .00 & .00 & .00 & .00 & .00 & .00 & .00 & .05 & .00 & .00 & .00 \\
\hline 11 & .00 & .00 & .00 & .00 & .00 & .10 & .00 & .10 & .00 & .00 & .70 & .00 \\
\hline 12 & .00 & .00 & .00 & .00 & .10 & .10 & .00 & .00 & .00 & .00 & .00 & .00 \\
\hline 13 & .00 & .00 & .00 & .80 & .00 & .00 & .00 & .00 & .00 & .30 & .00 & .00 \\
\hline 14 & .00 & .00 & .00 & .30 & .05 & .00 & .00 & .00 & .20 & .30 & .00 & .00 \\
\hline 15 & .00 & .00 & .00 & .00 & .00 & .00 & .00 & .00 & .00 & .00 & 1.25 & .00 \\
\hline 16 & .00 & .00 & .00 & .00 & .25 & .00 & .00 & .00 & .00 & .70 & .00 & .00 \\
\hline 17 & .00 & .00 & .00 & .20 & .90 & .00 & .65 & .20 & .00 & .00 & .00 & .00 \\
\hline 18 & .00 & .00 & .00 & .00 & .75 & .00 & .00 & .00 & .00 & .00 & .25 & .15 \\
\hline 19 & .00 & .00 & .00 & .00 & .00 & .00 & .00 & .00 & .00 & .00 & .20 & .00 \\
\hline 20 & .00 & .00 & .00 & .00 & .00 & .00 & .20 & .00 & .00 & .00 & .10 & .00 \\
\hline 21 & .00 & .00 & .00 & .00 & .00 & .00 & .20 & .00 & .00 & .00 & .05 & .10 \\
\hline 22 & .00 & .00 & .00 & .00 & .00 & .00 & .00 & .00 & .00 & .00 & .00 & .30 \\
\hline 23 & .00 & .00 & .00 & .00 & .00 & .00 & .35 & .20 & .00 & .00 & .00 & .35 \\
\hline 24 & .40 & .20 & .00 & .00 & .00 & .00 & .35 & .00 & .00 & .00 & .00 & .00 \\
\hline 25 & .35 & .20 & .00 & .00 & .00 & .00 & .00 & .00 & .00 & .00 & .00 & .00 \\
\hline 26 & .00 & .35 & .00 & .00 & .00 & .00 & .00 & .00 & .00 & .00 & .00 & .00 \\
\hline 27 & .00 & .00 & .00 & .00 & .00 & .00 & .00 & .40 & .00 & .00 & .00 & .00 \\
\hline 28 & .00 & .00 & .30 & .00 & .00 & .10 & .20 & 1.30 & .00 & .00 & .00 & .20 \\
\hline 29 & .00 & .00 & .00 & .00 & --- & .00 & .00 & .30 & .00 & .00 & .00 & .00 \\
\hline 30 & .00 & .00 & .00 & .20 & --- & .00 & .00 & .00 & .00 & .00 & .00 & .00 \\
\hline 31 & .00 & --- & .00 & .30 & --- & .00 & --- & .00 & --- & .00 & .00 & --- \\
\hline TOTAL & 1.05 & 0.75 & 0.30 & 2.20 & 2.75 & 1.00 & 3.45 & 2.50 & 0.55 & 1.35 & 2.65 & 1.15 \\
\hline
\end{tabular}

CAL YR 1989 TOTAL 12.50

WTR YR 1990 TOTAL 19.70 
EAST STEWART BASIN

VEG SPRING NEAR IONE, NV

DAILY TOTAL PRECIPITATION, WATER YEAR OCTOBER 1990 TO SEPTEMBER 1991 INCHES

\begin{tabular}{|c|c|c|c|c|c|c|c|c|c|c|c|c|}
\hline DAY & OCT & NOV & $D E C$ & JAN & FEB & MAR & APR & MAY & JUN & JUL & AUG & SEP \\
\hline 1 & .00 & .17 & .00 & .00 & .00 & .15 & .20 & .72 & .00 & .00 & .00 & .08 \\
\hline 2 & .00 & .00 & .00 & .00 & .00 & .00 & .00 & .28 & .00 & .00 & .20 & .00 \\
\hline 3 & .00 & .00 & .00 & .20 & .00 & .80 & .00 & .00 & .00 & .00 & .00 & .12 \\
\hline 4 & .00 & .00 & .00 & .10 & .00 & .80 & .00 & .00 & .00 & .00 & .00 & .00 \\
\hline 5 & .00 & .03 & .00 & .00 & .30 & .00 & .00 & .00 & .00 & .00 & .00 & .08 \\
\hline 6 & .00 & .00 & .00 & .10 & .00 & .00 & .00 & .00 & .00 & .00 & .00 & .12 \\
\hline 7 & .00 & .00 & .00 & .00 & .00 & .00 & .00 & .00 & .00 & .00 & .00 & .00 \\
\hline 8 & .00 & .00 & .00 & .00 & .00 & .00 & .00 & .00 & .00 & .00 & .00 & .10 \\
\hline 9 & .00 & .00 & .00 & .00 & .00 & .00 & .00 & .30 & .00 & .00 & .00 & .60 \\
\hline 10 & .00 & .00 & .05 & .00 & .00 & .20 & .15 & .18 & .00 & .00 & .00 & .00 \\
\hline 11 & .00 & .00 & .20 & .00 & .00 & .00 & .20 & .04 & .00 & .00 & .00 & .00 \\
\hline 12 & .00 & .00 & .00 & .00 & .00 & .00 & .40 & .00 & .00 & .00 & .00 & .00 \\
\hline 13 & .00 & .00 & .25 & .10 & .00 & .40 & .00 & 1.00 & .00 & .00 & .07 & .00 \\
\hline 14 & .00 & .00 & .00 & .00 & .00 & .70 & .00 & .00 & .00 & .00 & .00 & .00 \\
\hline 15 & .00 & .00 & .10 & .10 & .35 & .35 & .00 & .00 & .00 & .00 & .00 & .00 \\
\hline 16 & .00 & .00 & .00 & .00 & .00 & .00 & .00 & .00 & .00 & .00 & .00 & .00 \\
\hline 17 & .00 & .00 & .00 & .00 & .00 & .20 & .00 & .10 & .00 & .00 & .00 & .00 \\
\hline 18 & .30 & .00 & .20 & .00 & .00 & .75 & .00 & .30 & .00 & .00 & .00 & .00 \\
\hline 19 & .10 & .25 & .30 & .00 & .00 & .25 & .00 & .05 & .00 & .00 & .00 & .00 \\
\hline 20 & .00 & .00 & .00 & .00 & .00 & .55 & .00 & 1.05 & .00 & .00 & .00 & .00 \\
\hline 21 & .00 & .00 & .00 & .00 & .00 & .40 & .00 & .00 & .00 & .00 & .00 & .00 \\
\hline 22 & .00 & .00 & .00 & .00 & .00 & .00 & .20 & .00 & .00 & .00 & .00 & .00 \\
\hline 23 & .00 & .00 & .00 & .00 & .00 & .00 & .00 & .00 & .00 & .00 & .00 & .00 \\
\hline 24 & .00 & .00 & .00 & .00 & .00 & .50 & .20 & .00 & .20 & .00 & .00 & .00 \\
\hline 25 & .00 & .40 & .00 & .00 & .00 & .30 & .20 & .00 & .30 & .00 & .25 & .00 \\
\hline 26 & .00 & .00 & .00 & .00 & .00 & .20 & .00 & .00 & .00 & .00 & .00 & .00 \\
\hline 27 & .00 & .00 & .00 & .00 & .30 & .00 & .00 & .00 & .15 & .00 & .00 & .20 \\
\hline 28 & .00 & .00 & .00 & .00 & .20 & .00 & .00 & .00 & .30 & .00 & .00 & .05 \\
\hline 29 & .00 & .00 & .00 & .00 & --- & .00 & .00 & .00 & .00 & .00 & .00 & .00 \\
\hline 30 & .00 & .00 & .00 & .00 & $-\infty$ & .00 & .00 & .40 & .00 & .00 & .00 & .00 \\
\hline 31 & .10 & --- & .00 & .00 & $-\infty$ & .00 & $-\infty$ & .00 & -- & .00 & .00 & -- \\
\hline TOTAL & 0.50 & 0.85 & 1.10 & 0.60 & 1.15 & 6.55 & 1.55 & 4.42 & 0.95 & 0.00 & 0.52 & 1.35 \\
\hline
\end{tabular}

CAL YR 1990 TOTAL 20.05

WTR YR 1991 TOTAL 19.54 
EAST STEWART BASIN

VEG SPRING NEAR IONE, NV

WATER-QUALITY ANALYSIS FROM THE UNSATURATED-ZONE LYSIMETER \#1, WATER YEARS OCTOBER 1987 TO SEPTEMBER 1991

\begin{tabular}{|c|c|c|c|c|c|c|c|c|}
\hline & SPE- & $\begin{array}{l}\text { SPE- } \\
\text { CIFIC }\end{array}$ & $\begin{array}{c}\text { PH } \\
\text { WATER }\end{array}$ & $\begin{array}{c}\text { PH } \\
\text { WATER }\end{array}$ & & $\begin{array}{l}\text { ALKA- } \\
\text { LINITY }\end{array}$ & ALKA- & \\
\hline & CIFIC & $\mathrm{CON}-$ & WHOLE & WHOLE & & WAT WH & LINITY & CALCIUM \\
\hline TIME & $\begin{array}{l}\text { CON- } \\
\text { DUCT- } \\
\text { ANCE }\end{array}$ & $\begin{array}{l}\text { DUCT- } \\
\text { ANCE } \\
\text { LAB }\end{array}$ & $\begin{array}{l}\text { FIELD } \\
\text { (STAND- } \\
\text { ARD }\end{array}$ & $\begin{array}{c}\text { LAB } \\
\text { (STAND- } \\
\text { ARD }\end{array}$ & $\begin{array}{c}\text { TEMPER- } \\
\text { ATURE } \\
\text { WATER }\end{array}$ & $\begin{array}{l}\text { TOT FET } \\
\text { FIELD } \\
\text { MG/L AS }\end{array}$ & $\begin{array}{l}\text { LAB } \\
\text { (MG/L } \\
\text { AS }\end{array}$ & $\begin{array}{l}\text { DIS- } \\
\text { SOLVED } \\
\text { (MG/L }\end{array}$ \\
\hline & $(\mu \mathrm{s} / \mathrm{CM})$ & $(\mu \mathrm{s} / \mathrm{CM})$ & UNITS) & UNITS) & (DEG C) & $\mathrm{CACO} 3$ & (ACO3) & AS (A) \\
\hline
\end{tabular}

\begin{tabular}{|c|c|c|c|c|c|c|c|c|c|}
\hline $\begin{array}{l}\text { JUN } 1988 \\
29 \ldots \\
\text { SEP }\end{array}$ & 1030 & 56 & -- & 7.2 & -- & 11.0 & 21 & -- & 5.0 \\
\hline $\begin{array}{l}27 \ldots \\
\text { MAY } 1989\end{array}$ & 1430 & -- & -- & -- & -- & -- & -- & -- & -- \\
\hline $\operatorname{SEP}_{\operatorname{SEP}}^{25 \ldots}$ & 1000 & -- & -- & -- & -- & -- & -- & -- & -- \\
\hline MAY 14990 & 1230 & -- & -- & -- & -- & -- & -- & -- & -- \\
\hline$\underset{\text { JUL }}{24 \ldots}$ & 1330 & -- & -- & -- & -- & -- & -- & 8.4 & 2.7 \\
\hline $\operatorname{sEP}^{12} \cdots$ & 1205 & -- & -- & -- & -- & -- & -- & -- & -- \\
\hline JUL $25 . .991$ & ${ }^{1} 0900$ & -- & -- & -- & -- & -- & -- & -- & -- \\
\hline $\operatorname{sEP}^{24 \ldots}$ & 1430 & 55 & 59 & 7.4 & 7.5 & -- & 21 & -- & 4.9 \\
\hline $24 \ldots$ & ${ }^{1} 1305$ & -- & -- & -- & -- & -- & -- & -- & -- \\
\hline DATE & $\begin{array}{l}\text { MAGNE- } \\
\text { SIUM, } \\
\text { DIS- } \\
\text { SOLVED } \\
\text { (MG/L } \\
\text { AS MG) }\end{array}$ & $\begin{array}{l}\text { SODIUM, } \\
\text { DIS- } \\
\text { SOLVED } \\
\text { (MG /L } \\
\text { AS NA) }\end{array}$ & $\begin{array}{l}\text { POTAS- } \\
\text { SIUM, } \\
\text { DIS- } \\
\text { SOLVED } \\
\text { (MG/L } \\
\text { AS K) }\end{array}$ & $\begin{array}{l}\text { SULFATE } \\
\text { DIS- } \\
\text { SOLVED } \\
\text { (MG/L } \\
\text { AS SO4) }\end{array}$ & $\begin{array}{l}\text { CHLO- } \\
\text { RIDE, } \\
\text { DIS- } \\
\text { SOLVED } \\
\text { (MG/L } \\
\text { AS CL) }\end{array}$ & $\begin{array}{l}\text { FLUO- } \\
\text { RIDE, } \\
\text { DIS- } \\
\text { SOLVED } \\
\text { (MG/L } \\
\text { AS F) }\end{array}$ & $\begin{array}{c}\text { BROMIDE } \\
\text { DIS- } \\
\text { SOLVED } \\
\text { (MG/L } \\
\text { AS BR) }\end{array}$ & $\begin{array}{l}\text { SILICA, } \\
\text { DIS- } \\
\text { SOLVED } \\
\text { (MG/L } \\
\text { AS } \\
\text { SIO2) }\end{array}$ & $\begin{array}{c}\text { NITRO- } \\
\text { GEN, } \\
\text { NITRATE } \\
\text { DIS- } \\
\text { SOLVED } \\
\text { (MG/L } \\
\text { AS N) }\end{array}$ \\
\hline
\end{tabular}

JUN 1988

$29 \ldots$

0.95

2.8

4.0

1.5

0.69

$--\quad--$

6.3

$27 \ldots$

MAY 1989

$25 \ldots$

SEP

$14 \ldots$

MAY 1990

$24 \ldots$

JUL

$12 \ldots$

SEP

$25 \ldots$

JUL 1991

$24 \ldots$

-- -

7.2

9.8

0.93

--

$-$

$<0.010$

6.8

7.1

$0.24<0.010$

$--$

$<0.010$

$\begin{array}{lllll}- & - & - & - & -\end{array}$

22

0.77

$<0.010$

$<0.010$

$\begin{array}{lll}0.46 & 2.1 & 2.6\end{array}$

2.5

2.2

0.09

$<0.010$

7.1

0.170

SEP

$24 \ldots$

1.1

2.6

1.4

2.6

$<0.10$

$--$

$--\quad 33.0$

-- $\quad--$

4.2

3.2

0.79

0.12

0.030

9.4

0.070 
EAST STEWART BASIN

VEG SPRING NEAR IONE, NV

WATER-QUALITY ANALYSIS FROM THE UNSATURATED-ZONE LYSIMETER \#1, WATER YEARS OCTOBER 1987 TO SEPTEMBER 1991

\begin{tabular}{|c|c|c|c|c|c|c|c|c|}
\hline & $\begin{array}{l}\text { PHOS- } \\
\text { PHORUS }\end{array}$ & & MANGA- & STRON- & & TRITIUM & $\begin{array}{l}\mathrm{H}-2 / \\
\mathrm{H}-1\end{array}$ & $\begin{array}{l}0-18 \\
0-16\end{array}$ \\
\hline & ORTHO, & IRON, & NESE, & TIUM, & & 2 SIGMA & STABLE & STABLE \\
\hline & DIS- & DIS- & DIS- & DIS- & & WATER, & ISOTOPE & ISOTOPE \\
\hline & SOLVED & SOLVED & SOLVED & SOLVED & TRITIUM & WHOLE, & RATIO & RATIO \\
\hline ATE & (MG /L & $(\mu \mathrm{G} / \mathrm{L}$ & $(\mu \mathrm{G} / \mathrm{L}$ & $(\mu \mathrm{G} / \mathrm{L}$ & TOTAL & TOTAL & PER & PER \\
\hline & AS P) & AS FE) & AS $M N$ ) & AS SR) & (PCI/L) & (PCI/L) & MIL & MIL \\
\hline
\end{tabular}

\section{JUN 1988}

$29 \ldots$

SEP

SEP

JUL

SEP

AS FE)

AS MN)

AS SR)

$\begin{array}{ccccc}27 & 48 & -- & -114.0 & -15.35 \\ -- & -- & - & -- & -- \\ -- & -- & -- & -96.0 & -12.80 \\ -- & 65 & 5.0 & -- & -- \\ -- & -- & - & -105.0 & -13.60 \\ -- & -- & -- & -118.0 & -17.25 \\ -- & -- & -- & -116.0 & -16.05 \\ -- & -- & -- & - & -\end{array}$

27 ...

MAY 1989

25 ...

$14 \ldots$

MAY 1990

$24 \ldots$

$12 \ldots$

$25 \ldots$

JUL 1991

$24 \ldots$

24 ...
17

1.50

3.00

53.0

0.020

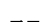

0.030

11

$-$

$-$

$-$

$-$

23

$--$

$-$

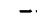

1 Sample collector dry. 
EAST STEWART BASIN

VEG SPRING NEAR IONE, NV WATER-QUALITY ANALYSIS FROM THE UNSATURATED-ZONE LYSIMETER \#2, WATER YEARS OCTOBER 1988 TO SEPTEMBER 1991

\begin{tabular}{|c|c|c|c|c|c|c|c|c|}
\hline TIME & $\begin{array}{l}\text { SPE- } \\
\text { CIF IC } \\
\text { CON- } \\
\text { DUCT- } \\
\text { ANCE } \\
\text { LAB }\end{array}$ & $\begin{array}{c}\text { ALKA- } \\
\text { LINITY } \\
\text { LAB } \\
\text { (MG/L } \\
\text { AS }\end{array}$ & $\begin{array}{l}\text { CALCIUM } \\
\text { DIS- } \\
\text { SOLVED } \\
\text { (MG/L }\end{array}$ & $\begin{array}{l}\text { MAGNE- } \\
\text { SIUM, } \\
\text { DIS- } \\
\text { SOLVED } \\
\text { (MG / L }\end{array}$ & $\begin{array}{l}\text { SODIUM, } \\
\text { DIS- } \\
\text { SOLVED } \\
\text { (MG/L }\end{array}$ & $\begin{array}{l}\text { POTAS- } \\
\text { SIUM, } \\
\text { DIS- } \\
\text { SOLVED } \\
\text { (MG/L }\end{array}$ & $\begin{array}{l}\text { SULFATE } \\
\text { DIS- } \\
\text { SOLVED } \\
\text { (MG/L }\end{array}$ & $\begin{array}{l}\text { CHLO- } \\
\text { RIDE, } \\
\text { DIS- } \\
\text { SOLVED } \\
\text { (MG / L }\end{array}$ \\
\hline & $(\mu \mathrm{S} / \mathrm{CM})$ & (ACO3) & AS CA) & AS MG) & AS NA) & AS K) & AS SO4) & AS CL) \\
\hline
\end{tabular}

\begin{tabular}{|c|c|c|c|c|c|c|c|c|c|}
\hline $\begin{array}{l}\text { MAY } 1989 \\
25 \ldots \\
\text { SEP }\end{array}$ & 1020 & -- & -- & -- & -- & -- & -- & 6.6 & 7.1 \\
\hline $\begin{array}{l}14 \ldots \\
\text { JUN } 1990\end{array}$ & 1245 & -- & -- & -- & -- & -- & -- & 2.9 & 2.4 \\
\hline${ }_{\text {JUL }}^{19} \cdots$ & 1330 & -- & $<0.5$ & 4.0 & 0.76 & 2.6 & 3.8 & 4.4 & 2.8 \\
\hline $\operatorname{SEP}^{12 \ldots}$ & 1235 & -- & -- & -- & -- & -- & -- & 1.5 & 2.6 \\
\hline JUL 25.1991 & ${ }^{1} 1245$ & -- & -- & -- & -- & -- & -- & -- & -- \\
\hline$\stackrel{24 \ldots}{S E P}$ & 1450 & 71 & -- & 4.0 & 0.68 & 3.8 & -- & 2.4 & 3.2 \\
\hline $24 \ldots$ & ${ }^{1} 1340$ & -- & -- & -- & -- & -- & -- & -- & -- \\
\hline DATE & $\begin{array}{l}\text { FLUO- } \\
\text { RIDE, } \\
\text { DIS- } \\
\text { SOLVED } \\
\text { (MG/L } \\
\text { AS F) }\end{array}$ & $\begin{array}{c}\text { BROMIDE } \\
\text { DIS- } \\
\text { SOLVED } \\
\text { (MG/L } \\
\text { AS BR) }\end{array}$ & $\begin{array}{l}\text { SILICA, } \\
\text { DIS- } \\
\text { SOLVED } \\
\text { (MG/L } \\
\text { AS } \\
\text { SIO2) }\end{array}$ & $\begin{array}{c}\text { NITRO- } \\
\text { GEN, } \\
\text { NITRATE } \\
\text { DIS- } \\
\text { SOLVED } \\
\text { (MG/L } \\
\text { AS N) }\end{array}$ & $\begin{array}{l}\text { PHOS- } \\
\text { PHORUS } \\
\text { ORTHO, } \\
\text { DIS- } \\
\text { SOLVED } \\
\text { (MG/L } \\
\text { AS P) }\end{array}$ & $\begin{array}{l}\text { IRON, } \\
\text { DIS- } \\
\text { SOLVED } \\
(\mu G / L \\
\text { AS FE) }\end{array}$ & $\begin{array}{l}\text { MANGA- } \\
\text { NESE, } \\
\text { DIS- } \\
\text { SOLVED } \\
(\mu G / L \\
\text { AS MN) }\end{array}$ & $\begin{array}{l}\text { H-2 / } \\
\text { H-1 } \\
\text { STABLE } \\
\text { ISOTOPE } \\
\text { RATIO } \\
\text { PER } \\
\text { MIL }\end{array}$ & $\begin{array}{c}\text { O-18 } \\
\text { O-16 } \\
\text { STABLE } \\
\text { ISOTOPI } \\
\text { RATIO } \\
\text { PER } \\
\text { MIL }\end{array}$ \\
\hline
\end{tabular}

MAY 1989

\begin{tabular}{|c|c|c|c|c|c|c|c|c|c|}
\hline $25 \ldots$ & 0.14 & $<0.010$ & -- & 8.70 & 0.490 & -- & -- & -- & -- \\
\hline $\begin{array}{l}14 \ldots \\
\text { JUN } 1990\end{array}$ & 0.09 & $<0.010$ & -- & 6.90 & 0.690 & -- & -- & -116.0 & -16.50 \\
\hline $19 \ldots$ & 0.10 & $<0.010$ & 4.5 & 0.690 & 0.190 & 52 & 7 & -124.0 & -16.50 \\
\hline$\underset{\operatorname{seP}}{12} \ldots$ & $<0.10$ & -- & -- & 37.0 & -- & -- & -- & -164.0 & -23.15 \\
\hline JUL 19991 & -- & -- & -- & -- & -- & -- & -- & -- & -- \\
\hline$\underset{S E P}{24 \ldots}$ & 0.09 & $<0.010$ & 4.8 & 3.70 & $<0.010$ & 33 & 6 & -- & -- \\
\hline $24 \ldots$ & -- & -- & -- & -- & -- & -- & -- & -- & -- \\
\hline
\end{tabular}

1 Sample collector dry. 
EAST STEWART BASIN

VEG SPRING NEAR IONE, NV

SURFACE-WATER QUALITY, WATER YEARS OCTOBER 1983 TO SEPTEMBER 1991

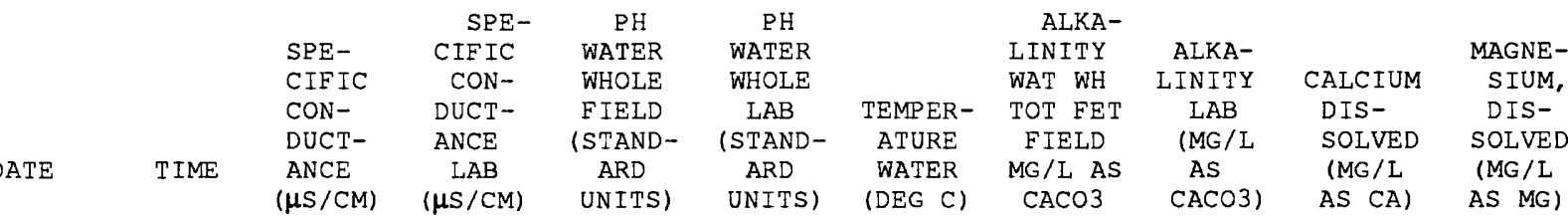

SEP 1984

$19 \ldots$

JUL $19 \dot{985}$

$09 \ldots$

SEP

27 ...

JUL 1986

$10 \ldots$

SEP $30 .$.

DEC

$30 \ldots$

MAY 1987

$06 \ldots$

JUN

$30 .$.

SEP

25 ...

JUN 1988

$28 \ldots$

SEP

$27 \ldots$

MAY 1989

25 ...

SEP

$14 \ldots$

NOV

$07 \ldots$

JAN 1990

$31 \ldots$

APR
$03 \ldots$

MAY $24 \ldots$

SEP

$25 \ldots$

JUL 1991

24 ...

SEP

24 ...
1730
1324
1702
1000
1330
0920
1230
1640
1420
1430
1420
1000
1325
1246
1130
1430
1630
1215
130
130

\begin{tabular}{|c|c|}
\hline 38 & -- \\
\hline-- & -- \\
\hline 23 & -- \\
\hline 31 & 30 \\
\hline 34 & 31 \\
\hline 32 & 31 \\
\hline-- & 40 \\
\hline 33 & 34 \\
\hline 33 & 35 \\
\hline 29 & 32 \\
\hline 28 & 31 \\
\hline 30 & 31 \\
\hline 29 & 31 \\
\hline 28 & 31 \\
\hline 23 & 30 \\
\hline 27 & 29 \\
\hline 31 & 33 \\
\hline 33 & 31 \\
\hline 26 & 30 \\
\hline 28 & 30 \\
\hline
\end{tabular}

$7.1 \quad--$

$5.8 \quad--$

$6.1 \quad-$

$7.1 \quad 7.4$

$6.7 \quad 7.6$

6.7

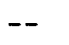

6.7

7.0

7.0

6.3

7.0

6.9

6.9

6.9

6.9

7.0

7.0

6.6

6.3

7.0

8.0

7.7

7.7

7.5

6.9

7.7

7.5

7.1

7.3

7.5

7.3

7.4

7.0
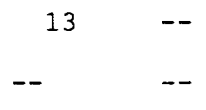

4. 0

4.0

5.0

7.0
4.5

4.5

4.5

4.0

4.5

4.5

4.5

$8.5 \quad--\quad 13$

5.0

4.0

3.0

4.0

4.0

11

$12 \quad 13$

$12 \quad 12$

1211

1212

1313

129.6

$12 \quad 12$
2.6

2.4

2.5

2.2

2.1

2.3

3.0

2.3

2.4

2.6

2.6

2.5

2.6

2.3

2.2

2.4

2.7

2.4

2.0

2.3

0.35

0.29 
EAST STEWART BASIN

VEG SPRING NEAR IONE, NV

SURFACE-WATER QUALITY, WATER YEARS OCTOBER 1983 TO SEPTEMBER 1991

\begin{tabular}{|c|c|c|c|c|c|c|c|c|}
\hline SODIUM, & $\begin{array}{l}\text { POTAS- } \\
\text { SIUM, }\end{array}$ & SULFATE & $\begin{array}{l}\text { CHLO- } \\
\text { RIDE, }\end{array}$ & $\begin{array}{l}\text { FLUO- } \\
\text { RIDE, }\end{array}$ & BROMIDE & $\begin{array}{l}\text { SILICA, } \\
\text { DIS- }\end{array}$ & $\begin{array}{l}\text { NITRO- } \\
\text { GEN, } \\
\text { NITRATE }\end{array}$ & $\begin{array}{l}\text { NITRO- } \\
\text { GEN, } \\
\text { NO2+NO3 }\end{array}$ \\
\hline DIS- & DIS- & DIS- & DIS- & DIS- & DIS- & SOLVED & DIS- & DIS- \\
\hline $\begin{array}{l}\text { SOLVED } \\
\text { (MG/L }\end{array}$ & $\begin{array}{l}\text { SOLVED } \\
\text { (MG/L }\end{array}$ & $\begin{array}{l}\text { SOLVED } \\
\text { (MG/L }\end{array}$ & $\begin{array}{l}\text { SOLVED } \\
\text { (MG/L }\end{array}$ & $\begin{array}{l}\text { SOLVED } \\
\text { (MG/L }\end{array}$ & $\begin{array}{l}\text { SOLVED } \\
\text { (MG/L }\end{array}$ & $\begin{array}{l}\text { (MG/L } \\
\mathrm{AS}\end{array}$ & $\begin{array}{l}\text { SOLVED } \\
\text { (MG/L }\end{array}$ & $\begin{array}{l}\text { SOLVED } \\
\text { (MG/L }\end{array}$ \\
\hline AS NA) & AS $\mathrm{K}$ ) & AS SO4) & AS CL) & AS F) & AS BR) & SIO2) & AS N) & AS N) \\
\hline
\end{tabular}

SEP 1984

$19 \ldots$

JUL 1985

$09 \ldots$

SEP

$27 \ldots$

JUL 1986

$10 \ldots$

$30 \ldots$

DEC

$30 \ldots$

MAY 1987

$06 \ldots$

JUN

$30 \ldots$

SEP

$25 \ldots$

JUN 1988

$28 \ldots$

SEP

27 ...

MAY 1989

$25 \ldots$

SEP

$14 \ldots$

$07 \ldots$

JAN 1990

$31 \ldots$

$\mathrm{APR}$
$03 . .$.

MAY

24 ...

SEP

$25 \ldots$

JUL 1991

$24 \ldots$

SEP

$24 \ldots$

\begin{tabular}{|c|c|c|c|c|c|c|c|c|}
\hline 3.1 & -- & 1.2 & 0.76 & 0.04 & 0.008 & 16 & -- & -- \\
\hline 3.2 & -- & 1.2 & 0.61 & 0.03 & $<0.050$ & 16 & -- & -- \\
\hline 2.8 & -- & 1.2 & 0.56 & 0.04 & 0.007 & 16 & -- & -- \\
\hline 3.3 & 0.46 & 1.2 & 0.63 & 0.03 & $<0.010$ & 17 & -- & -- \\
\hline 3.4 & 0.50 & 1.1 & 0.59 & -- & -- & -- & -- & -- \\
\hline 3.4 & 0.51 & 1.1 & 0.62 & 0.01 & -- & 17 & -- & -- \\
\hline 3.7 & 0.63 & 1.7 & 1.2 & 0.04 & -- & 16 & -- & -- \\
\hline 3.0 & 0.50 & 1.4 & 0.72 & 0.05 & -- & 16 & -- & -- \\
\hline 3.1 & 0.50 & 1.3 & 0.64 & -- & -- & 17 & -- & -- \\
\hline 3.2 & 0.45 & 1.3 & 0.65 & -- & -- & 16 & -- & -- \\
\hline 3.3 & 0.58 & 1.2 & 0.65 & -- & -- & 16 & -- & -- \\
\hline 3.1 & 0.51 & 1.0 & 0.60 & $<0.10$ & $<0.010$ & 16 & -- & 0.100 \\
\hline 3.3 & -- & 1.3 & 0.65 & 0.05 & $<0.010$ & 16 & 0.100 & -- \\
\hline 3.5 & -- & 1.2 & 0.68 & 0.05 & $<0.010$ & 17 & 0.170 & -- \\
\hline 3.4 & -- & 1.2 & 0.60 & 0.05 & $<0.010$ & 17 & 0.200 & -- \\
\hline 3.1 & -- & 1.4 & 0.73 & 0.04 & 0.030 & 15 & 0.210 & -- \\
\hline 3.4 & 0.50 & 1.9 & 0.97 & 0.06 & $<0.010$ & 17 & 0.110 & -- \\
\hline 3.4 & 0.50 & 1.2 & 0.66 & 0.05 & $<0.010$ & 16 & 0.180 & -- \\
\hline 2.6 & 0.50 & 1.3 & 0.59 & 0.06 & $<0.010$ & 15 & $<0.010$ & -- \\
\hline 3.2 & 0.50 & 1.2 & 0.62 & 0.05 & $<0.010$ & 16 & 0.150 & -- \\
\hline
\end{tabular}


EAST STEWART BASIN

VEG SPRING NEAR IONE, NV

SURFACE-WATER QUALITY, WATER YEARS OCTOBER 1983 TO SEPTEMBER 1991

\begin{tabular}{|c|c|c|c|c|c|c|c|c|c|}
\hline & PHOS- & & & & & & & $\mathrm{H}-2 /$ & $0-18$ \\
\hline & PHORUS & & MANGA- & STRON- & ALUM- & & TRITIUM & $\mathrm{H}-1$ & $0-16$ \\
\hline & ORTHO, & IRON, & NESE, & TIUM, & INUM, & & 2 SIGMA & STABLE & STABLE \\
\hline & DIS- & DIS- & DIS- & DIS- & DIS- & & WATER, & ISOTOPE & ISOTOPE \\
\hline & SOLVED & SOLVED & SOLVED & SOLVED & SOLVED & TRIT IUM & WHOLE, & RATIO & RATIO \\
\hline ATE & (MG / L & $(\mu \mathrm{G} / \mathrm{L}$ & $(\mu \mathrm{G} / \mathrm{L}$ & $(\mu \mathrm{G} / \mathrm{L}$ & $(\mu \mathrm{G} / \mathrm{L}$ & TOTAL & TOTAL & PER & PER \\
\hline & AS P) & AS FE) & AS MN) & AS SR) & AS AI) & (PCI/L) & $(P C I / I)$ & MIL & MIL \\
\hline
\end{tabular}

\begin{tabular}{|c|c|c|c|c|c|c|c|c|c|}
\hline $\begin{array}{c}\text { SEP } 1984 \\
19 \ldots \\
\text { JUL } 1985\end{array}$ & -- & $<3$ & 1 & 19 & -- & 110 & 12 & -122.0 & -16.40 \\
\hline $\begin{array}{l}09 . \cdots \\
\text { SEP }\end{array}$ & -- & 10 & 1 & 17 & -- & -- & -- & -118.5 & -16.55 \\
\hline JUL 27.386 & -- & 52 & 3 & 21 & -- & -- & -- & -122.0 & -16.55 \\
\hline $\begin{array}{l}10 \ldots \\
\mathrm{SEP}\end{array}$ & $\cdots$ & 8 & -- & 17 & -- & -- & -- & -121.0 & -16.50 \\
\hline $\begin{array}{c}30 \ldots \\
\mathrm{DEC}\end{array}$ & - & $<10$ & -- & 70 & -- & -- & - & -121.0 & -16.50 \\
\hline $\begin{array}{l}30 \ldots \\
\text { MAY } 1987\end{array}$ & -- & 4 & -- & 16 & -- & -- & -- & -121.5 & -16.60 \\
\hline JUN & -- & $<10$ & -- & 10 & 10 & -- & - & -- & -- \\
\hline $\begin{array}{l}30 \ldots \\
\operatorname{SEP}\end{array}$ & -- & $<3$ & -- & 1 & -- & -- & -- & -120.5 & -16.40 \\
\hline $\begin{array}{l}25 \ldots \\
\text { JUN } 1988\end{array}$ & -- & $<3$ & -- & 17 & -- & -- & -- & -122.0 & -16.55 \\
\hline$\stackrel{28 \ldots}{\operatorname{SEP}}$ & -- & 4 & -- & 18 & -- & -- & -- & -120.5 & -16.45 \\
\hline $\begin{array}{l}27 \\
\text { MAY } 1989\end{array}$ & -- & 8 & -- & 17 & $<10$ & 84 & 9.0 & -122.5 & -16.60 \\
\hline$\stackrel{25 \ldots}{S E P}$ & -- & 4 & -- & 18 & -- & -- & -- & -122.0 & -16.65 \\
\hline Nov $14 \ldots$ & $<0.010$ & $<3$ & $<1$ & -- & -- & 65 & 5.0 & $-124 \cdot 5$ & -16.65 \\
\hline $\begin{array}{l}07 \\
\text { JAN }\end{array}$ & 0.010 & $<3$ & $<1$ & -- & -- & 78 & 7.0 & -123.0 & -16.60 \\
\hline $\begin{array}{l}31 \ldots \\
A P R\end{array}$ & 0.030 & $<3$ & $<1$ & -- & -- & -- & -- & -121.0 & -16.65 \\
\hline$\underset{\text { MAY }}{03 \ldots}$ & $<0.010$ & 9 & $<1$ & -- & -- & -- & - & -121.0 & -16.65 \\
\hline$\stackrel{24 \ldots}{S E P}$ & $<0.010$ & 75 & $<1$ & -- & -- & -- & -- & -119.0 & -16.30 \\
\hline JUL 25 i991 & $<0.010$ & $<2$ & $<1$ & -- & -- & - & -- & -120.0 & -16.50 \\
\hline$\underset{\operatorname{SEP}}{24 \ldots}$ & $<0.010$ & 7 & 1 & -- & -- & -- & -- & -121.0 & -16.60 \\
\hline $24 \ldots$ & $<0.010$ & 4 & $<1$ & -- & -- & -- & - & -122.0 & -16.45 \\
\hline
\end{tabular}




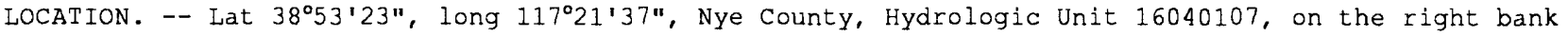
$1.3 \mathrm{mi}$ upstream from the Columbine campground, $13.3 \mathrm{mi}$ east of Ione on state Route 21.

DRAINAGE AREA. -- $0.36 \mathrm{mi}^{2}$.

PERIOD OF RECORD. -- September 1984 to current year.

GAGE. -- Two water-stage recorders on a 9 in Parshall flume and a standard 90 degree v-notch weir, and a crest stage gage. The flume is considered the primary gage. See remarks for more information.

REMARKS.-- The weather equipment is located approximately 150 feet northeast of the surface-water equipment. The surface-water-quality samples are taken upstream from the weir pool and the unsaturated-zone water-quality samples are taken approximately 100 feet upstream on the left bank from the trail crossing or about 700 feet upstream from the surface-water site. Daily discharges for 1987 and 1988 are determined from data collected at the v-notch weir while discharge records for 1989, 1990, and 1991 are determined from the 9 in Parshall flume. Accuracy of measurement by the flume diminishes below a discharge of $0.25 \mathrm{ft}^{3} / \mathrm{s}$. Any daily discharges below $0.25 \mathrm{ft}^{3} / \mathrm{s}$ reported during the years 1989, 1990, and 1991 are considered fair; discharges above $0.25 \mathrm{ft}^{3} / \mathrm{s}$ are considered good to excellent. Ice affects are considered minimal if they occurred at all during the winter months. During visits when the unsaturated-zone lysimeter was checked and found empty, the container was cleaned and replaced. Estimated periods of daily record are considered poor. Values for the daily maximum and minimum air temperature were obtained from the 40 minute average values.

PERIOD OF DAILY RECORD. --

AIR TEMPERATURE: January 1986 to current year.

SOIL TEMPERATURE: January 1986 to current year.

SOLAR RADIATION: January 1986 to current year.

RELATIVE HUMIDITY: October 1990 to current year.

TOTAL PRECIPITATION: October 1985 to current year.

DISCHARGE: MaY 1987 to current year.

EXTREMES FOR PERIOD OF RECORD. --

AIR TEMPERATURE: Maximum daily, $25.6^{\circ} \mathrm{C}$, July 4, 1991; minimum daily, $-28.2^{\circ} \mathrm{C}$, Dec. 22, 1990. See remarks for more information.

SOIL TEMPERATURE: Maximum daily mean, $21.2^{\circ} \mathrm{C}$, July 18, 1991; minimum daily mean, $-5.6^{\circ} \mathrm{C}$, Dec. $20-$ 21, 1989.

SOLAR RADIATION: Maximum daily total, $809 \mathrm{cal} / \mathrm{cm}^{2}$, June 29, 1990; minimum daily total, $7 \mathrm{cal} / \mathrm{cm}^{2}$, Nov. 2, 1987.

RELATIVE HUMIDITy: Maximum daily mean, 99 pct, May 2, 1991 and May 30, 1991; minimum daily mean, 15 pet, Aug. 4 and 30, 1991.

TOTAL PRECIPITATION: Maximum daily, 1.40 in, May 26, 1987; no precipitation, most days of record. DISCHARGE: Maximum daily mean, $3.4 \mathrm{ft}^{3} / \mathrm{s}$, June 13-14, 1991; minimum daily mean, $0.03 \mathrm{ft} / \mathrm{s}$,

Feb. 3-4, 1991 . 
EAST STEWART BASIN

EAST STEWART CREEK NEAR IONE, NV

DAILY MEAN AIR TEMPERATURE, WATER YEAR OCTOBER 1985 TO SEPTEMBER 1986 DEGREES CELSIUS

\begin{tabular}{|c|c|c|c|c|c|c|c|c|c|c|c|c|}
\hline DAY & OCT & NOV & DEC & JAN & FEB & MAR & APR & MAY & JUN & JUL & AUG & SEP \\
\hline 1 & -- & -- & --- & e1.2 & -3.4 & 4.3 & -.2 & 7.3 & 12.4 & 16.7 & 17.4 & 9.9 \\
\hline 2 & $\ldots$ &.- & $\ldots$ & e1.2 & -3.4 & -.2 & -10.8 & 8.3 & 13.1 & 16.6 & 18.0 & 10.7 \\
\hline 3 & -- & --- & -- & e2.3 & -6.5 & 2.4 & -4.6 & 4.0 & 12.7 & 15.1 & 16.0 & 11.9 \\
\hline 4 & -- & --- & --- & $e-1.1$ & -8.7 & 1.9 & -.1 & -6.7 & 13.5 & 13.1 & 16.4 & 13.5 \\
\hline 5 & -- & --- & --- & $e-2.1$ & -9.9 & 1.3 & .0 & -3.6 & 8.9 & 8.9 & 16.0 & 15.1 \\
\hline 6 & --- & -- & -- & $e-2.3$ & -14.2 & 1.8 & -1.0 & -7.5 & 10.2 & 11.5 & 16.9 & 14.3 \\
\hline 7 & -- & --- & -- & e. 0 & -13.7 & 2.0 & -3.8 & -6.4 & 6.3 & 10.9 & 16.6 & 13.3 \\
\hline 8 & -- & -- & -- & e5.7 & -14.8 & -2.6 & -3.4 & -3.5 & 4.9 & 11.6 & 16.5 & 9.1 \\
\hline 9 & -- & -- & -- & e5.8 & -12.3 & -6.2 & -.2 & .4 & 6.3 & e11.7 & 17.3 & 2.8 \\
\hline 10 & --- & --- & --- & e 5.9 & -7.8 & -4.5 & .7 & 1.4 & 9.8 & e 12.4 & 14.3 & 2.7 \\
\hline 11 & $\cdots$ & $\cdots$ & -- & e 4.7 & -6.0 & -6.6 & 2.4 & .2 & 13.8 & e14.5 & 13.8 & 8.7 \\
\hline 12 & -- & -- & -- & 3.7 & -2.8 & -8.7 & -3.0 & 2.2 & 8.7 & el6.1 & 13.7 & 8.8 \\
\hline 13 & $-\cdots$ & -- & -- & 6.7 & -2.6 & -7.6 & -7.8 & 4.8 & 11.4 & e 15.7 & 14.1 & 6.1 \\
\hline 14 & -- & -- & -- & .6 & .4 & -8.8 & .0 & 2.5 & 12.5 & e15.5 & 15.6 & 4.3 \\
\hline 15 & --- & $\cdots$ & --- & -3.0 & -3.7 & -5.9 & .6 & 2.8 & 12.5 & e10.3 & 17.7 & .6 \\
\hline 16 & -- & --- & -- & -.3 & -3.4 & -8.1 & -7.7 & 1.5 & 14.5 & e 8.4 & 16.6 & 3.0 \\
\hline 17 & $\cdots$ & -- & --- & -.3 & -.4 & -9.7 & -7.5 & 5.6 & 15.3 & e9.8 & 16.5 & 1.9 \\
\hline 18 & $\cdots$ & $\cdots$ & -- & 4.5 & -.1 & -7.8 & -4.9 & 9.7 & 10.9 & e11.0 & 15.5 & .2 \\
\hline 19 & -- & -- & --- & 5.0 & -3.8 & -2.6 & 1.0 & 11.4 & 7.6 & e13.5 & 11.8 & -.4 \\
\hline 20 & -- & -- & --- & -6.9 & -7.0 & 2.2 & 7.1 & 7.6 & 8.8 & e14.3 & 10.7 & -2.4 \\
\hline 21 & -- & -- & --- & -3.8 & -4.9 & 3.7 & 8.9 & .6 & 12.2 & e11.4 & 12.0 & 1.9 \\
\hline 22 & -- & --- & --- & 1.9 & -1.8 & 3.1 & 6.8 & .7 & 14.2 & e9.6 & 15.2 & 3.2 \\
\hline 23 & -- & -- & --- & -5.8 & 1.3 & 3.9 & 1.0 & 7.6 & 16.0 & e 8.0 & 16.3 & 1.0 \\
\hline 24 & -- & -- & -- & -3.1 & 6.6 & 1.6 & -1.4 & 10.2 & 14.8 & e9.1 & 16.7 & -.3 \\
\hline 25 & --- & --- & --- & 1.6 & 5.1 & .0 & -3.9 & 13.8 & 13.6 & e9.6 & 14.5 & -4.2 \\
\hline 26 & -- & -- & -- & 1.4 & 4.4 & 4.7 & -6.3 & 14.9 & 17.4 & e8.9 & 14.7 & -2.1 \\
\hline 27 & -- & -- & --- & 3.2 & 5.5 & 5.9 & 2.6 & 14.2 & 17.3 & e11.9 & 12.0 & -4.1 \\
\hline 28 & -- & -- & -- & 5.6 & 5.9 & 6.3 & 4.0 & 13.6 & 12.3 & e12.3 & 12.0 & -3.8 \\
\hline 29 & -- & -- & --- & 3.5 & -- & 5.0 & .9 & 14.6 & 9.9 & e10.8 & 12.5 & -.7 \\
\hline 30 & -- & -- & -- & -.9 & -- & 3.5 & 6.2 & 15.3 & 13.4 & e12.8 & 10.5 & 1.2 \\
\hline 31 & -- & $-\cdots$ & $\cdots$ & -2.2 & $\cdots$ & 2.1 & -- & 14.5 & - & e15.2 & 10.2 & $\cdots$ \\
\hline MEAN & --- & -- & -- & 1.1 & -3.6 & -.8 & -.8 & 5.2 & 11.8 & 12.2 & 14.8 & 4.2 \\
\hline MAX & $\cdots$ & $\cdots$ & -- & 6.7 & 6.6 & 6.3 & 8.9 & 15.3 & 17.4 & 16.7 & 18.0 & 15.1 \\
\hline MIN & -- & -- & -- & -6.9 & -14.8 & -9.7 & -10.8 & -7.5 & 4.9 & 8.0 & 10.2 & -4.2 \\
\hline MED & -- & -- & -- & 1.2 & -3.4 & 1.6 & -.2 & 4.8 & 12.4 & 11.7 & 15.5 & 2.7 \\
\hline
\end{tabular}

e Estimated 
EAST STEWART BASIN

EAST STEWART CREEK NEAR IONE, NV

DAILY MEAN AIR TEMPERATURE, WATER YEAR OCTOBER 1986 TO SEPTEMBER 1987 DEGREES CELSIUS

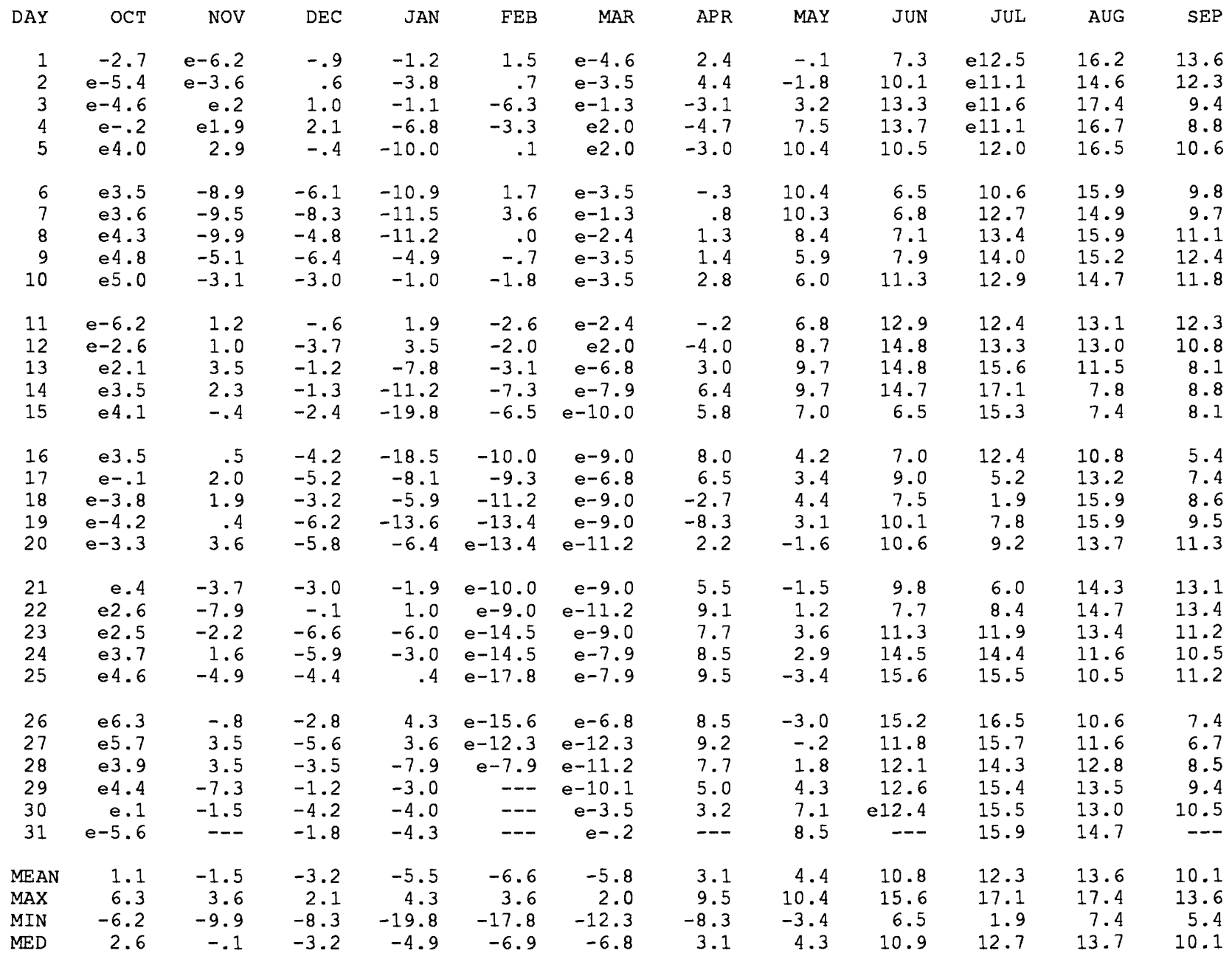

CAL YR 1986 MEAN 3.4 MAX 18.0 MIN -14.8 MED 2.3

WTR YR 1987 MEAN 2.8 MAX 17.4 MIN -19.8 MED 2.9

e Estimated 
EAST STEWART BASIN

EAST STEWART CREEK NEAR IONE, NV

DAILY MEAN AIR TEMPERATURE, WATER YEAR OCTOBER 1987 TO SEPTEMBER 1988 DEGREES CELSIUS

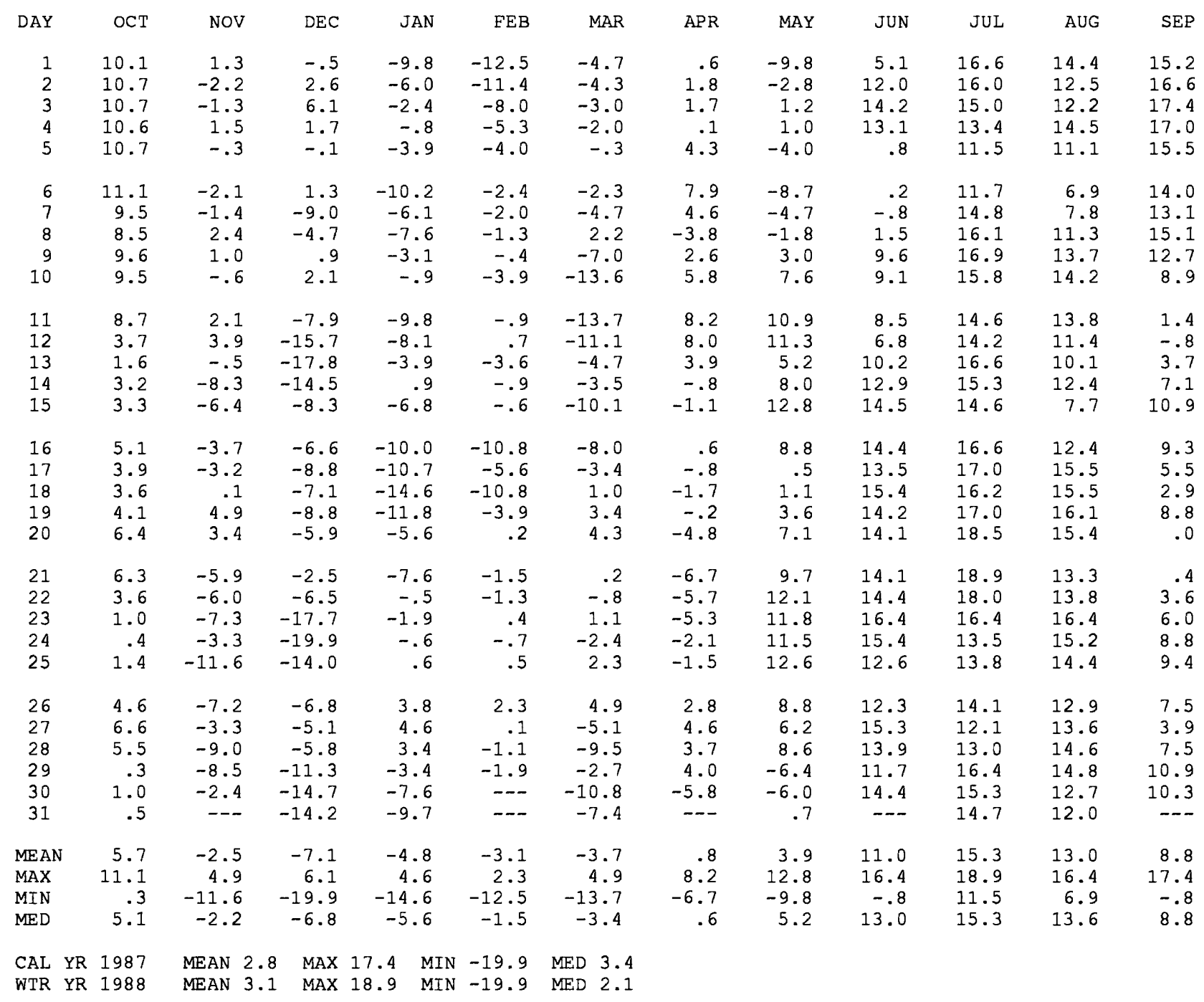


EAST STEWART BASIN

EAST STEWART CREEK NEAR IONE, NV DAILY MEAN AIR TEMPERATURE, WATER YEAR OCTOBER 1988 TO SEPTEMBER 1989 DEGREES CELSIUS

\begin{tabular}{|c|c|c|c|c|c|c|c|c|c|c|c|c|}
\hline DAY & $O C T$ & NOV & DEC & JAN & FEB & MAR & APR & MAY & JUN & JUL & AUG & SEP \\
\hline $\begin{array}{l}1 \\
2 \\
3 \\
4 \\
5\end{array}$ & $\begin{array}{r}11.2 \\
12.6 \\
12.6 \\
10.8 \\
8.7\end{array}$ & $\begin{array}{l}5.8 \\
2.7 \\
5.1 \\
2.7 \\
6.8\end{array}$ & $\begin{array}{r}3.4 \\
.8 \\
-2.0 \\
-2.2 \\
.0\end{array}$ & $\begin{array}{r}-11.8 \\
-3.4 \\
-.1 \\
-2.8 \\
-7.1\end{array}$ & $\begin{array}{r}-3.2 \\
-8.8 \\
-10.8 \\
-18.4 \\
-21.9\end{array}$ & $\begin{array}{r}-.7 \\
-3.9 \\
-15.0 \\
-3.8 \\
1.6\end{array}$ & $\begin{array}{r}-2.3 \\
-.8 \\
-.9 \\
1.5 \\
5.9\end{array}$ & $\begin{array}{r}5.3 \\
4.7 \\
5.6 \\
7.7 \\
12.2\end{array}$ & $\begin{array}{l}6.5 \\
8.4 \\
4.3 \\
4.1 \\
4.2\end{array}$ & $\begin{array}{l}11.9 \\
15.1 \\
17.8 \\
14.7 \\
15.0\end{array}$ & $\begin{array}{l}13.9 \\
12.3 \\
13.0 \\
14.0 \\
14.2\end{array}$ & $\begin{array}{l}11.8 \\
11.3 \\
11.4 \\
13.7 \\
12.7\end{array}$ \\
\hline $\begin{array}{r}6 \\
7 \\
8 \\
9 \\
10\end{array}$ & $\begin{array}{l}7.6 \\
7.9 \\
7.8 \\
7.9 \\
6.8\end{array}$ & $\begin{array}{r}2.4 \\
2.3 \\
-4.1 \\
-2.3 \\
-.2\end{array}$ & $\begin{array}{r}.0 \\
-7.4 \\
-4.6 \\
.4 \\
.4\end{array}$ & $\begin{array}{r}-15.8 \\
-15.6 \\
-11.8 \\
-4.6 \\
-7.6\end{array}$ & $\begin{array}{r}-18.9 \\
-15.9 \\
-7.6 \\
-3.5 \\
-8.2\end{array}$ & $\begin{array}{l}3.2 \\
2.5 \\
3.4 \\
4.6 \\
5.0\end{array}$ & $\begin{array}{l}7.9 \\
8.3 \\
6.9 \\
6.3 \\
7.9\end{array}$ & $\begin{array}{r}13.0 \\
11.1 \\
9.1 \\
6.3 \\
-1.3\end{array}$ & $\begin{array}{l}7.2 \\
8.2 \\
8.1 \\
6.4 \\
8.0\end{array}$ & $\begin{array}{l}15.7 \\
17.5 \\
15.8 \\
14.4 \\
11.5\end{array}$ & $\begin{array}{l}15.3 \\
14.0 \\
12.2 \\
12.5 \\
12.2\end{array}$ & $\begin{array}{r}10.5 \\
3.7 \\
6.9 \\
8.5 \\
8.5\end{array}$ \\
\hline $\begin{array}{l}11 \\
12 \\
13 \\
14 \\
15\end{array}$ & $\begin{array}{l}3.6 \\
4.7 \\
7.0 \\
2.2 \\
6.9\end{array}$ & $\begin{array}{r}-3.7 \\
1.7 \\
1.6 \\
-9.1 \\
-9.5\end{array}$ & $\begin{array}{r}2.0 \\
3.1 \\
2.0 \\
-7.0 \\
-13.0\end{array}$ & $\begin{array}{r}-15.2 \\
-8.2 \\
-3.5 \\
-12.2 \\
-9.8\end{array}$ & $\begin{array}{r}-10.1 \\
-12.4 \\
-14.9 \\
-12.2 \\
-7.0\end{array}$ & $\begin{array}{r}2.9 \\
2.2 \\
-4.9 \\
-6.2 \\
-.6\end{array}$ & $\begin{array}{l}2.8 \\
3.1 \\
5.3 \\
7.5 \\
5.1\end{array}$ & $\begin{array}{r}-2.4 \\
-2.4 \\
-2.2 \\
-1.5 \\
.2\end{array}$ & $\begin{array}{r}8.1 \\
11.3 \\
13.0 \\
14.5 \\
14.4\end{array}$ & $\begin{array}{l}14.4 \\
11.2 \\
14.4 \\
16.6 \\
15.0\end{array}$ & $\begin{array}{l}12.8 \\
14.4 \\
15.6 \\
13.9 \\
14.5\end{array}$ & $\begin{array}{r}7.3 \\
5.8 \\
8.6 \\
11.2 \\
12.6\end{array}$ \\
\hline $\begin{array}{l}16 \\
17 \\
18 \\
19 \\
20\end{array}$ & $\begin{array}{l}9.6 \\
8.0 \\
9.0 \\
7.6 \\
8.1\end{array}$ & $\begin{array}{r}-5.7 \\
-10.2 \\
-11.7 \\
-7.4 \\
-6.9\end{array}$ & $\begin{array}{l}-7.0 \\
-4.2 \\
-6.7 \\
-9.1 \\
-6.4\end{array}$ & $\begin{array}{r}-5.8 \\
-2.5 \\
1.0 \\
-.4 \\
-.9\end{array}$ & $\begin{array}{r}-3.7 \\
-.1 \\
.1 \\
-5.5 \\
-6.9\end{array}$ & $\begin{array}{r}-3.6 \\
-3.9 \\
.0 \\
-3.9 \\
-4.0\end{array}$ & $\begin{array}{r}5.6 \\
7.9 \\
9.6 \\
10.4 \\
9.8\end{array}$ & $\begin{array}{l}2.7 \\
6.9 \\
5.2 \\
3.2 \\
8.7\end{array}$ & $\begin{array}{r}9.2 \\
11.5 \\
14.6 \\
15.0 \\
8.3\end{array}$ & $\begin{array}{l}11.6 \\
13.8 \\
17.6 \\
20.2 \\
18.1\end{array}$ & $\begin{array}{r}14.3 \\
12.8 \\
11.4 \\
11.2 \\
8.4\end{array}$ & $\begin{array}{r}11.2 \\
2.9 \\
2.1 \\
3.1 \\
5.7\end{array}$ \\
\hline $\begin{array}{l}21 \\
22 \\
23 \\
24 \\
25\end{array}$ & $\begin{array}{l}8.4 \\
7.1 \\
7.6 \\
6.9 \\
6.8\end{array}$ & $\begin{array}{r}-3.0 \\
1.5 \\
-3.2 \\
-11.7 \\
-7.8\end{array}$ & $\begin{array}{r}-12.0 \\
-10.4 \\
-13.3 \\
-8.2 \\
-15.8\end{array}$ & $\begin{array}{r}-.4 \\
-.8 \\
-6.7 \\
-9.0 \\
-7.7\end{array}$ & $\begin{array}{r}-1.0 \\
2.7 \\
2.4 \\
3.9 \\
3.1\end{array}$ & $\begin{array}{r}1.7 \\
.5 \\
.5 \\
1.9 \\
-3.6\end{array}$ & $\begin{array}{r}7.0 \\
-1.1 \\
-1.9 \\
-8.0 \\
-6.9\end{array}$ & $\begin{array}{r}6.7 \\
9.6 \\
2.8 \\
-2.2 \\
.5\end{array}$ & $\begin{array}{l}6.9 \\
9.5 \\
7.4 \\
7.4 \\
8.2\end{array}$ & $\begin{array}{l}14.8 \\
13.1 \\
12.2 \\
13.4 \\
16.0\end{array}$ & $\begin{array}{r}9.9 \\
12.8 \\
6.5 \\
3.2 \\
6.5\end{array}$ & $\begin{array}{r}7.9 \\
9.3 \\
11.6 \\
13.5 \\
11.8\end{array}$ \\
\hline $\begin{array}{l}26 \\
27 \\
28 \\
29 \\
30 \\
31\end{array}$ & $\begin{array}{l}7.6 \\
6.7 \\
5.6 \\
4.9 \\
4.6 \\
8.9\end{array}$ & $\begin{array}{r}-9.8 \\
-2.0 \\
-3.1 \\
-2.6 \\
.5 \\
---\end{array}$ & $\begin{array}{r}-17.9 \\
-12.2 \\
-16.4 \\
-9.1 \\
-4.0 \\
-10.7\end{array}$ & $\begin{array}{r}-4.2 \\
-4.6 \\
-2.5 \\
.7 \\
4.4 \\
2.0\end{array}$ & $\begin{array}{r}.4 \\
-4.3 \\
-4.3 \\
--- \\
---\end{array}$ & $\begin{array}{r}-6.4 \\
-2.1 \\
1.0 \\
-2.2 \\
.8 \\
1.1\end{array}$ & $\begin{array}{r}-6.1 \\
-5.6 \\
-4.5 \\
.1 \\
4.3 \\
-.-\end{array}$ & $\begin{array}{r}5.8 \\
8.2 \\
2.3 \\
-3.3 \\
-2.3 \\
3.0\end{array}$ & $\begin{array}{r}11.4 \\
9.0 \\
11.3 \\
13.5 \\
11.0 \\
-\ldots\end{array}$ & $\begin{array}{l}18.4 \\
16.8 \\
16.6 \\
16.7 \\
18.0 \\
17.0\end{array}$ & $\begin{array}{r}10.6 \\
10.7 \\
13.4 \\
15.3 \\
9.9 \\
8.8\end{array}$ & $\begin{array}{r}12.2 \\
11.3 \\
11.3 \\
9.8 \\
3.1 \\
-\end{array}$ \\
\hline $\begin{array}{l}\text { MEAN } \\
\text { MAX } \\
\text { MIN } \\
\text { MED }\end{array}$ & $\begin{array}{r}7.6 \\
12.6 \\
2.2 \\
7.6\end{array}$ & $\begin{array}{r}-2.7 \\
6.8 \\
-11.7 \\
-2.8\end{array}$ & $\begin{array}{r}-6.0 \\
3.4 \\
-17.9 \\
-6.7\end{array}$ & $\begin{array}{r}-5.4 \\
4.4 \\
-15.8 \\
-4.6\end{array}$ & $\begin{array}{r}-6.7 \\
3.9 \\
-21.9 \\
-6.2\end{array}$ & $\begin{array}{r}-1.0 \\
5.0 \\
-15.0 \\
.0\end{array}$ & $\begin{array}{r}2.8 \\
10.4 \\
-8.0 \\
4.7\end{array}$ & $\begin{array}{r}4.0 \\
13.0 \\
-3.3 \\
4.7\end{array}$ & $\begin{array}{r}9.4 \\
15.0 \\
4.1 \\
8.3\end{array}$ & $\begin{array}{l}15.3 \\
20.2 \\
11.2 \\
15.1\end{array}$ & $\begin{array}{r}12.0 \\
15.6 \\
3.2 \\
12.8\end{array}$ & $\begin{array}{r}9.0 \\
13.7 \\
2.1 \\
10.1\end{array}$ \\
\hline
\end{tabular}

CAL YR 1988 MEAN 3.3 MAX 18.9 MIN -17.9 MED 2.8 WTR YR 1989 MEAN 3.3 MAX 20.2 MIN -21.9 MED 4.1 
EAST STEWART BASIN

EAST STEWART CREEK NEAR IONE, NV

DAILY MEAN AIR TEMPERATURE, WATER YEAR OCTOBER 1989 TO SEPTEMBER 1990 DEGREES CELSIUS

\begin{tabular}{|c|c|c|c|c|c|c|c|c|c|c|c|c|}
\hline DAY & OCT & NOV & DEC & JAN & FEB & MAR & $\mathrm{APR}$ & MAY & JUN & JUL & AUG & SEP \\
\hline 1 & 5.0 & -1.3 & -1.0 & -2.8 & -10.8 & -.2 & 4.5 & -1.6 & .6 & 17.0 & 12.8 & 14.7 \\
\hline 2 & .5 & -.3 & .6 & -12.1 & -10.2 & -.4 & 3.9 & 1.1 & 9.3 & 13.2 & 14.0 & 15.2 \\
\hline 3 & -2.6 & 1.7 & 1.2 & -11.4 & -2.8 & -.3 & 3.7 & 3.6 & 13.6 & 11.8 & 15.8 & 14.9 \\
\hline 4 & -.7 & 2.2 & 1.4 & -8.9 & -9.7 & -1.5 & 3.0 & 8.1 & 13.1 & 15.4 & 16.8 & 14.1 \\
\hline 5 & 1.9 & 1.4 & 1.1 & -4.5 & -6.6 & -8.2 & 1.7 & 10.4 & 9.5 & 15.0 & 18.3 & 13.7 \\
\hline 6 & 3.7 & -2.0 & -2.8 & -2.1 & -5.3 & -6.0 & 1.6 & 10.5 & 7.7 & 15.2 & 19.4 & 13.3 \\
\hline 7 & 5.0 & -3.4 & .8 & -.3 & -14.0 & .3 & .3 & 5.3 & 10.1 & 15.8 & 17.0 & 14.4 \\
\hline 8 & 6.7 & -.5 & 3.9 & 2.2 & -11.0 & .8 & -3.6 & 1.4 & 13.0 & 14.9 & 13.6 & 15.2 \\
\hline 9 & 9.2 & 3.7 & -.4 & 6.2 & -5.1 & .8 & -1.5 & 6.3 & 12.2 & 15.3 & 14.9 & 15.6 \\
\hline 10 & 10.3 & 5.7 & $-10 \cdot 9$ & 6.6 & -1.0 & -2.1 & 2.9 & 3.1 & 6.5 & 14.8 & 15.0 & 17.3 \\
\hline 11 & 8.1 & 5.9 & -6.9 & 3.0 & 2.8 & -12.5 & 4.3 & .9 & 6.4 & 15.9 & 12.4 & 16.8 \\
\hline 12 & 11.0 & 5.2 & -1.5 & .0 & -3.6 & -14.0 & 3.6 & 4.4 & 4.5 & 16.7 & 13.9 & 16.3 \\
\hline 13 & 9.4 & 3.3 & -3.3 & $-3 \cdot 3$ & -16.2 & -13.2 & 6.5 & 3.6 & 3.5 & 14.3 & 14.4 & 15.8 \\
\hline 14 & 5.9 & -.9 & -1.2 & -8.5 & -21.6 & -6.6 & 9.3 & 2.6 & 2.3 & 13.0 & 15.5 & 15.6 \\
\hline 15 & 1.7 & 1.1 & -.1 & -9.3 & -14.9 & -4.3 & 9.0 & .1 & 3.2 & 12.8 & 9.5 & 12.3 \\
\hline 16 & 2.1 & .2 & -4.2 & -8.8 & -10.9 & .8 & 4.1 & 4.5 & 6.3 & 12.7 & 10.9 & 9.1 \\
\hline 17 & 4.3 & -.9 & -4.0 & -9.6 & -11.3 & 1.1 & -1.3 & 5.6 & 10.6 & 12.9 & 10.8 & 8.0 \\
\hline 18 & 6.3 & 1.8 & -7.6 & -9.7 & -13.7 & 2.6 & .9 & 2.7 & 6.3 & 13.4 & 5.0 & 5.6 \\
\hline 19 & 9.2 & 6.0 & $-5 \cdot 3$ & -9.7 & -9.8 & 4.9 & 4.6 & .4 & 10.2 & 13.4 & 1.8 & 5.4 \\
\hline 20 & 5.7 & 6.0 & -3.9 & -5.0 & -4.9 & 4.3 & 2.4 & 4.1 & 13.8 & 14.0 & 3.4 & 8.1 \\
\hline 21 & 3.7 & 2.3 & -3.1 & -2.0 & -2.1 & 3.0 & .7 & 8.0 & 17.7 & 15.7 & 7.8 & 8.6 \\
\hline 22 & 4.0 & 2.5 & 1.1 & -2.2 & 1.0 & 3.2 & 1.5 & 7.2 & 18.2 & 16.3 & 9.4 & 6.6 \\
\hline 23 & 4.4 & 1.7 & .4 & $-4 \cdot 5$ & 2.0 & 1.4 & -1.4 & 3.0 & 17.1 & 14.8 & 10.9 & 4.8 \\
\hline 24 & 3.3 & -2.7 & .8 & -5.8 & 2.0 & 1.9 & -4.8 & -3.0 & 16.7 & 12.2 & 8.5 & 6.2 \\
\hline 25 & -6.7 & -1.9 & .7 & -.2 & .7 & 2.8 & .4 & 3.7 & 16.7 & 10.7 & 8.0 & 7.6 \\
\hline 26 & -5.6 & -9.0 & .5 & -8.3 & .4 & 2.1 & 3.0 & 4.2 & 14.7 & 10.8 & 9.8 & 7.1 \\
\hline 27 & -2.7 & -10.5 & .3 & -11.0 & -1.5 & -3.4 & 5.6 & 4.9 & 12.9 & 14.1 & 12.8 & 5.4 \\
\hline 28 & -9.3 & -3.9 & -5.7 & -6.6 & -.2 & -5.6 & 2.3 & -.3 & 14.8 & 15.6 & 12.8 & 4.1 \\
\hline 29 & -5.4 & -1.0 & -7.3 & -5.1 & -- & -5.2 & -4.8 & 2.0 & 18.2 & 17.6 & 15.6 & 7.1 \\
\hline 30 & -1.5 & -3.4 & -2.9 & -6.4 & --- & -1.1 & -5.0 & 1.9 & 17.9 & 16.9 & 12.3 & 8.4 \\
\hline 31 & -1.5 & --- & -1.0 & -9.9 & --- & 1.7 & --- & -.2 & --- & 15.0 & 13.0 & --- \\
\hline MEAN & 2.8 & .3 & -1.9 & -4.8 & -6.4 & -1.7 & 1.9 & 3.5 & 10.9 & 14.4 & 12.1 & 10.9 \\
\hline MAX & 11.0 & 6.0 & 3.9 & 6.6 & 2.8 & 4.9 & 9.3 & 10.5 & 18.2 & 17.6 & 19.4 & 17.3 \\
\hline MIN & -9.3 & -10.5 & -10.9 & -12.1 & -21.6 & -14.0 & -5.0 & -3.0 & .6 & 10.7 & 1.8 & 4.1 \\
\hline MED & 3.7 & .6 & -1.0 & -5.1 & -5.2 & -.2 & 2.3 & 3.6 & 11.4 & 14.8 & 12.8 & 10.7 \\
\hline
\end{tabular}

CAL YR 1989 MEAN 3.4 MAX 20.2 MIN -21.9 MED 3.2

WTR YR 1990

MEAN 3.6 MAX 19.4 MIN -21.6 MED 3.0 
EAST STEWART BASIN

EAST STEWART CREEK NEAR IONE, NV

DAILY MEAN AIR TEMPERATURE, WATER YEAR OCTOBER 1990 TO SEPTEMBER 1991 DEGREES CELSIUS

\begin{tabular}{|c|c|c|c|c|c|c|c|c|c|c|c|c|}
\hline DAY & OCT & NOV & DEC & JAN & FEB & MAR & $\mathrm{APR}$ & MAY & JUN & JUL & AUG & SEP \\
\hline $\begin{array}{l}1 \\
2 \\
3 \\
4 \\
5\end{array}$ & $\begin{array}{r}8.5 \\
5.5 \\
6.3 \\
11.3 \\
9.4\end{array}$ & $\begin{array}{r}-6.9 \\
-10.2 \\
-2.9 \\
1.3 \\
-4.6\end{array}$ & $\begin{array}{r}-6.4 \\
-7.7 \\
-3.7 \\
2.1 \\
-1.4\end{array}$ & $\begin{array}{l}-2.4 \\
-3.6 \\
-2.3 \\
-3.9 \\
-5.1\end{array}$ & $\begin{array}{r}1.6 \\
-2.3 \\
2.3 \\
-1.2\end{array}$ & $\begin{array}{r}-4.3 \\
-4.5 \\
-.9 \\
-.5 \\
-8.0\end{array}$ & $\begin{array}{r}-2.3 \\
-2.4 \\
.2 \\
6.1 \\
7.9\end{array}$ & $\begin{array}{r}-4.5 \\
-5.8 \\
-4.4 \\
-.4 \\
5.0\end{array}$ & $\begin{array}{l}6.1 \\
8.5 \\
9.5 \\
8.7 \\
4.4\end{array}$ & $\begin{array}{l}13.5 \\
16.4 \\
18.3 \\
19.1 \\
17.6\end{array}$ & $\begin{array}{l}14.1 \\
12.1 \\
13.7 \\
13.8 \\
13.5\end{array}$ & $\begin{array}{l}12.5 \\
14.2 \\
12.9 \\
12.8 \\
11.6\end{array}$ \\
\hline $\begin{array}{r}6 \\
7 \\
8 \\
9 \\
10\end{array}$ & $\begin{array}{r}3.0 \\
-2.5 \\
-.6 \\
4.7 \\
5.9\end{array}$ & $\begin{array}{r}-10.4 \\
-3.4 \\
.8 \\
1.9 \\
4.5\end{array}$ & $\begin{array}{r}-1.6 \\
.8 \\
1.1 \\
6.2 \\
4.2\end{array}$ & $\begin{array}{l}-2.2 \\
-4.2 \\
-7.2 \\
-6.9 \\
-4.8\end{array}$ & $\begin{array}{r}-1.2 \\
.5 \\
-.4 \\
.5 \\
.0\end{array}$ & $\begin{array}{r}-11.9 \\
-8.2 \\
-3.3 \\
-1.2 \\
-3.2\end{array}$ & $\begin{array}{r}3.4 \\
-4.4 \\
-4.3 \\
.4 \\
-10.7\end{array}$ & $\begin{array}{r}2.4 \\
6.6 \\
4.7 \\
-7.5 \\
-7.2\end{array}$ & $\begin{array}{r}8.8 \\
8.4 \\
10.1 \\
12.9 \\
14.3\end{array}$ & $\begin{array}{l}16.3 \\
15.5 \\
13.9 \\
13.4 \\
13.3\end{array}$ & $\begin{array}{l}14.1 \\
13.7 \\
14.1 \\
16.9 \\
15.5\end{array}$ & $\begin{array}{l}8.8 \\
8.0 \\
8.1 \\
2.8 \\
1.4\end{array}$ \\
\hline $\begin{array}{l}11 \\
12 \\
13 \\
14 \\
15\end{array}$ & $\begin{array}{l}2.6 \\
6.3 \\
6.2 \\
7.4 \\
8.4\end{array}$ & $\begin{array}{l}7.0 \\
7.4 \\
9.3 \\
6.0 \\
2.0\end{array}$ & $\begin{array}{r}-.9 \\
-5.1 \\
-9.2 \\
-11.3 \\
-7.4\end{array}$ & $\begin{array}{r}-1.8 \\
-.7 \\
-3.0 \\
-2.8 \\
-5.9\end{array}$ & $\begin{array}{r}.8 \\
-.9 \\
-.8 \\
1.9 \\
2.9\end{array}$ & $\begin{array}{r}-11.5 \\
-6.8 \\
-6.3 \\
-7.8 \\
-8.9\end{array}$ & $\begin{array}{r}-11.5 \\
-6.8 \\
-4.4 \\
-.1 \\
-2.2\end{array}$ & $\begin{array}{r}-4.1 \\
2.0 \\
1.0 \\
-2.1 \\
3.0\end{array}$ & $\begin{array}{r}14.3 \\
13.8 \\
11.0 \\
7.1 \\
10.5\end{array}$ & $\begin{array}{l}15.8 \\
18.2 \\
18.7 \\
16.7 \\
15.8\end{array}$ & $\begin{array}{l}13.7 \\
13.2 \\
12.6 \\
14.9 \\
12.3\end{array}$ & $\begin{array}{l}4.6 \\
5.5 \\
7.7 \\
7.9 \\
8.1\end{array}$ \\
\hline $\begin{array}{l}16 \\
17 \\
18 \\
19 \\
20\end{array}$ & $\begin{array}{r}4.3 \\
5.7 \\
4.9 \\
-4.4 \\
-4.5\end{array}$ & $\begin{array}{r}5.8 \\
1.0 \\
1.4 \\
-1.8 \\
-10.1\end{array}$ & $\begin{array}{r}-12.7 \\
-9.3 \\
-6.4 \\
-15.0 \\
-21.5\end{array}$ & $\begin{array}{r}-7.2 \\
-2.0 \\
-.7 \\
-3.9 \\
-8.7\end{array}$ & $\begin{array}{l}-3.1 \\
-7.9 \\
-7.4 \\
-1.1 \\
-.2\end{array}$ & $\begin{array}{l}-6.3 \\
-4.5 \\
-5.4 \\
-7.3 \\
-7.7\end{array}$ & $\begin{array}{r}-5.4 \\
-5.4 \\
-2.0 \\
2.1 \\
.1\end{array}$ & $\begin{array}{r}5.5 \\
-4.7 \\
-6.6 \\
-3.3 \\
-.8\end{array}$ & $\begin{array}{r}11.3 \\
11.1 \\
12.8 \\
7.9 \\
6.1\end{array}$ & $\begin{array}{l}16.3 \\
15.5 \\
16.1 \\
13.4 \\
11.6\end{array}$ & $\begin{array}{l}12.3 \\
15.4 \\
16.5 \\
16.9 \\
16.8\end{array}$ & $\begin{array}{l}10.4 \\
12.1 \\
13.0 \\
12.8 \\
12.5\end{array}$ \\
\hline $\begin{array}{l}21 \\
22 \\
23 \\
24 \\
25\end{array}$ & $\begin{array}{r}2.6 \\
3.6 \\
5.8 \\
8.0 \\
10.4\end{array}$ & $\begin{array}{r}-7.3 \\
-.9 \\
2.2 \\
2.7 \\
3.8\end{array}$ & $\begin{array}{r}-24.8 \\
-21.3 \\
-16.1 \\
-11.4 \\
-9.0\end{array}$ & $\begin{array}{l}-9.0 \\
-3.5 \\
-4.7 \\
-5.9 \\
-6.9\end{array}$ & $\begin{array}{r}2.2 \\
1.2 \\
-2.1 \\
-2.6 \\
-1.0\end{array}$ & $\begin{array}{l}-7.8 \\
-6.6 \\
-3.5 \\
-4.1 \\
-8.7\end{array}$ & $\begin{array}{r}-2.7 \\
-2.8 \\
1.2 \\
-.4 \\
-7.6\end{array}$ & $\begin{array}{l}.7 \\
4.8 \\
7.5 \\
7.2 \\
7.0\end{array}$ & $\begin{array}{l}8.6 \\
8.8 \\
9.1 \\
5.9 \\
4.5\end{array}$ & $\begin{array}{l}12.0 \\
15.0 \\
16.2 \\
15.1 \\
14.9\end{array}$ & $\begin{array}{l}17.8 \\
16.3 \\
16.1 \\
16.8 \\
16.1\end{array}$ & $\begin{array}{l}10.3 \\
10.1 \\
10.5 \\
10.9 \\
13.8\end{array}$ \\
\hline $\begin{array}{l}26 \\
27 \\
28 \\
29 \\
30 \\
31\end{array}$ & $\begin{array}{r}8.1 \\
11.0 \\
8.6 \\
7.5 \\
6.9 \\
1.5\end{array}$ & $\begin{array}{r}-13.3 \\
-10.3 \\
-5.2 \\
2.9 \\
-3.7 \\
---\end{array}$ & $\begin{array}{r}-10.2 \\
-5.0 \\
-6.6 \\
-15.5 \\
-7.0 \\
-5.0\end{array}$ & $\begin{array}{l}-6.5 \\
-7.9 \\
-8.4 \\
-8.3 \\
-3.2 \\
1.0\end{array}$ & $\begin{array}{r}-.5 \\
-1.0 \\
-2.6 \\
--- \\
--- \\
---\end{array}$ & $\begin{array}{r}-8.6 \\
-7.5 \\
-5.2 \\
-3.9 \\
.1 \\
1.3\end{array}$ & $\begin{array}{r}-5.9 \\
-6.0 \\
-2.3 \\
-1.4 \\
1.0 \\
---\end{array}$ & $\begin{array}{r}3.3 \\
2.6 \\
4.2 \\
3.7 \\
-3.1 \\
-1.7\end{array}$ & $\begin{array}{l}5.9 \\
4.2 \\
5.8 \\
3.0 \\
8.7 \\
---\end{array}$ & $\begin{array}{l}15.0 \\
16.6 \\
17.2 \\
18.7 \\
15.3 \\
12.9\end{array}$ & $\begin{array}{r}14.5 \\
9.3 \\
8.9 \\
15.3 \\
17.0 \\
15.4\end{array}$ & $\begin{array}{r}10.2 \\
8.3 \\
6.3 \\
8.1 \\
8.0 \\
---\end{array}$ \\
\hline $\begin{array}{l}\text { MEAN } \\
\text { MAX } \\
\text { MIN } \\
\text { MED }\end{array}$ & $\begin{array}{r}5.2 \\
11.3 \\
-4.5 \\
5.9\end{array}$ & $\begin{array}{r}-1.0 \\
9.3 \\
-13.3 \\
.9\end{array}$ & $\begin{array}{r}-7.6 \\
6.2 \\
-24.8 \\
-7.0\end{array}$ & $\begin{array}{r}-4.6 \\
1.0 \\
-9.0 \\
-4.2\end{array}$ & $\begin{array}{r}-.8 \\
2.9 \\
-7.9 \\
-.6\end{array}$ & $\begin{array}{r}-5.6 \\
1.3 \\
-11.9 \\
-6.3\end{array}$ & $\begin{array}{r}-2.3 \\
7.9 \\
-11.5 \\
-2.3\end{array}$ & $\begin{array}{r}.5 \\
7.5 \\
-7.5 \\
1.0\end{array}$ & $\begin{array}{r}8.7 \\
14.3 \\
3.0 \\
8.7\end{array}$ & $\begin{array}{l}15.6 \\
19.1 \\
11.6 \\
15.8\end{array}$ & $\begin{array}{r}14.5 \\
17.8 \\
8.9 \\
14.5\end{array}$ & $\begin{array}{r}9.5 \\
14.2 \\
1.4 \\
10.1\end{array}$ \\
\hline
\end{tabular}

CAL YR 1990 MEAN 3.2 MAX 19.4 MIN -24.8 MED 3.2

WTR YR 1991 MEAN 2.7 MAX 19.1 MIN -24.8 MED 2.0 
EAST STEWART BASIN

EAST STEWART CREEK NEAR IONE, NV

DAILY MAXIMUM AIR TEMPERATURE, WATER YEAR OCTOBER 1985 TO SEPTEMBER 1986 DEGREES CELSIUS

\begin{tabular}{|c|c|c|c|c|c|c|c|c|c|c|c|c|}
\hline DAY & OCT & NOV & DEC & JAN & FEB & MAR & APR & MAY & JUN & JUL & AUG & SEP \\
\hline $\begin{array}{l}1 \\
2 \\
3 \\
4 \\
5\end{array}$ & $\begin{array}{l}--- \\
--- \\
--- \\
--- \\
---\end{array}$ & $\begin{array}{l}--- \\
--- \\
--- \\
--- \\
---\end{array}$ & $\begin{array}{l}--- \\
--- \\
--- \\
--- \\
---\end{array}$ & $\begin{array}{l}--- \\
--- \\
--- \\
--- \\
---\end{array}$ & $\begin{array}{r}2.7 \\
-1.3 \\
-3.2 \\
-5.6 \\
-5.9\end{array}$ & $\begin{array}{r}10.1 \\
1.9 \\
11.2 \\
5.8 \\
7.3\end{array}$ & $\begin{array}{r}4.4 \\
-9.2 \\
1.5 \\
3.6 \\
2.6\end{array}$ & $\begin{array}{r}10.4 \\
12.1 \\
10.0 \\
-2.5 \\
1.4\end{array}$ & $\begin{array}{l}16.3 \\
17.2 \\
19.0 \\
18.7 \\
14.2\end{array}$ & $\begin{array}{l}22.4 \\
21.2 \\
21.0 \\
18.2 \\
15.4\end{array}$ & $\begin{array}{l}23.2 \\
23.1 \\
22.6 \\
23.0 \\
21.7\end{array}$ & $\begin{array}{l}16.8 \\
17.1 \\
19.3 \\
20.5 \\
21.3\end{array}$ \\
\hline $\begin{array}{r}6 \\
7 \\
8 \\
9 \\
10\end{array}$ & $\begin{array}{l}--- \\
--- \\
--- \\
--- \\
---\end{array}$ & $\begin{array}{l}--- \\
--- \\
--- \\
--- \\
---\end{array}$ & $\begin{array}{l}--- \\
--- \\
--- \\
--- \\
---\end{array}$ & $\begin{array}{l}--- \\
--- \\
--- \\
--- \\
---\end{array}$ & $\begin{array}{r}-11.5 \\
-7.3 \\
-8.5 \\
-7.7 \\
-3.7\end{array}$ & $\begin{array}{r}5.1 \\
4.8 \\
1.5 \\
-2.1 \\
-2.5\end{array}$ & $\begin{array}{r}2.6 \\
-2.0 \\
-2.2 \\
4.0 \\
4.3\end{array}$ & $\begin{array}{r}-4.4 \\
-2.8 \\
-.6 \\
5.6 \\
5.0\end{array}$ & $\begin{array}{l}16.1 \\
11.4 \\
10.4 \\
11.8 \\
17.2\end{array}$ & $\begin{array}{r}16.7 \\
17.2 \\
17.0 \\
--. \\
-.-\end{array}$ & $\begin{array}{l}22.3 \\
22.6 \\
23.0 \\
23.3 \\
19.3\end{array}$ & $\begin{array}{r}20.1 \\
18.7 \\
14.4 \\
7.1 \\
11.0\end{array}$ \\
\hline $\begin{array}{l}11 \\
12 \\
13 \\
14 \\
15\end{array}$ & $\begin{array}{l}--- \\
--- \\
--- \\
---\end{array}$ & $\begin{array}{l}--- \\
--- \\
--- \\
--- \\
---\end{array}$ & $\begin{array}{l}--- \\
--- \\
--- \\
--- \\
---\end{array}$ & $\begin{array}{l}--- \\
9.2 \\
8.7 \\
4.3 \\
-.8\end{array}$ & $\begin{array}{r}-2.7 \\
-.4 \\
-.3 \\
2.2 \\
-1.9\end{array}$ & $\begin{array}{l}-5.4 \\
-5.6 \\
-4.8 \\
-6.6 \\
-3.1\end{array}$ & $\begin{array}{r}7.3 \\
1.3 \\
-2.4 \\
3.3 \\
4.4\end{array}$ & $\begin{array}{l}5.4 \\
8.2 \\
9.6 \\
6.9 \\
7.4\end{array}$ & $\begin{array}{l}18.4 \\
14.1 \\
17.5 \\
18.3 \\
18.8\end{array}$ & $\begin{array}{l}--- \\
--- \\
--- \\
---\end{array}$ & $\begin{array}{l}20.5 \\
20.5 \\
20.5 \\
21.8 \\
21.9\end{array}$ & $\begin{array}{r}15.1 \\
12.6 \\
12.1 \\
9.6 \\
7.6\end{array}$ \\
\hline $\begin{array}{l}16 \\
17 \\
18 \\
19 \\
20\end{array}$ & $\begin{array}{l}--- \\
--- \\
--- \\
---\end{array}$ & $\begin{array}{l}--- \\
--- \\
--- \\
--- \\
---\end{array}$ & $\begin{array}{l}--- \\
--- \\
--- \\
--- \\
---\end{array}$ & $\begin{array}{r}2.6 \\
.9 \\
10.3 \\
8.0 \\
-1.3\end{array}$ & $\begin{array}{r}.0 \\
1.4 \\
2.0 \\
.4 \\
-4.3\end{array}$ & $\begin{array}{r}-3.3 \\
-8.7 \\
-2.9 \\
2.1 \\
9.8\end{array}$ & $\begin{array}{r}-6.2 \\
-4.9 \\
.3 \\
6.4 \\
11.6\end{array}$ & $\begin{array}{r}5.9 \\
12.7 \\
15.9 \\
16.8 \\
13.4\end{array}$ & $\begin{array}{l}19.6 \\
19.6 \\
15.7 \\
14.7 \\
15.9\end{array}$ & $\begin{array}{l}--- \\
--- \\
--- \\
--- \\
---\end{array}$ & $\begin{array}{l}21.2 \\
22.4 \\
19.1 \\
15.0 \\
13.5\end{array}$ & $\begin{array}{l}8.6 \\
6.9 \\
5.8 \\
4.6 \\
4.9\end{array}$ \\
\hline $\begin{array}{l}21 \\
22 \\
23 \\
24 \\
25\end{array}$ & $\begin{array}{l}--- \\
--- \\
--- \\
---\end{array}$ & $\begin{array}{l}--- \\
--- \\
--- \\
--- \\
---\end{array}$ & $\begin{array}{l}--- \\
--- \\
--- \\
---\end{array}$ & $\begin{array}{r}1.7 \\
5.3 \\
-1.0 \\
4.7 \\
7.7\end{array}$ & $\begin{array}{r}.9 \\
.2 \\
5.7 \\
14.1 \\
11.8\end{array}$ & $\begin{array}{l}6.6 \\
7.1 \\
7.4 \\
4.4 \\
5.7\end{array}$ & $\begin{array}{r}14.4 \\
11.6 \\
4.2 \\
2.1 \\
-. .2\end{array}$ & $\begin{array}{r}4.3 \\
9.4 \\
12.3 \\
17.1 \\
19.4\end{array}$ & $\begin{array}{l}18.6 \\
20.4 \\
21.6 \\
19.8 \\
20.1\end{array}$ & $\begin{array}{l}--- \\
--- \\
--- \\
--- \\
---\end{array}$ & $\begin{array}{l}18.1 \\
20.5 \\
21.2 \\
20.8 \\
19.2\end{array}$ & $\begin{array}{r}7.6 \\
7.9 \\
4.8 \\
3.3 \\
-1.8\end{array}$ \\
\hline $\begin{array}{l}26 \\
27 \\
28 \\
29 \\
30 \\
31\end{array}$ & $\begin{array}{l}--- \\
--- \\
--- \\
--- \\
--- \\
---\end{array}$ & $\begin{array}{l}--- \\
--- \\
--- \\
--- \\
--- \\
---\end{array}$ & $\begin{array}{l}--- \\
--- \\
--- \\
--- \\
---\end{array}$ & $\begin{array}{r}8.0 \\
10.5 \\
9.7 \\
5.6 \\
1.5 \\
.0\end{array}$ & $\begin{array}{r}13.0 \\
14.5 \\
13.3 \\
--- \\
--- \\
---\end{array}$ & $\begin{array}{r}11.6 \\
10.8 \\
9.5 \\
9.7 \\
7.3 \\
7.0\end{array}$ & $\begin{array}{r}-1.7 \\
7.8 \\
9.0 \\
6.3 \\
10.9 \\
---\end{array}$ & $\begin{array}{l}19.4 \\
19.8 \\
18.0 \\
19.4 \\
19.7 \\
19.6\end{array}$ & $\begin{array}{r}23.2 \\
20.7 \\
18.3 \\
17.2 \\
19.9 \\
-.-\end{array}$ & $\begin{array}{l}--- \\
--- \\
--- \\
--- \\
--- \\
---\end{array}$ & $\begin{array}{l}20.0 \\
15.1 \\
17.1 \\
18.2 \\
16.9 \\
16.6\end{array}$ & $\begin{array}{l}2.1 \\
-.3 \\
3.0 \\
5.5 \\
5.7 \\
-.--\end{array}$ \\
\hline MAX & --- & --- & -- & --- & 14.5 & 11.6 & 14.4 & 19.8 & 23.2 & -- & 23.3 & 21.3 \\
\hline
\end{tabular}


EAST STEWART BASIN

EAST STEWART CREEK NEAR IONE, NV

DAILY MAXIMUM AIR TEMPERATURE, WATER YEAR OCTOBER 1986 TO SEPTEMBER 1987 DEGREES CELSIUS

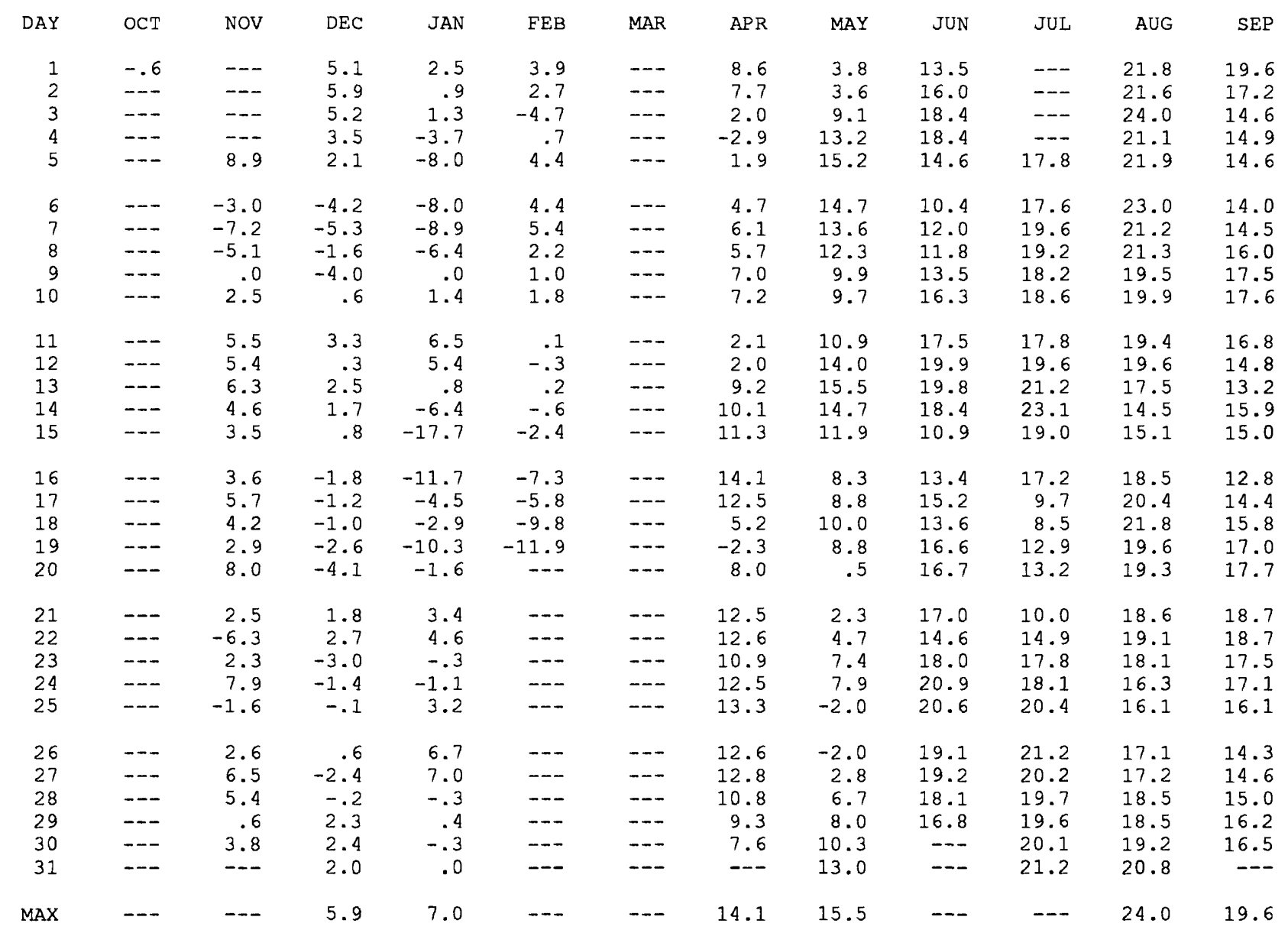


EAST STEWART BASIN

EAST STEWART CREEK NEAR IONE, NV

DAILY MAXIMUM AIR TEMPERATURE, WATER YEAR OCTOBER 1987 TO SEPTEMBER 1988 DEGREES CELSIUS

\begin{tabular}{|c|c|c|c|c|c|c|c|c|c|c|c|c|}
\hline DAY & OCT & NOV & $\mathrm{DEC}$ & JAN & FEB & MAR & $\mathrm{APR}$ & MAY & JUN & JUL & AUG & SEP \\
\hline $\begin{array}{l}1 \\
2 \\
3 \\
4 \\
5\end{array}$ & $\begin{array}{l}16.7 \\
17.1 \\
17.2 \\
17.0 \\
16.7\end{array}$ & $\begin{array}{r}3.2 \\
-.6 \\
.2 \\
4.2 \\
2.1\end{array}$ & $\begin{array}{l}1.6 \\
4.7 \\
8.1 \\
5.6 \\
3.0\end{array}$ & $\begin{array}{r}-6.3 \\
-2.5 \\
-1.5 \\
.7 \\
-.5\end{array}$ & $\begin{array}{r}-8.7 \\
-8.0 \\
-3.9 \\
-2.2 \\
.8\end{array}$ & $\begin{array}{r}-2.3 \\
-2.5 \\
1.0 \\
2.1 \\
2.7\end{array}$ & $\begin{array}{r}6.6 \\
7.8 \\
4.5 \\
3.4 \\
11.2\end{array}$ & $\begin{array}{r}-5.7 \\
2.3 \\
5.9 \\
2.6 \\
.4\end{array}$ & $\begin{array}{r}9.3 \\
18.1 \\
19.4 \\
16.7 \\
7.2\end{array}$ & $\begin{array}{l}21.5 \\
21.7 \\
21.0 \\
19.3 \\
18.0\end{array}$ & $\begin{array}{l}20.4 \\
18.2 \\
18.2 \\
21.0 \\
16.3\end{array}$ & $\begin{array}{l}19.9 \\
21.6 \\
22.7 \\
22.5 \\
21.5\end{array}$ \\
\hline $\begin{array}{r}6 \\
7 \\
8 \\
9 \\
10\end{array}$ & $\begin{array}{l}17.1 \\
15.5 \\
14.7 \\
14.3 \\
13.7\end{array}$ & $\begin{array}{r}-1.4 \\
.4 \\
5.7 \\
4.6 \\
4.6\end{array}$ & $\begin{array}{r}4.3 \\
-4.2 \\
-3.0 \\
3.2 \\
3.8\end{array}$ & $\begin{array}{r}-8.3 \\
-3.8 \\
-6.0 \\
-1.7 \\
1.7\end{array}$ & $\begin{array}{r}.5 \\
1.3 \\
2.0 \\
1.7 \\
-2.3\end{array}$ & $\begin{array}{r}-.6 \\
-.5 \\
7.4 \\
1.3 \\
-11.8\end{array}$ & $\begin{array}{r}10.7 \\
9.3 \\
1.4 \\
8.9 \\
12.1\end{array}$ & $\begin{array}{r}-5.0 \\
-1.2 \\
2.7 \\
7.1 \\
12.6\end{array}$ & $\begin{array}{r}6.1 \\
5.3 \\
10.0 \\
14.0 \\
13.0\end{array}$ & $\begin{array}{l}18.0 \\
20.5 \\
21.5 \\
22.2 \\
21.6\end{array}$ & $\begin{array}{l}11.4 \\
13.0 \\
16.8 \\
17.8 \\
18.7\end{array}$ & $\begin{array}{l}20.9 \\
20.3 \\
20.1 \\
15.7 \\
15.3\end{array}$ \\
\hline $\begin{array}{l}11 \\
12 \\
13 \\
14 \\
15\end{array}$ & $\begin{array}{r}12.4 \\
7.1 \\
4.4 \\
9.2 \\
9.3\end{array}$ & $\begin{array}{r}8.8 \\
5.9 \\
2.9 \\
-5.4 \\
-1.6\end{array}$ & $\begin{array}{r}-1.8 \\
-12.7 \\
-15.2 \\
-10.8 \\
-4.6\end{array}$ & $\begin{array}{r}-2.5 \\
-2.9 \\
-2.0 \\
5.7 \\
.6\end{array}$ & $\begin{array}{r}3.3 \\
5.8 \\
.1 \\
2.9 \\
2.0\end{array}$ & $\begin{array}{r}-10.7 \\
-6.0 \\
1.9 \\
-.1 \\
-4.7\end{array}$ & $\begin{array}{r}13.7 \\
11.0 \\
7.3 \\
1.1 \\
1.6\end{array}$ & $\begin{array}{r}16.5 \\
15.0 \\
9.1 \\
14.7 \\
16.2\end{array}$ & $\begin{array}{l}12.1 \\
12.0 \\
15.9 \\
17.4 \\
19.4\end{array}$ & $\begin{array}{l}21.1 \\
21.5 \\
22.4 \\
22.2 \\
21.2\end{array}$ & $\begin{array}{l}18.0 \\
16.7 \\
15.4 \\
17.1 \\
13.4\end{array}$ & $\begin{array}{r}6.8 \\
4.2 \\
11.0 \\
15.0 \\
16.1\end{array}$ \\
\hline $\begin{array}{l}16 \\
17 \\
18 \\
19 \\
20\end{array}$ & $\begin{array}{r}10.8 \\
10.7 \\
10.6 \\
9.3 \\
10.2\end{array}$ & $\begin{array}{r}-1.8 \\
.2 \\
4.7 \\
8.2 \\
8.1\end{array}$ & $\begin{array}{l}-5.4 \\
-7.4 \\
-4.1 \\
-6.7 \\
-2.0\end{array}$ & $\begin{array}{r}-7.7 \\
-9.1 \\
-11.4 \\
-7.3 \\
-2.6\end{array}$ & $\begin{array}{r}-5.9 \\
-.2 \\
-6.5 \\
1.7 \\
2.3\end{array}$ & $\begin{array}{r}-3.6 \\
1.7 \\
7.4 \\
8.7 \\
8.1\end{array}$ & $\begin{array}{r}2.0 \\
.4 \\
1.2 \\
1.8 \\
-1.8\end{array}$ & $\begin{array}{r}11.9 \\
1.8 \\
5.5 \\
9.1 \\
13.5\end{array}$ & $\begin{array}{l}18.3 \\
16.5 \\
19.5 \\
18.6 \\
20.3\end{array}$ & $\begin{array}{l}22.3 \\
23.4 \\
22.6 \\
22.9 \\
24.6\end{array}$ & $\begin{array}{l}18.7 \\
20.2 \\
21.0 \\
20.9 \\
19.0\end{array}$ & $\begin{array}{r}15.4 \\
12.7 \\
10.5 \\
13.0 \\
1.6\end{array}$ \\
\hline $\begin{array}{l}21 \\
22 \\
23 \\
24 \\
25\end{array}$ & $\begin{array}{l}9.8 \\
5.4 \\
3.3 \\
4.2 \\
5.6\end{array}$ & $\begin{array}{r}-2.8 \\
-1.9 \\
-4.0 \\
2.2 \\
-7.1\end{array}$ & $\begin{array}{r}-1.0 \\
-.8 \\
-15.7 \\
-18.2 \\
-10.0\end{array}$ & $\begin{array}{r}-2.6 \\
3.4 \\
1.7 \\
3.0 \\
5.4\end{array}$ & $\begin{array}{l}4.3 \\
4.3 \\
6.1 \\
4.8 \\
6.4\end{array}$ & $\begin{array}{l}3.2 \\
3.4 \\
5.0 \\
2.7 \\
8.5\end{array}$ & $\begin{array}{r}-2.5 \\
-1.7 \\
-3.6 \\
1.9 \\
3.1\end{array}$ & $\begin{array}{l}15.9 \\
15.9 \\
16.8 \\
16.3 \\
16.8\end{array}$ & $\begin{array}{l}19.3 \\
18.1 \\
20.7 \\
21.2 \\
17.3\end{array}$ & $\begin{array}{l}24.7 \\
23.4 \\
21.8 \\
18.5 \\
18.7\end{array}$ & $\begin{array}{l}19.0 \\
17.3 \\
21.0 \\
17.3 \\
19.7\end{array}$ & $\begin{array}{r}2.8 \\
10.5 \\
12.7 \\
13.5 \\
13.9\end{array}$ \\
\hline $\begin{array}{l}26 \\
27 \\
28 \\
29 \\
30 \\
31\end{array}$ & $\begin{array}{l}9.0 \\
9.9 \\
7.7 \\
3.1 \\
4.0 \\
2.9\end{array}$ & $\begin{array}{r}-1.1 \\
1.8 \\
-6.1 \\
-3.7 \\
-.6 \\
---\end{array}$ & $\begin{array}{r}-2.9 \\
-1.4 \\
-1.8 \\
-9.1 \\
-11.7 \\
-9.9\end{array}$ & $\begin{array}{r}8.2 \\
7.6 \\
6.0 \\
.7 \\
-5.6 \\
-7.3\end{array}$ & $\begin{array}{r}6.3 \\
1.9 \\
.0 \\
.3 \\
---- \\
----\end{array}$ & $\begin{array}{r}9.3 \\
1.8 \\
-3.2 \\
.0 \\
-7.3 \\
-1.9\end{array}$ & $\begin{array}{r}7.2 \\
7.1 \\
6.9 \\
9.1 \\
-1.1 \\
---\end{array}$ & $\begin{array}{r}13.5 \\
11.3 \\
12.3 \\
-1.5 \\
-1.8 \\
6.1\end{array}$ & $\begin{array}{l}17.7 \\
20.6 \\
20.2 \\
18.3 \\
20.3 \\
-.-\end{array}$ & $\begin{array}{l}20.2 \\
16.7 \\
19.2 \\
22.2 \\
20.0 \\
20.1\end{array}$ & $\begin{array}{l}17.7 \\
20.7 \\
21.3 \\
20.6 \\
18.7 \\
17.2\end{array}$ & $\begin{array}{r}13.5 \\
8.4 \\
13.3 \\
16.5 \\
17.5 \\
---\end{array}$ \\
\hline MAX & 17.2 & 8.8 & 8.1 & 8.2 & 6.4 & 9.3 & 13.7 & 16.8 & 21.2 & 24.7 & 21.3 & 22.7 \\
\hline
\end{tabular}

WTR YR 1988 MAX 24.7 
EAST STEWART BASIN

EAST STEWART CREEK NEAR IONE, NV

DAILY MAXIMUM AIR TEMPERATURE, WATER YEAR OCTOBER 1988 TO SEPTEMBER 1989 DEGREES CELSIUS

\begin{tabular}{|c|c|c|c|c|c|c|c|c|c|c|c|c|}
\hline$D A Y$ & OCT & NOV & DEC & JAN & EEB & MAR & APR & MAY & JUN & JUL & AUG & SEP \\
\hline $\begin{array}{l}1 \\
2 \\
3 \\
4 \\
5\end{array}$ & $\begin{array}{l}17.7 \\
17.3 \\
16.1 \\
15.6 \\
12.5\end{array}$ & $\begin{array}{r}9.8 \\
5.2 \\
9.2 \\
6.3 \\
11.9\end{array}$ & $\begin{array}{l}8.0 \\
5.8 \\
2.5 \\
2.8 \\
4.0\end{array}$ & $\begin{array}{r}-7.1 \\
1.5 \\
3.1 \\
.9 \\
-2.8\end{array}$ & $\begin{array}{r}.5 \\
-6.4 \\
-8.7 \\
-15.7 \\
-18.9\end{array}$ & $\begin{array}{r}3.1 \\
.8 \\
-8.1 \\
.8 \\
4.9\end{array}$ & $\begin{array}{r}-.1 \\
1.2 \\
2.5 \\
7.7 \\
13.0\end{array}$ & $\begin{array}{r}10.5 \\
9.9 \\
11.2 \\
14.4 \\
16.3\end{array}$ & $\begin{array}{r}11.6 \\
13.6 \\
9.6 \\
6.8 \\
8.0\end{array}$ & $\begin{array}{l}18.9 \\
21.1 \\
22.6 \\
21.9 \\
22.5\end{array}$ & $\begin{array}{l}19.4 \\
18.8 \\
19.3 \\
19.8 \\
20.2\end{array}$ & $\begin{array}{l}17.1 \\
17.8 \\
18.5 \\
19.4 \\
18.5\end{array}$ \\
\hline $\begin{array}{r}6 \\
7 \\
8 \\
9 \\
10\end{array}$ & $\begin{array}{l}11.1 \\
12.4 \\
13.6 \\
12.5 \\
10.0\end{array}$ & $\begin{array}{l}7.7 \\
6.9 \\
-.3 \\
3.6 \\
2.7\end{array}$ & $\begin{array}{r}4.1 \\
-6.1 \\
-1.0 \\
2.8 \\
3.7\end{array}$ & $\begin{array}{r}-14.5 \\
-11.6 \\
-6.4 \\
-3.0 \\
-2.3\end{array}$ & $\begin{array}{r}-15.5 \\
-11.8 \\
-3.0 \\
-. .2 \\
-5.0\end{array}$ & $\begin{array}{l}5.3 \\
5.1 \\
4.8 \\
6.6 \\
6.6\end{array}$ & $\begin{array}{l}15.0 \\
13.9 \\
13.1 \\
11.6 \\
14.0\end{array}$ & $\begin{array}{r}17.0 \\
16.0 \\
14.4 \\
11.4 \\
2.4\end{array}$ & $\begin{array}{l}14.3 \\
12.2 \\
13.3 \\
10.4 \\
14.2\end{array}$ & $\begin{array}{l}23.4 \\
24.9 \\
22.0 \\
19.4 \\
19.0\end{array}$ & $\begin{array}{l}21.3 \\
21.8 \\
16.6 \\
15.7 \\
17.3\end{array}$ & $\begin{array}{r}17.6 \\
9.0 \\
13.9 \\
15.0 \\
14.3\end{array}$ \\
\hline $\begin{array}{l}11 \\
12 \\
13 \\
14 \\
15\end{array}$ & $\begin{array}{r}6.5 \\
8.4 \\
9.5 \\
7.7 \\
11.2\end{array}$ & $\begin{array}{r}-.6 \\
5.2 \\
5.1 \\
-6.1 \\
-7.0\end{array}$ & $\begin{array}{r}5.8 \\
7.2 \\
6.5 \\
-.1 \\
-7.7\end{array}$ & $\begin{array}{r}-12.2 \\
-5.3 \\
-1.0 \\
-9.1 \\
-4.9\end{array}$ & $\begin{array}{r}-7.3 \\
-9.3 \\
-12.3 \\
-6.4 \\
.1\end{array}$ & $\begin{array}{r}6.5 \\
6.1 \\
-1.0 \\
-1.1 \\
4.0\end{array}$ & $\begin{array}{r}8.3 \\
8.1 \\
12.2 \\
13.1 \\
9.7\end{array}$ & $\begin{array}{r}-1.5 \\
1.5 \\
.4 \\
.8 \\
3.4\end{array}$ & $\begin{array}{l}13.0 \\
17.1 \\
19.3 \\
21.2 \\
20.8\end{array}$ & $\begin{array}{l}18.4 \\
14.1 \\
19.7 \\
21.5 \\
20.2\end{array}$ & $\begin{array}{l}18.0 \\
19.4 \\
21.4 \\
20.4 \\
21.1\end{array}$ & $\begin{array}{l}12.1 \\
12.5 \\
15.2 \\
16.1 \\
18.7\end{array}$ \\
\hline $\begin{array}{l}16 \\
17 \\
18 \\
19 \\
20\end{array}$ & $\begin{array}{l}14.4 \\
14.8 \\
15.5 \\
13.1 \\
13.7\end{array}$ & $\begin{array}{l}-2.6 \\
-8.4 \\
-8.9 \\
-3.1 \\
-3.3\end{array}$ & $\begin{array}{l}-4.4 \\
-3.0 \\
-4.9 \\
-7.9 \\
-2.7\end{array}$ & $\begin{array}{l}-.6 \\
1.3 \\
5.1 \\
2.1 \\
4.0\end{array}$ & $\begin{array}{r}-2.0 \\
2.8 \\
3.1 \\
-2.4 \\
-2.7\end{array}$ & $\begin{array}{r}2.1 \\
.9 \\
2.0 \\
-.3 \\
2.7\end{array}$ & $\begin{array}{l}11.6 \\
13.8 \\
15.1 \\
14.8 \\
14.4\end{array}$ & $\begin{array}{r}7.5 \\
13.2 \\
9.6 \\
10.4 \\
13.8\end{array}$ & $\begin{array}{l}14.3 \\
18.4 \\
21.7 \\
20.0 \\
12.9\end{array}$ & $\begin{array}{l}18.8 \\
21.0 \\
23.5 \\
24.5 \\
23.3\end{array}$ & $\begin{array}{l}20.4 \\
17.1 \\
17.3 \\
17.4 \\
13.2\end{array}$ & $\begin{array}{r}15.5 \\
7.3 \\
7.5 \\
8.6 \\
12.4\end{array}$ \\
\hline $\begin{array}{l}21 \\
22 \\
23 \\
24 \\
25\end{array}$ & $\begin{array}{l}14.6 \\
13.7 \\
14.5 \\
13.3 \\
13.0\end{array}$ & $\begin{array}{r}1.2 \\
3.5 \\
3.3 \\
-7.2 \\
-6.8\end{array}$ & $\begin{array}{r}-6.4 \\
-6.9 \\
-10.0 \\
-6.5 \\
-14.0\end{array}$ & $\begin{array}{r}3.5 \\
.8 \\
-3.4 \\
-4.9 \\
-5.2\end{array}$ & $\begin{array}{l}1.1 \\
5.8 \\
5.1 \\
6.2 \\
5.2\end{array}$ & $\begin{array}{l}5.4 \\
5.6 \\
3.1 \\
4.9 \\
1.3\end{array}$ & $\begin{array}{r}12.9 \\
3.7 \\
3.3 \\
-3.8 \\
-2.5\end{array}$ & $\begin{array}{r}13.5 \\
14.7 \\
7.0 \\
2.5 \\
6.7\end{array}$ & $\begin{array}{l}13.0 \\
16.0 \\
12.3 \\
11.6 \\
14.6\end{array}$ & $\begin{array}{l}19.3 \\
20.7 \\
17.4 \\
18.5 \\
22.2\end{array}$ & $\begin{array}{l}16.1 \\
17.9 \\
10.0 \\
11.3 \\
13.5\end{array}$ & $\begin{array}{l}14.3 \\
16.4 \\
17.9 \\
18.0 \\
14.7\end{array}$ \\
\hline $\begin{array}{l}26 \\
27 \\
28 \\
29 \\
30 \\
31\end{array}$ & $\begin{array}{r}12.2 \\
11.6 \\
10.2 \\
9.6 \\
10.4 \\
11.7\end{array}$ & $\begin{array}{r}-6.5 \\
2.9 \\
2.5 \\
.6 \\
2.7 \\
---\end{array}$ & $\begin{array}{r}-13.6 \\
-6.6 \\
-15.3 \\
-4.8 \\
-.4 \\
-8.1\end{array}$ & $\begin{array}{r}.1 \\
-.3 \\
.2 \\
2.9 \\
8.6 \\
3.5\end{array}$ & $\begin{array}{l}2.8 \\
-.8 \\
1.2 \\
--- \\
--- \\
---\end{array}$ & $\begin{array}{r}-3.1 \\
1.4 \\
5.3 \\
1.3 \\
6.0 \\
4.3\end{array}$ & $\begin{array}{r}-2.3 \\
-2.2 \\
-.7 \\
6.3 \\
7.4 \\
---\end{array}$ & $\begin{array}{r}11.9 \\
13.7 \\
6.8 \\
.5 \\
2.1 \\
7.6\end{array}$ & $\begin{array}{l}16.0 \\
13.2 \\
16.0 \\
17.6 \\
16.2 \\
-.-\end{array}$ & $\begin{array}{l}22.4 \\
21.8 \\
21.8 \\
22.5 \\
22.2 \\
21.7\end{array}$ & $\begin{array}{l}16.4 \\
17.8 \\
19.0 \\
19.8 \\
16.2 \\
16.3\end{array}$ & $\begin{array}{r}16.6 \\
15.3 \\
15.4 \\
13.8 \\
6.6 \\
-\end{array}$ \\
\hline MAX & 17.7 & 11.9 & 8.0 & 8.6 & 6.2 & 6.6 & 15.1 & 17.0 & 21.7 & 24.9 & 21.8 & 19.4 \\
\hline
\end{tabular}

CAL YR 1988 MAX 24.7

WTR YR 1989 MAX 24.9 
EAST STEWART BASIN

EAST STEWART CREEK NEAR IONE, NV

DAILY MAXIMUM AIR TEMPERATURE, WATER YEAR OCTOBER 1989 TO SEPTEMBER 1990 DEGREES CELSIUS

\begin{tabular}{|c|c|c|c|c|c|c|c|c|c|c|c|c|}
\hline DAY & OCT & NOV & DEC & JAN & FEB & MAR & APR & MAY & JUN & JUL & AUG & SEP \\
\hline $\begin{array}{l}1 \\
2 \\
3 \\
4 \\
5\end{array}$ & $\begin{array}{r}11.5 \\
6.6 \\
.5 \\
6.3 \\
10.2\end{array}$ & $\begin{array}{l}4.5 \\
4.5 \\
6.3 \\
7.7 \\
5.3\end{array}$ & $\begin{array}{l}3.7 \\
7.3 \\
8.3 \\
7.6 \\
4.7\end{array}$ & $\begin{array}{r}.2 \\
-8.8 \\
-6.5 \\
-3.9 \\
-.4\end{array}$ & $\begin{array}{r}-8.3 \\
-5.9 \\
1.2 \\
-5.9 \\
-1.5\end{array}$ & $\begin{array}{r}2.6 \\
2.4 \\
3.1 \\
3.2 \\
-6.9\end{array}$ & $\begin{array}{r}10.4 \\
8.9 \\
8.0 \\
8.1 \\
7.2\end{array}$ & $\begin{array}{r}4.0 \\
4.1 \\
8.5 \\
13.9 \\
17.0\end{array}$ & $\begin{array}{r}7.8 \\
15.1 \\
19.3 \\
17.8 \\
15.8\end{array}$ & $\begin{array}{l}22.1 \\
18.5 \\
18.5 \\
20.5 \\
21.6\end{array}$ & $\begin{array}{l}19.4 \\
21.3 \\
22.3 \\
22.5 \\
24.0\end{array}$ & $\begin{array}{l}20.1 \\
19.6 \\
19.1 \\
18.5 \\
19.1\end{array}$ \\
\hline $\begin{array}{r}6 \\
7 \\
8 \\
9 \\
10\end{array}$ & $\begin{array}{l}10.4 \\
12.3 \\
15.1 \\
16.7 \\
15.1\end{array}$ & $\begin{array}{r}3.7 \\
2.5 \\
5.9 \\
8.9 \\
11.8\end{array}$ & $\begin{array}{r}1.4 \\
5.8 \\
6.4 \\
2.2 \\
-7.6\end{array}$ & $\begin{array}{l}1.8 \\
1.8 \\
4.2 \\
8.8 \\
9.2\end{array}$ & $\begin{array}{r}-2.6 \\
-11.5 \\
-5.8 \\
-.6 \\
2.5\end{array}$ & $\begin{array}{l}-.3 \\
6.6 \\
3.6 \\
5.2 \\
2.0\end{array}$ & $\begin{array}{r}5.4 \\
6.5 \\
-2.1 \\
5.5 \\
10.3\end{array}$ & $\begin{array}{r}15.7 \\
10.3 \\
7.5 \\
12.2 \\
6.4\end{array}$ & $\begin{array}{l}12.3 \\
17.0 \\
19.3 \\
17.4 \\
10.8\end{array}$ & $\begin{array}{l}21.4 \\
20.8 \\
19.7 \\
20.4 \\
19.2\end{array}$ & $\begin{array}{l}24.7 \\
20.9 \\
20.9 \\
20.1 \\
21.3\end{array}$ & $\begin{array}{l}18.3 \\
19.9 \\
20.7 \\
22.2 \\
22.7\end{array}$ \\
\hline $\begin{array}{l}11 \\
12 \\
13 \\
14 \\
15\end{array}$ & $\begin{array}{r}14.8 \\
15.2 \\
14.6 \\
11.1 \\
7.6\end{array}$ & $\begin{array}{r}12.1 \\
9.0 \\
7.3 \\
2.3 \\
6.9\end{array}$ & $\begin{array}{r}-2.9 \\
1.7 \\
.5 \\
2.4 \\
4.8\end{array}$ & $\begin{array}{r}7.0 \\
1.8 \\
-2.2 \\
-5.1 \\
-6.4\end{array}$ & $\begin{array}{r}8.3 \\
.5 \\
-11.5 \\
-19.2 \\
-10.1\end{array}$ & $\begin{array}{r}-10.5 \\
-10.4 \\
-8.3 \\
-2.5 \\
.6\end{array}$ & $\begin{array}{r}9.3 \\
10.3 \\
13.8 \\
14.3 \\
13.2\end{array}$ & $\begin{array}{r}5.3 \\
11.4 \\
10.7 \\
7.6 \\
5.4\end{array}$ & $\begin{array}{r}12.1 \\
10.0 \\
11.1 \\
7.8 \\
7.2\end{array}$ & $\begin{array}{l}22.1 \\
21.2 \\
20.4 \\
19.0 \\
17.8\end{array}$ & $\begin{array}{l}20.0 \\
20.0 \\
21.7 \\
21.6 \\
15.5\end{array}$ & $\begin{array}{l}22.8 \\
22.6 \\
21.8 \\
20.1 \\
18.2\end{array}$ \\
\hline $\begin{array}{l}16 \\
17 \\
18 \\
19 \\
20\end{array}$ & $\begin{array}{r}8.5 \\
11.4 \\
13.7 \\
12.9 \\
8.7\end{array}$ & $\begin{array}{r}4.8 \\
2.3 \\
9.4 \\
13.3 \\
14.1\end{array}$ & $\begin{array}{r}.2 \\
-1.4 \\
-3.3 \\
-.1 \\
2.5\end{array}$ & $\begin{array}{r}-5.5 \\
-8.4 \\
-7.3 \\
-4.3 \\
-.9\end{array}$ & $\begin{array}{r}-9.1 \\
-7.4 \\
-11.3 \\
-5.3 \\
1.8\end{array}$ & $\begin{array}{l}6.7 \\
5.4 \\
8.9 \\
9.0 \\
9.8\end{array}$ & $\begin{array}{l}8.5 \\
1.4 \\
5.4 \\
8.2 \\
5.7\end{array}$ & $\begin{array}{r}11.2 \\
10.7 \\
9.4 \\
5.3 \\
9.4\end{array}$ & $\begin{array}{l}13.0 \\
16.4 \\
10.2 \\
17.0 \\
20.7\end{array}$ & $\begin{array}{l}17.2 \\
19.0 \\
19.0 \\
18.8 \\
21.0\end{array}$ & $\begin{array}{r}18.4 \\
16.5 \\
11.0 \\
6.3 \\
10.2\end{array}$ & $\begin{array}{r}15.7 \\
12.5 \\
9.8 \\
10.5 \\
13.6\end{array}$ \\
\hline $\begin{array}{l}21 \\
22 \\
23 \\
24 \\
25\end{array}$ & $\begin{array}{r}5.7 \\
8.7 \\
6.8 \\
7.6 \\
-2.5\end{array}$ & $\begin{array}{r}7.9 \\
8.6 \\
4.2 \\
.1 \\
1.6\end{array}$ & $\begin{array}{l}1.5 \\
5.7 \\
5.9 \\
6.4 \\
5.7\end{array}$ & $\begin{array}{r}3.2 \\
1.9 \\
-.3 \\
-1.7 \\
2.2\end{array}$ & $\begin{array}{l}1.4 \\
7.1 \\
9.5 \\
7.9 \\
6.6\end{array}$ & $\begin{array}{l}8.9 \\
7.5 \\
7.4 \\
8.4 \\
8.6\end{array}$ & $\begin{array}{r}4.9 \\
5.9 \\
3.5 \\
-3.0 \\
6.7\end{array}$ & $\begin{array}{r}13.6 \\
13.5 \\
10.3 \\
3.3 \\
9.1\end{array}$ & $\begin{array}{l}22.9 \\
23.6 \\
22.0 \\
22.2 \\
22.5\end{array}$ & $\begin{array}{l}22.9 \\
23.9 \\
22.1 \\
19.6 \\
18.7\end{array}$ & $\begin{array}{l}14.2 \\
17.5 \\
16.4 \\
14.3 \\
14.6\end{array}$ & $\begin{array}{r}13.1 \\
11.1 \\
8.6 \\
10.5 \\
12.7\end{array}$ \\
\hline $\begin{array}{l}26 \\
27 \\
28 \\
29 \\
30 \\
31\end{array}$ & $\begin{array}{r}.9 \\
1.3 \\
-5.5 \\
.4 \\
5.5 \\
3.9\end{array}$ & $\begin{array}{r}-2.8 \\
-6.5 \\
.0 \\
2.7 \\
2.6 \\
---\end{array}$ & $\begin{array}{r}6.7 \\
4.2 \\
-3.3 \\
-4.0 \\
-.5 \\
3.5\end{array}$ & $\begin{array}{l}-.7 \\
-5.1 \\
-3.3 \\
-2.6 \\
-2.9 \\
-5.3\end{array}$ & $\begin{array}{l}5.6 \\
4.1 \\
4.2 \\
--- \\
--- \\
---\end{array}$ & $\begin{array}{r}7.7 \\
1.9 \\
-2.3 \\
-1.4 \\
5.3 \\
8.2\end{array}$ & $\begin{array}{r}7.4 \\
10.0 \\
8.0 \\
-.7 \\
-.8 \\
---\end{array}$ & $\begin{array}{l}9.1 \\
8.2 \\
1.4 \\
5.6 \\
7.2 \\
3.4\end{array}$ & $\begin{array}{l}20.8 \\
20.7 \\
22.0 \\
23.3 \\
21.8 \\
---\end{array}$ & $\begin{array}{l}19.1 \\
21.9 \\
21.6 \\
23.1 \\
22.7 \\
21.2\end{array}$ & $\begin{array}{l}15.0 \\
18.9 \\
20.0 \\
21.1 \\
18.3 \\
20.0\end{array}$ & $\begin{array}{r}12.4 \\
11.7 \\
7.6 \\
13.2 \\
15.4 \\
---\end{array}$ \\
\hline MAX & 16.7 & 14.1 & 8.3 & 9.2 & 9.5 & 9.8 & 14.3 & 17.0 & 23.6 & 23.9 & 24.7 & 22.8 \\
\hline
\end{tabular}

CAL YR 1989 MAX 24.9

WTR YR 1990 MAX 24.7 
EAST STEWART BASIN

EAST STEWART CREEK NEAR IONE, NV

DAILY MAXIMUM AIR TEMPERATURE, WATER YEAR OCTOBER 1990 TO SEPTEMBER 1991 DEGREES CELSIUS

\begin{tabular}{|c|c|c|c|c|c|c|c|c|c|c|c|c|}
\hline DAY & OCT & NOV & $\mathrm{DEC}$ & JAN & FEB & MAR & APR & MAY & JUN & JUL & AUG & SEP \\
\hline $\begin{array}{l}1 \\
2 \\
3 \\
4 \\
5\end{array}$ & $\begin{array}{l}17.2 \\
11.4 \\
13.6 \\
16.6 \\
14.5\end{array}$ & $\begin{array}{r}-3.1 \\
-6.7 \\
.9 \\
7.0 \\
-.1\end{array}$ & $\begin{array}{r}-.8 \\
-1.8 \\
3.1 \\
6.3 \\
2.2\end{array}$ & $\begin{array}{l}5.7 \\
4.6 \\
1.3 \\
-.3 \\
3.7\end{array}$ & $\begin{array}{l}7.5 \\
5.1 \\
2.1 \\
8.1 \\
3.7\end{array}$ & $\begin{array}{r}-2.4 \\
-.5 \\
1.4 \\
1.2 \\
-4.1\end{array}$ & $\begin{array}{r}1.2 \\
4.4 \\
8.2 \\
12.0 \\
12.0\end{array}$ & $\begin{array}{r}1.1 \\
-4.5 \\
-1.3 \\
7.3 \\
11.9\end{array}$ & $\begin{array}{l}10.9 \\
14.8 \\
15.0 \\
14.5 \\
10.8\end{array}$ & $\begin{array}{l}20.8 \\
22.7 \\
24.1 \\
25.6 \\
24.9\end{array}$ & $\begin{array}{l}21.5 \\
19.2 \\
19.9 \\
19.5 \\
19.2\end{array}$ & $\begin{array}{l}20.2 \\
20.9 \\
19.0 \\
19.2 \\
15.6\end{array}$ \\
\hline $\begin{array}{r}6 \\
7 \\
8 \\
9 \\
10\end{array}$ & $\begin{array}{r}7.7 \\
2.0 \\
7.1 \\
13.7 \\
12.8\end{array}$ & $\begin{array}{r}-7.3 \\
1.4 \\
4.6 \\
10.5 \\
13.9\end{array}$ & $\begin{array}{r}5.4 \\
8.5 \\
9.0 \\
11.2 \\
9.2\end{array}$ & $\begin{array}{r}1.7 \\
-.2 \\
-1.0 \\
-2.3 \\
-2.4\end{array}$ & $\begin{array}{l}5.1 \\
7.5 \\
6.3 \\
6.8 \\
6.8\end{array}$ & $\begin{array}{r}-7.0 \\
.2 \\
3.6 \\
.8 \\
.6\end{array}$ & $\begin{array}{r}8.1 \\
.1 \\
4.0 \\
5.2 \\
-5.8\end{array}$ & $\begin{array}{r}6.9 \\
12.5 \\
9.5 \\
-4.8 \\
-.1\end{array}$ & $\begin{array}{l}16.3 \\
15.7 \\
16.5 \\
18.8 \\
20.0\end{array}$ & $\begin{array}{l}24.2 \\
23.0 \\
19.4 \\
18.2 \\
21.0\end{array}$ & $\begin{array}{l}20.1 \\
19.9 \\
23.0 \\
23.1 \\
21.7\end{array}$ & $\begin{array}{r}11.1 \\
13.5 \\
13.4 \\
9.1 \\
6.3\end{array}$ \\
\hline $\begin{array}{l}11 \\
12 \\
13 \\
14 \\
15\end{array}$ & $\begin{array}{l}10.3 \\
12.1 \\
11.4 \\
14.1 \\
13.5\end{array}$ & $\begin{array}{r}11.5 \\
12.6 \\
13.2 \\
9.3 \\
5.9\end{array}$ & $\begin{array}{r}3.2 \\
-1.9 \\
-5.9 \\
-5.6 \\
-4.3\end{array}$ & $\begin{array}{r}3.4 \\
3.1 \\
-1.4 \\
1.7 \\
-3.0\end{array}$ & $\begin{array}{l}6.6 \\
3.0 \\
3.6 \\
9.5 \\
6.2\end{array}$ & $\begin{array}{r}-8.0 \\
-1.2 \\
-.6 \\
-2.3 \\
-3.5\end{array}$ & $\begin{array}{r}-8.0 \\
-2.5 \\
2.0 \\
5.7 \\
3.3\end{array}$ & $\begin{array}{r}1.9 \\
8.9 \\
6.0 \\
2.9 \\
11.5\end{array}$ & $\begin{array}{l}21.7 \\
21.8 \\
18.0 \\
13.6 \\
17.6\end{array}$ & $\begin{array}{l}21.5 \\
22.6 \\
23.2 \\
22.2 \\
20.8\end{array}$ & $\begin{array}{l}20.5 \\
19.4 \\
19.8 \\
20.4 \\
16.6\end{array}$ & $\begin{array}{r}9.8 \\
13.4 \\
15.8 \\
14.7 \\
16.7\end{array}$ \\
\hline $\begin{array}{l}16 \\
17 \\
18 \\
19 \\
20\end{array}$ & $\begin{array}{r}8.9 \\
11.9 \\
8.0 \\
-1.3 \\
2.2\end{array}$ & $\begin{array}{r}9.4 \\
4.8 \\
5.6 \\
1.5 \\
-4.3\end{array}$ & $\begin{array}{r}-9.3 \\
-5.0 \\
-2.7 \\
-8.9 \\
-20.0\end{array}$ & $\begin{array}{r}-2.1 \\
.4 \\
6.6 \\
.4 \\
-3.9\end{array}$ & $\begin{array}{r}-1.4 \\
-6.2 \\
-3.0 \\
5.5 \\
7.6\end{array}$ & $\begin{array}{r}.8 \\
-2.1 \\
-3.2 \\
-3.4 \\
-4.2\end{array}$ & $\begin{array}{r}.8 \\
-.6 \\
3.9 \\
6.3 \\
4.4\end{array}$ & $\begin{array}{r}11.5 \\
1.6 \\
-4.1 \\
1.5 \\
4.1\end{array}$ & $\begin{array}{l}18.2 \\
17.1 \\
18.0 \\
14.2 \\
13.3\end{array}$ & $\begin{array}{l}21.6 \\
21.5 \\
21.3 \\
19.2 \\
17.1\end{array}$ & $\begin{array}{l}19.5 \\
21.9 \\
21.1 \\
22.4 \\
22.3\end{array}$ & $\begin{array}{l}19.7 \\
20.5 \\
20.1 \\
20.1 \\
20.3\end{array}$ \\
\hline $\begin{array}{l}21 \\
22 \\
23 \\
24 \\
25\end{array}$ & $\begin{array}{r}10.1 \\
8.9 \\
12.1 \\
15.4 \\
14.9\end{array}$ & $\begin{array}{r}-3.4 \\
8.8 \\
9.0 \\
11.3 \\
7.8\end{array}$ & $\begin{array}{r}-19.0 \\
-15.9 \\
-9.8 \\
-3.1 \\
-3.8\end{array}$ & $\begin{array}{r}-5.8 \\
-.3 \\
.7 \\
-.8 \\
.3\end{array}$ & $\begin{array}{l}9.3 \\
6.3 \\
3.4 \\
3.6 \\
7.0\end{array}$ & $\begin{array}{r}-5.3 \\
-1.7 \\
.0 \\
.2 \\
-5.5\end{array}$ & $\begin{array}{r}2.5 \\
2.8 \\
7.3 \\
5.7 \\
-4.9\end{array}$ & $\begin{array}{r}7.4 \\
10.1 \\
14.5 \\
14.3 \\
13.4\end{array}$ & $\begin{array}{l}14.1 \\
15.9 \\
16.4 \\
11.5 \\
10.8\end{array}$ & $\begin{array}{l}17.8 \\
20.3 \\
22.3 \\
21.8 \\
21.8\end{array}$ & $\begin{array}{l}22.9 \\
23.6 \\
22.1 \\
21.9 \\
22.0\end{array}$ & $\begin{array}{l}17.9 \\
18.4 \\
18.2 \\
20.5 \\
20.2\end{array}$ \\
\hline $\begin{array}{l}26 \\
27 \\
28 \\
29 \\
30 \\
31\end{array}$ & $\begin{array}{r}14.2 \\
15.8 \\
13.1 \\
11.9 \\
12.2 \\
4.3\end{array}$ & $\begin{array}{r}-8.5 \\
-3.3 \\
2.5 \\
9.1 \\
.5 \\
----\end{array}$ & $\begin{array}{r}-7.1 \\
1.9 \\
-3.0 \\
-9.4 \\
.5 \\
3.2\end{array}$ & $\begin{array}{r}1.2 \\
-1.5 \\
-2.1 \\
-1.8 \\
5.1 \\
5.4\end{array}$ & $\begin{array}{l}5.7 \\
4.5 \\
1.7 \\
--- \\
--- \\
---\end{array}$ & $\begin{array}{r}-3.2 \\
-2.6 \\
1.8 \\
1.4 \\
7.2 \\
4.9\end{array}$ & $\begin{array}{r}-2.0 \\
-1.3 \\
5.1 \\
2.5 \\
7.0 \\
---\end{array}$ & $\begin{array}{r}8.5 \\
8.8 \\
10.2 \\
9.5 \\
-.7 \\
2.8\end{array}$ & $\begin{array}{r}12.1 \\
6.4 \\
11.1 \\
8.4 \\
16.1 \\
-.-\end{array}$ & $\begin{array}{l}21.6 \\
23.8 \\
22.5 \\
24.2 \\
22.9 \\
16.0\end{array}$ & $\begin{array}{l}19.1 \\
13.8 \\
18.2 \\
22.1 \\
21.8 \\
19.9\end{array}$ & $\begin{array}{r}16.7 \\
13.6 \\
13.5 \\
15.5 \\
17.0 \\
---\end{array}$ \\
\hline MAX & 17.2 & 13.9 & 11.2 & 6.6 & 9.5 & 7.2 & 12.0 & 14.5 & 21.8 & 25.6 & 23.6 & 20.9 \\
\hline
\end{tabular}

CAL YR 1990 MAX 24.7

WTR YR 1991 MAX 25.6 
EAST STEWART BASIN

EAST STEWART CREEK NEAR IONE, NV

DAILY MINIMUM AIR TEMPERATURE, WATER YEAR OCTOBER 1985 TO SEPTEMBER 1986 DEGREES CELSIUS

\begin{tabular}{|c|c|c|c|c|c|c|c|c|c|c|c|c|}
\hline DAY & OCT & NOV & DEC & JAN & EEB & MAR & APR & MAY & JUN & JUL & AUG & SEP \\
\hline $\begin{array}{l}1 \\
2 \\
3 \\
4 \\
5\end{array}$ & $\begin{array}{l}--- \\
--- \\
--- \\
--- \\
---\end{array}$ & $\begin{array}{l}--- \\
--- \\
--- \\
--- \\
---\end{array}$ & $\begin{array}{l}--- \\
--- \\
--- \\
--- \\
---\end{array}$ & $\begin{array}{l}--- \\
--- \\
--- \\
--- \\
---\end{array}$ & $\begin{array}{r}-9.0 \\
-6.4 \\
-8.8 \\
-12.6 \\
-15.0\end{array}$ & $\begin{array}{r}1.4 \\
-1.8 \\
-1.5 \\
-1.3 \\
-2.5\end{array}$ & $\begin{array}{r}-10.8 \\
-13.2 \\
-10.7 \\
-4.0 \\
-2.9\end{array}$ & $\begin{array}{r}4.4 \\
5.3 \\
-4.2 \\
-10.0 \\
-9.0\end{array}$ & $\begin{array}{l}7.7 \\
8.0 \\
6.9 \\
6.7 \\
3.1\end{array}$ & $\begin{array}{r}9.5 \\
9.0 \\
8.3 \\
3.3 \\
.8\end{array}$ & $\begin{array}{r}11.3 \\
11.8 \\
9.8 \\
9.8 \\
9.7\end{array}$ & $\begin{array}{r}3.4 \\
4.7 \\
4.4 \\
6.8 \\
10.0\end{array}$ \\
\hline $\begin{array}{r}6 \\
7 \\
8 \\
9 \\
10\end{array}$ & $\begin{array}{l}--- \\
--- \\
--- \\
--- \\
---\end{array}$ & $\begin{array}{l}--- \\
--- \\
--- \\
--- \\
---\end{array}$ & $\begin{array}{l}--- \\
--- \\
--- \\
--- \\
---\end{array}$ & $\begin{array}{l}--- \\
--- \\
--- \\
--- \\
---\end{array}$ & $\begin{array}{l}-16.0 \\
-17.8 \\
-18.5 \\
-17.5 \\
-11.3\end{array}$ & $\begin{array}{l}-1.1 \\
-.2 \\
-9.0 \\
-9.8 \\
-6.8\end{array}$ & $\begin{array}{l}-4.3 \\
-5.6 \\
-5.6 \\
-3.6 \\
-2.3\end{array}$ & $\begin{array}{l}-9.5 \\
-9.8 \\
-6.1 \\
-5.5 \\
-2.8\end{array}$ & $\begin{array}{r}4.2 \\
1.5 \\
.2 \\
.4 \\
1.2\end{array}$ & $\begin{array}{l}4.7 \\
4.2 \\
4.7 \\
--- \\
---\end{array}$ & $\begin{array}{r}11.1 \\
10.2 \\
10.0 \\
10.7 \\
7.7\end{array}$ & $\begin{array}{r}9.1 \\
7.3 \\
2.0 \\
-2.8 \\
-3.4\end{array}$ \\
\hline $\begin{array}{l}11 \\
12 \\
13 \\
14 \\
15\end{array}$ & $\begin{array}{l}--- \\
--- \\
--- \\
--- \\
---\end{array}$ & $\begin{array}{l}--- \\
--- \\
--- \\
--- \\
---\end{array}$ & $\begin{array}{l}--- \\
--- \\
--- \\
--- \\
---\end{array}$ & $\begin{array}{r}--- \\
-.9 \\
4.3 \\
-3.4 \\
-5.4\end{array}$ & $\begin{array}{l}-8.7 \\
-5.9 \\
-5.3 \\
-1.6 \\
-4.9\end{array}$ & $\begin{array}{r}-7.9 \\
-13.6 \\
-11.5 \\
-11.0 \\
-7.7\end{array}$ & $\begin{array}{r}-2.5 \\
-10.3 \\
-11.9 \\
-3.5 \\
-6.1\end{array}$ & $\begin{array}{l}-6.7 \\
-3.3 \\
-1.5 \\
-3.0 \\
-1.3\end{array}$ & $\begin{array}{l}8.2 \\
3.4 \\
6.2 \\
3.5 \\
2.6\end{array}$ & $\begin{array}{l}--- \\
--- \\
--- \\
--- \\
---\end{array}$ & $\begin{array}{r}6.8 \\
7.3 \\
7.9 \\
8.4 \\
13.2\end{array}$ & $\begin{array}{r}1.5 \\
3.8 \\
1.1 \\
-2.1 \\
-4.3\end{array}$ \\
\hline $\begin{array}{l}16 \\
17 \\
18 \\
19 \\
20\end{array}$ & $\begin{array}{l}--- \\
--- \\
--- \\
--- \\
---\end{array}$ & $\begin{array}{l}--- \\
--- \\
--- \\
--- \\
---\end{array}$ & $\begin{array}{l}--- \\
--- \\
--- \\
--- \\
---\end{array}$ & $\begin{array}{r}-2.7 \\
-2.4 \\
-2.8 \\
-.6 \\
-10.6\end{array}$ & $\begin{array}{l}-5.6 \\
-2.0 \\
-1.3 \\
-7.6 \\
-8.7\end{array}$ & $\begin{array}{r}-12.6 \\
-10.5 \\
-12.4 \\
-6.8 \\
-2.6\end{array}$ & $\begin{array}{r}-8.9 \\
-9.1 \\
-10.2 \\
-5.4 \\
2.8\end{array}$ & $\begin{array}{r}-2.0 \\
-2.5 \\
2.3 \\
5.8 \\
3.1\end{array}$ & $\begin{array}{r}6.7 \\
11.8 \\
1.5 \\
.3 \\
1.5\end{array}$ & $\begin{array}{l}--- \\
--- \\
--- \\
--- \\
---\end{array}$ & $\begin{array}{r}12.1 \\
10.5 \\
11.0 \\
9.2 \\
6.5\end{array}$ & $\begin{array}{l}-2.1 \\
-1.8 \\
-5.1 \\
-5.6 \\
-8.2\end{array}$ \\
\hline $\begin{array}{l}21 \\
22 \\
23 \\
24 \\
25\end{array}$ & $\begin{array}{l}--- \\
--- \\
--- \\
--- \\
---\end{array}$ & $\begin{array}{l}--- \\
--- \\
--- \\
--- \\
---\end{array}$ & $\begin{array}{l}--- \\
--- \\
--- \\
--- \\
---\end{array}$ & $\begin{array}{r}-10.8 \\
-2.1 \\
-9.9 \\
-8.9 \\
-1.8\end{array}$ & $\begin{array}{r}-10.1 \\
-3.6 \\
-2.9 \\
.2 \\
.5\end{array}$ & $\begin{array}{r}1.0 \\
-.1 \\
.3 \\
-2.3 \\
-5.0\end{array}$ & $\begin{array}{r}4.0 \\
1.8 \\
-2.0 \\
-4.9 \\
-8.0\end{array}$ & $\begin{array}{r}-7.0 \\
-9.5 \\
3.9 \\
3.1 \\
7.3\end{array}$ & $\begin{array}{l}3.6 \\
8.2 \\
9.3 \\
8.3 \\
6.0\end{array}$ & $\begin{array}{l}--- \\
--- \\
--- \\
--- \\
---\end{array}$ & $\begin{array}{r}5.2 \\
7.9 \\
11.6 \\
14.3 \\
8.3\end{array}$ & $\begin{array}{r}-3.9 \\
-.8 \\
-2.6 \\
-4.8 \\
-6.0\end{array}$ \\
\hline $\begin{array}{l}26 \\
27 \\
28 \\
29 \\
30 \\
31\end{array}$ & $\begin{array}{l}--- \\
--- \\
--- \\
--- \\
--- \\
---\end{array}$ & $\begin{array}{l}--- \\
--- \\
--- \\
--- \\
--- \\
---\end{array}$ & $\begin{array}{l}--- \\
--- \\
--- \\
--- \\
--- \\
---\end{array}$ & $\begin{array}{r}-2.2 \\
-.3 \\
1.3 \\
-.8 \\
-4.0 \\
-4.8\end{array}$ & $\begin{array}{r}-1.0 \\
.7 \\
.2 \\
--- \\
--- \\
---\end{array}$ & $\begin{array}{r}-1.3 \\
2.8 \\
3.2 \\
.2 \\
-.7 \\
-1.4\end{array}$ & $\begin{array}{r}-10.3 \\
-4.6 \\
-.3 \\
-2.8 \\
-.6 \\
--.\end{array}$ & $\begin{array}{r}8.3 \\
7.1 \\
9.8 \\
9.2 \\
10.7 \\
9.3\end{array}$ & $\begin{array}{l}9.9 \\
9.9 \\
3.4 \\
1.9 \\
6.7 \\
---\end{array}$ & $\begin{array}{l}--- \\
--- \\
--- \\
--- \\
--- \\
---\end{array}$ & $\begin{array}{r}10.1 \\
8.7 \\
7.1 \\
7.1 \\
4.9 \\
4.7\end{array}$ & $\begin{array}{l}-5.5 \\
-8.7 \\
-8.9 \\
-5.9 \\
-2.8 \\
---\end{array}$ \\
\hline MIN & --- & --- & --- & -- & -18.5 & -13.6 & -13.2 & -10.0 & .2 & -- & 4.7 & -8.9 \\
\hline
\end{tabular}


EAST STEWART BASIN

EAST STEWART CREEK NEAR IONE, NV

DAILY MINIMUM AIR TEMPERATURE, WATER YEAR OCTOBER 1986 TO SEPTEMBER 1987 DEGREES CELSIUS

\begin{tabular}{|c|c|c|c|c|c|c|c|c|c|c|c|c|}
\hline DAY & OCT & NOV & DEC & JAN & FEB & MAR & APR & MAY & JUN & JUL & AUG & SEP \\
\hline $\begin{array}{l}1 \\
2 \\
3 \\
4 \\
5\end{array}$ & $\begin{array}{r}-4.5 \\
--- \\
--- \\
--- \\
---\end{array}$ & $\begin{array}{r}--- \\
--- \\
--- \\
--- \\
-1.7\end{array}$ & $\begin{array}{r}-4.5 \\
-2.9 \\
-2.1 \\
-.3 \\
-5.2\end{array}$ & $\begin{array}{r}-6.6 \\
-10.4 \\
-4.3 \\
-10.1 \\
-12.1\end{array}$ & $\begin{array}{l}-1.1 \\
-5.2 \\
-9.5 \\
-8.5 \\
-5.8\end{array}$ & $\begin{array}{l}--- \\
--- \\
--- \\
--- \\
---\end{array}$ & $\begin{array}{r}-3.4 \\
2.1 \\
-7.1 \\
-7.0 \\
-7.3\end{array}$ & $\begin{array}{r}-6.1 \\
-7.2 \\
-2.7 \\
2.1 \\
6.6\end{array}$ & $\begin{array}{l}1.4 \\
2.9 \\
5.3 \\
7.2 \\
5.3\end{array}$ & $\begin{array}{l}--- \\
--- \\
--- \\
--- \\
6.1\end{array}$ & $\begin{array}{r}9.6 \\
7.5 \\
10.5 \\
9.7 \\
10.5\end{array}$ & $\begin{array}{l}8.5 \\
6.5 \\
4.5 \\
2.7 \\
7.8\end{array}$ \\
\hline $\begin{array}{r}6 \\
7 \\
8 \\
9 \\
10\end{array}$ & $\begin{array}{l}--- \\
--- \\
--- \\
--- \\
---\end{array}$ & $\begin{array}{r}-11.5 \\
-12.7 \\
-13.5 \\
-8.5 \\
-6.5\end{array}$ & $\begin{array}{l}-8.6 \\
-9.8 \\
-7.5 \\
-9.3 \\
-7.3\end{array}$ & $\begin{array}{r}-15.5 \\
-15.0 \\
-16.3 \\
-11.0 \\
-5.0\end{array}$ & $\begin{array}{r}-.6 \\
.7 \\
-2.1 \\
-1.9 \\
-5.5\end{array}$ & $\begin{array}{l}--- \\
--- \\
--- \\
--- \\
---\end{array}$ & $\begin{array}{l}-5.7 \\
-4.2 \\
-3.4 \\
-2.8 \\
-2.2\end{array}$ & $\begin{array}{l}5.6 \\
6.6 \\
5.3 \\
2.8 \\
2.3\end{array}$ & $\begin{array}{l}3.6 \\
2.4 \\
2.6 \\
1.8 \\
7.1\end{array}$ & $\begin{array}{l}4.4 \\
6.1 \\
6.9 \\
9.6 \\
7.5\end{array}$ & $\begin{array}{r}9.6 \\
8.5 \\
10.5 \\
9.8 \\
7.6\end{array}$ & $\begin{array}{l}6.0 \\
4.4 \\
6.1 \\
7.3 \\
6.0\end{array}$ \\
\hline $\begin{array}{l}11 \\
12 \\
13 \\
14 \\
15\end{array}$ & $\begin{array}{l}--- \\
--- \\
--- \\
--- \\
---\end{array}$ & $\begin{array}{r}-3.1 \\
-3.0 \\
.1 \\
-1.2 \\
-4.8\end{array}$ & $\begin{array}{l}-3.9 \\
-6.4 \\
-5.3 \\
-3.9 \\
-5.1\end{array}$ & $\begin{array}{r}-1.8 \\
1.1 \\
-11.9 \\
-17.4 \\
-22.4\end{array}$ & $\begin{array}{r}-4.9 \\
-4.6 \\
-9.2 \\
-11.5 \\
-10.3\end{array}$ & $\begin{array}{l}--- \\
--- \\
--- \\
--- \\
---\end{array}$ & $\begin{array}{r}-5.8 \\
-9.5 \\
-3.4 \\
1.1 \\
.5\end{array}$ & $\begin{array}{l}2.7 \\
3.3 \\
2.4 \\
4.4 \\
4.1\end{array}$ & $\begin{array}{r}6.5 \\
10.0 \\
10.7 \\
11.2 \\
-.5\end{array}$ & $\begin{array}{r}7.6 \\
7.0 \\
8.2 \\
12.4 \\
10.5\end{array}$ & $\begin{array}{r}7.0 \\
6.7 \\
6.1 \\
1.3 \\
.4\end{array}$ & $\begin{array}{l}7.8 \\
5.9 \\
1.9 \\
3.2 \\
2.7\end{array}$ \\
\hline $\begin{array}{l}16 \\
17 \\
18 \\
19 \\
20\end{array}$ & $\begin{array}{l}--- \\
--- \\
--- \\
--- \\
---\end{array}$ & $\begin{array}{r}-4.4 \\
-.5 \\
-.4 \\
-2.2 \\
-1.0\end{array}$ & $\begin{array}{l}-7.1 \\
-8.2 \\
-7.1 \\
-9.4 \\
-6.8\end{array}$ & $\begin{array}{r}-22.2 \\
-13.7 \\
-9.5 \\
-16.4 \\
-15.4\end{array}$ & $\begin{array}{r}-12.4 \\
-13.0 \\
-14.2 \\
-15.8 \\
---\end{array}$ & $\begin{array}{l}--- \\
--- \\
--- \\
--- \\
---\end{array}$ & $\begin{array}{r}1.0 \\
1.8 \\
-11.7 \\
-13.4 \\
-5.1\end{array}$ & $\begin{array}{r}1.4 \\
.7 \\
.0 \\
-1.5 \\
-4.9\end{array}$ & $\begin{array}{r}-.9 \\
2.4 \\
.9 \\
3.2 \\
4.4\end{array}$ & $\begin{array}{r}7.7 \\
-3.7 \\
-5.0 \\
3.2 \\
6.0\end{array}$ & $\begin{array}{r}2.9 \\
5.6 \\
7.7 \\
12.3 \\
8.9\end{array}$ & $\begin{array}{l}-.2 \\
1.6 \\
2.4 \\
3.1 \\
4.3\end{array}$ \\
\hline $\begin{array}{l}21 \\
22 \\
23 \\
24 \\
25\end{array}$ & $\begin{array}{l}--- \\
--- \\
--- \\
--- \\
---\end{array}$ & $\begin{array}{r}-9.9 \\
-10.5 \\
-8.7 \\
-5.4 \\
-7.4\end{array}$ & $\begin{array}{r}-9.9 \\
-4.6 \\
-10.7 \\
-10.0 \\
-10.8\end{array}$ & $\begin{array}{r}-5.5 \\
-2.3 \\
-10.2 \\
-8.0 \\
-3.2\end{array}$ & $\begin{array}{l}--- \\
--- \\
--- \\
--- \\
---\end{array}$ & $\begin{array}{l}--- \\
--- \\
--- \\
--- \\
---\end{array}$ & $\begin{array}{r}-1.6 \\
6.3 \\
3.5 \\
4.4 \\
5.6\end{array}$ & $\begin{array}{r}-4.4 \\
-1.8 \\
-.3 \\
-2.1 \\
-4.9\end{array}$ & $\begin{array}{l}2.0 \\
-.3 \\
3.9 \\
6.6 \\
9.7\end{array}$ & $\begin{array}{r}.2 \\
.0 \\
5.8 \\
10.6 \\
11.6\end{array}$ & $\begin{array}{r}9.9 \\
11.5 \\
6.9 \\
6.9 \\
4.6\end{array}$ & $\begin{array}{l}8.4 \\
8.8 \\
5.8 \\
5.1 \\
4.8\end{array}$ \\
\hline $\begin{array}{l}26 \\
27 \\
28 \\
29 \\
30 \\
31\end{array}$ & $\begin{array}{l}--- \\
--- \\
--- \\
--- \\
--- \\
---\end{array}$ & $\begin{array}{r}-5.5 \\
1.3 \\
-.4 \\
-11.3 \\
-6.3 \\
----\end{array}$ & $\begin{array}{l}-5.6 \\
-8.3 \\
-7.5 \\
-7.3 \\
-7.7 \\
-5.4\end{array}$ & $\begin{array}{r}1.9 \\
-1.5 \\
-11.0 \\
-7.1 \\
-9.3 \\
-9.3\end{array}$ & $\begin{array}{l}--- \\
--- \\
--- \\
--- \\
--- \\
---\end{array}$ & $\begin{array}{l}--- \\
--- \\
--- \\
--- \\
--- \\
---\end{array}$ & $\begin{array}{r}6.0 \\
5.7 \\
3.9 \\
2.3 \\
-1.3 \\
----\end{array}$ & $\begin{array}{r}-4.5 \\
-2.2 \\
-2.3 \\
-.8 \\
4.2 \\
1.1\end{array}$ & $\begin{array}{l}9.2 \\
7.5 \\
6.9 \\
7.3 \\
--- \\
---\end{array}$ & $\begin{array}{r}13.1 \\
11.6 \\
7.8 \\
11.8 \\
8.0 \\
9.2\end{array}$ & $\begin{array}{l}4.9 \\
6.0 \\
6.9 \\
8.4 \\
8.6 \\
8.8\end{array}$ & $\begin{array}{l}2.2 \\
-.1 \\
1.9 \\
3.6 \\
5.5 \\
-.--\end{array}$ \\
\hline MIN & --- & --- & -10.8 & -22.4 & --- & --- & -13.4 & -7.2 & --- & --- & .4 & -.2 \\
\hline
\end{tabular}


EAST STEWART BASIN

EAST STEWART CREEK NEAR IONE, NV

DAILY MINIMUM AIR TEMPERATURE, WATER YEAR OCTOBER 1987 TO SEPTEMBER 1988 DEGREES CELSIUS

\begin{tabular}{|c|c|c|c|c|c|c|c|c|c|c|c|c|}
\hline DAY & OCT & NOV & $\mathrm{DEC}$ & JAN & FEB & MAR & APR & MAY & JUN & JUL & AUG & SEP \\
\hline $\begin{array}{l}1 \\
2 \\
3 \\
4 \\
5\end{array}$ & $\begin{array}{l}4.4 \\
5.3 \\
6.2 \\
4.6 \\
5.0\end{array}$ & $\begin{array}{r}-.8 \\
-3.4 \\
-2.4 \\
-.4 \\
-2.6\end{array}$ & $\begin{array}{r}-3.0 \\
.6 \\
3.9 \\
-1.6 \\
-5.4\end{array}$ & $\begin{array}{r}-12.7 \\
-11.1 \\
-3.4 \\
-2.7 \\
-8.5\end{array}$ & $\begin{array}{r}-16.2 \\
-15.1 \\
-14.0 \\
-9.0 \\
-7.4\end{array}$ & $\begin{array}{l}-6.0 \\
-5.6 \\
-7.9 \\
-5.1 \\
-3.0\end{array}$ & $\begin{array}{l}-7.0 \\
-3.5 \\
-1.7 \\
-2.4 \\
-3.2\end{array}$ & $\begin{array}{r}-13.0 \\
-9.7 \\
-4.3 \\
-1.8 \\
-9.7\end{array}$ & $\begin{array}{r}1.5 \\
4.6 \\
10.6 \\
8.0 \\
-4.0\end{array}$ & $\begin{array}{r}10.3 \\
9.3 \\
8.2 \\
6.4 \\
5.3\end{array}$ & $\begin{array}{l}8.1 \\
7.0 \\
6.9 \\
7.3 \\
6.7\end{array}$ & $\begin{array}{r}9.7 \\
13.4 \\
14.0 \\
10.2 \\
9.2\end{array}$ \\
\hline $\begin{array}{r}6 \\
7 \\
8 \\
9 \\
10\end{array}$ & $\begin{array}{l}5.5 \\
5.0 \\
3.3 \\
4.5 \\
6.2\end{array}$ & $\begin{array}{l}-2.7 \\
-3.7 \\
-3.7 \\
-3.8 \\
-4.2\end{array}$ & $\begin{array}{r}-3.9 \\
-12.5 \\
-7.1 \\
-3.9 \\
-1.8\end{array}$ & $\begin{array}{r}-12.7 \\
-9.3 \\
-9.3 \\
-5.7 \\
-5.9\end{array}$ & $\begin{array}{l}-4.9 \\
-5.7 \\
-4.3 \\
-3.3 \\
-5.7\end{array}$ & $\begin{array}{r}-6.0 \\
-8.7 \\
-3.6 \\
-13.0 \\
-16.0\end{array}$ & $\begin{array}{r}5.2 \\
-5.3 \\
-8.3 \\
-4.1 \\
-.3\end{array}$ & $\begin{array}{r}-10.6 \\
-8.8 \\
-8.3 \\
-2.2 \\
3.7\end{array}$ & $\begin{array}{r}-4.0 \\
-7.0 \\
-9.0 \\
4.5 \\
5.4\end{array}$ & $\begin{array}{r}4.4 \\
9.2 \\
10.5 \\
12.1 \\
9.0\end{array}$ & $\begin{array}{r}2.7 \\
2.6 \\
4.0 \\
10.4 \\
7.7\end{array}$ & $\begin{array}{l}8.1 \\
7.4 \\
8.5 \\
8.6 \\
1.2\end{array}$ \\
\hline $\begin{array}{l}11 \\
12 \\
13 \\
14 \\
15\end{array}$ & $\begin{array}{r}6.4 \\
.3 \\
-.9 \\
-.3 \\
-.8\end{array}$ & $\begin{array}{r}-1.0 \\
2.2 \\
-5.3 \\
-13.5 \\
-13.5\end{array}$ & $\begin{array}{l}-13.6 \\
-17.8 \\
-19.2 \\
-20.5 \\
-14.6\end{array}$ & $\begin{array}{r}-14.2 \\
-15.9 \\
-6.3 \\
-2.1 \\
-12.9\end{array}$ & $\begin{array}{l}-3.5 \\
-4.8 \\
-6.8 \\
-5.1 \\
-5.6\end{array}$ & $\begin{array}{r}-15.9 \\
-14.7 \\
-10.1 \\
-7.0 \\
-12.5\end{array}$ & $\begin{array}{r}2.1 \\
4.7 \\
.8 \\
-2.5 \\
-2.4\end{array}$ & $\begin{array}{r}3.7 \\
5.5 \\
1.4 \\
1.0 \\
10.1\end{array}$ & $\begin{array}{r}3.3 \\
2.2 \\
5.4 \\
9.0 \\
10.1\end{array}$ & $\begin{array}{l}8.1 \\
6.8 \\
9.5 \\
7.2 \\
7.6\end{array}$ & $\begin{array}{r}10.4 \\
4.1 \\
2.1 \\
6.6 \\
1.4\end{array}$ & $\begin{array}{r}-2.2 \\
-5.6 \\
-3.0 \\
.0 \\
5.3\end{array}$ \\
\hline $\begin{array}{l}16 \\
17 \\
18 \\
19 \\
20\end{array}$ & $\begin{array}{r}.6 \\
-.3 \\
-1.6 \\
-.3 \\
4.1\end{array}$ & $\begin{array}{l}-5.6 \\
-7.2 \\
-6.5 \\
-1.4 \\
-6.8\end{array}$ & $\begin{array}{r}-8.3 \\
-11.1 \\
-11.4 \\
-11.2 \\
-10.9\end{array}$ & $\begin{array}{r}-13.1 \\
-14.0 \\
-18.3 \\
-17.7 \\
-9.3\end{array}$ & $\begin{array}{r}-14.0 \\
-13.3 \\
-12.6 \\
-11.0 \\
-1.6\end{array}$ & $\begin{array}{r}-11.9 \\
-10.4 \\
-5.0 \\
-2.1 \\
-.4\end{array}$ & $\begin{array}{l}-.4 \\
-2.7 \\
-6.5 \\
-2.6 \\
-9.5\end{array}$ & $\begin{array}{r}1.3 \\
-1.7 \\
-4.0 \\
-1.9 \\
-.2\end{array}$ & $\begin{array}{r}10.9 \\
10.5 \\
10.9 \\
10.8 \\
9.0\end{array}$ & $\begin{array}{r}10.2 \\
9.7 \\
8.4 \\
10.7 \\
13.3\end{array}$ & $\begin{array}{r}3.0 \\
11.8 \\
10.4 \\
11.5 \\
12.9\end{array}$ & $\begin{array}{r}2.6 \\
-1.2 \\
-3.3 \\
-.5 \\
-1.4\end{array}$ \\
\hline $\begin{array}{l}21 \\
22 \\
23 \\
24 \\
25\end{array}$ & $\begin{array}{r}3.4 \\
1.1 \\
-.7 \\
-2.9 \\
-2.2\end{array}$ & $\begin{array}{r}-8.5 \\
-8.4 \\
-10.7 \\
-10.4 \\
-15.5\end{array}$ & $\begin{array}{r}-4.9 \\
-15.6 \\
-19.8 \\
-22.1 \\
-21.1\end{array}$ & $\begin{array}{r}-12.2 \\
-6.9 \\
-4.9 \\
-3.7 \\
-3.3\end{array}$ & $\begin{array}{l}-5.3 \\
-5.3 \\
-3.0 \\
-4.6 \\
-4.2\end{array}$ & $\begin{array}{l}-5.2 \\
-6.4 \\
-5.9 \\
-7.4 \\
-2.9\end{array}$ & $\begin{array}{r}-11.6 \\
-9.8 \\
-7.4 \\
-6.2 \\
-4.8\end{array}$ & $\begin{array}{l}1.5 \\
6.2 \\
5.7 \\
5.3 \\
7.9\end{array}$ & $\begin{array}{r}11.1 \\
11.4 \\
11.4 \\
11.0 \\
7.8\end{array}$ & $\begin{array}{r}10.8 \\
11.4 \\
10.0 \\
9.7 \\
8.4\end{array}$ & $\begin{array}{r}8.2 \\
8.2 \\
13.1 \\
13.5 \\
9.6\end{array}$ & $\begin{array}{r}-1.8 \\
-.7 \\
1.0 \\
4.0 \\
6.1\end{array}$ \\
\hline $\begin{array}{l}26 \\
27 \\
28 \\
29 \\
30 \\
31\end{array}$ & $\begin{array}{r}.6 \\
1.7 \\
2.7 \\
-1.2 \\
-1.9 \\
-.6\end{array}$ & $\begin{array}{r}-16.1 \\
-6.7 \\
-12.2 \\
-12.8 \\
-5.5 \\
---\end{array}$ & $\begin{array}{r}-9.5 \\
-9.2 \\
-9.0 \\
-14.1 \\
-18.1 \\
-17.1\end{array}$ & $\begin{array}{r}-.8 \\
1.9 \\
.0 \\
-8.3 \\
-8.7 \\
-11.9\end{array}$ & $\begin{array}{r}-2.1 \\
-.8 \\
-1.8 \\
-4.3 \\
--- \\
---\end{array}$ & $\begin{array}{r}1.0 \\
-12.5 \\
-14.9 \\
-9.8 \\
-13.5 \\
-12.4\end{array}$ & $\begin{array}{r}-2.5 \\
3.1 \\
-1.4 \\
-1.6 \\
-11.7 \\
---\end{array}$ & $\begin{array}{r}3.3 \\
1.1 \\
-.2 \\
-7.9 \\
-8.5 \\
-6.5\end{array}$ & $\begin{array}{r}5.9 \\
10.6 \\
7.5 \\
5.5 \\
6.7 \\
---\end{array}$ & $\begin{array}{r}8.9 \\
7.4 \\
6.8 \\
11.1 \\
10.9 \\
10.0\end{array}$ & $\begin{array}{l}9.7 \\
7.5 \\
7.8 \\
8.8 \\
8.7 \\
7.5\end{array}$ & $\begin{array}{l}1.8 \\
-.3 \\
1.9 \\
4.6 \\
4.0 \\
---\end{array}$ \\
\hline MIN & -2.9 & -16.1 & -22.1 & -18.3 & -16.2 & -16.0 & -11.7 & -13.0 & -9.0 & 4.4 & 1.4 & -5.6 \\
\hline
\end{tabular}

WTR YR 1988 MIN -22.1 
EAST STEWART BASIN

EAST STEWART CREEK NEAR IONE, NV

DAILY MINIMUM AIR TEMPERATURE, WATER YEAR OCTOBER 1988 TO SEPTEMBER 1989 DEGREES CELSIUS

\begin{tabular}{|c|c|c|c|c|c|c|c|c|c|c|c|c|}
\hline DAY & OCT & NOV & DEC & JAN & FEB & MAR & APR & MAY & JUN & JUL & AUG & SER \\
\hline $\begin{array}{l}1 \\
2 \\
3 \\
4 \\
5\end{array}$ & $\begin{array}{l}5.0 \\
6.8 \\
9.4 \\
5.8 \\
4.1\end{array}$ & $\begin{array}{r}-1.3 \\
-2.0 \\
.6 \\
.5 \\
.8\end{array}$ & $\begin{array}{l}-4.0 \\
-4.9 \\
-5.0 \\
-4.9 \\
-3.7\end{array}$ & $\begin{array}{r}-16.1 \\
-9.1 \\
-5.3 \\
-5.7 \\
-14.4\end{array}$ & $\begin{array}{r}-9.5 \\
-11.4 \\
-15.3 \\
-23.7 \\
-24.1\end{array}$ & $\begin{array}{r}-5.3 \\
-10.8 \\
-17.6 \\
-9.1 \\
-3.4\end{array}$ & $\begin{array}{l}-5.5 \\
-2.5 \\
-3.9 \\
-3.5 \\
-.5\end{array}$ & $\begin{array}{r}.8 \\
-1.1 \\
.7 \\
1.2 \\
6.2\end{array}$ & $\begin{array}{r}1.6 \\
1.7 \\
1.4 \\
2.0 \\
.4\end{array}$ & $\begin{array}{r}3.3 \\
7.4 \\
11.2 \\
6.7 \\
7.6\end{array}$ & $\begin{array}{l}6.7 \\
6.8 \\
7.0 \\
6.5 \\
7.1\end{array}$ & $\begin{array}{l}5.0 \\
4.9 \\
4.8 \\
6.3 \\
5.6\end{array}$ \\
\hline $\begin{array}{r}6 \\
7 \\
8 \\
9 \\
10\end{array}$ & $\begin{array}{l}5.1 \\
3.3 \\
2.7 \\
2.6 \\
5.5\end{array}$ & $\begin{array}{l}-2.7 \\
-3.6 \\
-7.8 \\
-9.2 \\
-4.4\end{array}$ & $\begin{array}{l}-6.4 \\
-8.4 \\
-8.8 \\
-1.8 \\
-4.3\end{array}$ & $\begin{array}{r}-16.9 \\
-19.3 \\
-20.1 \\
-6.1 \\
-16.3\end{array}$ & $\begin{array}{r}-21.3 \\
-21.9 \\
-14.6 \\
-8.1 \\
-11.9\end{array}$ & $\begin{array}{r}.6 \\
.3 \\
1.2 \\
3.1 \\
3.9\end{array}$ & $\begin{array}{l}1.9 \\
3.6 \\
2.5 \\
1.4 \\
1.7\end{array}$ & $\begin{array}{r}7.9 \\
6.5 \\
5.4 \\
2.4 \\
-5.4\end{array}$ & $\begin{array}{l}.3 \\
3.4 \\
4.1 \\
2.4 \\
1.9\end{array}$ & $\begin{array}{l}7.2 \\
9.9 \\
9.9 \\
5.1 \\
2.8\end{array}$ & $\begin{array}{r}9.2 \\
9.3 \\
8.1 \\
10.5 \\
8.1\end{array}$ & $\begin{array}{r}3.8 \\
-1.3 \\
.5 \\
1.9 \\
2.9\end{array}$ \\
\hline $\begin{array}{l}11 \\
12 \\
13 \\
14 \\
15\end{array}$ & $\begin{array}{r}-.2 \\
.1 \\
1.0 \\
-.5 \\
.5\end{array}$ & $\begin{array}{r}-8.3 \\
-1.7 \\
-4.9 \\
-12.5 \\
-13.8\end{array}$ & $\begin{array}{r}-1.6 \\
.0 \\
-3.3 \\
-16.7 \\
-16.5\end{array}$ & $\begin{array}{r}-16.5 \\
-15.2 \\
-8.2 \\
-16.2 \\
-15.4\end{array}$ & $\begin{array}{l}-12.2 \\
-15.8 \\
-18.5 \\
-16.6 \\
-13.3\end{array}$ & $\begin{array}{r}.2 \\
-2.2 \\
-9.1 \\
-11.2 \\
-6.8\end{array}$ & $\begin{array}{r}-.9 \\
-.9 \\
-1.3 \\
2.0 \\
.5\end{array}$ & $\begin{array}{l}-4.5 \\
-5.6 \\
-5.7 \\
-2.9 \\
-2.2\end{array}$ & $\begin{array}{l}3.8 \\
6.2 \\
6.3 \\
7.1 \\
7.2\end{array}$ & $\begin{array}{r}10.5 \\
7.6 \\
8.1 \\
9.5 \\
6.9\end{array}$ & $\begin{array}{r}6.1 \\
9.7 \\
10.3 \\
8.2 \\
7.7\end{array}$ & $\begin{array}{l}2.7 \\
-.9 \\
3.3 \\
5.5 \\
6.4\end{array}$ \\
\hline $\begin{array}{l}16 \\
17 \\
18 \\
19 \\
20\end{array}$ & $\begin{array}{l}4.7 \\
3.8 \\
3.8 \\
2.8 \\
2.9\end{array}$ & $\begin{array}{l}-11.2 \\
-11.9 \\
-14.1 \\
-10.9 \\
-10.3\end{array}$ & $\begin{array}{r}-8.7 \\
-5.1 \\
-10.5 \\
-12.0 \\
-11.4\end{array}$ & $\begin{array}{l}-9.8 \\
-6.0 \\
-3.0 \\
-2.5 \\
-4.1\end{array}$ & $\begin{array}{r}-6.4 \\
-6.1 \\
-4.2 \\
-7.6 \\
-11.4\end{array}$ & $\begin{array}{r}-10.3 \\
-11.3 \\
-1.7 \\
-10.0 \\
-11.5\end{array}$ & $\begin{array}{l}.4 \\
1.6 \\
3.3 \\
4.7 \\
6.0\end{array}$ & $\begin{array}{r}-1.7 \\
-.5 \\
-3.4 \\
-5.0 \\
1.6\end{array}$ & $\begin{array}{l}3.4 \\
3.3 \\
7.3 \\
7.7 \\
2.0\end{array}$ & $\begin{array}{r}3.5 \\
6.1 \\
10.5 \\
15.8 \\
13.4\end{array}$ & $\begin{array}{l}6.5 \\
5.2 \\
4.8 \\
6.6 \\
3.9\end{array}$ & $\begin{array}{r}7.4 \\
-2.0 \\
-.8 \\
-.3 \\
.7\end{array}$ \\
\hline $\begin{array}{l}21 \\
22 \\
23 \\
24 \\
25\end{array}$ & $\begin{array}{l}4.2 \\
2.4 \\
3.4 \\
3.1 \\
2.6\end{array}$ & $\begin{array}{r}-8.2 \\
-1.2 \\
-11.7 \\
-14.5 \\
-9.5\end{array}$ & $\begin{array}{l}-14.8 \\
-13.4 \\
-17.4 \\
-13.4 \\
-17.7\end{array}$ & $\begin{array}{r}-5.3 \\
-3.5 \\
-12.3 \\
-12.6 \\
-10.4\end{array}$ & $\begin{array}{r}-4.8 \\
1.1 \\
.6 \\
1.8 \\
1.1\end{array}$ & $\begin{array}{r}-1.3 \\
-4.5 \\
-2.4 \\
-.3 \\
-7.9\end{array}$ & $\begin{array}{r}-1.4 \\
-4.9 \\
-5.3 \\
-12.5 \\
-9.3\end{array}$ & $\begin{array}{r}-.9 \\
4.1 \\
-5.0 \\
-7.9 \\
-5.6\end{array}$ & $\begin{array}{r}.8 \\
1.9 \\
2.6 \\
3.6 \\
.5\end{array}$ & $\begin{array}{l}8.8 \\
8.3 \\
7.1 \\
8.4 \\
7.9\end{array}$ & $\begin{array}{r}3.3 \\
6.4 \\
.2 \\
-3.6 \\
-.6\end{array}$ & $\begin{array}{r}2.1 \\
1.6 \\
3.6 \\
10.3 \\
8.3\end{array}$ \\
\hline $\begin{array}{l}26 \\
27 \\
28 \\
29 \\
30 \\
31\end{array}$ & $\begin{array}{r}2.9 \\
1.8 \\
.9 \\
.3 \\
-.9 \\
4.5\end{array}$ & $\begin{array}{r}-12.3 \\
-11.3 \\
-7.3 \\
-7.5 \\
-1.4 \\
---\end{array}$ & $\begin{array}{r}-22.5 \\
-15.5 \\
-18.1 \\
-14.9 \\
-8.0 \\
-14.7\end{array}$ & $\begin{array}{r}-8.0 \\
-8.1 \\
-4.0 \\
-3.2 \\
1.1 \\
.4\end{array}$ & $\begin{array}{r}-4.2 \\
-7.3 \\
-8.4 \\
--- \\
--- \\
---\end{array}$ & $\begin{array}{l}-9.6 \\
-6.4 \\
-3.9 \\
-4.8 \\
-4.5 \\
-3.0\end{array}$ & $\begin{array}{r}-10.8 \\
-8.2 \\
-9.8 \\
-7.2 \\
-1.0 \\
---\end{array}$ & $\begin{array}{r}-3.5 \\
3.1 \\
-5.1 \\
-5.9 \\
-5.6 \\
-2.5\end{array}$ & $\begin{array}{r}5.0 \\
4.9 \\
5.5 \\
10.7 \\
3.9 \\
---\end{array}$ & $\begin{array}{r}15.7 \\
11.8 \\
10.8 \\
9.8 \\
15.0 \\
11.0\end{array}$ & $\begin{array}{r}3.9 \\
4.1 \\
5.7 \\
10.6 \\
2.8 \\
1.4\end{array}$ & $\begin{array}{r}10.0 \\
9.2 \\
8.5 \\
6.6 \\
-1.4 \\
---\end{array}$ \\
\hline MIN & -.9 & -14.5 & -22.5 & -20.1 & -24.1 & -17.6 & -12.5 & -7.9 & .3 & 2.8 & -3.6 & -2.0 \\
\hline
\end{tabular}

CAL YR 1988 MIN -22.5

WTR YR 1989 MIN -24.1 
EAST STEWART BASIN

EAST STEWART CREEK NEAR IONE, NV

DAILY MINIMUM AIR TEMPERATURE, WATER YEAR OCTOBER 1989 TO SEPTEMBER 1990 DEGREES CELSIUS

\begin{tabular}{|c|c|c|c|c|c|c|c|c|c|c|c|c|}
\hline DAY & OCT & NOV & DEC & JAN & EEB & MAR & APR & MAY & JUN & JUL & AUG & SEP \\
\hline $\begin{array}{l}1 \\
2 \\
3 \\
4 \\
5\end{array}$ & $\begin{array}{l}-2.1 \\
-3.2 \\
-4.4 \\
-6.4 \\
-4.2\end{array}$ & $\begin{array}{l}-6.3 \\
-5.0 \\
-2.3 \\
-1.5 \\
-4.5\end{array}$ & $\begin{array}{l}-7.5 \\
-2.6 \\
-2.1 \\
-1.7 \\
-3.1\end{array}$ & $\begin{array}{r}-8.7 \\
-15.5 \\
-16.5 \\
-14.3 \\
-7.9\end{array}$ & $\begin{array}{r}-13.1 \\
-14.9 \\
-8.0 \\
-15.1 \\
-15.5\end{array}$ & $\begin{array}{l}-2.8 \\
-2.6 \\
-4.4 \\
-6.9 \\
-9.5\end{array}$ & $\begin{array}{r}-.9 \\
-1.7 \\
-1.1 \\
-1.2 \\
-2.6\end{array}$ & $\begin{array}{r}-7.2 \\
-2.6 \\
-1.0 \\
2.2 \\
4.7\end{array}$ & $\begin{array}{r}-6.9 \\
4.2 \\
8.9 \\
6.0 \\
2.9\end{array}$ & $\begin{array}{r}12.0 \\
5.1 \\
4.0 \\
10.7 \\
7.6\end{array}$ & $\begin{array}{r}8.2 \\
6.8 \\
9.5 \\
10.7 \\
12.1\end{array}$ & $\begin{array}{r}8.6 \\
11.9 \\
11.8 \\
10.7 \\
7.7\end{array}$ \\
\hline $\begin{array}{r}6 \\
7 \\
8 \\
9 \\
10\end{array}$ & $\begin{array}{r}-1.6 \\
1.2 \\
.8 \\
2.6 \\
4.6\end{array}$ & $\begin{array}{r}-6.4 \\
-6.8 \\
-5.2 \\
-.2 \\
1.9\end{array}$ & $\begin{array}{r}-6.8 \\
-5.0 \\
1.0 \\
-6.7 \\
-13.8\end{array}$ & $\begin{array}{r}-7.6 \\
-3.5 \\
.2 \\
3.9 \\
3.5\end{array}$ & $\begin{array}{r}-10.5 \\
-16.4 \\
-17.7 \\
-10.3 \\
-3.5\end{array}$ & $\begin{array}{r}-11.1 \\
-6.4 \\
-1.7 \\
-4.2 \\
-11.5\end{array}$ & $\begin{array}{l}-2.2 \\
-3.9 \\
-5.3 \\
-6.7 \\
-4.0\end{array}$ & $\begin{array}{r}3.7 \\
1.2 \\
-4.2 \\
-2.6 \\
-.4\end{array}$ & $\begin{array}{l}2.2 \\
2.8 \\
5.0 \\
5.7 \\
1.5\end{array}$ & $\begin{array}{r}9.1 \\
10.0 \\
9.0 \\
10.7 \\
8.8\end{array}$ & $\begin{array}{r}15.1 \\
10.3 \\
8.6 \\
8.9 \\
8.8\end{array}$ & $\begin{array}{r}8.0 \\
10.3 \\
10.3 \\
9.5 \\
12.5\end{array}$ \\
\hline $\begin{array}{l}11 \\
12 \\
13 \\
14 \\
15\end{array}$ & $\begin{array}{r}3.0 \\
6.0 \\
3.2 \\
.0 \\
-2.6\end{array}$ & $\begin{array}{r}1.4 \\
.5 \\
-1.3 \\
-4.6 \\
-3.4\end{array}$ & $\begin{array}{r}-13.7 \\
-4.3 \\
-6.6 \\
-5.8 \\
-3.8\end{array}$ & $\begin{array}{r}-1.3 \\
-2.5 \\
-7.0 \\
-12.7 \\
-13.1\end{array}$ & $\begin{array}{r}-3.8 \\
-10.7 \\
-21.0 \\
-23.5 \\
-23.2\end{array}$ & $\begin{array}{r}-14.7 \\
-19.0 \\
-17.2 \\
-12.4 \\
-9.4\end{array}$ & $\begin{array}{r}-1.0 \\
-2.0 \\
-.3 \\
3.9 \\
6.0\end{array}$ & $\begin{array}{l}-1.9 \\
-1.9 \\
-4.0 \\
-3.2 \\
-5.6\end{array}$ & $\begin{array}{r}-.7 \\
-1.1 \\
-2.4 \\
-1.7 \\
-.1\end{array}$ & $\begin{array}{r}9.7 \\
11.9 \\
9.9 \\
7.2 \\
7.7\end{array}$ & $\begin{array}{l}7.6 \\
7.6 \\
6.7 \\
8.4 \\
4.8\end{array}$ & $\begin{array}{r}11.1 \\
10.7 \\
9.4 \\
12.3 \\
4.9\end{array}$ \\
\hline $\begin{array}{l}16 \\
17 \\
18 \\
19 \\
20\end{array}$ & $\begin{array}{r}-2.8 \\
-.7 \\
1.7 \\
6.8 \\
1.5\end{array}$ & $\begin{array}{r}-2.9 \\
-3.4 \\
-4.2 \\
1.8 \\
2.0\end{array}$ & $\begin{array}{r}-8.5 \\
-8.2 \\
-10.3 \\
-9.2 \\
-7.8\end{array}$ & $\begin{array}{l}-10.7 \\
-10.5 \\
-11.8 \\
-14.3 \\
-10.0\end{array}$ & $\begin{array}{l}-12.4 \\
-15.7 \\
-15.9 \\
-15.6 \\
-11.4\end{array}$ & $\begin{array}{r}-4.2 \\
-1.7 \\
-3.4 \\
1.6 \\
-.5\end{array}$ & $\begin{array}{r}-1.9 \\
-2.8 \\
-2.5 \\
.9 \\
-.5\end{array}$ & $\begin{array}{l}-4.0 \\
-1.6 \\
-5.0 \\
-3.9 \\
-2.0\end{array}$ & $\begin{array}{r}-1.7 \\
5.4 \\
.8 \\
1.7 \\
5.6\end{array}$ & $\begin{array}{l}8.3 \\
7.1 \\
7.9 \\
8.4 \\
7.4\end{array}$ & $\begin{array}{r}4.3 \\
4.5 \\
.5 \\
-.6 \\
-2.5\end{array}$ & $\begin{array}{r}3.4 \\
4.3 \\
1.6 \\
.3 \\
4.0\end{array}$ \\
\hline $\begin{array}{l}21 \\
22 \\
23 \\
24 \\
25\end{array}$ & $\begin{array}{r}1.7 \\
1.7 \\
2.6 \\
-2.0 \\
-7.9\end{array}$ & $\begin{array}{l}-1.3 \\
-1.4 \\
-1.8 \\
-6.7 \\
-7.1\end{array}$ & $\begin{array}{l}-6.9 \\
-1.6 \\
-2.9 \\
-2.8 \\
-2.5\end{array}$ & $\begin{array}{r}-5.2 \\
-8.3 \\
-8.5 \\
-11.1 \\
-2.6\end{array}$ & $\begin{array}{l}-6.3 \\
-2.9 \\
-2.7 \\
-2.3 \\
-3.0\end{array}$ & $\begin{array}{r}-1.2 \\
-.6 \\
-2.6 \\
-3.1 \\
-2.2\end{array}$ & $\begin{array}{l}-2.1 \\
-1.0 \\
-5.3 \\
-6.9 \\
-6.5\end{array}$ & $\begin{array}{r}2.8 \\
.2 \\
-6.8 \\
-7.0 \\
-3.6\end{array}$ & $\begin{array}{r}12.0 \\
10.8 \\
10.4 \\
9.5 \\
9.9\end{array}$ & $\begin{array}{l}8.1 \\
9.2 \\
7.4 \\
4.4 \\
3.1\end{array}$ & $\begin{array}{r}3.0 \\
2.6 \\
4.3 \\
3.1 \\
.5\end{array}$ & $\begin{array}{l}4.8 \\
4.0 \\
2.6 \\
3.3 \\
4.6\end{array}$ \\
\hline $\begin{array}{l}26 \\
27 \\
28 \\
29 \\
30 \\
31\end{array}$ & $\begin{array}{r}-12.9 \\
-9.0 \\
-12.6 \\
-8.8 \\
-6.8 \\
-5.8\end{array}$ & $\begin{array}{r}-14.2 \\
-15.9 \\
-7.0 \\
-4.0 \\
-7.3 \\
----\end{array}$ & $\begin{array}{r}-2.9 \\
-3.6 \\
-8.5 \\
-11.2 \\
-6.2 \\
-5.4\end{array}$ & $\begin{array}{r}-15.5 \\
-16.1 \\
-13.1 \\
-8.9 \\
-12.6 \\
-16.2\end{array}$ & $\begin{array}{r}-3.4 \\
-4.5 \\
-3.4 \\
--- \\
--- \\
---\end{array}$ & $\begin{array}{l}-4.4 \\
-6.9 \\
-8.9 \\
-9.2 \\
-5.9 \\
-3.8\end{array}$ & $\begin{array}{r}-.5 \\
-.2 \\
-5.6 \\
-7.8 \\
-8.8 \\
----\end{array}$ & $\begin{array}{r}.0 \\
1.3 \\
-1.6 \\
-.5 \\
-4.8 \\
-5.2\end{array}$ & $\begin{array}{r}7.1 \\
4.4 \\
6.1 \\
13.5 \\
12.4 \\
---\end{array}$ & $\begin{array}{r}2.6 \\
5.5 \\
9.9 \\
10.1 \\
10.9 \\
8.1\end{array}$ & $\begin{array}{l}4.7 \\
5.8 \\
4.7 \\
7.5 \\
7.1 \\
6.9\end{array}$ & $\begin{array}{l}4.0 \\
1.3 \\
1.2 \\
2.9 \\
1.8 \\
---\end{array}$ \\
\hline MIN & -12.9 & -15.9 & -13.8 & -16.5 & -23.5 & -19.0 & -8.8 & -7.2 & -6.9 & 2.6 & -2.5 & .3 \\
\hline
\end{tabular}

CAL YR 1989 MIN -24.1

WTR YR 1990 MIN -23.5 
EAST STEWART BASIN

EAST STEWART CREEK NEAR IONE, NV

DAILY MINIMUM AIR TEMPERATURE, WATER YEAR OCTOBER 1990 TO SEPTEMBER 1991 DEGREES CELSIUS

\begin{tabular}{|c|c|c|c|c|c|c|c|c|c|c|c|c|}
\hline DAY & OCT & NOV & DEC & JAN & FEB & MAR & APR & MAY & JUN & JUL & AUG & SEP \\
\hline $\begin{array}{l}1 \\
2 \\
3 \\
4 \\
5\end{array}$ & $\begin{array}{r}2.4 \\
-.5 \\
-1.4 \\
6.9 \\
1.7\end{array}$ & $\begin{array}{r}-11.2 \\
-14.3 \\
-11.5 \\
-3.5 \\
-10.5\end{array}$ & $\begin{array}{r}-9.0 \\
-13.3 \\
-8.7 \\
-1.4 \\
-4.5\end{array}$ & $\begin{array}{l}-6.4 \\
-7.8 \\
-4.7 \\
-7.9 \\
-8.7\end{array}$ & $\begin{array}{l}-4.2 \\
-5.1 \\
-7.5 \\
-4.1 \\
-4.8\end{array}$ & $\begin{array}{r}-7.4 \\
-10.2 \\
-2.6 \\
-3.6 \\
-12.7\end{array}$ & $\begin{array}{r}-6.5 \\
-8.2 \\
-7.0 \\
-.8 \\
5.9\end{array}$ & $\begin{array}{l}-6.6 \\
-6.4 \\
-8.1 \\
-8.1 \\
-2.7\end{array}$ & $\begin{array}{r}.8 \\
2.3 \\
2.4 \\
1.3 \\
-1.5\end{array}$ & $\begin{array}{r}5.5 \\
9.1 \\
13.5 \\
11.5 \\
10.0\end{array}$ & $\begin{array}{l}5.9 \\
7.1 \\
7.1 \\
6.7 \\
5.0\end{array}$ & $\begin{array}{l}5.7 \\
9.8 \\
9.8 \\
6.2 \\
7.5\end{array}$ \\
\hline $\begin{array}{r}6 \\
7 \\
8 \\
9 \\
10\end{array}$ & $\begin{array}{r}-.8 \\
-7.8 \\
-8.7 \\
-1.8 \\
-.2\end{array}$ & $\begin{array}{r}-12.1 \\
-11.2 \\
-2.9 \\
-3.7 \\
-1.5\end{array}$ & $\begin{array}{r}-6.1 \\
-3.6 \\
-3.9 \\
.2 \\
-1.4\end{array}$ & $\begin{array}{r}-5.1 \\
-9.9 \\
-11.5 \\
-9.8 \\
-8.8\end{array}$ & $\begin{array}{l}-4.7 \\
-4.0 \\
-6.6 \\
-4.6 \\
-5.8\end{array}$ & $\begin{array}{r}-17.6 \\
-14.7 \\
-9.6 \\
-5.1 \\
-9.8\end{array}$ & $\begin{array}{r}-4.6 \\
-8.6 \\
-11.3 \\
-5.4 \\
-14.7\end{array}$ & $\begin{array}{r}-.7 \\
-1.5 \\
-6.9 \\
-10.1 \\
-10.8\end{array}$ & $\begin{array}{r}1.0 \\
.8 \\
3.7 \\
7.3 \\
8.5\end{array}$ & $\begin{array}{l}8.6 \\
8.7 \\
8.1 \\
6.4 \\
4.6\end{array}$ & $\begin{array}{l}9.7 \\
7.1 \\
5.5 \\
9.8 \\
8.6\end{array}$ & $\begin{array}{r}5.8 \\
5.6 \\
3.4 \\
-2.7 \\
-2.9\end{array}$ \\
\hline $\begin{array}{l}11 \\
12 \\
13 \\
14 \\
15\end{array}$ & $\begin{array}{r}-2.0 \\
-.2 \\
2.1 \\
.8 \\
4.5\end{array}$ & $\begin{array}{r}4.9 \\
3.4 \\
6.9 \\
.2 \\
-.9\end{array}$ & $\begin{array}{r}-4.9 \\
-7.2 \\
-13.6 \\
-19.5 \\
-11.7\end{array}$ & $\begin{array}{r}-5.7 \\
-4.1 \\
-5.0 \\
-5.7 \\
-10.5\end{array}$ & $\begin{array}{l}-3.4 \\
-3.9 \\
-3.9 \\
-3.1 \\
-1.5\end{array}$ & $\begin{array}{l}-14 \cdot 4 \\
-14.5 \\
-10.3 \\
-10.4 \\
-12.2\end{array}$ & $\begin{array}{r}-15.5 \\
-9.2 \\
-11.7 \\
-5.8 \\
-10.6\end{array}$ & $\begin{array}{r}-11.8 \\
-5.3 \\
-4.9 \\
-5.0 \\
-5.8\end{array}$ & $\begin{array}{r}6.8 \\
6.4 \\
3.3 \\
.3 \\
2.0\end{array}$ & $\begin{array}{r}10.2 \\
14.0 \\
14.7 \\
10.8 \\
9.4\end{array}$ & $\begin{array}{r}6.5 \\
6.3 \\
6.9 \\
10.5 \\
7.1\end{array}$ & $\begin{array}{r}.7 \\
-.9 \\
.2 \\
2.1 \\
1.0\end{array}$ \\
\hline $\begin{array}{l}16 \\
17 \\
18 \\
19 \\
20\end{array}$ & $\begin{array}{r}-2.8 \\
-2.7 \\
-.8 \\
-9.3 \\
-10.8\end{array}$ & $\begin{array}{r}1.3 \\
-2.4 \\
-2.6 \\
-4.3 \\
-14.1\end{array}$ & $\begin{array}{r}-15.0 \\
-13.6 \\
-9.4 \\
-20.5 \\
-25.3\end{array}$ & $\begin{array}{r}-11.6 \\
-5.5 \\
-5.3 \\
-8.5 \\
-12.2\end{array}$ & $\begin{array}{r}-6.1 \\
-10.3 \\
-10.9 \\
-5.7 \\
-5.3\end{array}$ & $\begin{array}{r}-13.7 \\
-7.3 \\
-6.6 \\
-11.1 \\
-10.6\end{array}$ & $\begin{array}{r}-12.5 \\
-10.5 \\
-10.0 \\
-2.2 \\
-4.5\end{array}$ & $\begin{array}{r}-.6 \\
-9.2 \\
-9.0 \\
-10.0 \\
-3.4\end{array}$ & $\begin{array}{r}3.8 \\
2.7 \\
6.0 \\
.1 \\
-2.2\end{array}$ & $\begin{array}{r}11.8 \\
10.1 \\
11.0 \\
5.8 \\
5.2\end{array}$ & $\begin{array}{r}5.7 \\
8.2 \\
13.2 \\
12.6 \\
8.5\end{array}$ & $\begin{array}{l}3.0 \\
4.0 \\
5.8 \\
6.4 \\
6.2\end{array}$ \\
\hline $\begin{array}{l}21 \\
22 \\
23 \\
24 \\
25\end{array}$ & $\begin{array}{r}-5.6 \\
.5 \\
2.1 \\
1.7 \\
6.3\end{array}$ & $\begin{array}{r}-10.6 \\
-8.9 \\
-2.1 \\
-2.3 \\
-8.3\end{array}$ & $\begin{array}{l}-27.7 \\
-28.2 \\
-20.2 \\
-17.4 \\
-12.2\end{array}$ & $\begin{array}{r}-11.8 \\
-5.5 \\
-8.6 \\
-11.5 \\
-12.0\end{array}$ & $\begin{array}{l}-4.0 \\
-3.4 \\
-6.9 \\
-8.1 \\
-6.7\end{array}$ & $\begin{array}{r}-9.2 \\
-14.4 \\
-6.1 \\
-7.8 \\
-11.9\end{array}$ & $\begin{array}{l}-6.2 \\
-6.8 \\
-3.9 \\
-5.0 \\
-9.2\end{array}$ & $\begin{array}{r}-3.4 \\
-.3 \\
.3 \\
2.1 \\
.6\end{array}$ & $\begin{array}{r}2.5 \\
.6 \\
1.8 \\
-.7 \\
-1.3\end{array}$ & $\begin{array}{r}5.3 \\
7.3 \\
11.2 \\
8.2 \\
7.4\end{array}$ & $\begin{array}{r}12.4 \\
8.4 \\
9.8 \\
10.5 \\
9.4\end{array}$ & $\begin{array}{l}4.6 \\
4.0 \\
4.1 \\
3.7 \\
7.8\end{array}$ \\
\hline $\begin{array}{l}26 \\
27 \\
28 \\
29 \\
30 \\
31\end{array}$ & $\begin{array}{r}3.4 \\
5.0 \\
3.6 \\
4.8 \\
3.9 \\
-2.9\end{array}$ & $\begin{array}{r}-16.5 \\
-16.0 \\
-10.8 \\
-2.0 \\
-9.6 \\
---\end{array}$ & $\begin{array}{r}-12.7 \\
-10.5 \\
-13.7 \\
-21.7 \\
-13.7 \\
-9.5\end{array}$ & $\begin{array}{r}-10.2 \\
-11.7 \\
-14.9 \\
-15.1 \\
-9.2 \\
-3.9\end{array}$ & $\begin{array}{r}-4.8 \\
-5.4 \\
-4.4 \\
--- \\
--- \\
---\end{array}$ & $\begin{array}{r}-13.5 \\
-10.5 \\
-11.9 \\
-8.5 \\
-7.0 \\
-1.1\end{array}$ & $\begin{array}{r}-10.2 \\
-11.8 \\
-9.7 \\
-7.3 \\
-7.7 \\
---\end{array}$ & $\begin{array}{l}-2.0 \\
-2.4 \\
-3.5 \\
-2.4 \\
-5.8 \\
-5.4\end{array}$ & $\begin{array}{r}-2.2 \\
1.5 \\
.3 \\
-.9 \\
.1 \\
---\end{array}$ & $\begin{array}{r}8.8 \\
10.8 \\
11.2 \\
12.2 \\
9.0 \\
7.9\end{array}$ & $\begin{array}{r}11.0 \\
-.3 \\
-1.7 \\
9.3 \\
14.0 \\
12.6\end{array}$ & $\begin{array}{l}5.9 \\
3.5 \\
1.3 \\
2.5 \\
1.8 \\
---\end{array}$ \\
\hline MIN & -10.8 & -16.5 & -28.2 & -15.1 & -10.9 & -17.6 & -15.5 & -11.8 & -2.2 & 4.6 & -1.7 & -2.9 \\
\hline
\end{tabular}

CAL YR 1990 MIN -28.2

WTR YR 1991 MIN -28.2 
EAST STEWART BASIN

EAST STEWART CREEK NEAR IONE, NV

DAILY MEAN SOIL TEMPERATURE, WATER YEAR OCTOBER 1985 TO SEPTEMBER 1986 DEGREES CELSIUS

\begin{tabular}{|c|c|c|c|c|c|c|c|c|c|c|c|c|}
\hline DAY & OCT & NOV & DEC & JAN & FEB & MAR & APR & MAY & JUN & JUL & AUG & SEP \\
\hline 1 & --- & -- & --- & e. 7 & .6 & .1 & .4 & .5 & 12.4 & 15.6 & 19.2 & 15.6 \\
\hline 2 & --- & --- & --- & e. 7 & .6 & .1 & .4 & .5 & 11.8 & 16.2 & 19.5 & 15.7 \\
\hline 3 & -- & -- & -- & e. 8 & .5 & .1 & .4 & .5 & 11.5 & 16.2 & 19.6 & 15.8 \\
\hline 4 & --- & --- & --- & e. 8 & .5 & .1 & .4 & .5 & 11.6 & 16.1 & 19.7 & 16.3 \\
\hline 5 & --- & -- & --- & e. 8 & .4 & .1 & .4 & .5 & 11.8 & 15.9 & 19.2 & 16.4 \\
\hline 6 & --- & -- & --- & e. 8 & .4 & .1 & .4 & .5 & 12.2 & 16.0 & 19.0 & 16.5 \\
\hline 7 & --- & --- & --- & e. 8 & .3 & .1 & .4 & .5 & 12.2 & 15.9 & 19.4 & 16.6 \\
\hline 8 & --- & --- & --- & e. 8 & .3 & .2 & .4 & .5 & 11.8 & 15.5 & 19.7 & 16.2 \\
\hline 9 & --- & --- & --- & e. 7 & .2 & .2 & .4 & .6 & 11.8 & e 15.6 & 19.4 & 14.5 \\
\hline 10 & --- & --- & --- & e. 6 & .1 & .2 & .4 & .6 & 11.9 & e 15.6 & 18.5 & 13.4 \\
\hline 11 & --- & --- & --- & e. 6 & .0 & .2 & .4 & .6 & 12.5 & e15. 8 & 16.5 & 12.8 \\
\hline 12 & --- & --- & --- & .5 & -.1 & .2 & .4 & .6 & 12.7 & e 16.3 & 17.1 & 13.6 \\
\hline 13 & --- & -- & --- & .5 & -.2 & .2 & .4 & .6 & 11.5 & e 16.6 & 17.7 & 13.4 \\
\hline 14 & -- & -- & -- & .5 & -.2 & .2 & .4 & .6 & 11.6 & e16.7 & 17.9 & 12.2 \\
\hline 15 & --- & --- & --- & .6 & -.2 & .2 & .5 & .6 & 12.2 & e 16.2 & 18.7 & 10.6 \\
\hline 16 & --- & --- & --- & .6 & -.2 & .2 & .5 & .8 & 13.0 & e 13.2 & 19.4 & 9.9 \\
\hline 17 & --- & -- & -- & .7 & -.1 & .3 & .5 & 1.6 & 13.7 & e13.1 & 19.5 & 9.6 \\
\hline 18 & --- & --- & --- & .7 & -.1 & .3 & .5 & 3.1 & 14.1 & e 13.5 & 19.1 & 8.9 \\
\hline 19 & --- & --- & --- & .8 & -.1 & .3 & .5 & 4.4 & 13.9 & e13.9 & 17.9 & 9.2 \\
\hline 20 & --- & --- & --- & .8 & .0 & .3 & .5 & 5.4 & 13.7 & e14. 2 & 16.1 & 8.3 \\
\hline 21 & --- & --- & --- & .9 & .0 & .3 & .5 & 5.4 & 13.7 & e13.6 & 15.1 & 7.9 \\
\hline 22 & --- & --- & --- & .9 & .0 & .3 & .4 & 4.9 & 14.2 & e 12.0 & 15.5 & 8.1 \\
\hline 23 & --- & --- & --- & .8 & .0 & .3 & .4 & 5.4 & 14.4 & e 11.0 & 16.9 & 7.9 \\
\hline 24 & --- & --- & --- & .8 & .0 & .3 & .4 & 6.2 & 14.8 & e 10.8 & 17.9 & 7.1 \\
\hline 25 & --- & --- & --- & .7 & .0 & .3 & .5 & 7.7 & 14.7 & e 10.9 & 17.7 & 6.6 \\
\hline 26 & -- & --- & --- & .7 & .0 & .3 & .5 & 9.1 & 15.1 & e11. 6 & 16.7 & 6.0 \\
\hline 27 & --- & --- & --- & .7 & .0 & .3 & .5 & 10.2 & 15.6 & e 12.1 & 16.4 & 5.5 \\
\hline 28 & --- & --- & --- & .6 & .0 & .3 & .5 & 10.8 & 15.8 & e 12.8 & 15.4 & 4.7 \\
\hline 29 & --- & --- & --- & .6 & --- & .3 & .5 & 10.4 & 15.2 & e 13.2 & 15.7 & 4.1 \\
\hline 30 & --- & --- & --- & .6 & --- & .3 & .5 & 11.4 & 15.3 & e 13.5 & 15.9 & 4.3 \\
\hline 31 & --- & --- & --- & .6 & --- & .4 & --- & 12.3 & --- & e14.0 & 15.7 & --- \\
\hline MEAN & -- & --- & -- & .7 & .1 & .2 & .4 & 3.8 & 13.2 & 14.3 & 17.8 & 10.9 \\
\hline MAX & -- & --- & --- & .9 & .6 & .4 & .5 & 12.3 & 15.8 & 16.7 & 19.7 & 16.6 \\
\hline MIN & --- & --- & -- & .5 & -.2 & .1 & .4 & .5 & 11.5 & 10.8 & 15.1 & 4.1 \\
\hline MED & --- & --- & --- & .7 & .0 & .2 & .4 & .8 & 12.9 & 14.2 & 17.9 & 10.2 \\
\hline
\end{tabular}


EAST STEWART BASIN

EAST STEWART CREEK NEAR IONE, NV

DAILY MEAN SOIL TEMPERATURE, WATER YEAR OCTOBER 1986 TO SEPTEMBER 1987 DEGREES CELSIUS

\begin{tabular}{|c|c|c|c|c|c|c|c|c|c|c|c|c|}
\hline DAY & OCT & NOV & $\mathrm{DEC}$ & JAN & FEB & MAR & APR & MAY & JUN & JUL & AUG & SEP \\
\hline $\begin{array}{l}1 \\
2 \\
3 \\
4 \\
5\end{array}$ & $\begin{array}{r}4.9 \\
\text { e } 4.6 \\
\text { e } 4.1 \\
\text { e } 4.2 \\
\text { e } 4.4\end{array}$ & $\begin{array}{r}\text { e3.1 } \\
\text { e2 } .7 \\
\text { e2 } .6 \\
\mathrm{e} 2.6 \\
2.3\end{array}$ & $\begin{array}{r}-1.0 \\
-1.2 \\
-1.1 \\
-.8 \\
-.2\end{array}$ & $\begin{array}{l}-2.7 \\
-2.2 \\
-1.9 \\
-1.5 \\
-1.6\end{array}$ & $\begin{array}{r}-1.1 \\
-.9 \\
-.6 \\
-.6 \\
-.8\end{array}$ & $\begin{array}{l}e-.1 \\
e-.1 \\
e-.1 \\
e-.1 \\
e-.1\end{array}$ & $\begin{array}{l}2.0 \\
2.5 \\
2.7 \\
2.8 \\
2.8\end{array}$ & $\begin{array}{l}6.6 \\
6.2 \\
6.7 \\
7.9 \\
9.0\end{array}$ & $\begin{array}{r}7.1 \\
9.1 \\
11.1 \\
12.6 \\
12.6\end{array}$ & $\begin{array}{r}\text { e } 16.2 \\
\text { e } 16.6 \\
\text { e17.0 } \\
17.3 \\
17.4\end{array}$ & $\begin{array}{l}20.5 \\
20.4 \\
20.4 \\
20.2 \\
19.8\end{array}$ & $\begin{array}{l}16.5 \\
16.1 \\
15.6 \\
15.3 \\
15.5\end{array}$ \\
\hline $\begin{array}{r}6 \\
7 \\
8 \\
9 \\
10\end{array}$ & $\begin{array}{l}\text { e } 4.6 \\
\text { e4.9 } \\
\text { e4.9 } \\
\text { e } 4.9 \\
\text { e4.8 }\end{array}$ & $\begin{array}{r}2.0 \\
1.7 \\
1.7 \\
1.2 \\
.9\end{array}$ & $\begin{array}{r}-.1 \\
-.2 \\
-1.0 \\
-1.9 \\
-2.6\end{array}$ & $\begin{array}{l}-1.6 \\
-1.6 \\
-1.7 \\
-2.2 \\
-2.5\end{array}$ & $\begin{array}{l}-.9 \\
-.7 \\
-.5 \\
-.4 \\
-.2\end{array}$ & $\begin{array}{r}\mathrm{e}-.1 \\
\mathrm{e}-.1 \\
\mathrm{e}-.1 \\
\mathrm{e} .0 \\
\mathrm{e} .1\end{array}$ & $\begin{array}{l}2.8 \\
2.8 \\
2.8 \\
2.8 \\
2.8\end{array}$ & $\begin{array}{r}9.9 \\
10.0 \\
10.2 \\
9.3 \\
8.4\end{array}$ & $\begin{array}{l}12.3 \\
11.7 \\
11.6 \\
11.2 \\
12.5\end{array}$ & $\begin{array}{l}17.4 \\
17.5 \\
17.8 \\
17.9 \\
18.0\end{array}$ & $\begin{array}{l}20.3 \\
20.3 \\
20.5 \\
19.9 \\
18.9\end{array}$ & $\begin{array}{l}14.8 \\
14.0 \\
14.0 \\
14.7 \\
14.8\end{array}$ \\
\hline $\begin{array}{l}11 \\
12 \\
13 \\
14 \\
15\end{array}$ & $\begin{array}{l}\text { e } 4.8 \\
\text { e } 4.3 \\
\text { e } 4.0 \\
\text { e } 4.0 \\
\text { e } 4.0\end{array}$ & $\begin{array}{l}.8 \\
.7 \\
.6 \\
.8 \\
.6\end{array}$ & $\begin{array}{l}-2.7 \\
-2.8 \\
-2.9 \\
-2.7 \\
-2.9\end{array}$ & $\begin{array}{l}-2.5 \\
-2.4 \\
-2.2 \\
-2.8 \\
-3.2\end{array}$ & $\begin{array}{r}-.1 \\
.0 \\
.2 \\
.4 \\
.4\end{array}$ & $\begin{array}{r}\text { e. } 3 \\
\text { e. } 8 \\
\text { el. } 2 \\
\text { e } 1.2 \\
\text { e1.1 }\end{array}$ & $\begin{array}{l}2.8 \\
2.8 \\
2.8 \\
2.8 \\
2.8\end{array}$ & $\begin{array}{r}8.7 \\
9.5 \\
10.6 \\
11.1 \\
10.9\end{array}$ & $\begin{array}{l}13.7 \\
14.8 \\
15.2 \\
15.3 \\
14.9\end{array}$ & $\begin{array}{l}18.4 \\
18.1 \\
18.2 \\
18.5 \\
18.2\end{array}$ & $\begin{array}{l}19.3 \\
19.4 \\
19.2 \\
18.5 \\
18.2\end{array}$ & $\begin{array}{l}14.6 \\
14.4 \\
14.3 \\
13.9 \\
14.2\end{array}$ \\
\hline $\begin{array}{l}16 \\
17 \\
18 \\
19 \\
20\end{array}$ & $\begin{array}{l}\text { e } 4.1 \\
\text { e } 4.4 \\
\text { e } 3.9 \\
\text { e } 3.0 \\
\text { e2 } .5\end{array}$ & $\begin{array}{r}.2 \\
.4 \\
1.0 \\
1.9 \\
1.5\end{array}$ & $\begin{array}{l}-3.1 \\
-3.3 \\
-3.2 \\
-2.8 \\
-2.3\end{array}$ & $\begin{array}{l}-3.5 \\
-3.9 \\
-3.8 \\
-3.6 \\
-3.5\end{array}$ & $\begin{array}{r}.4 \\
.4 \\
.3 \\
.2 \\
\text { e. } 1\end{array}$ & $\begin{array}{l}\text { e. } 8 \\
\text { e. } 6 \\
\text { e. } 7 \\
\text { e. } 8 \\
\text { e. } 7\end{array}$ & $\begin{array}{l}2.8 \\
2.8 \\
2.8 \\
2.8 \\
2.8\end{array}$ & $\begin{array}{r}10.4 \\
9.7 \\
9.1 \\
9.0 \\
8.4\end{array}$ & $\begin{array}{l}14.0 \\
14.4 \\
14.7 \\
15.1 \\
15.6\end{array}$ & $\begin{array}{l}17.2 \\
16.6 \\
15.6 \\
15.7 \\
16.1\end{array}$ & $\begin{array}{l}18.1 \\
18.5 \\
18.9 \\
19.5 \\
18.9\end{array}$ & $\begin{array}{l}14.0 \\
13.6 \\
13.7 \\
13.7 \\
13.8\end{array}$ \\
\hline $\begin{array}{l}21 \\
22 \\
23 \\
24 \\
25\end{array}$ & $\begin{array}{l}\text { e2. } \\
\text { e2. } 8 \\
\text { e2. } \\
\text { e3.0 } \\
\text { e3.1 }\end{array}$ & $\begin{array}{l}1.7 \\
-.1 \\
-.5 \\
-.5 \\
-.7\end{array}$ & $\begin{array}{l}-2.3 \\
-2.4 \\
-2.0 \\
-2.5 \\
-2.7\end{array}$ & $\begin{array}{l}-3.6 \\
-3.4 \\
-2.8 \\
-2.5 \\
-2.0\end{array}$ & $\begin{array}{l}\text { e. } 0 \\
\text { e. } 0 \\
\text { e. } 0 \\
\text { e. } 0 \\
\text { e. } 0\end{array}$ & $\begin{array}{l}\text { e. } 7 \\
\text { e. } 5 \\
\text { e. } 5 \\
\text { e. } 5 \\
\text { e. } 8\end{array}$ & $\begin{array}{l}2.8 \\
2.8 \\
2.8 \\
2.8 \\
3.0\end{array}$ & $\begin{array}{l}7.1 \\
6.8 \\
7.4 \\
7.9 \\
7.2\end{array}$ & $\begin{array}{l}15.8 \\
15.5 \\
15.9 \\
16.6 \\
17.3\end{array}$ & $\begin{array}{l}15.7 \\
14.9 \\
16.0 \\
17.1 \\
17.6\end{array}$ & $\begin{array}{l}19.2 \\
19.7 \\
19.7 \\
18.8 \\
17.9\end{array}$ & $\begin{array}{l}14.3 \\
14.7 \\
14.6 \\
14.2 \\
14.3\end{array}$ \\
\hline $\begin{array}{l}26 \\
27 \\
28 \\
29 \\
30 \\
31\end{array}$ & $\begin{array}{l}\mathrm{e} 3.3 \\
\mathrm{e} 3.6 \\
\mathrm{e} 3.8 \\
\mathrm{e} 3.9 \\
\mathrm{e} 4.2 \\
\mathrm{e} 3.9\end{array}$ & $\begin{array}{r}-1.8 \\
-1.3 \\
-.5 \\
-.6 \\
-.7 \\
--.\end{array}$ & $\begin{array}{l}-2.7 \\
-2.4 \\
-2.7 \\
-3.0 \\
-3.5 \\
-3.7\end{array}$ & $\begin{array}{r}-1.7 \\
-1.4 \\
-1.0 \\
-1.0 \\
-.9 \\
-.9\end{array}$ & $\begin{array}{r}\text { e. } 0 \\
\text { e. } 0 \\
\text { e-.1 } \\
--- \\
--- \\
---\end{array}$ & $\begin{array}{l}\mathrm{e} 1.1 \\
\mathrm{e} 1.2 \\
\mathrm{e} 1.2 \\
\mathrm{e} 1.2 \\
\mathrm{e} 1.3 \\
\mathrm{e} 1.6\end{array}$ & $\begin{array}{l}3.4 \\
4.7 \\
5.8 \\
6.9 \\
6.8 \\
---\end{array}$ & $\begin{array}{l}5.8 \\
5.2 \\
4.8 \\
4.4 \\
4.2 \\
4.7\end{array}$ & $\begin{array}{r}16.7 \\
16.1 \\
15.9 \\
15.8 \\
\text { e15.9 } \\
\ldots\end{array}$ & $\begin{array}{l}18.7 \\
19.5 \\
19.7 \\
20.0 \\
20.3 \\
20.4\end{array}$ & $\begin{array}{l}17.7 \\
17.4 \\
16.9 \\
16.9 \\
16.6 \\
16.4\end{array}$ & $\begin{array}{r}14.0 \\
13.1 \\
12.4 \\
12.4 \\
12.5 \\
\ldots-\end{array}$ \\
\hline $\begin{array}{l}\text { MEAN } \\
\text { MAX } \\
\text { MIN } \\
\text { MED }\end{array}$ & $\begin{array}{l}4.0 \\
4.9 \\
2.5 \\
4.0\end{array}$ & $\begin{array}{r}.8 \\
3.1 \\
-1.8 \\
.8\end{array}$ & $\begin{array}{r}-2.2 \\
-.1 \\
-3.7 \\
-2.6\end{array}$ & $\begin{array}{r}-2.3 \\
-.9 \\
-3.9 \\
-2.2\end{array}$ & $\begin{array}{r}-.2 \\
.4 \\
-1.1 \\
.0\end{array}$ & $\begin{array}{r}.6 \\
1.6 \\
-.1 \\
.7\end{array}$ & $\begin{array}{l}3.2 \\
6.9 \\
2.0 \\
2.8\end{array}$ & $\begin{array}{r}8.0 \\
11.1 \\
4.2 \\
8.4\end{array}$ & $\begin{array}{r}14.0 \\
17.3 \\
7.1 \\
14.8\end{array}$ & $\begin{array}{l}17.6 \\
20.4 \\
14.9 \\
17.5\end{array}$ & $\begin{array}{l}18.9 \\
20.5 \\
16.4 \\
19.2\end{array}$ & $\begin{array}{l}14.3 \\
16.5 \\
12.4 \\
14.2\end{array}$ \\
\hline
\end{tabular}

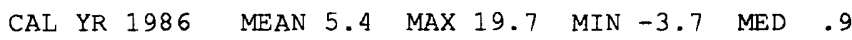
WTR YR 1987 MEAN 6.4 MAX 20.5 MIN -3.9 MED 3.1

e Estimated 
EAST STEWART BASIN

EAST STEWART CREEK NEAR IONE, NV

DAILY MEAN SOIL TEMPERATURE, WATER YEAR OCTOBER 1987 TO SEPTEMBER 1988 DEGREES CELSIUS

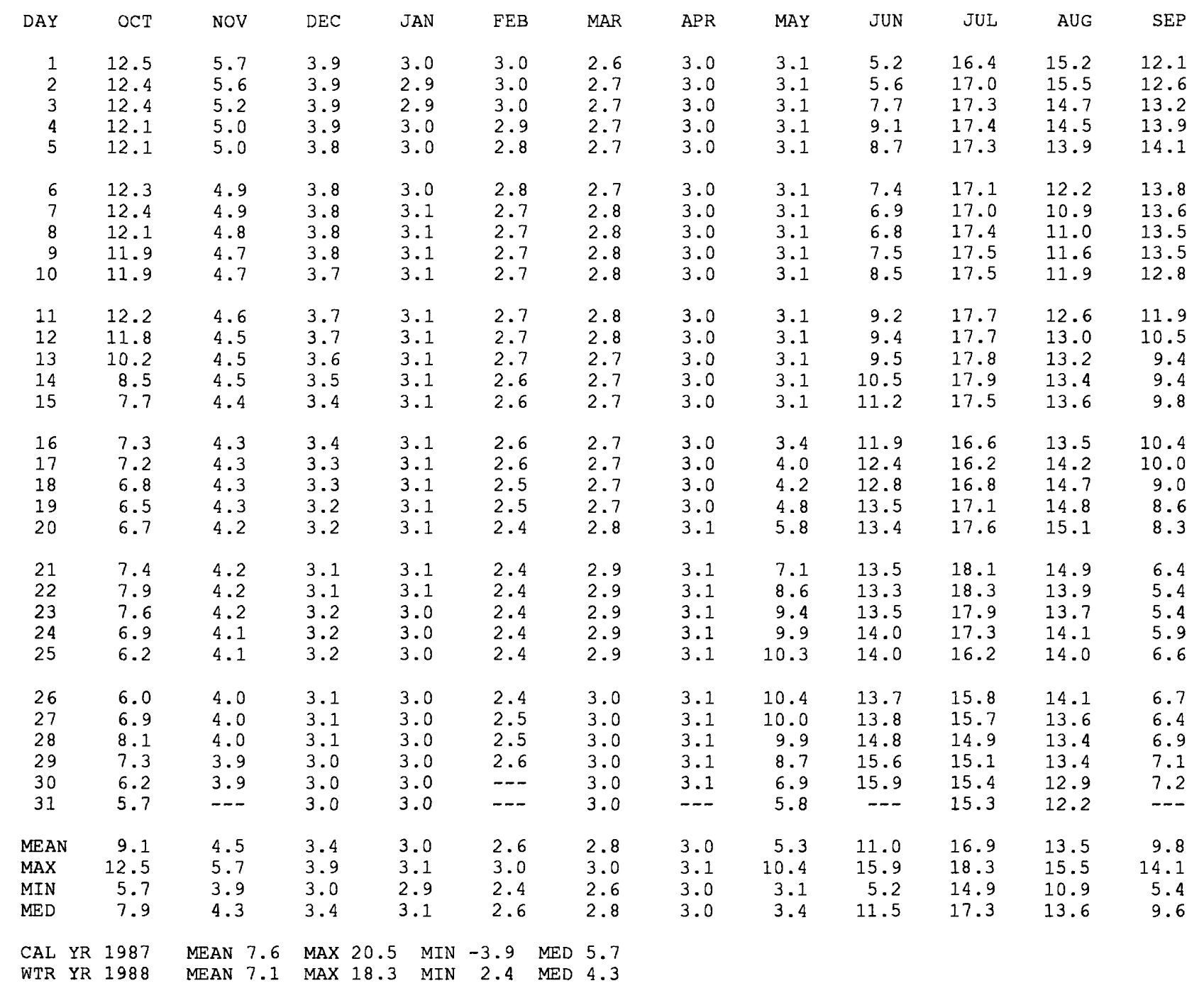


EAST STEWART BASIN

EAST STEWART CREEK NEAR IONE, NV

DAILY MEAN SOIL TEMPERATURE, WATER YEAR OCTOBER 1988 TO SEPTEMBER 1989 DEGREES CELSIUS

\begin{tabular}{|c|c|c|c|c|c|c|c|c|c|c|c|c|}
\hline DAY & OCT & NOV & DEC & JAN & FEB & MAR & $\mathrm{APR}$ & MAY & JUN & JUL & AUG & SEP \\
\hline $\begin{array}{l}1 \\
2 \\
3 \\
4 \\
5\end{array}$ & $\begin{array}{l}7.5 \\
7.7 \\
8.5 \\
8.2 \\
8.1\end{array}$ & $\begin{array}{l}2.9 \\
2.4 \\
3.5 \\
2.2 \\
1.7\end{array}$ & $\begin{array}{r}.3 \\
.2 \\
.0 \\
-.1 \\
-.4\end{array}$ & $\begin{array}{l}-.7 \\
-.7 \\
-.7 \\
-.7 \\
-.7\end{array}$ & $\begin{array}{l}-.8 \\
-.8 \\
-.8 \\
-.9 \\
-.9\end{array}$ & $\begin{array}{l}-.5 \\
-.6 \\
-.6 \\
-.6 \\
-.6\end{array}$ & $\begin{array}{l}-.2 \\
-.2 \\
-.2 \\
-.2 \\
-.2\end{array}$ & $\begin{array}{l}4.2 \\
5.8 \\
6.8 \\
8.2 \\
9.8\end{array}$ & $\begin{array}{l}6.7 \\
8.9 \\
9.6 \\
7.7 \\
7.4\end{array}$ & $\begin{array}{l}15.8 \\
16.5 \\
17.3 \\
17.7 \\
17.4\end{array}$ & $\begin{array}{l}19.9 \\
19.1 \\
19.0 \\
19.3 \\
19.0\end{array}$ & $\begin{array}{l}\mathrm{e} 15.4 \\
\mathrm{e} 15.2 \\
\mathrm{e} 15.7 \\
\mathrm{e} 16.1 \\
\mathrm{e} 16.2\end{array}$ \\
\hline $\begin{array}{r}6 \\
7 \\
8 \\
9 \\
10\end{array}$ & $\begin{array}{l}7.6 \\
7.2 \\
6.6 \\
6.0 \\
5.6\end{array}$ & $\begin{array}{r}2.3 \\
1.4 \\
.9 \\
.6 \\
.6\end{array}$ & $\begin{array}{l}-.6 \\
-.8 \\
-.9 \\
-.9 \\
-.9\end{array}$ & $\begin{array}{l}-.7 \\
-.7 \\
-.6 \\
-.6 \\
-.6\end{array}$ & $\begin{array}{r}-.9 \\
-1.0 \\
-1.1 \\
-1.2 \\
-1.2\end{array}$ & $\begin{array}{l}-.4 \\
-.2 \\
-.2 \\
-.2 \\
-.2\end{array}$ & $\begin{array}{l}-.2 \\
-.2 \\
-.2 \\
-.2 \\
-.2\end{array}$ & $\begin{array}{l}9.8 \\
9.8 \\
9.3 \\
8.5 \\
5.7\end{array}$ & $\begin{array}{r}8.4 \\
10.0 \\
10.0 \\
8.4 \\
7.7\end{array}$ & $\begin{array}{l}17.6 \\
18.1 \\
18.0 \\
17.4 \\
16.6\end{array}$ & $\begin{array}{l}19.1 \\
18.5 \\
16.5 \\
14.9 \\
15.2\end{array}$ & $\begin{array}{l}\mathrm{e} 15.3 \\
\mathrm{e} 13.8 \\
\mathrm{e} 13.3 \\
\mathrm{e} 13.9 \\
\mathrm{e} 13.3\end{array}$ \\
\hline $\begin{array}{l}11 \\
12 \\
13 \\
14 \\
15\end{array}$ & $\begin{array}{l}4.6 \\
3.2 \\
2.9 \\
3.3 \\
3.0\end{array}$ & $\begin{array}{l}.4 \\
.3 \\
.4 \\
.5 \\
.4\end{array}$ & $\begin{array}{l}-1.1 \\
-1.2 \\
-1.2 \\
-1.4 \\
-1.7\end{array}$ & $\begin{array}{l}-.6 \\
-.6 \\
-.6 \\
-.6 \\
-.6\end{array}$ & $\begin{array}{l}-1.2 \\
-1.3 \\
-1.3 \\
-1.3 \\
-1.2\end{array}$ & $\begin{array}{l}-.2 \\
-.2 \\
-.2 \\
-.2 \\
-.2\end{array}$ & $\begin{array}{l}-.2 \\
-.1 \\
-.1 \\
-.1 \\
-.1\end{array}$ & $\begin{array}{l}3.9 \\
2.7 \\
2.5 \\
3.0 \\
3.1\end{array}$ & $\begin{array}{r}8.6 \\
9.4 \\
11.9 \\
13.4 \\
14.5\end{array}$ & $\begin{array}{l}16.9 \\
16.4 \\
15.4 \\
16.2 \\
17.5\end{array}$ & $\begin{array}{l}16.2 \\
18.3 \\
17.9 \\
17.8 \\
17.9\end{array}$ & $\begin{array}{r}\mathrm{e} 12.7 \\
\mathrm{e} 11.8 \\
\mathrm{e} 11.2 \\
\mathrm{e} 12.9 \\
13.1\end{array}$ \\
\hline $\begin{array}{l}16 \\
17 \\
18 \\
19 \\
20\end{array}$ & $\begin{array}{l}3.7 \\
3.8 \\
3.6 \\
3.6 \\
3.1\end{array}$ & $\begin{array}{l}.4 \\
.3 \\
.3 \\
.3 \\
.3\end{array}$ & $\begin{array}{r}-1.6 \\
-1.3 \\
-1.1 \\
-1.0 \\
-.9\end{array}$ & $\begin{array}{l}-.6 \\
-.6 \\
-.7 \\
-.7 \\
-.7\end{array}$ & $\begin{array}{l}-1.2 \\
-1.1 \\
-1.1 \\
-1.0 \\
-1.0\end{array}$ & $\begin{array}{l}-.2 \\
-.2 \\
-.2 \\
-.2 \\
-.2\end{array}$ & $\begin{array}{r}-.1 \\
-.1 \\
1.9 \\
4.4 \\
6.1\end{array}$ & $\begin{array}{l}4.5 \\
6.8 \\
8.5 \\
8.4 \\
9.6\end{array}$ & $\begin{array}{l}14.6 \\
14.6 \\
15.6 \\
16.1 \\
15.5\end{array}$ & $\begin{array}{l}17.3 \\
17.8 \\
18.2 \\
18.7 \\
19.5\end{array}$ & $\begin{array}{l}17.8 \\
17.8 \\
17.0 \\
15.9 \\
14.1\end{array}$ & $\begin{array}{r}13.4 \\
10.3 \\
5.4 \\
5.8 \\
7.6\end{array}$ \\
\hline $\begin{array}{l}21 \\
22 \\
23 \\
24 \\
25\end{array}$ & $\begin{array}{l}2.9 \\
2.8 \\
2.5 \\
2.2 \\
2.0\end{array}$ & $\begin{array}{r}.3 \\
.3 \\
.3 \\
.3 \\
.3\end{array}$ & $\begin{array}{l}-.9 \\
-.8 \\
-.8 \\
-.8 \\
-.8\end{array}$ & $\begin{array}{l}-.7 \\
-.7 \\
-.7 \\
-.7 \\
-.7\end{array}$ & $\begin{array}{l}-.9 \\
-.9 \\
-.8 \\
-.8 \\
-.7\end{array}$ & $\begin{array}{l}-.2 \\
-.2 \\
-.2 \\
-.2 \\
-.2\end{array}$ & $\begin{array}{l}6.8 \\
5.6 \\
5.0 \\
3.0 \\
1.7\end{array}$ & $\begin{array}{r}9.9 \\
10.2 \\
10.0 \\
8.6 \\
8.4\end{array}$ & $\begin{array}{l}14.7 \\
14.8 \\
15.1 \\
14.1 \\
14.0\end{array}$ & $\begin{array}{l}18.6 \\
17.5 \\
16.0 \\
15.8 \\
16.4\end{array}$ & $\begin{array}{l}12.8 \\
14.0 \\
13.6 \\
12.2 \\
12.5\end{array}$ & $\begin{array}{r}8.3 \\
8.6 \\
9.2 \\
10.2 \\
10.5\end{array}$ \\
\hline $\begin{array}{l}26 \\
27 \\
28 \\
29 \\
30 \\
31\end{array}$ & $\begin{array}{l}2.1 \\
2.1 \\
2.2 \\
2.3 \\
1.6 \\
1.8\end{array}$ & $\begin{array}{r}.3 \\
.4 \\
.4 \\
.4 \\
.4 \\
----\end{array}$ & $\begin{array}{l}-.7 \\
-.7 \\
-.7 \\
-.7 \\
-.7 \\
-.7\end{array}$ & $\begin{array}{l}-.8 \\
-.8 \\
-.8 \\
-.9 \\
-.9 \\
-.9\end{array}$ & $\begin{array}{l}-.6 \\
-.5 \\
-.5 \\
--- \\
--- \\
---\end{array}$ & $\begin{array}{l}-.2 \\
-.2 \\
-.2 \\
-.2 \\
-.2 \\
-.2\end{array}$ & $\begin{array}{r}1.0 \\
.6 \\
.4 \\
.3 \\
1.3 \\
--.-\end{array}$ & $\begin{array}{r}9.3 \\
10.9 \\
10.8 \\
7.6 \\
5.8 \\
5.8\end{array}$ & $\begin{array}{r}14.5 \\
13.8 \\
13.9 \\
15.2 \\
15.7 \\
---\end{array}$ & $\begin{array}{l}18.7 \\
19.4 \\
19.5 \\
19.4 \\
20.0 \\
20.4\end{array}$ & $\begin{array}{r}11.2 \\
\mathrm{e} 15.1 \\
\mathrm{e} 15.7 \\
\mathrm{e} 16.3 \\
\mathrm{e} 16.0 \\
\mathrm{e} 15.5\end{array}$ & $\begin{array}{r}10.5 \\
10.8 \\
10.5 \\
9.8 \\
8.9 \\
---\end{array}$ \\
\hline $\begin{array}{l}\text { MEAN } \\
\text { MAX } \\
\text { MIN } \\
\text { MED }\end{array}$ & $\begin{array}{l}4.3 \\
8.5 \\
1.6 \\
3.3\end{array}$ & $\begin{array}{r}.8 \\
3.5 \\
.3 \\
.4\end{array}$ & $\begin{array}{r}-.8 \\
-1.3 \\
-1.7\end{array}$ & $\begin{array}{l}-.7 \\
-.6 \\
-.9 \\
-.7\end{array}$ & $\begin{array}{r}-1.0 \\
-.5 \\
-1.3 \\
-1.0\end{array}$ & $\begin{array}{l}-.3 \\
-.2 \\
-.6 \\
-.2\end{array}$ & $\begin{array}{r}1.2 \\
6.8 \\
-.2 \\
-.1\end{array}$ & $\begin{array}{r}7.4 \\
10.9 \\
2.5 \\
8.4\end{array}$ & $\begin{array}{r}12.2 \\
16.1 \\
6.7 \\
13.8\end{array}$ & $\begin{array}{l}17.7 \\
20.4 \\
15.4 \\
17.5\end{array}$ & $\begin{array}{l}16.3 \\
19.9 \\
11.2 \\
16.3\end{array}$ & $\begin{array}{r}11.7 \\
16.2 \\
5.4 \\
11.5\end{array}$ \\
\hline
\end{tabular}

CAL YR 1988 MEAN 6.1 MAX 18.3 MIN -1.7 MED 3.1 WTR YR 1989 MEAN 5.8 MAX 20.4 MIN -1.7 MED 2.5

e Estimated 
EAST STEWART BASIN

EAST STEWART CREEK NEAR IONE, NV

DAILY MEAN SOII TEMPERATURE, WATER YEAR OCTOBER 1989 TO SEPTEMBER 1990 DEGREES CEISIUS

\begin{tabular}{|c|c|c|c|c|c|c|c|c|c|c|c|c|}
\hline DAY & OCT & NOV & DEC & JAN & FEB & MAR & $\mathrm{APR}$ & MAY & JUN & JUI & AUG & SEP \\
\hline $\begin{array}{l}1 \\
2 \\
3 \\
4 \\
5\end{array}$ & $\begin{array}{l}7.0 \\
6.3 \\
5.0 \\
3.8 \\
3.2\end{array}$ & $\begin{array}{l}.4 \\
.2 \\
.1 \\
.1 \\
.1\end{array}$ & $\begin{array}{l}-3.0 \\
-3.2 \\
-3.4 \\
-3.4 \\
-3.3\end{array}$ & $\begin{array}{l}-4.2 \\
-3.9 \\
-3.7 \\
-3.6 \\
-3.6\end{array}$ & $\begin{array}{l}-3.3 \\
-3.2 \\
-3.1 \\
-3.1 \\
-3.1\end{array}$ & $\begin{array}{l}-2.3 \\
-2.2 \\
-2.1 \\
-1.9 \\
-1.9\end{array}$ & $\begin{array}{l}.0 \\
.0 \\
.0 \\
.0 \\
.0\end{array}$ & $\begin{array}{l}3.1 \\
3.6 \\
4.9 \\
7.0 \\
9.0\end{array}$ & $\begin{array}{r}4.7 \\
8.4 \\
10.9 \\
12.5 \\
13.0\end{array}$ & $\begin{array}{l}19.3 \\
18.9 \\
18.3 \\
19.2 \\
19.9\end{array}$ & $\begin{array}{l}17.8 \\
17.2 \\
17.7 \\
17.9 \\
18.6\end{array}$ & $\begin{array}{l}16.2 \\
16.9 \\
17.3 \\
17.5 \\
17.2\end{array}$ \\
\hline $\begin{array}{r}6 \\
7 \\
8 \\
9 \\
10\end{array}$ & $\begin{array}{l}3.1 \\
3.2 \\
3.3 \\
3.8 \\
4.6\end{array}$ & $\begin{array}{r}.0 \\
-. .2 \\
-.6 \\
-.8 \\
-.8\end{array}$ & $\begin{array}{l}-2.7 \\
-3.0 \\
-3.0 \\
-2.7 \\
-2.9\end{array}$ & $\begin{array}{l}-3.6 \\
-3.6 \\
-3.3 \\
-3.0 \\
-2.9\end{array}$ & $\begin{array}{l}-3.2 \\
-3.0 \\
-3.0 \\
-3.0 \\
-2.9\end{array}$ & $\begin{array}{l}-1.8 \\
-1.8 \\
-1.8 \\
-1.7 \\
-1.7\end{array}$ & $\begin{array}{l}.0 \\
.0 \\
.0 \\
.0 \\
.0\end{array}$ & $\begin{array}{l}9.8 \\
9.1 \\
8.9 \\
8.7 \\
7.5\end{array}$ & $\begin{array}{l}13.0 \\
13.3 \\
14.5 \\
14.7 \\
13.1\end{array}$ & $\begin{array}{l}20.2 \\
20.5 \\
20.9 \\
21.0 \\
20.2\end{array}$ & $\begin{array}{l}20.1 \\
19.6 \\
17.1 \\
16.7 \\
17.8\end{array}$ & $\begin{array}{l}16.9 \\
16.7 \\
16.7 \\
16.9 \\
17.1\end{array}$ \\
\hline $\begin{array}{l}11 \\
12 \\
13 \\
14 \\
15\end{array}$ & $\begin{array}{l}4.7 \\
4.9 \\
5.1 \\
5.4 \\
4.3\end{array}$ & $\begin{array}{r}-.8 \\
-.6 \\
-.8 \\
-1.4 \\
-2.0\end{array}$ & $\begin{array}{l}-3.8 \\
-4.3 \\
-4.3 \\
-4.5 \\
-4.5\end{array}$ & $\begin{array}{l}-2.8 \\
-2.7 \\
-2.5 \\
-2.4 \\
-2.5\end{array}$ & $\begin{array}{l}-2.7 \\
-2.6 \\
-2.5 \\
-2.7 \\
-3.1\end{array}$ & $\begin{array}{l}-1.6 \\
-1.5 \\
-1.5 \\
-1.5 \\
-1.5\end{array}$ & $\begin{array}{l}.0 \\
.0 \\
.0 \\
.0 \\
.0\end{array}$ & $\begin{array}{l}6.1 \\
7.0 \\
8.6 \\
9.0 \\
7.8\end{array}$ & $\begin{array}{l}12.1 \\
12.7 \\
12.3 \\
11.1 \\
10.0\end{array}$ & $\begin{array}{l}18.6 \\
18.4 \\
17.9 \\
17.3 \\
16.9\end{array}$ & $\begin{array}{l}17.6 \\
16.6 \\
18.0 \\
18.4 \\
15.0\end{array}$ & $\begin{array}{l}17.6 \\
17.3 \\
17.1 \\
17.4 \\
16.8\end{array}$ \\
\hline $\begin{array}{l}16 \\
17 \\
18 \\
19 \\
20\end{array}$ & $\begin{array}{l}3.4 \\
3.0 \\
3.1 \\
3.7 \\
4.1\end{array}$ & $\begin{array}{l}-2.2 \\
-2.2 \\
-2.7 \\
-3.0 \\
-2.9\end{array}$ & $\begin{array}{l}-4.5 \\
-4.8 \\
-4.9 \\
-5.3 \\
-5.6\end{array}$ & $\begin{array}{l}-2.6 \\
-2.6 \\
-2.6 \\
-2.6 \\
-2.8\end{array}$ & $\begin{array}{l}-3.4 \\
-3.5 \\
-3.4 \\
-3.3 \\
-3.1\end{array}$ & $\begin{array}{r}-1.5 \\
-1.5 \\
-1.3 \\
.0 \\
.0\end{array}$ & $\begin{array}{r}.0 \\
.0 \\
.0 \\
.1 \\
.1\end{array}$ & $\begin{array}{l}8.2 \\
9.4 \\
9.5 \\
8.5 \\
8.6\end{array}$ & $\begin{array}{r}9.9 \\
12.1 \\
12.7 \\
13.2 \\
14.8\end{array}$ & $\begin{array}{l}16.7 \\
16.8 \\
17.0 \\
16.7 \\
17.3\end{array}$ & $\begin{array}{l}13.4 \\
15.4 \\
14.2 \\
10.7 \\
10.4\end{array}$ & $\begin{array}{l}15.3 \\
14.3 \\
13.6 \\
11.5 \\
11.5\end{array}$ \\
\hline $\begin{array}{l}21 \\
22 \\
23 \\
24 \\
25\end{array}$ & $\begin{array}{l}4.8 \\
4.2 \\
4.3 \\
4.1 \\
3.5\end{array}$ & $\begin{array}{l}-3.1 \\
-3.4 \\
-3.3 \\
-2.3 \\
-2.0\end{array}$ & $\begin{array}{l}-5.6 \\
-5.5 \\
-5.3 \\
-5.2 \\
-5.2\end{array}$ & $\begin{array}{l}-2.9 \\
-3.0 \\
-3.1 \\
-3.2 \\
-3.3\end{array}$ & $\begin{array}{l}-3.0 \\
-2.9 \\
-2.8 \\
-2.7 \\
-2.6\end{array}$ & $\begin{array}{l}.0 \\
.0 \\
.0 \\
.0 \\
.0\end{array}$ & $\begin{array}{l}.1 \\
.1 \\
.1 \\
.1 \\
.1\end{array}$ & $\begin{array}{r}10.1 \\
11.3 \\
10.3 \\
6.9 \\
6.1\end{array}$ & $\begin{array}{l}16.2 \\
17.2 \\
17.5 \\
18.0 \\
18.2\end{array}$ & $\begin{array}{l}18.7 \\
19.2 \\
19.7 \\
19.4 \\
18.9\end{array}$ & $\begin{array}{l}11.6 \\
12.4 \\
12.9 \\
12.9 \\
13.2\end{array}$ & $\begin{array}{r}12.0 \\
12.1 \\
11.1 \\
11.0 \\
9.8\end{array}$ \\
\hline $\begin{array}{l}26 \\
27 \\
28 \\
29 \\
30 \\
31\end{array}$ & $\begin{array}{r}2.6 \\
2.1 \\
1.8 \\
1.3 \\
.9 \\
.6\end{array}$ & $\begin{array}{r}-1.8 \\
-1.9 \\
-2.2 \\
-2.5 \\
-2.7 \\
---\end{array}$ & $\begin{array}{l}-5.3 \\
-5.2 \\
-4.8 \\
-4.4 \\
-4.3 \\
-4.3\end{array}$ & $\begin{array}{l}-3.2 \\
-3.3 \\
-3.6 \\
-3.6 \\
-3.5 \\
-3.4\end{array}$ & $\begin{array}{l}-2.5 \\
-2.4 \\
-2.3 \\
--- \\
--- \\
---\end{array}$ & $\begin{array}{l}.0 \\
.0 \\
.0 \\
.0 \\
.0 \\
.0\end{array}$ & $\begin{array}{r}.1 \\
.1 \\
.7 \\
1.6 \\
2.8 \\
----\end{array}$ & $\begin{array}{l}7.7 \\
7.7 \\
5.4 \\
3.4 \\
2.5 \\
2.3\end{array}$ & $\begin{array}{r}18.1 \\
17.8 \\
18.0 \\
18.8 \\
19.1 \\
-\ldots\end{array}$ & $\begin{array}{l}18.6 \\
18.9 \\
18.7 \\
19.0 \\
20.0 \\
19.6\end{array}$ & $\begin{array}{l}14.0 \\
15.0 \\
15.2 \\
16.2 \\
16.3 \\
16.4\end{array}$ & $\begin{array}{l}9.7 \\
9.3 \\
8.0 \\
8.3 \\
8.2 \\
---\end{array}$ \\
\hline $\begin{array}{l}\text { MEAN } \\
\text { MAX } \\
\text { MIN } \\
\text { MED }\end{array}$ & $\begin{array}{r}3.7 \\
7.0 \\
.6 \\
3.8\end{array}$ & $\begin{array}{r}-1.5 \\
.4 \\
-3.4 \\
-1.9\end{array}$ & $\begin{array}{l}-4.3 \\
-2.7 \\
-5.6 \\
-4.4\end{array}$ & $\begin{array}{l}-3.1 \\
-2.4 \\
-4.2 \\
-3.2\end{array}$ & $\begin{array}{l}-2.9 \\
-2.3 \\
-3.5 \\
-3.0\end{array}$ & $\begin{array}{r}-1.0 \\
.0 \\
-2.3 \\
-1.5\end{array}$ & $\begin{array}{r}.2 \\
2.8 \\
.0 \\
.0\end{array}$ & $\begin{array}{r}7.4 \\
11.3 \\
2.3 \\
7.8\end{array}$ & $\begin{array}{r}13.9 \\
19.1 \\
4.7 \\
13.1\end{array}$ & $\begin{array}{l}18.8 \\
21.0 \\
16.7 \\
18.9\end{array}$ & $\begin{array}{l}15.7 \\
20.1 \\
10.4 \\
16.3\end{array}$ & $\begin{array}{r}14.0 \\
17.6 \\
8.0 \\
15.7\end{array}$ \\
\hline
\end{tabular}

CAL YR 1989 MEAN 5.2 MAX 20.4 MIN -5.6 MED 2.7 WTR YR 1990 MEAN 5.1 MAX 21.0 MIN -5.6 MED 1.6 
EAST STEWART BASIN

EAST STEWART CREEK NEAR IONE, NV

DAILY MEAN SOIL TEMPERATURE, WATER YEAR OCTOBER 1990 TO SEPTEMBER 1991 DEGREES CELSIUS

\begin{tabular}{|c|c|c|c|c|c|c|c|c|c|c|c|}
\hline DAY & OCT & NoV & DEC & JAN & FEB & MAR & APR & MAY & JUN & JUL & AUG \\
\hline $\begin{array}{l}1 \\
2 \\
3 \\
4 \\
5\end{array}$ & $\begin{array}{l}8.3 \\
8.1 \\
7.3 \\
7.6 \\
8.0\end{array}$ & $\begin{array}{r}1.3 \\
1.3 \\
1.1 \\
.9 \\
.9\end{array}$ & $\begin{array}{r}-.8 \\
-1.2 \\
-1.4 \\
-1.4 \\
-1.1\end{array}$ & $\begin{array}{l}-2.8 \\
-2.9 \\
-2.9 \\
-2.5 \\
-2.2\end{array}$ & $\begin{array}{l}-3.5 \\
-3.3 \\
-3.0 \\
-2.9 \\
-2.4\end{array}$ & $\begin{array}{r}-1.2 \\
-1.1 \\
-1.1 \\
-1.0 \\
-.9\end{array}$ & $\begin{array}{l}.8 \\
.9 \\
.9 \\
.9 \\
.9\end{array}$ & $\begin{array}{l}.9 \\
.9 \\
.9 \\
.9 \\
.9\end{array}$ & $\begin{array}{l}.9 \\
.9 \\
.9 \\
.9 \\
.9\end{array}$ & $\begin{array}{l}14.6 \\
16.4 \\
17.7 \\
18.4 \\
18.7\end{array}$ & $\begin{array}{l}17.9 \\
17.7 \\
18.2 \\
19.1 \\
18.9\end{array}$ \\
\hline $\begin{array}{r}6 \\
7 \\
8 \\
9 \\
10\end{array}$ & $\begin{array}{l}7.3 \\
6.6 \\
3.7 \\
3.1 \\
3.1\end{array}$ & $\begin{array}{r}.7 \\
.3 \\
.6 \\
.1 \\
-1.3\end{array}$ & $\begin{array}{l}-1.6 \\
-1.8 \\
-2.0 \\
-2.0 \\
-1.8\end{array}$ & $\begin{array}{l}-2.4 \\
-2.4 \\
-2.2 \\
-2.4 \\
-2.5\end{array}$ & $\begin{array}{l}-2.0 \\
-2.0 \\
-2.1 \\
-2.2 \\
-2.3\end{array}$ & $\begin{array}{r}-.9 \\
-1.0 \\
-1.2 \\
-1.4 \\
-1.4\end{array}$ & $\begin{array}{l}.9 \\
.9 \\
.9 \\
.9 \\
.9\end{array}$ & $\begin{array}{l}.9 \\
.9 \\
.9 \\
.9 \\
.9\end{array}$ & $\begin{array}{r}.9 \\
2.2 \\
4.3 \\
8.1 \\
10.9\end{array}$ & $\begin{array}{l}18.8 \\
18.5 \\
17.3 \\
16.5 \\
17.1\end{array}$ & $\begin{array}{l}19.6 \\
20.2 \\
20.1 \\
20.7 \\
20.7\end{array}$ \\
\hline $\begin{array}{l}11 \\
12 \\
13 \\
14 \\
15\end{array}$ & $\begin{array}{l}3.0 \\
2.6 \\
2.9 \\
3.1 \\
3.7\end{array}$ & $\begin{array}{r}-1.4 \\
-1.1 \\
-.8 \\
.3 \\
.5\end{array}$ & $\begin{array}{r}-1.3 \\
-.9 \\
-.9 \\
-.9 \\
-1.1\end{array}$ & $\begin{array}{l}-2.4 \\
-2.1 \\
-1.8 \\
-1.6 \\
-1.5\end{array}$ & $\begin{array}{l}-2.3 \\
-2.2 \\
-2.0 \\
-1.8 \\
-1.5\end{array}$ & $\begin{array}{l}-1.4 \\
-1.4 \\
-1.3 \\
-1.2 \\
-1.1\end{array}$ & $\begin{array}{l}.9 \\
.9 \\
.9 \\
.9 \\
.9\end{array}$ & $\begin{array}{l}.9 \\
.9 \\
.9 \\
.9 \\
.9\end{array}$ & $\begin{array}{l}12.5 \\
13.6 \\
14.2 \\
13.8 \\
13.8\end{array}$ & $\begin{array}{l}17.8 \\
19.1 \\
20.2 \\
20.6 \\
20.4\end{array}$ & $\begin{array}{l}19.8 \\
18.8 \\
18.2 \\
18.5 \\
16.8\end{array}$ \\
\hline $\begin{array}{l}16 \\
17 \\
18 \\
19 \\
20\end{array}$ & $\begin{array}{l}4.1 \\
3.0 \\
4.5 \\
3.9 \\
3.0\end{array}$ & $\begin{array}{l}.2 \\
.6 \\
.4 \\
.4 \\
.4\end{array}$ & $\begin{array}{r}-1.1 \\
-1.0 \\
-.9 \\
-1.0 \\
-1.1\end{array}$ & $\begin{array}{l}-1.4 \\
-1.4 \\
-1.4 \\
-1.5 \\
-1.6\end{array}$ & $\begin{array}{r}-1.1 \\
-.9 \\
-.9 \\
-1.0 \\
-1.0\end{array}$ & $\begin{array}{r}-1.0 \\
-.9 \\
-.9 \\
-.8 \\
-.7\end{array}$ & $\begin{array}{l}.9 \\
.9 \\
.9 \\
.9 \\
.9\end{array}$ & $\begin{array}{l}.9 \\
.9 \\
.9 \\
.9 \\
.9\end{array}$ & $\begin{array}{l}14.3 \\
14.2 \\
14.2 \\
13.8 \\
13.0\end{array}$ & $\begin{array}{l}20.9 \\
21.1 \\
21.2 \\
20.9 \\
19.7\end{array}$ & $\begin{array}{l}15.2 \\
15.8 \\
17.2 \\
17.6 \\
18.2\end{array}$ \\
\hline $\begin{array}{l}21 \\
22 \\
23 \\
24 \\
25\end{array}$ & $\begin{array}{l}2.4 \\
2.1 \\
1.9 \\
1.7 \\
1.7\end{array}$ & $\begin{array}{r}.1 \\
-.3 \\
-.6 \\
-1.0 \\
-1.0\end{array}$ & $\begin{array}{l}-1.2 \\
-1.6 \\
-2.0 \\
-2.2 \\
-2.4\end{array}$ & $\begin{array}{l}-1.8 \\
-2.0 \\
-2.2 \\
-2.3 \\
-2.5\end{array}$ & $\begin{array}{l}-1.0 \\
-1.0 \\
-1.0 \\
-1.1 \\
-1.3\end{array}$ & $\begin{array}{l}-.7 \\
-.6 \\
-.6 \\
-.5 \\
-.5\end{array}$ & $\begin{array}{l}.9 \\
.9 \\
.9 \\
.9 \\
.9\end{array}$ & $\begin{array}{l}.9 \\
.9 \\
.9 \\
.9 \\
.9\end{array}$ & $\begin{array}{l}13.1 \\
13.8 \\
14.3 \\
14.2 \\
12.6\end{array}$ & $\begin{array}{l}18.0 \\
18.2 \\
20.0 \\
19.6 \\
19.6\end{array}$ & $\begin{array}{l}18.7 \\
18.7 \\
18.3 \\
18.1 \\
18.7\end{array}$ \\
\hline $\begin{array}{l}26 \\
27 \\
28 \\
29 \\
30 \\
31\end{array}$ & $\begin{array}{l}2.1 \\
2.1 \\
1.9 \\
1.7 \\
1.4 \\
1.3\end{array}$ & $\begin{array}{l}-.6 \\
-.5 \\
-.6 \\
-.6 \\
-.4 \\
-.--\end{array}$ & $\begin{array}{l}-2.3 \\
-2.2 \\
-2.3 \\
-2.4 \\
-2.6 \\
-2.8\end{array}$ & $\begin{array}{l}-2.7 \\
-2.9 \\
-3.1 \\
-3.2 \\
-3.7 \\
-3.8\end{array}$ & $\begin{array}{r}-1.5 \\
-1.5 \\
-1.4 \\
--- \\
--- \\
---\end{array}$ & $\begin{array}{l}-.4 \\
-.4 \\
-.4 \\
-.4 \\
-.4 \\
-.2\end{array}$ & $\begin{array}{r}.9 \\
.9 \\
.9 \\
.9 \\
.9 \\
---\end{array}$ & $\begin{array}{l}.9 \\
.9 \\
.9 \\
.9 \\
.9 \\
.9\end{array}$ & $\begin{array}{c}12.2 \\
11.3 \\
11.3 \\
10.3 \\
11.9 \\
-\ldots\end{array}$ & $\begin{array}{l}19.1 \\
18.2 \\
18.2 \\
19.6 \\
19.2 \\
17.9\end{array}$ & $\begin{array}{l}17.8 \\
15.3 \\
13.5 \\
14.9 \\
16.2 \\
16.7\end{array}$ \\
\hline $\begin{array}{l}\text { MEAN } \\
\text { MAX } \\
\text { MIN } \\
\text { MED }\end{array}$ & $\begin{array}{l}3.8 \\
8.3 \\
1.3 \\
3.0\end{array}$ & $\begin{array}{r}.0 \\
1.3 \\
-1.4 \\
.1\end{array}$ & $\begin{array}{r}-1.6 \\
-.8 \\
-2.8 \\
-1.4\end{array}$ & $\begin{array}{l}-2.3 \\
-1.4 \\
-3.8 \\
-2.4\end{array}$ & $\begin{array}{r}-1.8 \\
-.9 \\
-3.5 \\
-1.7\end{array}$ & $\begin{array}{r}-.9 \\
-. .2 \\
-1.4 \\
-.9\end{array}$ & $\begin{array}{l}.9 \\
.9 \\
.8 \\
.9\end{array}$ & $\begin{array}{l}.9 \\
.9 \\
.9 \\
.9\end{array}$ & $\begin{array}{r}9.8 \\
14.3 \\
.9 \\
12.4\end{array}$ & $\begin{array}{l}18.8 \\
21.2 \\
14.6 \\
18.8\end{array}$ & $\begin{array}{l}17.9 \\
20.7 \\
13.5 \\
18.2\end{array}$ \\
\hline
\end{tabular}

CAL YR 1990 MEAN 5.5 MAX 21.0 MIN -4.2 MED 1.7 WTR YR 1991 MEAN 4.7 MAX 21.2 MIN -3.8 MED .9 
EAST STEWART BASIN

EAST STEWART CREEK NEAR IONE, NV

DAILY TOTAL SOLAR RADIATION, INCIDENTAL, WATER YEAR OCTOBER 1985 TO SEPTEMBER 1986 CALORIES PER SQUARE CENTIMETER

\begin{tabular}{|c|c|c|c|c|c|c|c|c|c|c|c|c|}
\hline DAY & OCT & NOV & DEC & JAN & FEB & MAR & APR & MAY & JUN & JUL & AUG & SEP \\
\hline 1 & -- & --- & -- & e 173 & 294 & 303 & 395 & 382 & 396 & 769 & 724 & 574 \\
\hline 2 & --- & -- & -- & e 175 & 161 & 111 & 246 & 587 & 450 & 643 & 701 & 575 \\
\hline 3 & --- & --- & -- & el 178 & 196 & 379 & 615 & 513 & 531 & 597 & 719 & 574 \\
\hline 4 & -- & --- & -- & e 180 & 190 & 212 & 497 & 245 & 601 & 757 & 695 & 578 \\
\hline 5 & -- & -- & --- & e 183 & 108 & 444 & 390 & 416 & 741 & 766 & 471 & 418 \\
\hline 6 & -- & -- & $-\infty$ & e186 & 196 & 368 & 440 & 445 & 687 & 712 & 626 & 495 \\
\hline 7 & -- & -- & --- & e 188 & 298 & 265 & 292 & 368 & 505 & 495 & 691 & 469 \\
\hline 8 & --- & --- & $-\sim$ & el91 & 280 & 89 & 111 & 414 & 765 & 732 & 649 & 393 \\
\hline 9 & --- & --- & -- & e 193 & 325 & 413 & 456 & 696 & 777 & e 781 & 422 & 486 \\
\hline 10 & -- & -- & --- & e196 & 321 & 345 & 384 & 498 & 776 & e779 & 285 & 537 \\
\hline 11 & -- & -- & --- & e198 & 245 & 278 & 631 & 708 & 738 & e 778 & 597 & 515 \\
\hline 12 & -- & --- & --- & 189 & 151 & 270 & 288 & 713 & 301 & e 777 & 663 & 468 \\
\hline 13 & -- & --- & -- & 171 & 264 & 228 & 575 & 572 & 445 & e 775 & 605 & 401 \\
\hline 14 & --- & --- & --- & 159 & 221 & 404 & 498 & 519 & 668 & e 774 & 621 & 383 \\
\hline 15 & --- & --- & -- & 174 & 153 & 292 & 427 & 674 & 770 & e 773 & 648 & 509 \\
\hline 16 & --- & --- & --- & 139 & 214 & 347 & 297 & 717 & 773 & e 772 & 636 & 469 \\
\hline 17 & --- & --- & -- & 104 & 165 & 272 & 324 & 710 & 780 & e 770 & 667 & 231 \\
\hline 18 & -- & -- & --- & 217 & 250 & 510 & 644 & 719 & 753 & e 768 & 376 & 405 \\
\hline 19 & -- & -- & -- & 171 & 229 & 520 & 648 & 634 & 777 & e766 & 211 & 454 \\
\hline 20 & --- & -- & -- & 123 & 211 & 527 & 656 & 519 & 779 & e764 & 210 & 474 \\
\hline 21 & --- & -- & -- & 158 & 370 & 493 & 472 & 587 & 792 & e761 & 451 & 474 \\
\hline 22 & --- & --- & --- & 166 & 158 & 499 & 440 & 715 & 717 & e759 & 531 & 358 \\
\hline 23 & -- & --- & --- & 175 & 334 & 384 & 295 & 541 & 683 & e 757 & 628 & 203 \\
\hline 24 & --- & --- & --- & 228 & 399 & 437 & 523 & 717 & 561 & e754 & 612 & 273 \\
\hline 25 & --- & --- & --- & 236 & 410 & 538 & 311 & 722 & 774 & e 752 & 301 & 233 \\
\hline 26 & --- & -- & -- & 250 & 403 & 547 & 614 & 706 & 728 & e749 & 456 & 391 \\
\hline 27 & -- & --- & --- & 243 & 401 & 556 & 612 & 688 & 697 & e 746 & 234 & 275 \\
\hline 28 & --- & --- & --- & 239 & 415 & 441 & 520 & 372 & 590 & e 743 & 491 & 460 \\
\hline 29 & --- & --- & --- & 223 & --- & 462 & 687 & 647 & 779 & e 740 & 562 & 404 \\
\hline 30 & -- & -- & -- & 163 & -- & 340 & 637 & 728 & 773 & e 737 & 594 & 348 \\
\hline 31 & -- & --- & --- & 129 & -- & 352 & --- & 636 & -- & e734 & 559 & -- \\
\hline TOTAL & --- & --- & --- & 5698 & 7362 & 11626 & 13925 & 18108 & 20107 & 22980 & 16636 & 12827 \\
\hline
\end{tabular}

e Estimated 
EAST STEWART BASIN

EAST STEWART CREEK NEAR IONE, NV

DAILY TOTAL SOLAR RADIATION, INCIDENTAL, WATER YEAR OCTOBER 1986 TO SEPTEMBER 1987 CALORIES PER SQUARE CENTIMETER

\begin{tabular}{|c|c|c|c|c|c|c|c|c|c|c|c|c|}
\hline DAY & OCT & NOV & $D E C$ & JAN & FEB & MAR & APR & MAY & JUN & JUL & AUG & SEP \\
\hline 1 & 151 & e291 & 193 & 95 & 243 & e 417 & 585 & 600 & 781 & e790 & 743 & 309 \\
\hline 2 & e 438 & e287 & 193 & 171 & 161 & e 423 & 586 & 612 & 772 & e789 & 731 & 335 \\
\hline 3 & e434 & $\mathrm{e} 282$ & 167 & 137 & 224 & e 428 & 167 & 703 & 759 & e 788 & 667 & 282 \\
\hline 4 & e 429 & e278 & 103 & 62 & 291 & e433 & 44 & 657 & 679 & e 787 & 341 & 527 \\
\hline 5 & e 425 & 284 & 114 & 16 & 295 & e 438 & 265 & 711 & 498 & 770 & 673 & 257 \\
\hline 6 & e 420 & 92 & 105 & 38 & 298 & e 443 & 606 & 490 & 251 & 773 & 648 & 250 \\
\hline 7 & e 416 & 128 & 137 & 38 & 208 & e 448 & 559 & 451 & 512 & 743 & 700 & 265 \\
\hline 8 & e 411 & 249 & 173 & 46 & 184 & e 453 & 525 & 452 & 346 & 730 & 557 & 326 \\
\hline 9 & e406 & 255 & 174 & 193 & 250 & e 459 & 606 & 216 & 505 & 553 & 287 & 505 \\
\hline 10 & e 401 & 255 & 171 & 198 & 227 & e 464 & 458 & 393 & 671 & 700 & 570 & 329 \\
\hline 11 & e395 & 249 & 177 & 201 & 80 & e 469 & 510 & 238 & 781 & 606 & 678 & 274 \\
\hline 12 & e390 & 250 & 169 & 205 & 155 & e 475 & 625 & 550 & 781 & 649 & 637 & 426 \\
\hline 13 & e 385 & 249 & 175 & 93 & 156 & e 480 & 628 & 666 & 534 & 605 & 553 & 317 \\
\hline 14 & e 380 & 128 & 170 & 205 & 321 & e 485 & 579 & 379 & 668 & 631 & 682 & 482 \\
\hline 15 & e 374 & 221 & 166 & 112 & 169 & e491 & 608 & 224 & 632 & 283 & 687 & 501 \\
\hline 16 & e 369 & 232 & 108 & 155 & 351 & e 497 & 666 & 183 & 745 & 439 & 689 & 494 \\
\hline 17 & e 364 & 227 & 140 & 209 & 319 & e 503 & 611 & 315 & 762 & 757 & 680 & 495 \\
\hline 18 & e 359 & 99 & 141 & 222 & 202 & e 508 & 503 & 484 & 790 & 665 & 682 & 492 \\
\hline 19 & e 354 & 212 & 170 & 112 & 191 & e 514 & 665 & 498 & 753 & 691 & 461 & 488 \\
\hline 20 & e 349 & 217 & 113 & 220 & e 367 & e 519 & 679 & 147 & 685 & 505 & 470 & 488 \\
\hline 21 & e 344 & 110 & 168 & 228 & e 373 & e 524 & 682 & 280 & 697 & 482 & 667 & 478 \\
\hline 22 & e339 & 30 & 168 & 153 & e379 & e 530 & 691 & 405 & 784 & 750 & 660 & 475 \\
\hline 23 & e 333 & 185 & 152 & 120 & e 386 & e 535 & 569 & 486 & 787 & 762 & 576 & 371 \\
\hline 24 & e 328 & 209 & 157 & 109 & e391 & e 540 & 695 & 508 & 793 & 596 & 412 & 381 \\
\hline 25 & e 323 & 204 & 161 & 223 & e397 & e 545 & 647 & 20 & 598 & 763 & 621 & 469 \\
\hline 26 & e 318 & 183 & 113 & 242 & e 402 & e 550 & 388 & 26 & 471 & 756 & 612 & 457 \\
\hline 27 & e 314 & 209 & 129 & 236 & e 407 & e 555 & 514 & 340 & 386 & 660 & 384 & 467 \\
\hline 28 & e 309 & 116 & 169 & 220 & e 412 & e 561 & 442 & 668 & 532 & 597 & 381 & 458 \\
\hline 29 & e 304 & 56 & 131 & 214 & -- & e 566 & 401 & 550 & 459 & 734 & 397 & 456 \\
\hline 30 & e 300 & 198 & 144 & 175 & --- & e 571 & 558 & 592 & e791 & 744 & 327 & 452 \\
\hline 31 & e295 & --- & 74 & 258 & -- & e 576 & -- & 775 & -- & 747 & 471 & --- \\
\hline TOTAL & 11157 & 5985 & 4625 & 4906 & 7839 & 15400 & 16062 & 13619 & 19203 & 20845 & 17644 & 12306 \\
\hline CAL YF & 1986 & TOTAL & 1036 & & & & & & & & & \\
\hline WTR YF & R 1987 & TOTAL & 9591 & & & & & & & & & \\
\hline
\end{tabular}

e Estimated 
EAST STEWART BASIN

EAST STEWART CREEK NEAR IONE, NV

DAILY TOTAL SOLAR RADIATION, INCIDENTAL, WATER YEAR OCTOBER 1987 TO SEPTEMBER 1988 CALORIES PER SQUARE CENTIMETER

\begin{tabular}{|c|c|c|c|c|c|c|c|c|c|c|c|c|}
\hline DAY & OCT & NOV & DEC & JAN & FEB & MAR & APR & MAY & JUN & JUL & AUG & $S E E$ \\
\hline 1 & 441 & 142 & 143 & 174 & 246 & 233 & 574 & 549 & 494 & 780 & 578 & 43 \\
\hline 2 & 457 & 7 & 133 & 156 & 213 & 225 & 550 & 562 & 744 & 775 & 480 & 50 \\
\hline 3 & 320 & 34 & 167 & 69 & 273 & 427 & 283 & 687 & 758 & 725 & 515 & 565 \\
\hline 4 & 402 & 245 & 141 & 137 & 283 & 297 & 588 & 449 & 685 & 713 & 574 & $53 \varepsilon$ \\
\hline 5 & 416 & 108 & 144 & 71 & 289 & 434 & 542 & 400 & 445 & 740 & 303 & 495 \\
\hline 6 & 415 & 20 & 173 & 123 & 290 & 319 & 397 & 327 & 711 & 765 & 248 & \\
\hline 7 & 407 & 278 & 114 & 185 & 288 & 443 & 504 & 571 & 678 & 766 & 348 & 52 \\
\hline 8 & 402 & 257 & 90 & 129 & 305 & 434 & 587 & 684 & 769 & 710 & 494 & 48 \\
\hline 9 & 397 & 257 & 124 & 40 & 283 & 249 & 611 & 519 & 781 & 646 & 476 & \\
\hline 10 & 374 & 244 & 124 & 178 & 196 & 380 & 595 & 724 & 603 & 741 & 591 & \\
\hline 11 & 403 & 233 & 137 & 129 & 316 & 304 & 556 & 750 & 562 & 734 & 700 & 52 \\
\hline 12 & 196 & 213 & 115 & 195 & 324 & 486 & 561 & 741 & 468 & 731 & 678 & \\
\hline 13 & 188 & 81 & 129 & 214 & 321 & 490 & 387 & 743 & 694 & 733 & 594 & \\
\hline 14 & 391 & 96 & 139 & 199 & 330 & 491 & 377 & 752 & 565 & 759 & 694 & \\
\hline 15 & 382 & 237 & 105 & 151 & 295 & 288 & 267 & 751 & 730 & 590 & 685 & 50. \\
\hline 16 & 362 & 191 & 165 & 118 & 331 & 422 & 118 & 471 & 766 & 470 & 684 & 49 \\
\hline 17 & 371 & 170 & 110 & 131 & 341 & 507 & 27 & 203 & 661 & 757 & 690 & 43 \\
\hline 18 & 370 & 225 & 162 & 223 & 173 & 508 & 325 & 565 & 758 & 774 & 682 & 5 \\
\hline 19 & 361 & 208 & 47 & 222 & 362 & 515 & 551 & 764 & 319 & 768 & 468 & \\
\hline 20 & 354 & 178 & 159 & 210 & 371 & 509 & 151 & 767 & 694 & 762 & 484 & 11 \\
\hline 21 & 301 & 201 & 45 & 216 & 369 & 324 & 359 & 753 & 608 & 766 & 317 & \\
\hline 22 & 156 & 211 & 54 & 234 & 383 & 459 & 471 & 710 & 426 & 532 & 342 & \\
\hline 23 & 162 & 197 & 96 & 219 & 368 & 502 & 77 & 706 & 704 & 513 & 420 & \\
\hline 24 & 203 & 194 & 53 & 230 & 377 & 526 & 561 & 694 & 378 & 340 & 322 & 4 \\
\hline 25 & 230 & 81 & 160 & 233 & 389 & 536 & 683 & 745 & 394 & 497 & 285 & \\
\hline 26 & 318 & 208 & 162 & 204 & 372 & 544 & 674 & 588 & 557 & 554 & 246 & \\
\hline 27 & 236 & 214 & 166 & 223 & 181 & 440 & 520 & 757 & 686 & 438 & 502 & \\
\hline 28 & 201 & 174 & 139 & 133 & 219 & 556 & 566 & 361 & 771 & 448 & 549 & \\
\hline 29 & 47 & 190 & 169 & 140 & 344 & 335 & 715 & 162 & 780 & 555 & 437 & 4 \\
\hline 30 & 220 & 130 & 37 & 245 & -- & 438 & 353 & 283 & 764 & 379 & 272 & 4 \\
\hline 31 & 130 & -- & 88 & 263 & -- & 573 & -- & 675 & -- & 405 & 285 & \\
\hline TOTAL & 9613 & 5224 & 3790 & 5394 & 8832 & 13194 & 13530 & 18413 & 18953 & 19866 & 14943 & 1307 \\
\hline CAL YR & 1987 & COTAL & & & & & & & & & & \\
\hline WTR YR & 1988 & TOTAL & & & & & & & & & & \\
\hline
\end{tabular}


EAST STEWART BASIN

EAST STEWART CREEK NEAR IONE, NV

DAILY TOTAL SOLAR RADIATION, INCIDENTAL, WATER YEAR OCTOBER 1988 TO SEPTEMBER 1989 CALORIES PER SQUARE CENTIMETER

\begin{tabular}{|c|c|c|c|c|c|c|c|c|c|c|c|c|}
\hline DAY & OCT & NOV & DEC & JAN & FEB & MAR & APR & MAY & JUN & JUL & AUG & SEP \\
\hline 1 & 435 & 261 & 180 & 162 & 115 & 395 & 244 & 682 & 526 & 798 & 737 & 540 \\
\hline 2 & 422 & 127 & 183 & 143 & 257 & 150 & 176 & 590 & 628 & 798 & 729 & 579 \\
\hline 3 & 421 & 202 & 175 & 170 & 105 & 375 & 535 & 629 & 352 & 804 & 715 & 573 \\
\hline 4 & 330 & 263 & 172 & 138 & 176 & 292 & 582 & 673 & 251 & 800 & 713 & 566 \\
\hline 5 & 313 & 255 & 172 & 156 & 268 & 244 & 597 & 591 & 450 & 803 & 620 & 565 \\
\hline 6 & 306 & 189 & 107 & 82 & 280 & 256 & 600 & 420 & 565 & 802 & 571 & 498 \\
\hline 7 & 409 & 180 & 80 & 65 & 287 & 358 & 586 & 442 & 432 & 802 & 571 & 494 \\
\hline 8 & 409 & 139 & 163 & 86 & 212 & 223 & 609 & 378 & 502 & 547 & 245 & 531 \\
\hline 9 & 274 & 197 & 148 & 45 & 274 & 365 & 608 & 386 & 170 & 580 & 378 & 499 \\
\hline 10 & 180 & 198 & 166 & 78 & 280 & 318 & 587 & 272 & 501 & 793 & 401 & 372 \\
\hline 11 & 135 & 209 & 160 & 133 & 141 & 372 & 581 & 84 & 272 & 554 & 635 & 278 \\
\hline 12 & 358 & 212 & 156 & 189 & 32 & 427 & 619 & 433 & 684 & 434 & 636 & 510 \\
\hline 13 & 303 & 235 & 157 & 172 & 35 & 358 & 601 & 399 & 688 & 580 & 526 & 503 \\
\hline 14 & 225 & 107 & 169 & 137 & 121 & 468 & 601 & 190 & 781 & 628 & 628 & 499 \\
\hline 15 & 366 & 83 & 99 & 192 & 322 & 473 & 587 & 281 & 737 & 774 & 680 & 491 \\
\hline 16 & 363 & 110 & 91 & 198 & 306 & 251 & 584 & 595 & 747 & 776 & 682 & 442 \\
\hline 17 & 357 & 93 & 85 & 200 & 270 & 349 & 651 & 740 & 790 & 763 & 618 & 128 \\
\hline 18 & 353 & 196 & 115 & 205 & 229 & 152 & 588 & 744 & 802 & 590 & 619 & 276 \\
\hline 19 & 352 & 181 & 40 & 208 & 166 & 113 & 464 & 756 & 704 & 709 & 339 & 431 \\
\hline 20 & 349 & 157 & 119 & 216 & 360 & 513 & 568 & 725 & 721 & 580 & 297 & 472 \\
\hline 21 & 342 & 199 & 71 & 217 & 205 & 393 & 630 & 761 & 794 & 434 & 430 & 476 \\
\hline 22 & 331 & 120 & 88 & 191 & 335 & 514 & 555 & 664 & 792 & 519 & 604 & 479 \\
\hline 23 & 326 & 70 & 116 & 141 & 352 & 310 & 584 & 721 & 652 & 357 & 343 & 473 \\
\hline 24 & 323 & 148 & 106 & 222 & 333 & 373 & 181 & 619 & 483 & 381 & 623 & 446 \\
\hline 25 & 310 & 42 & 71 & 221 & 259 & 218 & 510 & 764 & 776 & 661 & 557 & 315 \\
\hline 26 & 308 & 137 & 56 & 223 & 367 & 256 & 408 & 764 & 645 & 722 & 599 & 403 \\
\hline 27 & 258 & 197 & 175 & 225 & 396 & 465 & 152 & 718 & 437 & 747 & 598 & 415 \\
\hline 28 & 267 & 164 & 21 & 239 & 403 & 383 & 435 & 739 & 788 & 736 & 594 & 356 \\
\hline 29 & 279 & 191 & 61 & 226 & --- & 558 & 669 & 282 & 804 & 742 & 597 & 303 \\
\hline 30 & 286 & 183 & 136 & 245 & --- & 536 & 380 & 480 & 798 & 727 & 595 & 312 \\
\hline 31 & 282 & --- & 128 & 241 & -- & 224 & -- & 522 & --- & 702 & 585 & --- \\
\hline TOTAL & 9972 & 5045 & 3766 & 5366 & 6886 & 10682 & 15472 & 17044 & 18272 & 20643 & 17465 & 13225 \\
\hline CAL YR & 1988 & TOTAL & 4979 & & & & & & & & & \\
\hline WTR YR & 1989 & TOTAL & 3838 & & & & & & & & & \\
\hline
\end{tabular}


EAST STEWART BASIN

EAST STEWART CREEK NEAR IONE, NV

DAILY TOTAL SOLAR RADIATION, INCIDENTAL, WATER YEAR OCTOBER 1989 TO SEPTEMBER 1990 CALORIES PER SQUARE CENTIMETER

\begin{tabular}{|c|c|c|c|c|c|c|c|c|c|c|c|c|}
\hline DAY & OCT & NOV & DEC & JAN & FEB & MAR & $\mathrm{APR}$ & MAY & JUN & JUL & AUG & SEP \\
\hline 1 & 433 & 279 & 178 & 93 & 151 & 234 & 502 & 705 & 769 & 612 & 376 & 587 \\
\hline 2 & 211 & 275 & 173 & 13 & 247 & 234 & 470 & 413 & 752 & 602 & 574 & 563 \\
\hline 3 & 116 & 270 & 170 & 44 & 211 & 373 & 399 & 693 & 775 & 774 & 487 & 554 \\
\hline 4 & 415 & 263 & 160 & 161 & 128 & 306 & 367 & 723 & 783 & 790 & 484 & 570 \\
\hline 5 & 407 & 236 & 185 & 165 & 263 & 152 & 353 & 740 & 784 & 796 & 612 & 518 \\
\hline 6 & 402 & 246 & 164 & 137 & 179 & 432 & 262 & 622 & 637 & 792 & 709 & 368 \\
\hline 7 & 408 & 190 & 163 & 77 & 76 & 357 & 447 & 731 & 780 & 787 & 272 & 479 \\
\hline 8 & 402 & 240 & 151 & 182 & 278 & 384 & 83 & 735 & 774 & 744 & 305 & 548 \\
\hline 9 & 396 & 233 & 153 & 180 & 284 & 447 & 605 & 705 & 497 & 743 & 399 & 543 \\
\hline 10 & 346 & 229 & 160 & 161 & 291 & 359 & 598 & 199 & 399 & 424 & 551 & 527 \\
\hline 11 & 385 & 226 & 157 & 157 & 258 & 259 & 547 & 361 & 788 & 408 & 385 & 527 \\
\hline 12 & 369 & 228 & 152 & 144 & 269 & 318 & 603 & 694 & 788 & 304 & 558 & 525 \\
\hline 13 & 370 & 222 & 155 & 109 & 143 & 369 & 608 & 668 & 609 & 344 & 686 & 519 \\
\hline 14 & 247 & 218 & 153 & 176 & 244 & 341 & 517 & 489 & 323 & 517 & 525 & 515 \\
\hline 15 & 306 & 153 & 157 & 128 & 317 & 481 & 586 & 584 & 389 & 404 & 150 & 512 \\
\hline 16 & 355 & 216 & 152 & 195 & 154 & 357 & 451 & 721 & 597 & 340 & 666 & 505 \\
\hline 17 & 352 & 194 & 162 & 57 & 285 & 356 & 59 & 500 & 725 & 397 & 575 & 266 \\
\hline 18 & 350 & 204 & 152 & 207 & 43 & 456 & 397 & 697 & 548 & 509 & 275 & 304 \\
\hline 19 & 343 & 205 & 152 & 201 & 117 & 490 & 484 & 520 & 789 & 377 & 189 & 334 \\
\hline 20 & 263 & 202 & 150 & 204 & 293 & 477 & 337 & 602 & 719 & 734 & 525 & 455 \\
\hline 21 & 228 & 192 & 151 & 211 & 370 & 479 & 485 & 642 & 788 & 712 & 560 & 365 \\
\hline 22 & 334 & 191 & 151 & 166 & 371 & 393 & 341 & 674 & 788 & 661 & 566 & 267 \\
\hline 23 & 324 & 152 & 150 & 227 & 378 & 514 & 175 & 430 & 802 & 746 & 395 & 288 \\
\hline 24 & 311 & 39 & 146 & 215 & 301 & 501 & 321 & 554 & 800 & 756 & 402 & 254 \\
\hline 25 & 10 & 126 & 155 & 208 & 322 & 483 & 661 & 666 & 793 & 762 & 597 & 426 \\
\hline 26 & 136 & 60 & 152 & 79 & 286 & 521 & 669 & 363 & 802 & 759 & 610 & 388 \\
\hline 27 & 307 & 181 & 163 & 214 & 228 & 414 & 645 & 325 & 806 & 753 & 597 & 320 \\
\hline 28 & 279 & 186 & 35 & 197 & 324 & 354 & 545 & 26 & 806 & 454 & 602 & 120 \\
\hline 29 & 295 & 182 & 158 & 171 & --- & 300 & 507 & 439 & 809 & 747 & 604 & 442 \\
\hline 30 & 289 & 187 & 154 & 129 & --- & 503 & 468 & 556 & 609 & 694 & 595 & 449 \\
\hline 31 & 284 & -- & 157 & 200 & $-\cdots$ & 556 & --- & 544 & --- & 551 & 595 & --- \\
\hline TOTAL & 9673 & 6025 & 4771 & 4808 & 6811 & 12200 & 13492 & 17321 & 21028 & 18993 & 15426 & 13038 \\
\hline CAL YR & 1989 & TOTAL & 524 & & & & & & & & & \\
\hline WTR YR & 1990 & TOTAL & 586 & & & & & & & & & \\
\hline
\end{tabular}


EAST STEWART BASIN

EAST STEWART CREEK NEAR IONE, NV

DAILY TOTAL SOLAR RADIATION, INCIDENTAL, WATER YEAR OCTOBER 1990 TO SEPTEMBER 1991 CALORIES PER SQUARE CENTIMETER

\begin{tabular}{|c|c|c|c|c|c|c|c|c|c|c|c|c|}
\hline DAY & OCT & NOV & DEC & JAN & FEB & MAR & APR & MAY & JUN & JUL & AUG & SEP \\
\hline 1 & 442 & 119 & 199 & 177 & 270 & 204 & 234 & 124 & 535 & 717 & 616 & 299 \\
\hline 2 & 424 & 292 & 194 & 202 & 95 & 356 & 535 & 142 & 734 & 766 & 331 & 352 \\
\hline 3 & 414 & 287 & 188 & 86 & 228 & 126 & 571 & 456 & 556 & 770 & 625 & 198 \\
\hline 4 & 427 & 274 & 203 & 48 & 248 & 98 & 494 & 739 & 605 & 770 & 679 & 320 \\
\hline 5 & 324 & 109 & 188 & 185 & 211 & 239 & 525 & 660 & 771 & 724 & 644 & 218 \\
\hline 6 & 317 & 125 & 184 & 148 & 295 & 359 & 426 & 437 & 780 & 769 & 695 & 141 \\
\hline 7 & 403 & 262 & 184 & 154 & 300 & 473 & 623 & 604 & 773 & 642 & 630 & 266 \\
\hline 8 & 422 & 131 & 181 & 188 & 305 & 474 & 631 & 555 & 495 & 360 & 685 & 332 \\
\hline 9 & 411 & 257 & 179 & 200 & 308 & 332 & 537 & 228 & 580 & 478 & 686 & 167 \\
\hline 10 & 403 & 257 & 175 & 109 & 314 & 417 & 443 & 442 & 678 & 719 & 594 & 266 \\
\hline 11 & 402 & 225 & 94 & 155 & 290 & 364 & 423 & 528 & 685 & 655 & 481 & 333 \\
\hline 12 & 393 & 224 & 66 & 152 & 284 & 404 & 321 & 665 & 767 & 740 & 363 & 488 \\
\hline 13 & 377 & 188 & 28 & 86 & 214 & 294 & 518 & 363 & 747 & 727 & 368 & 486 \\
\hline 14 & 373 & 184 & 120 & 223 & 323 & 104 & 509 & 291 & 796 & 778 & 492 & 496 \\
\hline 15 & 368 & 219 & 175 & 67 & 236 & 45 & 518 & 753 & 762 & 769 & 343 & 500 \\
\hline 16 & 343 & 201 & 75 & 214 & 102 & 76 & 676 & 611 & 734 & 785 & 303 & 500 \\
\hline 17 & 370 & 155 & 93 & 204 & 190 & 483 & 372 & 362 & 656 & 755 & 527 & 496 \\
\hline 18 & 166 & 170 & 178 & 212 & 335 & 308 & 572 & 339 & 514 & 688 & 566 & 468 \\
\hline 19 & 62 & 94 & 59 & 221 & 356 & 273 & 557 & 244 & 753 & 653 & 572 & 478 \\
\hline 20 & 339 & 184 & 75 & 201 & 380 & 403 & 475 & 368 & 685 & 565 & 640 & 440 \\
\hline 21 & 349 & 182 & 69 & 230 & 307 & 42 & 363 & 301 & 677 & 431 & 639 & 479 \\
\hline 22 & 331 & 216 & 168 & 231 & 389 & 429 & 322 & 662 & 795 & 634 & 632 & 466 \\
\hline 23 & 332 & 204 & 177 & 189 & 390 & 557 & 564 & 637 & 748 & 733 & 528 & 473 \\
\hline 24 & 325 & 214 & 176 & 236 & 405 & 542 & 455 & 472 & 660 & 476 & 528 & 395 \\
\hline 25 & 319 & 216 & 190 & 243 & 410 & 207 & 214 & 685 & 391 & 628 & 517 & 437 \\
\hline 26 & 325 & 158 & 176 & 248 & 413 & 515 & 330 & 750 & 624 & 396 & 548 & 299 \\
\hline 27 & 315 & 212 & 179 & 247 & 286 & 215 & 701 & 738 & 339 & 375 & 370 & 213 \\
\hline 28 & 311 & 208 & 179 & 253 & 390 & 561 & 719 & 694 & 486 & 372 & 592 & 211 \\
\hline 29 & 288 & 193 & 180 & 263 & --- & 568 & 729 & 542 & 319 & 611 & 580 & 304 \\
\hline 30 & 292 & 205 & 179 & 262 & --- & 570 & 623 & 265 & 773 & 357 & 583 & 432 \\
\hline 31 & 179 & --- & 178 & 225 & -- & 511 & -- & 248 & --- & 205 & 494 & -- \\
\hline TOTAL & 10546 & 5965 & 4689 & 5859 & 8274 & 10549 & 14980 & 14905 & 19418 & 19048 & 16851 & 10953 \\
\hline CAL YR & 1990 & TOTAL & 4317 & & & & & & & & & \\
\hline WTR YR & 21991 & TOTAL & 2037 & & & & & & & & & \\
\hline
\end{tabular}


EAST STEWART BASIN

EAST STEWART CREEK NEAR IONE, NV

DAILY MEAN RELATIVE HUMIDITY, WATER YEAR OCTOBER 1990 TO SEPTEMBER 1991 PERCENT

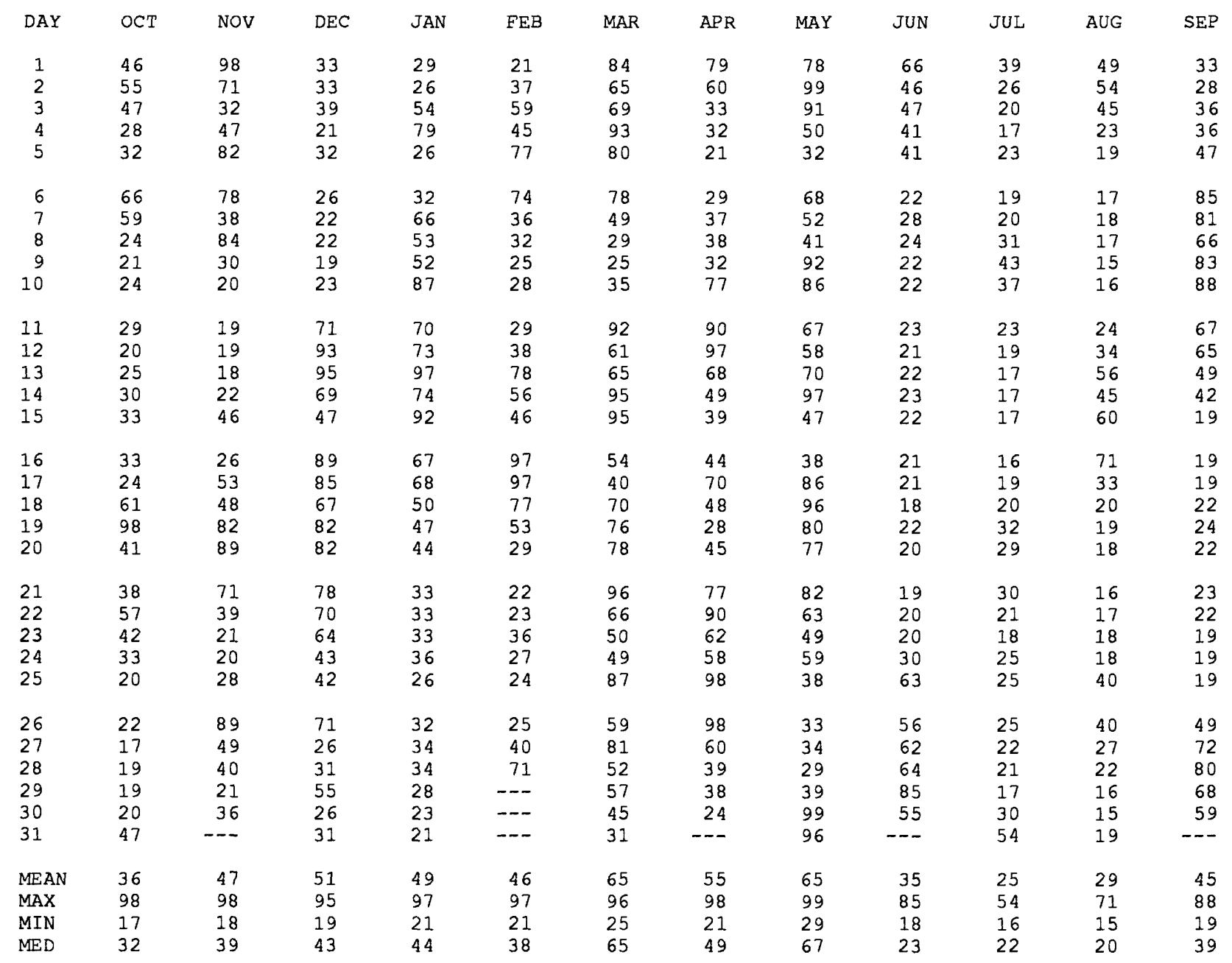

WTR YR 1991 MEAN 46 MAX 99 MIN 15 MED 38 
EAST STEWART BASIN

EAST STEWART CREEK NEAR IONE, NV

DAILY TOTAL PRECIPITATION, WATER YEAR OCTOBER 1985 TO SEPTEMBER 1986 INCHES

\begin{tabular}{|c|c|c|c|c|c|c|c|c|c|c|c|c|}
\hline DAY & OCT & NOV & DEC & JAN & FEB & MAR & APR & MAY & JUN & JUL & AUG & SEP \\
\hline $\begin{array}{l}1 \\
2 \\
3 \\
4 \\
5\end{array}$ & $\begin{array}{l}.00 \\
.00 \\
.00 \\
.00 \\
.00\end{array}$ & $\begin{array}{l}.00 \\
.00 \\
.00 \\
.00 \\
.00\end{array}$ & $\begin{array}{l}.00 \\
.30 \\
.00 \\
.00 \\
.00\end{array}$ & $\begin{array}{l}.00 \\
.00 \\
.00 \\
.00 \\
.00\end{array}$ & $\begin{array}{r}.00 \\
e .05 \\
.00 \\
e .31 \\
e .11\end{array}$ & $\begin{array}{l}.00 \\
.35 \\
.00 \\
.00 \\
.00\end{array}$ & $\begin{array}{l}.30 \\
.00 \\
.00 \\
.00 \\
.12\end{array}$ & $\begin{array}{l}.00 \\
.20 \\
.00 \\
.00 \\
.00\end{array}$ & $\begin{array}{l}.00 \\
.00 \\
.30 \\
.00 \\
.00\end{array}$ & $\begin{array}{l}.00 \\
.00 \\
.00 \\
.00 \\
.00\end{array}$ & $\begin{array}{l}.00 \\
.00 \\
.00 \\
.00 \\
.00\end{array}$ & $\begin{array}{l}.00 \\
.00 \\
.00 \\
.00 \\
.00\end{array}$ \\
\hline $\begin{array}{r}6 \\
7 \\
8 \\
9 \\
10\end{array}$ & $\begin{array}{l}.55 \\
.15 \\
.65 \\
.00 \\
.00\end{array}$ & $\begin{array}{l}.00 \\
.00 \\
.00 \\
.10 \\
.75\end{array}$ & $\begin{array}{l}.00 \\
.00 \\
.15 \\
.05 \\
.20\end{array}$ & $\begin{array}{l}.00 \\
.00 \\
.00 \\
.00 \\
.00\end{array}$ & $\begin{array}{r}e .33 \\
.00 \\
.00 \\
.00 \\
.00\end{array}$ & $\begin{array}{l}.00 \\
.00 \\
.15 \\
.25 \\
.10\end{array}$ & $\begin{array}{l}.10 \\
.00 \\
.00 \\
.00 \\
.00\end{array}$ & $\begin{array}{l}.00 \\
.00 \\
.00 \\
.00 \\
.00\end{array}$ & $\begin{array}{l}.00 \\
.00 \\
.00 \\
.00 \\
.00\end{array}$ & $\begin{array}{l}.00 \\
.00 \\
.00 \\
.00 \\
.00\end{array}$ & $\begin{array}{l}.00 \\
.00 \\
.00 \\
.00 \\
.20\end{array}$ & $\begin{array}{l}.00 \\
.00 \\
.00 \\
.00 \\
.00\end{array}$ \\
\hline $\begin{array}{l}11 \\
12 \\
13 \\
14 \\
15\end{array}$ & $\begin{array}{l}.00 \\
.00 \\
.00 \\
.00 \\
.00\end{array}$ & $\begin{array}{l}.45 \\
.00 \\
.00 \\
.00 \\
.00\end{array}$ & $\begin{array}{l}.00 \\
.00 \\
.00 \\
.00 \\
.00\end{array}$ & $\begin{array}{l}.00 \\
.00 \\
.00 \\
.00 \\
.00\end{array}$ & $\begin{array}{r}.00 \\
\mathrm{e} .28 \\
\mathrm{e} .07 \\
.00 \\
.00\end{array}$ & $\begin{array}{l}.15 \\
.20 \\
.20 \\
.10 \\
.00\end{array}$ & $\begin{array}{l}.00 \\
.18 \\
.00 \\
.00 \\
.00\end{array}$ & $\begin{array}{l}.00 \\
.00 \\
.00 \\
.00 \\
.00\end{array}$ & $\begin{array}{l}.00 \\
.00 \\
.00 \\
.00 \\
.00\end{array}$ & $\begin{array}{l}.00 \\
.00 \\
.00 \\
.00 \\
.00\end{array}$ & $\begin{array}{l}.20 \\
.00 \\
.00 \\
.00 \\
.00\end{array}$ & $\begin{array}{l}.00 \\
.00 \\
.00 \\
.35 \\
.00\end{array}$ \\
\hline $\begin{array}{l}16 \\
17 \\
18 \\
19 \\
20\end{array}$ & $\begin{array}{l}.00 \\
.00 \\
.00 \\
.00 \\
.00\end{array}$ & $\begin{array}{l}.20 \\
.40 \\
.20 \\
.00 \\
.15\end{array}$ & $\begin{array}{l}.00 \\
.00 \\
.00 \\
.00 \\
.00\end{array}$ & $\begin{array}{l}.00 \\
.00 \\
.00 \\
.00 \\
.00\end{array}$ & $\begin{array}{r}e .02 \\
e .02 \\
.00 \\
e .91 \\
.00\end{array}$ & $\begin{array}{l}.10 \\
.05 \\
.00 \\
.00 \\
.00\end{array}$ & $\begin{array}{l}.00 \\
.00 \\
.00 \\
.00 \\
.00\end{array}$ & $\begin{array}{l}.00 \\
.00 \\
.00 \\
.00 \\
.10\end{array}$ & $\begin{array}{l}.00 \\
.00 \\
.00 \\
.00 \\
.00\end{array}$ & $\begin{array}{l}.00 \\
.00 \\
.00 \\
.00 \\
.00\end{array}$ & $\begin{array}{l}.00 \\
.00 \\
.00 \\
.00 \\
.20\end{array}$ & $\begin{array}{l}.00 \\
.05 \\
.00 \\
.05 \\
.05\end{array}$ \\
\hline $\begin{array}{l}21 \\
22 \\
23 \\
24 \\
25\end{array}$ & $\begin{array}{l}.40 \\
.00 \\
.00 \\
.00 \\
.00\end{array}$ & $\begin{array}{l}.10 \\
.00 \\
.00 \\
.00 \\
.00\end{array}$ & $\begin{array}{l}.00 \\
.00 \\
.00 \\
.00 \\
.00\end{array}$ & $\begin{array}{l}.00 \\
.00 \\
.00 \\
.00 \\
.00\end{array}$ & $\begin{array}{r}e .06 \\
.00 \\
.00 \\
.00 \\
.00\end{array}$ & $\begin{array}{l}.00 \\
.00 \\
.00 \\
.00 \\
.00\end{array}$ & $\begin{array}{l}.00 \\
.00 \\
.00 \\
.00 \\
.00\end{array}$ & $\begin{array}{l}.00 \\
.00 \\
.00 \\
.00 \\
.00\end{array}$ & $\begin{array}{l}.00 \\
.00 \\
.00 \\
.00 \\
.00\end{array}$ & $\begin{array}{l}.00 \\
.16 \\
.84 \\
.00 \\
.00\end{array}$ & $\begin{array}{l}.00 \\
.00 \\
.00 \\
.00 \\
.00\end{array}$ & $\begin{array}{l}.00 \\
.00 \\
.00 \\
.15 \\
.15\end{array}$ \\
\hline $\begin{array}{l}26 \\
27 \\
28 \\
29 \\
30 \\
31\end{array}$ & $\begin{array}{l}.00 \\
.00 \\
.00 \\
.00 \\
.00 \\
.00\end{array}$ & $\begin{array}{l}.00 \\
.30 \\
.45 \\
.45 \\
.15 \\
----\end{array}$ & $\begin{array}{l}.00 \\
.00 \\
.00 \\
.00 \\
.10 \\
.00\end{array}$ & $\begin{array}{r}.00 \\
.00 \\
.00 \\
.00 \\
e .04 \\
.00\end{array}$ & $\begin{array}{l}.00 \\
.00 \\
.00 \\
--- \\
--- \\
---\end{array}$ & $\begin{array}{l}.00 \\
.00 \\
.00 \\
.00 \\
.00 \\
.10\end{array}$ & $\begin{array}{l}.00 \\
.00 \\
.00 \\
.00 \\
.00 \\
-.-\end{array}$ & $\begin{array}{l}.00 \\
.00 \\
.00 \\
.00 \\
.00 \\
.00\end{array}$ & $\begin{array}{l}.00 \\
.00 \\
.00 \\
.00 \\
.00 \\
----\end{array}$ & $\begin{array}{l}.00 \\
.00 \\
.00 \\
.00 \\
.00 \\
.00\end{array}$ & $\begin{array}{l}.00 \\
.00 \\
.20 \\
.00 \\
.00 \\
.00\end{array}$ & $\begin{array}{l}.20 \\
.00 \\
.00 \\
.00 \\
.00 \\
----\end{array}$ \\
\hline TOTAL & 1.75 & 3.70 & 0.80 & 0.04 & 2.16 & 1.75 & 0.70 & 0.30 & 0.30 & 1.00 & 0.80 & 1.00 \\
\hline
\end{tabular}

WTR YR 1986 TOTAL 14.30

e Estimated 
EAST STEWART BASIN

EAST STEWART CREEK NEAR IONE, NV

DAILY TOTAL PRECIPITATION, WATER YEAR OCTOBER 1986 TO SEPTEMBER 1987 INCHES

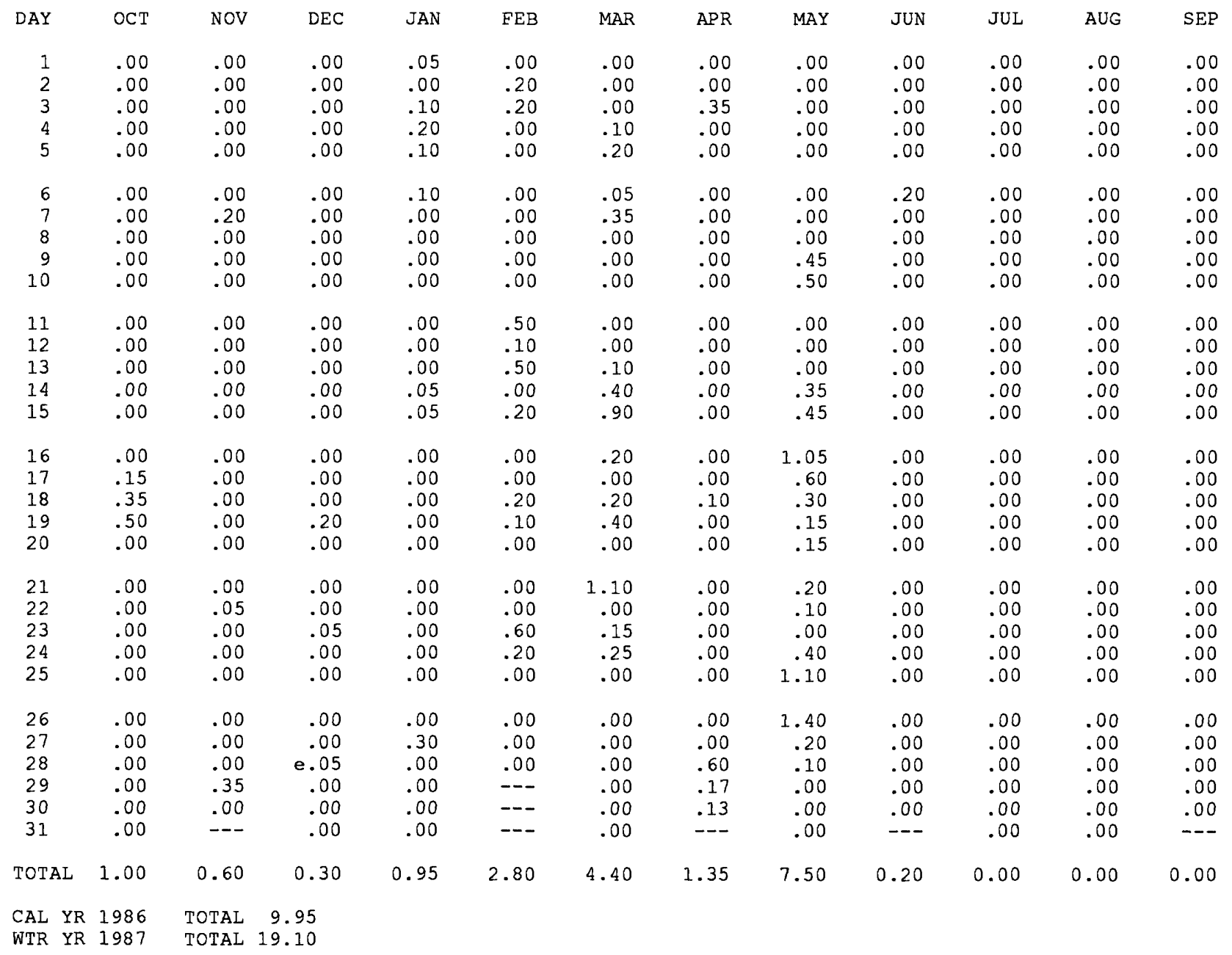

e Estimated 
EAST STEWART BASIN

EAST STEWART CREEK NEAR IONE, NV

DAILY TOTAL PRECIPITATION, WATER YEAR OCTOBER 1987 TO SEPTEMBER 1988 INCHES

\begin{tabular}{|c|c|c|c|c|c|c|c|c|c|c|c|c|}
\hline DAY & OCT & NOV & $\mathrm{DEC}$ & JAN & FEB & MAR & APR & MAY & JUN & JUL & AUG & SEP \\
\hline $\begin{array}{l}1 \\
2 \\
3 \\
4 \\
5\end{array}$ & $\begin{array}{l}.00 \\
.00 \\
.00 \\
.00 \\
.00\end{array}$ & $\begin{array}{l}.30 \\
.45 \\
.50 \\
.00 \\
.40\end{array}$ & $\begin{array}{l}.00 \\
.00 \\
.00 \\
.00 \\
.00\end{array}$ & $\begin{array}{r}.00 \\
.10 \\
1.35 \\
.15 \\
.20\end{array}$ & $\begin{array}{l}.00 \\
.00 \\
.00 \\
.00 \\
.00\end{array}$ & $\begin{array}{l}.20 \\
.00 \\
.05 \\
.00 \\
.00\end{array}$ & $\begin{array}{l}.00 \\
.00 \\
.00 \\
.00 \\
.00\end{array}$ & $\begin{array}{l}.00 \\
.00 \\
.00 \\
.00 \\
.20\end{array}$ & $\begin{array}{l}.00 \\
.00 \\
.00 \\
.00 \\
.00\end{array}$ & $\begin{array}{l}.00 \\
.00 \\
.00 \\
.00 \\
.00\end{array}$ & $\begin{array}{l}.00 \\
.10 \\
.10 \\
.00 \\
.70\end{array}$ & $\begin{array}{l}.00 \\
.00 \\
.00 \\
.00 \\
.00\end{array}$ \\
\hline $\begin{array}{r}6 \\
7 \\
8 \\
9 \\
10\end{array}$ & $\begin{array}{l}.00 \\
.00 \\
.00 \\
.00 \\
.00\end{array}$ & $\begin{array}{l}.85 \\
.20 \\
.00 \\
.00 \\
.00\end{array}$ & $\begin{array}{l}.00 \\
.00 \\
.20 \\
.00 \\
.00\end{array}$ & $\begin{array}{l}.15 \\
.00 \\
.00 \\
.40 \\
.20\end{array}$ & $\begin{array}{l}.00 \\
.00 \\
.00 \\
.00 \\
.00\end{array}$ & $\begin{array}{l}.00 \\
.00 \\
.00 \\
.00 \\
.00\end{array}$ & $\begin{array}{l}.00 \\
.00 \\
.00 \\
.00 \\
.00\end{array}$ & $\begin{array}{l}.90 \\
.00 \\
.00 \\
.00 \\
.00\end{array}$ & $\begin{array}{l}.00 \\
.00 \\
.10 \\
.00 \\
.00\end{array}$ & $\begin{array}{l}.00 \\
.00 \\
.00 \\
.00 \\
.00\end{array}$ & $\begin{array}{l}.65 \\
.10 \\
.00 \\
.00 \\
.00\end{array}$ & $\begin{array}{l}.00 \\
.00 \\
.00 \\
.00 \\
.00\end{array}$ \\
\hline $\begin{array}{l}11 \\
12 \\
13 \\
14 \\
15\end{array}$ & $\begin{array}{l}.00 \\
.25 \\
.25 \\
.00 \\
.00\end{array}$ & $\begin{array}{l}.00 \\
.00 \\
.40 \\
.30 \\
.00\end{array}$ & $\begin{array}{l}.10 \\
.00 \\
.00 \\
.00 \\
.00\end{array}$ & $\begin{array}{l}.00 \\
.00 \\
.00 \\
.00 \\
.00\end{array}$ & $\begin{array}{l}.00 \\
.00 \\
.00 \\
.00 \\
.00\end{array}$ & $\begin{array}{l}.00 \\
.00 \\
.00 \\
.00 \\
.00\end{array}$ & $\begin{array}{l}.00 \\
.00 \\
.00 \\
.50 \\
.90\end{array}$ & $\begin{array}{l}.00 \\
.00 \\
.00 \\
.00 \\
.00\end{array}$ & $\begin{array}{l}.00 \\
.00 \\
.00 \\
.00 \\
.00\end{array}$ & $\begin{array}{l}.00 \\
.00 \\
.00 \\
.00 \\
.00\end{array}$ & $\begin{array}{l}.00 \\
.00 \\
.00 \\
.00 \\
.00\end{array}$ & $\begin{array}{l}.00 \\
.00 \\
.00 \\
.00 \\
.00\end{array}$ \\
\hline $\begin{array}{l}16 \\
17 \\
18 \\
19 \\
20\end{array}$ & $\begin{array}{l}.00 \\
.00 \\
.00 \\
.00 \\
.00\end{array}$ & $\begin{array}{l}.15 \\
.10 \\
.00 \\
.00 \\
.00\end{array}$ & $\begin{array}{l}.20 \\
.00 \\
.00 \\
.10 \\
.00\end{array}$ & $\begin{array}{l}.00 \\
.20 \\
.00 \\
.00 \\
.00\end{array}$ & $\begin{array}{l}.00 \\
.00 \\
.00 \\
.00 \\
.00\end{array}$ & $\begin{array}{l}.00 \\
.00 \\
.00 \\
.00 \\
.00\end{array}$ & $\begin{array}{r}.20 \\
1.00 \\
.20 \\
.20 \\
.95\end{array}$ & $\begin{array}{l}.00 \\
.20 \\
.00 \\
.00 \\
.00\end{array}$ & $\begin{array}{l}.00 \\
.00 \\
.00 \\
.00 \\
.00\end{array}$ & $\begin{array}{l}.00 \\
.00 \\
.00 \\
.00 \\
.00\end{array}$ & $\begin{array}{l}.00 \\
.00 \\
.00 \\
.00 \\
.00\end{array}$ & $\begin{array}{l}.00 \\
.00 \\
.00 \\
.00 \\
.00\end{array}$ \\
\hline $\begin{array}{l}21 \\
22 \\
23 \\
24 \\
25\end{array}$ & $\begin{array}{l}.00 \\
.10 \\
.30 \\
.10 \\
.00\end{array}$ & $\begin{array}{l}.00 \\
.00 \\
.00 \\
.10 \\
.20\end{array}$ & $\begin{array}{l}.15 \\
.70 \\
.10 \\
.20 \\
.00\end{array}$ & $\begin{array}{l}.00 \\
.00 \\
.00 \\
.00 \\
.00\end{array}$ & $\begin{array}{l}.00 \\
.00 \\
.00 \\
.00 \\
.00\end{array}$ & $\begin{array}{l}.00 \\
.00 \\
.00 \\
.00 \\
.00\end{array}$ & $\begin{array}{l}.35 \\
.00 \\
.70 \\
.30 \\
.00\end{array}$ & $\begin{array}{l}.00 \\
.00 \\
.00 \\
.00 \\
.00\end{array}$ & $\begin{array}{l}.20 \\
.00 \\
.00 \\
.00 \\
.00\end{array}$ & $\begin{array}{l}.00 \\
.00 \\
.00 \\
.05 \\
.15\end{array}$ & $\begin{array}{l}.15 \\
.00 \\
.05 \\
.00 \\
.00\end{array}$ & $\begin{array}{l}.45 \\
.15 \\
.00 \\
.00 \\
.00\end{array}$ \\
\hline $\begin{array}{l}26 \\
27 \\
28 \\
29 \\
30 \\
31\end{array}$ & $\begin{array}{l}.00 \\
.00 \\
.00 \\
.50 \\
.20 \\
.00\end{array}$ & $\begin{array}{l}.00 \\
.00 \\
.00 \\
.00 \\
.00 \\
.--\end{array}$ & $\begin{array}{l}.00 \\
.00 \\
.00 \\
.00 \\
.10 \\
.00\end{array}$ & $\begin{array}{l}.00 \\
.00 \\
.00 \\
.00 \\
.00 \\
.00\end{array}$ & $\begin{array}{l}.00 \\
.20 \\
.40 \\
.10 \\
--- \\
---\end{array}$ & $\begin{array}{l}.00 \\
.00 \\
.00 \\
.00 \\
.00 \\
.00\end{array}$ & $\begin{array}{l}.00 \\
.00 \\
.00 \\
.00 \\
.00 \\
.--\end{array}$ & $\begin{array}{l}.00 \\
.00 \\
.70 \\
.74 \\
.38 \\
.00\end{array}$ & $\begin{array}{l}.00 \\
.00 \\
.00 \\
.00 \\
.00 \\
. .-\end{array}$ & $\begin{array}{l}.05 \\
.05 \\
.00 \\
.00 \\
.00 \\
.00\end{array}$ & $\begin{array}{l}.30 \\
.10 \\
.00 \\
.00 \\
.05 \\
.00\end{array}$ & $\begin{array}{l}.00 \\
.00 \\
.00 \\
.00 \\
.00 \\
-.-\end{array}$ \\
\hline TOTAL & 1.70 & 3.95 & 1.85 & 2.75 & 0.70 & 0.25 & 5.30 & 3.12 & 0.30 & 0.30 & 2.30 & 0.60 \\
\hline
\end{tabular}

CAL YR 1987 TOTAL 24.70

WTR YR 1988 TOTAL 23.12 
EAST STEWART BASIN

EAST STEWART CREEK NEAR IONE, NV

DAILY TOTAL PRECIPITATION, WATER YEAR OCTOBER 1988 TO SEPTEMBER 1989 INCHES

\begin{tabular}{|c|c|c|c|c|c|c|c|c|c|c|c|c|}
\hline DAY & OCT & NOV & $\mathrm{DEC}$ & JAN & FEB & MAR & APR & MAY & JUN & JUL & AUG & SEP \\
\hline 1 & .00 & .00 & .00 & .00 & .00 & .00 & .20 & .00 & .00 & e. 00 & .00 & .00 \\
\hline 2 & .00 & .00 & .00 & .00 & .00 & .55 & .10 & .00 & .00 & e. 00 & .00 & .00 \\
\hline 3 & .00 & .00 & .00 & .00 & .05 & .00 & .00 & .00 & .60 & e.00 & .00 & .00 \\
\hline 4 & .00 & .00 & .00 & .00 & .10 & .00 & .00 & .00 & .45 & e. 00 & .00 & .00 \\
\hline 5 & .00 & .00 & .00 & .25 & .00 & .00 & .00 & .00 & .10 & e.00 & .00 & .00 \\
\hline 6 & .00 & .10 & .00 & .10 & .00 & .00 & .00 & .00 & .00 & e. 00 & .10 & .00 \\
\hline 7 & .00 & .00 & .00 & .00 & .00 & .00 & .00 & .00 & .00 & e.00 & .00 & .00 \\
\hline 8 & .00 & .00 & .00 & .00 & .00 & .05 & .00 & .00 & .15 & e. 00 & .30 & .00 \\
\hline 9 & .00 & .00 & .00 & .30 & .00 & .00 & .00 & .10 & .55 & e. 00 & .00 & .00 \\
\hline 10 & .00 & .00 & .00 & .20 & .00 & .00 & .00 & .40 & .00 & e. 00 & .05 & .00 \\
\hline 11 & .00 & .00 & .00 & .05 & .00 & .00 & .00 & .35 & .30 & e.00 & .00 & .00 \\
\hline 12 & .00 & .00 & .00 & .00 & .50 & .00 & .00 & .15 & .05 & e. 10 & .00 & .00 \\
\hline 13 & .00 & .15 & .00 & .05 & .10 & .00 & .00 & .05 & .00 & e.00 & .00 & .00 \\
\hline 14 & .00 & .40 & .05 & .00 & .00 & .00 & .00 & .20 & .00 & e. 00 & .00 & e. 00 \\
\hline 15 & .00 & .00 & .05 & .00 & .00 & .00 & .00 & .20 & .05 & e. 00 & .00 & e. 00 \\
\hline 16 & .70 & .45 & .00 & .00 & .00 & .00 & .00 & .00 & .00 & .00 & .00 & e. 00 \\
\hline 17 & .30 & .45 & .05 & .00 & .00 & .00 & .00 & .00 & .00 & .00 & .00 & e1.00 \\
\hline 18 & .00 & .15 & .20 & .00 & .00 & .10 & .00 & .10 & .00 & .00 & .00 & e. 00 \\
\hline 19 & .00 & .00 & .15 & .00 & .10 & .35 & .00 & .00 & .00 & .00 & .00 & e.00 \\
\hline 20 & .00 & .00 & .10 & .00 & .00 & .00 & .00 & .00 & .00 & .00 & .00 & e.00 \\
\hline 21 & .00 & .00 & .25 & .00 & .00 & .00 & .00 & .00 & .00 & .00 & .00 & e.00 \\
\hline 22 & .00 & .10 & .55 & .00 & .00 & .00 & .00 & .00 & .00 & .25 & .00 & e. 00 \\
\hline 23 & .00 & .70 & .10 & .00 & .00 & .00 & .00 & .05 & .00 & .15 & .05 & e. 00 \\
\hline 24 & .00 & .10 & .60 & .00 & .00 & .05 & .30 & .00 & .00 & .00 & .00 & e. 00 \\
\hline 25 & .00 & .30 & .05 & .00 & .00 & .65 & .00 & .00 & .00 & .00 & .00 & e.00 \\
\hline 26 & .00 & .20 & .10 & .00 & .00 & .10 & .00 & .00 & e. 00 & .05 & .00 & e. 00 \\
\hline 27 & .00 & .00 & .20 & .00 & .00 & .05 & .15 & .00 & e. 00 & .00 & .00 & e. 00 \\
\hline 28 & .00 & .05 & .05 & .00 & .00 & .05 & .15 & .00 & e. 00 & .00 & .00 & e. 00 \\
\hline 29 & .00 & .00 & .10 & .00 & -- & .00 & .00 & .15 & e. 00 & .05 & .00 & e.00 \\
\hline 30 & .00 & .00 & .20 & .00 & --- & .00 & .00 & .15 & e. 00 & .00 & .00 & e. 05 \\
\hline 31 & .00 & --- & .00 & .00 & --- & .50 & --- & .00 & --- & .00 & .00 & --- \\
\hline TOTAL & 1.00 & 3.15 & 2.80 & 0.95 & 0.85 & 2.45 & 0.90 & 1.90 & 2.25 & 0.60 & 0.50 & 1.05 \\
\hline CAL YR & 1988 & TOTAL & & & & & & & & & & \\
\hline WTR YR & 1989 & TOTAL & & & & & & & & & & \\
\hline
\end{tabular}

e Estimated 
EAST STEWART BASIN

EAST STEWART CREEK NEAR IONE, NV

DAILY TOTAL PRECIPITATION, WATER YEAR OCTOBER 1989 TO SEPTEMBER 1990 INCHES

\begin{tabular}{|c|c|c|c|c|c|c|c|c|c|c|c|c|}
\hline DAY & OCT & NOV & DEC & JAN & FEB & MAR & APR & MAY & JUN & JUL & AUG & SEP \\
\hline 1 & e. 00 & e. 00 & .00 & .05 & .30 & .00 & .00 & .00 & .00 & .00 & .05 & .00 \\
\hline 2 & e. 15 & .00 & .00 & .25 & .00 & .00 & .00 & .00 & .00 & .10 & .00 & .00 \\
\hline 3 & e. 10 & .00 & .00 & .05 & .10 & .00 & .00 & .00 & .00 & .00 & .00 & .00 \\
\hline 4 & e.00 & .00 & .00 & .00 & .00 & .10 & .00 & .00 & .00 & .00 & .00 & .00 \\
\hline 5 & e. 00 & .00 & .00 & .00 & .00 & .60 & .00 & .00 & .00 & .00 & .00 & .00 \\
\hline 6 & e.00 & .00 & .10 & .00 & .10 & .10 & .20 & .05 & .00 & .00 & .00 & .00 \\
\hline 7 & e.00 & .00 & .00 & .00 & .15 & .00 & 1.00 & .00 & .00 & .00 & .00 & .05 \\
\hline 8 & e. 00 & .00 & .00 & .00 & .00 & .00 & .65 & .00 & .00 & .00 & .00 & .00 \\
\hline 9 & e.00 & .00 & .00 & .00 & .00 & .00 & .25 & .00 & .15 & .00 & .00 & .00 \\
\hline 10 & e. 00 & .00 & .00 & .00 & .00 & .10 & .00 & .00 & .25 & .00 & .00 & .00 \\
\hline 11 & e.00 & .00 & .00 & .00 & .00 & .10 & .00 & .05 & .00 & .00 & .50 & .00 \\
\hline 12 & e. 00 & .00 & .00 & .00 & .10 & .10 & .00 & .00 & .00 & .00 & .20 & .00 \\
\hline 13 & e.00 & .00 & .00 & .10 & .00 & .00 & .00 & .00 & .05 & .40 & .00 & .00 \\
\hline 14 & e.00 & .00 & .00 & .65 & .00 & .00 & .00 & .00 & .05 & .20 & .00 & .00 \\
\hline 15 & e.00 & .00 & .00 & .05 & .00 & .00 & .00 & .00 & .05 & .00 & .75 & .00 \\
\hline 16 & e.00 & .00 & .00 & .00 & .10 & .00 & .05 & .00 & .00 & .30 & .25 & .00 \\
\hline 17 & e.0o & .00 & .00 & .30 & .30 & .00 & .30 & .10 & .00 & .25 & .00 & .00 \\
\hline 18 & e.00 & .00 & .00 & .00 & .60 & .00 & .15 & .00 & .00 & .00 & .15 & .10 \\
\hline 19 & e.00 & .00 & .00 & .00 & .30 & .00 & .00 & .00 & .00 & .05 & .15 & .00 \\
\hline 20 & e. 00 & .00 & .00 & .00 & .00 & .00 & .10 & .00 & .00 & .00 & .25 & .00 \\
\hline 21 & e. 00 & .00 & .00 & .00 & .00 & .00 & .15 & .00 & .00 & .00 & .10 & .05 \\
\hline 22 & e. 00 & .00 & .00 & .00 & .00 & .00 & .10 & .00 & .00 & .00 & .00 & .20 \\
\hline 23 & e. 00 & .00 & .00 & .00 & .00 & .00 & .90 & .30 & .00 & .00 & .00 & .55 \\
\hline 24 & e. 00 & .35 & .00 & .00 & .00 & .00 & .25 & .00 & .00 & .05 & .00 & .00 \\
\hline 25 & e1.00 & .55 & .00 & .00 & .00 & .00 & .00 & .00 & .00 & .00 & .00 & .00 \\
\hline 26 & e. 00 & .00 & .00 & .00 & .00 & .00 & .00 & .00 & .00 & .00 & .00 & .00 \\
\hline 27 & e.00 & .00 & .00 & .00 & .00 & .00 & .00 & .00 & .00 & .00 & .00 & .00 \\
\hline 28 & e. 00 & .00 & .20 & .00 & .00 & .10 & .20 & .90 & .00 & .00 & .00 & .40 \\
\hline 29 & e. 00 & .00 & .00 & .00 & --- & .00 & .00 & .80 & .00 & .00 & .00 & .10 \\
\hline 30 & e. 00 & .00 & .00 & .20 & --- & .00 & .00 & .00 & .00 & .00 & .00 & .00 \\
\hline 31 & e. 00 & --- & .00 & .10 & --- & .00 & --- & .10 & -- & .00 & .00 & --- \\
\hline TOTAL & 1.25 & 0.90 & 0.30 & 1.75 & 2.05 & 1.20 & 4.30 & 2.30 & 0.55 & 1.35 & 2.40 & 1.45 \\
\hline
\end{tabular}

CAL YR 1989 TOTAL 13.90

WTR YR 1990 TOTAL 19.80

e Estimated 
EAST STEWART BASIN

EAST STEWART CREEK NEAR IONE, NV

DAILY TOTAL PRECIPITATION, WATER YEAR OCTOBER 1990 TO SEPTEMBER 1991 INCHES

\begin{tabular}{|c|c|c|c|c|c|c|c|c|c|c|c|c|}
\hline DAY & OCT & NOV & DEC & JAN & FEB & MAR & APR & MAY & JUN & JUL & AUG & SEP \\
\hline 1 & .00 & .20 & .00 & .00 & .00 & .10 & e.17 & e1.10 & .00 & .00 & .00 & .00 \\
\hline 2 & .00 & .00 & .00 & .00 & .00 & .10 & .00 & e. 40 & .00 & .00 & .10 & .00 \\
\hline 3 & .00 & .00 & .00 & .10 & .00 & .25 & .00 & .00 & .00 & .00 & .00 & .07 \\
\hline 4 & .00 & .00 & .00 & .10 & .00 & .25 & .00 & .00 & .00 & .00 & .00 & .00 \\
\hline 5 & .00 & .10 & .00 & .00 & .15 & .00 & .00 & .00 & .00 & .00 & .00 & .06 \\
\hline 6 & .00 & .00 & .00 & .05 & .00 & .00 & .00 & .00 & .00 & .00 & .00 & .10 \\
\hline 7 & .00 & .00 & .00 & .00 & .00 & .00 & .00 & .00 & .00 & .00 & .00 & .02 \\
\hline 8 & .00 & .00 & .00 & .00 & .00 & .00 & .00 & .00 & .00 & .00 & .00 & .12 \\
\hline 9 & .00 & .00 & .00 & .00 & .00 & .00 & .00 & .63 & .00 & .00 & .00 & .60 \\
\hline 10 & .00 & .00 & .00 & .00 & .00 & e.17 & .20 & .38 & .00 & .00 & .00 & .00 \\
\hline 11 & .00 & .00 & .10 & .00 & .00 & .00 & .20 & .08 & .00 & .00 & .00 & .00 \\
\hline 12 & .00 & .00 & .00 & .00 & .00 & .00 & e. 52 & .00 & .00 & .00 & .00 & .00 \\
\hline 13 & .00 & .00 & .30 & .10 & .00 & e. 34 & .00 & 1.30 & .00 & .00 & .15 & .00 \\
\hline 14 & .00 & .00 & .05 & .00 & .00 & e. 60 & .00 & .00 & .00 & .00 & .00 & .00 \\
\hline 15 & .00 & .00 & .15 & .10 & .35 & e. 30 & .00 & .00 & .00 & .00 & .00 & .00 \\
\hline 16 & .00 & .00 & .00 & .05 & .00 & .00 & .00 & .10 & .00 & .00 & .00 & .00 \\
\hline 17 & .00 & .00 & .00 & .00 & .00 & e. 17 & .00 & .20 & .00 & .00 & .00 & .00 \\
\hline 18 & .00 & .00 & .00 & .00 & .00 & e. 65 & .00 & e.33 & .00 & .00 & .00 & .00 \\
\hline 19 & .50 & .20 & .30 & .00 & .00 & e. 22 & .00 & e. 05 & .00 & .00 & .00 & .00 \\
\hline 20 & .00 & .20 & .25 & .00 & .00 & e. 47 & .00 & e1.17 & .00 & .00 & .00 & .00 \\
\hline 21 & .00 & .00 & .05 & .00 & .00 & e. 34 & .00 & .00 & .00 & .00 & .00 & .00 \\
\hline 22 & .00 & .00 & .00 & .00 & .00 & .00 & e. 26 & .00 & .00 & .00 & .00 & .00 \\
\hline 23 & .00 & .00 & .00 & .00 & .00 & .00 & .00 & .00 & .00 & .00 & .00 & .00 \\
\hline 24 & .00 & .00 & .00 & .00 & .00 & e. 43 & e. 26 & .00 & .10 & .00 & .00 & .00 \\
\hline 25 & .00 & .40 & .00 & .00 & .00 & e. 26 & e. 26 & .00 & .20 & .00 & .25 & .00 \\
\hline 26 & .00 & .10 & .00 & .00 & .00 & e.17 & .00 & .00 & .00 & .00 & .00 & .00 \\
\hline 27 & .00 & .00 & .00 & .00 & .30 & .00 & .00 & .00 & .10 & .00 & .00 & .80 \\
\hline 28 & .00 & .00 & .00 & .00 & .10 & .00 & .00 & .00 & .25 & .00 & .00 & .10 \\
\hline 29 & .00 & .00 & .00 & .00 & --- & .00 & .00 & .00 & .15 & .00 & .00 & .00 \\
\hline 30 & .00 & .00 & .00 & .00 & -- & .00 & .00 & e. 44 & .00 & .00 & .00 & .00 \\
\hline 31 & .00 & -- & .00 & .00 & --- & .00 & -- & .00 & -- & .00 & .00 & -- \\
\hline TOTAL & 0.50 & 1.20 & 1.20 & 0.50 & 0.90 & 4.82 & 1.87 & 6.18 & 0.80 & 0.00 & 0.50 & 1.87 \\
\hline
\end{tabular}

CAL YR 1990 TOTAL 20.25

WTR YR 1991 TOTAL 20.34

e Estimated 
EAST STEWART BASIN

EAST STEWART CREEK NEAR IONE, NV

PRECIPITATION WATER QUALITY, WATER YEARS OCTOBER 1984 TO SEPTEMBER 1991

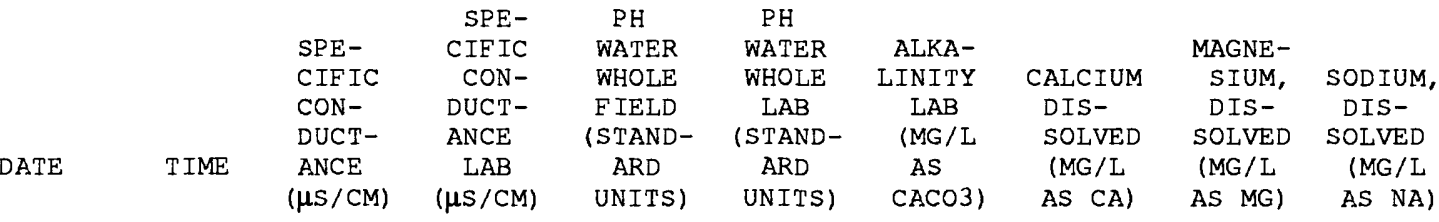

\begin{tabular}{|c|c|c|c|c|c|c|c|c|c|}
\hline $\begin{array}{c}\text { JAN } 1985 \\
11 \ldots \\
\text { APR }\end{array}$ & 0900 & -- & -- & -- & -- & -- & 0.33 & 0.06 & 0.16 \\
\hline$\underset{\text { JUL }}{03 \ldots}$ & 0945 & -- & -- & -- & -- & -- & 0.50 & 0.08 & 2.7 \\
\hline $\begin{array}{l}08 \ldots \\
\mathrm{SEP}\end{array}$ & 1015 & -- & -- & -- & -- & -- & 2.7 & 0.30 & 3.1 \\
\hline JAN 27986 & 1600 & 33 & -- & -- & -- & -- & 1.4 & 0.20 & 0.50 \\
\hline $\operatorname{APR}^{11 \ldots}$ & 1015 & 7 & 15 & -- & 7.0 & 2.0 & 0.90 & 0.05 & 1.1 \\
\hline$\stackrel{01 \ldots}{\text { JUL }}$ & 0900 & 9 & 13 & 6.3 & 6.6 & 4.0 & 0.35 & 0.03 & 0.73 \\
\hline $\begin{array}{l}10 \cdots \\
\operatorname{SEP}\end{array}$ & 0830 & 74 & 62 & -- & 7.2 & -- & 5.3 & 0.39 & 4.6 \\
\hline $\begin{array}{c}30 \\
\mathrm{DEC}\end{array}$ & 1030 & 28 & 26 & -- & 6.5 & 4.0 & 1.3 & 0.14 & 0.97 \\
\hline $\begin{array}{l}30 \ldots \\
\text { MAR } 1987\end{array}$ & 1300 & 13 & 14 & -- & 6.1 & 4.0 & 0.60 & 0.11 & 0.35 \\
\hline$\underset{\text { JUN }}{31 \ldots}$ & 0900 & 9 & 5 & -- & 7.1 & 2.0 & 0.35 & 0.04 & 0.26 \\
\hline $\begin{array}{l}30 \ldots \\
\operatorname{SEP}\end{array}$ & 1300 & 7 & 15 & 6.2 & 7.2 & 4.0 & 0.34 & 0.07 & 0.20 \\
\hline $\begin{array}{l}24 \ldots \\
\text { JAN } 1988\end{array}$ & 1230 & -- & -- & -- & -- & -- & -- & -- & -- \\
\hline$\underset{A P R}{09} \cdots$ & 1630 & -- & 6 & -- & 6.0 & 3.0 & 0.57 & 0.13 & 0.22 \\
\hline$\stackrel{05 \ldots}{\text { JUN }}$ & 1230 & -- & -- & -- & -- & -- & 0.52 & 0.07 & 0.98 \\
\hline $\begin{array}{l}28 \ldots \\
\operatorname{SEP}\end{array}$ & 1614 & 8 & 11 & 6.4 & 6.9 & 3.0 & 0.85 & 0.08 & 0.70 \\
\hline $\begin{array}{l}28 \ldots \\
\text { FEB } 1989\end{array}$ & 1550 & 8 & 12 & 6.1 & 6.2 & 3.0 & 0.69 & 0.13 & 0.19 \\
\hline $\begin{array}{l}22 \\
\mathrm{APR}\end{array}$ & 1400 & 12 & 13 & 6.6 & 6.6 & 5.0 & 1.3 & 0.28 & 0.20 \\
\hline $\operatorname{MAY}_{11 \ldots}^{11 . .}$ & 1010 & 6 & 8 & 6.9 & 6.9 & 2.0 & 0.41 & $<0.01$ & 0.80 \\
\hline $\operatorname{SEP}^{25 \ldots}$ & 1400 & 8 & 10 & 6.3 & 6.6 & 2.0 & 0.67 & 0.07 & $<0.58$ \\
\hline $\begin{array}{l}14 \ldots \\
\text { Nov }\end{array}$ & 1520 & 12 & 12 & 5.8 & 6.2 & -- & 0.82 & 0.04 & 0.40 \\
\hline JAN 1990 & 1324 & 7 & 9 & 6.2 & 6.2 & 1.6 & 0.86 & 0.09 & 0.40 \\
\hline $\begin{array}{l}31 \ldots \\
\mathrm{APR}\end{array}$ & 1415 & 4 & 6 & 5.3 & 6.6 & 1.0 & 0.22 & 0.05 & 0.30 \\
\hline${ }_{M A Y}^{03} \cdots$ & 1100 & 5 & 9 & 6.2 & 6.4 & $<0.5$ & 0.55 & 0.04 & 0.70 \\
\hline $\mathrm{JUL}^{24 \ldots}$ & 1240 & 7 & 9 & 6.4 & 6.4 & $<0.5$ & 0.56 & 0.05 & 0.70 \\
\hline $\operatorname{SEP}^{12} \cdots$ & 0800 & 8 & 15 & 6.4 & 6.3 & $<0.5$ & 0.67 & 0.11 & 0.50 \\
\hline JAN $25 . \ddot{9} 91$ & 0915 & 9 & 9 & 6.0 & 6.3 & 1.0 & 0.56 & 0.08 & 0.20 \\
\hline$\underset{\text { JUN }}{29 . \cdots}$ & 1400 & 23 & 29 & 6.3 & 6.6 & 6.7 & 2.4 & 0.49 & 0.40 \\
\hline$\underset{\text { JUL }}{01 \ldots}$ & 1400 & 3 & 4 & 6.1 & 6.1 & $<0.5$ & 0.35 & 0.04 & 0.30 \\
\hline $\operatorname{SEP}^{24 \ldots}$ & 1016 & 12 & 22 & 5.8 & 5.0 & $<0.5$ & 0.94 & 0.12 & 0.90 \\
\hline $24 \ldots$ & 0915 & 12 & 13 & 6.1 & 5.9 & 1.4 & 0.89 & 0.12 & 0.30 \\
\hline
\end{tabular}


EAST STEWART BASIN

EAST STEWART CREEK NEAR IONE, NV

PRECIPITATION WATER QUALITY, WATER YEARS OCTOBER 1984 TO SEPTEMBER 1991

\begin{tabular}{|c|c|c|c|c|c|c|c|c|}
\hline DATE & $\begin{array}{l}\text { POTAS- } \\
\text { SIUM, } \\
\text { DIS- } \\
\text { SOLVED } \\
\text { (MG/L } \\
\text { AS K) }\end{array}$ & $\begin{array}{l}\text { SULFATE } \\
\text { DIS- } \\
\text { SOLVED } \\
\text { (MG/L } \\
\text { AS SO4) }\end{array}$ & $\begin{array}{l}\text { CHLO- } \\
\text { RIDE, } \\
\text { DIS- } \\
\text { SOLVED } \\
\text { (MG/L } \\
\text { AS CL) }\end{array}$ & $\begin{array}{l}\text { FLUO- } \\
\text { RIDE, } \\
\text { DIS- } \\
\text { SOLVED } \\
\text { (MG/L } \\
\text { AS F) }\end{array}$ & $\begin{array}{c}\text { BROMIDE } \\
\text { DIS- } \\
\text { SOLVED } \\
\text { (MG/L } \\
\text { AS BR) }\end{array}$ & $\begin{array}{l}\text { SILICA, } \\
\text { DIS- } \\
\text { SOLVED } \\
\text { (MG/L } \\
\text { AS } \\
\text { SIO2) }\end{array}$ & $\begin{array}{c}\text { NITRO- } \\
\text { GEN, } \\
\text { NITRATE } \\
\text { DIS- } \\
\text { SOLVED } \\
\text { (MG/L } \\
\text { AS N) }\end{array}$ & $\begin{array}{l}\text { PHOS- } \\
\text { PHORUS } \\
\text { ORTHO, } \\
\text { DIS- } \\
\text { SOLVED } \\
\text { (MG/L } \\
\text { AS P) }\end{array}$ \\
\hline JAN 1985 & & & & & & & & \\
\hline $\operatorname{APR}^{11 \ldots}$ & 0.06 & 0.42 & 0.17 & 0.01 & -- & $<1.0$ & -- & -- \\
\hline $\begin{array}{l}03 \ldots \\
\text { JUL }\end{array}$ & 0.10 & 1.8 & 0.67 & -- & -- & $<1.0$ & -- & -- \\
\hline $\begin{array}{l}08 \ldots \\
\text { SEP }\end{array}$ & -- & 2.5 & 0.76 & 0.03 & -- & 15 & -- & -- \\
\hline $\begin{array}{c}27 \\
\text { JAN } 1986\end{array}$ & -- & 1.9 & 0.52 & 0.02 & 0.010 & 0.20 & -- & -- \\
\hline$\underset{A P R}{11 \ldots}$ & -- & 0.76 & 0.55 & 0.03 & -- & $<0.01$ & -- & -- \\
\hline$\underset{\text { JUL }}{01 \ldots}$ & 0.24 & 0.77 & 0.46 & 0.04 & 0.009 & 35 & -- & -- \\
\hline $\operatorname{SEP}^{10} \ldots$ & 1.0 & 3.7 & 4.3 & 0.09 & $<0.010$ & -- & 0.540 & $<0.010$ \\
\hline $\begin{array}{c}30 \\
D E C\end{array}$ & 1.1 & 1.9 & 1.1 & 0.02 & -- & -- & -- & -- \\
\hline $\begin{array}{l}30 \\
\operatorname{MAR} 1987\end{array}$ & 1.5 & 0.72 & 0.76 & 0.07 & -- & 22 & -- & -- \\
\hline $\begin{array}{l}31 \ldots \\
\text { JUN }\end{array}$ & 0.08 & 0.25 & 0.19 & 0.02 & -- & 34 & -- & -- \\
\hline $\begin{array}{l}30 \ldots \\
\operatorname{SEP}\end{array}$ & 0.12 & 0.70 & 0.16 & 0.04 & -- & 0.0 & -- & -- \\
\hline JAN 1988 & -- & 6.8 & 3.8 & -- & 0.025 & -- & -- & -- \\
\hline $\begin{array}{c}09 \\
A P R\end{array}$ & 0.36 & 0.17 & 0.33 & -- & -- & 0.04 & -- & -- \\
\hline$\underset{\text { JUN }}{05}$ & 0.10 & 1.5 & 0.61 & -- & 0.015 & -- & -- & -- \\
\hline$\stackrel{28 \ldots}{\operatorname{SEP}}$ & 0.21 & 0.89 & 0.61 & -- & -- & 0.06 & -- & -- \\
\hline FEB 28.1989 & 0.30 & 0.97 & 0.24 & -- & -- & 0.09 & -- & -- \\
\hline$\underset{A P R}{22} \cdots$ & 1.1 & 0.85 & 0.59 & 0.04 & $<0.010$ & $<0.01$ & $<0.010$ & $<0.010$ \\
\hline $\operatorname{MAY}^{11} \ldots$ & 0.13 & 1.0 & 0.38 & 0.15 & 0.41 & 0.03 & $<0.010$ & $<0.010$ \\
\hline $\begin{array}{l}25 \ldots \\
\text { SEP }\end{array}$ & 0.09 & 1.0 & 0.30 & $<0.01$ & $<0.010$ & 0.12 & 0.240 & $<0.010$ \\
\hline $\begin{array}{l}14 \ldots \\
\text { NoV }\end{array}$ & -- & 1.2 & 0.41 & $<0.01$ & -- & 0.07 & -- & -- \\
\hline JAN 079 & -- & 0.58 & 0.50 & 0.01 & $<0.050$ & $<0.01$ & 0.380 & $<0.010$ \\
\hline $\begin{array}{l}31 \ldots \\
A P R\end{array}$ & -- & 0.54 & 0.17 & $<0.01$ & $<0.010$ & 0.04 & 0.160 & $<0.010$ \\
\hline$\underset{M A Y}{03}$ & -- & 0.64 & 0.64 & 0.01 & $<0.010$ & $<0.01$ & 0.150 & $<0.010$ \\
\hline${ }_{\text {JUL }} 24 \ldots$ & 0.10 & 0.81 & 0.43 & $<0.01$ & $<0.010$ & $<0.01$ & 0.270 & $<0.010$ \\
\hline $\operatorname{sEP}^{12} \cdots$ & 0.90 & 0.73 & 0.51 & 0.05 & $<0.010$ & $<0.01$ & 3.26 & 0.010 \\
\hline JAN 1991 & 0.40 & 0.68 & 0.22 & $<0.01$ & $<0.010$ & $<0.01$ & 0.290 & $<0.010$ \\
\hline $\begin{array}{l}29 \ldots \\
\text { JUN }\end{array}$ & 3.2 & 0.97 & 1.4 & 0.25 & $<0.010$ & $<0.10$ & 0.020 & $<0.010$ \\
\hline$\underset{\text { JUL }}{01 \ldots}$ & $<0.10$ & 0.48 & 0.10 & 0.03 & $<0.010$ & $<0.10$ & 0.150 & $<0.010$ \\
\hline $\begin{array}{l}24 \ldots \\
\text { SEP }\end{array}$ & 0.60 & 1.1 & 0.65 & 0.08 & $<0.010$ & 0.20 & 1.20 & $<0.010$ \\
\hline $24 \ldots$ & 1.0 & 1.5 & 0.35 & 0.05 & $<0.010$ & 0.10 & 0.380 & $<0.010$ \\
\hline
\end{tabular}


EAST STEWART BASIN

EAST STEWART CREEK NEAR IONE, NV

PRECIPITATION WATER QUALITY, WATER YEARS OCTOBER 1984 TO SEPTEMBER 1991

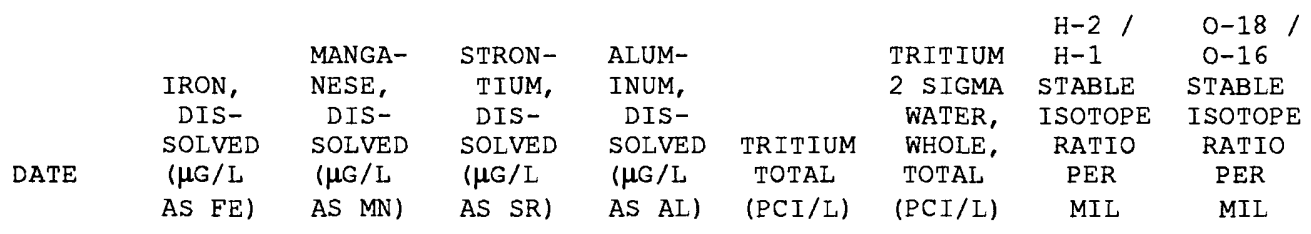

\begin{tabular}{|c|c|c|c|c|c|c|c|c|}
\hline $\begin{array}{c}\text { JAN } 1985 \\
11 \ldots\end{array}$ & -- & -- & -- & -- & -- & -- & -130.0 & -17.80 \\
\hline $\mathrm{APR}$ & & & & & & & & \\
\hline$\underset{\text { JUL }}{03 \ldots}$ & -- & -- & -- & -- & -- & -- & -- & -- \\
\hline $\begin{array}{c}08 \ldots \\
\operatorname{SEP}\end{array}$ & 4 & $<1$ & 23 & -- & -- & -- & -71.0 & -10.25 \\
\hline${ }_{\text {JAN }}^{27} \ddot{1986}$ & $<3$ & 4 & 19 & -- & -- & -- & -71.5 & -10.25 \\
\hline${ }_{\mathrm{APR}}^{11 \cdots}$ & 12 & 7 & 9 & -- & -- & -- & -125.5 & -17.25 \\
\hline JUL $^{01 \ldots}$ & 15 & -- & -- & -- & -- & -- & -105.0 & -14.20 \\
\hline $\operatorname{SEP}_{10} \cdots$ & 10 & -- & 130 & -- & -- & -- & -57.5 & -7.40 \\
\hline${ }_{\mathrm{DEC}}^{30} \ldots$ & $<10$ & -- & 80 & -- & -- & -- & -81.5 & -11.00 \\
\hline $\begin{array}{l}30 \ldots \\
\operatorname{MAR} 1987\end{array}$ & 11 & -- & 4 & -- & -- & -- & -99.5 & $-14 \cdot 20$ \\
\hline$\underset{\text { JUN }}{31 \ldots}$ & $<3$ & -- & 3 & -- & 20 & 2.0 & -142.5 & -19.20 \\
\hline $\begin{array}{l}30 \ldots \\
\operatorname{SEP}\end{array}$ & $<3$ & - & 3 & -- & 60 & 6.0 & -78.4 & -11.50 \\
\hline JAN 1988 & -- & -- & -- & -- & -- & -- & -56.5 & -4.70 \\
\hline $\begin{array}{c}09 \ldots \\
A P R\end{array}$ & 3 & -- & 4 & -- & 19 & 3.0 & -131.5 & -18.15 \\
\hline$\underset{\text { JUN }}{05 \ldots}$ & $<10$ & -- & $<10$ & -- & -- & -- & -89.0 & -11.05 \\
\hline $\operatorname{seP}^{28} \cdots$ & 4 & -- & 9 & -- & 43 & 4.0 & -106.9 & -14.40 \\
\hline FEB 1989 & $<3$ & -- & 5 & $<10$ & 40 & 5.8 & -81.0 & -12.20 \\
\hline $\begin{array}{l}22 \cdots \\
\text { APR }\end{array}$ & 10 & 11 & $<10$ & -- & 34 & 3.0 & -117.0 & -16.25 \\
\hline${ }_{M A Y}^{11} \ldots$ & $<3$ & 2 & $<10$ & -- & -- & -- & -127.5 & -17.10 \\
\hline $\operatorname{SEP}^{25 \ldots}$ & 5 & -- & 6 & -- & -- & -- & -111.5 & -15.55 \\
\hline $\begin{array}{l}14 \ldots \\
\text { Nov }\end{array}$ & $<3$ & $<1$ & -- & -- & -- & -- & -86.5 & -12.05 \\
\hline $\begin{array}{l}07 \\
\text { JAN }\end{array}$ & $<3$ & 3 & -- & -- & -- & -- & -106.5 & $-15 \cdot 10$ \\
\hline$\underset{A P R}{31} \cdots$ & 3 & 3 & -- & -- & 16 & 2.0 & -123.5 & -16.90 \\
\hline $\begin{array}{c}03 \\
\text { MAY }\end{array}$ & 4 & 4 & -- & -- & 29 & 3.0 & -144.0 & -19.40 \\
\hline$\underset{\text { JUL }}{24 \ldots}$ & 9 & 4 & -- & -- & -- & -- & -112.0 & $-15 \cdot 20$ \\
\hline $\operatorname{SEP}_{\mathrm{SEP}}^{12 \ldots}$ & 2 & 5 & -- & -- & -- & -- & -105.0 & -8.35 \\
\hline JAN 1991 & $<2$ & 3 & -- & -- & - & -- & -77.5 & -11.60 \\
\hline$\underset{\text { JUN }}{29 \ldots}$ & 3 & 29 & -- & -- & -- & -- & -128.0 & -17.35 \\
\hline$\underset{\text { JUI }}{01 \ldots}$ & 5 & 4 & -- & -- & 41 & 3.0 & -132.0 & -18.00 \\
\hline$\underset{\text { SEP }}{24} \ldots$ & 15 & 11 & -- & -- & -- & -- & -129.0 & -17.65 \\
\hline $24 \ldots$ & $<3$ & 13 & -- & -- & -- & -- & -50.0 & -7.70 \\
\hline
\end{tabular}


EAST STEWART BASIN

EAST STEWART CREEK NEAR IONE, NV

WATER-QUALITY ANALYSIS FROM THE UNSATURATED-ZONE LYSIMETER, WATER YEARS OCTOBER 1986 TO SEPTEMBER 1991

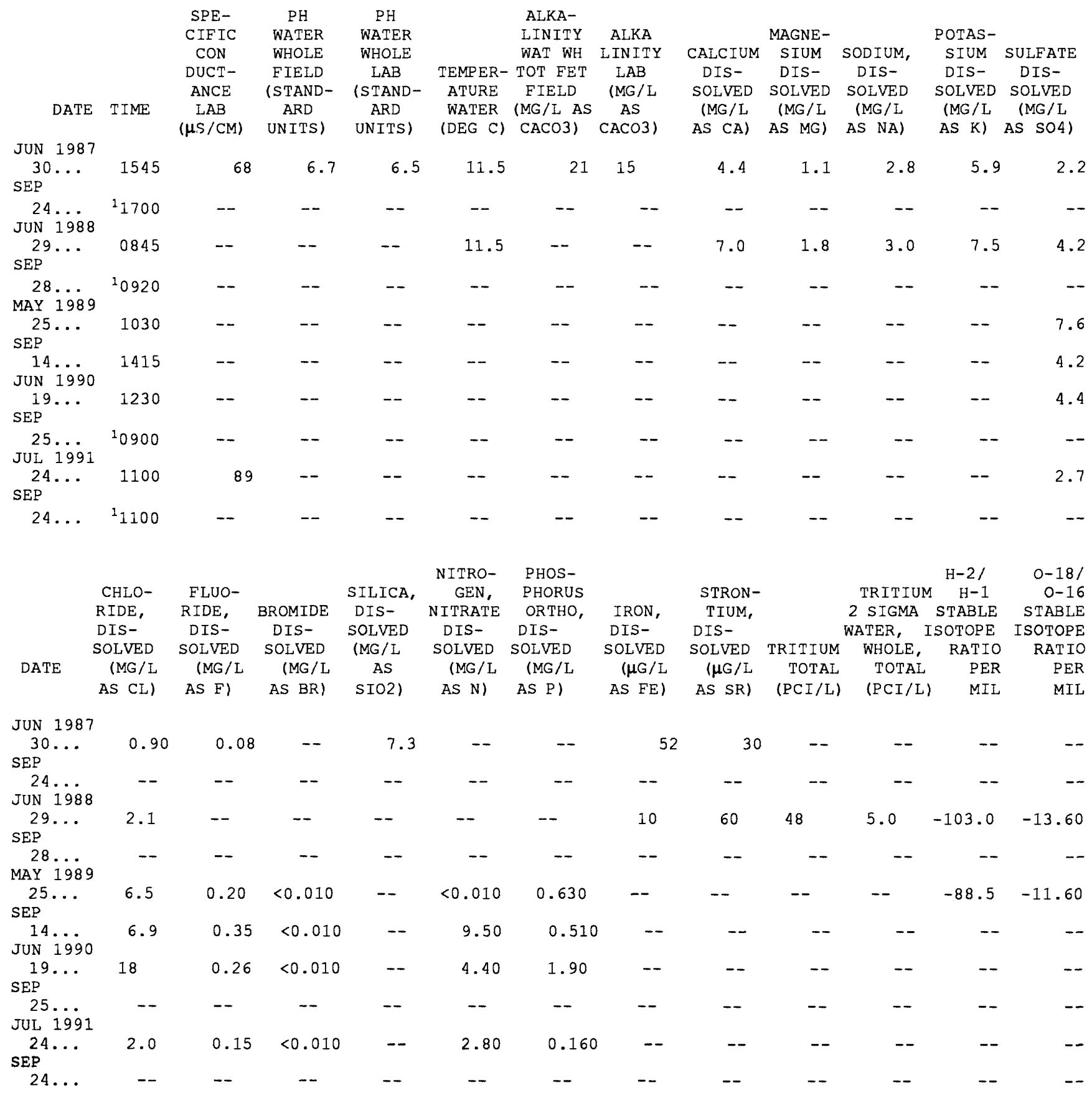

1 Sample collector dry. 
EAST STEWART BASIN

EAST STEWART CREEK NEAR IONE, NV

DAILY MEAN DISCHARGE, WATER YEAR OCTOBER 1986 TO SEPTEMBER 1987 CUBIC FEET PER SECOND

\begin{tabular}{|c|c|c|c|c|c|c|c|c|c|c|c|c|}
\hline DAY & OCT & NOV & DEC & JAN & FEB & MAR & APR & MAY & JUN & JUL & AUG & SEP \\
\hline 1 & -- & --- & --- & --- & --- & --- & -- & -- & 1.7 & .67 & .30 & .20 \\
\hline 2 & -- & -- & --- & --- & --- & --- & --- & -- & 1.9 & .65 & .30 & .21 \\
\hline 3 & --- & -- & --- & --- & --- & --- & --- & -- & 2.2 & .63 & .29 & .22 \\
\hline 4 & --- & --- & --- & --- & --- & --- & --- & --- & 2.4 & .61 & .29 & .22 \\
\hline 5 & -- & --- & -- & --- & --- & --- & -- & -- & 2.5 & .59 & .28 & .21 \\
\hline 6 & -- & -- & -- & -- & --- & -- & -- & -- & 2.7 & .56 & .28 & .21 \\
\hline 7 & -- & $\cdots$ & -- & -- & -- & $\cdots$ & -- & .32 & 2.7 & .55 & .27 & .21 \\
\hline 8 & -- & $-\cdots$ & -- & -- & -- & --- & -- & .34 & 2.6 & .53 & .27 & .21 \\
\hline 9 & -- & -- & -- & -- & -- & -- & -- & .39 & 2.4 & .51 & .28 & .21 \\
\hline 10 & --- & --- & --- & --- & --- & --- & -- & .45 & 2.2 & .49 & .28 & .20 \\
\hline 11 & --- & --- & --- & --- & --- & --- & -- & .48 & 2.1 & .46 & .28 & .20 \\
\hline 12 & -- & -- & -- & -- & -- & -- & -- & .51 & 2.1 & .43 & .27 & .20 \\
\hline 13 & -- & -- & -- & -- & -- & -- & -- & .58 & 2.0 & .41 & .27 & .20 \\
\hline 14 & --- & --- & --- & --- & -- & $\cdots$ & -- & .65 & 1.8 & .40 & .27 & .20 \\
\hline 15 & --- & --- & --- & --- & --- & --- & --- & .75 & 1.7 & .39 & .27 & .20 \\
\hline 16 & -- & -- & -- & -- & -- & --- &.- & .92 & 1.6 & .39 & .26 & .20 \\
\hline 17 & -- & --- & -- & --- & -- & -- & -- & 1.3 & 1.4 & .38 & .25 & .19 \\
\hline 18 & -- & -- & -- & -- & -- & -- & -- & 1.5 & 1.3 & .38 & .25 & .19 \\
\hline 19 & -- & --- & --- & --- & -- & --- & -- & 1.7 & 1.2 & .37 & .24 & .19 \\
\hline 20 & --- & --- & --- & --- & --- & --- & --- & 1.8 & 1.2 & .36 & .24 & .19 \\
\hline 21 & --- & --- & --- & --- & --- & --- & --- & 1.8 & 1.1 & .36 & .23 & .19 \\
\hline 22 & -- & --- & --- & --- & --- & --- & -- & 1.9 & 1.1 & .37 & .23 & .19 \\
\hline 23 & -- & -- & -- & -- & -- & --- & -- & 1.9 & 1.0 & .36 & .23 & .18 \\
\hline 24 & -- & --- & -- & -- & -- & --- & -- & 1.9 & .97 & .35 & .23 & .18 \\
\hline 25 & --- & --- & --- & --- & --- & --- & --- & 1.8 & .91 & .35 & .22 & .18 \\
\hline 26 & -- & -- & -- & -- & -- & -- & -- & 1.8 & .8 & .3 & .2 & .18 \\
\hline 27 & -- & -- & -- & -- & --- & --- & -- & 1.7 & .80 & .33 & .22 & .18 \\
\hline 28 & -- & -- & -- & -- & --- & --- & -- & 1.6 & .77 & .33 & .21 & .18 \\
\hline 29 & -- & --- & --- & -- & -- & -- & -- & 1.5 & .74 & .32 & .21 & .18 \\
\hline 30 & -- & --- & --- & -- & --- & --- & --- & 1.4 & .70 & .31 & .21 & .18 \\
\hline 31 & -- & --- & --- & --- & --- & --- & -- & 1.5 & -- & .31 & .21 & --- \\
\hline TOTAL & -- & -- & --- & -- & --- & --- & -- & -- & 48.64 & 13.49 & 7.86 & 5.88 \\
\hline MEAN & --- & -- & -- & --- & --- & --- & --- & --- & 1.62 & .44 & .25 & .20 \\
\hline MAX & -- & -- & -- & -- & -- & -- & -- & --- & 2.7 & .67 & .30 & .22 \\
\hline MIN & $\cdots$ & -- & -- & -- & --- & --- & -- & -- & .70 & .31 & .21 & .18 \\
\hline$A C-E T$ & -- & -- & -- & --- & -- & -- & $\cdots$ & --- & 96 & 27 & 16 & 12 \\
\hline
\end{tabular}


EAST STEWART BASIN

EAST STEWART CREEK NEAR IONE, NV

DAILY MEAN DISCHARGE, WATER YEAR OCTOBER 1987 TO SEPTEMBER 1988 CUBIC FEET PER SECOND

\begin{tabular}{|c|c|c|c|c|c|c|c|c|c|c|c|c|}
\hline DAY & OCT & NOV & DEC & JAN & FEB & MAR & APR & MAY & JUN & JUL & AUG & SEP \\
\hline $\begin{array}{l}1 \\
2 \\
3 \\
4 \\
5\end{array}$ & $\begin{array}{l}.18 \\
.18 \\
.18 \\
.17 \\
.17\end{array}$ & $\begin{array}{l}.16 \\
.16 \\
.16 \\
.15 \\
.15\end{array}$ & $\begin{array}{l}.12 \\
.12 \\
.12 \\
.12 \\
.12\end{array}$ & $\begin{array}{l}.11 \\
.11 \\
.11 \\
.11 \\
.11\end{array}$ & $\begin{array}{l}.08 \\
.08 \\
.08 \\
.08 \\
.08\end{array}$ & $\begin{array}{l}.07 \\
.07 \\
.07 \\
.07 \\
.07\end{array}$ & $\begin{array}{l}.09 \\
.09 \\
.09 \\
.09 \\
.09\end{array}$ & $\begin{array}{l}.31 \\
.28 \\
.27 \\
.25 \\
.25\end{array}$ & $\begin{array}{l}2.0 \\
1.9 \\
1.9 \\
2.1 \\
2.3\end{array}$ & $\begin{array}{l}1.2 \\
1.1 \\
1.1 \\
1.0 \\
.96\end{array}$ & $\begin{array}{l}.43 \\
.42 \\
.42 \\
.41 \\
.46\end{array}$ & $\begin{array}{l}.27 \\
.26 \\
.26 \\
.26 \\
.26\end{array}$ \\
\hline $\begin{array}{r}6 \\
7 \\
8 \\
9 \\
10\end{array}$ & $\begin{array}{l}.17 \\
.17 \\
.17 \\
.17 \\
.17\end{array}$ & $\begin{array}{l}.15 \\
.15 \\
.15 \\
.15 \\
.14\end{array}$ & $\begin{array}{l}.12 \\
.12 \\
.12 \\
.11 \\
.11\end{array}$ & $\begin{array}{l}.11 \\
.11 \\
.11 \\
.10 \\
.08\end{array}$ & $\begin{array}{l}.08 \\
.08 \\
.08 \\
.08 \\
.08\end{array}$ & $\begin{array}{l}.07 \\
.07 \\
.07 \\
.07 \\
.07\end{array}$ & $\begin{array}{l}.10 \\
.11 \\
.11 \\
.11 \\
.12\end{array}$ & $\begin{array}{l}.25 \\
.24 \\
.23 \\
.22 \\
.25\end{array}$ & $\begin{array}{l}2.4 \\
2.4 \\
2.3 \\
2.2 \\
2.1\end{array}$ & $\begin{array}{l}.92 \\
.89 \\
.85 \\
.81 \\
.77\end{array}$ & $\begin{array}{l}.48 \\
.46 \\
.43 \\
.41 \\
.40\end{array}$ & $\begin{array}{l}.26 \\
.26 \\
.25 \\
.25 \\
.25\end{array}$ \\
\hline $\begin{array}{l}11 \\
12 \\
13 \\
14 \\
15\end{array}$ & $\begin{array}{l}.17 \\
.17 \\
.17 \\
.17 \\
.17\end{array}$ & $\begin{array}{l}.14 \\
.14 \\
.14 \\
.14 \\
.14\end{array}$ & $\begin{array}{l}.11 \\
.11 \\
.11 \\
.11 \\
.11\end{array}$ & $\begin{array}{l}.08 \\
.08 \\
.09 \\
.09 \\
.09\end{array}$ & $\begin{array}{l}.08 \\
.08 \\
.08 \\
.08 \\
.08\end{array}$ & $\begin{array}{l}.08 \\
.07 \\
.07 \\
.07 \\
.07\end{array}$ & $\begin{array}{l}.13 \\
.15 \\
.20 \\
.20 \\
.19\end{array}$ & $\begin{array}{l}.33 \\
.46 \\
.59 \\
.66 \\
.83\end{array}$ & $\begin{array}{l}2.0 \\
1.9 \\
1.9 \\
1.9 \\
1.9\end{array}$ & $\begin{array}{l}.75 \\
.72 \\
.70 \\
.67 \\
.65\end{array}$ & $\begin{array}{l}.39 \\
.38 \\
.37 \\
.34 \\
.34\end{array}$ & $\begin{array}{l}.26 \\
.26 \\
.26 \\
.25 \\
.25\end{array}$ \\
\hline $\begin{array}{l}16 \\
17 \\
18 \\
19 \\
20\end{array}$ & $\begin{array}{l}.17 \\
.17 \\
.17 \\
.17 \\
.17\end{array}$ & $\begin{array}{l}.14 \\
.14 \\
.13 \\
.13 \\
.13\end{array}$ & $\begin{array}{l}.11 \\
.11 \\
.10 \\
.10 \\
.10\end{array}$ & $\begin{array}{l}.09 \\
.09 \\
.09 \\
.09 \\
.09\end{array}$ & $\begin{array}{l}.08 \\
.08 \\
.07 \\
.07 \\
.07\end{array}$ & $\begin{array}{l}.07 \\
.07 \\
.07 \\
.07 \\
.07\end{array}$ & $\begin{array}{l}.18 \\
.18 \\
.17 \\
.17 \\
.17\end{array}$ & $\begin{array}{l}.98 \\
.99 \\
.98 \\
.98 \\
.99\end{array}$ & $\begin{array}{l}1.9 \\
1.9 \\
1.9 \\
1.9 \\
1.8\end{array}$ & $\begin{array}{l}.63 \\
.60 \\
.59 \\
.57 \\
.55\end{array}$ & $\begin{array}{l}.33 \\
.33 \\
.32 \\
.31 \\
.31\end{array}$ & $\begin{array}{l}.25 \\
.25 \\
.24 \\
.24 \\
.24\end{array}$ \\
\hline $\begin{array}{l}21 \\
22 \\
23 \\
24 \\
25\end{array}$ & $\begin{array}{l}.17 \\
.17 \\
.17 \\
.17 \\
.17\end{array}$ & $\begin{array}{l}.13 \\
.13 \\
.13 \\
.13 \\
.13\end{array}$ & $\begin{array}{l}.10 \\
.10 \\
.10 \\
.10 \\
.11\end{array}$ & $\begin{array}{l}.09 \\
.09 \\
.09 \\
.09 \\
.09\end{array}$ & $\begin{array}{l}.07 \\
.07 \\
.07 \\
.07 \\
.07\end{array}$ & $\begin{array}{l}.08 \\
.08 \\
.08 \\
.08 \\
.08\end{array}$ & $\begin{array}{l}.17 \\
.19 \\
.19 \\
.19 \\
.20\end{array}$ & $\begin{array}{l}1.1 \\
1.2 \\
1.4 \\
1.7 \\
1.9\end{array}$ & $\begin{array}{l}1.8 \\
1.7 \\
1.7 \\
1.6 \\
1.6\end{array}$ & $\begin{array}{l}.53 \\
.51 \\
.50 \\
.50 \\
.49\end{array}$ & $\begin{array}{l}.32 \\
.32 \\
.31 \\
.30 \\
.30\end{array}$ & $\begin{array}{l}.25 \\
.25 \\
.25 \\
.24 \\
.23\end{array}$ \\
\hline $\begin{array}{l}26 \\
27 \\
28 \\
29 \\
30 \\
31\end{array}$ & $\begin{array}{r}.16 \\
.16 \\
.16 \\
.16 \\
.16 \\
.16\end{array}$ & $\begin{array}{l}.12 \\
.12 \\
.12 \\
.12 \\
.12 \\
----\end{array}$ & $\begin{array}{l}.11 \\
.11 \\
.11 \\
.11 \\
.11 \\
.11\end{array}$ & $\begin{array}{l}.09 \\
.08 \\
.08 \\
.08 \\
.09 \\
.08\end{array}$ & $\begin{array}{l}.07 \\
.07 \\
.07 \\
.07 \\
--- \\
---\end{array}$ & $\begin{array}{l}.09 \\
.10 \\
.10 \\
.10 \\
.09 \\
.09\end{array}$ & $\begin{array}{l}.21 \\
.25 \\
.26 \\
.29 \\
.33 \\
.---\end{array}$ & $\begin{array}{l}2.0 \\
2.2 \\
2.2 \\
2.2 \\
2.2 \\
2.1\end{array}$ & $\begin{array}{l}1.5 \\
1.4 \\
1.3 \\
1.2 \\
1.2 \\
---\end{array}$ & $\begin{array}{r}.48 \\
.47 \\
.46 \\
.45 \\
.44 \\
.43\end{array}$ & $\begin{array}{r}.30 \\
.29 \\
.29 \\
.28 \\
.28 \\
.27\end{array}$ & $\begin{array}{r}.22 \\
.22 \\
.24 \\
.24 \\
.24 \\
-.--\end{array}$ \\
\hline $\begin{array}{l}\text { TOTAL } \\
\text { MEAN } \\
\text { MAX } \\
\text { MIN } \\
\text { AC-FT }\end{array}$ & $\begin{array}{r}5.24 \\
.17 \\
.18 \\
.16 \\
10\end{array}$ & $\begin{array}{r}4.14 \\
.14 \\
.16 \\
.12 \\
8.2\end{array}$ & $\begin{array}{r}3.42 \\
.11 \\
.12 \\
.10 \\
6.8\end{array}$ & $\begin{array}{r}2.89 \\
.093 \\
.11 \\
.08 \\
5.7\end{array}$ & $\begin{array}{r}2.20 \\
.076 \\
.08 \\
.07 \\
4.4\end{array}$ & $\begin{array}{r}2.38 \\
.077 \\
.10 \\
.07 \\
4.7\end{array}$ & $\begin{array}{r}5.02 \\
.17 \\
.33 \\
.09 \\
10\end{array}$ & $\begin{array}{r}30.54 \\
.99 \\
2.2 \\
.22 \\
61\end{array}$ & $\begin{array}{r}55.6 \\
1.85 \\
2.4 \\
1.2 \\
110\end{array}$ & $\begin{array}{r}21.29 \\
.69 \\
1.2 \\
.43 \\
42\end{array}$ & $\begin{array}{r}11.00 \\
.35 \\
.48 \\
.27 \\
22\end{array}$ & $\begin{array}{r}7.46 \\
.25 \\
.27 \\
.22 \\
15\end{array}$ \\
\hline
\end{tabular}

$\begin{array}{llllllllll}\text { WTR YR } 1988 \text { TOTAL } & 151.18 & \text { MEAN } & 41 & \text { MAX 2.4 } & \text { MIN } & .07 & \text { AC-FT } & 300\end{array}$ 
EAST STEWART BASIN

EAST STEWART CREEK NEAR IONE, NV

DAILY MEAN DISCHARGE, WATER YEAR OCTOBER 1988 TO SEPTEMBER 1989 CUBIC EEET PER SECOND

\begin{tabular}{|c|c|c|c|c|c|c|c|c|c|c|c|c|}
\hline DAY & OCT & NOV & DEC & JAN & FEB & MAR & APR & MAY & JUN & JUL & AUG & SEP \\
\hline 1 & .25 & .22 & .19 & .17 & .11 & .10 & .11 & .28 & .85 & .60 & .32 & .24 \\
\hline 2 & .25 & .21 & .19 & .17 & .10 & .10 & .11 & .30 & .86 & .58 & .32 & .24 \\
\hline 3 & .24 & .21 & .19 & .17 & .10 & .10 & .11 & .34 & .89 & .55 & .32 & .24 \\
\hline 4 & .23 & .21 & .19 & .17 & .10 & .10 & .12 & .39 & .90 & .53 & .31 & .23 \\
\hline 5 & .23 & .21 & .19 & .17 & .10 & .11 & .13 & .43 & .90 & .52 & .30 & .23 \\
\hline 6 & .23 & .21 & .19 & .17 & .10 & .12 & .16 & .46 & .92 & .50 & .30 & .22 \\
\hline 7 & .23 & .21 & .19 & .17 & .11 & .11 & .19 & .51 & .93 & .49 & .30 & .23 \\
\hline 8 & .23 & .21 & .19 & .17 & .11 & .11 & .21 & .56 & .96 & .48 & .32 & .23 \\
\hline 9 & .23 & .20 & .19 & .16 & .11 & .11 & .23 & .61 & .99 & .47 & .31 & .22 \\
\hline 10 & .23 & .21 & .19 & .16 & .11 & .11 & .24 & .64 & .99 & .46 & .30 & .23 \\
\hline 11 & .24 & .21 & .19 & .15 & .10 & .10 & .24 & .64 & 1.0 & .45 & .30 & .22 \\
\hline 12 & .25 & .21 & .19 & .14 & .10 & .11 & .24 & .63 & 1.0 & .45 & .29 & .22 \\
\hline 13 & .23 & .21 & .19 & .14 & .10 & .11 & .25 & .61 & 1.0 & .43 & .28 & .22 \\
\hline 14 & .23 & .18 & .18 & .14 & .10 & .10 & .26 & .59 & 1.0 & .43 & .28 & .22 \\
\hline 15 & .23 & .21 & .19 & .14 & .10 & .11 & .27 & .58 & 1.0 & .41 & .28 & .23 \\
\hline 16 & .23 & .19 & .19 & .14 & .10 & .10 & .27 & .57 & 1.0 & .40 & .27 & .23 \\
\hline 17 & .23 & .20 & .19 & .14 & .10 & .11 & .29 & .59 & .97 & .39 & .27 & .27 \\
\hline 18 & .23 & .20 & .19 & .14 & .10 & .10 & .32 & .61 & .95 & .38 & .26 & .25 \\
\hline 19 & .22 & .20 & .18 & .13 & .08 & .10 & .33 & .61 & .91 & .37 & .27 & .24 \\
\hline 20 & .21 & .20 & .18 & .13 & .09 & .10 & .35 & .65 & .88 & .36 & .27 & .23 \\
\hline 21 & .21 & .20 & .17 & .13 & .08 & .11 & .36 & .70 & .84 & .37 & .27 & .23 \\
\hline 22 & .21 & .20 & .17 & .13 & .09 & .11 & .35 & .76 & .81 & .38 & .26 & .23 \\
\hline 23 & .21 & .17 & .17 & .13 & .10 & .11 & .33 & .81 & .78 & .37 & .26 & .23 \\
\hline 24 & .21 & .20 & .18 & .13 & .10 & .11 & .32 & .84 & .76 & .36 & .26 & .23 \\
\hline 25 & .21 & .21 & .17 & .13 & .10 & .12 & .30 & .86 & .73 & .34 & .26 & .23 \\
\hline 26 & .21 & .20 & .17 & .13 & .10 & .10 & .29 & .88 & .70 & .35 & .26 & .23 \\
\hline 27 & .21 & .19 & .18 & .13 & .10 & .11 & .29 & .90 & .69 & .34 & .25 & .23 \\
\hline 28 & .21 & .19 & .17 & .13 & .10 & .11 & .28 & .90 & .66 & .34 & .24 & .23 \\
\hline 29 & .21 & .19 & .17 & .12 & --- & .10 & .28 & .91 & .63 & .33 & .24 & .23 \\
\hline 30 & .21 & .19 & .17 & .11 & --- & .10 & .28 & .90 & .61 & .32 & .24 & .23 \\
\hline 31 & .21 & --- & .17 & .11 & --- & .11 & --- & .87 & --- & .32 & .24 & --- \\
\hline TOTAL & 6.96 & 6.05 & 5.66 & 4.45 & 2.79 & 3.30 & 7.51 & 19.93 & 26.11 & 13.07 & 8.65 & 6.94 \\
\hline MEAN & .22 & .20 & .18 & .14 & .10 & .11 & .25 & .64 & .87 & .42 & .28 & .23 \\
\hline MAX & .25 & .22 & .19 & .17 & .11 & .12 & .36 & .91 & 1.0 & .60 & .32 & .27 \\
\hline MIN & .21 & .17 & .17 & .11 & .08 & .10 & .11 & .28 & .61 & .32 & .24 & .22 \\
\hline $\mathrm{AC}-\mathrm{ET}$ & 14 & 12 & 11 & 8.8 & 5.5 & 6.5 & 15 & 40 & 52 & 26 & 17 & 14 \\
\hline
\end{tabular}

WTR YR 1989 TOTAL 111.42 MEAN .31 MAX 1.0 MIN .08 AC-ET 221 
EAST STEWART BASIN

EAST STEWART CREEK NEAR IONE, NV

DAILY MEAN DISCHARGE, WATER YEAR OCTOBER 1989 TO SEPTEMBER 1990 CUBIC FEET PER SECOND

\begin{tabular}{|c|c|c|c|c|c|c|c|c|c|c|c|c|}
\hline DAY & OCT & NOV & $D E C$ & JAN & FEB & MAR & APR & MAY & JUN & JUL & AUG & SEP \\
\hline $\begin{array}{l}1 \\
2 \\
3 \\
4 \\
5\end{array}$ & $\begin{array}{l}.23 \\
.23 \\
.22 \\
.21 \\
.21\end{array}$ & $\begin{array}{l}.17 \\
.17 \\
.17 \\
.17 \\
.17\end{array}$ & $\begin{array}{l}.17 \\
.17 \\
.17 \\
.17 \\
.17\end{array}$ & $\begin{array}{l}.14 \\
.14 \\
.14 \\
.14 \\
.14\end{array}$ & $\begin{array}{l}.11 \\
.11 \\
.11 \\
.10 \\
.10\end{array}$ & $\begin{array}{l}.07 \\
.08 \\
.08 \\
.08 \\
.07\end{array}$ & $\begin{array}{l}.11 \\
.11 \\
.11 \\
.11 \\
.11\end{array}$ & $\begin{array}{l}.21 \\
.23 \\
.25 \\
.27 \\
.31\end{array}$ & $\begin{array}{l}.52 \\
.57 \\
.64 \\
.75 \\
.91\end{array}$ & $\begin{array}{l}.62 \\
.62 \\
.59 \\
.57 \\
.55\end{array}$ & $\begin{array}{l}.32 \\
.32 \\
.31 \\
.30 \\
.30\end{array}$ & $\begin{array}{l}.23 \\
.23 \\
.23 \\
.23 \\
.23\end{array}$ \\
\hline $\begin{array}{r}6 \\
7 \\
8 \\
9 \\
10\end{array}$ & $\begin{array}{l}.21 \\
.21 \\
.21 \\
.21 \\
.21\end{array}$ & $\begin{array}{l}.17 \\
.17 \\
.17 \\
.17 \\
.17\end{array}$ & $\begin{array}{l}.17 \\
.17 \\
.17 \\
.17 \\
.17\end{array}$ & $\begin{array}{l}.13 \\
.13 \\
.13 \\
.11 \\
.11\end{array}$ & $\begin{array}{l}.10 \\
.10 \\
.09 \\
.08 \\
.08\end{array}$ & $\begin{array}{l}.07 \\
.07 \\
.07 \\
.08 \\
.08\end{array}$ & $\begin{array}{l}.11 \\
.10 \\
.11 \\
.09 \\
.10\end{array}$ & $\begin{array}{l}.34 \\
.34 \\
.34 \\
.35 \\
.35\end{array}$ & $\begin{array}{l}1.0 \\
1.2 \\
1.3 \\
1.4 \\
1.5\end{array}$ & $\begin{array}{l}.54 \\
.52 \\
.50 \\
.50 \\
.49\end{array}$ & $\begin{array}{l}.29 \\
.29 \\
.29 \\
.28 \\
.28\end{array}$ & $\begin{array}{l}.23 \\
.22 \\
.22 \\
.22 \\
.22\end{array}$ \\
\hline $\begin{array}{l}11 \\
12 \\
13 \\
14 \\
15\end{array}$ & $\begin{array}{l}.20 \\
.19 \\
.18 \\
.18 \\
.18\end{array}$ & $\begin{array}{l}.17 \\
.17 \\
.17 \\
.16 \\
.16\end{array}$ & $\begin{array}{l}.17 \\
.17 \\
.16 \\
.16 \\
.16\end{array}$ & $\begin{array}{l}.11 \\
.11 \\
.11 \\
.11 \\
.11\end{array}$ & $\begin{array}{l}.08 \\
.08 \\
.08 \\
.08 \\
.08\end{array}$ & $\begin{array}{l}.08 \\
.08 \\
.08 \\
.08 \\
.08\end{array}$ & $\begin{array}{l}.11 \\
.12 \\
.14 \\
.15 \\
.16\end{array}$ & $\begin{array}{l}.35 \\
.35 \\
.36 \\
.36 \\
.35\end{array}$ & $\begin{array}{l}1.5 \\
1.4 \\
1.3 \\
1.3 \\
1.2\end{array}$ & $\begin{array}{l}.47 \\
.46 \\
.47 \\
.47 \\
.45\end{array}$ & $\begin{array}{l}.30 \\
.28 \\
.27 \\
.26 \\
.33\end{array}$ & $\begin{array}{l}.21 \\
.21 \\
.21 \\
.21 \\
.21\end{array}$ \\
\hline $\begin{array}{l}16 \\
17 \\
18 \\
19 \\
20\end{array}$ & $\begin{array}{l}.18 \\
.18 \\
.18 \\
.18 \\
.18\end{array}$ & $\begin{array}{l}.17 \\
.17 \\
.16 \\
.16 \\
.16\end{array}$ & $\begin{array}{l}.16 \\
.16 \\
.16 \\
.17 \\
.17\end{array}$ & $\begin{array}{l}.12 \\
.13 \\
.13 \\
.12 \\
.13\end{array}$ & $\begin{array}{l}.08 \\
.09 \\
.09 \\
.08 \\
.08\end{array}$ & $\begin{array}{l}.08 \\
.07 \\
.08 \\
.08 \\
.09\end{array}$ & $\begin{array}{l}.15 \\
.13 \\
.13 \\
.13 \\
.13\end{array}$ & $\begin{array}{l}.36 \\
.37 \\
.38 \\
.37 \\
.38\end{array}$ & $\begin{array}{l}1.1 \\
1.1 \\
1.0 \\
.99 \\
.96\end{array}$ & $\begin{array}{l}.45 \\
.45 \\
.43 \\
.42 \\
.41\end{array}$ & $\begin{array}{l}.29 \\
.28 \\
.27 \\
.28 \\
.28\end{array}$ & $\begin{array}{l}.21 \\
.21 \\
.21 \\
.21 \\
.20\end{array}$ \\
\hline $\begin{array}{l}21 \\
22 \\
23 \\
24 \\
25\end{array}$ & $\begin{array}{l}.18 \\
.18 \\
.17 \\
.17 \\
.18\end{array}$ & $\begin{array}{l}.17 \\
.18 \\
.18 \\
.18 \\
.18\end{array}$ & $\begin{array}{l}.17 \\
.17 \\
.17 \\
.16 \\
.16\end{array}$ & $\begin{array}{l}.13 \\
.13 \\
.13 \\
.13 \\
.13\end{array}$ & $\begin{array}{l}.07 \\
.07 \\
.07 \\
.07 \\
.07\end{array}$ & $\begin{array}{l}.09 \\
.10 \\
.11 \\
.11 \\
.12\end{array}$ & $\begin{array}{l}.15 \\
.16 \\
.15 \\
.12 \\
.16\end{array}$ & $\begin{array}{l}.41 \\
.44 \\
.43 \\
.47 \\
.48\end{array}$ & $\begin{array}{l}.92 \\
.88 \\
.86 \\
.82 \\
.78\end{array}$ & $\begin{array}{l}.39 \\
.38 \\
.36 \\
.36 \\
.36\end{array}$ & $\begin{array}{l}.27 \\
.26 \\
.26 \\
.25 \\
.25\end{array}$ & $\begin{array}{l}.20 \\
.22 \\
.24 \\
.22 \\
.21\end{array}$ \\
\hline $\begin{array}{l}26 \\
27 \\
28 \\
29 \\
30 \\
31\end{array}$ & $\begin{array}{l}.17 \\
.17 \\
.17 \\
.17 \\
.17 \\
.17\end{array}$ & $\begin{array}{l}.16 \\
.17 \\
.17 \\
.17 \\
.17 \\
---\end{array}$ & $\begin{array}{l}.15 \\
.14 \\
.14 \\
.13 \\
.14 \\
.14\end{array}$ & $\begin{array}{l}.12 \\
.12 \\
.12 \\
.13 \\
.13 \\
.11\end{array}$ & $\begin{array}{l}.07 \\
.07 \\
.07 \\
--- \\
-.- \\
---\end{array}$ & $\begin{array}{l}.12 \\
.12 \\
.10 \\
.10 \\
.10 \\
.10\end{array}$ & $\begin{array}{l}.18 \\
.22 \\
.25 \\
.23 \\
.22 \\
. .-\end{array}$ & $\begin{array}{l}.47 \\
.47 \\
.49 \\
.50 \\
.51 \\
.52\end{array}$ & $\begin{array}{l}.74 \\
.72 \\
.70 \\
.67 \\
.65 \\
.--\end{array}$ & $\begin{array}{r}.34 \\
.34 \\
.34 \\
.33 \\
.32 \\
.32\end{array}$ & $\begin{array}{l}.25 \\
.24 \\
.24 \\
.24 \\
.24 \\
.24\end{array}$ & $\begin{array}{l}.20 \\
.21 \\
.22 \\
.21 \\
.21 \\
. .-\end{array}$ \\
\hline $\begin{array}{l}\text { TOTAL } \\
\text { MEAN } \\
\text { MAX } \\
\text { MIN } \\
\text { AC-ET }\end{array}$ & $\begin{array}{r}5.88 \\
.19 \\
.23 \\
.17 \\
12\end{array}$ & $\begin{array}{r}5.08 \\
.17 \\
.18 \\
.16 \\
10\end{array}$ & $\begin{array}{r}5.01 \\
.16 \\
.17 \\
.13 \\
9.9\end{array}$ & $\begin{array}{r}3.87 \\
.12 \\
.14 \\
.11 \\
7.7\end{array}$ & $\begin{array}{r}2.36 \\
.084 \\
.11 \\
.07 \\
4.7\end{array}$ & $\begin{array}{r}2.72 \\
.088 \\
.12 \\
.07 \\
5.4\end{array}$ & $\begin{array}{r}4.25 \\
.14 \\
.25 \\
.09 \\
8.4\end{array}$ & $\begin{array}{r}11.81 \\
.38 \\
.52 \\
.21 \\
23\end{array}$ & $\begin{array}{r}29.38 \\
.98 \\
1.5 \\
.52 \\
58\end{array}$ & $\begin{array}{r}13.82 \\
.45 \\
.62 \\
.32 \\
27\end{array}$ & $\begin{array}{r}8.56 \\
.28 \\
.33 \\
.24 \\
17\end{array}$ & $\begin{array}{r}6.49 \\
.22 \\
.24 \\
.20 \\
13\end{array}$ \\
\hline $\begin{array}{ll}\text { CAL YR } \\
\text { WTR }\end{array}$ & $\begin{array}{l}1989 \\
1990\end{array}$ & $\begin{array}{l}\text { TOTAL } \\
\text { TOTAL }\end{array}$ & $\begin{array}{r}108.72 \\
99.23\end{array}$ & $\begin{array}{ll}\text { MEAN } & .30 \\
\text { MEAN } & .27\end{array}$ & $\begin{array}{ll}\operatorname{MAX} & 1.0 \\
\operatorname{MAX} & 1.5\end{array}$ & $\begin{array}{ll}\text { MIN } & .08 \\
\text { MIN } & .07\end{array}$ & $\begin{array}{l}A C-E T \\
A C-E T\end{array}$ & $\begin{array}{l}216 \\
197\end{array}$ & & & & \\
\hline
\end{tabular}


EAST STEWART BASIN

EAST STEWART CREEK NEAR IONE, NV

DAILY MEAN DISCHARGE, WATER YEAR OCTOBER 1990 TO SEPTEMBER 1991 CUBIC FEET PER SECOND

\begin{tabular}{|c|c|c|c|c|c|c|c|c|c|c|c|c|}
\hline DAY & OCT & NOV & DEC & JAN & FEB & MAR & APR & MAY & JUN & JUL & AUG & SEP \\
\hline $\begin{array}{l}1 \\
2 \\
3 \\
4 \\
5\end{array}$ & $\begin{array}{l}.20 \\
.20 \\
.20 \\
.19 \\
.19\end{array}$ & $\begin{array}{l}.17 \\
.17 \\
.18 \\
.19 \\
.20\end{array}$ & $\begin{array}{l}.13 \\
.13 \\
.13 \\
.13 \\
.13\end{array}$ & $\begin{array}{l}.11 \\
.11 \\
.11 \\
.11 \\
.10\end{array}$ & $\begin{array}{l}.05 \\
.04 \\
.03 \\
.03 \\
.04\end{array}$ & $\begin{array}{l}.06 \\
.06 \\
.06 \\
.06 \\
.06\end{array}$ & $\begin{array}{l}.06 \\
.07 \\
.08 \\
.09 \\
.10\end{array}$ & $\begin{array}{l}.10 \\
.09 \\
.08 \\
.10 \\
.11\end{array}$ & $\begin{array}{r}.47 \\
.59 \\
.74 \\
.99 \\
1.2\end{array}$ & $\begin{array}{l}1.3 \\
1.3 \\
1.2 \\
1.2 \\
1.2\end{array}$ & $\begin{array}{l}.48 \\
.48 \\
.47 \\
.46 \\
.45\end{array}$ & $\begin{array}{l}.31 \\
.30 \\
.31 \\
.30 \\
.30\end{array}$ \\
\hline $\begin{array}{r}6 \\
7 \\
8 \\
9 \\
10\end{array}$ & $\begin{array}{l}.19 \\
.19 \\
.19 \\
.19 \\
.19\end{array}$ & $\begin{array}{l}.20 \\
.18 \\
.15 \\
.15 \\
.15\end{array}$ & $\begin{array}{l}.13 \\
.13 \\
.13 \\
.13 \\
.13\end{array}$ & $\begin{array}{l}.10 \\
.10 \\
.10 \\
.10 \\
.10\end{array}$ & $\begin{array}{l}.04 \\
.04 \\
.04 \\
.05 \\
.05\end{array}$ & $\begin{array}{l}.06 \\
.06 \\
.06 \\
.06 \\
.06\end{array}$ & $\begin{array}{l}.10 \\
.09 \\
.09 \\
.09 \\
.08\end{array}$ & $\begin{array}{l}.12 \\
.16 \\
.20 \\
.17 \\
.14\end{array}$ & $\begin{array}{l}1.4 \\
1.6 \\
1.7 \\
1.9 \\
2.2\end{array}$ & $\begin{array}{r}1.1 \\
1.1 \\
1.0 \\
.99 \\
.96\end{array}$ & $\begin{array}{l}.43 \\
.43 \\
.42 \\
.41 \\
.40\end{array}$ & $\begin{array}{l}.31 \\
.30 \\
.30 \\
.31 \\
.31\end{array}$ \\
\hline $\begin{array}{l}11 \\
12 \\
13 \\
14 \\
15\end{array}$ & $\begin{array}{l}.18 \\
.18 \\
.18 \\
.18 \\
.18\end{array}$ & $\begin{array}{l}.15 \\
.16 \\
.16 \\
.16 \\
.16\end{array}$ & $\begin{array}{l}.13 \\
.13 \\
.13 \\
.13 \\
.13\end{array}$ & $\begin{array}{l}.09 \\
.08 \\
.08 \\
.07 \\
.07\end{array}$ & $\begin{array}{l}.05 \\
.05 \\
.05 \\
.05 \\
.05\end{array}$ & $\begin{array}{l}.06 \\
.06 \\
.06 \\
.11 \\
.17\end{array}$ & $\begin{array}{l}.08 \\
.08 \\
.08 \\
.08 \\
.08\end{array}$ & $\begin{array}{l}.13 \\
.14 \\
.13 \\
.13 \\
.15\end{array}$ & $\begin{array}{l}2.7 \\
3.2 \\
3.4 \\
3.4 \\
3.3\end{array}$ & $\begin{array}{l}.92 \\
.86 \\
.80 \\
.77 \\
.74\end{array}$ & $\begin{array}{l}.40 \\
.39 \\
.39 \\
.39 \\
.38\end{array}$ & $\begin{array}{l}.30 \\
.29 \\
.29 \\
.28 \\
.28\end{array}$ \\
\hline $\begin{array}{l}16 \\
17 \\
18 \\
19 \\
20\end{array}$ & $\begin{array}{l}.17 \\
.18 \\
.17 \\
.18 \\
.17\end{array}$ & $\begin{array}{l}.16 \\
.16 \\
.16 \\
.16 \\
.13\end{array}$ & $\begin{array}{l}.13 \\
.13 \\
.13 \\
.12 \\
.11\end{array}$ & $\begin{array}{l}.06 \\
.06 \\
.06 \\
.06 \\
.06\end{array}$ & $\begin{array}{l}.05 \\
.05 \\
.05 \\
.05 \\
.05\end{array}$ & $\begin{array}{l}.10 \\
.10 \\
.10 \\
.10 \\
.10\end{array}$ & $\begin{array}{l}.08 \\
.08 \\
.08 \\
.09 \\
.08\end{array}$ & $\begin{array}{l}.19 \\
.19 \\
.17 \\
.17 \\
.16\end{array}$ & $\begin{array}{l}3.2 \\
3.0 \\
2.9 \\
2.7 \\
2.5\end{array}$ & $\begin{array}{l}.71 \\
.69 \\
.66 \\
.66 \\
.64\end{array}$ & $\begin{array}{l}.38 \\
.37 \\
.36 \\
.36 \\
.34\end{array}$ & $\begin{array}{l}.27 \\
.27 \\
.27 \\
.26 \\
.26\end{array}$ \\
\hline $\begin{array}{l}21 \\
22 \\
23 \\
24 \\
25\end{array}$ & $\begin{array}{l}.18 \\
.18 \\
.17 \\
.17 \\
.17\end{array}$ & $\begin{array}{l}.14 \\
.13 \\
.13 \\
.13 \\
.12\end{array}$ & $\begin{array}{l}.11 \\
.11 \\
.11 \\
.11 \\
.11\end{array}$ & $\begin{array}{l}.06 \\
.06 \\
.06 \\
.06 \\
.06\end{array}$ & $\begin{array}{l}.05 \\
.05 \\
.06 \\
.06 \\
.06\end{array}$ & $\begin{array}{l}.10 \\
.10 \\
.09 \\
.08 \\
.08\end{array}$ & $\begin{array}{l}.08 \\
.08 \\
.09 \\
.09 \\
.08\end{array}$ & $\begin{array}{l}.15 \\
.19 \\
.24 \\
.29 \\
.36\end{array}$ & $\begin{array}{l}2.4 \\
2.2 \\
2.1 \\
2.0 \\
1.8\end{array}$ & $\begin{array}{l}.63 \\
.61 \\
.59 \\
.58 \\
.56\end{array}$ & $\begin{array}{l}.34 \\
.34 \\
.34 \\
.33 \\
.33\end{array}$ & $\begin{array}{l}.26 \\
.26 \\
.25 \\
.25 \\
.25\end{array}$ \\
\hline $\begin{array}{l}26 \\
27 \\
28 \\
29 \\
30 \\
31\end{array}$ & $\begin{array}{l}.17 \\
.17 \\
.17 \\
.17 \\
.17 \\
.17\end{array}$ & $\begin{array}{l}.12 \\
.13 \\
.13 \\
.13 \\
.13 \\
. .-\end{array}$ & $\begin{array}{l}.11 \\
.11 \\
.11 \\
.11 \\
.11 \\
.11\end{array}$ & $\begin{array}{l}.06 \\
.06 \\
.06 \\
.06 \\
.06 \\
.06\end{array}$ & $\begin{array}{l}.06 \\
.06 \\
.06 \\
--- \\
--- \\
---\end{array}$ & $\begin{array}{l}.07 \\
.05 \\
.05 \\
.06 \\
.06 \\
.07\end{array}$ & $\begin{array}{l}.08 \\
.08 \\
.09 \\
.09 \\
.09 \\
-.--\end{array}$ & $\begin{array}{l}.40 \\
.43 \\
.48 \\
.49 \\
.47 \\
.46\end{array}$ & $\begin{array}{l}1.7 \\
1.6 \\
1.5 \\
1.5 \\
1.4 \\
-.-\end{array}$ & $\begin{array}{l}.55 \\
.53 \\
.52 \\
.51 \\
.51 \\
.50\end{array}$ & $\begin{array}{l}.33 \\
.32 \\
.32 \\
.32 \\
.30 \\
.30\end{array}$ & $\begin{array}{l}.25 \\
.28 \\
.27 \\
.25 \\
.25 \\
-.--\end{array}$ \\
\hline $\begin{array}{l}\text { TOTAL } \\
\text { MEAN } \\
\text { MAX } \\
\text { MIN } \\
\text { AC-FT }\end{array}$ & $\begin{array}{r}5.59 \\
.18 \\
.20 \\
.17 \\
11\end{array}$ & $\begin{array}{r}4.59 \\
.15 \\
.20 \\
.12 \\
9.1\end{array}$ & $\begin{array}{r}3.78 \\
.12 \\
.13 \\
.11 \\
7.5\end{array}$ & $\begin{array}{r}2.39 \\
.077 \\
.11 \\
.06 \\
4.7\end{array}$ & $\begin{array}{r}1.37 \\
.049 \\
.06 \\
.03 \\
2.7\end{array}$ & $\begin{array}{r}2.37 \\
.076 \\
.17 \\
.05 \\
4.7\end{array}$ & $\begin{array}{r}2.51 \\
.084 \\
.10 \\
.06 \\
5.0\end{array}$ & $\begin{array}{r}6.79 \\
.22 \\
.49 \\
.08 \\
13\end{array}$ & $\begin{array}{r}61.29 \\
2.04 \\
3.4 \\
.47 \\
122\end{array}$ & $\begin{array}{r}24.89 \\
.80 \\
1.3 \\
.50 \\
49\end{array}$ & $\begin{array}{r}11.76 \\
.38 \\
.48 \\
.30 \\
23\end{array}$ & $\begin{array}{r}8.39 \\
.28 \\
.31 \\
.25 \\
17\end{array}$ \\
\hline $\begin{array}{ll}\text { CAL } & \text { YR } \\
\text { WTR } & \text { YR }\end{array}$ & $\begin{array}{l}1990 \\
1991\end{array}$ & $\begin{array}{l}\text { TOTAL } \\
\text { TOTAL }\end{array}$ & $\begin{array}{r}97.22 \\
135.72\end{array}$ & $\begin{array}{ll}\text { MEAN } & .27 \\
\text { MEAN } & .37\end{array}$ & $\begin{array}{ll}\text { MAX } & 1.5 \\
M A X & 3.4\end{array}$ & $\begin{array}{ll}\text { MIN } & .07 \\
\text { MIN } & .03\end{array}$ & $\begin{array}{l}A C-E T \\
A C-F T\end{array}$ & $\begin{array}{l}193 \\
269\end{array}$ & & & & \\
\hline
\end{tabular}


EAST STEWART CREEK BASIN

EAST STEWART CREEK NEAR IONE, NV

SURFACE-WATER QUALITY, WATER YEARS OCTOBER 1983 TO SEPTEMBER 1991

\begin{tabular}{|c|c|c|c|c|c|c|c|c|c|c|}
\hline & TIME & $\begin{array}{l}\text { SPE- } \\
\text { CIEIC } \\
\text { CON- } \\
\text { DUCT- } \\
\text { ANCE }\end{array}$ & $\begin{array}{l}\text { SPE- } \\
\text { CIFIC } \\
\text { CON- } \\
\text { DUCT- } \\
\text { ANCE } \\
\text { IAAB }\end{array}$ & $\begin{array}{c}\text { PH } \\
\text { WATER } \\
\text { WHOLE } \\
\text { FIELD } \\
\text { (STAND- } \\
\text { ARD }\end{array}$ & $\begin{array}{c}\text { PH } \\
\text { WATER } \\
\text { WHOLE } \\
\text { LAB } \\
\text { (STAND- } \\
\text { ARD }\end{array}$ & $\begin{array}{c}\text { TEMPER- } \\
\text { ATURE } \\
\text { WATER }\end{array}$ & $\begin{array}{l}\text { ALKA- } \\
\text { LINITY } \\
\text { WAT WH } \\
\text { TOT FET } \\
\text { FIELD } \\
\text { MG/L AS }\end{array}$ & $\begin{array}{c}\text { ALKA- } \\
\text { LINITY } \\
\text { LAB } \\
\text { (MG/L } \\
\text { AS }\end{array}$ & $\begin{array}{l}\text { CALCIUM } \\
\text { DIS- } \\
\text { SOLVED } \\
\text { (MG/L }\end{array}$ & $\begin{array}{c}\text { MAGNE- } \\
\text { SIUM, } \\
\text { DIS- } \\
\text { SOLVED } \\
\text { (MG/L }\end{array}$ \\
\hline & & $(\mu \mathrm{S} / \mathrm{CM})$ & $(\mu \mathrm{s} / \mathrm{CM})$ & UNITS) & UNITS) & (DEG C) & $\mathrm{CACO} 3$ & $(\mathrm{CACO} 3)$ & AS CA) & AS MG) \\
\hline
\end{tabular}

\begin{tabular}{|c|c|c|c|c|c|c|c|c|c|c|}
\hline $\begin{array}{c}\text { SEP } 1984 \\
19 \ldots \\
\text { JAN } 1985\end{array}$ & 1630 & 25 & -- & 7.6 & -- & 7.5 & 12 & -- & 3.2 & 0.50 \\
\hline${ }_{A P R}^{19} \ldots$ & 0930 & -- & -- & -- & -- & -- & 12 & -- & 2.9 & 0.43 \\
\hline JUL & 1000 & -- & -- & -- & -- & -- & -- & -- & 2.8 & 0.42 \\
\hline $\begin{array}{l}09 \ldots \\
\text { SEP }\end{array}$ & 1200 & -- & 32 & -- & 7.7 & -- & 13 & $\cdots$ & 2.3 & 0.24 \\
\hline JAN 1986 & 1630 & 52 & -- & 6.5 & -- & 5.0 & 14 & -- & 2.9 & 0.40 \\
\hline $\begin{array}{l}11 \ldots \\
\text { MAR }\end{array}$ & 1000 & 34 & 37 & 7.1 & 7.6 & 2.0 & 13 & 12 & 2.8 & $<0.40$ \\
\hline $\begin{array}{l}20 \ldots \\
A P R\end{array}$ & 1030 & 34 & 37 & 8.2 & 7.5 & 2.5 & 15 & 15 & 3.0 & 0.39 \\
\hline$\underset{\text { MAY }}{01}$ & 1000 & 30 & 37 & 7.1 & 7.6 & 3.0 & 14 & 15 & 3.0 & 0.38 \\
\hline JUL $29 .$. & 1430 & -- & 31 & 7.3 & 7.7 & 5.5 & -- & 12 & 2.3 & 0.28 \\
\hline $\operatorname{seP}^{10 \ldots}$ & 0900 & 36 & -- & 7.5 & -- & 5.5 & 13 & -- & 2.5 & 0.30 \\
\hline $\begin{array}{l}30 \ldots \\
\text { OCT }\end{array}$ & 1130 & 38 & 33 & 6.8 & 7.7 & 5.0 & 12 & 14 & 2.4 & 0.31 \\
\hline $\begin{array}{l}09 \ldots \\
\text { DEC }\end{array}$ & 1200 & -- & 33 & -- & 7.7 & -- & -- & 14 & 2.5 & 0.31 \\
\hline $\begin{array}{l}30 \ldots \\
\text { MAR } 1987\end{array}$ & 1330 & 35 & 34 & 6.6 & 6.9 & 1.0 & 13 & 12 & 2.7 & 0.41 \\
\hline $\begin{array}{l}31 \ldots \\
\text { MAY }\end{array}$ & 1330 & 23 & 33 & 6.7 & 7.9 & 2.0 & 12 & 13 & 2.7 & 0.39 \\
\hline $\begin{array}{l}06 \ldots \\
\text { JUN }\end{array}$ & 1300 & -- & 34 & - & 7.5 & 3.0 & -- & 13 & 2.6 & 0.35 \\
\hline $\begin{array}{l}30 \ldots \\
\text { SEP }\end{array}$ & 1730 & 33 & 23 & 6.9 & 7.6 & 6.5 & 12 & 7.0 & 2.4 & 0.38 \\
\hline JAN 1988 & 1530 & 38 & 39 & 6.9 & 7.7 & 8.0 & 14 & 14 & 3.0 & 0.41 \\
\hline $\begin{array}{l}09 \ldots \\
A P R\end{array}$ & 1615 & 36 & 36 & 6.8 & 7.6 & 2.0 & 12 & 14 & 2.8 & 0.41 \\
\hline$\underset{\text { JUN }}{05 \ldots}$ & 1400 & 28 & 35 & 6.7 & 7.1 & 4.0 & 12 & 13 & 2.8 & 0.41 \\
\hline $\operatorname{SEP}^{27 \ldots}$ & 1800 & 27 & 30 & 7.0 & 7.5 & 6.5 & 12 & 13 & 2.6 & 0.34 \\
\hline FEB 1989 & 1500 & 32 & 34 & 6.8 & 7.6 & 5.5 & 13 & 14 & 2.8 & 0.51 \\
\hline$\underset{A P R}{22 \ldots}$ & 1530 & 31 & 33 & 6.6 & 7.5 & 2.0 & 12 & 13 & 2.7 & 0.47 \\
\hline $\begin{array}{l}11 \ldots \\
\text { MAY }\end{array}$ & 1220 & 31 & 33 & 7.0 & 7.5 & 6.0 & 12 & 13 & 2.9 & 0.42 \\
\hline $\operatorname{SEP}^{25 \ldots}$ & 1250 & 30 & 30 & 7.3 & 7.9 & 6.5 & -- & 13 & 2.6 & 0.35 \\
\hline NoV $14 \ldots$ & 1500 & 33 & 28 & 7.0 & 7.4 & 7.5 & 15 & -- & 3.0 & 0.39 \\
\hline JAN 1990 & 1400 & 31 & 33 & 7.0 & 7.1 & 2.0 & 13 & 14 & 2.8 & 0.36 \\
\hline $\begin{array}{c}31 \ldots \\
A P R\end{array}$ & 1430 & 29 & 31 & 6.8 & 7.3 & 1.0 & 11 & 12 & 2.5 & 0.35 \\
\hline$\underset{\text { MAY }}{03 \ldots}$ & 1150 & 31 & 33 & 6.9 & 7.1 & 3.0 & 12 & 11 & 3.8 & 0.40 \\
\hline $\begin{array}{l}24 \ldots \\
\text { SEP }\end{array}$ & 1000 & 28 & 31 & 7.3 & 7.4 & 2.5 & 12 & 11 & 2.7 & 0.35 \\
\hline $\begin{array}{l}25 \\
\text { JAN } 1991\end{array}$ & 0830 & 37 & 35 & 7.1 & 7.0 & 4.0 & 13 & 13 & 3.0 & 0.40 \\
\hline$\underset{\text { JUN }}{29 . .}$ & 1200 & 32 & 32 & 6.9 & 7.1 & 0.5 & 14 & 12 & 2.7 & 0.38 \\
\hline JUL & 1430 & 32 & 33 & 7.2 & 8.1 & 3.5 & 12 & 12 & 2.8 & 0.41 \\
\hline$\underset{S E P}{24 \ldots}$ & 1100 & 27 & 30 & 6.8 & 7.0 & 6.5 & 12 & 11 & 2.0 & 0.34 \\
\hline $24 \ldots$ & 0945 & 31 & 33 & 6.4 & 7.2 & 7.0 & 13 & 13 & 2.8 & 0.38 \\
\hline
\end{tabular}


EAST STEWART CREEK BASIN

EAST STEWART CREEK NEAR IONE, NV

SURFACE-WATER QUALITY, WATER YEARS OCTOBER 1983 TO SEPTEMBER 1991

\begin{tabular}{|c|c|c|c|c|c|c|c|c|}
\hline & POTAS- & & & & & SI LICA, & $\begin{array}{l}\text { NI TRO- } \\
\text { GEN, }\end{array}$ & $\begin{array}{l}\text { PHOS- } \\
\text { PHORUS } \\
\text { ORTHO. }\end{array}$ \\
\hline ODIUM, & SIUM, & SULFATE & RIDE, & RIDE, & BROMIDE & DIS- & NITRATE & ORTHO, \\
\hline $\begin{array}{l}\text { DIS- } \\
\text { SOLVED }\end{array}$ & $\begin{array}{l}\text { DIS- } \\
\text { SOLVED }\end{array}$ & $\begin{array}{l}\text { DIS- } \\
\text { SOLVED }\end{array}$ & $\begin{array}{l}\text { DIS- } \\
\text { SOLVED }\end{array}$ & $\begin{array}{l}\text { DIS- } \\
\text { SOLVED }\end{array}$ & $\begin{array}{l}\text { DIS- } \\
\text { SOLVED }\end{array}$ & $\begin{array}{r}\text { SOLVED } \\
(\mathrm{MG} / \mathrm{L}\end{array}$ & $\begin{array}{l}\text { DIS- } \\
\text { SOLVED }\end{array}$ & $\begin{array}{l}\text { DIS- } \\
\text { SOLVED }\end{array}$ \\
\hline $\begin{array}{c}(M G / L \\
\text { AS NA) }\end{array}$ & (MG/L & $\begin{array}{c}(M G / L \\
\text { AS SO4) }\end{array}$ & $\begin{array}{c}(\mathrm{MG} / \mathrm{L} \\
\mathrm{AS} \text { CL) }\end{array}$ & $\begin{array}{l}\text { (MG/L } \\
A S F)\end{array}$ & $\begin{array}{l}\text { (MG/L } \\
A S \text { BR) }\end{array}$ & $\begin{array}{l}\text { AS } \\
\text { SIO2) }\end{array}$ & (MG/L & (MG / L \\
\hline
\end{tabular}

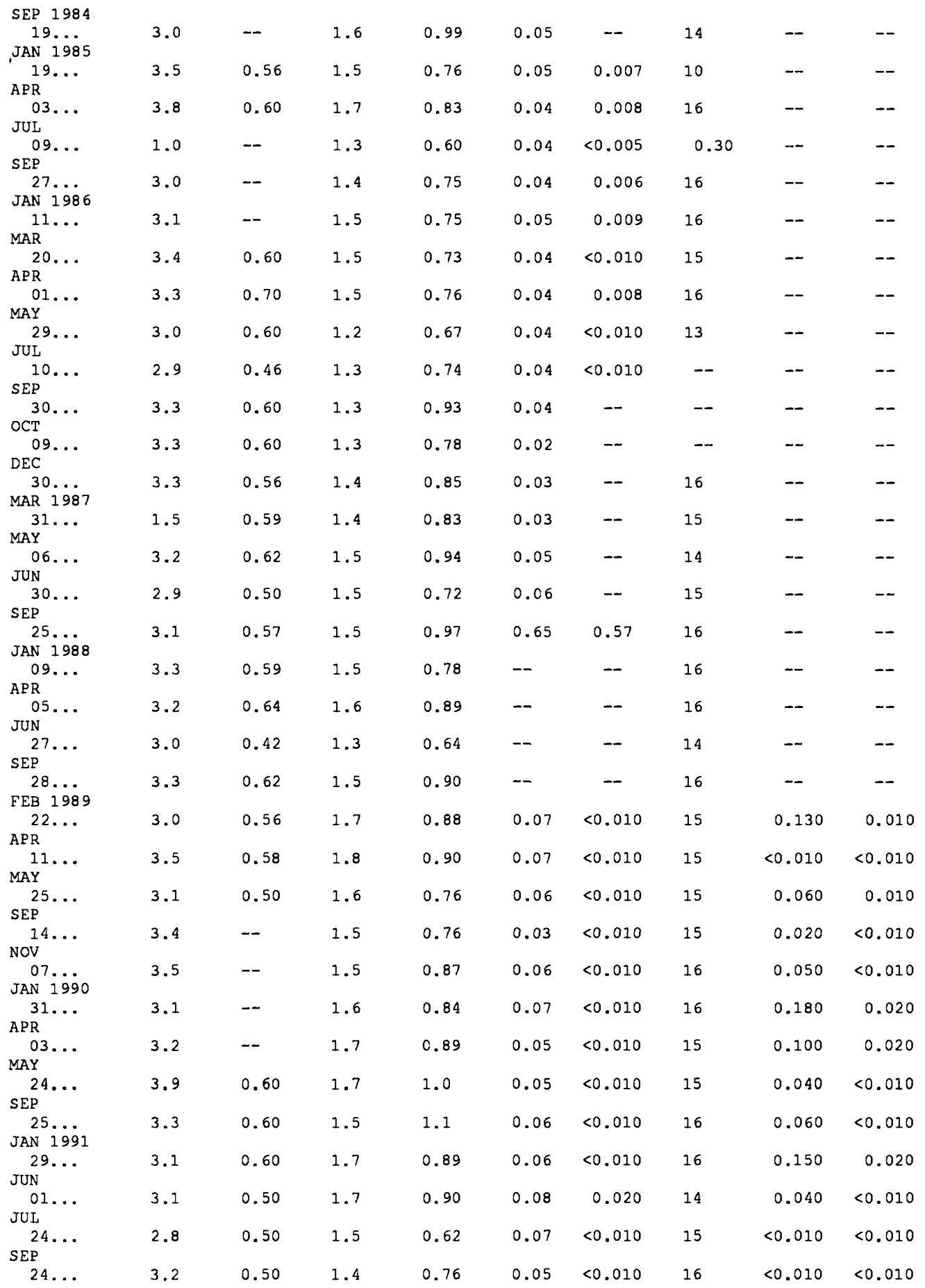


EAST STEWART CREEK BASIN

EAST STEWART CREEK NEAR IONE, NV

SURFACE-WATER QUALITY, WATER YEARS OCTOBER 1983 TO SEPTEMBER 1991

\begin{tabular}{|c|c|c|c|c|c|c|c|c|c|}
\hline & $\begin{array}{l}\text { BARIUM, } \\
\text { DIS- }\end{array}$ & $\begin{array}{l}\text { BERYI- } \\
\text { LIUM, } \\
\text { DIS- }\end{array}$ & $\begin{array}{l}\text { CADMIUM } \\
\text { DIS- }\end{array}$ & $\begin{array}{l}\text { COBALT, } \\
\text { DIS- }\end{array}$ & $\begin{array}{l}\text { COPPER, } \\
\text { DIS- }\end{array}$ & $\begin{array}{l}\text { IRON, } \\
\text { DIS- }\end{array}$ & $\begin{array}{l}\text { LEAD, } \\
\text { DIS- }\end{array}$ & $\begin{array}{l}\text { MANGA- } \\
\text { NESE, } \\
\text { DIS- }\end{array}$ & $\begin{array}{c}\text { MOLYB- } \\
\text { DENUM, } \\
\text { DIS- }\end{array}$ \\
\hline & $\begin{array}{r}\text { SOLVED } \\
(\mu \mathrm{G} / \mathrm{L}\end{array}$ & $\begin{array}{l}\text { SOLVED } \\
(\mu \mathrm{G} / \mathrm{L}\end{array}$ & $\begin{array}{l}\text { SOLVED } \\
(\mu G / L\end{array}$ & $\begin{array}{r}\text { SOLVED } \\
(\mu G / \mathrm{L}\end{array}$ & $\begin{array}{c}\text { SOLVED } \\
(\mu \mathrm{G} / \mathrm{L}\end{array}$ & $\begin{array}{c}\text { SOLVED } \\
(\mu \mathrm{G} / \mathrm{L}\end{array}$ & $\begin{array}{c}\text { SOLVED } \\
(\mu \mathrm{G} / \mathrm{L}\end{array}$ & $\begin{array}{c}\text { SOLVED } \\
(\mu \mathrm{H} / \mathrm{L}\end{array}$ & $\begin{array}{c}\text { SOLVED } \\
\langle\mu G / L\end{array}$ \\
\hline DATE & $A S$ BA) & AS BE) & AS $\quad$ D & AS $\mathrm{CO}$ & AS CU & $A S F E$ & $A S \quad P B$ & $\mathrm{AS} M N$ & AS MO) \\
\hline
\end{tabular}

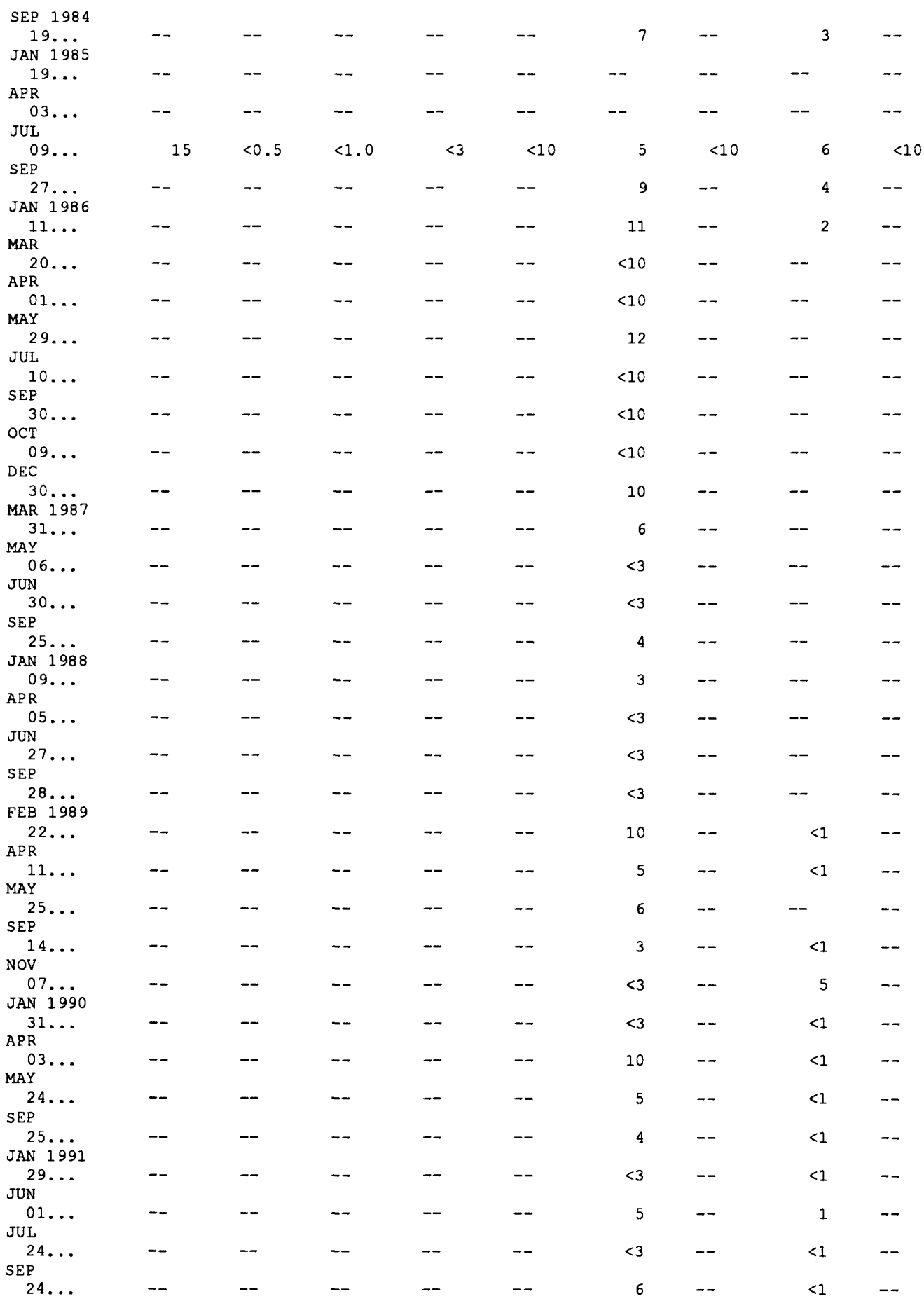


EAST STEWART CREEK BASIN

EAST STEWART CREEK NEAR IONE, NV

SURFACE-WATER QUALITY, WATER YEARS OCTOBER 1983 TO SEPTEMBER 1991

DATE

\begin{tabular}{|c|c|c|c|c|c|c|c|c|}
\hline & & & & & & & $\mathrm{H}-2$ / & $0-18 /$ \\
\hline $\begin{array}{l}\text { STRON- } \\
\text { TIUM, } \\
\text { DIS- } \\
\text { SOLVED }\end{array}$ & $\begin{array}{l}\text { ZINC, } \\
\text { DIS- } \\
\text { SOLVED }\end{array}$ & $\begin{array}{l}\text { ALUM- } \\
\text { INUM, } \\
\text { DIS- } \\
\text { SOLVED }\end{array}$ & $\begin{array}{r}\text { LITHIUM } \\
\text { DIS- } \\
\text { SOLVED }\end{array}$ & TRIT & $\begin{array}{c}\text { TRITIUM } \\
2 \text { SIGMA } \\
\text { WATER, } \\
\text { WHOLE, }\end{array}$ & $\begin{array}{r}\text { URANIUM } \\
\text { NATURAL } \\
\text { DIS- } \\
\text { SOLVED }\end{array}$ & $\begin{array}{l}\text { H-1 } \\
\text { STABLE } \\
\text { ISOTOPE } \\
\text { RATIO }\end{array}$ & $\begin{array}{l}0-16 \\
\text { STABLE } \\
\text { ISOTORE } \\
\text { RATIO }\end{array}$ \\
\hline$(\mu \mathrm{G} / \mathrm{L}$ & $\langle\mu \mathrm{G} / \mathrm{L}$ & $\langle\mu \mathrm{G} / \mathrm{L}$ & $(\mu \mathrm{G} / \mathrm{L}$ & TOTAL & TOTAL & $(\mu \mathrm{G} / \mathrm{L}$ & PER & PER \\
\hline AS SR) & AS $2 \mathrm{~N}$ ) & AS AL) & AS LI) & $(\mathrm{PCI} / \mathrm{L})$ & $(\mathrm{PCI} / \mathrm{L})$ & AS U) & MIL & MIL \\
\hline
\end{tabular}

\begin{tabular}{|c|c|c|c|c|c|c|c|c|c|}
\hline $\begin{array}{c}\text { SEP } 1984 \\
19 \ldots\end{array}$ & 26 & -- & -- & -- & 140 & 14 & -- & -117.0 & -15.70 \\
\hline $\begin{array}{c}\text { JAN } 1985 \\
19 . . .\end{array}$ & -- & -- & -- & -- & -- & -- & -- & -120.0 & -16.30 \\
\hline $\begin{array}{c}\text { APR } \\
03 . . .\end{array}$ & -- & - & -- & -- & -- & -- & -- & -119.8 & -- \\
\hline $\begin{array}{l}\text { JUL } \\
\text { O9.... } \\
\text { SEP }\end{array}$ & 25 & 16 & -- & $<4$ & -- & -- & -- & -118.2 & -16.29 \\
\hline JAN 1986 & 26 & -- & -- & -- & -- & -- & -- & -120.0 & -16.35 \\
\hline $\begin{array}{l}11 \ldots \\
\operatorname{MAR}\end{array}$ & 24 & -- & -- & -- & -- & -- & -- & -119.0 & -16.40 \\
\hline $\begin{array}{l}20 \ldots \\
A P R\end{array}$ & 150 & -- & -- & -- & -- & -- & -- & -122.0 & -16.45 \\
\hline${ }_{\operatorname{MAY}}^{01 \ldots}$ & 140 & -- & -- & -- & -- & -- & -- & -120.0 & -16.30 \\
\hline $\begin{array}{l}29 . . . \\
\text { JUL }\end{array}$ & 17 & -- & -- & -- & -- & -- & -- & -120.0 & -16.00 \\
\hline $10 \ldots$ & $<10$ & -- & -- & -- & -- & -- & 5.6 & -119.0 & -16.30 \\
\hline $\begin{array}{c}30 \ldots \\
\text { OCT }\end{array}$ & 70 & -- & -- & -- & -- & -- & -- & -120.5 & -16.20 \\
\hline $\begin{array}{c}09 . . . \\
\text { DEC }\end{array}$ & 70 & -- & -- & -- & -- & -- & -- & -- & -- \\
\hline $\begin{array}{l}30 \ldots \\
\operatorname{MAR} \\
1987\end{array}$ & 25 & -- & -- & -- & -- & -- & -- & -119.0 & -16.25 \\
\hline $\begin{array}{l}31 \ldots \\
\text { MAY }\end{array}$ & 21 & -- & -- & -- & -- & -- & -- & -119.0 & -16.25 \\
\hline JuN & 23 & -- & -- & -- & -- & -- & -- & -- & -- \\
\hline${ }_{\operatorname{SEP}}^{30 \ldots}$ & 20 & -- & -- & -- & -- & -- & -- & -119.0 & -16.25 \\
\hline$\stackrel{25 \ldots}{25}$ & 24 & -- & -- & -- & -- & -- & -- & -120.5 & -16.30 \\
\hline$\underset{A P R}{09 . \cdots}$ & $<1$ & -- & -- & -- & -- & -- & -- & -120.5 & -16.45 \\
\hline $\begin{array}{l}05 \ldots \\
\text { JUN }\end{array}$ & 26 & -- & -- & -- & -- & -- & -- & -121.5 & -16.35 \\
\hline $\operatorname{SEP}_{\operatorname{SEP}}^{27 .}$ & 19 & -- & -- & -- & -- & -- & -- & -120.0 & -16.40 \\
\hline $\begin{array}{c}28 \% \\
\text { FEB } 19899\end{array}$ & 25 & -- & $<10$ & -- & 110 & 11 & -- & -119.5 & -16.30 \\
\hline${ }_{A P R}^{22 \ldots}$ & $<10$ & -- & -- & -- & -- & -- & -- & -121.0 & -16.35 \\
\hline $11 \ldots$ & 20 & -- & -- & -- & -- & -- & -- & -120.0 & -16.60 \\
\hline $\begin{array}{l}M A Y \\
25 \ldots \\
\\
M A P\end{array}$ & 22 & -- & -- & -- & -- & -- & -- & -117.5 & -16.55 \\
\hline $\begin{array}{l}\text { SEP } \\
14 \ldots \\
\text { NOY }\end{array}$ & - & -- & -- & -- & 110 & 10 & -- & -122.0 & -16.25 \\
\hline $07 \ldots$ & -- & -- & -- & -- & 98 & 10 & -- & -120.5 & -16.35 \\
\hline $\begin{array}{c}\text { JAN } 1990 \\
31 \ldots\end{array}$ & -- . & -- & -- & -- & 100 & 9.0 & -- & -122.5 & -16.35 \\
\hline APR $03 . .$. & -- & -- & -- & -- & -- & -- & -- & -123.0 & -16.55 \\
\hline $\begin{array}{l}\text { MAY } \\
\quad 24 \ldots\end{array}$ & -- & -- & -- & -- & -- & -- & -- & -119.0 & -16.25 \\
\hline SEP $25 \ldots$ & -- & -- & -- & -- & -- & -- & -- & -119.0 & -16.20 \\
\hline $\begin{array}{l}\text { JAN } 1991 \\
29 . \\
\text { JUN }\end{array}$ & -- & -- & -- & -- & -- & - & -- & -122.0 & -16.35 \\
\hline JUL $01 \ldots$ & -- & -- & -- & -- & -- & -- & -- & -118.0 & -16.20 \\
\hline $24 \ldots$ & -- & -- & -- & -- & 89 & 6.0 & -- & -121.0 & -16.35 \\
\hline $24 \ldots$ & -- & -- & -- & -- & -- & -- & -- & -120.0 & -16.30 \\
\hline
\end{tabular}


EAST STEWART BASIN

HELLEBORE SPRING NEAR IONE, NV

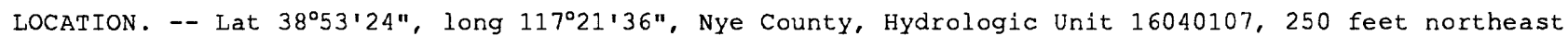
of the East Stewart Creek surface-water equipment, $1.2 \mathrm{mi}$ southeast of the Columbine campground, $13.3 \mathrm{mi}$ east of Ione on State Route 21 .

PERIOD OF RECORD. -- May 1986 to current year.

SURFACE-WATER QUALITY, WATER YEARS OCTOBER 1985 TO SEPTEMBER 1991

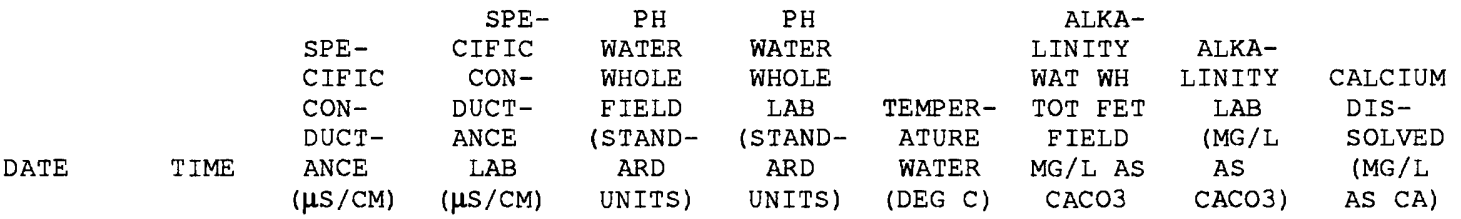

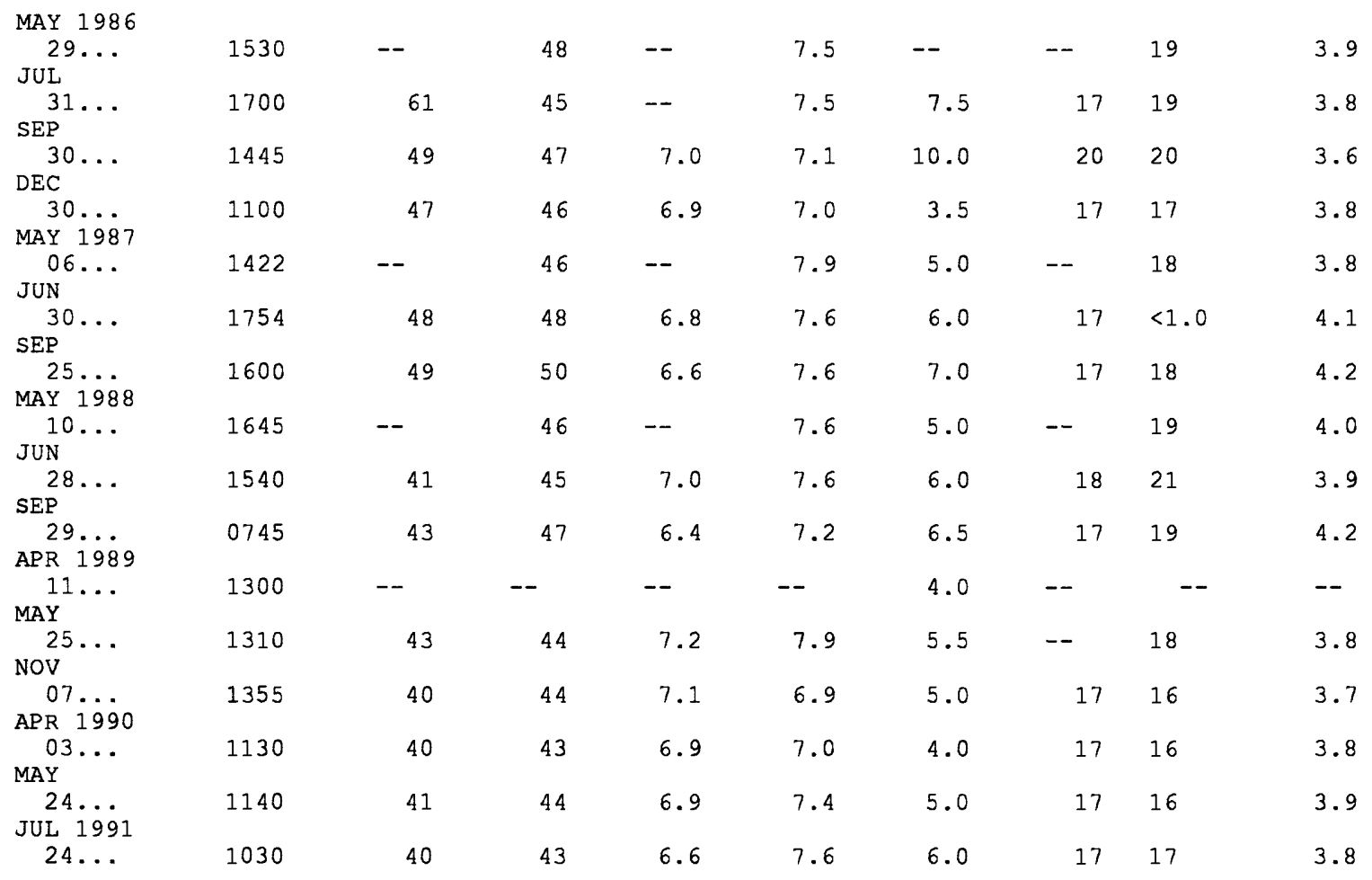


EAST STEWART BASIN

HELLEBORE SPRING NEAR IONE, NV

SURFACE-WATER QUALITY, WATER YEARS OCTOBER 1985 TO SEPTEMBER 1991

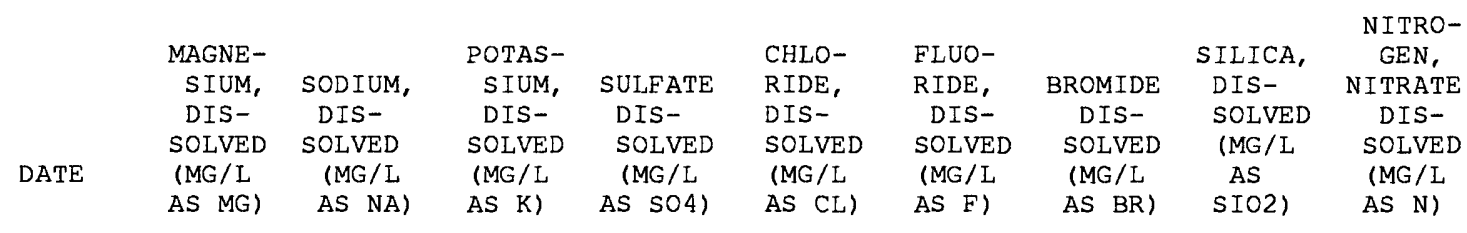

\begin{tabular}{|c|c|c|c|c|c|c|c|c|c|}
\hline $\begin{array}{c}\text { MAY } 1986 \\
29 \ldots \\
\text { JUL }\end{array}$ & 0.51 & 4.3 & 0.70 & 1.9 & 1.9 & 0.05 & 0.023 & 19 & -- \\
\hline $31 \ldots$ & 0.49 & 3.8 & 0.80 & 1.8 & 1.0 & 0.09 & $<0.010$ & 21 & 0.220 \\
\hline SEP & & & & & & & & & \\
\hline${ }_{\mathrm{DEC}}^{30} \cdots$ & 0.49 & 4.3 & 1.1 & 1.6 & 1.2 & 0.00 & -- & 20 & -- \\
\hline $\begin{array}{l}30 \\
\text { MAY } 1987\end{array}$ & 0.55 & 4.2 & 0.66 & 1.6 & 1.2 & 0.00 & -- & 20 & -- \\
\hline$\underset{\text { JUN }}{06 \ldots}$ & 0.52 & 4.2 & 0.68 & 1.8 & 1.1 & 0.09 & -- & 19 & -- \\
\hline $\operatorname{sEP}^{30} \ldots$ & 0.56 & 3.9 & 0.66 & 1.9 & 1.3 & 0.07 & -- & 20 & -- \\
\hline MAY 19288 & 0.56 & 3.9 & 0.68 & 2.0 & 1.1 & -- & -- & 20 & -- \\
\hline$\underset{\text { JUN }}{10}$ & 0.59 & 4.2 & 0.67 & 2.0 & 1.2 & 0.10 & $<0.010$ & 20 & $<0.010$ \\
\hline$\stackrel{28 \ldots}{\mathrm{SEP}}$ & 0.53 & 4.0 & 0.57 & 1.7 & 1.0 & -- & -- & 19 & -- \\
\hline $\begin{array}{l}29 \ldots \\
\text { APR } 1989\end{array}$ & 0.68 & 4.1 & 0.81 & 2.0 & 1.2 & -- & 0.010 & 20 & -- \\
\hline$\frac{11 \ldots}{\operatorname{MAY}}$ & -- & -- & -- & -- & -- & -- & -- & -- & -- \\
\hline $\begin{array}{l}25 \ldots \\
\text { NOV }\end{array}$ & 0.54 & 4.0 & 0.65 & 1.9 & 1.2 & 0.08 & $<0.010$ & 19 & 0.230 \\
\hline $\begin{array}{l}07 \\
\text { APR } 1990\end{array}$ & 0.50 & 4.2 & -- & 1.8 & 1.1 & 0.11 & $<0.010$ & 20 & 0.030 \\
\hline MAY & 0.55 & 4.0 & -- & 1.7 & 1.1 & 0.05 & 0.030 & 20 & 0.150 \\
\hline $\begin{array}{l}24 \ldots \\
\text { JUL } 1991\end{array}$ & 0.52 & 4.0 & 0.70 & 2.0 & 1.1 & 0.07 & $<0.010$ & 19 & 0.270 \\
\hline $24 \ldots$ & 0.57 & 4.0 & 0.60 & 1.9 & 1.1 & 0.08 & $<0.010$ & 20 & 0.170 \\
\hline
\end{tabular}


EAST STEWART BASIN

HELLEBORE SPRING NEAR IONE, NV

SURFACE-WATER QUALITY, WATER YEARS OCTOBER 1985 TO SEPTEMBER 1991

DATE

PHOS-
PHORUS
ORTHO,
DIS-
SOLVED
(MG / L
AS P)

IRON,
DIS-
SOLVED
( $\mu$ G / L
AS FE)

MANGA-
NESE,
DIS-
SOLVED
( $\mu G / L$
AS MN)

STRON-
TIUM,
DIS-
SOLVED
( $\mu G / L$
AS SR)

$\begin{array}{ll}\text { ALUM- } & \\ \text { INUM, } & \\ \text { DIS- } & \\ \text { SOLVED } & \text { TRITIUM } \\ (\mu G / L & \text { TOTAL } \\ \text { AS AL) } & (\mathrm{PCI} / L)\end{array}$

$\begin{array}{ccc} & \text { H-2 / } & 0-18 / \\ \text { TRITIUM } & \text { H-1 } & 0-16 \\ 2 \text { SIGMA } & \text { STABLE } & \text { STABLE } \\ \text { WATER, } & \text { ISOTOPE } & \text { ISOTOPE } \\ \text { WHOLE, } & \text { RATIO } & \text { RATIO } \\ \text { TOTAL } & \text { PER } & \text { PER } \\ \text { (PCI/L) } & \text { MIL } & \text { MIL }\end{array}$

MAY 1986

$29 \ldots$

JUE

$31 .$.

SEP

$30 \ldots$

DEC

$30 \ldots$

MAY 1987

$06 . .$.

JUN

$30 \ldots$

SEP

$25 \ldots$

MAY 1988

$10 \ldots$

JUN

$28 \ldots$

SEP

$29 \ldots$

APR 1989

$11 \ldots$

MAY

$25 \ldots$

NOV

$07 \ldots$

APR 1990

$03 \ldots$

MAY

$24 \ldots$

JUL 1991

24 ...

\begin{tabular}{|c|c|c|c|c|c|c|c|c|}
\hline-- & 6 & -- & 33 & -- & -- & -- & -120.0 & -16.00 \\
\hline 0.030 & 54 & -- & 37 & -- & -- & -- & -121.0 & -16.20 \\
\hline-- & $<10$ & -- & 140 & -- & -- & -- & $-121 \cdot 0$ & -16.20 \\
\hline-- & $<3$ & -- & 35 & -- & -- & -- & $-118 \cdot 0$ & -16.20 \\
\hline-- & $<3$ & -- & 33 & -- & -- & -- & -- & -- \\
\hline-- & 3 & -- & 36 & -- & -- & -- & -120.5 & -16.20 \\
\hline-- & $<3$ & -- & 36 & -- & -- & -- & -120.5 & -16.25 \\
\hline$<0.010$ & $<3$ & -- & 38 & -- & -- & -- & $-120 \cdot 0$ & -16.20 \\
\hline-- & $<3$ & -- & 34 & -- & -- & -- & -120.5 & -16.15 \\
\hline-- & $<3$ & -- & 37 & $<10$ & 110 & 10 & -119.5 & -16.20 \\
\hline-- & -- & -- & -- & -- & -- & -- & -121.0 & -16.25 \\
\hline 0.010 & 5 & -- & 35 & -- & -- & -- & -124.5 & -16.30 \\
\hline$<0.010$ & $<3$ & $<1$ & -- & -- & -- & -- & -120.0 & -16.25 \\
\hline$<0.010$ & 5 & 1 & -- & -- & -- & -- & -120.0 & -16.25 \\
\hline$<0.010$ & 3 & $<1$ & -- & -- & -- & -- & -118.0 & -16.10 \\
\hline$<0.010$ & 4 & $<1$ & -- & -- & -- & -- & $-121 \cdot 0$ & -16.25 \\
\hline
\end{tabular}

